\title{
Optimisation of the Ablation Profiles in CUSTOMISED TREATMENTS FOR LASER CORNEAL REFRACTIVE SURgERY
}

A project of dissertation submitted for evaluation at the University of Valladolid in partial fulfilment of the requirements for the academic degree of Doctor of Philosophy (PhD) in Sciences of Vision with the mention "Doctor europeus“

Research Group "Grupo de Investigación Reconocido (GIR) de Técnicas ópticas de Diagnóstico (TOD)". Universidad de Valladolid Preceptor and Director: Jesús M. Merayo-Lloves MD, PhD, MBA

by Samuel Arba Mosquera, MSc in Physics, MSc in Environmental Protection, MSc in Sciences of Vision, Student at the postgraduate program "Doctorado en Investigación en Ciencias de la Visión" Instituto de Oftalmobiología Aplicada (IOBA). University of Valladolid, Valladolid, December 14, 2010 

“...Por eso no será inútil insistir ante los párvulos en la historia del progreso científico, aprovechando la primera ocasión favorable, digamos el paso de un estrepitoso avión a reacción, a fin de mostrar a los jóvenes los admirables resultados del esfuerzo humano.

El ejemplo del "jet" es una de las mejores pruebas. Cualquiera sabe, aun sin haber viajado en ellos, lo que representan los aviones modernos: velocidad, silencio en la cabina, estabilidad, radio de acción. Pero la ciencia es por antonomasia una búsqueda sin término, y los "jets" no han tardado en quedar atrás, superados por nuevas y más portentosas muestras del ingenio humano. Con todos sus adelantos estos aviones tenían numerosas desventajas, hasta el día en que fueron sustituidos por los aviones de hélice. Esta conquista representó un importante progreso, pues al volar a poca velocidad y altura el piloto tenía mayores posibilidades de fijar el rumbo y de efectuar en buenas condiciones de seguridad las maniobras de despegue y aterrizaje. No obstante, los técnicos siguieron trabajando en busca de nuevos medios de comunicación aún más aventajados, y así dieron a conocer con breve intervalo dos descubrimientos capitales: nos referimos a los barcos de vapor y al ferrocarril. Por primera vez, y gracias a ellos, se logró la conquista extraordinaria de viajar al nivel del suelo, con el inapreciable margen de seguridad que ello representaba.

Sigamos paralelamente la evolución de estas técnicas, comenzando por la navegación marítima. El peligro de los incendios, tan frecuente en alta mar, incitó a los ingenieros a encontrar un sistema más seguro: así fueron naciendo la navegación a vela y más tarde (aunque la cronología no es segura) el remo como el medio más aventajado para propulsar las naves.

Este progreso era considerable, pero los naufragios se repetían de tiempo en tiempo por razones diversas, hasta que los adelantos técnicos proporcionaron un método seguro y perfeccionado para desplazarse en el agua. Nos referimos por supuesto a la natación, más allá de la cual no parece haber progreso posible, aunque desde luego la ciencia es pródiga en sorpresas.

Por lo que nos toca a los ferrocarriles, sus ventajas eran notorias con relación a los aviones, pero a su turno fueron superados por las diligencias, vehículos que no contaminaban el aire con el humo del petróleo o el carbón, y que permitían admirar las bellezas del paisaje y el vigor de los caballos de tiro. La bicicleta, medio de transporte altamente científico, se sitúa históricamente entre la diligencia y el ferrocarril, sin que pueda definirse exactamente el momento de su aparición. Se sabe en cambio, y ello constituye el último eslabón del progreso, que la incomodidad innegable de las diligencias aguzó el ingenio humano a tal punto que no tardó en inventarse un medio de viaje incomparable, el de andar a pie.

Peatones y nadadores constituyen así el coronamiento de la pirámide científica, como cabe comprobar en cualquier playa cuando se ve a los paseantes del malecón que a su vez observan complacidos las evoluciones de los bañistas..."

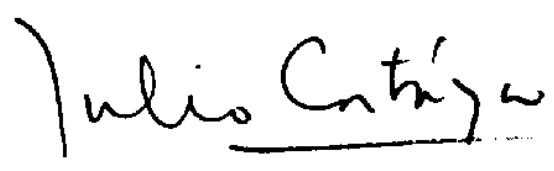



...nem todos estes nomes serão os próprios do tempo e do lugar [...], mas enquanto não se acabar quem trabalhe, não se acabarão os trabalhos, e alguns deles estarão no futuro de alguns daqueles, à espera de quem vier a ter o nome e a profissão...

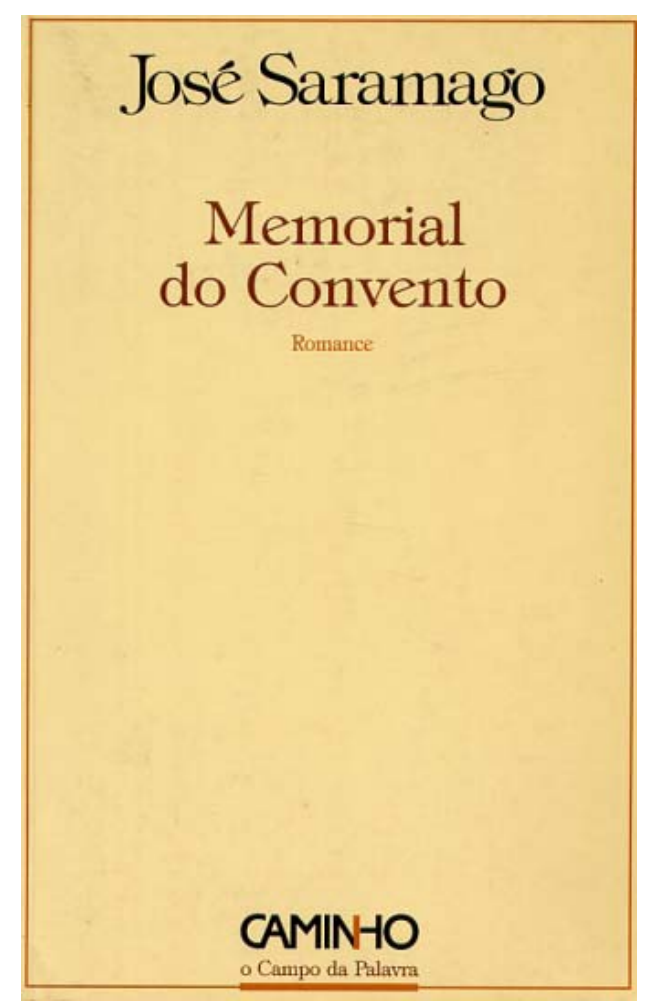



...qué podemos agregar...

...que no se haya dicho ya...

...o que sí se haya dicho...

LES LUTHIERS

It's not a hill, it's a mountain

as you start out the climb

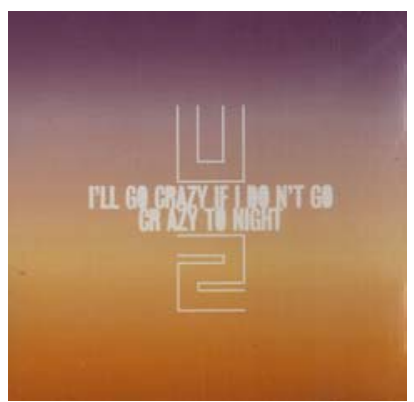





\section{ACKNOWLEDGEMENTS \& AGRADECIMIENTOS}

A Elena, mi amorín, por el pasado, por el presente y por el futuro; por todo, por ser guía, motivación y apoyo, por ser tú y por estar conmigo. Siempre fuiste mi espejo, quiero decir que para verme tenía que mirarte. Scis quia ego amo te.

A Míriel que viene y trae el por-venir...

...mientras esperábamos ha llegado.

All the coauthors of the different publications in which this thesis has resulted (Arbelaez MC, Vidal C, de Ortueta D, Magnago T, Merayo-Lloves J, Piñero D, Ortiz D, Alió JL, Baatz H, Al Jabri B, Gatell J, Ewering T, Camellin M, Aslanides IM, Triefenbach N, Shraiki M, Kolli S, Rosman M, Hollerbach T), as well as the anonymous reviewers, provided helpful suggestions, encouraged and supported this research.

Ablations on PMMA were performed at SCHWIND eye-tech-solutions, surgical treatments were performed at Augenzentrum Recklinghausen, Muscat Eye Laser Center and SEKAL Rovigo Microsurgery Centre.

I acknowledge many fruitful discussions with Carlos Dorronsoro, Alfonso Pérez-Escudero, Pablo Pérez Merino, and Enrique Gambra.

This research was granted by SCHWIND eye-tech-solutions. 
Tendría que dar las gracias (inexcusablemente) a montones de amigos, familiares, compañeros y ex-compañeros de aquí y de allá por miles de cosas distintas.

I have to thank my friend Cheng-Hao Huang for all the time you dedicated to discuss with me, and for the invaluable input that your comments taught me. Also thank you for your way of thinking, your illusion and your spirit. It has been a pleasure having worked for and with you for four years.

Großer Dank geht an Herrn Dr. Hartmut Vogelsang, der mich dazu gebracht hat über Forschungen tiefsinniger nachzudenken, meinen Beitrag zur Forschung ernst zu nehmen und dafür hart zu arbeiten.

Ich danke auch Herrn Rolf Schwind und Herrn Thomas Hollerbach, die es mir ermöglichten, diese Arbeit letztendlich schreiben zu können, und mich somit bei meiner weiteren Entwicklung als Forscher unterstützt haben.

Me gustaría agradecer al Dr. Jesús Merayo-Llovés y al Prof. Santiago Mar por su tiempo, experiencia y el incalculable valor de sus comentarios al evaluar mi trabajo. Por el tiempo dedicado a nuestras discusiones, vuestra experiencia y el valor de vuestros comentarios sobre cómo construir y organizar la tesis.

Mención especial al Dr. Diego de Ortueta por las fructíferas discusiones que tuvimos, así como por su generoso y desinteresado esfuerzo proporcionándome las historias clínicas utilizadas. Es un placer haber trabajado contigo para realizar esta Tesis Doctoral (y seguirá siéndolo el seguir trabajando juntos de cara a nuevos e interesantes proyectos).

A la Dr. María C Arbelaez por su colaboración en la evaluación clínica. 
Special mention to Dr. Massimo Camellin for the fruitful discussions we had, and for his generous and selfless effort by providing the medical records.

To Ricardo Toledo for his wonderful music-philosophy artworks.

¡Vivan mis amigos por antonomasia! A Jes, MariDí, Pedro, Jorge, Vi por ser los mejores, por ser necesarios mientras yo soy contingente, por nuestra cercanía desde el otro lado del mundo y del tiempo. También al resto de mis amigos, sin excepción, "a pesar de que el tiempo y la distancia nos hagan cambiar"... de golpe es Silvio y su guitarra.

Al socialismo de Cortázar y al comunismo de Saramago, así como también a Alonso Quijano (que no a Cervantes ni a Avellaneda) por no renunciar a los sueños. ¿Quién cogerá las estrellas cuando caigan?

También a mis compañeros (y a veces contrincantes en combate) de Jose, Óscar, Moncho, también a Máximo, y a David, Diego, Miguel y Marcos que además de hacerme pensar, me han destrozado el cuerpo a menudo...

To all my colleagues at SCHWIND eye-tech-solutions (with special mention to Kenny for your prelayed loads of music, to Mario and Rüdiger for your reactions in real time or faster, to Anita for having been the first one suffering the very first naïve versions of this work, and to Tobias for your necessary recalls back down to earth), to all for their patience and support. 
A mis compañeros de fatigas en el Master y el Doctorado: Alfonso por tu dominio del espacio y del tempo, Pablo por tu inagotable curiosidad, Enrique por tu adaptación para despejar la incertidumbre.

A los más de 50 que nos juntábamos en el Castro las fiestas y días de guardar. Y que la intersección nunca sea conjunto vacío. A los $20+$ de Villarrube. A Luis, que se fue sin decir nada.

Finalmente, mi más cálido reconocimiento con mucho cariño a mis padres Moncho y Marina y a mis hermanas Marta y Rut por su apoyo y por haber hecho de mí lo que ahora soy.

I fondly appreciate the interest that all you have shown to my work.

Un trabajo es el resultado de un cúmulo de contribuciones de mucha gente, es por ello que mis agradecimientos quizá sean un poco extensos, pero creo, de verdad, que sin el apoyo, más o menos desinteresado de todos y cada uno de ellos (de todos y cada uno de vosotros), el resultado no sería exitoso. 


\section{TABLE OF CONTENTS \\ (Índice)}

ACKNOWLEDGEMENTS \& AGRADECIMIENTOS .................................. ix

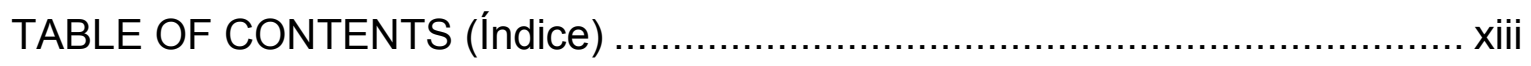

LIST OF TABLES (Listado de tablas) ..............................................xvii

LIST OF FIGURES (Listado de figuras) .......................................... xxix

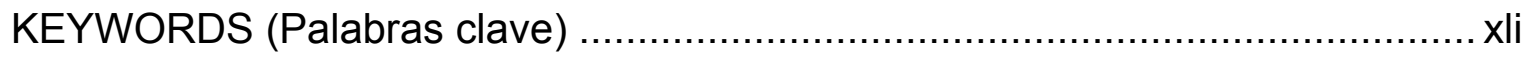

NOMENCLATURE (Nomenclatura) ................................................ xliii

GLOSSARY (Glosario) ..............................................................

FINANCIAL DISCLOSURES (Financiación y declaración de conflicto de

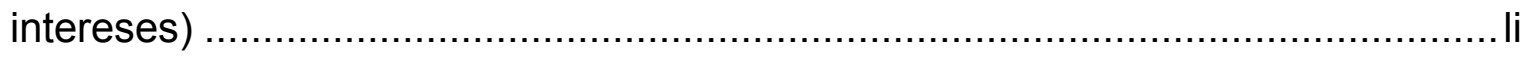

Part 1 INTRODUCTION (Introducción) .......................................... 53

Chapter 1 HYPOTHESIS OF THIS THESIS (Hipótesis de esta Tesis) ... 55

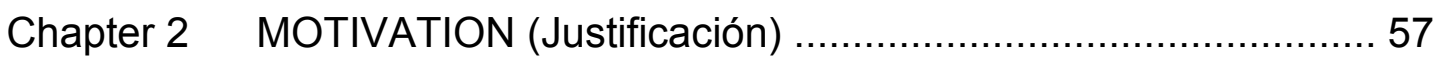

Chapter 3 LASER REFRACTIVE SURGERY (Cirugía refractiva laser) .. 59

3.1 The origins of refractive surgery (Los orígenes de la cirugía

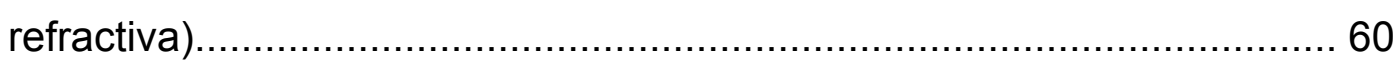

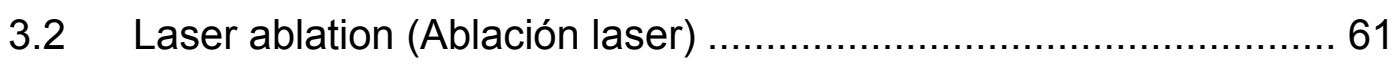

3.3 Zernike representation of aberrations (Representación de Zernike

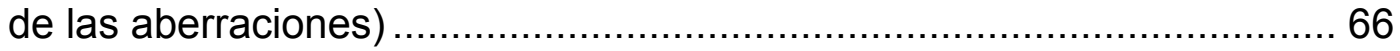

3.4 Measurement of Optical Aberrations (Medida de las aberraciones ópticas)

3.5 Theoretical ablation profiles (Perfiles de ablación teóricos) ...........68 68

3.6 The LASIK technique (La técnica LASIK) .................................6 69

Chapter 4 CORNEAL ABLATION AND OPTICAL ABERRATIONS (Ablación corneal y aberraciones ópticas) ........................................... 71 
4.1 Aberrations and visual performance after refractive surgery (Aberraciones y rendimiento visual tras cirugía refractiva) 71

4.2 Biological response of the cornea (Respuesta biológica de la córnea).

4.3 Visual degradation (Deterioro visual) 73

4.4 Current trends in refractive surgery (Tendencias actuales en cirugía refractiva).

Chapter 5 OPEN QUESTIONS IN CORNEAL REFRACTIVE SURGERY (Cuestiones actuales en cirugía refractiva corneal)... 77

Chapter 6 SPECIFIC GOALS OF THIS THESIS (Objetivos específicos) 79 Chapter 7 THESIS SYNOPSIS (Sinopsis de esta Tesis).................... 81

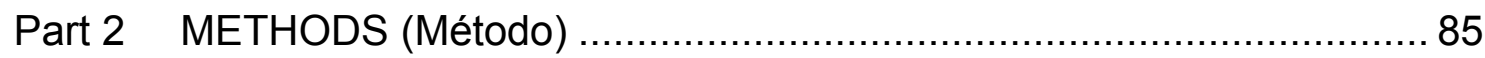

Chapter 8 CONTRIBUTION OF THE WAVE ABERRATION INTO THE MANIFEST REFRACTION (Contribución de la aberración de onda sobre la refracción manifiesta)

8.1 Wavefront refraction from low order Zernike modes at full pupil size (Refracción del frente de onda a partir de los modos de Zernike de bajo orden considerados para la pupila completa) 86

8.2 Objective wavefront refraction from Seidel aberrations at full pupil size (Refracción objetiva del frente de onda a partir de las aberraciones de Seidel consideradas para la pupila completa) 87

8.3 Objective wavefront refraction from low order Zernike modes at subpupil size (Refracción objetiva del frente de onda a partir de los modos de Zernike de bajo orden para un diámetro subpupilar) 88

8.4 Objective wavefront refraction from Seidel aberrations at subpupil size (Refracción objetiva del frente de onda a partir de las aberraciones de Seidel para un diámetro subpupilar). 88

8.5 Objective wavefront refraction from paraxial curvature (Refracción objetiva del frente de onda a partir de la curvatura paraxial) 88 
8.6 Objective wavefront refraction from wavefront axial refraction (Refracción objetiva del frente de onda a partir de la refracción axial del frente de onda) 89

8.7 Automatic Manifest Refraction Balance (Compensación automática de la refracción manifiesta).

Chapter 9 DETERMINATION OF THE ACTUAL CLINICAL RELEVANCE OF THE WAVE ABERRATION (Determinación de la relevancia clínica de la aberración de onda). 95

9.1 Clinical relevance of the single terms in a Zernike expansion (Relevancia clínica de términos individuales de Zernike) 95

9.2 Global clinical relevance of the Zernike expansion (Relevancia clínica global de la expansión de Zernike) 101

9.3 Classification of the clinical relevance (Clasificación de la relevancia clínica) 101

Chapter 10 PROTOCOLS FOR MEASUREMENTS ON SUBJECTS (Protocolos de las medidas realizadas en sujetos) 105

Part 3 TOPICAL REVIEW (Revisión temática) 111

Topic A ANALYSIS OF THE CORNEAL ASPHERICITY (Análisis de la asfericidad corneal)

Section A.1 ABSTRACT (Resumen)

Section A.2 INTRODUCTION (Introducción)

Section A.3 METHODS (Método)

A.3.1 Clinical evaluation (Evaluación clínica)

A.3.2 Repeatability of the methods (Repitibilidad de los métodos)

A.3.3 Statistical analysis (Análisis estadístico) 116

Section A.4 RESULTS (Resultados)

A.4.1 Refractive outcomes (Resultados refractivos). 
A.4.2 Corneal spherical aberrations (Aberración esférica corneal) 118

A.4.3 Corneal asphericity (Asfericidad corneal) 119

A.4.4 Corneal Asphericity Changes (Cambios en la asfericidad corneal) 121

A.4.5 Repeatability of the Corneal Asphericity (Repitibilidad de las determinaciones de asfericidad corneal)..... 123

Section A.5 DISCUSSION (Discusión) 123

Section A.6 CONCLUSIONS (Conclusiones) 128

Section A.7 OUTLOOK (Perspectiva) 128

Topic B MODEL OF AN ABERRATION-FREE PROFILE (Modelo de un perfil libre de aberraciones) 129

Section B.1 ABSTRACT (Resumen) ….................................. 129

Section B.2 INTRODUCTION (Introducción) …………............. 131

Section B.3 METHODS (Método) ………………………...... 132

B.3.1 Theoretical aberration-free profile (Perfil teóricamente libre de aberraciones). 132

B.3.2 Compensation for the focus shift (Compensación del desplazamiento del foco). 134

B.3.3 Optical simulation (Simulaciones ópticas) 135

B.3.4 Clinical evaluation (Evaluación clínica) 136

B.3.5 Ablation centre (Centrado de la ablación) 137

B.3.6 Comparison to Munnerlyn based profiles (Comparación con perfiles directamente basados en Munnerlyn) 139

B.3.7 Bilateral symmetry (Simetría bilateral) 139

B.3.7.1 Correlations for bilateral symmetry of Zernike terms across subjects (Correlaciones de la simetría bilateral para los términos de Zernike) 140 
B.3.7.2 Correlations for symmetry of aberrations in right and left eye of the same subjects (Correlaciones de la simetría interocular de los sujetos)

B.3.7.3 Differences for symmetry of aberrations in right and left eye of the same subjects (Diferencias en la simetría interocular de los sujetos)

B.3.7.4 Dioptrical differences in corneal wavefront aberration between the right and left eyes of the same subjects (Diferencias dióptricas interoculares de la aberración del frente de onda corneal de los sujetos) 141

B.3.7.5 Changes in bilateral symmetry of Zernike terms as a result of refractive surgery (Cambios en la simetría bilateral de términos de Zernike provocados por la cirugía refractiva) .... 142

B.3.7.6 Changes in bilateral symmetry of wavefront aberration as a result of refractive surgery (Cambios en la simetría bilateral interocular provocados por la cirugía refractiva) ..... 142

B.3.7.7 Statistical analysis (Análisis estadístico) 142

Section B.4 RESULTS (Resultados) 142

B.4.1 Simulation of the surgical performance of the profile (Simulación del rendimiento quirúrgico del perfil). 142

B.4.2 Clinical evaluation (Evaluación clínica) 144

B.4.3 Ablation centre (Centrado de la ablación) 148

B.4.4 Comparison to Munnerlyn based profiles (Comparación con los perfiles directamente basados en Munnerlyn) 150

B.4.5 Bilateral symmetry (Simetría bilateral)... 151

B.4.5.1 Changes in bilateral symmetry of Zernike terms as a result of refractive surgery (Cambios en la simetría bilateral de términos de Zernike provocados por la cirugía refractiva) .... 151 
B.4.5.2 Changes in bilateral symmetry of wavefront aberration as a result of refractive surgery (Cambios en la simetría bilateral interocular provocados por la cirugía refractiva)...... 151

Section B.5 DISCUSSION (Discusión) ..................................... 152

B.5.1 Aberration-free pattern (Perfiles libres de aberración).. 152

B.5.2 Ablation centre (Centrado de la ablación)..................... 155

B.5.3 Bilateral symmetry (Simetría bilateral) ….................... 158

Section B.6 CONCLUSIONS (Conclusiones) …........................160

Section B.7 OUTLOOK (Perspectiva)........................................ 161

Topic C DECISION TREE ANALYSIS SYSTEM TO OPTIMISE REFRACTIVE SURGERY OUTCOMES (Análisis por árbol de decisión para la optimización de resultados en cirugía refractiva)................. 163

Section C.1 ABSTRACT (Resumen) ……………………........ 163

Section C.2 INTRODUCTION (Introducción).............................. 164

Section C.3 METHODS (Método) ……………………........... 166

C.3.1 Videokeratoscopy (Videoqueratoscopía)...................... 166

C.3.2 Aberrometry (Aberrometría) …………………........... 166

C.3.3 Manifest refraction (Refracción manifiesta) .................. 167

C.3.4 Decision process (Proceso de decisión)....................... 167

Section C.4 RESULTS (Resultados) …………….................. 169

C.4.1 Distribution of treatments (Distribución de los

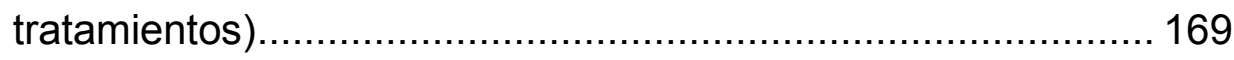

C.4.2 Rate of retreatments (Índice de retratamientos) ........... 169

Section C.5 DISCUSSION (Discusión) ……………................ 170

Section C.6 CONCLUSIONS (Conclusiones) ........................... 172

Section C.7 OUTLOOK (Perspectiva) ……………………..... 172 
Topic D ANALYSIS OF THE LOSS OF ABLATION EFFICIENCY AT NON-NORMAL INCIDENCE (Análisis de la pérdida de eficiencia de ablación para incidencia no-normal).

Section D.1 ABSTRACT (Resumen) ……………….............. 173

Section D.2 INTRODUCTION (Introducción) ………………..... 173

Section D.3 METHODS (Método) ………………….............. 175

D.3.1 Determination of the ablation efficiency at non-normal incidence (Determinación de la eficiencia de la ablación para incidencia no-normal) ........................................................... 175

Section D.4 RESULTS (Resultados) ...................................... 183

Section D.5 DISCUSSION (Discusión)..................................... 189

Section D.6 CONCLUSIONS (Conclusiones)............................ 193

Section D.7 OUTLOOK (Perspectiva) ....................................... 194

Topic E CLINICAL EFFECTS OF CYCLOTORSIONAL ERRORS DURING REFRACTIVE SURGERY (Efectos clínicos de los errores de ciclotorsión durante cirugía refractiva).......................................... 195

Section E.1 ABSTRACT (Resumen) …………………........... 195

Section E.2 INTRODUCTION (Introducción)............................. 196

Section E.3 METHODS (Método)............................................ 198

E.3.1 Determination of Cyclotorsion during Refractive Surgery (Determinación de la ciclotorsión durante cirugía refractiva).... 198

E.3.2 Residual Aberration after Cyclotorsional Errors during Refractive Surgery (Aberración residual tras errores de ciclotorsión durante cirugía refractiva)................................... 200

E.3.3 Derivation of a Mathematic Condition to Determine an Optical Benefit (Derivación de una condición matemática para determinar un beneficio óptico) 202 
E.3.4 Derivation of a Mathematic Condition to Determine a Visual Benefit (Derivación de una condición matemática para determinar un beneficio visual). 203

E.3.5 Derivation of a Mathematic Condition to Determine an Absolute Benefit (Derivación de una condición matemática para determinar un beneficio absoluto) 205

Section E.4 RESULTS (Resultados) 206

E.4.1 Static Cyclotorsion during Laser Refractive Surgery (Ciclotorsión estática durante cirugía refractiva laser)..... 206

E.4.2 Theoretical Ranges to Obtain Optical, Visual, and Absolute Benefits (Rangos teóricos para la obtención de beneficios ópticos, visuales o absolutos)................................ 207

E.4.3 Clinical Optical Benefit (Beneficio óptico clínico) ..........210

E.4.4 Clinical Visual Benefit (Beneficio visual clínico)........... 211

E.4.5 Clinical Absolute Benefit (Beneficio absoluto clínico) ...211

E.4.6 Clinical Ranges to Obtain Optical, Visual, and Absolute Benefits (Rangos clínicos para la obtención de beneficios ópticos,

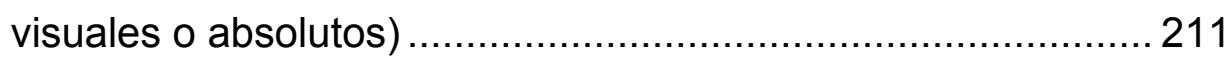

Section E.5 DISCUSSION (Discusión) …………………….... 212

Section E.6 CONCLUSIONS (Conclusiones) ………................. 217

Section E.7 OUTLOOK (Perspectiva) ……………………...... 217

Topic $F \quad$ THE EFFECTIVE OPTICAL ZONE AFTER REFRACTIVE SURGERY (La zona óptica efectiva tras cirugía refractiva)............. 219

Section F.1 ABSTRACT (Resumen)..................................... 219

Section F.2 INTRODUCTION (Introducción) ............................ 220

Section F.3 METHODS (Método) ………………………....... 221

F.3.1 Subjects (Sujetos) .................................................. 221

F.3.2 Ablation profiles (Perfiles de ablación) .......................... 222 
F.3.3 Ablation zones (Zonas de ablación) 222

F.3.4 Analysis of the effective optical zone (Análisis de la zona óptica efectiva) 222

F.3.4.1 Change Of Root-Mean-Square Of Higher Order Wavefront Aberration Method (Método del cambio de la raíz cuadrática media de la aberración de onda de alto orden)... 223

F.3.4.2 Change In Spherical Aberration Method (Método del cambio de la aberración esférica) 223

F.3.4.3 Root-Mean-Square Of The Change Of Higher Order Wavefront Aberration Method (Método de la raíz cuadrática media del cambio de la aberración de onda de alto orden) .. 224

F.3.5 Mean value analyses (Análisis de valores promedio)... 225

F.3.6 Regression analyses (Análisis de regresión)................ 226

F.3.7 Calculation of isometric lines (Cálculo de líneas isométricas). 226

F.3.8 Calculation of proposed nomogram for OZ (Cálculo de una propuesta de nomograma para ZO) 227

Section F.4 RESULTS (Resultados) 227

F.4.1 Adverse events (Complicaciones) 227

F.4.2 Refractive Outcomes (Resultados refractivos) 227

F.4.3 Changes in corneal wavefront aberration at 6- $\mathrm{mm}$ analysis diameter (Cambios en la aberración del frente de onda corneal analizado para 6-mm de diámetro) 228

F.4.4 Mean value analyses for EOZ (Análisis de valores promedio para ZOE) 228

F.4.5 Regression analyses for EOZ (Análisis de regresión de la zona óptica efectiva) 229

F.4.6 Isometric lines for OZ (Líneas isométricas para ZO) .... 232

F.4.7 Proposed nomogram for OZ (Nomograma para ZO).... 232 
Section F.5 DISCUSSION (Discusión) ……………............... 234

Section F.6 CONCLUSIONS (Conclusiones) ........................... 238

Section F.7 OUTLOOK (Perspectiva) ……….......................... 239

Topic G METHOD TO OBJECTIVELY MINIMISE THE ABLATED TISSUE OF A CUSTOMISED ABLATION BASED ON THE ZERNIKE EXPANSION OF THE WAVEFRONT ABERRATION (Método para minimizar objetivamente la cantidad de tejido resecado en una ablación personalizada basada en la expansión de Zernike de la

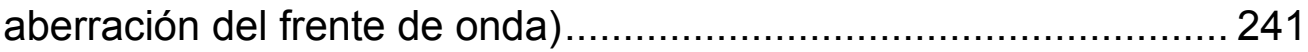

Section G.1 ABSTRACT (Resumen) ………….................... 241

Section G.2 INTRODUCTION (Introducción)............................. 243

G.2.1 Multizonal treatments (Tratamientos multizonales) ......243

G.2.2 Smaller optical zone treatments with large transition zone (Tratamientos en menor zona óptica con mayor zona de transición)

G.2.3 Smaller optical zone for the astigmatic correction (Zonas ópticas menores para la corrección astígmata) 244

G.2.4 Boost slider method (El modulador incremental)......... 245

G.2.5 Simplified profile method (El perfil simplificado) .......... 246

G.2.6 Z-Clipping method (Método de la poda en Z) .............. 246

G.2.7 Z-Shifting method (Método del recorte en Z)............... 247

Section G.3 METHODS (Método)........................................ 247

G.3.1 The "Minimise Depth" and "Minimise Depth+" functions (Las funciones „Minimizar profundidad“ y "Minimizar profundidad+")

G.3.2 The "Minimise Volume" and "Minimise Volume+" functions (Las funciones „Minimizar volumen“ $\mathrm{y}$ „Minimizar volumen+“).. 251

G.3.3 Simulation of the tissue-saving capabilities of such methods for minimising the required ablation tissue (Simulación 
de la capacidad de ahorro de tejido de dichos métodos para minimizar la cantidad de tejido de ablación). 255

G.3.4 Evaluation of the clinical application of such methods for minimising the required ablation tissue (Evaluación de la aplicación clínica de dichos métodos para minimizar la cantidad de tejido de ablación) 256

G.3.4.1 Treatment selection criteria (Criterios de selección de tratamiento) 257

G.3.4.2 Evaluation of the tissue-savings of such methods for minimising the required ablation tissue (Evaluación del ahorro de tejido de tales métodos para minimizar la cantidad de tejido de ablación) 259

G.3.4.3 Direct comparison (Comparación directa) ............. 260

G.3.4.4 Statistical analysis (Análisis estadístico) .............. 266

Section G.4 RESULTS (Resultados)..................................... 266

G.4.1 Simulations (Simulaciones) ……………………........ 266

G.4.1.1 Objective determination of the actual clinical relevance of the single terms in a Zernike expansion of the wavefront aberration (Determinación objetiva de la relevancia clínica de términos individuales de la expansión de Zernike de la aberración del frente de onda) 266

G.4.1.2 Objective minimisation of the maximum depth or volume of a customised ablation based on the Zernike expansion of the wavefront aberration (Minimización objetiva de la profundidad máxima o el volumen de ablación de tratamientos personalizados basados en la expansión de Zernike de la aberración del frente de onda). 268

G.4.2 Evaluation of the clinical application of such methods for minimising the required ablation tissue (Evaluación de la aplicación clínica de tales métodos para minimizar la cantidad de tejido de ablación) 
G.4.2.1 Case report (Caso de estudio) 271

G.4.2.2 Comparative series: Preoperative evaluation (Series comparativas: evaluación preoperatoria) 279 G.4.2.3 Comparative series: Refractive outcomes (Series comparativas: resultados refractivos) 281

G.4.2.4 Comparative series: Evaluation of the tissue-saving capabilities for minimising the required ablation tissue (Series comparativas: evaluación de la capacidad de ahorro de tejido para minimizar la cantidad de tejido de ablación) 285

G.4.2.5 Comparative series: Direct comparison (Series comparativas: comparación directa) ................................... 287

Section G.5 DISCUSSION (Discusión).................................... 288

G.5.1 Clinical relevance of wave aberrations (Relevancia clínica de las aberraciones de onda).... 288

G.5.2 Minimisation of the ablated tissue (Minimización del tejido de ablación). 289

G.5.3 Simulations (Simulaciones) …………….................. 293

G.5.4 Clinical evaluations (Evaluaciones clínicas) .................2294

Section G.6 CONCLUSIONS (Conclusiones)............................297

Section G.7 OUTLOOK (Perspectiva) .......................................298

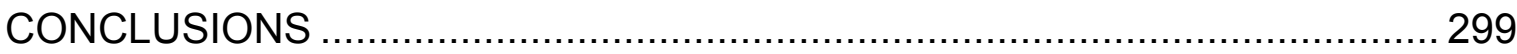

ACHIEVED GOALS AND SPECIFIC CONCLUSIONS ..................................... 301

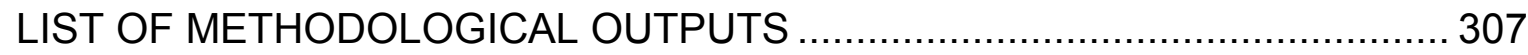

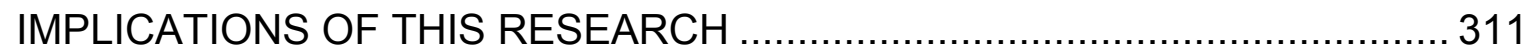

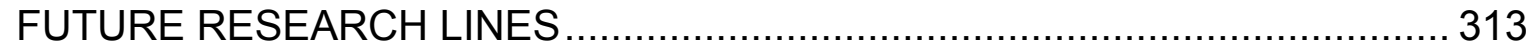

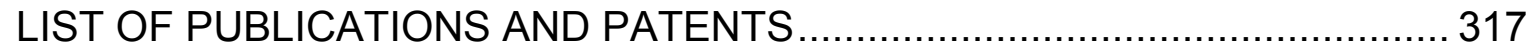

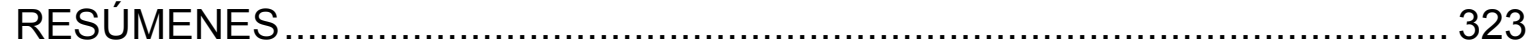

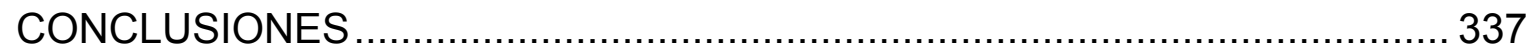


LOGROS ALCANZADOS Y CONCLUSIONES ESPECÍFICAS 339

LISTA DE RESULTADOS METODOLÓGICOS 345

IMPLICACIONES DE ESTA INVESTIGACIÓN 347

LÍNEAS DE INVESTIGACIÓN FUTURA. 349 REFERENCES (Bibliografía) 353 



\section{LIST OF TABLES}

(Listado de tablas)

Table 1: Relative optical blur of the Zernike polynomials up to $7^{\text {th }}$ order. 101

Table 2: Preoperative and postoperative data.

Table 3: Corneal wavefront aberration data reported for $6 \mathrm{~mm}$ analysis diameter.

Table 4: Asphericity data. 119

Table 5: Indications chart. 169

Table 6: Maximum Treatable Magnitude for Different Aberration Components and Different Cyclotorsional Errors for the $<0.50$ DEQ Criterion. 208

Table 7: Maximum Allowable Cyclotorsional Errors for Different Aberration Components and Different Criteria 209

Table 8: Residual Aberration Ratios and Relative Orientations for Different Cyclotorsional Errors. The percentage is the amount of postoperative residual in magnitude, whereas the angle is the relative orientation of the postoperative residual 210

Table 9: Percentage of Treatments That Could Have Been Planned to Achieve an Optical and a Visual Benefit as a Function of the Highest Included Zernike Mode 212

Table 10: Refractive outcomes and induced corneal aberrations after refractive surgery. 228

Table 11: Effective optical zone after refractive surgery vs. planned optical zone 229

Table 12: Mean effective optical zone after refractive surgery vs. planned optical zone 231 
Table 13: Mean nomogrammed optical zone vs. intended effective optical zone 234

Table 14: Summary properties of the four minimisation approaches. 255

Table 15: Patient information 261

Table 16: Treatment information 261

Table 17: Normal light questionnaire 262

Table 18: Dim light questionnaire 263

Table 19: Preoperative diagnosis 264

Table 20: Scheduled diagnosis during follow-up 265

Table 21: Preoperative data of the patient K.S. 272

Table 22: Comparative treatment plans and savings of the patient K.S. 274

Table 23: 3-month postoperative data of the patient K.S 276

Table 24: Demographic data, preoperative and postoperative data for the three groups 280

Table 25: Savings in depth and time of the minimise depth approach. 286

Table 26: Savings in depth and time of the minimise volume approach 287 


\section{LIST OF FIGURES \\ (Listado de figuras)}

Figure 1: Beam profiles for different beam geometries, where $N$ is the Gaussian or supergaussian order. Gaussian profile $(N=1)$ in blue, supergaussian profile $(N=2)$ in pink, Flat-Top profile $(N=\infty)$ in yellow.

Figure 2: Spot profiles for different beam geometries. Parabolic spot profile (from Gaussian beams, $N=1$ ) in blue, quartic spot profile (from supergaussian beams with $N=2$ ) in pink, Flat-Top spot profile (from Flat-Top beams, $N=\infty$ ) in yellow.

Figure 3: Spot profiles for different radiant exposures. Quartic spot profiles (from supergaussian beams with $\mathrm{N}=2$ ) for a peak radiant exposure of $150 \mathrm{~mJ} / \mathrm{cm}^{2}$ in blue and for a peak radiant exposure of $300 \mathrm{~mJ} / \mathrm{cm}^{2}$ in pink. 64

Figure 4: Zernike pyramid showing the Zernike terms up to $7^{\text {th }}$ order. 67

Figure 5: Induced Spherical aberration vs. achieved correction using classical profiles for different treatment strategies: LASIK (in blue), PRK (in purple). Notice that LASIK induced Spherical aberration does not go across the origin, representing the isolated effect due to the flap cut treatments. Notice as well that the induced Spherical aberration was more pronounced for hyperopic treatments than for myopic ones. 72

Figure 6: Biomechanical changes due to the ablation, depending on the stromal layer where the tissue will be removed. 73

Figure 7: Representative axes of the human eye. 76

Figure 8: Representation of the axial refractive error. The line of sight represents a chief ray; the wavefront aberration is zero at the pupil centre, and perpendicular to the line of sight. Each point of the wavefront propagates perpendicular to the local surface of the wavefront. The axial distance from the pupil centre to the intercept between the propagated local wavefront and 
the line of sight expressed in dioptres corresponds to the axial refractive error $^{143}$. 89

Figure 9: Comparison of the different quadric methods described here for the determination of the objective wavefront refraction for a given pupil size..... 90

Figure 10: Zernike refraction of a pure Spherical Aberration (at $6 \mathrm{~mm}$ ) is per definition 0 because Spherical Aberration is a High Order Aberration mode, when analysed for a smaller diameter $(4 \mathrm{~mm})$ produces Defocus.

Figure 11: Zernike refraction of a pure High Order Astigmatism (at $6 \mathrm{~mm}$ ) is per definition 0 because of High Order Aberration mode, when analysed for a smaller diameter (4 $\mathrm{mm}$ ) produces Astigmatism. 92

Figure 12: Zernike refraction of a pure Coma (at $6 \mathrm{~mm}$ ) is per definition 0 because Coma is a High Order Aberration mode, when analysed for a smaller diameter $(4 \mathrm{~mm})$ produces only tilt. Notice that coma may have "visual effect" if the visual axis changes producing Astigmatism.

Figure 13: Zernike refraction of a general wavefront aberration analysed at $6 \mathrm{~mm}$ and analysed for a smaller diameter $(4 \mathrm{~mm})$.

Figure 14: Automatic Refraction Balance. Optical impact of the HOAb the refraction is calculated and balanced from input refraction. Notice that the same wavefront aberration is analysed for two different diameters. The difference in the refraction provided at the two different analysis diameters correspond to the manifest refraction provided by the high order aberration.

Figure 15: Zernike pyramid showing the effects on vision produced by 1 dioptre of equivalent defocus of different Zernike terms up to $7^{\text {th }}$ order.

Figure 16: List of Zernike coefficients classified and colour coded by their dioptric equivalent relative to optical blur (DEq). 102

Figure 17: Zernike pyramid classified and colour coded by the dioptric equivalent relative to optical blur (DEq) of the single Zernike terms. 103

Figure 18: The SCHWIND Combi Workstation for comprehensive corneal and ocular analysis. 106 
Figure 19: The SCHWIND Carriazo-Pendular Console and Handpiece. 106

Figure 20: The SCHWIND AMARIS Total-Tech Laser. 108

Figure 21: The SCHWIND ESIRIS excimer laser system. 109

Figure 22: Predictability scattergram. 117

Figure 23: Induced spherical aberration. 119

Figure 24: Preoperative asphericity. 120

Figure 25: Postoperative asphericity. 121

Figure 26: Ideally expected postoperative asphericity. 122

Figure 27: Bland-Altman plot for p-value calculated from meridians vs. $p$-value calculated from corneal wavefront. 124

Figure 28: Analysis of the induced aberration at $6.50 \mathrm{~mm}$ for $-6.00 \mathrm{D}$ for a balanced corneal model, for 4 different ablation profiles: A) Munnerlyn based profiles, B) Parabolic based profiles, C) Asphericity preserving profiles, D) Aberration-free profiles. Notice the pre-op aberrated status (in blue), the post-op aberrated status (in red), and the induced aberrations (in green). Note the multifocality range ( $x$-axis) running from $-2 D$ to $+1 D$ in all graphs.

Figure 29: Theoretical analysis of the induced corneal spherical aberration analyzed at $6 \mathrm{~mm}$ vs. refractive power change (MRSEq) for 4 different asphericities: "Free-of-aberrations" cornea (Q-Val -0.53, in blue), balancedeye model cornea (Q-Val -0.25, in magenta), spherical cornea (Q-Val 0.00, in yellow), parabolic cornea (Q-Val -1.00, in cyan). 143

Figure 30: Theoretical analysis of the induced corneal spherical aberration at $6.50 \mathrm{~mm}$ using Aberration-Free profiles for $-6.00 \mathrm{D}$ for 2 anterior corneal surfaces: $7.87 \mathrm{~mm}$, Q-factor -0.25 ( $A$ and $C$ ); and $7.87 \mathrm{~mm}, \mathrm{Q}$-factor +0.30 $(B$ and $D)$; and for 2 posterior corneal surfaces and pachymetries: $525 \mu \mathrm{m}$ central pachymetry, $775 \mu \mathrm{m}$ peripheral pachymetry at $5 \mathrm{~mm}$ radial distance ( $A$ and $B$ ); and $550 \mu m$ central pachymetry, $550 \mu m$ peripheral pachymetry at $5 \mathrm{~mm}$ radial distance ( $C$ and $D)$. Notice the pre-op aberrated status (in blue), the post-op aberrated status (in red), and the induced aberrations (in green). 
Note the multifocality range (x-axis) running from $-3.5 D$ to $+0.5 D$ in all graphs. 144

Figure 31: Induced corneal and ocular spherical aberration at 3 months follow-up analysed at $6.0 \mathrm{~mm}$ pupil (ocular: ORK-Wavefront Analyzer (purple squares); corneal: Keratron Scout (blue diamonds)). 145

Figure 32: Change in corneal and ocular coma aberration magnitude at 3 months follow-up analysed at $6.0 \mathrm{~mm}$ pupil (ocular: ORK-Wavefront Analyzer (purple squares); corneal: Keratron Scout (blue diamonds)). 146

Figure 33: Change in corneal and ocular high-order aberrations (HO-RMS) aberrations magnitude at 3 months follow-up analysed at $6.0 \mathrm{~mm}$ pupil (ocular: ORK-Wavefront Analyzer (purple squares); corneal: Keratron Scout (blue diamonds)).

Figure 34: Induced ocular coma/defocus diopter ratio for the CV group (blue) and the PC group (magenta). 148

Figure 35: Induced ocular trefoil/defocus diopter ratio for the CV group (blue) and the PC group (magenta). 149

Figure 36: Induced spherical ocular aberration/defocus diopter ratio for the CV group (blue) and the PC group (magenta). 150

Figure 37: Decision-Tree applied for selecting the treatment mode (Aspheric aberration neutral, Corneal-Wavefront-Guided, or Ocular-Wavefront-Guided).

Figure 38: Hyperopic shift and coupling factor. Ablating a simple myopic astigmatism, the neutral axis became refractive, and the ablation depth in the periphery was smaller than in the centre. 175

Figure 39: Loss on reflection (Fresnel's equations) dependent on the angle of incidence, and losses also dependent on the geometric distortion (angle of incidence). 176

Figure 40: The radius of corneal curvature changes during treatment, efficiency also varies over treatment; the values at $50 \%$ of the treatment represent a reasonable compromise to consider both the correction applied and the preoperative curvature. 
Figure 41: The offset of the galvoscanners from the axis of the system is considered in the calculation of the angle of incidence of the beam onto a flat surface perpendicular to the axis of the laser. 178

Figure 42: Ablation efficiency at $3 \mathrm{~mm}$ radial distance for a sphere with $7.97 \mathrm{~mm}$ radius of curvature. The ablation efficiency was simulated for an excimer laser with a peak radiant exposure of $120 \mathrm{~mJ} / \mathrm{cm}^{2}$ and a full-width-halfmaximum (FWHM) beam size of $2 \mathrm{~mm}$. The radius of corneal curvature changes during treatment, accordingly also the efficiency varies over treatment. Note the improvement of ablation efficiency during myopic corrections as opposed to the increased loss of ablation efficiency during hyperopic corrections. 183

Figure 43: Contribution of the asphericity quotient to the ablation efficiency for a radius of $7.97 \mathrm{~mm}$ curvature. The ablation efficiency at the cornea was simulated for an excimer laser with a peak radiant exposure of $120 \mathrm{~mJ} / \mathrm{cm}^{2}$ and a beam size of $2 \mathrm{~mm}$ (FWHM). Note the identical ablation efficiency close to the vertex as opposed to differences in ablation efficiency at the periphery. A parabolic surface provides higher peripheral ablation efficiency (due to prolate peripheral flattening) compared to an oblate surface (with peripheral steepening).

Figure 44: Contribution of the reflection and distortion losses to ablation efficiency for a sphere with $7.97 \mathrm{~mm}$ radius of curvature. Note that the reflection losses already exist with normal incidence and decrease very slightly towards the periphery. Although normal reflection losses approximately amount to 5\%, they do not increase excessively for non-normal incidence. As our calculation defined the ablation efficiency for a general incidence as the ratio between the spot volume for general incidence and the spot volume for normal incidence, it is evident that the so-defined efficiency equals 1 for normal incidences. 185

Figure 45: Ablation efficiency at $3 \mathrm{~mm}$ radial distance for a sphere with $7.97 \mathrm{~mm}$ radius of curvature. The ablation efficiency was simulated for an excimer laser with a peak radiant exposure up to $400 \mathrm{~mJ} / \mathrm{cm}^{2}$ and FWHM beam size of $2 \mathrm{~mm}$. 186 
Figure 46: Efficiency obtained with the proposed model for the conditions reported by Dorronsoro et al. Ablation efficiency for a sphere with $7.97 \mathrm{~mm}$ radius of curvature. The ablation efficiency was simulated for an excimer laser with a peak radiant exposure of $120 \mathrm{~mJ} / \mathrm{cm}^{2}$ and FWHM beam size of 2 $m m$.

Figure 47: Efficiency obtained with the proposed model for the conditions reported by Dorronsoro et al. ${ }^{214}$ Average ablation efficiency for a sphere with $7.97 \mathrm{~mm}$ preoperative radius of curvature and a correction of $-12 \mathrm{D}$. The ablation efficiency was simulated for an excimer laser with a peak radiant exposure of $120 \mathrm{~mJ} / \mathrm{cm}^{2}$ and $F W H M$ beam size of $2 \mathrm{~mm}$. The radius of corneal curvature changes during treatment, consequently, also the efficiency varies over treatment. Note the improvement of ablation efficiency. 188

Figure 48: Efficiency obtained with the proposed model for the conditions reported by Dorronsoro et al. ${ }^{214}$ Average ablation efficiency for a sphere with $7.97 \mathrm{~mm}$ preoperative radius of curvature and a correction of $+6 \mathrm{D}$. The ablation efficiency was simulated for an excimer laser with a peak radiant exposure of $120 \mathrm{~mJ} / \mathrm{cm}^{2}$ and $F W H M$ beam size of $2 \mathrm{~mm}$. The radius of corneal curvature changes during treatment, consequently also the efficiency varies over treatment. Note the increased loss of ablation efficiency during hyperopic corrections. 188

Figure 49: (Top) Original wavefront error, (middle) $15^{\circ}$ clockwise torted wavefront error, and (bottom) residual wavefront error (all in two-dimension and threedimensions)

Figure 50: The difference between the postoperative and preoperative topographies compared to the intended correction (the difference in the orientation of the astigmatism defines the cyclotorsional error). Preoperative topography. (B) Postoperative topography. (C) Differential topography. (D) Planned correction. Counterclockwise torsion of the astigmatism can be seen 199

Figure 51: The percentage of residual aberrations vs. cyclotorsional error. Modulation of the cyclotorsional error by the angular frequency $(m)$ is seen; the higher the angular frequency, the faster the residual aberration varies. 
For $m=1$ (coma), the maximum residual error is achieved for $180^{\circ}$ torsion; for $m=2$ (cylinder), the maximum residual error would be achieved for $90^{\circ}$ torsion; for $m=3$ (trefoil), the maximum residual error would be achieved for $60^{\circ}$ torsion, and so on 201

Figure 52: The relative orientation of residual aberrations vs. cyclotorsion error. Modulation of the cyclotorsional error and the relative orientation by the angular frequency $(m)$ are seen 202

Figure 53: Matching factor vs. relative orientation of residual aberrations. 204

Figure 54: Distribution of the magnitudes of the attempted astigmatic correction 206

Figure 55: Distribution of the retrospectively calculated cyclotorsional errors .. 207

Figure 56: The maximum allowable cyclotorsional errors vs. angular frequency for different criteria 208

Figure 57: Concept of the $\triangle R M S h o$ method: By comparing postoperative and preoperative corneal wavefront aberrations analysed for a common diameter starting from 4-mm, we have increased the analysis diameter in $10 \mu \mathrm{m}$ steps, until the difference of the corneal RMSho was above $0.25 D$ for the first time. This diameter minus $10 \mu \mathrm{m}$ was determining the EOZ...... 223

Figure 58: Concept of the $\triangle S p h A b$ method: By analysing the differential corneal wavefront aberrations for a diameter starting from 4-mm, we have increased the analysis diameter in $10 \mu \mathrm{m}$ steps, until the differential corneal spherical aberration was above $0.25 \mathrm{D}$ for the first time. This diameter minus $10 \mu \mathrm{m}$ was determining the EOZ. 224

Figure 59: Concept of the RMS( $\triangle H O A b)$ method: By analysing the differential corneal wavefront aberrations for a diameter starting from 4- $\mathrm{mm}$, we have increased the analysis diameter in $10 \mu \mathrm{m}$ steps, until the root-mean-square of the differential corneal wavefront aberration was above $0.25 D$ for the first time. This diameter minus $10 \mu \mathrm{m}$ was determining the EOZ. 225

Figure 60: Bilinear regression analyses for the correlations of EOZ with POZ and with defocus correction for each of the methods: $\triangle R M S h o$ method $\left(r^{2}=.5\right.$, $p<.005)$ (top), $\triangle S p h A b$ method $\left(r^{2}=.7, p<.0001\right)$ (middle), and RMS $(\triangle H O A b)$ 
method $\left(r^{2}=.7, p<.0005\right)$ (bottom). EOZ correlates positively with POZ, and declines steadily with increasing defocus corrections. EOZ depends stronger on POZ than on SEq. Example of double-entry graphs: A treatment of $-5 D$ in $6.5 \mathrm{~mm} \mathrm{POZ}$ results in green when analysed with the $\triangle R M S h o$ and $\triangle S p h A b$ methods ( 6.5 mm EOZ), but in yellow when analysed with the $R M S(\triangle H O A b)$ method ( $6.0 \mathrm{~mm} E O Z)$. 230

Figure 61: Isometric optical zones: $\triangle R M S h o$ method (red), $\triangle S p h A b$ method (blue), and RMS( $\triangle H O A b)$ method (green). For $P O Z<I O Z \Leftrightarrow E O Z<P O Z$, for $P O Z=I O Z \Leftrightarrow E O Z=P O Z$, and for $P O Z>I O Z \Leftrightarrow E O Z>P O Z$. POZ larger than $6.75 \mathrm{~mm}$ result in EOZ, at least, as large as POZ. 232

Figure 62: Calculated the nomogram planned OZ (NPOZ) required to achieve an intended EOZ (IEOZ) for defocus correction for each of the methods: $\triangle R M S h o$ method (top), $\triangle S p h A b$ method (middle), and RMS( $\triangle H O A b)$ method (bottom). Example of double-entry graphs: A treatment of $-5 D$ with intended EOZ of $6.5 \mathrm{~mm}$ results in green when planned for the $\triangle R M S h o$ and $\triangle S p h A b$ methods ( 6.5 mm nomogrammed OZ), but in yellow when planned for the RMS $(\triangle H O A b)$ method ( 7.0 mm nomogrammed OZ). 233

Figure 63: Minimisation by multizonal treatments. 243

Figure 64: Minimisation with smaller optical zone treatments with large transition zone.

Figure 65: Minimisation with smaller optical zone for the astigmatic correction 245 Figure 66: Minimisation by a boost slider (down-slided) 245

Figure 67: Minimisation by a Z-Clipping method........................................ 246

Figure 68: Minimisation by a Z-Shifting method.......................................... 247

Figure 69: Example of a patient with a normal WFAb and his preoperative visus 249

Figure 70: Manual analysis of the optical effects (visus) of the different aberration modes for the same WFAb 249

Figure 71: Diffraction limited visus (all aberration modes are corrected, ideal case) 250 
Figure 72: Objective analysis (Optimised Aberration modes selection) of the optical and ablative effects of the different aberration modes for the same WFAb: Notice that the aberration modes to be selected are not trivial: Not all the modes in green are unselected (not corrected) because some of them may help to save tissue. Not all aberration modes in yellow are selected (corrected) because some of them may have low impact on vision. Notice, as well, that $8 \mu \mathrm{m}$ tissue are saved (16\% of the ablation), but that the overall shape of the ablation remains 250

Figure 73: Analysis of the optical effects (visus) of the objective analysis (Optimised Aberration modes selection) for the same WFAb 251

Figure 74: Optimised Aberration Modes Selection. Based on the wavefront aberration map, the software is able to recommend the best possible aberration modes selection to minimise tissue and time, without compromising the visual quality. Notice that the wavefront aberration is analysed by the software showing the original ablation for a full wavefront correction and the suggested set of aberration modes to be corrected. Notice the difference in required tissue, but notice as well that the most representative characteristics of the wavefront map are still presented in the minimised tissue selection. 253

Figure 75: Optimised Aberration Modes Selection. Based on the wavefront aberration map, the software is able to recommend the best possible aberration modes selection to minimise tissue and time, without compromising the visual quality. Notice that the wavefront aberration is analysed by the software showing the original ablation for a full wavefront correction and the suggested set of aberration modes to be corrected. Notice the difference in required tissue, but notice as well that the most representative characteristics of the wavefront map are still presented in the minimised tissue selection. 254

Figure 76: Zernike coefficients distribution for the sample population of wavefront maps: natural arithmetic average, average considering bilateral symmetry, average considering absolute value, root-mean-square respect to zero.... 267 
Figure 77: Ablation depth for OZTS vs. Ablation depth for full-customised correction for: Aberration-Free correction (all HOAb disabled) (in blue), minimise depth (in magenta), minimise volume (in yellow), minimise depth+ (in cyan), and minimise volume+ (in purple). 270

Figure 78: Ablation time for OZTS vs. Ablation time for full-customised correction for: Aberration-Free correction (all HOAb disabled) (in blue), minimise depth (in magenta), minimise volume (in yellow), minimise depth+ (in cyan), and minimise volume+ (in purple). 270

Figure 79: Preoperative topography and corneal wavefront maps of the patient K.S. 273

Figure 80: Preoperative corneal wavefront map of the patient K.S. determining a functional optical zone (with threshold of $0.38 D$ ) of $4.69 \mathrm{~mm} \varnothing$ 273

Figure 81: Preoperative corneal wavefront map of the patient K.S. determining a functional optical zone (with threshold of $0.50 D$ ) of $5.85 \mathrm{~mm} \varnothing$ 274

Figure 82: Comparative treatment plans and savings of the patient K.S. 275

Figure 83: 3-month postoperative topography and corneal wavefront maps of the patient K.S.

Figure 84: 3-month postoperative corneal wavefront map of the patient K.S. determining a functional optical zone (with threshold of $0.38 \mathrm{D}$ ) of $4.69 \mathrm{~mm} \varnothing$ 277

Figure 85: 3-month postoperative corneal wavefront map of the patient K.S. determining a functional optical zone (with threshold of $0.50 \mathrm{D}$ ) of $5.85 \mathrm{~mm} \varnothing$ 278

Figure 86: Comparative corneal wavefront maps of the patient K.S. simulating UCVA conditions..... 278

Figure 87: Comparative corneal wavefront maps of the patient K.S. simulating UCVA conditions ...... 279

Figure 88: Comparison of the refractive outcome in SEq for CW group (green bars), MD group (blue bars), and MV group (yellow bars) 281 
Figure 89: Comparison of the predictability scattergram for SEq for CW group (green diamonds), MD group (blue triangles), and MV group (yellow squares) 282

Figure 90: Comparison of the predictability for astigmatism for $\mathrm{CW}$ group (green diamonds), MD group (blue triangles), and MV group (yellow squares).... 283

Figure 91: Comparison of the change in BSCVA (Safety) for CW group (green bars), MD group (blue bars), and MV group (yellow bars) 284

Figure 92: Comparison of the contrast sensitivity for preoperative status (dark blue triangles), CW group (green squares), MD group (blue circles), and MV group (yellow diamonds) 284

Figure 93: Comparison of the ablation depths for CW group (green diamonds), MD group (blue squares), and MV group (yellow triangles) 285

Figure 94: Comparison of the ablation times for CW group (green diamonds), $M D$ group (blue squares), and MV group (yellow triangles) 286 



\section{KEYWORDS}

\section{(Palabras clave)}

Aberration-Free Automatic Refraction Balance Dioptres

Equivalent Defocus Dioptric equivalent

Weight coefficients of the Zernike expansion Custom Ablation Manager

Corneal wavefront

Zernike terms

Epi-LASIK

Laser Keratomileusis LASAK LASEK Laser Keratectomy

LASIK Angular frequency Meridional index

Minimise Depth Minimise Volume Radial order

Optimised Refractive Keratectomy Optical Society of America

$\begin{array}{llll}\text { Ocular wavefront } & \text { Optical Zone } & \text { PRK } & \text { PTK }\end{array}$

Root-Mean-Square Refractive Zone Spherical Equivalent

Total Ablation Zone Tissue-Saving Transition Zone

Optical blur Wave aberration Zernike polynomials

Aberration Optical system Blurring Image

$\begin{array}{lll}\text { Point source } \quad \text { Ablation } & \text { Wavefront }\end{array}$

Cylinder HOA HOAb defocus optical degradation

high order aberration higher-order aberrations

Zernike expansion refractive properties cornea

wavefront error

maximum depth customisation

customised ablation

eye Zernike mode

functional Optical Zone

wave of light phase

Wavefront aberration

unit disk

Frits Zernike 



\section{NOMENCLATURE}

\section{(Nomenclatura)}

\begin{tabular}{|c|c|}
\hline$A b$ & Aberration(s) \\
\hline $\mathrm{Abl}$ & Ablation \\
\hline AF & Aberration-Free \\
\hline ARB & Automatic Refraction Balance \\
\hline D & Dioptres \\
\hline DEQ & Equivalent Defocus \\
\hline DEq & Dioptric equivalent \\
\hline \multicolumn{2}{|c|}{$\mathrm{C}^{\mathrm{m}}{ }_{n}$ or $\mathrm{C}[\mathrm{n}, \mathrm{m}]$ Weight coefficients of the Zernike expansion } \\
\hline CAM & Custom Ablation Manager \\
\hline CW & Corneal wavefront \\
\hline$E_{n}^{m}$ & Factor for enabling/disabling specific Zernike terms \\
\hline Epi-LASIK & Epithelial Laser ASsisted In-situ Keratomileusis \\
\hline HOAb & High Order Aberration(s) \\
\hline LASAK & Laser ASsisted Anterior Keratomileusis \\
\hline LASEK & Laser Assisted Sub-Epithelial Keratectomy \\
\hline LASIK & Laser ASsisted In-situ Keratomileusis \\
\hline LOAb & Low Order Aberration(s) \\
\hline
\end{tabular}


m

Angular frequency or meridional index

MD

MD+ $\quad$ Minimise Depth Plus

$M_{e} \quad$ Equivalent Defocus

MV Minimise Volume

MV+ $\quad$ Minimise Volume Plus

n Radial order

OD Oculus dexter (right eye)

ORK Optimised Refractive Keratectomy

OS Oculus sinister (left eye)

OSA Optical Society of America

OW Ocular wavefront

OZ Optical Zone

OZTS Optimised Zernike Terms Selection

PRK Photo-Refractive Keratectomy

PTK Photo-Therapeutic Keratectomy

RMS Root-Mean-Square

RZ Refractive Zone

SEq Spherical Equivalent

TAZ Total Ablation Zone 
TS

Tissue-Saving

TZ

Transition Zone

$U_{G} \quad$ Optical blur

WA Wavefront aberration

WAb Wavefront aberration

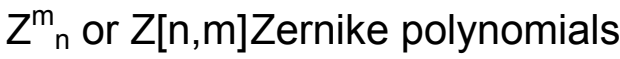





\title{
GLOSSARY \\ (Glosario)
}

\begin{abstract}
Aberration: In optical systems, a condition that leads to blurring of the image. It occurs when light from infinity or from a point source after transmission through the system does not converge into (or does not diverge from) a single point.
\end{abstract}

Aberration-Free: An ablation profile with which the Wavefront aberration (within Optical Zone) after surgery equals the Wavefront aberration (within Optical Zone) prior to the surgery after balancing Sphere and Cylinder components, i.e. there is no induced change in Wavefront aberration (within Optical Zone) other than Sphere and Cylinder components.

Angular frequency: Sinusoidal velocity with which the Zernike terms complete a cycle over a circumference at a radial distance.

Automatic Refraction Balance: Objective combination of the planned refraction into the wavefront aberration, to correct, in a single step, the decided $\mathrm{HOAb}$ and the planned refraction.

Dioptric equivalent: the amount of ordinary defocus needed to produce a similar optical degradation that is produced by one or more higher-order aberrations.

Custom Ablation Manager: A module to select for ablation subsets of Zernike terms included in the Zernike expansion of the wavefront aberration.

Corneal wavefront: The wavefront aberration corresponding solely to the refractive properties of the cornea. 
Equivalent Defocus: the amount of ordinary defocus needed to produce the same RMS wavefront error that is produced by one or more higher-order aberrations.

Meridional index: See Angular frequency.

Minimise Depth: A method to objectively minimise the maximum depth of a customised ablation based on the Zernike expansion of the wavefront aberration.

Minimise Depth Plus: Another method to objectively minimise the maximum depth of a customised ablation based on the Zernike expansion of the wavefront aberration.

Minimise Volume: A method to objectively minimise the ablation volume of a customised ablation based on the Zernike expansion of the wavefront aberration.

Minimise Volume Plus: Another method to objectively minimise the ablation volume of a customised ablation based on the Zernike expansion of the wavefront aberration.

Optical Zone: The zone for which the change of refraction or HOAb is planned.

Ocular wavefront: The wavefront aberration corresponding to the refractive properties of the eye as a whole.

Optimised Zernike Terms Selection: A method to objectively select for ablation subsets of Zernike terms included in the Zernike expansion of the wavefront aberration.

Radial order: The highest power found in a Zernike term.

Refractive Zone: The zone for which the change of refraction shall match the planned refractive correction. 
Total Ablation Zone: The zone that receives laser pulses.

Transition Zone: The zone ablated with a profile to smooth the ablated area towards the non-treated cornea, responsible for providing the actual functional Optical Zone.

\section{Wave aberration: see Wavefront error.}

Wavefront: In a wave of light propagating in a medium, a surface containing the locus of points having the same phase.

\section{Wavefront aberration: see Wavefront error.}

Wavefront error: The deviation of a wavefront in an optical system from a desired perfect planar (or perfect spherical) wavefront is called the wavefront aberration. Wavefront aberrations are usually described as either a sampled image or a collection of two-dimensional polynomial terms.

Zernike mode: Each pair of Zernike polynomials structured in two complementary symmetrical sets, one governed by the cosine, and the other governed by the sine functions.

Zernike polynomials: A series of polynomials that are orthogonal on the unit disk. Named after Frits Zernike. 



\section{FINANCIAL DISCLOSURES \\ (Financiación y declaración de conflicto de intereses)}

This work has been financed by SCHWIND eye-tech-solutions without any other source of external funding.

Maria C Arbelaez, Diego de Ortueta, Jorge L Alió, Massimo Camellin, and loannis M Aslanides are consultants for SCHWIND eye-tech-solutions.

Thomas Magnago, Tobias Ewering, Nico Triefenbach, Mario Shraiki, Thomas Hollerbach, Anita Grimm, and Thomas Klinner are employees of SCHWIND eye-tech-solutions.

Samuel Arba-Mosquera is an employee of SCHWIND eye-tech-solutions.

This project of dissertation represents the personal views of its author, and was not written as a work for hire within the terms of the author's employment with SCHWIND eye-tech-solutions. The work described in the project of dissertation itself (as opposed to the work done writing the article) was conducted as part of the author's work for SCHWIND eye-tech-solutions. Content attributed to the author was vetted by a standard SCHWIND eye-tech-solutions approval process for third-party publications. 



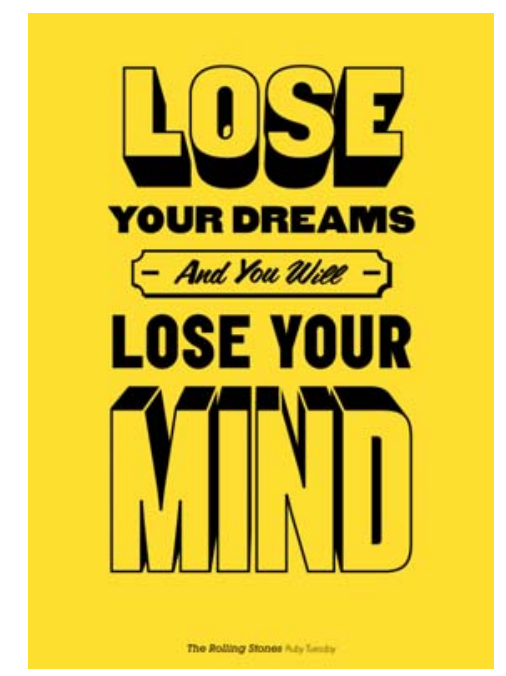

\section{Part 1 INTRODUCTION \\ (Introducción)}

Laser corneal refractive surgery has become a revolution in ophthalmology, allowing surgeons to correct precisely and safely refractive defects in a stable manner. Until the introduction of laser corneal refractive surgery about 20 years ago, these refractive defects could only be corrected by ophthalmic prostheses such as spectacles or contact lenses, or they could be approached by a restricted elite of pioneer surgeons in the form mechanical keratomileusis or intraocular surgery with all the complications and risks that these techniques imply.

Since the introduction by Trokel $^{1}$ of the excimer laser surgery of the cornea in 1983, more than 50 millions of treatments have been successfully performed. As the prevalence of myopic defects in western societies is about $30 \%{ }^{2}$ and of above $50 \%$ in Asian countries, the potential of the surgical techniques reaches more than 1 billion people (without consideration of their economic means).

Despite its predictability and safety, laser corneal refractive surgery is not a free of risk technique. Nowadays, the limits of the laser refractive corrections are imposed by the number of dioptres that can be corrected (typically from $-12 \mathrm{D}$ to +6D) combined with the pupil size (larger pupils may receive only smaller dioptric corrections), the age of the patients (typically 18 years or older with records of 
stable refractions), corneal thickness (thinner corneas may receive only smaller dioptric corrections).

Further, specific aspects of the ablation profiles, the ablation procedure, and the biomechanical reactions of etching a new corneal shape by removing a corneal lenticule may affect the quality of vision postoperatively. Illustrative examples include over- and under-corrections, induction of haze and other corneal opacities, induction of aberrations and reduction of the contrast sensitivity, perception of halos, glare, or visual disturbance at night.

Over and under-corrections can be approached by secondary refractive treatments (at the expense of extra tissue removal) and subsequently prevented by the use of nomograms. For the induced haze, mytomicin $C$ has opened up a successful therapy.

These problems among other challenges are the basis research conducted by the "Grupo de investigación en cirugía refractiva y calidad de visión." Remarkable are the efforts to face the problems observed in refractive surgery by developing adequate experimental animal models for analysing $\mathrm{PRK}^{3}$, LASIK ${ }^{4}$, LASEK, $\mathrm{CXL}^{5}$, corneal additive surgery ${ }^{6}$, as well as the investigations of the corneal cauterization ${ }^{7}$ and induction of corneal haze ${ }^{8}, 9$. From biophysics, the cooperations with the University of Valladolid ${ }^{10}$ and the Consejo Superior de Investigaciones Científicas ${ }^{11}$ have demonstrated be very fruitful.

At the light of the unresolved issues mentioned at the introduction, it is pertinent and justified to attempt to address some of the previously raised questions using the scientific method and engineered developed tools. 


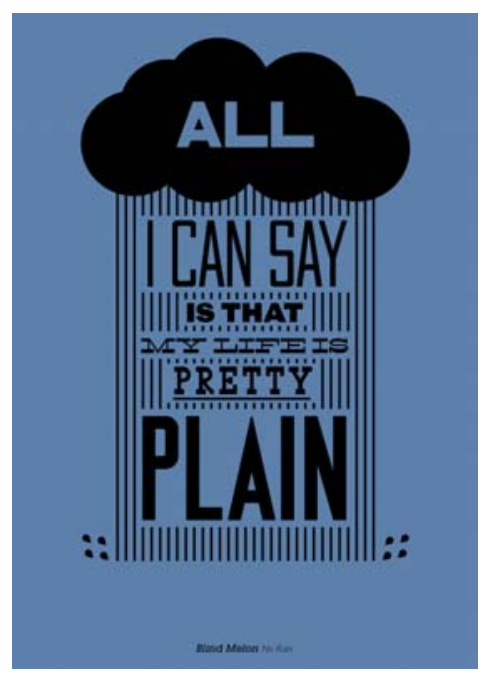

\section{Chapter 1 HYPOTHESIS OF THIS THESIS}

(Hipótesis de esta Tesis)

The starting hypothesis is that it is possible to develop new algorithms and ablation strategies for efficiently performing laser corneal refractive surgery in a customised form minimising the amount of ablated tissue without compromising the visual quality. The availability of such profiles, potentially maximising visual performance without increasing the factors of risk, would be of great value for the refractive surgery community and ultimately for the health and safety of the patients.

It is possible to improve the application of laser cornea refractive surgical treatments by properly compensating the loss of ablation efficiency for non-normal incidences, enhancing the systems to track eye movements and by optimised ablation profiles for customised refractive surgery.

The results and improvements derived out of this work can be directly applied to the laser systems for corneal refractive surgery, as well as to the algorithms and computer programmes, which control and monitor the ablation procedures. 



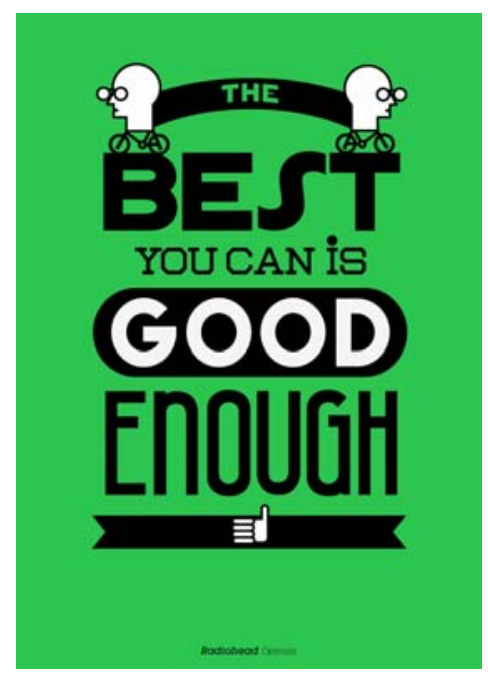

\section{Chapter 2 MOTIVATION \\ (Justificación)}

Laser corneal refractive surgery presents yet unresolved problems e.g. loss of ablation efficiency, cyclotorsional movements, as well as the induction of aberrations. A research work to approach these issues may help reducing the complications and occurrence of adverse events during and after refractive surgery, improving the postoperative quality of vision, as well as reducing the ratio of retreatments and reoperations.

The intention of this dissertation is to describe in detail the theoretical framework, explaining a possible method of tissue-saving optimisation and exploring its tissue-saving capabilities, as well as evaluating its clinical application. This analysis considers the results in terms of predictability, refractive outcome, efficacy, safety, and wavefront aberration. 



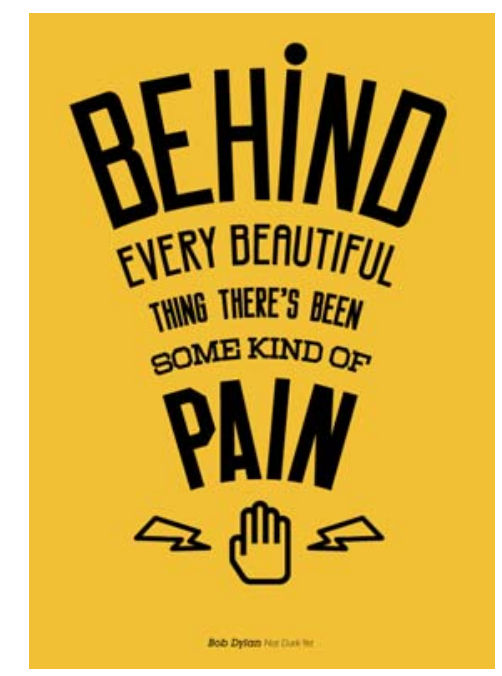

\section{Chapter 3 LASER REFRACTIVE SURGERY}

(Cirugía refractiva laser)

With the introduction of the laser technologies ${ }^{12},{ }^{13}$ for refractive surgery ${ }^{14}$, the change of the corneal curvature to compensate in a controlled manner for refractive errors of the ey $\mathrm{e}^{15}$ is more accurate than ever. The procedure is nowadays a successful technique, due to its sub micrometric precision and the high predictability and repeatability of corneal ablation ${ }^{16}$ accompanied by minimal side effects ${ }^{17}$. Standard ablation profiles based on the removal of convexconcave tissue lenticules with spherocylindrical surfaces proved to be effective in the compensation of primary refractive errors ${ }^{18}$. However, the quality of vision deteriorated significantly, especially under mesopic and low-contrast conditions ${ }^{19}$.

Furthermore, there is still controversy concerning the optimal technique for corneal refractive procedure ${ }^{20}$, and about in which corneal layer to perform refractive procedures to maximise patients' visual outcomes. 


\subsection{The origins of refractive surgery}

\section{(Los orígenes de la cirugía refractiva)}

Historically, several attempts have been developed with two clearly different approaches: surface ablations and lamellar ablations. Late in the 80's Photo-Refractive Keratectomy ${ }^{21}$ (PRK) was performed with broad beam lasers, mechanical debridement, small optical zones $5 \mathrm{~mm}$, without transition zones, and the surgery used to be unilateral; at the same time, excimer laser keratomileusis ${ }^{22}$ was performed with thick free caps $(240 \mu \mathrm{m})$, ablated on the underside of the flap and then sutured in place. In the early 90's, Laser-Assisted in Situ Keratomileusis $^{23}$ (LASIK) was developed, by creating a hinged flap (180 $\left.\mu \mathrm{m}\right)$; ablating on the stromal surface, and no suture was needed. In the mid 90's the first scanning lasers were used ${ }^{24}$, and the ablation zones were increased up to 7 $\mathrm{mm}$, moreover alcohol debridement was slowly replacing mechanical debridement in the surfaces treatments. Late in the 90's laser systems were enhanced by adapting Eye-Tracking technologies ${ }^{25}$. In the early 2000's, Laser-Assisted SubEpithelial Keratectomy ${ }^{26}$ (LASEK) was introduced by creating epithelial flaps, and the laser technology improved introducing flying spot patterns ${ }^{27}$. Recently, Epithelial Laser in Situ Keratomileusis ${ }^{28}$ (Epi-LASIK), and Epithelial LaserAssisted Sub-Epithelial Keratectomy ${ }^{29}$ (Epi-LASEK) were introduced by creating a truly epithelial flap at the Bowman's layer level, and Femtosecond-Laser-Assisted Laser in Situ Keratomileusis ${ }^{30}$ (Femto-LASIK), Thin-Flap and Ultra-Thin-Flap Laser in Situ Keratomileusis (Ultra-Thin-Flap-LASIK) were introduced by creating a flap slightly beneath Bowman's layer level (Sub-Bowman's Keratomileusis ${ }^{31}$ ). Nowadays, the technique selection is really wide-ranged, and the surgeons can decide, based on different criteria, which technique they will apply for each specific treatment. In surface ablations one can decide how to remove the epithelium (mechanically ${ }^{32}$, with alcohol, transepithelially with a PhotoTherapeutic Keratectomy (PTK), or with a truly epithelial flap), whereas in stromal ablation one can decide how to cut the flap (hinge position, flap thickness, mechanically or with a femtosecond laser). 


\subsection{Laser ablation}

\section{(Ablación laser)}

Laser corneal refractive surgery is based on the use of a Laser (typically an excimer one) to change the corneal curvature to compensate for refractive errors of the eye. It has become the most successful technique, mainly due to the submicron precision and the high repeatability of the ablation of the cornea accompanied by minimal side effects. Laser refractive surgery is based upon the sequential delivery of a multiplicity of laser pulses each one removing (ablating) a small amount of corneal tissue.

Corneal remodelling is essentially similar to any other form of micromachining. The lasers used in micro-machining are normally pulsed excimer lasers, where the time length of the pulses is very short compared to the period between the pulses. Although the pulses contain little energy, given the small size of the beams, energy density can be high for this reason; and given the short pulse duration, the peak power provided can be high.

Many parameters have to be considered in designing an efficient laser ablation. One is the selection of the appropriate wavelength $(193.3 \pm 0.8 \mathrm{~nm}$ for ArF) with optimum depth of absorption in tissue, which results in a high-energy deposition in a small volume for a speedy and complete ablation. The second parameter is a short pulse duration to maximize peak power and minimize thermal conductivity to the adjacent tissue (ArF excimer based $\mathrm{T}<20 \mathrm{~ns}$ ).

The radiant exposure is a measure of the density of energy that governs the amount of corneal tissue removed by a single pulse. In excimer laser refractive surgery, this energy density must exceed $40-50 \mathrm{~mJ} / \mathrm{cm}^{2}$. The depth of a single impact relates to the fluence, and also the thermal load per pulse increases with increasing fluence. Knowing the fluence and details of the energy profile of the beam (size, profile, and symmetry), we can estimate the depth, diameter and volume of the ablation impact. Assuming a super-Gaussian beam energy profile, the following equation applies:

$$
I(r)=I_{0} e^{-2\left(r / R_{0}\right)^{2 N}}
$$


where $\mathrm{I}$ is the radiant exposure at a radial distance $\mathrm{r}$ of the axis of the laser beam, $\mathrm{I}_{0}$ is the peak radiant exposure (at the axis of the laser beam), $R_{0}$ is the beam size when the radiant exposure falls to $1 / \mathrm{e}^{2}$ its peak value, and $\mathrm{N}$ is the superGaussian order of the beam profile (where $\mathrm{N}=1$ represents a pure Gaussian beam profile, and $\mathrm{N} \rightarrow \infty$ represents a flat-top beam profile).

From the blow-off model ${ }^{33}$ (derived form the Beer-Lambert's law ${ }^{34}$ ), the real energy density absorbed at that point determines the ablation depth as:

$$
d_{i j}=\frac{\ln \left(\frac{I_{i j}\left(1-R_{i j}\right)}{I_{T h}}\right)}{\alpha_{\text {Correa }}}
$$

where $\mathrm{d}_{\mathrm{ij}}$ : Actual depth per pulse at the location $\mathrm{i}, \mathrm{j}$; $\mathrm{I}_{\mathrm{ij}}$ : Radiant Exposure of pulse at the location i,j; $\mathrm{R}_{\mathrm{ij}}$ : Reflectivity at the location $\mathrm{i}, \mathrm{j} ; \mathrm{I}_{\mathrm{Th}}$ : Corneal Threshold; $\alpha_{\text {Cornea }}$ : Corneal Absorption Coefficient.

For human corneal tissue, the ablation threshold takes values of about 40$50 \mathrm{~mJ} / \mathrm{cm}^{2}$, and the absorption coefficient is about 3.33-3.99 $\mu \mathrm{m}^{-1}$. We chose values of $46 \mathrm{~mJ} / \mathrm{cm}^{2}$ for the ablation threshold and $3.49 \mu \mathrm{m}^{-1}$ as absorption coefficient of the human corneal tissue. For PMMA, the ablation threshold takes values of about $70-80 \mathrm{~mJ} / \mathrm{cm}^{2}$, and the absorption coefficient is about $3.7-4.4 \mu \mathrm{m}^{-1}$. We chose values of $76 \mathrm{~mJ} / \mathrm{cm}^{2}$ for the ablation threshold and $4.0 \mu \mathrm{m}^{-1}$ as absorption coefficient for PMMA. Calculating the volume of a single spot for the cornea, and dividing it by the volume of a single impact on PMMA, we get the socalled "cornea-to-PMMA-ratio."

In general:

$$
d(r)=\frac{\ln \left(\frac{I(r)(1-R(r))}{I_{T h}}\right)}{\alpha_{\text {Cornea }}}
$$

For different beam profiles, we get different spot profiles as depicted in Figure 1 and Figure 2. 


\section{Beam energy profiles}

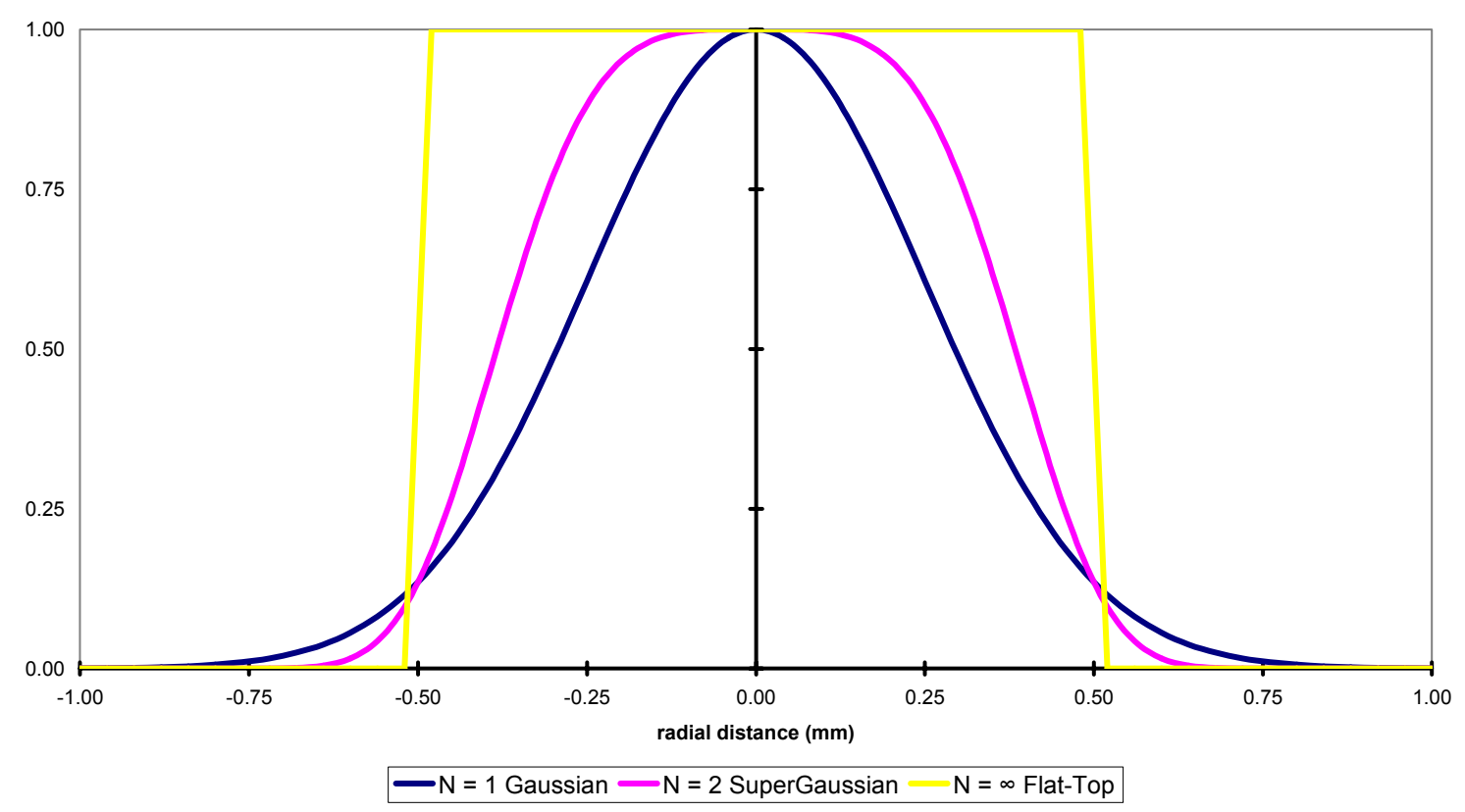

Figure 1: Beam profiles for different beam geometries, where $N$ is the Gaussian or supergaussian order. Gaussian profile $(\mathrm{N}=1)$ in blue, supergaussian profile $(\mathrm{N}=2)$ in pink, Flat-Top profile $(\mathrm{N}=\infty)$ in yellow.

\section{Ablative spot profiles}

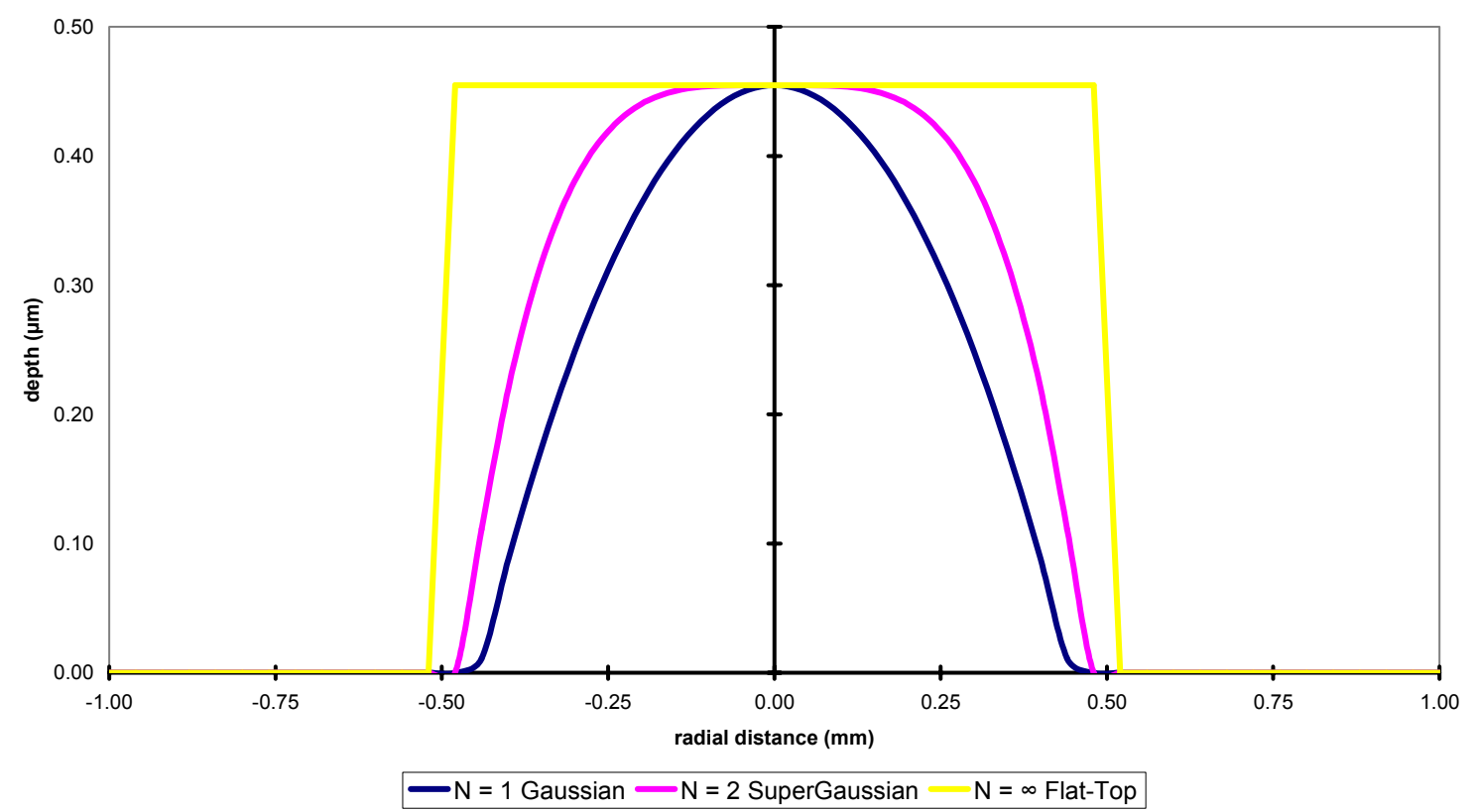

Figure 2: Spot profiles for different beam geometries. Parabolic spot profile (from Gaussian beams, $\mathrm{N}=1$ ) in blue, quartic spot profile (from supergaussian 
beams with $\mathrm{N}=2$ ) in pink, Flat-Top spot profile (from Flat-Top beams, $\mathrm{N}=\infty$ ) in yellow.

For different radiant exposures with the same beam profiles we get different spot profiles, as well (Figure 3).

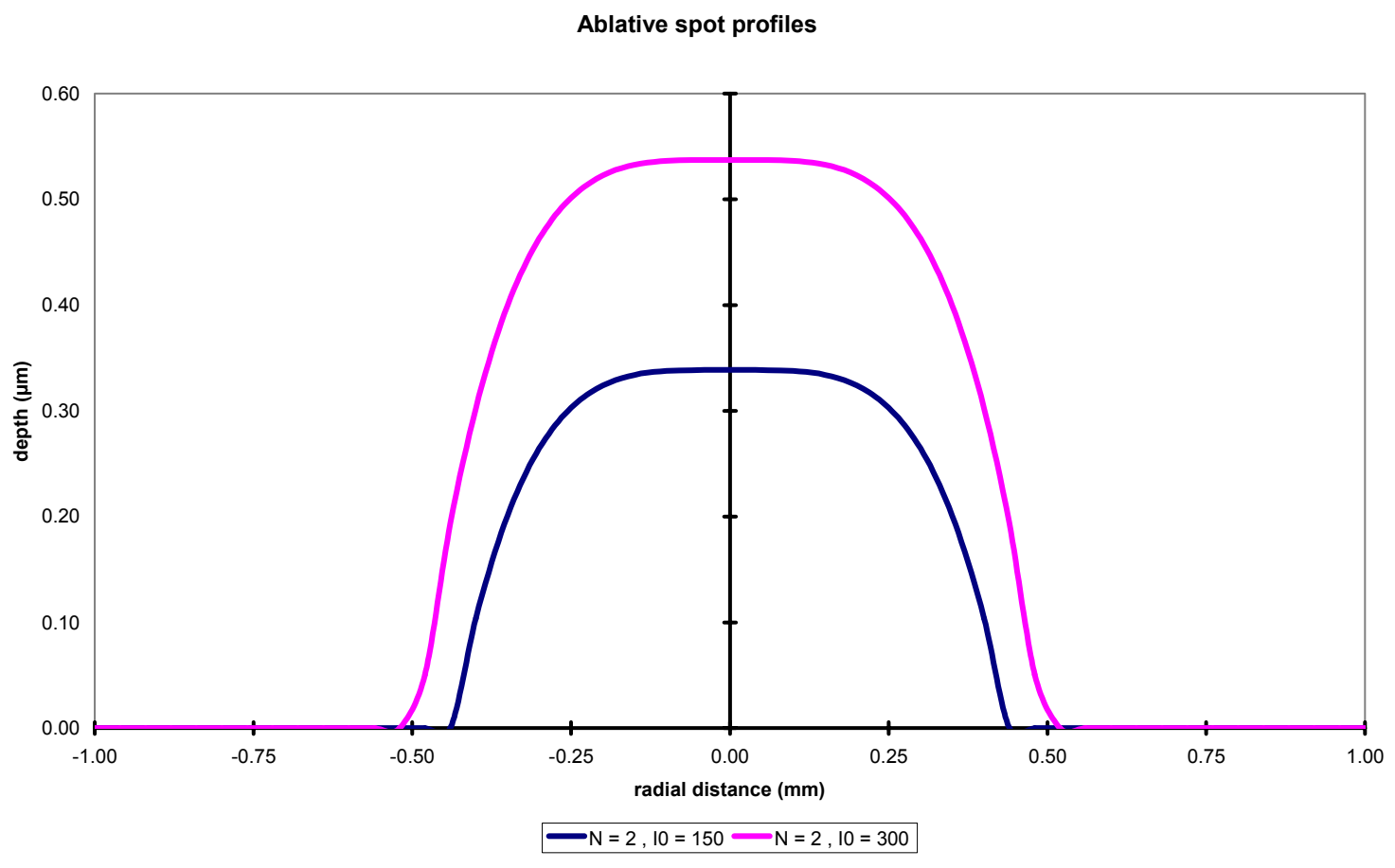

Figure 3: Spot profiles for different radiant exposures. Quartic spot profiles (from supergaussian beams with $\mathrm{N}=2$ ) for a peak radiant exposure of $150 \mathrm{~mJ} / \mathrm{cm}^{2}$ in blue and for a peak radiant exposure of $300 \mathrm{~mJ} / \mathrm{cm}^{2}$ in pink.

Applying Lambert-Beer's law (blow-off model), the footprint (diameter) of the impact is:

$$
F P=2 R_{0}\left(\frac{\ln \left(\frac{I_{0}}{I_{T h}}\right)}{2}\right)^{1 / 2 N}
$$

where FP is the footprint (diameter) of the ablative spot and $\mathrm{I}_{\mathrm{Th}}$ is the ablation threshold for radiant exposure for the irradiated tissue or material below which no ablation occurs. 
From these data (and the beam symmetry: square, hexagonal, circular), we can calculate the volume of ablation impact:

$$
V_{S}=\int_{0}^{2 \pi} \int_{0}^{\frac{F P}{2}} \frac{\ln \left(\frac{I_{0}}{I_{T h}}\right)-2\left(r / R_{0}\right)^{2 N}}{\alpha} r d r d \theta
$$

where $V_{S}$ is the volume of a single spot, and $\alpha$ the absorption coefficient of the irradiated tissue or material.

$$
V_{S}=\frac{\pi}{\alpha} \frac{N}{N+1} \ln \left(\frac{I_{0}}{I_{T h}}\right)^{\frac{N+1}{N}} \frac{R_{0}^{2}}{2^{1 / N}}
$$

The problem of the spot profile and the radiant exposure of the system relies on the sequential delivery of a multiplicity of laser pulses each one ablating locally a small amount of corneal tissue ${ }^{35}$. Being the global process an integral effect. The higher the spot profile is, the higher the ablated volume per pulse limiting the resolution of the treatment.

There are several ways to avoid that problem:

- $\quad$ Reducing the radiant exposure improving the vertical resolution of the treatment

- $\quad$ Reducing the spot diameter improving the horizontal resolution of the treatment

The problem of both alternatives is that they need extra time for the ablation procedure, which may produce other inconveniences.

The gained ablation volume has to be applied onto the cornea by thousands of single laser shots at different but partly repeated corneal positions, because the ablated volume of a single spot is much smaller than the ablation volume.

SCHWIND eye-tech-solutions has introduced as well some innovations concerning ablation shot file (sequences of pulses needed to carry out a refractive procedure) generation, to optimally remove the tissue corresponding to these state-of-the-art treatments, generating the sequence of laser shot coordinates in a way that: 
- guarantees a high fidelity reproduction of the given ablation volume line shape

- avoids vacancies and roughness of the cornea

In this context, two opposed requirements define the fluence level:

- A short ablation time (favouring high fluence levels)

- A high fidelity ablation (favouring low fluence levels)

\subsection{Zernike representation of aberrations}

\section{(Representación de Zernike de las aberraciones)}

A wavefront aberration expressed as expansion in series of Zernike polynomials ${ }^{36}$ takes the form:

$$
W A(\rho, \theta)=\sum_{n=0}^{\infty} \sum_{m=-n}^{+n} C_{n}^{m} Z_{n}^{m}(\rho, \theta)
$$

Where WA is the wavefront aberration described in polar coordinates, $Z[n, m]$ are the Zernike polynomials (Figure 4) in polar coordinates and C[n,m] are weight coefficients for the Zernike polynomials. 


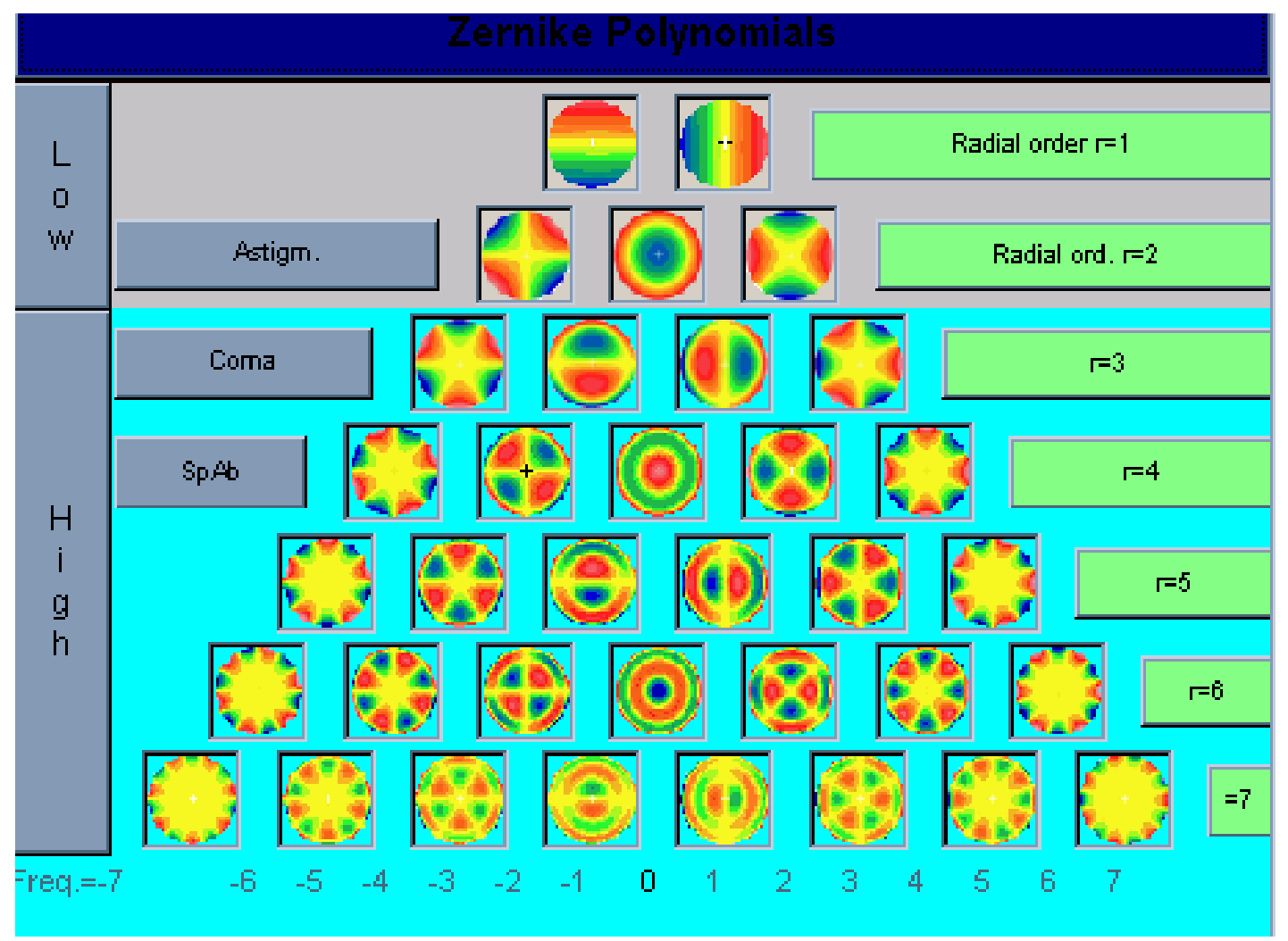

Figure 4: Zernike pyramid showing the Zernike terms up to $7^{\text {th }}$ order.

The wavefront is defined as the multidimensional surface of the points with equal phase. The difference from each of the points having length units to a wavefront reference surface (typically either a planar wavefront surface or a spherical wavefront surface) determines the wavefront aberration. Therefore, the wavefront error is described in units of length. As the $C[n, m]$ are just weight coefficients, they are non-dimensional, and the $Z[n, m]$ are the Zernike polynomials describe units of length.

\subsection{Measurement of Optical Aberrations (Medida de las aberraciones ópticas)}

To avoid inducing aberrations, as well as to eliminate the existing aberrations, "customized" treatments were developed ${ }^{37}$. Customisation of the 
ablation is possible, either using wavefront measurements of the whole eye ${ }^{38}, 39$ (obtained, e.g., by Scheiner aberroscopes ${ }^{40}$, Tscherning aberroscopes ${ }^{41}, 42$, Hartmann screens ${ }^{43}$, 44 , Slit skiascopic refractometer ${ }^{45}$, Hartmann-Shack ${ }^{46}$ or other type of wavefront sensors $47,48,49,50,51,52,53$ ) or by using corneal topography-derived wavefront analyses ${ }^{54,55}$. Topographic-guided ${ }^{56}$, Wavefront-driven ${ }^{37}$, Wavefrontoptimized $^{57}$, Asphericity preserving ${ }^{58}$, and Q-factor profiles ${ }^{59}$ have all been put forward as solutions. A customized treatment is the treatment of choice in highly aberrated eyes and for retreatments, especially for those performed ex domo, where the original preoperative aberrations are unknown.

Throughout this thesis, optical errors, represented by wave aberration, are described by the weight coefficients of the Zernike polynomials ${ }^{36}$ in OSA standard $^{60}$.

\subsection{Theoretical ablation profiles}

\section{(Perfiles de ablación teóricos)}

A correcting method in laser refractive surgery that corrects higher-order aberrations as well as defocus and astigmatism could improve vision. In first approximation to laser refractive surgery case where tissue removal occurs, this volume can be expressed as $^{37}$ :

Wavefront correction can be achieved by applying the reverse wavefront. Because a refractive surgery laser system can remove tissue rather than add tissue, the wavefront correction must also be taken into consideration by shifting the ablation profile from negative values to only positive values. Furthermore, the correction will be performed by modifying the anterior front surface of the cornea by photoablation. Thus, the change in the refractive index of air $(n=1)$ and the cornea $(n=1.376)$ boundary must be included.

Applying these considerations, one will get:

$$
\operatorname{Abl}(\rho, \theta)=\frac{W A(\rho, \theta)-\min [W A(\rho, \theta)]}{n_{\text {Cornea }}-n_{\text {Air }}}
$$


where $\operatorname{Abl}(\rho, \theta)$ is the ablation at a given point (in polar coordinates), WA the wave aberration, and $n_{\text {Cornea }}$ and $n_{\text {Air }}$ the refractive indices of the cornea and the air, respectively.

\subsection{The LASIK technique (La técnica LASIK)}

With the LASIK (Laser in Situ Keratomileusis ${ }^{23}$ ) treatment, we have an accepted method for correcting refractive errors such myopia ${ }^{22}$, hyperopia ${ }^{61}$, and astigmatism $^{62}, 63$. One of the most significant side effects in myopic LASIK, is the induction of spherical aberration ${ }^{64}$, which causes halos and a reduction of contrast sensitivity ${ }^{19}$. However, the different laser platforms are always introducing new concepts and optimising their ablation profiles. 



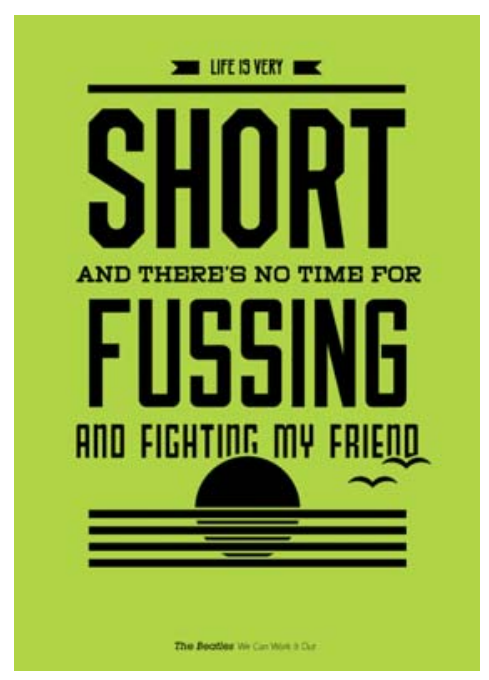

Chapter 4 CORNEAL ABLATION AND OPTICAL ABERRATIONS (Ablación corneal y aberraciones ópticas)

\subsection{Aberrations and visual performance after refractive surgery (Aberraciones y rendimiento visual tras cirugía refractiva) \\ With the introduction of wavefront analysis, it was proved that the conventional refractive LASER techniques were far from ideal, by measuring the aberrations induced by conventional algorithms ${ }^{65}$, and the aberrations induced by the LASIK flap cut itself ${ }^{66}$ (Figure 5).}


Induced SphAb vs. MRSEq (for "Classical" profiles)

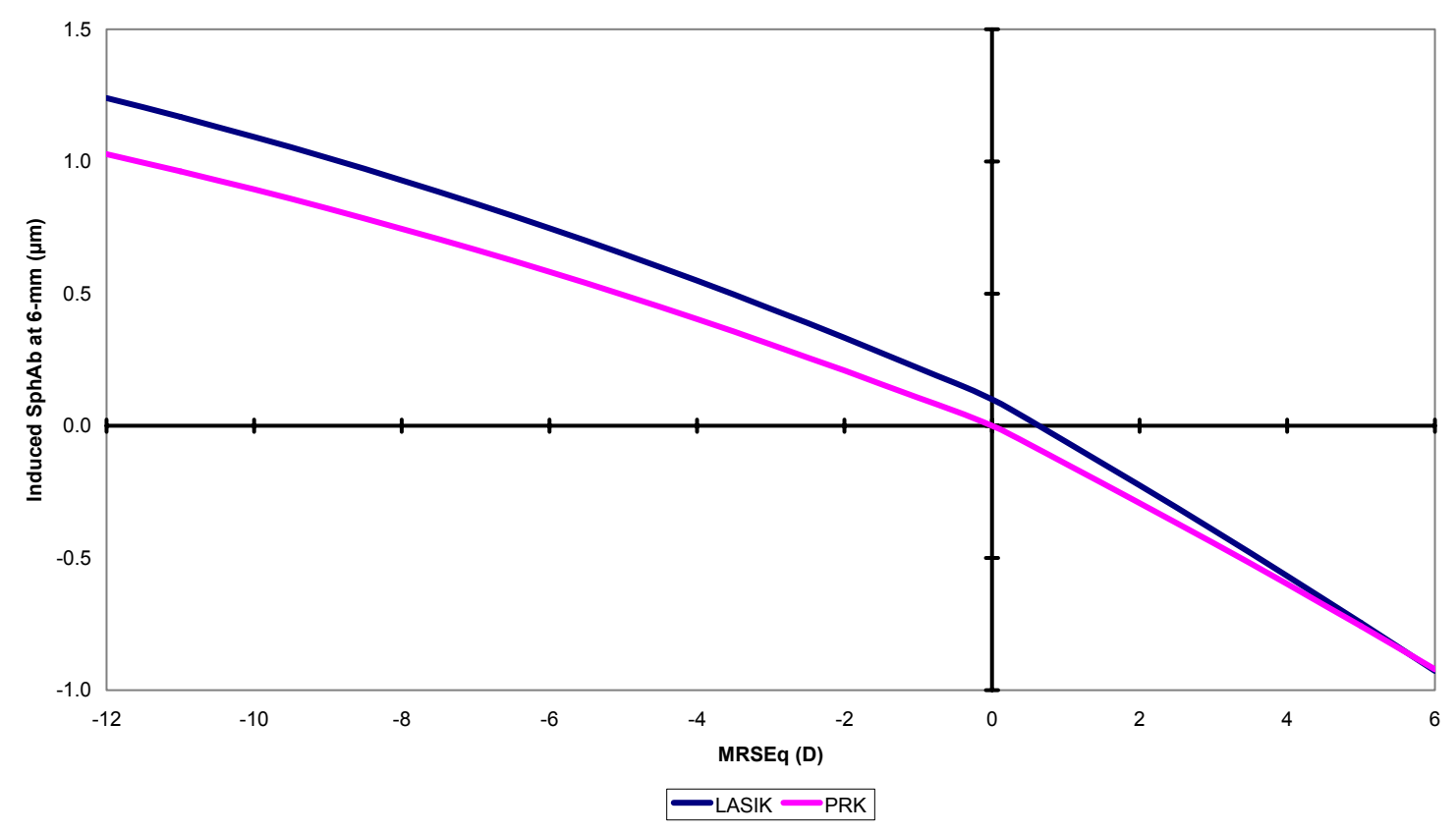

Figure 5: Induced Spherical aberration vs. achieved correction using classical profiles for different treatment strategies: LASIK (in blue), PRK (in purple). Notice that LASIK induced Spherical aberration does not go across the origin, representing the isolated effect due to the flap cut treatments. Notice as well that the induced Spherical aberration was more pronounced for hyperopic treatments than for myopic ones.

\subsection{Biological response of the cornea (Respuesta biológica de la córnea)}

Recent studies reporting LASIK induced optical aberrations have analysed the different variables affecting the LASIK flap cut, and their influence to the aberration induction ${ }^{67}$.

In 2004, Carriazo reported one study revealing the biomechanical response of the cornea after creating a flap cut, showing a clear correlation between amount of aberrations induced and flap thickness (Figure 6). 


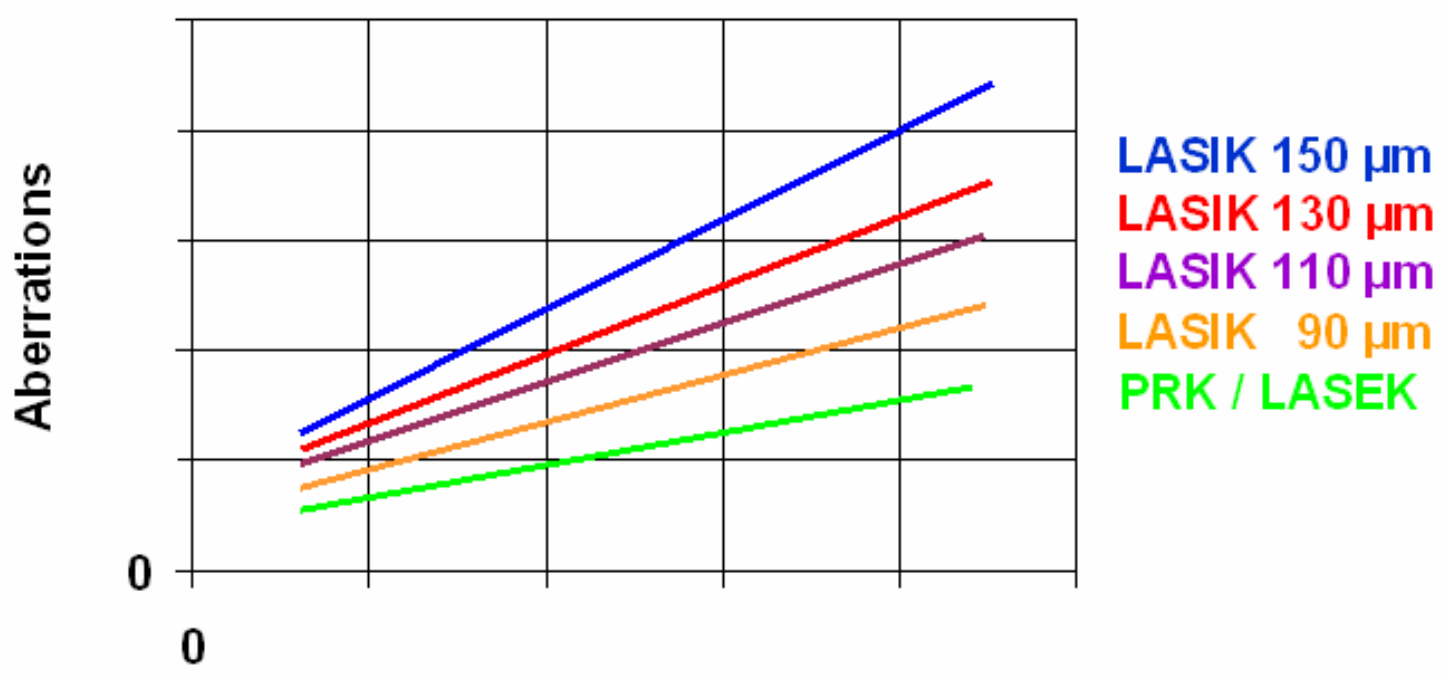

SEQ

Figure 6: Biomechanical changes due to the ablation, depending on the stromal layer where the tissue will be removed.

Flaps thinner than 130 microns induce much less optical aberration than flaps thicker than 140 microns. A clear difference should be established between LASIK (traditionally getting flaps averaging $170 \pm 30$ microns), and thin flap LASIK $^{68}$ (flap thickness averaging $110 \pm 30$ microns), sometimes named LASAK (LASER Anterior-stromal Keratomileusis).

\subsection{Visual degradation \\ (Deterioro visual)}

It is still not known precisely whether and when an "optically perfect eye" after surgery is better than preserving the aberrations that the eye had before surgery. Although the optical quality of the eye can be described in terms of the aberration of its wavefront, in healthy individuals with a certain degree of 
supervision was observed that they presented a measurable degree of aberration in their wavefront ${ }^{69}$. Still more, it was observed that the individuals with smaller aberration in their wavefront were not always those scoring the best visual qualities. Thus, the optical quality of the human eye does not determine in a univocal way its visual quality.

However, the induction of aberrations, such as spherical aberrations and coma, has been related to a loss of visual acuity ${ }^{70}$, accommodative lag ${ }^{71}$ or, in general, visual quality. Finally, the concept of neural compensation suggests that the neural visual system is adapted to the eye's own aberration pattern. ${ }^{72}$. A study by Artal et al. ${ }^{73}$, on the effects that this neural compensation causes on the visual function, indicates that the visual quality we have is somewhat superior to the optical quality that our eye provides.

\subsection{Current trends in refractive surgery (Tendencias actuales en cirugía refractiva)}

The trends to correct refractive errors using LASER refractive surgery are evolving nowadays towards searching where to target the LASER ablation to optimise the photo-ablated tissue layer.

Historically, the first approaches were using PRK (Photo-Refractive Keratomileusis) in most of the patients. But finally, this technique was mainly discarded due to post-op pain, long recovery times, and clinically due to often post-op haze as undesired effect ${ }^{74}$. Later on, LASIK was getting more and more common in clinical practice using flap cuts around 160 microns, and it was soon the leading refractive surgery preferred technique ${ }^{75}$.

Afterwards, two new competing techniques were introduced in the standard clinical practice: LASEK concept (Epithelium removal by using alcohol, and LASER photo-ablation just over Bowman's layer), and just recently, Epi-LASIK (Epithelium mechanically lifting up without weakener agents and LASER photoablation also over Bowman's layer). These two techniques are relatively new, 
and there are controversial reports, reporting on average diminished post-op pain and less often haze complications compared to PRK ${ }^{76},{ }^{77}$. Nevertheless, these techniques still produce post-op pain and sometimes haze complications ${ }^{76,78}$.

Since laser refractive surgery was introduced, the technology rapidly improved. With the beginning of photo-ablation, the goal was to achieve predictable and stable results for myopic, hyperopic, and astigmatic corrections. Today's technology is far more advanced since sophisticated diagnostic instruments, such as aberrometers and topography systems, offer the challenge of improving the postoperative results in terms of visual acuity and night vision. At the same time, the better knowledge and understanding on refractive surgery by potential patients upgrades the required standard outcomes. Making more challenge finding new approaches towards the close-to-zero aberrations target results in several senses: a) finding the sources of the induced aberrations due to laser refractive surgery ${ }^{79}$, b) developing "free-of-aberrations" ablation profiles, c) developing ablation profiles to compensate the natural aberrations of any single eye to get a close-to-zero aberrations result ${ }^{80}$.

The pupil centre $(P C)$ considered for a patient who fixates properly defines the line-of-sight, which is the reference axis recommended by the OSA for representing the wavefront aberration.

Nevertheless, because the pupil centre is unstable, a morphologic reference is more advisable. The pupil centre shifts with changes in the pupil size, moreover, because the entrance pupil we see is a virtual image of the real one.

The corneal vertex $(\mathrm{CV})$ in different modalities is the other major choice as the centering reference. In perfectly acquired topography, if the human optical system were truly coaxial, the corneal vertex would represent the corneal intercept of the visual axis. Although the human optical system is not truly coaxial, the cornea is the main refractive surface. Thus, the corneal vertex represents a stable preferable morphologic reference (Figure 7). 


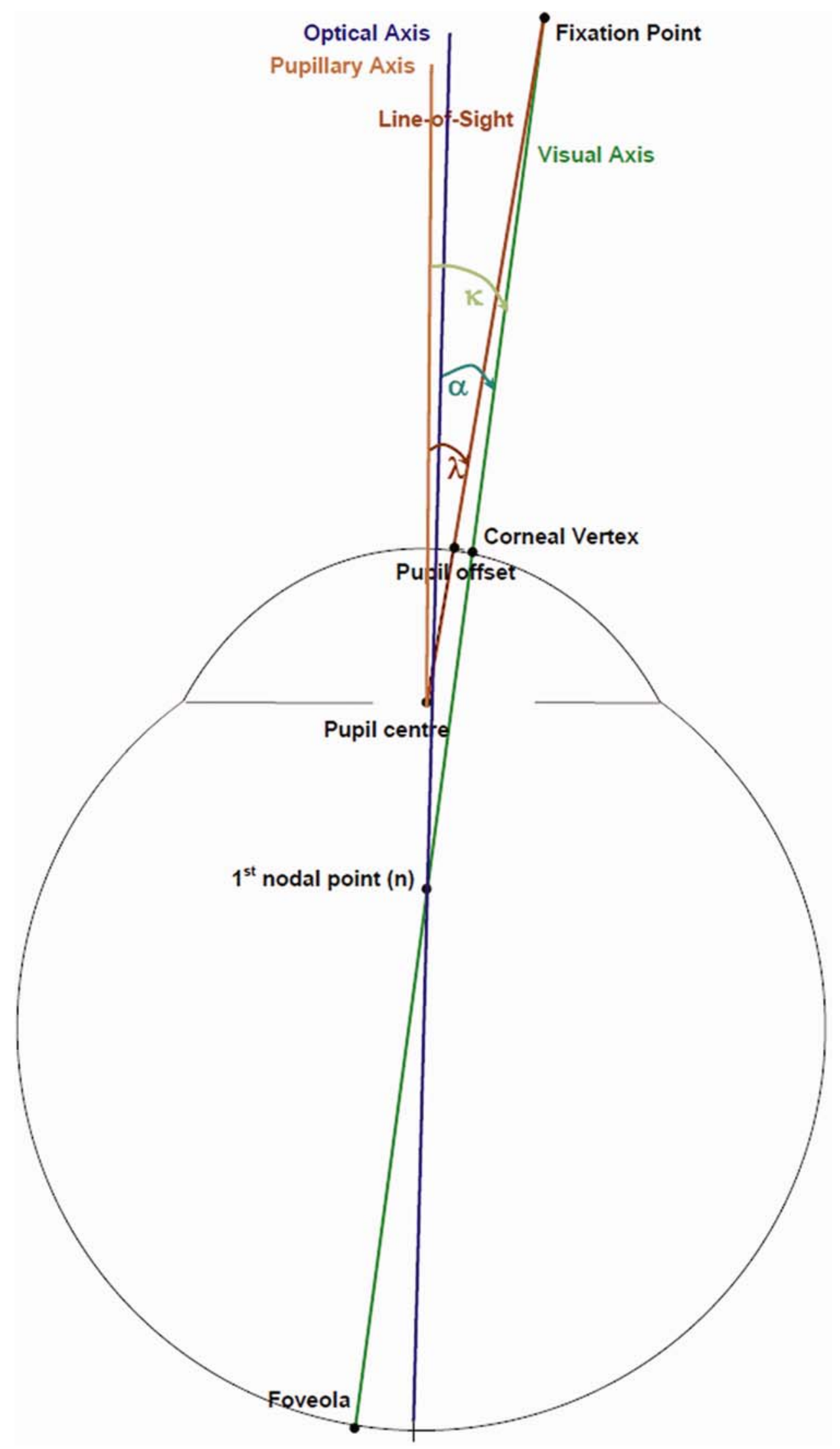

Figure 7: Representative axes of the human eye. 


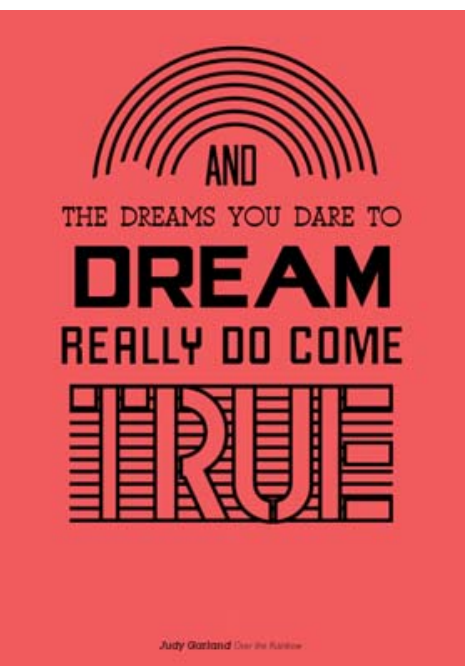

\section{Chapter 5 OPEN QUESTIONS IN CORNEAL REFRACTIVE SURGERY (Cuestiones actuales en cirugía refractiva corneal)}

Controversy remains over the proper definition of an optimal ablation profile for corneal refractive surgery ${ }^{81}$. Considerations such as the duration of the treatment, removal of tissue ${ }^{82}$, remodelling of tissue ${ }^{83}$, and, in general, the overall surgical outcome have made it difficult to establish a universal optimal profile. These considerations are actually interrelated in a multifactorial way, and may lead to clinical problems ${ }^{84}$ as corneal dehydration, ectasia or regression.

In laser corneal refractive surgery, one always aims to reduce the ablated tissue thickness (and, to a minor degree, to reduce the intervention time), the principal risk being an ectasia of the cornea due to excess thinning. Customised laser corneal refractive surgery on aberrated eyes may yield better results than the standard procedure ${ }^{85,86}$, but generally results in higher ablation depth, volume and time. The idea to optimise the customised treatment to reduce the ablated thickness while retaining the positive aspects is therefore pertinent.

The development of new algorithms or ablation strategies for efficiently performing laser corneal refractive surgery in a customised form minimising the amount of ablated tissue without compromising the visual quality becomes an important challenge. 
The availability of such profiles, potentially maximising visual performance without increasing the factors of risk, would be of great value for the refractive surgery community and, ultimately, for the patients' health and safety. Therefore, the topic "Optimised Zernike terms selection in customised treatments for laser corneal refractive surgery" is worth to be analysed, because its clinical implications are not yet deeply explored.

The real impact of tissue-saving algorithms in customised treatments is still discussed in a controversial way. Disagreement remains about optimum profile definitions. The aim of this work is to provide a simple and understandable theoretical frame explaining a possible method of tissue-saving optimisation.

Most of the systems available for laser refractive surgery include possibilities to customise the ablation, either based on topographical elevation or on corneal or ocular wavefront aberration. The problem of minimising the amount of tissue that is removed is that it must be done in such a way that:
a) does not compromise the refractive correction
b) does not compromise the visual performance
c) is safe, reliable and reproducible 


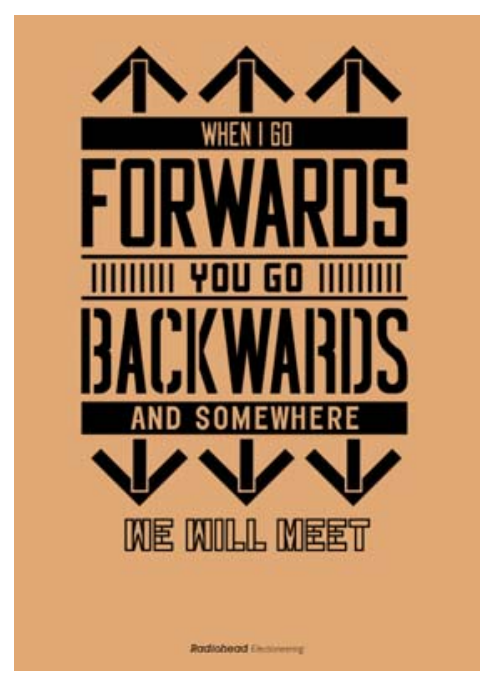

\section{Chapter 6 SPECIFIC GOALS OF THIS THESIS (Objetivos específicos)}

This dissertation deals with multiple purposes and goals:

A. To analyse the corneal asphericity using corneal wavefront and topographic meridional fits ${ }^{87,88}, 89$

B. To provide a model of an aberration-free profile and to clinically evaluate the impact of treatments based upon these theoretical profiles in the

post-operative cornea $9091,9293949596979899100101,102,103,104,105,106,107,108$

C. To assess a decision tree analysis system to further optimize refractive surgery outcomes ${ }^{109,110,111,112,113,114,115,116}$

D. To develop a geometrical analysis of the loss of ablation efficiency at non-normal incidence ${ }^{117},{ }^{118},{ }^{119},{ }^{120}$

E. To analyze the clinical effects of pure cyclotorsional errors during refractive surgery ${ }^{121,122,123,124,125,126}$

F. To evaluate the effective optical zone (the part of the ablation that receives full correction) after refractive surgery ${ }^{127},{ }^{128},{ }^{129}$ 
G. To develop a method to objectively minimise the ablated tissue of a customised ablation based on the Zernike expansion of the wavefront aberration ${ }^{130},{ }^{131},{ }^{132},{ }^{133},{ }^{134},{ }^{135},{ }^{136}$ 


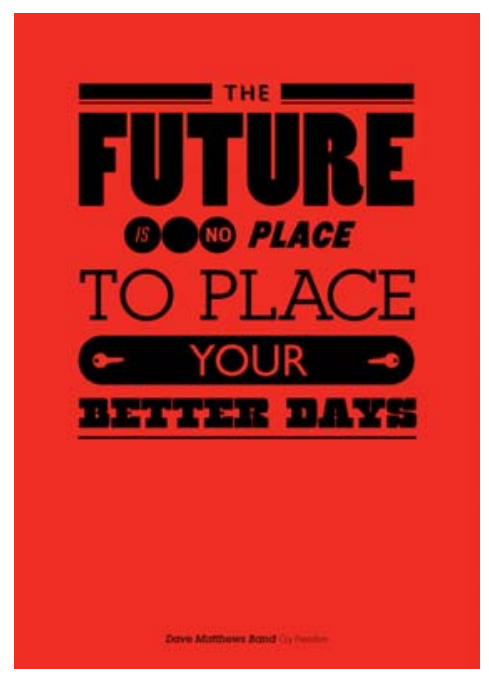

\section{Chapter 7 THESIS SYNOPSIS}

(Sinopsis de esta Tesis)

In this thesis, we studied the physical implications of corneal ablation as well as several possibilities for improving the clinical outcomes. Both are complex phenomena, entangled with biological effects, and with many mixed (and often yet unbounded) physical causes. Our approach was to first study the major patterns of induction of aberrations, second to analyse isolated sources of aberrations compatible with the observed patterns, and finally to develop ablation profiles of increasing complexity, by adding solutions for each and any of the identified sources of aberrations.

Besides, during this thesis different clinical evaluations of the specific solutions studied theoretically were performed on patients.

The body of this thesis is structured as follows:

Part 1 provides the introductory framework for this dissertation.

Part 2 describes the common methods used throughout this thesis: 
Methods for determination of the optical properties of the wave aberration, methods for combination of manifest refraction into the wave aberration, methods for determination of the actual clinical relevance of the wave aberration, as well as, the protocols in evaluations on subjects.

Part 3 introduces a topical review of the own works related to this thesis:

For Topic A, the corneal asphericity was analysed using corneal wavefront and topographic meridional fits, in an attempt to determine whether changes in asphericity were fully described by the induced corneal spherical aberration. The calculation of corneal asphericity as a three-dimensional fit renders more accurate results when it is based on corneal wavefront aberrations rather than on corneal topography of the principal meridians. A more accurate prediction could be obtained for hyperopic treatments compared to myopic treatments.

Topic B presents a model of an aberration-free profile, as well as a clinical evaluation of the impact of such treatments in the post-operative cornea, in an attempt to determine whether simple definitions of an ablation profile could suffice to come closer to the goal „zero aberrations induced.“

Since higher complexity might be related to larger potential hazards, Topic $\mathrm{C}$ describes a systematic analysis in the form of a decision tree to further optimize refractive surgery outcomes.

Topic D reports our findings with a geometrical analysis of the loss of ablation efficiency at non-normal incidence, in an attempt to determine whether how much of the induced spherical-like aberration in refractive surgery could be explained and avoided by the use of a simple cost-effective compensation.

The movements of the eye during refractive surgery and particularly the clinical impact of cyclotorsional movements are addressed in Topic E.

Evaluate of the effective optical zone (the part of the ablation that receives full correction) after refractive surgery is approached in Topic F. 
Finally, a method to objectively minimise the ablated tissue of a customised ablation based on the Zernike expansion of the wavefront aberration is developed in Topic G.

Finally, the major findings of this work, and their implications, are summarized in the Conclusions. 



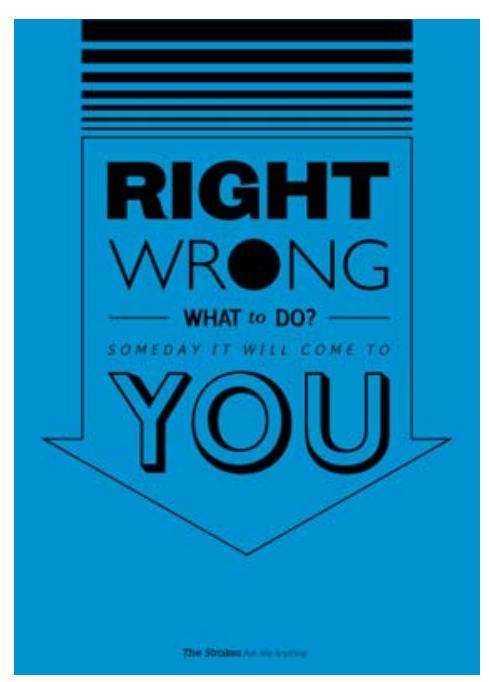

\section{Part 2 METHODS \\ (Método)}

\section{Chapter 8 CONTRIBUTION OF THE WAVE ABERRATION INTO THE MANIFEST REFRACTION \\ (Contribución de la aberración de onda sobre la refracción manifiesta)}

Classical ametropias (myopia, hyperopia and astigmatism) are, as well, difference to a reference surface, and are included in the, more general, wavefront error. However, classical ametropias are used to be described, not in units of length, but in units of optical refractive power.

It is, then, necessary to find a relationship between wavefront error magnitudes and classical ametropias ${ }^{137,138,139,140,141}$. This relationship is often called "objective wavefront refraction:"

In our study, the quadratic equivalent of a wave-aberration map was used as a relationship between wavefront-error magnitudes and classical ametropias. That quadratic is a sphero-cylindrical surface, which approximates the wave aberration map. The idea of approximating an arbitrary surface with a quadratic equivalent is a simple extension of the ophthalmic technique of approximating a sphero-cylindrical surface with an equivalent sphere. 
Several possibilities to define this relationship can be found in the literature:

- Objective wavefront refraction from low order Zernike modes at full pupil size ${ }^{142}$

- Objective wavefront refraction from Seidel aberrations at full pupil size $^{142}$

- Objective wavefront refraction from low order Zernike modes at subpupil size $e^{142}$

- Objective wavefront refraction from Seidel aberrations at subpupil size $^{142}$

- Objective wavefront refraction from paraxial curvature ${ }^{39}$

- Objective wavefront refraction from wavefront axial refraction ${ }^{143}$

\subsection{Wavefront refraction from low order Zernike modes at full pupil size}

\section{(Refracción del frente de onda a partir de los modos de Zernike de bajo orden considerados para la pupila completa)}

A common way to fit an arbitrarily aberrated wavefront with a quadratic surface is to find the surface that minimizes the sum of the squared deviations between the two surfaces.

The least-square fitting method is the basis of the Zernike wavefront expansion. Since the Zernike expansion employs an orthogonal set of basic functions, the least-square solution is simply given by the second-order Zernike coefficients of the aberrated wavefront, regardless of the values of the other coefficients. These second-order Zernike coefficients can be converted into a sphero-cylindrical prescription in power vector notation of the form [J0, M, J45].

$$
J_{0}=\frac{-8 \sqrt{6} C_{2}^{+2}}{P D^{2}}
$$




$$
\begin{aligned}
& M=\frac{-16 \sqrt{3} C_{2}^{0}}{P D^{2}} \\
& J_{45}=\frac{-8 \sqrt{6} C_{2}^{-2}}{P D^{2}}
\end{aligned}
$$

where $P D$ is the pupil diameter, $M$ is the spherical equivalent, $J_{0}$, the cardinal astigmatism and $J_{45}$ the oblique astigmatism. The components $J_{0}, M$, and $\mathrm{J}_{45}$ represent the power of a Jackson crossed cylinder with axes at 0 and $90^{\circ}$, the spherical equivalent power, and the power of a Jackson crossed cylinder with axes at 45 and $135^{\circ}$, respectively.

The power-vector notation is a cross-cylinder convention that is easily transposed into conventional refractions in terms of sphere, cylinder, and axis in the minus-cylinder or plus-cylinder formats used by clinicians.

$$
\begin{aligned}
& S=M-\frac{C}{2} \\
& C=2 \sqrt{J_{0}^{2}+J_{45}^{2}} \\
& A=\frac{\arctan \left(\frac{J_{45}}{J_{0}}\right)}{2}
\end{aligned}
$$

\subsection{Objective wavefront refraction from Seidel aberrations at full}

$$
\text { pupil size }
$$

\section{(Refracción objetiva del frente de onda a partir de las}

\section{aberraciones de Seidel consideradas para la pupila completa)}

The Seidel sphere adds a value for the primary spherical aberration to improve, in theory, the fit of the wavefront to a sphere and improve accuracy of the spherical equivalent power.

$$
M=\frac{-16 \sqrt{3} C_{2}^{0}+48 \sqrt{5} C_{4}^{0}}{P D^{2}}
$$


8.3 Objective wavefront refraction from low order Zernike modes at subpupil size

(Refracción objetiva del frente de onda a partir de los modos de Zernike de bajo orden para un diámetro subpupilar)

The same low-order Zernike modes can be used to calculate the refraction for any given smaller pupil size, either by refitting the raw wave-aberration data to a smaller diameter, or by mathematically performing the so-called radius transformation ${ }^{144}$ of the Zernike expansion to a smaller diameter.

\subsection{Objective wavefront refraction from Seidel aberrations at subpupil size}

(Refracción objetiva del frente de onda a partir de las aberraciones de Seidel para un diámetro subpupilar)

In the same way, Seidel aberrations can be used to calculate the refraction for any subpupil size.

\subsection{Objective wavefront refraction from paraxial curvature (Refracción objetiva del frente de onda a partir de la curvatura paraxial)}

Curvature is the property of wavefronts that determines how they focus. Thus, another reasonable way to fit an arbitrary wavefront with a quadratic surface is to match the curvature of the two surfaces at some reference point.

A variety of reference points could be selected, but the natural choice is the pupil center. Two surfaces that are tangent at a point and have the same curvature in every meridian are said to osculate. Thus, the surface we seek is the osculating quadric.

Fortunately, a closed-form solution exists for the problem of deriving the power vector parameters of the osculating quadratic from the Zernike coefficients of the wavefront. This solution is obtained by computing the curvature at the 
origin of the Zernike expansion of the Seidel formulae for defocus and astigmatism. This process effectively collects all $r^{2}$ terms from the various Zernike modes.

$$
\begin{gathered}
J_{0}=\frac{-8 \sqrt{6} C_{2}^{-2}+24 \sqrt{10} C_{4}^{-2}-48 \sqrt{14} C_{6}^{-2}+240 \sqrt{2} C_{8}^{-2}-120 \sqrt{22} C_{10}^{-2}+\ldots}{P D^{2}}( \\
M=\frac{-16 \sqrt{3} C_{2}^{0}+48 \sqrt{5} C_{4}^{0}-96 \sqrt{7} C_{6}^{0}+480 C_{8}^{0}-240 \sqrt{11} C_{10}^{0}+\ldots}{P D^{2}} \\
J_{45}=\frac{-8 \sqrt{6} C_{2}^{+2}+24 \sqrt{10} C_{4}^{+2}-48 \sqrt{14} C_{6}^{+2}+240 \sqrt{2} C_{8}^{+2}-120 \sqrt{22} C_{10}^{+2}+\ldots}{P D^{2}}(
\end{gathered}
$$

\subsection{Objective wavefront refraction from wavefront axial refraction (Refracción objetiva del frente de onda a partir de la refracción axial del frente de onda)}

It is also possible to represent the wavefront aberration in optical refractive power, without the need of simplifying it to a quadric surface, and, therefore, providing a higher level of detail. Straightforward approach for the problem is to use the concept of axial refractive error (vergence maps ${ }^{145}$ ) (Figure 8).

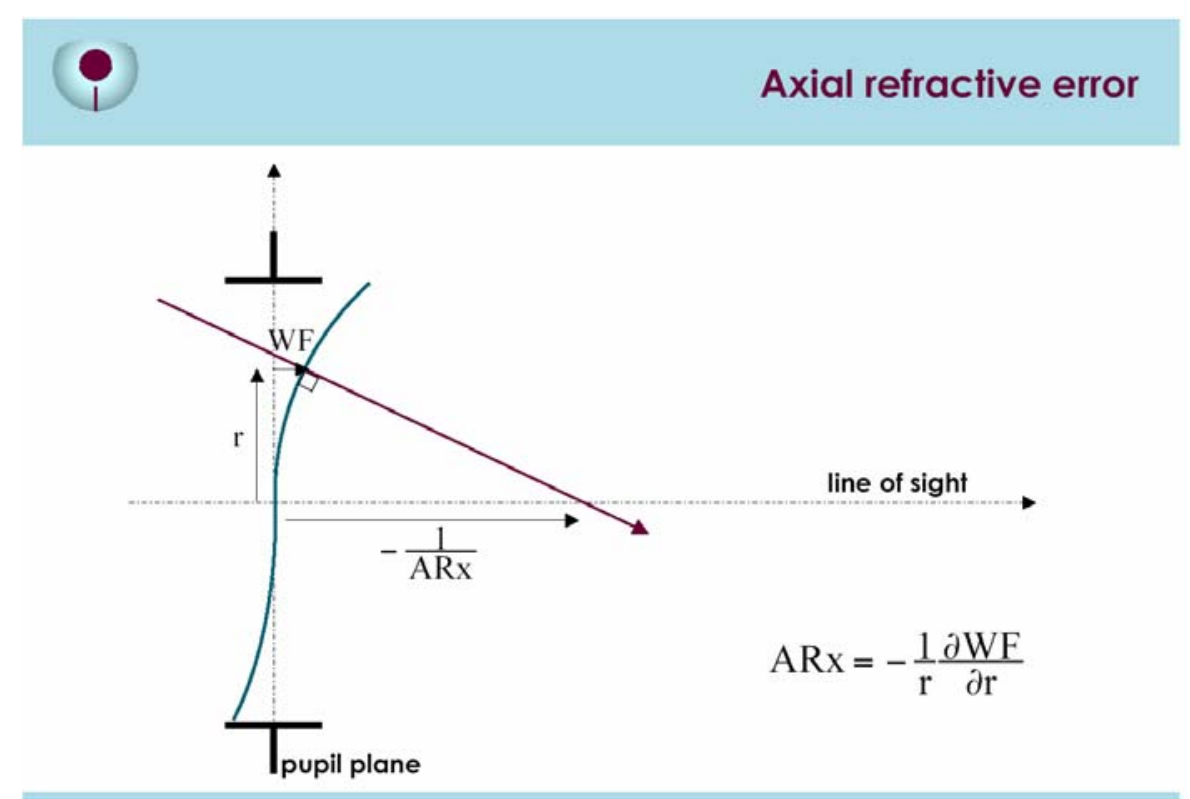

Figure 8: Representation of the axial refractive error. The line of sight represents a chief ray; the wavefront aberration is zero at the pupil centre, and 
perpendicular to the line of sight. Each point of the wavefront propagates perpendicular to the local surface of the wavefront. The axial distance from the pupil centre to the intercept between the propagated local wavefront and the line of sight expressed in dioptres corresponds to the axial refractive error ${ }^{143}$.

The line of sight represents a chief ray; the wavefront aberration is zero at the pupil centre, and perpendicular to the line of sight. Each point of the wavefront propagates perpendicular to the local surface of the wavefront. The axial distance from the pupil centre to the intercept between the propagated local wavefront and the line of sight expressed in dioptres corresponds to the axial refractive error.

$$
A R x(\rho, \theta)=\frac{-1}{r} \frac{\partial W(\rho, \theta)}{\partial \rho}
$$

A schematic comparison of the different quadric methods described here for the determination of the objective wavefront refraction for a given pupil size is depicted in Figure 9.

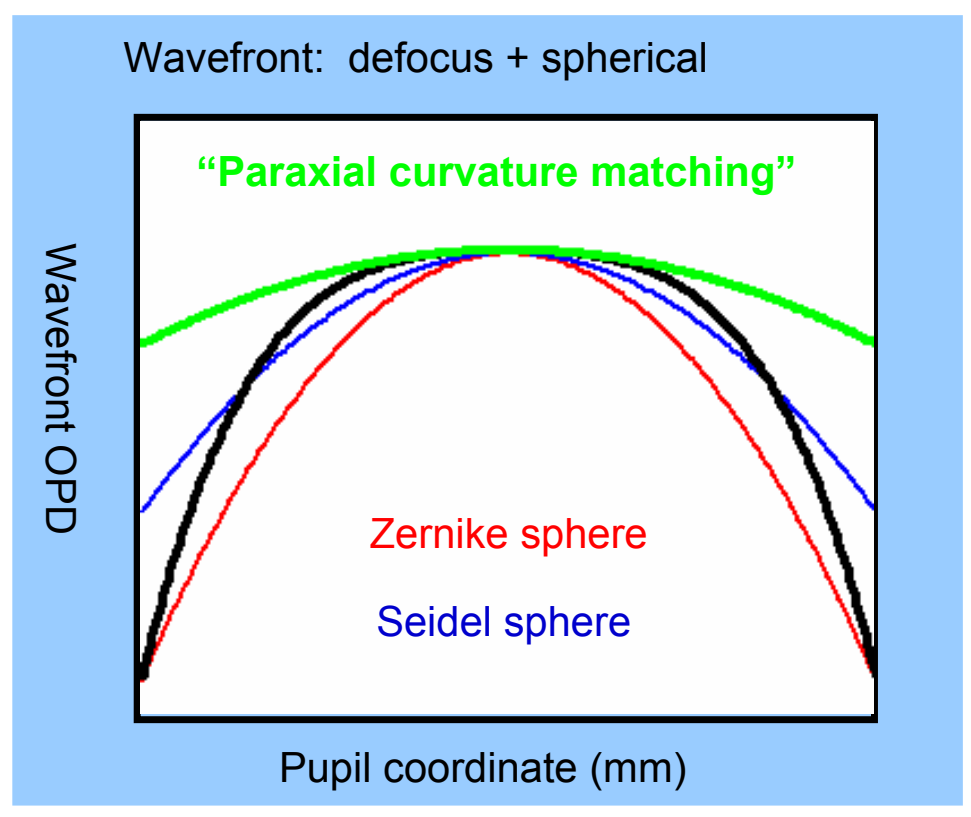

Figure 9: Comparison of the different quadric methods described here for the determination of the objective wavefront refraction for a given pupil size. 


\subsection{Automatic Manifest Refraction Balance (Compensación automática de la refracción manifiesta)}

These objective methods for calculating the refraction are optically correct but have some practical limitations in clinical practice ${ }^{39,146,147}$.

The devices to obtain the wavefront aberration of an eye use to work in the infrared range (IR), which is invisible for the human eye and avoid undesired miotic effects in the pupil size. The refractive indices of the different optical elements in our visual system depend on the wavelength of the illumination light. In this way, the propagated wavefront (and the corresponding wavefront aberration) ingoing to (or outcoming from) our visual system depends on the wavelength of the illumination light, leading to the so-called chromatic aberration $^{148}$.

The different methods provide "slightly" different results, depending on how they are compared to the subjective manifest refraction, one or another correlates better with manifest refraction ${ }^{142}$.

$\mathrm{HOAb}$ influence LOAb (refraction) when analysed for smaller diameters: For full pupil (e.g. $6 \mathrm{~mm}$ ) the eye sees the world through HOAb producing some multifocality but without defocus, for a smaller pupil (e.g. $4 \mathrm{~mm}$ ), the optical aberration of the eye is the same but the outer ring is blocked, thereby the eye sees the world through the central part of the HOAb, which may producing some defocus or astigmatism (LOAb, refraction).

For this work, a variation of the objective wavefront refraction from loworder Zernike modes at a fixed subpupil diameter of $4 \mathrm{~mm}$ was chosen as starting point to objectively include the measured subjective manifest refraction in the wave aberration (Figure 10 to Figure 13). 


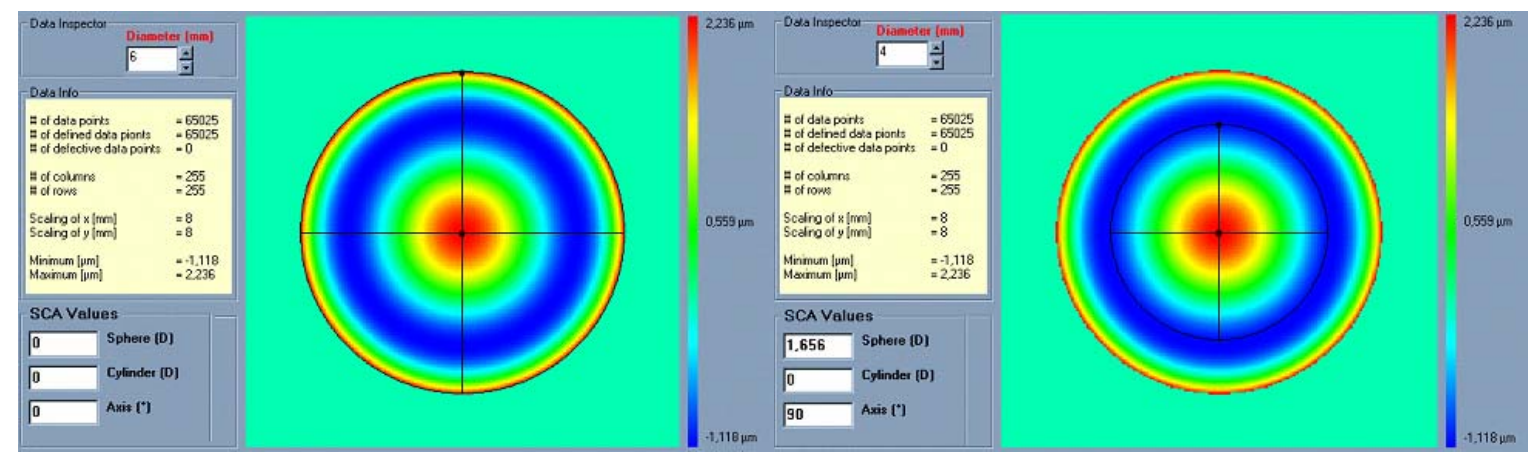

Figure 10: Zernike refraction of a pure Spherical Aberration (at $6 \mathrm{~mm}$ ) is per definition 0 because Spherical Aberration is a High Order Aberration mode, when analysed for a smaller diameter $(4 \mathrm{~mm})$ produces Defocus.

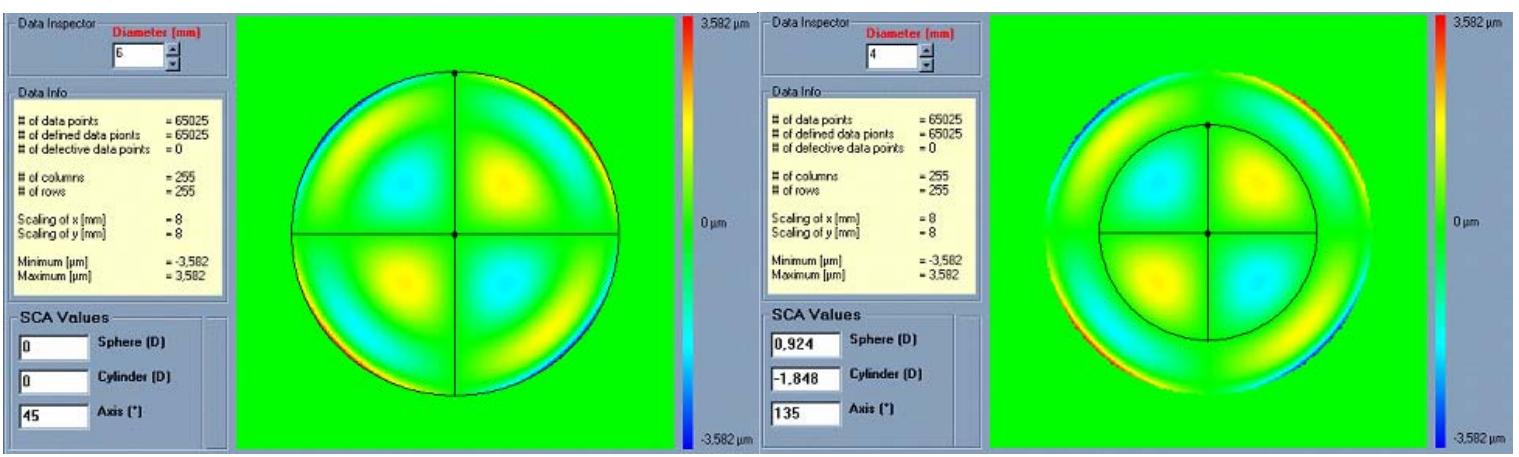

Figure 11: Zernike refraction of a pure High Order Astigmatism (at $6 \mathrm{~mm}$ ) is per definition 0 because of High Order Aberration mode, when analysed for a smaller diameter $(4 \mathrm{~mm})$ produces Astigmatism.

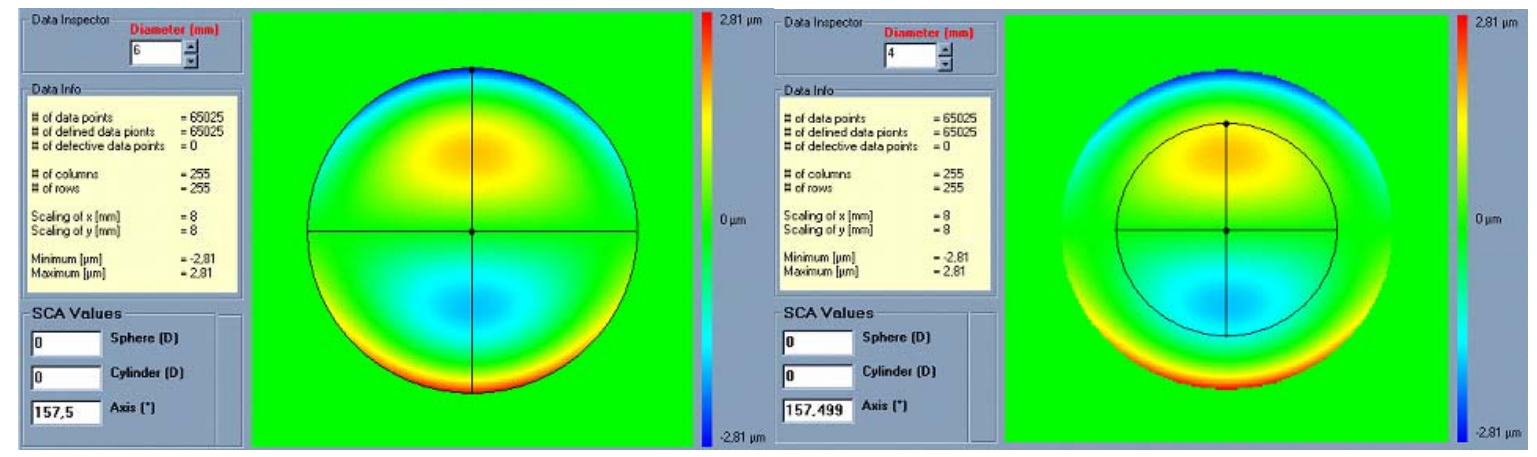

Figure 12: Zernike refraction of a pure Coma (at $6 \mathrm{~mm}$ ) is per definition 0 because Coma is a High Order Aberration mode, when analysed for a smaller diameter $(4 \mathrm{~mm})$ produces only tilt. Notice that coma may have "visual effect" if the visual axis changes producing Astigmatism. 


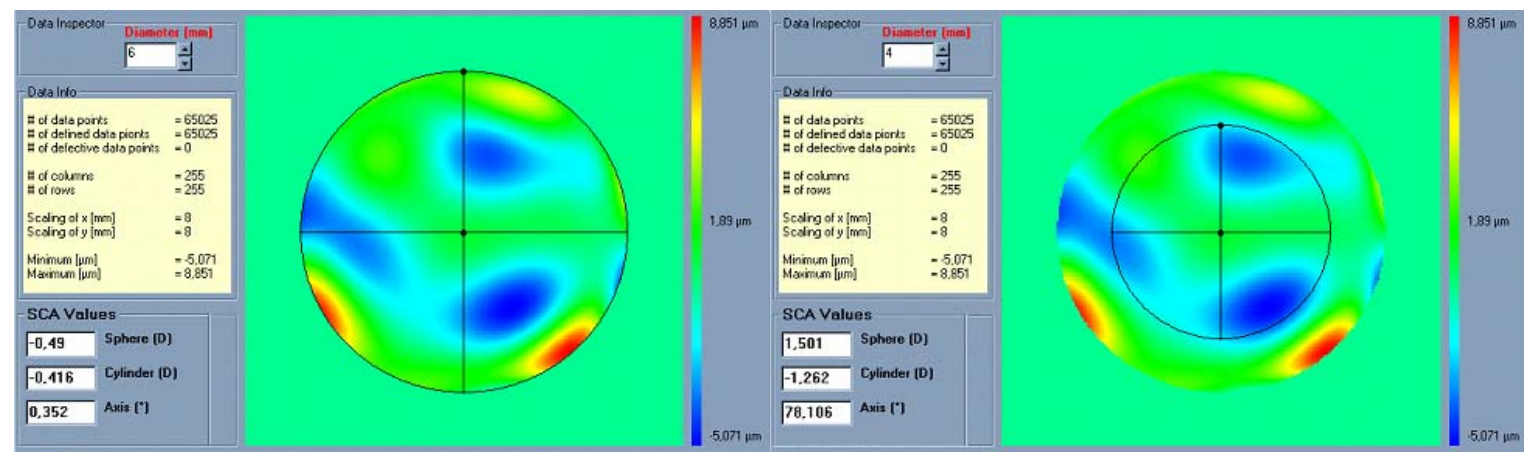

Figure 13: Zernike refraction of a general wavefront aberration analysed at $6 \mathrm{~mm}$ and analysed for a smaller diameter $(4 \mathrm{~mm})$.

The expected optical impact of high-order aberrations in the refraction is calculated and modified from the input manifest refraction. The same wave aberration is analysed for two different diameters: for the full wavefront area (6 $\mathrm{mm}$ in this study) and for a fixed subpupil diameter of $4 \mathrm{~mm}$. The difference in refraction obtained for each of the two diameters corresponds to the manifest refraction associated to the high-order aberrations (Figure 14).

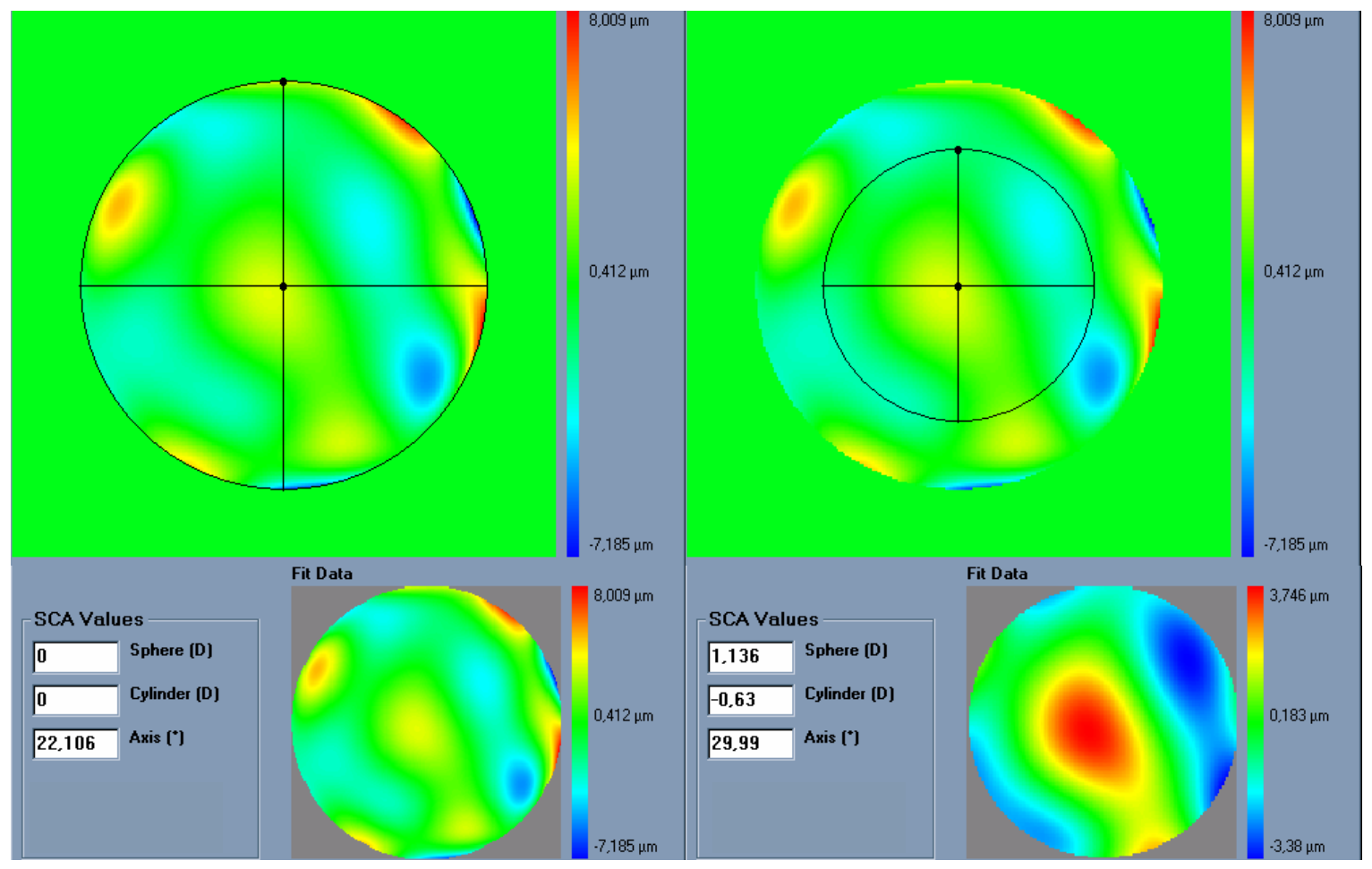

Figure 14: Automatic Refraction Balance. Optical impact of the HOAb the refraction is calculated and balanced from input refraction. Notice that the same 
wavefront aberration is analysed for two different diameters. The difference in the refraction provided at the two different analysis diameters correspond to the manifest refraction provided by the high order aberration.

The condition is to re-obtain the input manifest refraction for the subpupil diameter of $4 \mathrm{~mm}$. This way, the low-order parabolic terms of the modified wave aberration for the full wavefront area can be determined. 


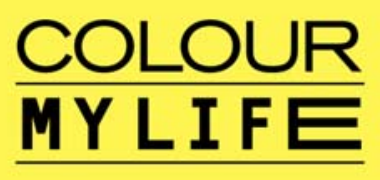

O०O WITH THE OOO

\section{Chapter 9 DETERMINATION OF THE ACTUAL CLINICAL RELEVANCE OF THE WAVE ABERRATION}

(Determinación de la relevancia clínica de la aberración de onda)

\subsection{Clinical relevance of the single terms in a Zernike expansion (Relevancia clínica de términos individuales de Zernike)}

A wavefront aberration can be expressed as a linear combination of weighted Zernike polynomials.

In the previous section, several methods were described to generally represent wavefront aberrations in a simplified way as classical ametropias in units of optical refractive power.

These methods considered the wavefront aberrations as a whole and determine by different means the classically equivalent optical refractive power in terms of sphere, cylinder and axis as "objective wavefront refraction."

However, due to the nature of the Zernike polynomials, these relationships were always functions of the terms $\mathrm{C}[\mathrm{n}, 0]$ for the defocus, functions of the terms $\mathrm{C}[\mathrm{n},+2]$ for the cardinal astigmatism, and functions of the terms $\mathrm{C}[\mathrm{n},-2]$ for the oblique astigmatism. 
It would be as well of interest to get a sort of classification of the clinical relevance of single aberration terms.

The first inconvenience, as mentioned before, is that wavefront error is expressed in units of length, whereas clinical refraction is expressed in units of optical refractive power.

A simple approach for classification of the clinical relevance of single aberration terms was proposed by Thibos et al., ${ }^{149}$ by introducing the concept of equivalent defocus (DEQ) as a metric to minimise the differences in the Zernike coefficients due to different pupil sizes.

Equivalent defocus is defined as the amount of defocus required to produce the same wavefront variance as found in one or more higher-order aberrations. A simple formula allows us to compute equivalent defocus in dioptres if we know the total wavefront variance in the Zernike modes in question:

$$
M_{e}=\frac{16 \sqrt{3} R M S}{P D^{2}}
$$

One could apply this concept of equivalent defocus to each individual Zernike mode to compute its clinical relevance. Of course, we must keep in mind that the kind of optical blur produced by higher-order aberrations is not the same as the blur produced by defocus.

Nevertheless, this concept of equivalent defocus is helpful when it comes to interpreting the Zernike coefficients in familiar dioptric terms. The basis of the equivalent defocus concept is the notion that the imaging quality of an eye is determined primarily by wavefront variance, and that it does not matter which Zernike mode produces that variance. 
It is important to bear in mind that 1 dioptre of ordinary defocus does not necessarily have the same effect as 1 dioptre of equivalent defocus because different types of aberrations affect the retinal image in different ways (Figure 15). Nevertheless, by expressing RMS error in terms of equivalent defocus the data are put into familiar units that help us judge the order of magnitude of the effect.

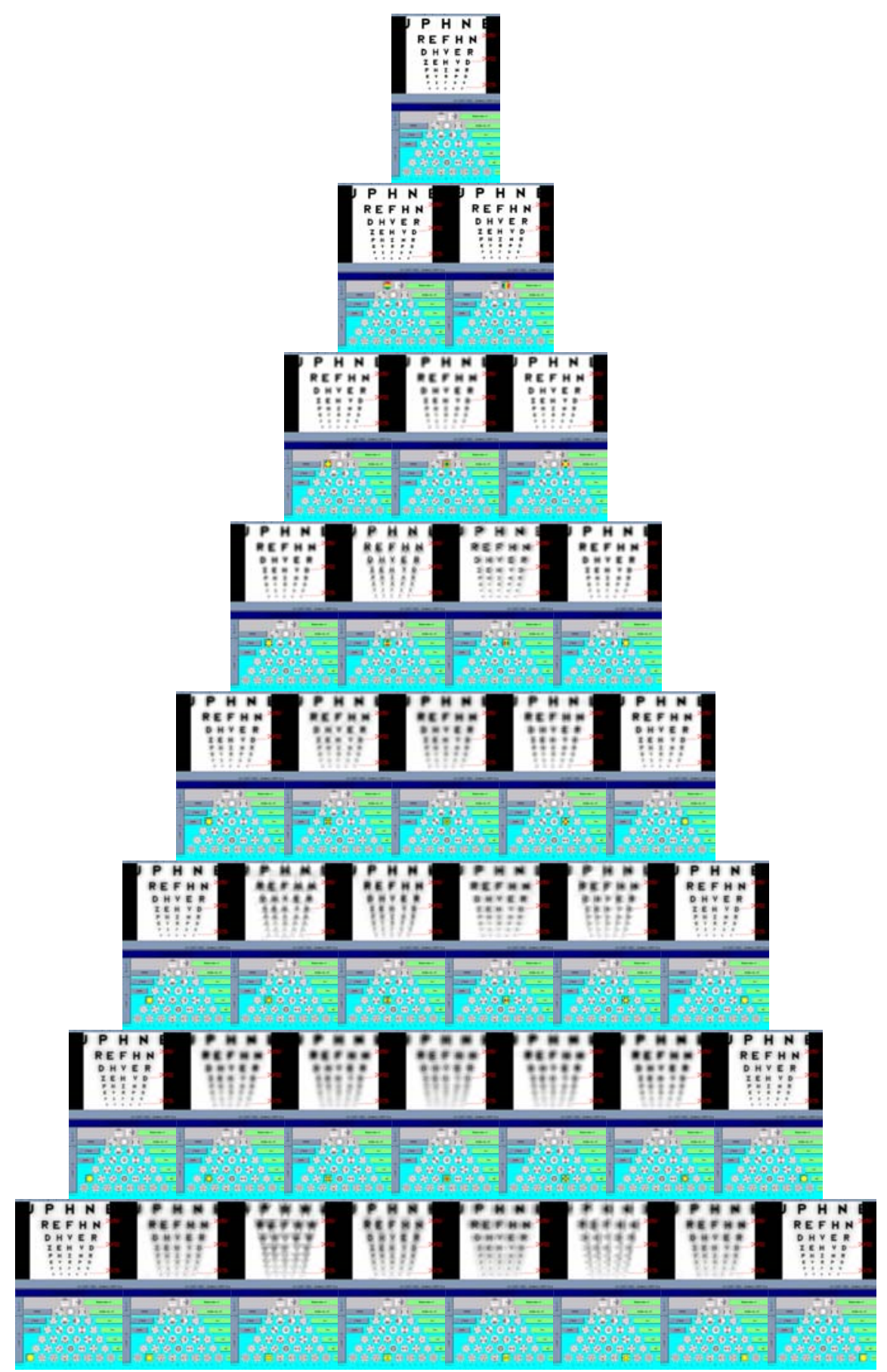

Figure 15: Zernike pyramid showing the effects on vision produced by 1 dioptre of equivalent defocus of different Zernike terms up to $7^{\text {th }}$ order. 
Wavefront aberration leads to blurring of the image. When it occurs, light from infinity or from a point source after transmission through the system does not converge into (or does not diverge from) a single point. The shape of the blurred spot at the retina is called point-spread-function (PSF). The PSF is the image that an optical system forms of a point source. Even if wave aberration does not exist for the system, the PSF is still affected by diffraction effects coming from the size of the pupil (aperture) $)^{150}$.

From the PSF, the blur of an image can be simulated. This can be done because an object can be considered as composed of an infinite array of point sources, each with its respective intensity, position, and colour. Giving each of these points the shape of the PSF the blurred image can be simulated ${ }^{151}$.

In general, for the same amount of equivalent defocus, the optical blur produced by higher-order aberrations increases with increasing radial order and decreases with increasing angular frequencies. Based on this blur effect of the individual Zernike terms we have defined a dioptric equivalent (DEq) of the form:

$$
D E q_{n}^{m}=\frac{16 \sqrt{\frac{2(n+1)}{\left(1+\delta_{m 0}\right)\left(\delta_{m 0}+m^{2}\right)}} C_{n}^{m}}{P D^{2}}
$$

where $D E q[n, m]$ is the optical blur for the individual Zernike term, $n$ is the radial order of the Zernike term, $m$ the meridional frequency of the Zernike term, $\delta_{m 0}$ a delta function, $P D$ the analysis diameter, and $C[n, m]$ the weight coefficient of the Zernike term.

In such a way, the dioptric equivalent produced by higher order aberrations increases with increasing radial order and decreases with increasing angular frequencies (Table 1). 


\begin{tabular}{|c|c|c|c|}
\hline j-index & n-order & m-frequency & $\begin{array}{l}\text { Relative Optical Blur } \\
\text { (Defocus }=1)\end{array}$ \\
\hline 3 & 2 & -2 & $\frac{1}{\sqrt{2}}=0.707$ \\
\hline 4 & 2 & 0 & 1 \\
\hline 5 & 2 & 2 & $\frac{1}{\sqrt{2}}=0.707$ \\
\hline 6 & 3 & -3 & $\frac{2}{3} \sqrt{\frac{2}{3}}=0.544$ \\
\hline 7 & 3 & -1 & $2 \sqrt{\frac{2}{3}}=1.633$ \\
\hline 8 & 3 & 1 & $2 \sqrt{\frac{2}{3}}=1.633$ \\
\hline 9 & 3 & 3 & $\frac{2}{3} \sqrt{\frac{2}{3}}=0.544$ \\
\hline 10 & 4 & -4 & $\frac{1}{2} \sqrt{\frac{5}{6}}=0.456$ \\
\hline 11 & 4 & -2 & $\sqrt{\frac{5}{6}}=0.913$ \\
\hline 12 & 4 & 0 & $\sqrt{\frac{5}{3}}=1.291$ \\
\hline 13 & 4 & 2 & $\sqrt{\frac{5}{6}}=0.913$ \\
\hline 14 & 4 & 4 & $\frac{1}{2} \sqrt{\frac{5}{6}}=0.456$ \\
\hline 15 & 5 & -5 & $\frac{2}{5}=0.400$ \\
\hline 16 & 5 & -3 & $\frac{2}{3}=0.667$ \\
\hline 17 & 5 & -1 & 2 \\
\hline 18 & 5 & 1 & 2 \\
\hline
\end{tabular}




\begin{tabular}{|c|c|c|c|}
\hline j-index & n-order & m-frequency & $\begin{array}{c}\text { Relative Optical Blur } \\
(\text { Defocus }=1 \text { ) }\end{array}$ \\
\hline 19 & 5 & 3 & $\frac{2}{3}=0.667$ \\
\hline 20 & 5 & 5 & $\frac{2}{5}=0.400$ \\
\hline 21 & 6 & -6 & $\frac{1}{3} \sqrt{\frac{7}{6}}=0.360$ \\
\hline 22 & 6 & -4 & $\frac{1}{2} \sqrt{\frac{7}{6}}=0.540$ \\
\hline 23 & 6 & -2 & $\sqrt{\frac{7}{6}}=1.080$ \\
\hline 24 & 6 & 0 & $\sqrt{\frac{7}{3}}=1.528$ \\
\hline 25 & 6 & 2 & $\sqrt{\frac{7}{6}}=1.080$ \\
\hline 26 & 6 & 4 & $\frac{1}{2} \sqrt{\frac{7}{6}}=0.540$ \\
\hline 27 & 6 & 6 & $\frac{1}{3} \sqrt{\frac{7}{6}}=0.360$ \\
\hline 28 & 7 & -7 & $\frac{4}{7 \sqrt{3}}=0.330$ \\
\hline 29 & 7 & -5 & $\frac{4}{5 \sqrt{3}}=0.462$ \\
\hline 30 & 7 & -3 & $\frac{4}{3 \sqrt{3}}=0.770$ \\
\hline 31 & 7 & -1 & $\frac{4}{\sqrt{3}}=2.309$ \\
\hline 32 & 7 & 1 & $\frac{4}{\sqrt{3}}=2.309$ \\
\hline 33 & 7 & 3 & $\frac{4}{3 \sqrt{3}}=0.770$ \\
\hline
\end{tabular}




\begin{tabular}{|c|c|c|c|}
\hline j-index & n-order & m-frequency & $\begin{array}{c}\text { Relative Optical Blur } \\
\text { (Defocus = 1) }\end{array}$ \\
\hline \hline 34 & 7 & 5 & $\frac{4}{5 \sqrt{3}}=0.462$ \\
\hline 35 & 7 & 7 & $\frac{4}{7 \sqrt{3}}=0.330$ \\
\hline
\end{tabular}

Table 1: Relative optical blur of the Zernike polynomials up to $7^{\text {th }}$ order.

\subsection{Global clinical relevance of the Zernike expansion} (Relevancia clínica global de la expansión de Zernike)

This dioptric equivalent metric is identical to the power vector notation for the low orders, and makes it possible to define a general optical blur of the form:

$$
U_{G}=\sqrt{\sum D E q[n, m]^{2}}
$$

as a generalization of the expression proposed by Thibos et al. ${ }^{152}$

Wave aberrations with similar general optical blur will show similar global optical performance, irrespective of which specific Zernike modes are responsible for this optical blur.

\subsection{Classification of the clinical relevance (Clasificación de la relevancia clínica)}

We have expressed each of the Zernike terms as a dioptric equivalent into familiar units that help us judge the order of magnitude of the effect.

Using common clinician limits, the following classification is proposed:

$$
\begin{gathered}
D E q_{n}^{m} \leq 0.25 D \Rightarrow \text { Not clinically relevant } \\
0.25 D<D E q_{n}^{m} \leq 0.50 D \Rightarrow \text { Might be clinically relevant } \\
D E q_{n}^{m}>0.50 D \Rightarrow \text { Clinically relevant }
\end{gathered}
$$


this represents the proposed objective determination of the actual clinical relevance of the single terms in a Zernike expansion of the wavefront aberration.

According to this classification, Zernike terms considered not clinically relevant (DEq $\leq 0.25 \mathrm{D}$ ) will be marked in green, Zernike terms that might be considered clinically relevant $(0.25 \mathrm{D}<\mathrm{DEq} \leq 0.50 \mathrm{D})$ will be marked in yellow, and Zernike terms considered clinically relevant (DEq $>0.50 D)$ will be marked in red (Figure 16 and Figure 17).

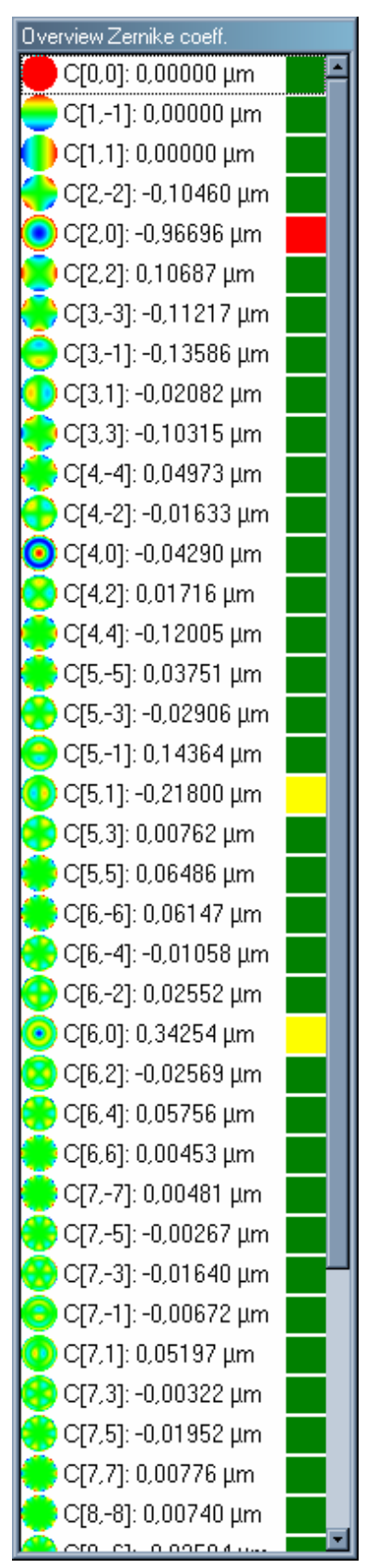

Figure 16: List of Zernike coefficients classified and colour coded by their dioptric equivalent relative to optical blur (DEq). 


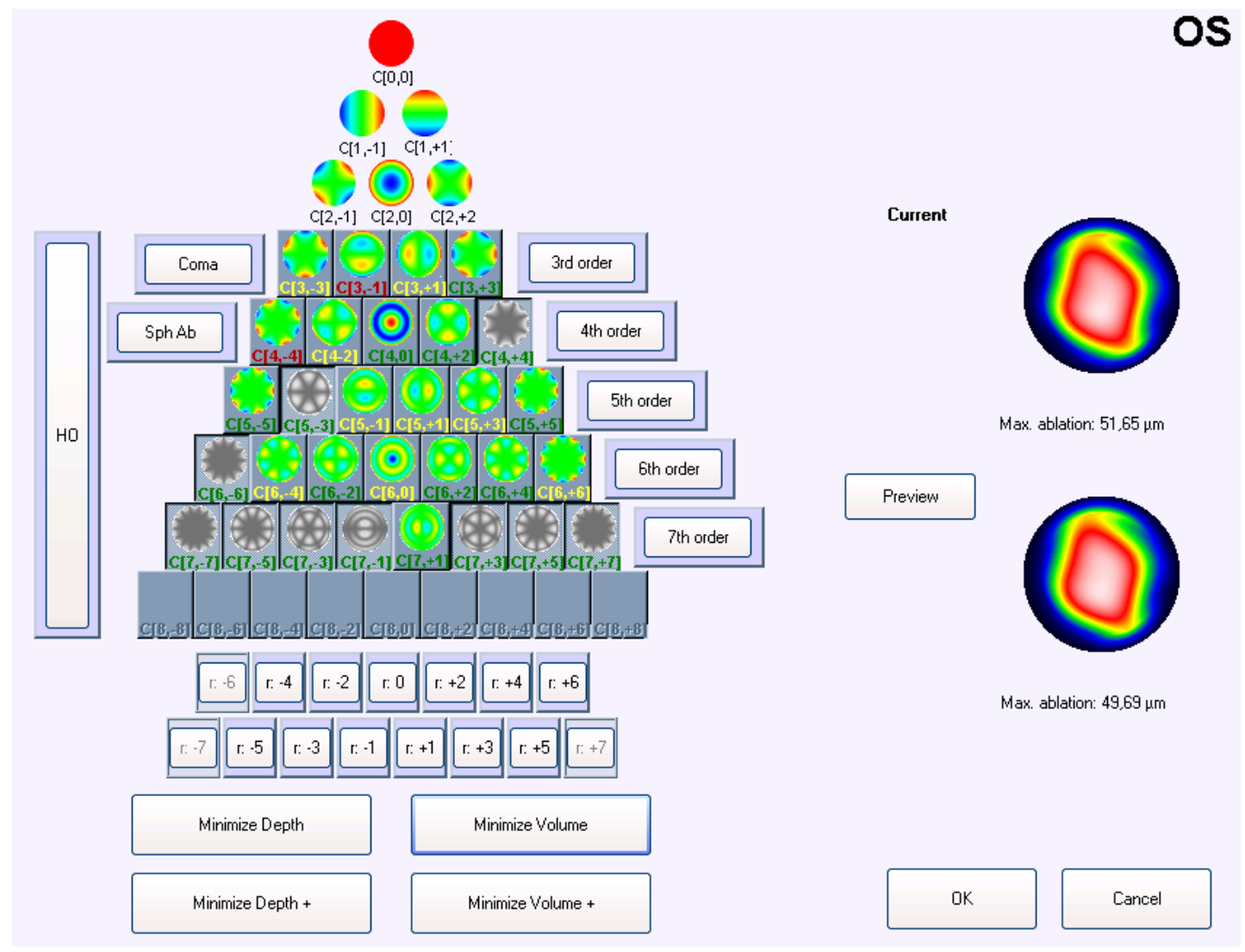

Figure 17: Zernike pyramid classified and colour coded by the dioptric equivalent relative to optical blur (DEq) of the single Zernike terms. 



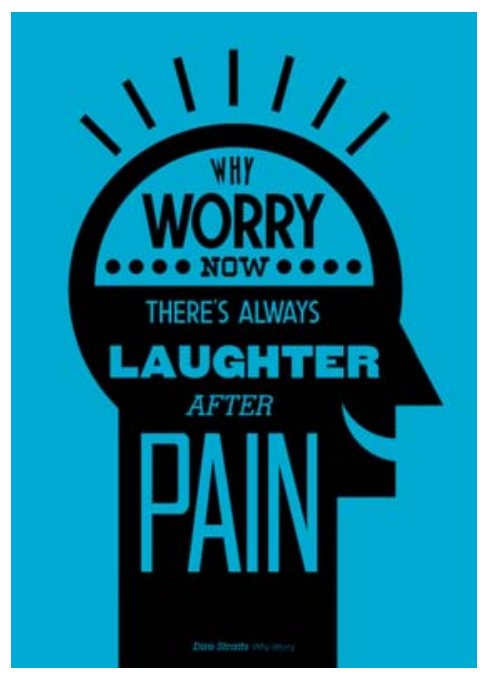

\section{Chapter 10 PROTOCOLS FOR MEASUREMENTS ON SUBJECTS (Protocolos de las medidas realizadas en sujetos)}

The procedures involving subjects were reviewed and approved by Institutional Bioethical Committees of SCHWIND eye-tech-solutions, and the clinic where measurements or procedures were performed and met the tenets of the Declaration of Helsinki.

All patients were fully informed and understood and signed an informed consent before enrolment in any of the studies.

All refractive procedures were planned and ablated with the available systems of SCHWIND eye-tech-solutions.

Using the Keratron-Scout (Figure 18) videokeratoscope ${ }^{153}$ (Optikon2000, Rome, Italy), corneal wavefront aberration before and after refractive surgery was analysed.

Using the Complete Ophthalmic Analysis System (COAS, Figure 18) aberrometer (Wavefront Sciences, Albuquerque, USA), ocular wavefront aberrations before and after refractive surgery was analysed. 


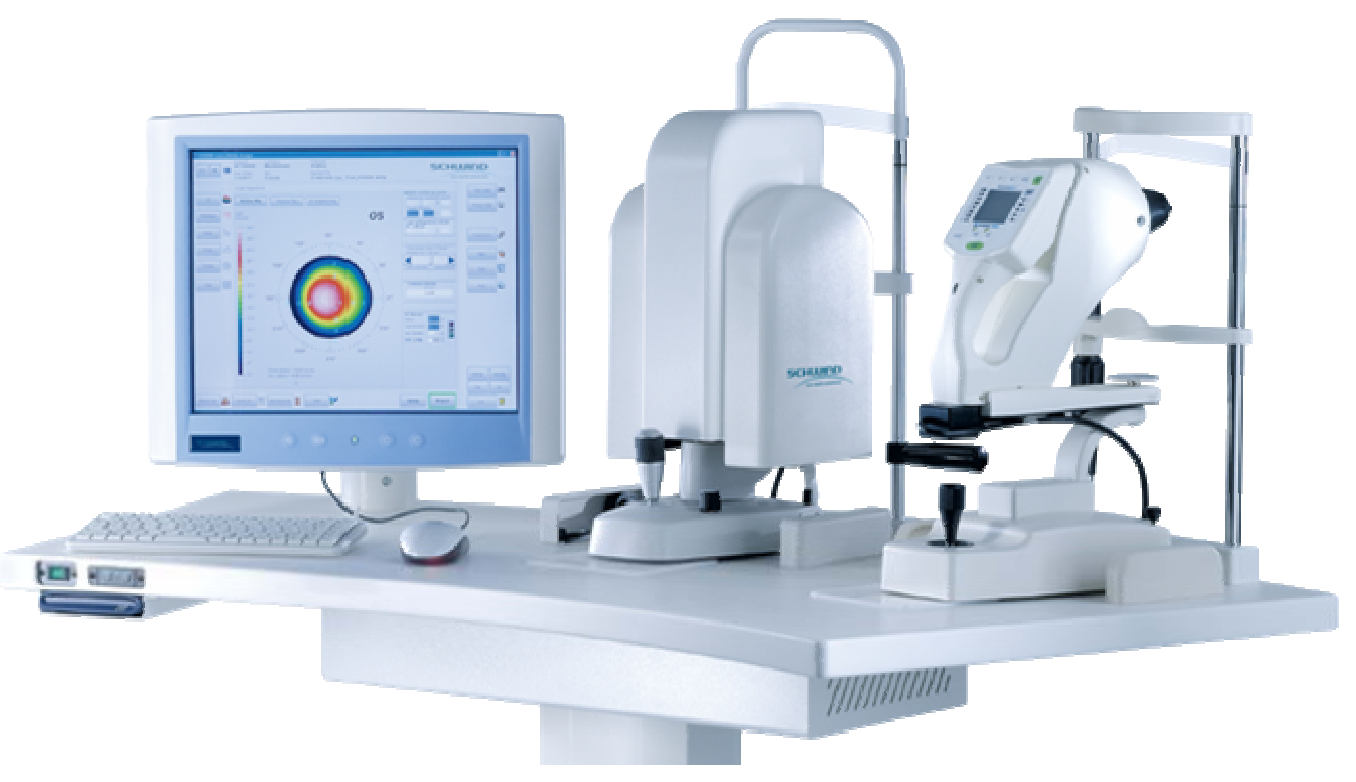

Figure 18: The SCHWIND Combi Workstation for comprehensive corneal and ocular analysis.

LASIK flaps were created with a superior hinge using a Carriazo-Pendular microkeratome ${ }^{154}$ (SCHWIND eye-tech-solutions $\mathrm{GmbH}$, Kleinostheim, Germany) (Figure 19).
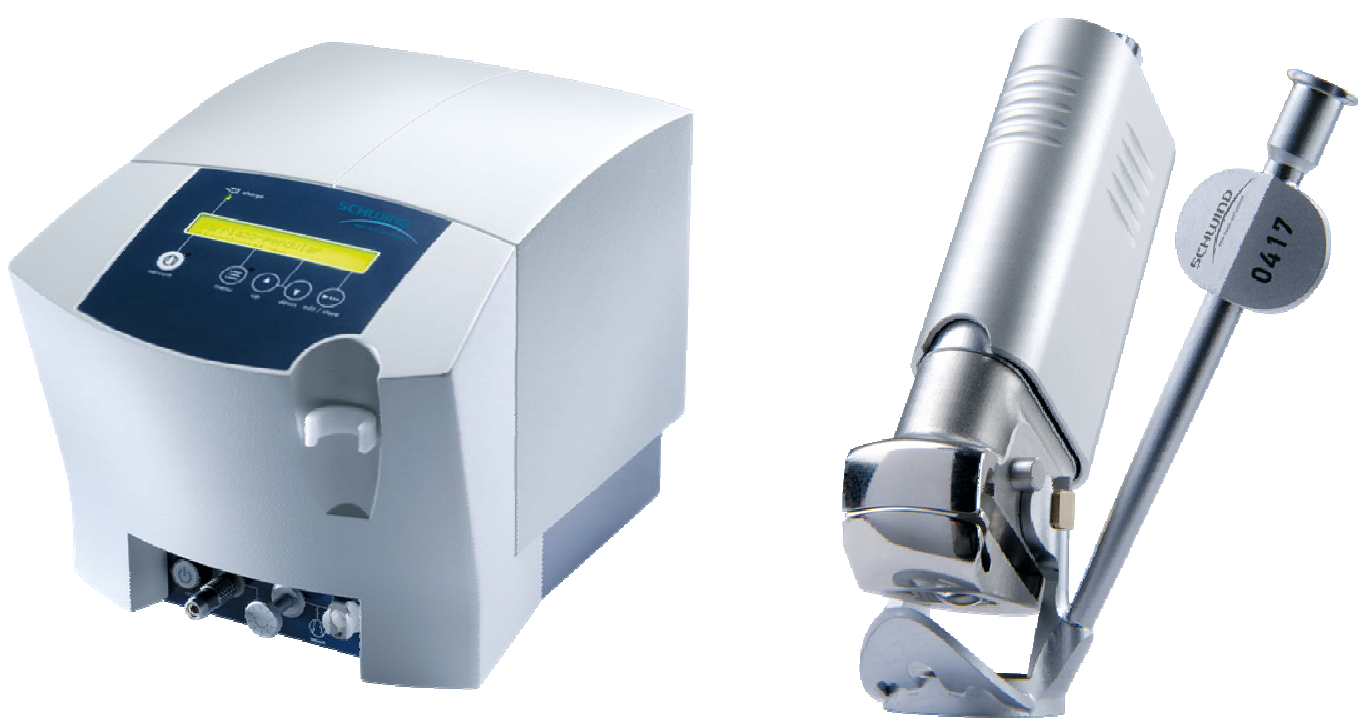

Figure 19: The SCHWIND Carriazo-Pendular Console and Handpiece. 
The CAM software was used to plan the ablations, which were first simulated, ablated onto flat PMMA plates ${ }^{155}$, and finally applied onto the patients corneas with either an AMARIS excimer laser ${ }^{156}$ (Figure 20) or an ESIRIS excimer laser $^{157}$ (Figure 21) (both SCHWIND eye-tech-solutions $\mathrm{GmbH}$, Kleinostheim, Germany) both delivering aspheric wavefront-customised profiles.

The aspheric profiles go beyond the Munnerlyn proposed profiles, and add some aspheric characteristics to balance the induction of spherical aberration (prolateness optimisation).

These particular case of aspheric profiles compensate for the aberrations induction observed with other types of profile definitions ${ }^{158}$, some of those sources of aberrations are those ones related to the loss of efficiency of the laser ablation for non-normal incidence $159,160,161,162$. Optimisation consisted to take into account the loss of efficiency at the periphery of the cornea in relation to the centre as there is a tangential effect of the spot in relation to the curvature of the cornea (Keratometry (K-reading)). The software provides K-reading compensation, which considers the change in spot geometry and reflection losses of ablation efficiency.

Real ablative spot shape (volume) is considered through a selfconstructing algorithm. In addition, there is a randomised flying-spot ablation pattern, and controls the local repetition rates to minimise the thermal load of the treatment (smooth ablation, no risk of thermal damage ${ }^{163}$ ). Therefore, the ablated surface after aspheric wavefront-customised profiles is very smooth, so that there are some benefits in higher order aberrations. 


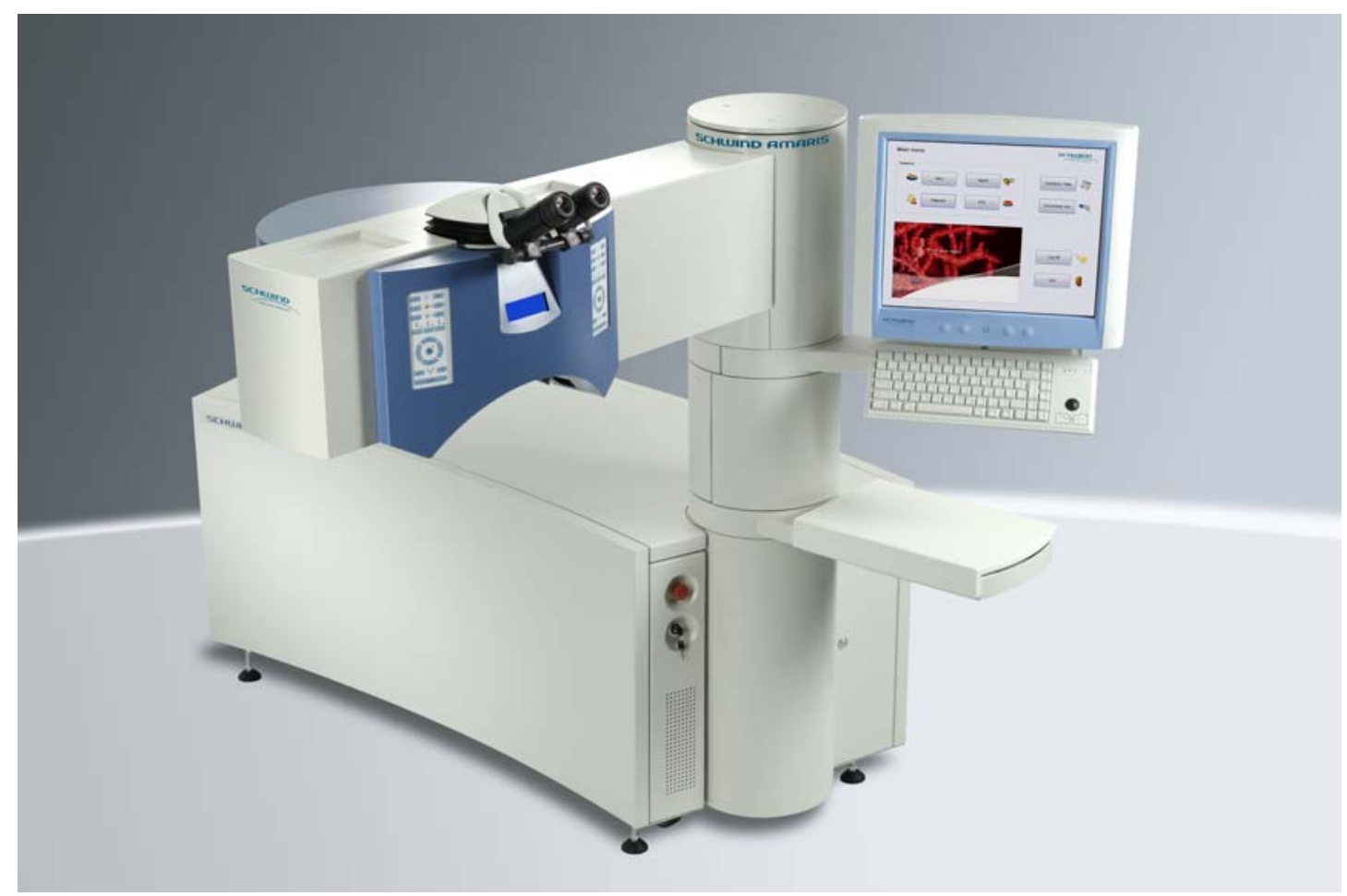

Figure 20: The SCHWIND AMARIS Total-Tech Laser.

The AMARIS laser system works at a repetition rate of $500 \mathrm{~Hz}$, produces a spot size of $0.54 \mathrm{~mm}$ (full width at half maximum (FWHM)) with a superGaussian ablative spot profile ${ }^{164,165}$. High-speed eye-tracking ${ }^{166,167}$ with $1050 \mathrm{~Hz}$ acquisition rate is accomplished with 3-ms latency period ${ }^{168}$. 


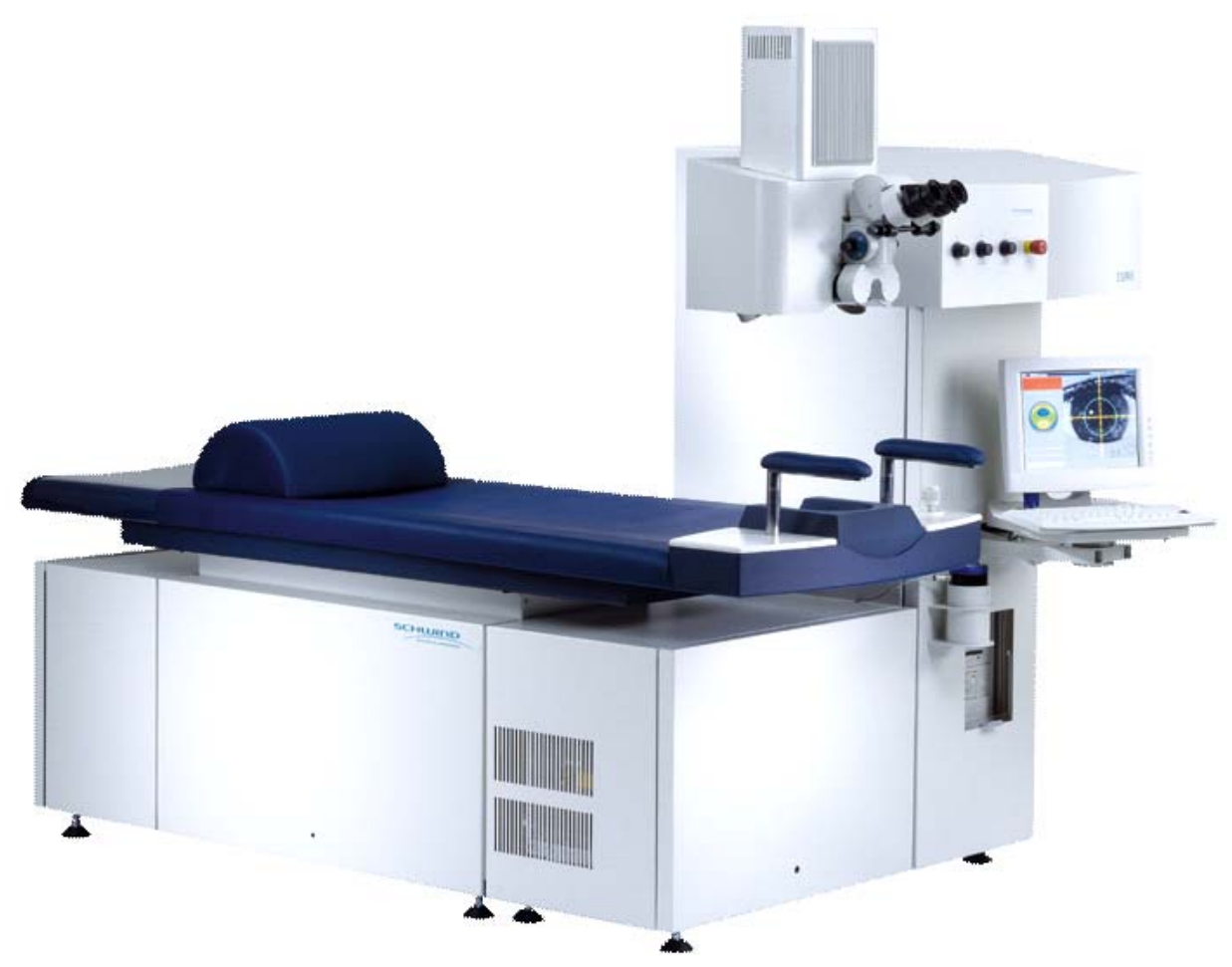

Figure 21: The SCHWIND ESIRIS excimer laser system.

The ESIRIS laser system works at a repetition rate of $200 \mathrm{~Hz}$, produces a spot size of $0.8 \mathrm{~mm}$ (full width at half maximum (FWHM)) with a para-Gaussian ablative spot profile. High-speed eye-tracking with $330 \mathrm{~Hz}$ acquisition rate is accomplished with a 5-ms latency period. 



\title{
Part 3 TOPICAL REVIEW \\ (Revisión temática)
}

\section{Topic A ANALYSIS OF THE CORNEAL ASPHERICITY}

(Análisis de la asfericidad corneal)

\begin{abstract}
AUTHOR CONTRIBUTIONS
Study concept and design (S.A.M.); data collection (D.O.); analysis and interpretation of data (S.A.M.); drafting (D.O., S.A.M.); critical revision (T.M., J.M.); statistical expertise (S.A.M.).
\end{abstract}

Section A.1 ABSTRACT

(Resumen)

PURPOSE: Evaluation of a method to calculate corneal asphericity and asphericity changes after refractive surgery.

METHODS: 60 eyes of 15 consecutive myopic patients and 15 consecutive hyperopic patients ( $n=30$ each) were retrospectively evaluated. Preoperative and 3-month postoperative topographic and corneal wavefront analyses were performed using corneal topography. Ablations were performed using a laser with an aberration-free profile. Topographic changes in asphericity and corneal aberrations were evaluated for a $6 \mathrm{~mm}$ corneal diameter.

RESULTS: The induction of corneal spherical aberrations and asphericity changes correlated with the achieved defocus correction. Preoperatively as well as postoperatively, asphericity calculated from the topography meridians correlated with asphericity calculated from the corneal wavefront in myopic and hyperopic treatments. A stronger correlation between postoperative asphericity and the ideally expected/predicted asphericity could be obtained based on aberration-free assumptions calculated from corneal wavefront values rather than 
from the meridians. In hyperopic treatments, a better correlation could be obtained compared to the correlation in myopic treatments.

CONCLUSIONS: Corneal asphericity calculated from corneal wavefront aberrations represents a three-dimensional fit of the corneal surface; asphericity calculated from the main topographic meridians represents a two-dimensional fit of the principal corneal meridians. Postoperative corneal asphericity can be calculated from corneal wavefront aberrations with higher fidelity than from corneal topography of the principal meridians. Hyperopic treatments showed a greater accuracy than myopic treatments.

\section{Section A.2 INTRODUCTION}

(Introducción)

A strong tendency towards use of asphericity parameters in refractive surgery can be observed ${ }^{169},{ }^{170}$, reporting measurements ${ }^{171},{ }^{172}$, mean values $^{173},{ }^{174},{ }^{175}$, using different descriptors (asphericity quotient [Q], conic constant $[\mathrm{K}]$, eccentricity $[\mathrm{e}]$, $\mathrm{p}$-value $[\mathrm{p}]$, or shape-factor $[E])$ or measuring the effects of refractive treatments on corneal asphericity ${ }^{176},{ }^{177}$.

Analysis of corneal topography involves fitting of the measured data to geometric models, usually by inclusion of a simple regular surface and a polynomial adjustment of the extra components not covered by the simple regular surface basis.

In this study, two simple methods to calculate corneal asphericity - based on corneal wavefront, and based on asphericity of the two principal meridians are compared and the question whether corneal wavefront alone is a useful metric to evaluate the corneal asphericity in refractive surgery is addressed. For this study, the methods presented were applied to a patient population treated with laser in situ keratomileusis (LASIK). 


\section{Section A.3 METHODS}

(Método)

Retrospective analysis of 60 eyes, including 15 consecutive patients each with myopia and hyperopia, treated at Augenzentrum-Recklinghausen was performed. Preoperative and 3-month postoperative data are reported.

All operations were performed by one surgeon (DdO). LASIK flaps were created with a Carriazo-Pendular microkeratome (SCHWIND eye-tech-solutions GmbH, Kleinostheim, Germany). An ESIRIS system (SCHWIND eye-techsolutions $\mathrm{GmbH}$ ) set for an optical zone of $6.25 \mathrm{~mm}$ was used to perform the ablations with aberration-free ${ }^{\mathrm{TM}}$ profiles without nomogram adjustments.

Using the Keratron-Scout (Optikon2000, Rome, Italy), topographical analysis of the radii of curvature and asphericities of the principal meridians and the corneal wavefront aberrations to seventh Zernike order was performed preoperatively and 3 months postoperatively.

Classical relationships between different asphericity descriptors ${ }^{178}$ were calculated using the formulae:

$$
\begin{aligned}
& p \equiv Q+1 \equiv 1-E=1-e^{2} \\
& Q \equiv p-1 \equiv-E=-e^{2} \\
& E \equiv 1-p \equiv-Q=e^{2} \\
& e=\sqrt{1-p} \equiv \sqrt{-Q} \equiv \sqrt{S F} \\
& p<0 \quad \Rightarrow \text { Hyperbola } \\
& p=0 \quad \Rightarrow \text { Parabola }
\end{aligned}
$$

where $0<p<1 \Rightarrow$ Prolate ellipse

$$
\begin{array}{ll}
p=1 & \Rightarrow \text { Sphere } \\
p>1 & \Rightarrow \text { Oblate ellipse }
\end{array}
$$

However, asphericity is a dependent parameter with "non-linear" behaviour, i.e. it has no meaning, if the apical curvature is not taken into consideration. Any asphericity descriptor can be used, however, to obtain consistent results and interpretations, computing cannot be reduced to linear arithmetic.

$p$-value (p) was the asphericity descriptor used throughout the study. 
Topographic asphericity was computed using two methods. The first method was the topographic method based on the principal meridians. Considering the mean corneal asphericity of a series of corneal asphericities, the mean asphericity was computed as

$$
\bar{p}=\frac{\sum_{i=1}^{m} \frac{p_{i}}{R_{i}^{3}}}{\left(\sum_{i=1}^{m} \frac{1}{R_{i}}\right)^{3}} m^{2}
$$

where $\bar{p}$ is the mean asphericity, $\mathrm{p}_{\mathrm{i}}$ the asphericity factors, $\mathrm{R}_{\mathrm{i}}$ the apical radii of curvature, and $\mathrm{m}$ the sample size.

For averaging the asphericity of the two main meridians under consideration of their curvature, equation reduces to:

$$
p=4 \frac{\frac{p_{s}}{R_{s}^{3}}+\frac{p_{f}}{R_{f}^{3}}}{\left(\frac{1}{R_{s}}+\frac{1}{R_{f}}\right)^{3}}
$$

where $p$ is the corneal $p$-value, $p_{s}$ and $p_{f}$ are the $p$-values of the steep and flat principal meridian, respectively, and $R_{s}$ and $R_{f}$ the apical radius of curvature of the steep and flat principal meridian.

This method represents a calculation of mean asphericity derived from $\mathrm{m}$ meridional radii and asphericities obtained from two-dimensional fits of the corneal meridians.

The second method investigated was the corneal wavefront method.

$$
p=\frac{768 R^{3}\left(C_{4}^{0} \sqrt{5}-5 C_{6}^{0} \sqrt{7}+45 C_{8}^{0}\right)}{O Z^{4}(1-n)}
$$

where $p$ is the corneal $p$-value; $C[4,0], C[6,0]$, and $C[8,0]$ are the radiallysymmetric terms of the corneal Zernike expansion; $R$ is the apical radius of the corneal curvature; $n$ the corneal refractive index; and $\mathrm{OZ}$ the analyzed diameter of the corneal Zernike expansion. 
This method represents a calculation of the mean asphericity derived from corneal wavefront data obtained from a three-dimensional fit of the corneal surface. The radial-symmetric terms of the corneal Zernike expansion, C[4,0], $\mathrm{C}[6,0]$, and $\mathrm{C}[8,0]$, were calculated from the radial-symmetric terms of the corneal Zernike expansion of the surface elevation of a Cartesian oval $\left(\mathrm{C}_{\mathrm{co}}[4,0], \mathrm{C}_{\mathrm{co}}[6,0]\right.$, and $\left.\mathrm{C}_{\mathrm{co}}[8,0]\right)$ plus the radial-symmetric terms of the corneal wavefront aberration as provided by the videokeratoscope $\left(C_{c w}[4,0]\right.$ and $\left.C_{c w}[6,0]\right)$.

Also, the ideally expected topographic asphericity assumed from aberration-neutral conditions was calculated using two methods: First, the ideally expected principal meridians of the topographic method

$$
p_{\text {exp }}=p_{c o}+\frac{p_{p r e}-p_{c o}}{\left(1+\frac{R \cdot S E q_{c p}}{n-1}\right)^{3}}
$$

where $p_{\text {exp }}$ is the predicted corneal $p$-value; $p_{c o}$ and $p_{\text {pre }}$ are the $p$-values of a Cartesian oval and the preoperative cornea, respectively; $\mathrm{R}$ is the apical radius of curvature of the preoperative cornea, and $S E q_{c p}$ the spherical equivalent to be corrected at the corneal plane.

In this paper, the term "ideally expected" is understood to mean "predicted values," if the aberration-free condition were strictly fulfilled.

The second method employed was the ideally expected corneal wavefront method, with $\mathrm{R}$ as the postoperative predicted apical radius of curvature.

Note that the ideally expected corneal wavefront method can easily be further applied to any target condition, simply by setting the radial-symmetric terms of the corneal wavefront aberration $\left(\mathrm{C}_{\mathrm{cw}}[4,0]\right.$ and $\left.\mathrm{C}_{\mathrm{cw}}[6,0]\right)$ to the desired value.

Optical errors, represented by wavefront aberrations, were analyzed for 6 $\mathrm{mm}$ diameters. 


\section{A.3.1 Clinical evaluation \\ (Evaluación clínica)}

Each cornea underwent 4 consecutive measurements preoperatively as well as at the 3-month follow-up examination, summing up to a total of 240 measurements. For every cornea, the 4 corresponding topographies were analyzed using both methods, and the corresponding mean value was used as representative asphericity of that cornea with each method.

\section{A.3.2 Repeatability of the methods \\ (Repitibilidad de los métodos)}

Following preoperative calculation of the p-values with both methods, global analysis of the behaviour of the term $p \cdot R^{-3}$ was performed. It constitutes a term to be operated in a simple linear way. The four corresponding values of each cornea were averaged for both methods, and a global standard deviation value was calculated across the 240 measurements for each method using the formula:

$$
S t d D E v_{\text {Global }}=\sqrt{\frac{\sum_{a=1}^{A} \sum_{b=1}^{B}\left(p_{a, b} \cdot R_{a, b}^{-3}-\frac{\sum_{c=1}^{B} p_{a, c} \cdot R_{a, c}^{-3}}{B}\right)^{2}}{A \cdot B-1}}
$$

where a runs over the number of corneas of the sample $(A=60)$, and $b$ and $c$ run over the number of corresponding measurements for each cornea $(B=4)$.

\section{A.3.3 Statistical analysis \\ (Análisis estadístico)}

$t$-tests were used for statistical analysis, with $\mathrm{P}<.05$ being considered significant. 
Section A.4 RESULTS

(Resultados)

\section{A.4.1 Refractive outcomes \\ (Resultados refractivos)}

In both myopic and hyperopic eyes, spherical equivalent (SEq) and cylinder were reduced to subclinical values at 3 months postoperatively (range -0.50 to $+0.75 \mathrm{D}$ for defocus and 0.00 to $0.75 \mathrm{D}$ for astigmatism), and $95 \%$ of eyes ( $n=57$ ) were within $\pm 0.50 \mathrm{D}$ of the attempted correction (Table 2 and Figure 22).

\begin{tabular}{|l|r|r|r|}
\hline & Myopic group & \multicolumn{1}{c|}{$\begin{array}{c}\text { Hyperopic } \\
\text { group }\end{array}$} & \multicolumn{1}{c|}{$\begin{array}{c}\text { All } \\
\text { treatments }\end{array}$} \\
\hline No. of treated eyes (patients) & $30(15)$ & $30(15)$ & $60(30)$ \\
Preoperative SEq \pm StdDev (D) & $-3.18 \pm 1.21$ & $+2.48 \pm 1.41$ & $-0.32 \pm 3.15$ \\
Preoperative cylinder \pm StdDev (D) & $0.50 \pm 0.48$ & $0.65 \pm 0.79$ & $0.58 \pm 0.66$ \\
Postoperative SEq \pm StdDev (D) & $+0.19 \pm 0.28$ & $+0.04 \pm 0.35$ & $+0.11 \pm 0.32$ \\
Postoperative cylinder \pm StdDev (D) & $0.05 \pm 0.08$ & $0.11 \pm 0.15$ & $0.08 \pm 0.13$ \\
Predictability < 0.50 D (\%) & $90 \%$ & $100 \%$ & $95 \%$ \\
Predictability < 1.00 D (\%) & $100 \%$ & $100 \%$ & $100 \%$
\end{tabular}

Table 2: Preoperative and postoperative data.

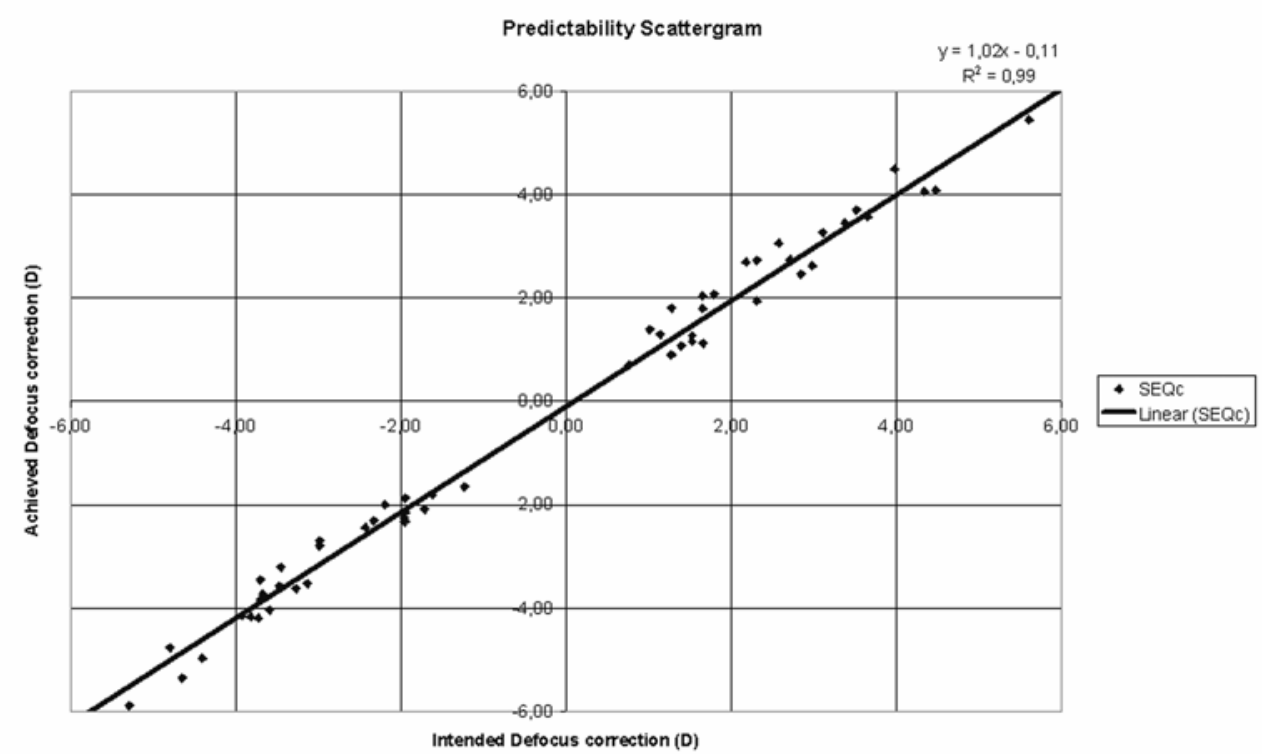

Figure 22: Predictability scattergram. 


\section{A.4.2 Corneal spherical aberrations \\ (Aberración esférica corneal)}

In the myopic group, the preoperative primary corneal spherical aberration $(\mathrm{C}[4,0])$ was $+0.243 \pm 0.098 \mu \mathrm{m}$ (mean \pm standard deviation), and changed to $+0.319 \pm 0.132 \mu \mathrm{m}$ at 3 months postoperatively $(P<.01)$. In the hyperopic group, $\mathrm{C}[4,0]$ was $+0.201 \pm 0.118 \mu \mathrm{m}$ and changed to $-0.006 \pm 0.139 \mu \mathrm{m}$ at 3 months postoperatively $(\mathrm{P}<.001)$ (Table 3$)$.

\begin{tabular}{|l|r|r|r|}
\hline Preoperative primary SphAb $\pm \operatorname{StdDev}(\mu \mathrm{m})$ & Myopic group & Hyperopic group & All treatments \\
Preoperative secondary SphAb $\pm \operatorname{StdDev}(\mu \mathrm{m})$ & $+0.243 \pm 0.098$ & $+0.201 \pm 0.118$ & $+0.221 \pm 0.111$ \\
Postoperative primary SphAb $\pm \operatorname{StdDev}(\mu \mathrm{m})$ & $0.000 \pm 0.003$ & $0.000 \pm 0.002$ & $0.000 \pm 0.002$ \\
Postoperative secondary SphAb $\pm \operatorname{StdDev}(\mu \mathrm{m})$ & $+0.319 \pm 0.132$ & $-0.006 \pm 0.139$ & $+0.154 \pm 0.214$ \\
Induced primary SphAb per diopter $(\mu \mathrm{m})$ & $-0.003 \pm 0.003$ & $-0.004 \pm 0.004$ & $-0.0001 \pm 0.005$ \\
Induced secondary SphAb per diopter $(\mu \mathrm{m})$ & -0.001 & -0.048 & -0.043 \\
& & -0.001 & -0.001
\end{tabular}

Table 3: Corneal wavefront aberration data reported for $6 \mathrm{~mm}$ analysis diameter.

Induced corneal spherical aberration, defined as the difference in postoperative corneal spherical aberration minus the preoperative value, was significant for primary and secondary spherical aberrations $(P<.001$ for both) and significantly correlated with the achieved defocus correction for primary and secondary spherical aberrations $\left(r^{2}=0.65, P<.001\right.$ for primary spherical aberration and $r^{2}=0.59, P<.001$ for secondary spherical aberration, Figure 23). The rates of induced corneal spherical aberration per defocus (regression slope) were $-0.045 \mu \mathrm{m} / \mathrm{D}$ for primary spherical aberration and $-0.001 \mu \mathrm{m} / \mathrm{D}$ for secondary spherical aberration at $6 \mathrm{~mm}$. 


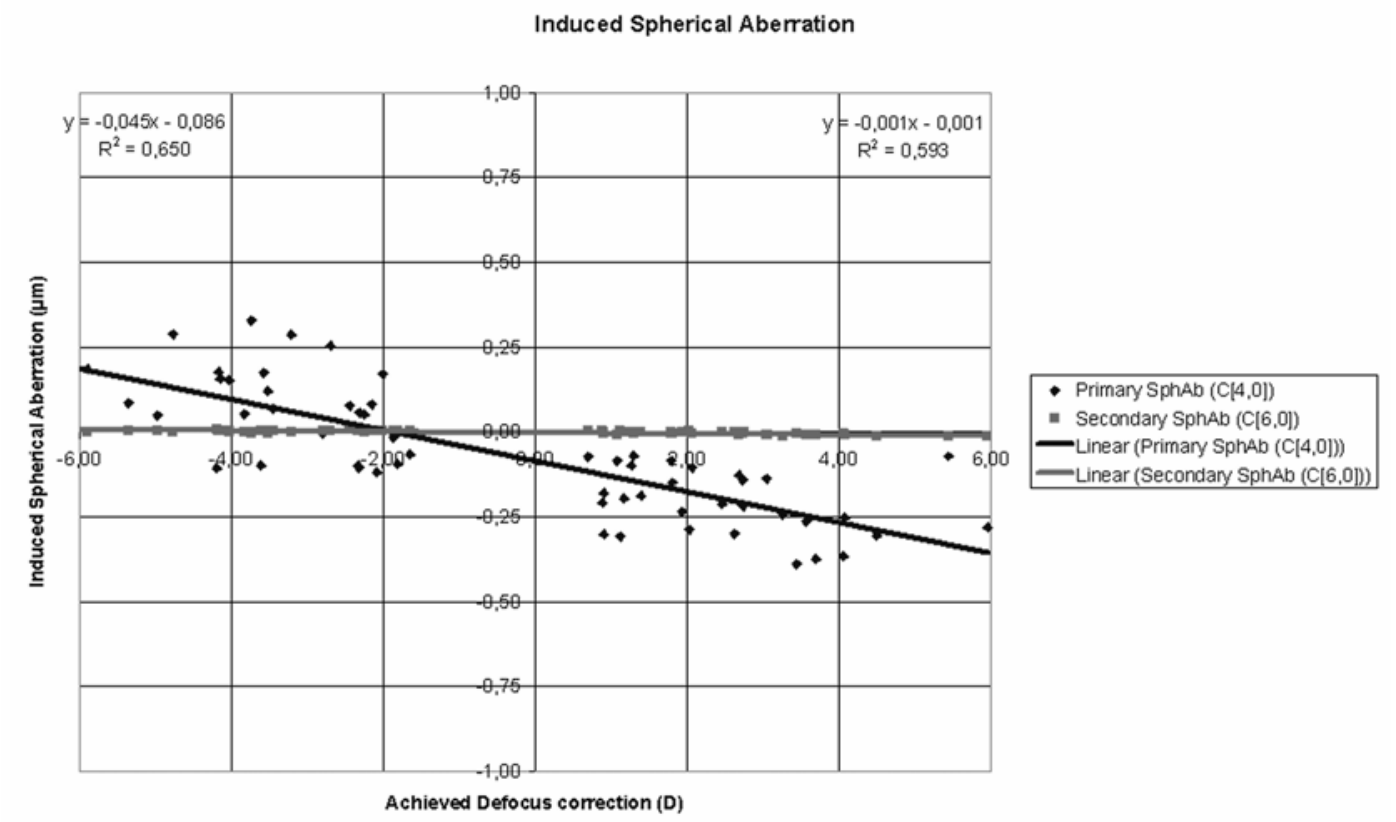

Figure 23: Induced spherical aberration.

\section{A.4.3 Corneal asphericity \\ (Asfericidad corneal)}

In the myopic group, the mean preoperative corneal asphericity calculated from the principal meridians was +0.79 , whereas the mean corneal asphericity calculated from corneal wavefront was +0.89 . In the hyperopic group, the mean preoperative corneal asphericity calculated from the principal meridians was +0.81 , whereas the mean corneal asphericity calculated from corneal wavefront was +0.82 (Table 4).

\begin{tabular}{|l|r|r|r|} 
& \multicolumn{1}{|c|}{$\begin{array}{c}\text { Myopic } \\
\text { group }\end{array}$} & \multicolumn{1}{c|}{$\begin{array}{c}\text { Ayperopic } \\
\text { group }\end{array}$} \\
\hline Preoperative p-value from meridians & +0.79 & +0.81 & +0.80 \\
Preoperative p-value from corneal wavefront & +0.89 & +0.82 & +0.85 \\
Postoperative p-value from meridians & +1.24 & +0.39 & +0.73 \\
Postoperative p-value from corneal wavefront & +1.13 & +0.47 & +0.74 \\
Expected/predicted p-value from meridians & +0.87 & +0.76 & +0.81 \\
Expected/predicted p-value from corneal & & & \\
wavefront & +0.98 & +0.75 & +0.85
\end{tabular}

Table 4: Asphericity data. 
The preoperative corneal asphericity calculated from corneal wavefront significantly correlated with corneal asphericity calculated from the principal meridians in both the myopic and the hyperopic group $\left(r^{2}=0.84, P<.001\right.$ for the myopic group; $r^{2}=0.87, P<.001$ for the hyperopic group, Figure 24). Further, the regression slope was 1.01 for the myopic group and 1.09 for the hyperopic group.

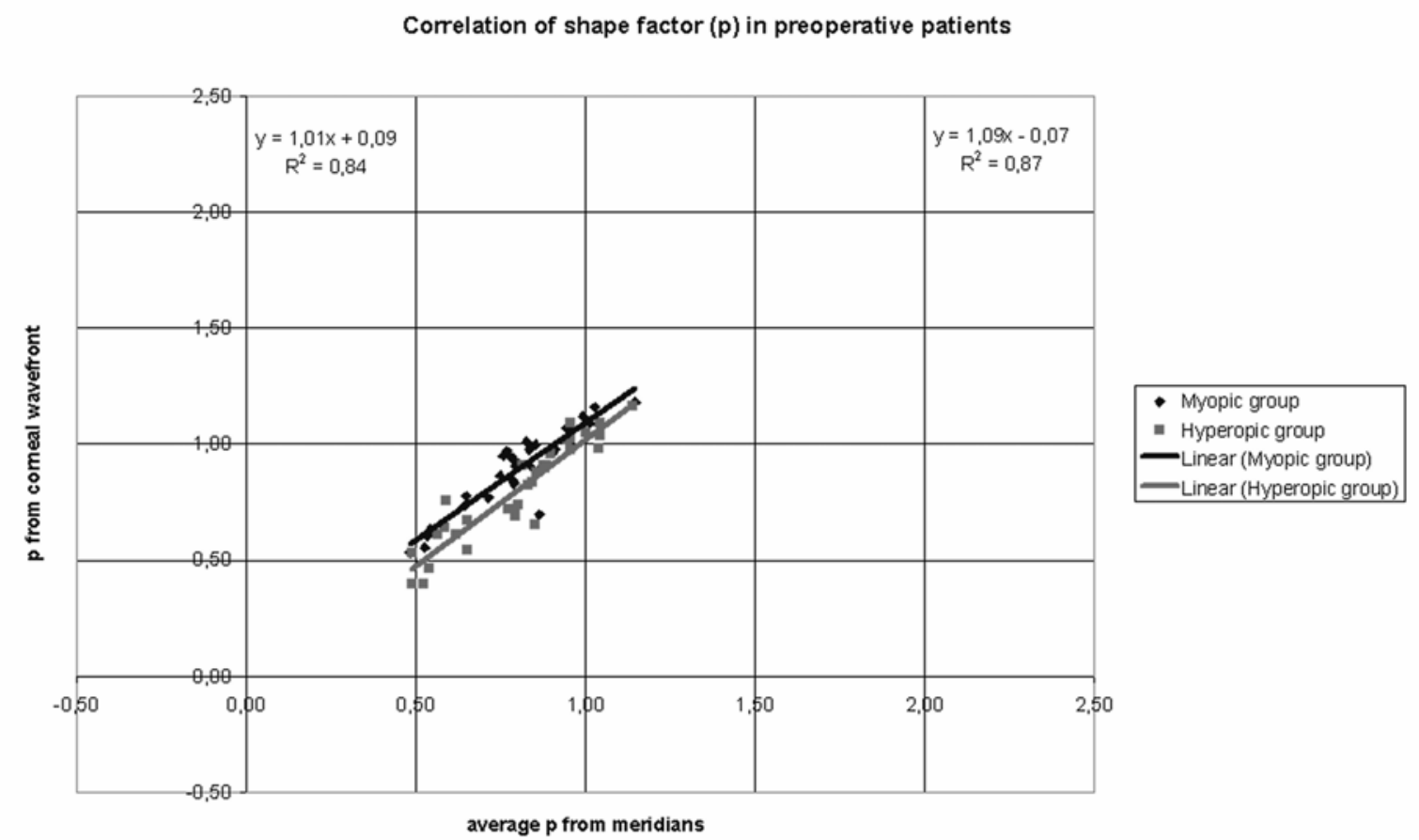

Figure 24: Preoperative asphericity.

In the myopic group, the mean postoperative corneal asphericity calculated from the principal meridians was +1.24 , whereas the mean corneal asphericity calculated from corneal wavefront was +1.13 (Table 4). In the hyperopic group, the mean postoperative corneal asphericity calculated from the principal meridians was +0.39 , whereas the mean corneal asphericity calculated from corneal wavefront was +0.47 (Table 4 ). 
Postoperatively, the corneal asphericity calculated from corneal wavefront values significantly correlated with corneal asphericity calculated from principal meridians in both the myopic and the hyperopic group $\left(r^{2}=0.81, P<.001\right.$ for the myopic group; $r^{2}=0.85, P<.001$ for the hyperopic group, Figure 25). Further, the regression slope was 0.51 for the myopic group and 0.88 for the hyperopic group.

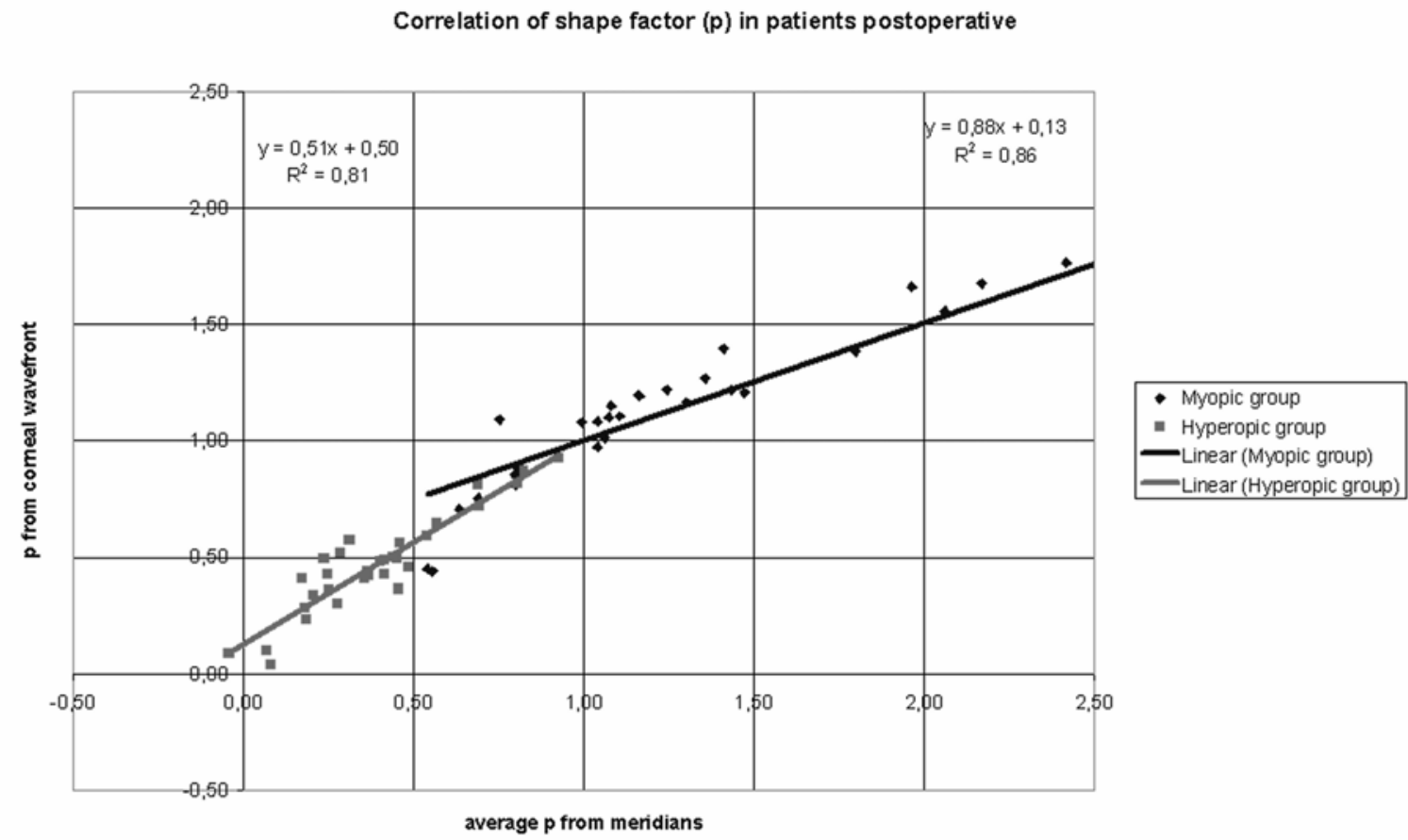

Figure 25: Postoperative asphericity.

For myopia, the ideally expected postoperative $p$-value calculated from the principal meridians was +0.87 , compared to +0.98 in wavefront based calculation (Table 4). The postoperative asphericity did not correlate with the predicted asphericity when calculated from meridians $\left(r^{2}=0.07, P=.2\right)$, and showed a weak but significant correlation with the ideally expected asphericity when calculated from wavefront $\left(r^{2}=0.12, P=.05\right)$. Further, the regression slope was +0.68 in corneal wavefront based calculation. 
For hyperopia, the predicted postoperative asphericity calculated from the principal meridians was +0.76 , compared to +0.75 in wavefront based calculation (Table 4). The postoperative asphericity was significantly correlated with the ideally expected asphericity when calculated from meridians $\left(r^{2}=0.39, P<.001\right)$, and strongly correlated with the predicted asphericity when calculated from wavefront $\left(r^{2}=0.51, P<.001\right)$. Further, the regression slope was +0.67 when calculated from principal meridians and +0.71 when calculated from corneal wavefront.

Combining the results of both groups, the ideally expected postoperative asphericity calculated from the principal meridians was +0.81 and that calculated from corneal wavefront +0.85 . The postoperative asphericity was significantly but weakly correlated with the predicted asphericity when calculated from the principal meridians $\left(r^{2}=0.17, P<.05\right)$, and showed a strong correlation with the ideally expected corneal asphericity when calculated from corneal wavefront $\left(r^{2}=0.37, P<.001\right)$ (Figure 26). Further, the regression slope was +1.44 in principal meridians based calculation and +1.19 in corneal wavefront based calculation.

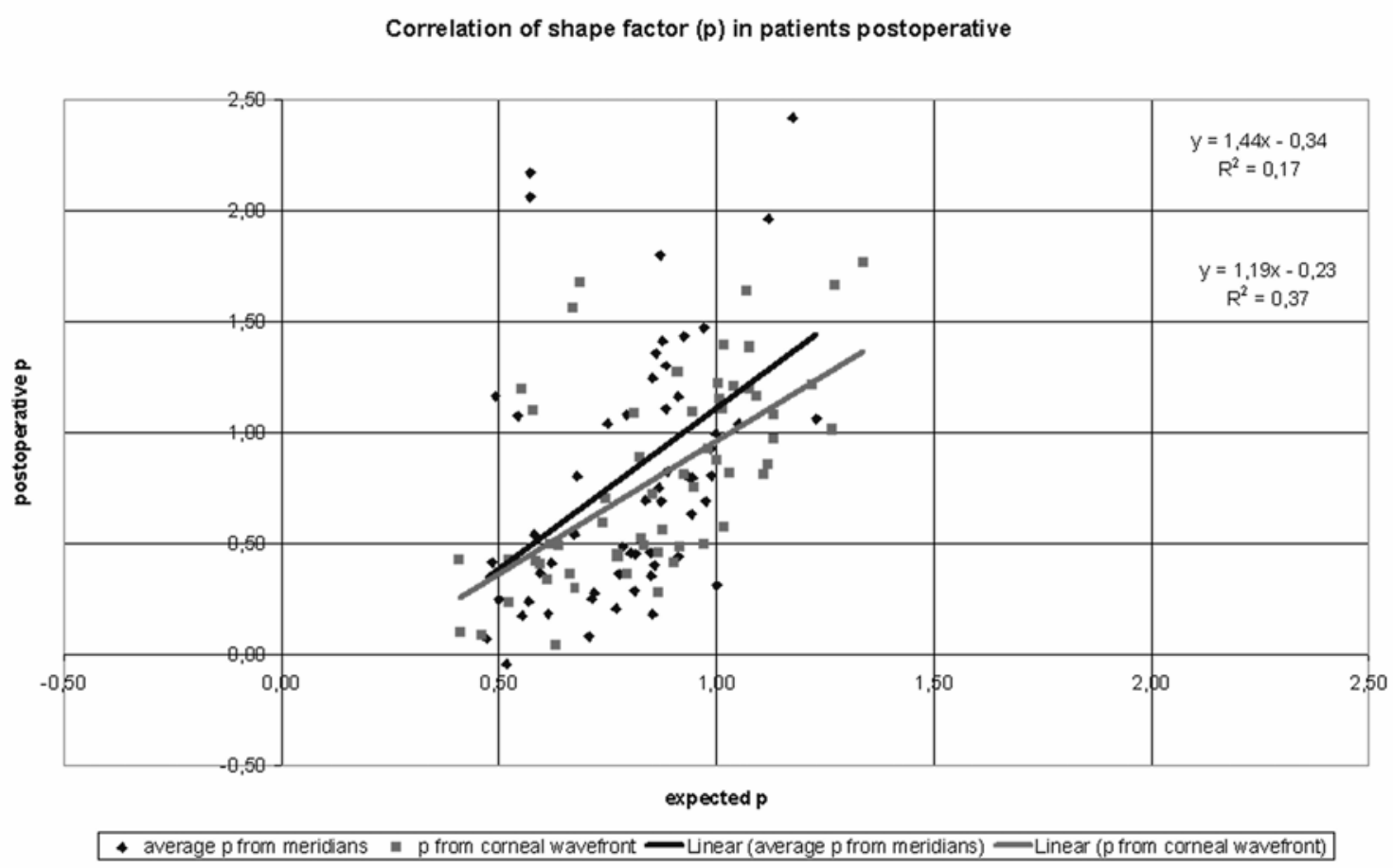

Figure 26: Ideally expected postoperative asphericity. 


\section{A.4.5 Repeatability of the Corneal Asphericity \\ (Repitibilidad de las determinaciones de asfericidad corneal)}

The global standard deviation was $0.0003 \mathrm{~mm}^{-3}$ for the meridional method, compared to $0.0001 \mathrm{~mm}^{-3}$ for the corneal wavefront method $(P<.05)$.

\section{Section A.5 DISCUSSION}

(Discusión)

p-value was the asphericity descriptor used throughout this study. The reason for this choice was not a preference of $p$-value over other asphericity descriptors. In fact, using the identities and equalities described, similar equations could have been derived for any asphericity descriptor. Our aim was the consistent use of one descriptor and to use the classical relationships between descriptors to derive descriptor-specific-equations for the computing of mean values, asphericity out of corneal wavefront, or estimation of the postoperative asphericity, respectively. Note that using simple arithmetic the average of a parabola $(p=0)$ with an apical curvature of $7 \mathrm{~mm}$ and a sphere $(p=1)$ with a radius of curvature of $8 \mathrm{~mm}$ would be $p=0.5$ (i.e. $e=0.71$ ). For the same surfaces, however, an averaged parabola $(e=1)$ and an averaged sphere $(e=0)$ would be $e=0.5$ (i.e. $p=0.75$ ) and not 0.71 . Using our model, the result would always be $\mathrm{p}=0.41$ or $\mathrm{e}=0.77$.

In particular, the corneal wavefront method benefits from avoidance of complicated non-linear effects in the analysis. Once the Zernike expansion of the corneal wavefront aberration is known, corresponding coefficients can be linearly averaged, added, or subtracted, or any other linear operation can be performed, and finally the asphericity value can be computed in the desired descriptor.

By analyzing topographic changes, a highly significant correlation between the asphericity calculated from corneal wavefront and from the principal meridians could be observed in both the myopic and the hyperopic group preoperatively as well as postoperatively. 
To assess the agreement between the methods, a Bland-Altman plot was created $^{179}$ that showed that asphericity calculation with the two methods does not produce equivalent results. Corneal wavefront based calculation showed asphericity with an average of 0.05 units higher compared to calculation based on the principal meridians. Moreover, the difference between the two methods correlated weakly but significantly with the measured value $\left(r^{2}=0.11 ; P<.05\right.$, Figure 27).

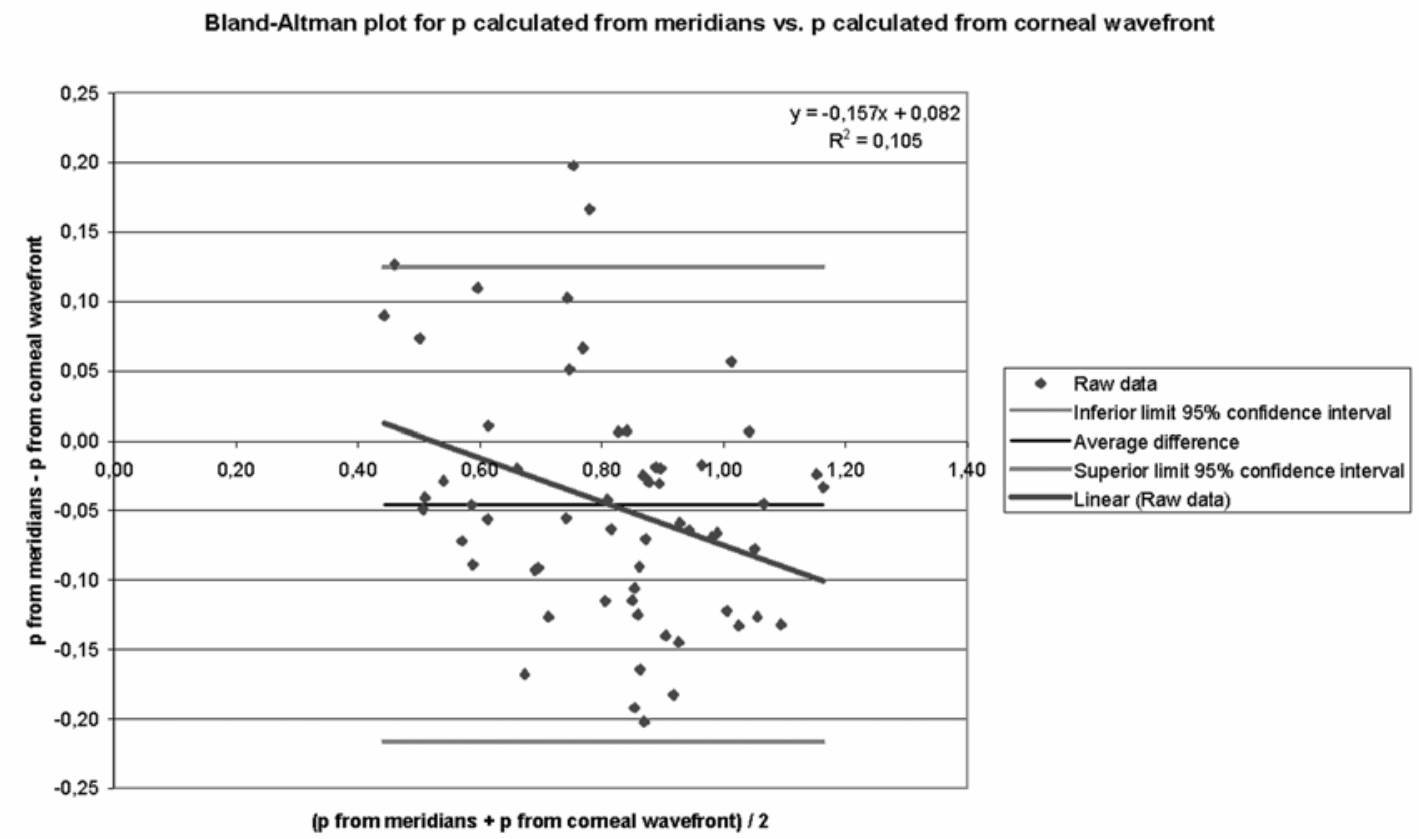

Figure 27: Bland-Altman plot for $p$-value calculated from meridians vs. p-value calculated from corneal wavefront.

The wavefront method proved to be superior to the meridional method, since the aberration coefficients were computed from much denser data sampling (all corneal points within a disk with a $6 \mathrm{~mm}$ diameter), and not only from two meridians. However, the conclusion that if many meridians were included in the "meridional" method, the results would approach those of the "wavefront" method is misleading.

Another weakness of the "two meridians method" is that both meridians are usually selected based upon their respective curvature, i.e. the main origin of astigmatism. These two meridians closely represent the highest and lowest meridional curvature of a cornea, but their corresponding asphericities do not 
necessarily represent the highest and lowest meridional asphericities of that cornea.

In the groups to this study, the postoperative asphericity deviated more from the preoperative asphericity than predicted by aberration-neutral assumptions calculated from the principal meridians as well as corneal wavefront. Also, the postoperative asphericity showed a stronger correlation with asphericity predicted from aberration-neutral assumptions when calculated from corneal wavefront than from the meridians.

The preoperative mean corneal asphericity in myopic eyes calculated with the two methods showed a similar result, which, however, was not as consistent as the result found in the hyperopic group. The fact that both the amount of corneal astigmatism, which was larger in the hyperopic group, as well as the offset between the corneal vertex and the pupil centre, which was also larger in the hyperopic group, may play a role here.

Note that also the Zernike decomposition only predicted $37 \%$ of the variance of asphericity change, i.e. there is high scatter and there is a tendency towards higher asphericity, which is also reflected by the induction of spherical aberrations.

A possible cause of measured differences in induced asphericity between calculated and real postoperative corneas could be the fact that changes in radius and changes in asphericity were analyzed separately. This is only strictly valid, if both parameters are independent, however, there is a very strong correlation between changes in asphericity and changes in radius. This correlation may have two origins: (1) artefacts of the measurement or the fitting procedure, or (2) a real correlation in changes of radius and asphericity in the cornea, possibly due to biomechanical constraints. Similar to Pérez-Escudero et al. ${ }^{180}$ and to the findings of a paper presented earlier by the authors ${ }^{89}$, a topography describing a perfect rotationally symmetric ellipsoid with radius $R=7.87 \mathrm{~mm}$ and asphericity $p=0.75$, which are typical values for the anterior corneal surface, was created. Subsequently, random noise was added to the elevation. Normally distributed random noise with a standard deviation of $3 \mu \mathrm{m}$ was employed, which is the same order of magnitude observed in measurements with the Scout videokeratoscope. This results in a data set similar to the experimental data sets, however, without 
the particularities, which may be specific to our setup. 100 such surfaces were created, using the same base ellipsoid and changing the noise only. Subsequently, this surface was fitted. The results show that the parameters of the base ellipsoid are well recovered by the mean, but that there is a strong correlation between changes in $\mathrm{R}$ and changes in $\mathrm{p}$. The same applies to correlations between changes in $1 / R$ and changes in $p / R^{3}$. These correlations are not particular to our specific fitting procedure, rather are they a general characteristic of fits to surfaces that derive from ellipses. These correlations are an artefact caused by the fit's sensitivity to measurement noise and are probably common to all fits of ellipse-based surfaces. Both the biomechanical response of the stroma and wound healing could contribute to this phenomenon, as well.

Navarro et al. ${ }^{181}$ proposed a relatively simple general model to represent the corneal surface in its canonical form with respect to the axes of corneal symmetry. One limitation of Navarro's model is that it assumes that the orientations of the principal curvatures, i.e. steepest and flattest radii, related to corneal toricity, correspond to the orientations of the principal asphericities. Kiely et al. ${ }^{171}$ investigated this problem in 1982, using a model more general than an ellipsoid, which was oriented according to the instrument axes.

The mean asphericity is a convenient parameter for comparison of different eyes and characterization of spherical aberration of a conicoid, but it cannot be a substitute for corneal topography. There are circumstances where knowledge of the asphericity in the two principle meridians might be more useful for vision correction than the mean asphericity. However, as already mentioned, the asphericity of the two principle meridians might not be the minimum and maximum meridional asphericity for that cornea. In this respect, Navarro's corneal model presents a good basis for corneal topography, representing a realistic anatomic situation and employing additional terms of Zernike expansion to describe extra surface deformation of real corneas. Zernike terms would resolve the issue, with the strongest asphericity not being along the principal meridians. On the other hand, the quadratic surface basis for the corneal surface will only provide an aberration free basis with the instrument on axis and will not be as realistic as Navarro's ellipsoid. As a consequence, the quadratic surface 
will require larger additional Zernike terms to represent the real corneal topography.

Corneal description should not be limited to the mean asphericity, related to spherical aberration, when corneal topography in Zernike terms gives much more general information on corneal aberrations. However, if a simple corneal model based on asphericity is of interest for reasons of simplicity, we advocate for calculation of the mean asphericity from the corneal wavefront rather than from the asphericity of the two principle meridians. This simplification is less complicated but essentially similar to reduction of the wavefront aberration map to a generic description based on $\mathrm{n}$ weight coefficients of the Zernike expansion. This approach is no attempt to discredit the full details of corneal topography or the optical description provided by Zernike polynomials. Rather is the aim to reduce the complexity of the description to an appropriate minimal set of parameters $^{182},{ }^{183}$.

In particular cases, spherical aberration could be described by way of comparison of the Zernike terms with radial symmetry, such as $C[4,0]$ and $C[6,0]$, to be more accurate, the contribution from the power terms with pure $\rho^{4}$ and $\rho^{6}$ in the corneal topography expansion ( $\rho$ - normalized pupil radius). In this way, a higher-order aspheric surface could be characterized rather than limiting analysis to the mean asphericity that corresponds to a conicoid surface, which in some cases is a poor approximation for high-order aspheric corneas.

Another possible model, which is also direct and simple and combines the advantages of different other models is that of a quadric surface free on the space, i.e. oriented according to the natural corneal axes, however, with a fixed constant asphericity corresponding to the Cartesian oval for the refractive index ( $p$-value of +0.472 with a corneal refractive index of 1.376), without astigmatism, to determine the apical curvature and the corneal axis. The modelled surface would always be a surface free of on-axis aberrations for any particular apical curvature. The residual component would be adjusted to a Zernike polynomial expansion, because it would directly represent the surface aberration of the corneal wavefront. 


\section{Section A.6 CONCLUSIONS}

(Conclusiones)

This study suggests that the corneal wavefront alone is a useful metric to evaluate the optical quality of an ablation in refractive surgery, and a useful metric to evaluate corneal asphericity. Corneal wavefront can be used effectively to analyze laser refractive surgery, avoiding complicated non-linear effects in the analysis. On these grounds, this method has the potential to replace or perhaps supplement currently used methods of asphericity analysis based on simple averaging of asphericity values.

\section{Section A.7 OUTLOOK}

(Perspectiva)

In this study we have used corneal wave aberration as a basis for the determination of corneal asphericity. However, as the OSA recommends, corneal wave aberration was based on the line of sight. Thus, larger offsets between pupil centre and corneal vertex may have negatively affected the power of the correlations. In further studies, we will include the offsets between pupil centre and corneal vertex for improving the accuracy of the method.

This chapter was limited to a laser system (and ablation algorithm). In further studies, newer state-of-the-art laser systems and algorithms will be evaluated as well. 


\title{
Topic B MODEL OF AN ABERRATION-FREE PROFILE
}

(Modelo de un perfil libre de aberraciones)

\begin{abstract}
AUTHOR CONTRIBUTIONS
Study concept and design (S.A.M.); data collection (D.P., C.V., D.O., J.G.); analysis and interpretation of data (S.A.M.); drafting (D.O., S.A.M.); critical revision (J.L.A., M.C.A., H.B., T.M., J.G.); statistical expertise (S.A.M.).
\end{abstract}

\section{Section B.1 ABSTRACT}

(Resumen)

Purpose: To provide a model of an aberration-free profile and to clinically evaluate the impact of treatments based upon these theoretical profiles in the post-op cornea and to evaluate the clinical outcomes of treatments using the optimized Aberration-Free ablation profiles of the ESIRIS and AMARIS platforms comparing the outcomes of ablations based on the normal corneal vertex and the pupil centre, as well as to compare the induced corneal wavefront aberration using the aspheric aberration neutral ablation profile versus a classical Munnerlyn standard profile.

Methods: Aberration-free profiles were deducted from the Zernike expansion of the difference between two corneal Cartesian-ovals. Compensation for the focusshift effects of removing corneal tissue were incorporated by preserving the location of the optical focus of the anterior corneal surface. Simulation of the surgical performance of the profile was performed by simulated ray-tracing through a cornea described by its anterior surface and pachymetry. Two groups (using normal corneal vertex and using the pupil centre) with pupillary offset $>200$ microns were compared. Clinical outcomes were evaluated in terms of predictability, refractive outcome, safety, and wavefront aberration. OD/OS bilateral symmetry was evaluated in terms of corneal wavefront aberration. 
Results: The proposed „aberration-free” profiles theoretically preserve aberrations, becoming more oblate asphericity after myopic treatments, and more prolate after hyperopic ones. Induced corneal aberrations at 6-mm were below clinically relevant levels: $0.061 \pm 0.129 \mu \mathrm{m}$ for HO-RMS ( $p<.001), 0.058 \pm 0.128 \mu \mathrm{m}$ for spherical aberration $(p<.001)$ and $0.053 \pm 0.128 \mu \mathrm{m}$ for coma $(p<.01)$, whereas the rate of induced aberrations per achieved $D$ of correction were $-0.042 \mu \mathrm{m} / \mathrm{D}$, $0.031 \mu \mathrm{m} / \mathrm{D}$, and $-0.030 \mu \mathrm{m} / \mathrm{D}$ for HO-RMS, SphAb, and coma (all $p<.001$ ). No other Zernike mode was significantly correlated. Induction of positive asphericity correlated to achieved correction $(p<.001)$ at a rate $3 x$ theoretical prediction. $38 \%$ of the CV eyes improved BSCVA compared with $24 \%$ of the PC eyes (comparison $\mathrm{CV} / \mathrm{PC} \mathrm{P}=0.38$ ). Induced ocular coma was on average 0.17 micron for the $\mathrm{CV}$ group and 0.26 micron for the $P C$ group (comparison $C V / P C P=0.01$ favouring $\mathrm{CV}$ ). Induced ocular spherical aberration was on average +0.01 micron for the $\mathrm{CV}$ group and +0.07 micron for the $\mathrm{PC}$ group (comparison CV/PC $\mathrm{P}=0.05$ favouring $\mathrm{CV}$ ). At $6.00 \mathrm{~mm}$, corneal aberrations changed in a higher amount after Munnerlyn based profiles than after aspheric aberration neutral profiles.

Conclusions: "Aberration-free" patterns for refractive surgery as defined here together with consideration of other sources of aberrations such as blending zones, eye-tracking, and corneal biomechanics yielded results comparable to those of customisation approaches. CV-centred treatments performed better in terms of induced ocular aberrations and asphericity, but both centrations were identical in terms of photopic visual acuity. Aberration-Free Treatments with the SCHWIND AMARIS did not induce clinically significant aberrations, maintained the global OD-vs.-OS bilateral symmetry, as well as the bilateral symmetry between corresponding Zernike terms (which influences binocular summation). The induced corneal aberrations were less than compared with the classical profile or other publications. Having close-to-ideal profiles should improve clinical outcomes decreasing the need for nomograms, and diminishing induced aberrations after surgery. 


\section{Section B.2 INTRODUCTION}

(Introducción)

Previous studies have shown that spherical aberration shows a consistent increase after excimer laser ablation directly proportional to the achieved refractive correction. It has been suggested that almost half of the induced spherical aberration is due to the lower delivery of excimer laser energy in the peripheral cornea due to corneal curvature.

The recent advances in excimer laser technology, such has the use of aspheric ablation profiles, incorporation of HOAb treatment and eye trackers have presumably led to better refractive outcomes and reduced HOAb induction postoperatively that have been recently reported ${ }^{184,185}$.

Ocular wavefront-guided and wavefront-optimized treatments can increase HOAb by $100 \%$ postoperatively ${ }^{184}$. A significant number of refractive surgery patients may not benefit from ocular wavefront guided treatment as the induction of $\mathrm{HOAb}$ is related to baseline levels of HOAb ${ }^{184,186}$. For example, HOAb tend to be induced in patients with less than $0.30 \mu \mathrm{m}$ and reduced in patients with greater than $0.30 \mu \mathrm{m}$ of HOAb. ${ }^{184,186}$ Furthermore, physiologic optical aberrations may be warranted to maintain the optical quality of the eye. ${ }^{73,187}$ Based on these studies, ${ }^{184,186,187}$ it seems the custom ablation algorithm may not be appropriate for the entire refractive surgery population.

Mclellan and colleagues have reported a beneficial effect on the visual quality of pre-existing higher order aberrations ${ }^{187}$. There is evidence of neural adaption to the baseline wavefront profile ${ }^{69,72,73}$. The interaction between higher order aberrations can be beneficial to visual quality regardless of the magnitude $\mathrm{HOAb}^{188,189}$. Furthermore, higher order aberrations seem to be induced in patients with $0.30 \mu \mathrm{m}$ or less of preoperative $\mathrm{HOAb}^{184,186}$. Approximately half the patients that present for refractive surgery have HOAb of $0.30 \mu \mathrm{m}$ or less ${ }^{184}$. To date, the induction of wavefront aberrations postoperatively is random and the wavefront profile postoperatively cannot be predicted. Based on the random nature of the HOAb induction and current research, it maybe beneficial to maintain the preoperative wavefront profile for a significant number of refractive surgery candidates. 
Excimer laser refractive surgery has evolved from simple myopic ablations ${ }^{15}$ to the most sophisticated topography-guided ${ }^{56}$ and wavefront-driven ${ }^{37}$, either using wavefront measurements of the whole eye ${ }^{38}$ (obtained, e.g., by Hartman-Shack wavefront sensors ${ }^{46}$ ) or by using corneal topography-derived wavefront analyses ${ }^{54}$, customised ablation patterns ${ }^{80}$. Because the corneal ablations for refractive surgery treatments induce aberrations (one of the most significant side-effects in myopic LASIK is the induction of spherical aberration, which causes halos and reduced contrast sensitivity), special ablation patterns were designed to preserve the preoperative level of high order aberrations. Not to forget the fact that astigmatism (especially high ones) has its main origin in the anterior corneal surface, and topographically is usually found located 2-fold symmetrically from the normal corneal vertex and not at the pupil centre. Patient satisfaction in any refractive surgery, wavefront-guided or not, is primarily dependent on successful treatment of the lower order aberrations (LOA) of the eye (sphere and cylinder). Achieving accurate clinical outcomes and reducing the likelihood of a retreatment procedure are major goals of refractive surgery.

Section B.3 METHODS

(Método)

\section{B.3.1 Theoretical aberration-free profile}

(Perfil teóricamente libre de aberraciones)

We generated a rotationally symmetric cornea satisfying Baker's equation (1943) for conicoids:

$$
r^{2}+p z^{2}-2 z R=0
$$

where, $z$-axis is the axis of revolution, $r$ the radial distance to the corneal vertex, $p$ the aspherical factor, and $R$ the apical radius of curvature. $p$ represents how fast the surface deviates from a paraboloid, while the quotient of asphericity $(Q)$ represents how fast the surface deviates from a sphere. The relationship between them both is simply:

$$
Q \equiv p-1
$$


We expressed this 3-dimensional surface in terms of Zernike polynomials ${ }^{36}$, where $Z[j, k]$ are the Zernike polynomials, $C[j, k]$ their coefficients, $\mathrm{OZ}$ the optical zone (i.e. the physical size of the unit disc) and $\mathrm{n}$ the corneal refractive index:

$$
\begin{aligned}
& C_{8}^{0}=\frac{(1-n)}{5376} R p^{3}\left(\frac{O Z}{2 R}\right)^{8} \\
& C_{6}^{0} \sqrt{7}=\frac{(1-n)}{320} R p^{2}\left(\frac{O Z}{2 R}\right)^{6}+21 C_{8}^{0} \\
& C_{4}^{0} \sqrt{5}=\frac{(1-n)}{48} R p\left(\frac{O Z}{2 R}\right)^{4}+5 C_{6}^{0} \sqrt{7}-45 C_{8}^{0} \\
& C_{2}^{0} \sqrt{3}=\frac{(1-n)}{4} R\left(\frac{O Z}{2 R}\right)^{2}+3 C_{4}^{0} \sqrt{5}-6 C_{6}^{0} \sqrt{7}+30 C_{8}^{0}
\end{aligned}
$$

These equations were deducted by identification in a "term-by-term of the same radial order" fashion. Meaning that, if a higher (or lower) order for the Taylor's expansion were used, the obtained set of equations would have been slightly different by including more (or less) terms. Given the orthogonal properties of Zernike polynomials, inner-products between the conic and Zernike functions may as well be used to correlate conic parameters $(R, Q)$ with Zernike coefficients $(\mathrm{C}[\mathrm{j}, 0])$.

A Cartesian-oval (an aspherical surface with Q-factor $-1 / \mathrm{n}^{2}$ ) results from the condition of stigmatism and represents the free-of-aberrations surface for the infinity-point (far-point in a person). If the anterior corneal surface were a Cartesian-oval it would have no aberrations. The anterior cornea is typically different from this shape and posses its own aberration pattern, but the optical aberrations of a cornea can be calculated as:

$$
C_{\text {CornealWavefrontaberration }}[j, k]=C_{\text {CartesianOval }}[j, k]-C_{\text {Cornea }}[j, k]
$$

From that, a preoperative cornea with Q-factor $-1 / n^{2}(\sim-0.528$ for corneal refractive index of 1.376 ) will not manifest "corneal-wavefront-aberration," and also a postoperative cornea with Q-factor -0.528 will not manifest "cornealwavefront-aberration." Following this analysis, a treatment whose application on 
a Cartesian-oval cornea would result in a new Cartesian-oval cornea with different dioptric power defines the „aberration-free” profile:

$$
z=\frac{n^{2} R}{n^{2}-1}\left(\frac{(n-1) R}{n-1+D_{C} R}-1+\sqrt{1-\frac{n^{2}-1}{n^{2}}\left(\frac{r}{R}\right)^{2}}-\sqrt{\left(\frac{(n-1) R}{n-1+D_{C} R}\right)^{2}-\frac{n^{2}-1}{n^{2}}\left(\frac{r}{R}\right)^{2}}\right)(
$$

where $D_{C}$ is refractive power change.

Such profile is expected to fulfill the "aberration-free" condition:

$$
\begin{array}{ll}
H_{O W F A} b_{\text {Post }}(\rho, \theta)=H O W F A b_{\text {Pre }}(\rho, \theta) & \forall \rho, \theta \in O Z \\
\Delta H O W F A b(\rho, \theta)=0 & \forall \rho, \theta \in O Z
\end{array}
$$

where HOWFAb means High-order Wavefront-aberration.

\section{B.3.2 Compensation for the focus shift}

(Compensación del desplazamiento del foco)

The approach to theoretically balance the focus-shift due to tissue removal bases on preserving the location of the optical focus of the anterior corneal surface after removing the tissue.

Corneal refractive power is given by:

$$
P_{A C S}=\frac{n_{\text {Cornea }}-n_{\text {Air }}}{R_{A C S}}
$$

where $\mathrm{P}_{\mathrm{ACS}}$ is the refractive power of the anterior corneal surface, $\mathrm{R}_{A C S}$ the radius of curvature of the anterior corneal surface, $\mathrm{n}_{\text {cornea }}$ the refractive index of the cornea, and $\mathrm{n}_{\text {Air }}$ the refractive index of air.

Preserving the location of the optical focus of the anterior corneal surface after removing tissue means:

$$
F_{\text {CorneaPostAblation }}=F_{\text {ACS }}-Z_{\text {Ablation }}
$$

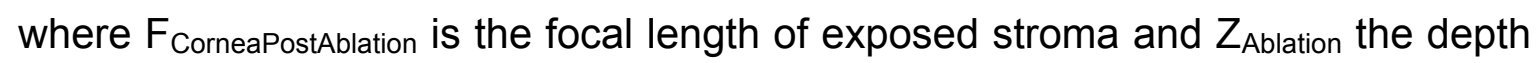
of ablation. 
This way, concerning refractive surgery and considering only the amount of tissue removed but not the corneal biomechanics, or wound healing, and assuming that the refractive index remains constant throughout the procedure and at the postoperative stage (i.e. $n_{\text {Cornea, preoperative }}=n_{\text {Cornea, postoperative }}=n_{\text {Cornea }}$ ), the approximate refractive shift due only to tissue removal corresponds to combining all equations into one:

$$
P_{\text {CorneaPostAblation }}-P_{A C S}=\frac{P_{A C S} \cdot n_{\text {Cornea }}}{\left(n_{\text {Cornea }}-n_{\text {Air }} P_{A C S} Z_{\text {Ablation }}\right)}-P_{A C S}
$$

where $\mathrm{P}_{\text {CorneaPostAblation }}$ is the refractive power of the exposed stroma.

\section{B.3.3 Optical simulation \\ (Simulaciones ópticas)}

The "aberration-free" profile as proposed here is built on a single surface eye model whose anterior surface does not induce spherical aberration. The fact that the actual cornea consists of a double surface optic whose posterior surface partly balances the effect of its anterior surface raises the question whether implementing such profile would result in excessive negative spherical aberration in a real eye. For this reason, we simulated ray-tracing through a cornea described by its anterior surface $(\mathrm{Ra}, \mathrm{Qa})$, and its pachymetry. We took 1 as refractive index for air, 1.376 for cornea, and 1.336 for aqueous humour.

For the pachymetry, we selected a simple radially symmetric model that approaches the mean pachymetry of the human population, by defining a parabolic increasing pachymetry:

$$
\operatorname{Pachy}(r)=\text { CPachy }+(\text { PPachy }- \text { CPachy })\left(\frac{r}{5}\right)^{2}
$$

where CPachy represents the central pachymetry and PPachy the pachymetry at $5 \mathrm{~mm}$ radial distance.

We consider the cornea (the added contributions of anterior and posterior surfaces) as the only element in the optical system, and we consider optical effects of changing its anterior surface. 
An „aberration-free” profile as defined here is only valid in a theoretical frame and/or for educational purposes, if other inherent sources of aberrations such as biomechanical reactions due to the flap cut or to the ablation process itself, blending zones, loss of efficiency considerations, spot-size limitations, or active eye-tracking capabilities are not accounted for.

\section{B.3.4 Clinical evaluation (Evaluación clínica)}

A total of 250 eyes (125 patients) were consecutively treated the using ORK-CAM „Aberration neutral“ Aspheric ablation profiles and retrospectively analysed. Three-months follow up was available in 232 of these eyes (93\%), and their preoperative data were as follows: mean sphere $-3.64 \pm 1.03 \mathrm{D}$ (range, 0 to $9.25 \mathrm{D}$ ); mean cylinder $-0.97 \pm 0.87 \mathrm{D}$ (range, 0 to $-3.00 \mathrm{D}$ ); mean spherical equivalent refraction $-4.12 \pm 2.26 \mathrm{D}$ (range, -0.37 to $-9.50 \mathrm{D}$ ). In all eyes, we measured corneal topography and derived corneal wavefront analyses (Keratron Scout, Optikon2000 S.p.A., Rome, Italy), ocular wavefront with a high resolution Hartmann-Shack sensor (ORK-Wavefront Analyzer, SCHWIND eye-techsolutions, Kleinostheim, Germany), manifest refraction, and uncorrected and best spectacle-corrected Snellen visual acuity. Measurements were performed preoperatively and at one and three months after surgery.

A $6.5 \mathrm{~mm}$ central fully corrected ablation zone was used in all eyes with a variable transition size automatically provided by the laser related to the planned refractive correction $(6.7 \mathrm{~mm}$ to $8.2 \mathrm{~mm})$.

Since we have available corneal wavefront information, not limited by the pupil boundaries, we have reported the topographic wavefront findings for 6,7 and $8 \mathrm{~mm}$ diameter zones, to include the total treatment zone and transition zone and junction zone, as well. 


\section{B.3.5 Ablation centre \\ (Centrado de la ablación)}

Thirty five patients (53 eyes) seeking laser correction at the Muscat Eye Laser Center, Sultanate of Oman, were enrolled for this analysis. The patients were divided into two myopic astigmatism groups. In the CV group (24 eyes, 16 patients, 8 patients with both eyes enrolled in the study and 8 patients with only 1 eye enrolled); the ablation was centred using the pupillary offset, i.e., the distance between the pupil centre and the normal CV measured by videokeratoscopy (Keratron Scout topographer, Optikon2000 s.p.a., Rome, Italy). The measurement was performed under photopic conditions of 1,500 lux, similar to the conditions under the operating microscope. The excimer laser allows for modification of the ablation centration from the pupillary centre with an offset by entering either $X$ and $Y$ Cartesian values or $R$ and $\theta$ polar values in a regular treatment. The measurement of the pupillary offset was translated into the treatment planning as polar coordinates to be manually entered in the excimer laser computer.

In the PC group (29 eyes, 19 patients, 10 patients with both eyes enrolled in the study and 9 patients with only 1 eye enrolled), the ablation was centred using the pupil centre as observed by the eye-tracking module.

Eyes were enrolled in the study groups only if they had no symptomatic aberrations $(<0.65 \mu \mathrm{m}$ root mean square HOAb measured by the Ocular Wavefront Analyzer and the Optikon Keratron Scout for $6.00 \mathrm{~mm}$ analysis diameter (<0.50 DEq)) and moderate-to-large pupillary offset (>200 microns). Patients were randomly assigned to the $\mathrm{CV}$ or $\mathrm{PC}$ centration groups based on a coin toss. In the patients with only one eye fulfilling the enrolling criteria, both eyes were treated with the randomly assigned centration method, but only eye was included for analysis.

The exclusion criteria included unstable refraction during the previous 6 months; signs of keratoconus or abnormal corneal topography; collagen vascular, autoimmune or immunodeficiency diseases; severe local infective or allergic conditions; severe dry eye disease; monocularity or severe amblyopia; or cataracts. 
To determine the ablation profile of the CAM, the manifest refraction was measured in each eye and cross checked with the objective refraction from the SCHWIND Ocular Wavefront Analyzer. Preoperative topography and aberrometry measurements were taken, and the VA and mesopic pupil size (SCHWIND Ocular Wavefront Analyzer) were measured. In both groups, we used an optical zone of 6.50 millimetres with a variable transition zone provided automatically by the software in relation to the planned refraction.

In all cases, one surgeon (MCA) performed all standard LASIK procedures at the Muscat Eye Laser Center. Immediately before the ablation, the laser was calibrated according to the manufacturer's instructions and the calibration settings were recorded.

The manifest refraction, VA, topography, and aberrometry measurements were recorded for each eye at 1, 3, and 6 months and 1 year postoperatively.

At the preoperative stage, as well as, at any of the follow-ups after the treatments, the pupillary offset was measured directly at the topographical map displayed by the videokeratoscope, and corresponds to the distance between the pupil centre under photopic conditions of 1,500 lux and the normal CV.

In particular, we analysed the possible correlations between induced ocular aberrations with defocus correction and with pupillary offset.

As the used profiles are aspherical based aiming for effects "neutral for aberration," correlations between induced ocular spherical aberration and defocus assess how close (or how far) the profiles are from the targeted neutral effect when centred according to the different references, whereas correlations between induced ocular coma aberration and defocus assess whether the profiles suffer from a systematic decentration (a spherical aberration analysed off-axis results in coma aberration) when referred according to different points.

For statistical analysis, paired $t$-tests were used to compare postoperative vs. preoperative results within each group, and unpaired $t$-tests were used to compare results between groups. For correlation tests, the Coefficient of Determination $\left(r^{2}\right)$ was used and the significance of the correlations has been evaluated using p-values calculated considering a metric distributed 
approximately as $\mathrm{t}$ with $\mathrm{N}-2$ degrees of freedom where $\mathrm{N}$ is the size of the sample. For all test, $P<0.05$ was considered statistically significant.

\section{B.3.6 Comparison to Munnerlyn based profiles \\ (Comparación con perfiles directamente basados en Munnerlyn)}

For this comparison, we retrospectively analyzed 2 consecutive groups, 70 eyes each, treated for myopia and myopic astigmatism with LASIK technique. One group was treated for myopic LASIK with a classical Munnerlyn standard profile and compared to the first 70 eyes treated for myopic LASIK using the aspheric aberration neutral (Aberration-Free ${ }^{\mathrm{TM}}$ ) profile.

We analyzed the visual outcome, the corneal wavefront aberration and the topographical changes of these two consecutive groups of eyes after 3 months. All patients were examined preoperatively and 1 day, 1 week, 1 month, and 3 months postoperatively.

In the case of the classical standard profile we used an own nomogram calculated from previously treated eyes, which had resulted in some undercorrection. In the case of the aspheric aberration neutral (AberrationFree $^{\mathrm{TM}}$ ) profile, we did not use any nomogram.

For statistical analysis, unpaired t-tests were used to test statistical differences with $p$ values of less than 0.05 being considered statistically significant.

\section{B.3.7 Bilateral symmetry \\ (Simetría bilateral)}

For the evaluation of the influence of the ablation profile on the bilateral symmetry, 50 eyes (25 patients) that had been treated with the AMARIS „aberration neutral“ (Aberration-Free ${ }^{\mathrm{TM}}$ ) aspheric ablation profiles were retrospectively analysed.

Inclusion criteria for review were bilateral surgery on the same day targeted for emmetropia, preoperative best spectacle corrected visual acuity (BCVA) $\geq$ 
$20 / 25$ (logMAR $\leq 0.1)$ in both eyes, no signs of amblyopia, and successful completion of the 6-month follow-up.

Six-months follow-up data were available for all 50 eyes $(100 \%)$, and their preoperative data were as follows: mean manifest defocus refraction: $-2.47 \pm$ $2.51 \mathrm{D}$ (range, -8.13 to $+5.63 \mathrm{D}$ ) and mean manifest astigmatism magnitude: $2.02 \pm 0.91 \mathrm{D}$ (range, 0.00 to $4.75 \mathrm{D}$ ). For all eyes, we measured corneal topography and derived corneal wavefront aberrations up to the $7^{\text {th }}$ Zernike order (36 terms) (Keratron-Scout, OPTIKON2000, Rome, Italy). Measurements were performed preoperatively and also 1,3 , and 6 months after surgery.

A $6.5 \mathrm{~mm}$ central and fully corrected ablation zone was used in all eyes, together with a variable transition size that was automatically provided by the laser depending on the planned refractive correction $(6.7 \mathrm{~mm}$ to $8.9 \mathrm{~mm})$.

\section{B.3.7.1 Correlations for bilateral symmetry of Zernike terms across subjects (Correlaciones de la simetría bilateral para los términos de Zernike)}

To test this hypothesis, we plotted left-vs.-right-eye scatter graphs for each Zernike term to analyse the predicted correlations between the two eyes. These plots reveal, for our sample, which Zernike modes show symmetry and which type of symmetry they show (pre- and postoperatively). What is expected is that 0 modes show even symmetry; -odd modes show even symmetry; -even modes show odd symmetry; +odd modes show odd symmetry and +even modes show even symmetry. The slope and intercept of the linear regression (least-square fitting) were calculated for each Zernike term up to the seventh radial order (36 coefficients). We assessed the statistical significance of the correlations using Student's T-test; the Coefficient of Determination $\left(\mathrm{r}^{2}\right)$ was also employed and the significance of the correlations has been evaluated assuming a metric that is distributed approximately as $\mathrm{t}$ with $\mathrm{N}-2$ degrees of freedom, where $\mathrm{N}$ is the size of the sample. 
B.3.7.2 Correlations for symmetry of aberrations in right and left eye of the same subjects

(Correlaciones de la simetría interocular de los sujetos)

Taking symmetry into account, we plotted for each subject left-vs.-right eye scatter graphs of the Zernike coefficients. These plots reveal which patients in our sample show symmetry (pre- and postoperatively). The slope and intercept of the linear regression (least-square fitting) were calculated for each subject (25 patients). We assessed the statistical significance of the correlations using Student's T-tests; the Coefficient of Determination $\left(r^{2}\right)$ was also employed and the significance of the correlations has been evaluated assuming a metric that is distributed approximately as $\mathrm{t}$ with $\mathrm{N}-2$ degrees of freedom, where $\mathrm{N}$ is the size of the sample.

B.3.7.3 Differences for symmetry of aberrations in right and left eye of the same subjects

(Diferencias en la simetría interocular de los sujetos)

Taking symmetry into account, we compared the Zernike coefficients obtained for the left and right eyes of the same subjects. We assessed the statistical significance using paired Student's T-tests.

B.3.7.4 Dioptrical differences in corneal wavefront aberration between the right and left eyes of the same subjects

(Diferencias dióptricas interoculares de la aberración del frente de onda corneal de los sujetos)

For our analysis, the concept of equivalent defocus (DEQ) has been used as metric to be able to associate a dioptric power with the RMS of the Zernike coefficients.

We have set a threshold value of $0.25 \mathrm{D}$ to establish whether or not the differential corneal wavefront aberration between the left and the right eye was clinically relevant. 
B.3.7.5 Changes in bilateral symmetry of Zernike terms as a result of refractive surgery

(Cambios en la simetría bilateral de términos de Zernike provocados por la cirugía refractiva)

We analysed the number of Zernike terms that postoperatively lost, gained or preserved symmetry, compared to the preoperative baseline.

B.3.7.6 Changes in bilateral symmetry of wavefront aberration as a result of refractive surgery

(Cambios en la simetría bilateral interocular provocados por la cirugía refractiva)

We analysed the number of patients that postoperatively lost, gained or preserved symmetry, compared to the preoperative baseline.

Statistical analysis

(Análisis estadístico)

The level of statistical significance was taken to be $p<0.05$.

Section B.4 RESULTS

(Resultados)

B.4.1

Simulation of the surgical performance of the profile

(Simulación del rendimiento quirúrgico del perfil)

From Figure 28 to Figure 30, the previously defined „aberration-free” profile effectively preserves existing aberrations, even when a wide range of anterior and posterior corneal surfaces are considered, whereas "asphericity preserving" profiles induce aberrations. The graphs were obtained by simulated ray-tracing of the preoperative and postoperative corneas and calculation of the difference. 

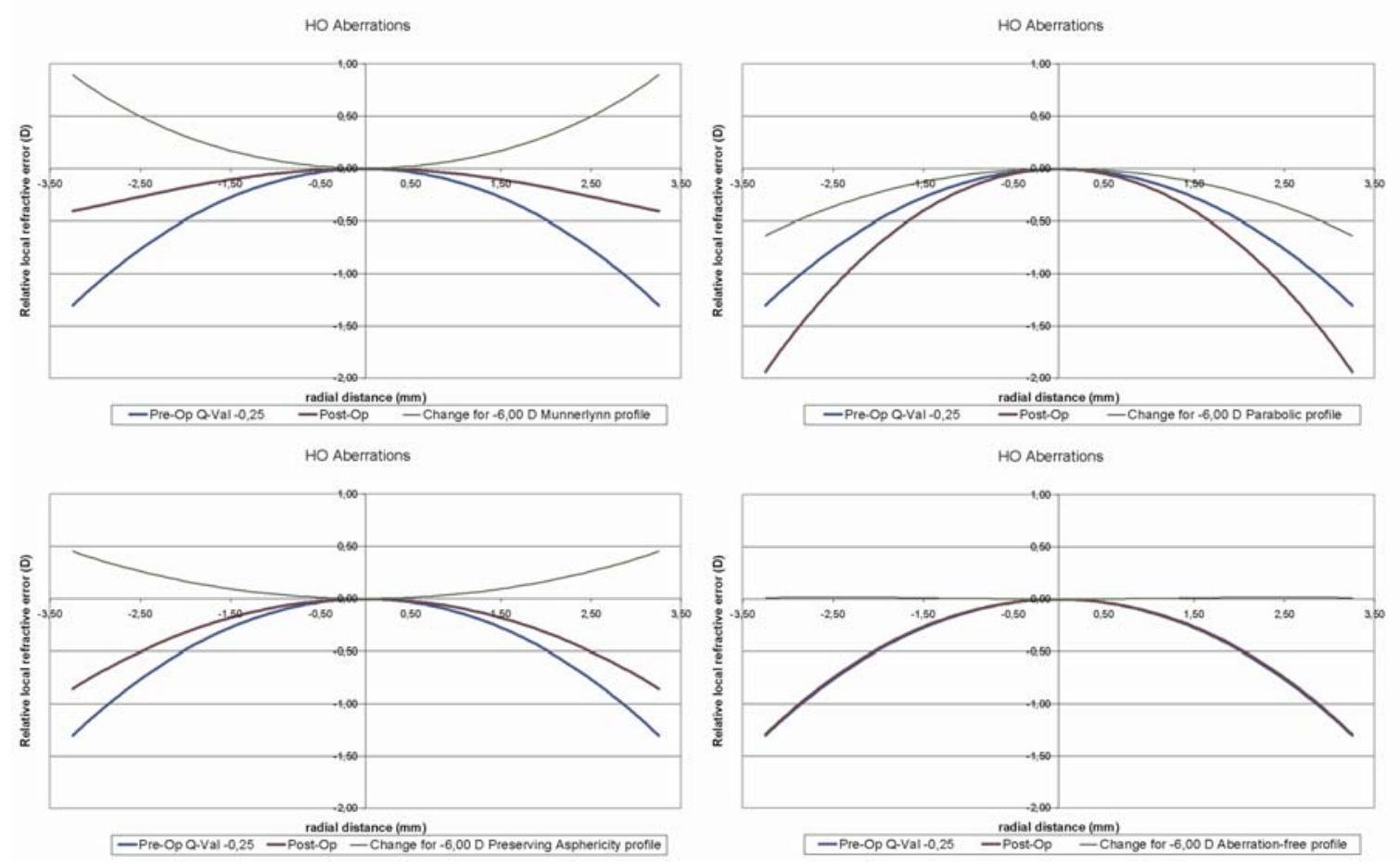

Figure 28: Analysis of the induced aberration at $6.50 \mathrm{~mm}$ for $-6.00 \mathrm{D}$ for a balanced corneal model, for 4 different ablation profiles: A) Munnerlyn based profiles, B) Parabolic based profiles, C) Asphericity preserving profiles, D) Aberration-free profiles. Notice the pre-op aberrated status (in blue), the post-op aberrated status (in red), and the induced aberrations (in green). Note the multifocality range ( $x$-axis) running from $-2 \mathrm{D}$ to $+1 \mathrm{D}$ in all graphs.

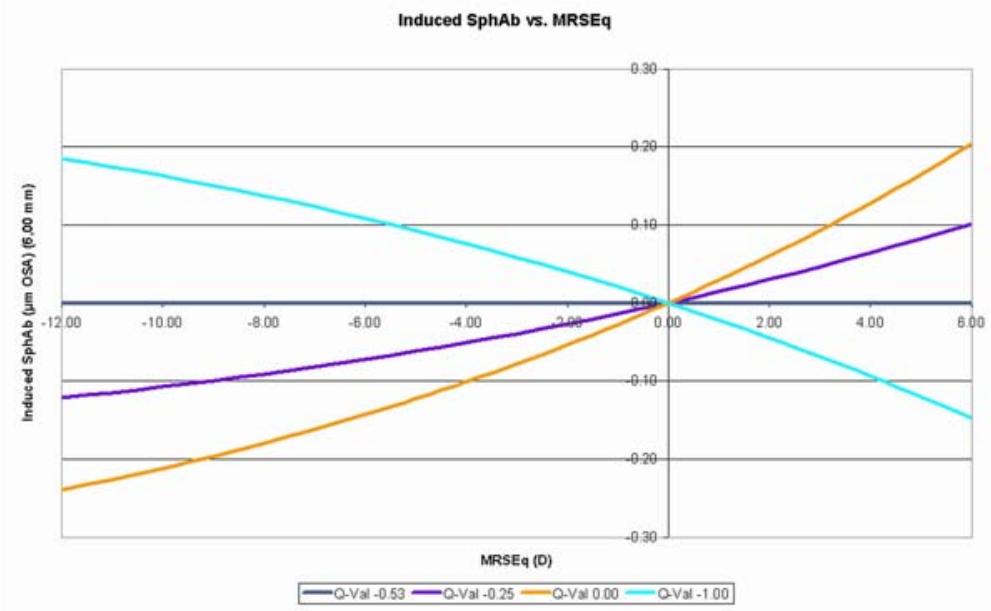

Figure 29: Theoretical analysis of the induced corneal spherical aberration analyzed at $6 \mathrm{~mm}$ vs. refractive power change (MRSEq) for 4 different asphericities: "Free-of-aberrations" cornea (Q-Val -0.53, in blue), balanced-eye 
model cornea (Q-Val -0.25, in magenta), spherical cornea (Q-Val 0.00, in yellow), parabolic cornea (Q-Val -1.00, in cyan).
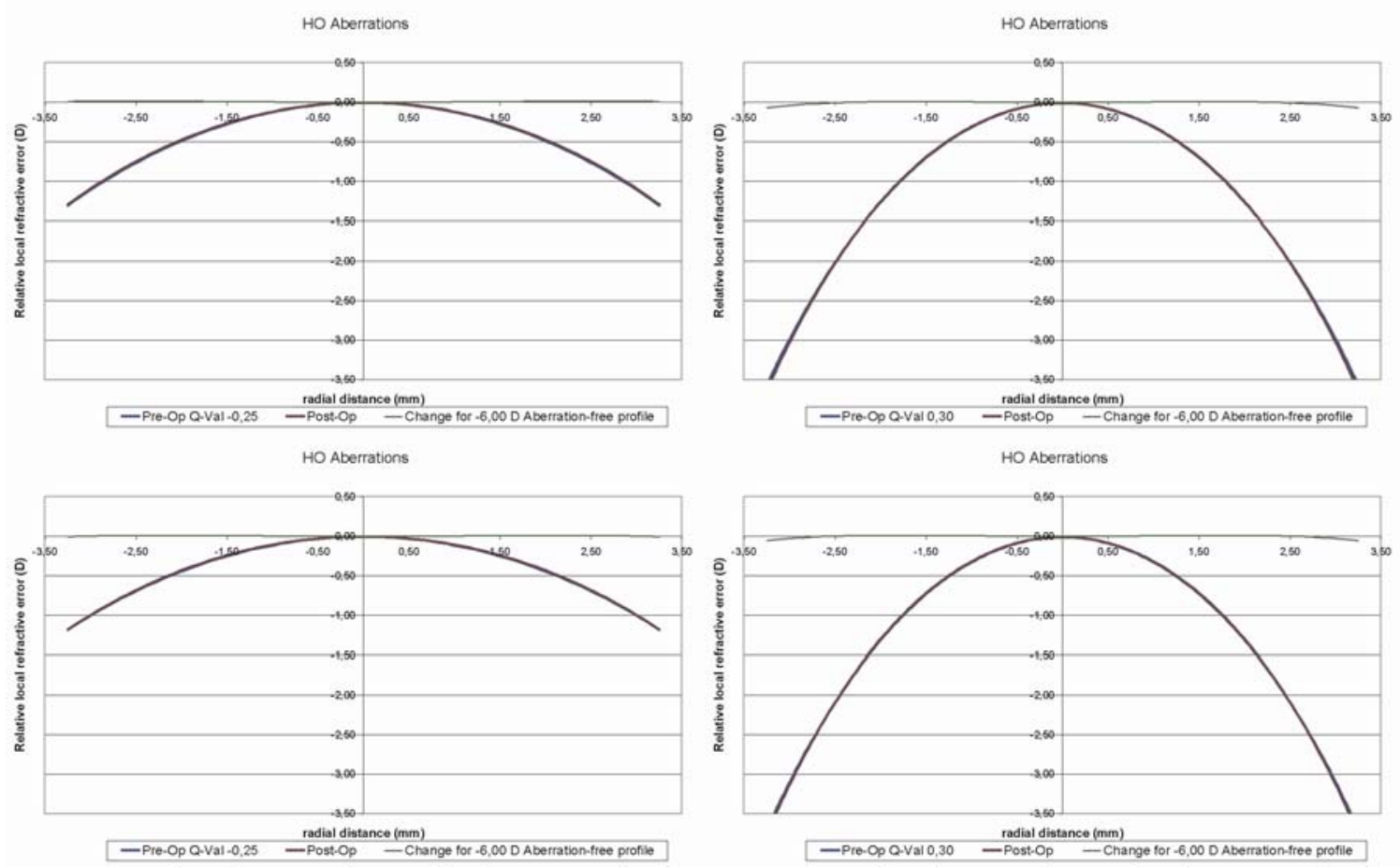

Figure 30: Theoretical analysis of the induced corneal spherical aberration at $6.50 \mathrm{~mm}$ using Aberration-Free profiles for $-6.00 \mathrm{D}$ for 2 anterior corneal surfaces: $7.87 \mathrm{~mm}, \mathrm{Q}$-factor -0.25 (A and C); and $7.87 \mathrm{~mm}, \mathrm{Q}$-factor +0.30 (B and D); and for 2 posterior corneal surfaces and pachymetries: $525 \mu \mathrm{m}$ central pachymetry, $775 \mu \mathrm{m}$ peripheral pachymetry at $5 \mathrm{~mm}$ radial distance (A and $\mathrm{B}$ ); and $550 \mu \mathrm{m}$ central pachymetry, $550 \mu \mathrm{m}$ peripheral pachymetry at $5 \mathrm{~mm}$ radial distance $(\mathrm{C}$ and D). Notice the pre-op aberrated status (in blue), the post-op aberrated status (in red), and the induced aberrations (in green). Note the multifocality range ( $x$ axis) running from $-3.5 \mathrm{D}$ to $+0.5 \mathrm{D}$ in all graphs.

\section{B.4.2 Clinical evaluation (Evaluación clínica)}

We have included 232 treatments for this evaluation, all of them without adverse events. At three months, mean manifest spherical equivalent was $0.10 \pm 0.33 \mathrm{D}$ (range, +0.86 to $-1.18 \mathrm{D}$ ) and mean cylinder $0.23 \pm 0.26 \mathrm{D}$ (range, 0 to 
1.50 D). Eighty-eight percent eyes (202) were within $\pm 0.50 \mathrm{D}$ of attempted correction.

Preoperatively, mean ocular spherical aberration was $+0.03 \pm 0.11 \mu \mathrm{m}$ (range -0.19 to +0.25 ) and corneal spherical aberration was $+0.33 \pm 0.10 \mu \mathrm{m}$ (range +0.14 to +0.52 ). Postoperatively, the values were $+0.07 \pm 0.16 \mu \mathrm{m}$ (range 0.25 to +0.38$)$ for ocular spherical aberration $(P<0.0001)$, and $+0.40 \pm 0.13 \mu \mathrm{m}$ (range +0.14 to +0.67$)$ for corneal spherical aberration $(P<0.0001)$. Ocular spherical aberration increased on average by $0.028 \mu \mathrm{m}$ per dioptre of achieved defocus correction for a $6-\mathrm{mm}$ pupil $(P<0.0001)$ and $0.030 \mu \mathrm{m}$ per dioptre of achieved defocus correction for corneal spherical aberration $(P<0.0001)$, the difference between both measurements was not statistically significant $(P=0.47)$ (Figure 31).

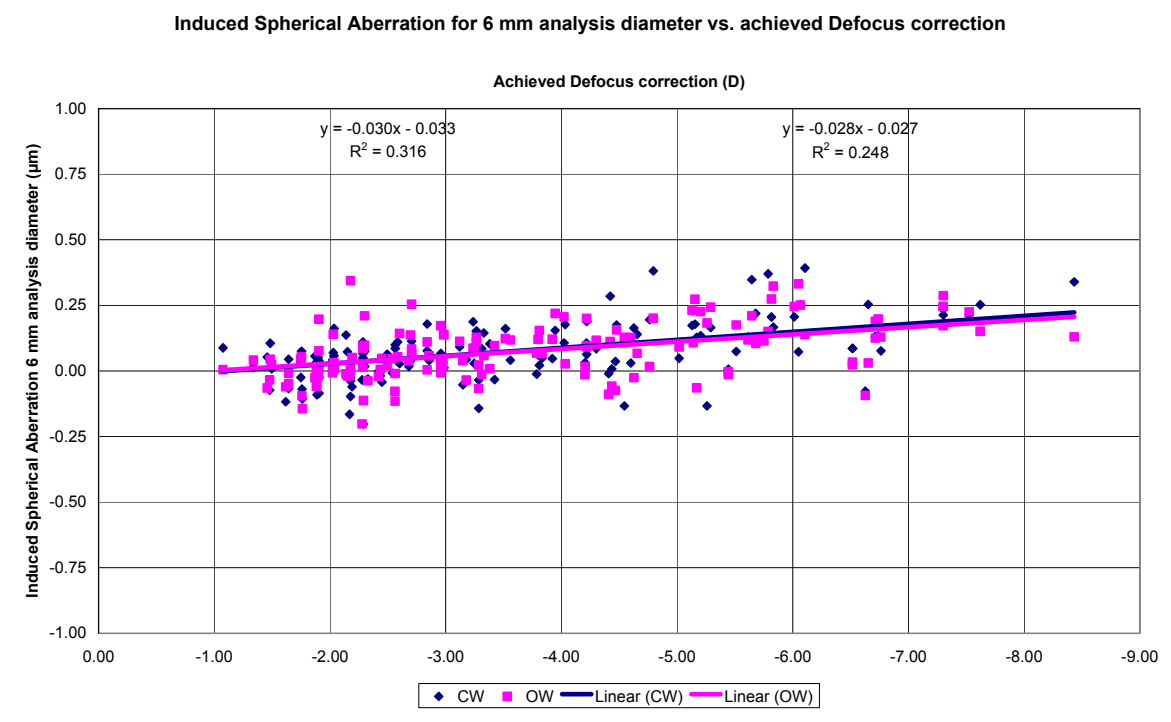

Figure 31: Induced corneal and ocular spherical aberration at 3 months follow-up analysed at $6.0 \mathrm{~mm}$ pupil (ocular: ORK-Wavefront Analyzer (purple squares); corneal: Keratron Scout (blue diamonds)).

Preoperatively, mean ocular coma was $0.18 \pm 0.09 \mu \mathrm{m}$ (range 0.01 to 0.36 ) and corneal coma was $0.26 \pm 0.12 \mu \mathrm{m}$ (range 0.02 to 0.50 ). Postoperatively, the values were $0.22 \pm 0.12 \mu \mathrm{m}$ (range 0.02 to 0.45 ) for ocular coma $(P=0.01$ ), and $0.31 \pm 0.16 \mu \mathrm{m}$ (range 0.01 to 0.62 ) for corneal coma $(P=0.09)$. 
Preoperatively, ocular RMSho was, on average, $0.32 \pm 0.13 \mu \mathrm{m}$ (range 0.07 to 0.57 ) and corneal RMSho was $0.54 \pm 0.14 \mu \mathrm{m}$ (range 0.26 to 0.82). Postoperatively, the values were $0.37 \pm 0.14 \mu \mathrm{m}$ (range 0.10 to 0.65 ) for ocular RMSho $(P=0.002)$, and $0.60 \pm 0.14 \mu \mathrm{m}$ (range 0.32 to 0.88 ) for corneal RMSho ( $P$ $<0.0001)$.

On average, induced coma, defined as the difference in absolute value in coma aberration magnitude postoperatively minus its preoperative magnitude excluding orientation, was $0.016 \mu \mathrm{m}$ per dioptre (Figure 32), whereas induced high-order aberrations, defined as the difference in absolute value of the rootmean square postoperatively minus its preoperative value, was $0.014 \mu \mathrm{m}$ per dioptre for ocular aberration and $0.035 \mu \mathrm{m}$ per dioptre for corneal aberration, both for a 6-mm pupil (Figure 33).

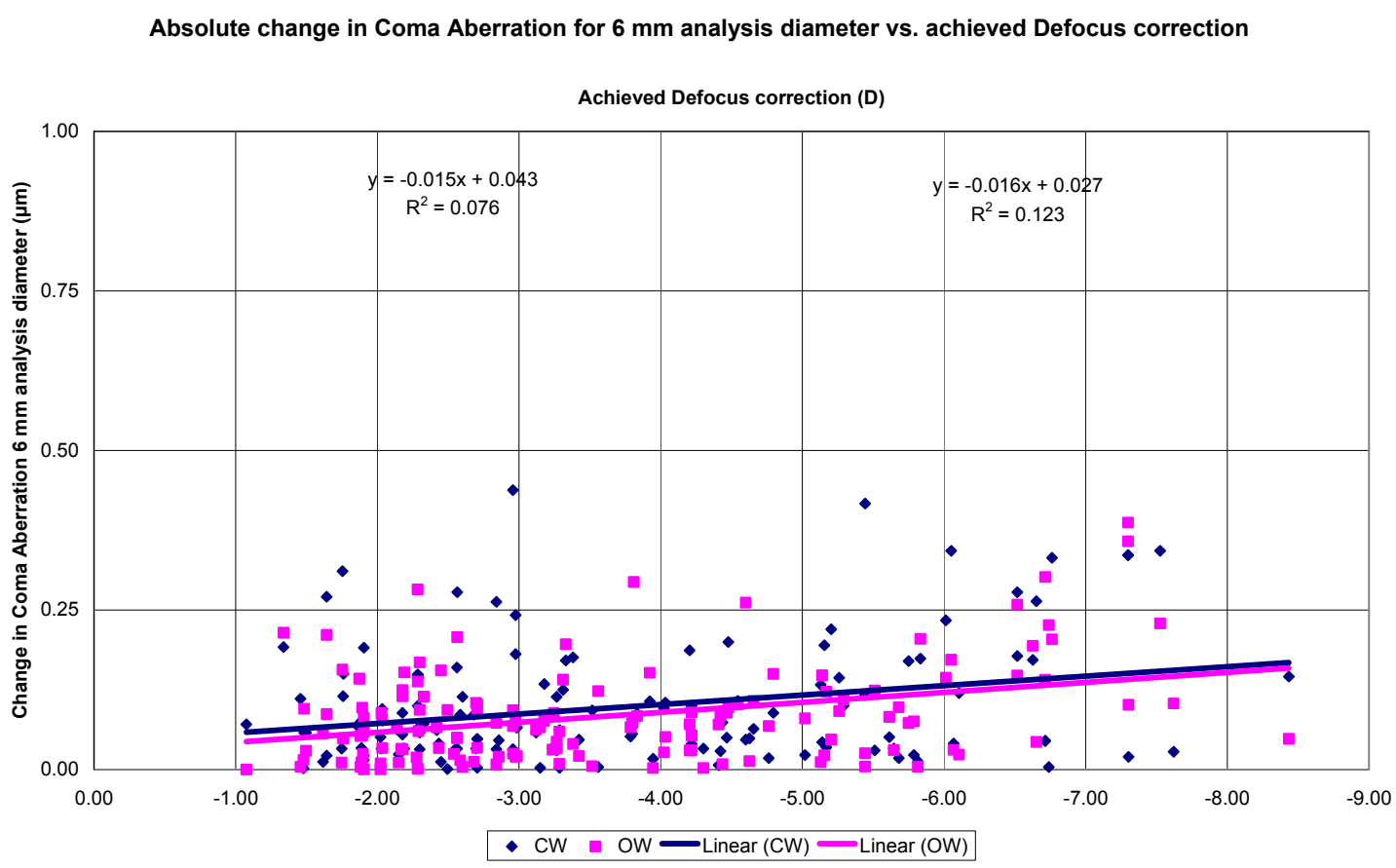

Figure 32: Change in corneal and ocular coma aberration magnitude at 3 months follow-up analysed at $6.0 \mathrm{~mm}$ pupil (ocular: ORK-Wavefront Analyzer (purple squares); corneal: Keratron Scout (blue diamonds)). 
Absolute change in RMSho Aberration for $6 \mathrm{~mm}$ analysis diameter vs. achieved Defocus correction

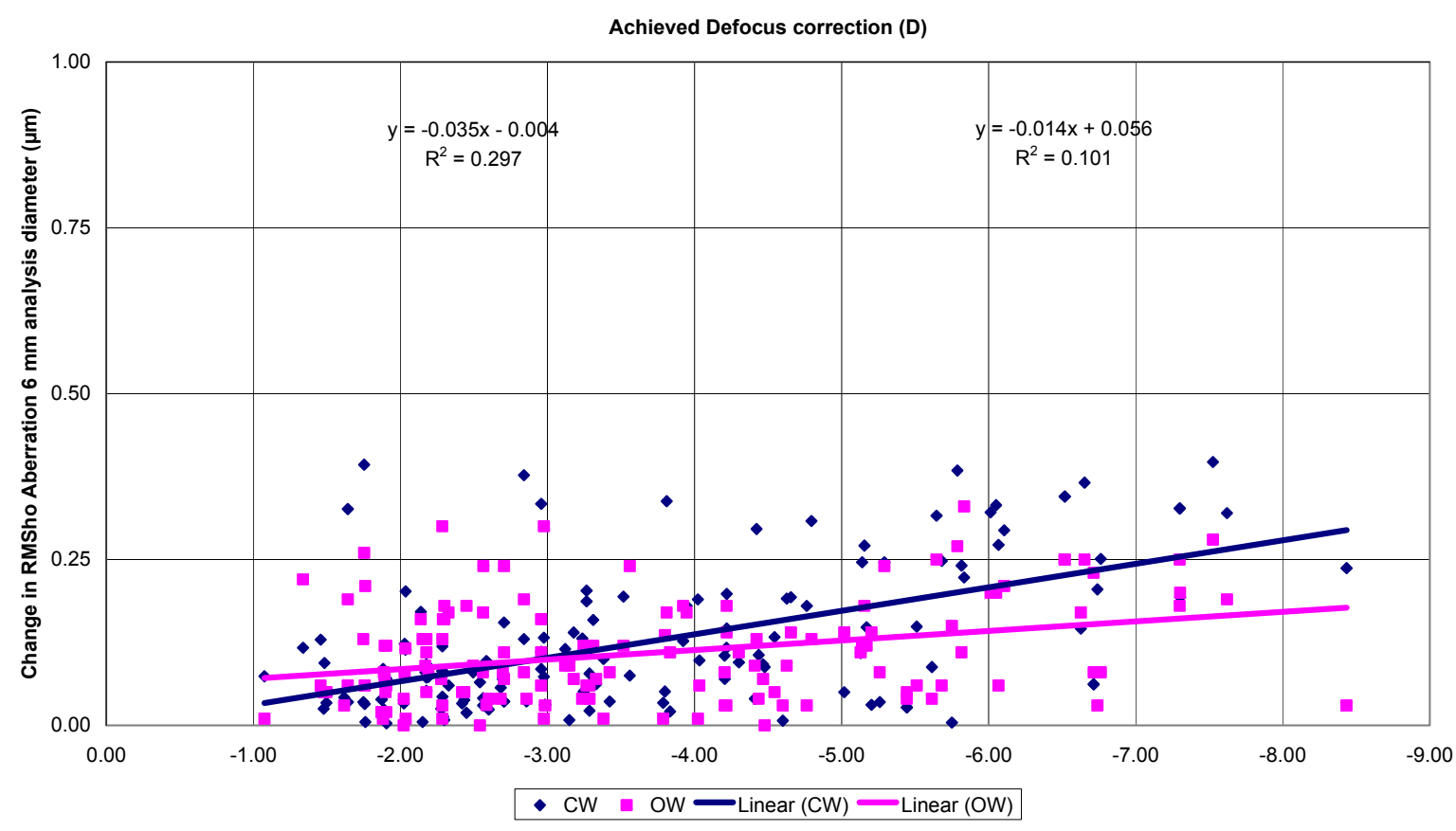

Figure 33: Change in corneal and ocular high-order aberrations (HO-RMS) aberrations magnitude at 3 months follow-up analysed at $6.0 \mathrm{~mm}$ pupil (ocular: ORK-Wavefront Analyzer (purple squares); corneal: Keratron Scout (blue diamonds)).

At $7 \mathrm{~mm}$, preoperatively, mean corneal spherical aberration was $+0.51 \pm 0.15 \mu \mathrm{m}$ (range +0.22 to +0.81 ). Postoperatively, the values were $+0.68 \pm 0.25 \mu \mathrm{m}$ (range +0.20 to +1.16$)(P<0.0001)$. Preoperatively, mean corneal coma was $0.38 \pm 0.18 \mu \mathrm{m}$ (range 0.02 to 0.73 ). Postoperatively, the values were $0.50 \pm 0.25 \mu \mathrm{m}$ (range 0.01 to 0.98$)(P=0.003)$. Preoperatively, corneal RMSho was, on average, $0.80 \pm 0.21 \mu \mathrm{m}$ (range 0.38 to 1.22). Postoperatively, the values were $1.02 \pm 0.25 \mu \mathrm{m}$ (range 0.54 to 1.50$)(P<0.0001)$.

At $8 \mathrm{~mm}$, preoperatively, mean corneal spherical aberration was $+0.75 \pm 0.22 \mu \mathrm{m}$ (range +0.32 to +1.18 ). Postoperatively, the values were $+1.13 \pm 0.41 \mu \mathrm{m}$ (range +0.32 to +1.93$)(P<0.0001)$. Preoperatively, mean corneal coma was $0.53 \pm 0.25 \mu \mathrm{m}$ (range 0.03 to 1.02 ). Postoperatively, the values were $0.74 \pm 0.37 \mu \mathrm{m}$ (range 0.02 to 1.46$)(P<0.0001)$. Preoperatively, 
corneal RMSho was, on average, 1.14 $\pm 0.31 \mu \mathrm{m}$ (range 0.55 to 1.74). Postoperatively, the values were $1.62 \pm 0.39 \mu \mathrm{m}$ (range 0.85 to 2.38$)(P<0.0001)$.

\section{B.4.3 Ablation centre \\ (Centrado de la ablación)}

The amount of induced ocular coma was small for both centration strategies: an average 0.17 micron (range, 0.03-0.32 micron) for the CV group and 0.26 micron (range, 0.01-0.72 micron) for the PC group. The difference in induced ocular coma between groups favouring CV was significant (unpaired $t$ test $P=0.01)$. Furthermore, the induced ocular coma was not correlated with achieved defocus correction for the eyes treated with the CV strategy $\left(r^{2}=0.004\right.$, $P=0.78$ for $C V$ group), but it was correlated with achieved defocus correction for the eyes treated with the PC strategy $\left(r^{2}=0.24, P=0.01\right.$ for the PC group) (Figure 34). The induced ocular coma/dioptres of achieved defocus correction ratio (the slope of the regression) was -0.004 micron of induced ocular coma/diopter for the CV group and -0.049 micron of induced ocular coma/diopter for the PC group.

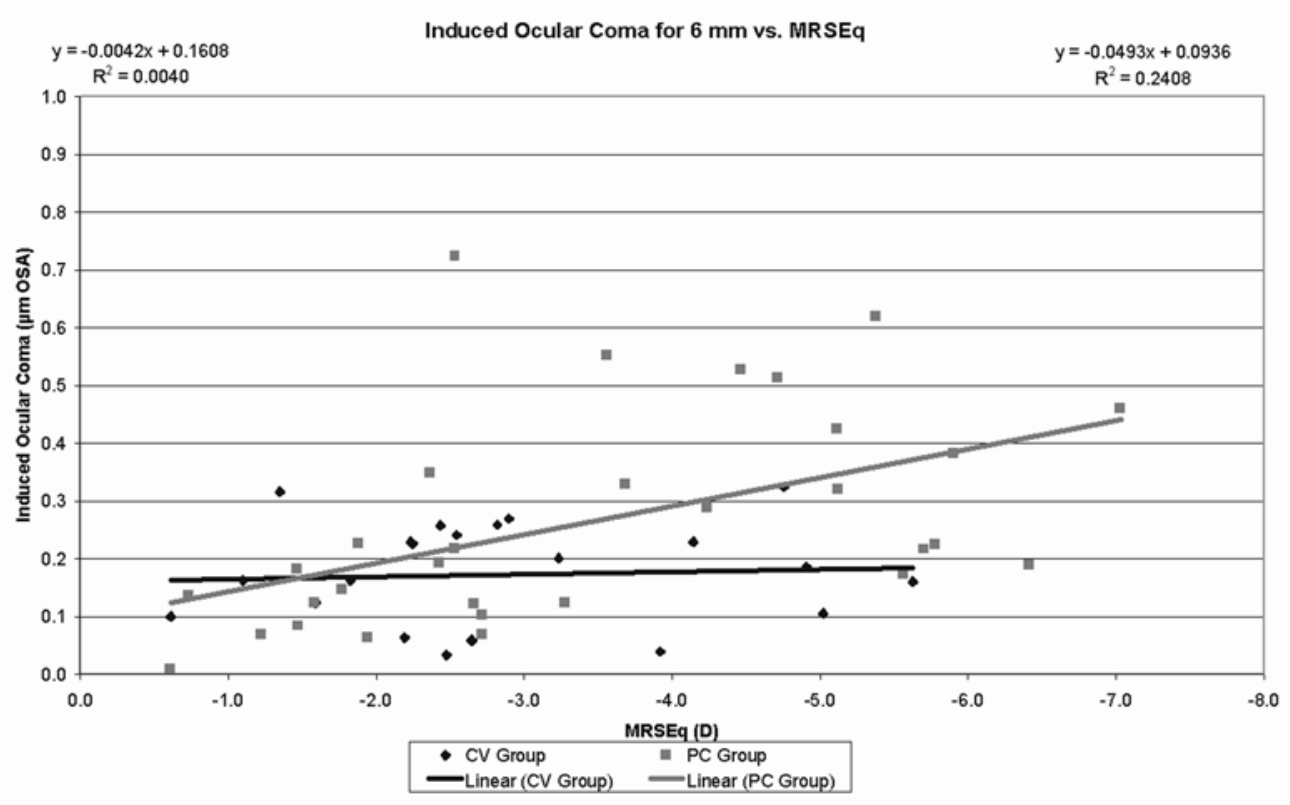

Figure 34: Induced ocular coma/defocus diopter ratio for the CV group (blue) and the PC group (magenta). 
The induced ocular trefoil was small for both centration strategies, i.e., an average $0.09 \mu \mathrm{m}$ (range, 0.01-0.34 micron) for the CV group and $0.13 \mu \mathrm{m}$ (range, 0.01-0.49 micron) for the PC group. The difference in induced ocular trefoil between groups favouring the $\mathrm{CV}$ strategy was not significant (unpaired $t$-test $P=0.07$ ). Further, the induced ocular trefoil was not correlated with the achieved defocus correction for the eyes treated with either centration strategy $\left(r^{2}=0.01\right.$, $P=0.69$ for $C V$ group, $r^{2}=0.11, P=0.07$ for the $P C$ group) (Figure 35). The induced ocular trefoil/dioptres of achieved defocus correction ratio (the slope of the regression) was -0.005 induced ocular trefoil/diopter for the CV group, and -0.019 induced ocular trefoil/diopter for the PC group.

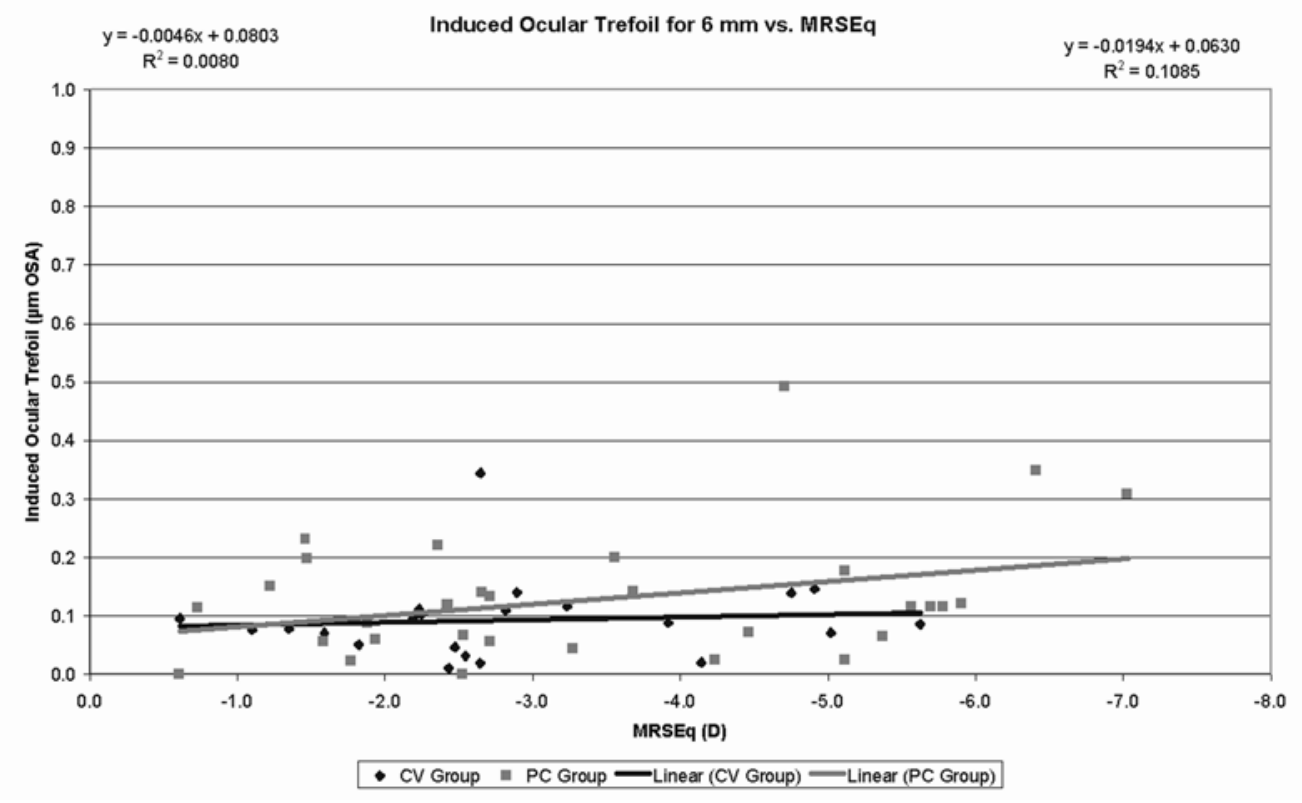

Figure 35: Induced ocular trefoil/defocus diopter ratio for the CV group (blue) and the PC group (magenta).

The induced ocular spherical aberration was minute for both centration strategies, i.e., an average +0.01 micron(range, -0.25 to +0.34 micron) for the $\mathrm{CV}$ group and +0.07 micron (range, -0.01 to +0.46 micron) for the PC group. The difference in induced ocular coma between groups favouring the CV strategy was significant (unpaired $t$-test $P=0.05$ ). Further, the induced ocular spherical 
aberration was not correlated with achieved defocus correction for the eyes treated with the CV strategy $\left(r^{2}=0.13, P=0.09\right.$ for the $C V$ group), but it was correlated with the achieved defocus correction for the eyes treated with the PC strategy $\left(r^{2}=0.17, P=0.02\right.$ for the PC group) (Figure 36$)$. The induced ocular spherical aberration/dioptres of achieved defocus correction ratio (the slope of the regression) was $-0.028 \mu \mathrm{m}$ of induced ocular spherical aberration/diopter for the CV group and $-0.035 \mu \mathrm{m}$ of induced ocular spherical aberration/diopter for the PC group.

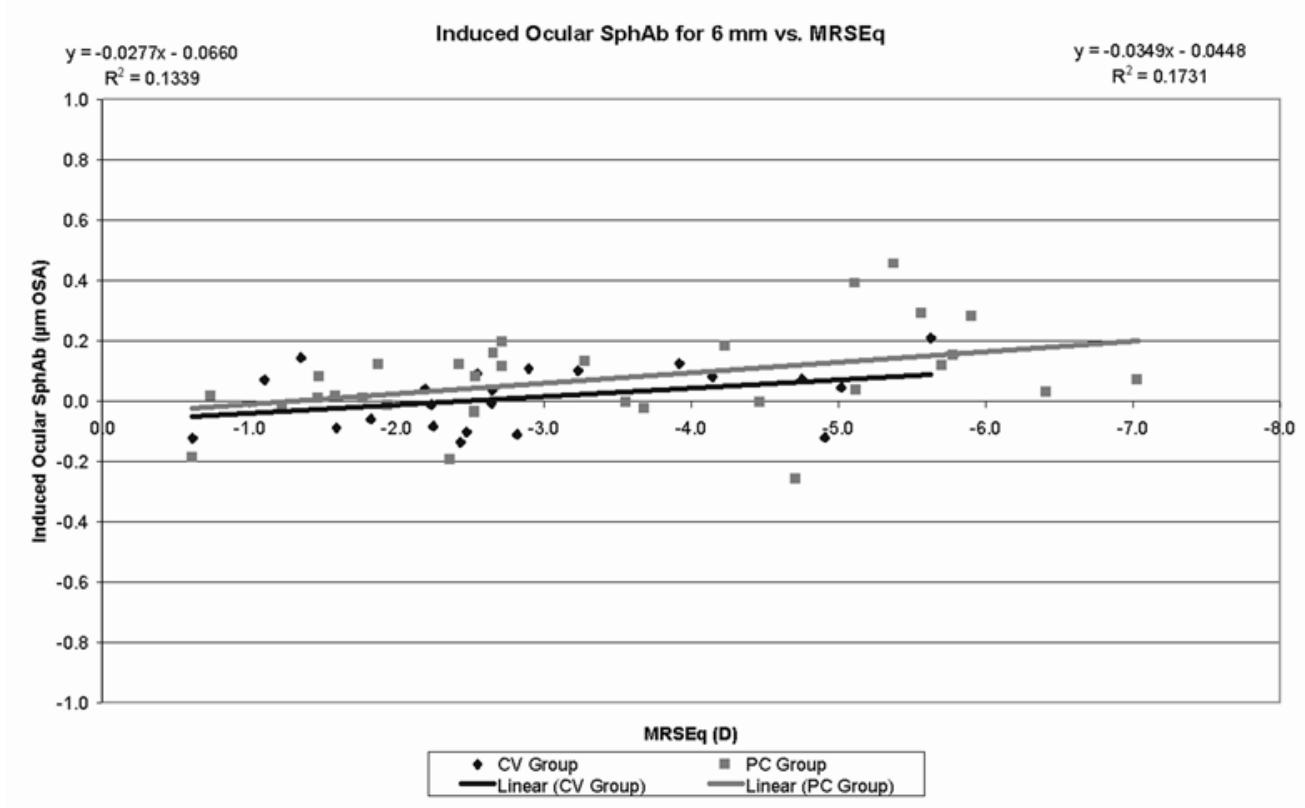

Figure 36: Induced spherical ocular aberration/defocus diopter ratio for the $\mathrm{CV}$ group (blue) and the PC group (magenta).

The induced corneal spherical aberrations at $6.00 \mathrm{~mm}$ measured $0.17 \pm 0.10$ $\mu \mathrm{m}$ in the classical group and $0.09 \pm 0.16 \mu \mathrm{m}$ in the aspheric aberration neutral (Aberration-Free ${ }^{\mathrm{TM}}$ ) group, the difference in induced SphAb in both groups were 
statistically significant favouring the aberration neutral (Aberration-Free ${ }^{\mathrm{TM}}$ ) group $(p<0.005)$.

\section{B.4.5 Bilateral symmetry \\ (Simetría bilateral)}

B.4.5.1 Changes in bilateral symmetry of Zernike terms as a result of refractive surgery

(Cambios en la simetría bilateral de términos de Zernike provocados por la cirugía refractiva)

Six months postoperatively, 3 Zernike terms $(C[2,-2], C[4,+4], C[5,-5])$ had lost significant OS-vs.-OD correlation symmetry, 4 Zernike terms $(C[4,-4], C[5,-3]$, $C[6,0], C[7,-1])$ had gained significant correlation symmetry, and 29 Zernike terms preserved correlation symmetry OS vs. OD compared to the preoperative baseline.

Six months postoperatively, for 6 Zernike terms $(C[4,+4], C[5,+1], C[6,-6]$, $C[6,-4], C[6,+2], C[7,-5])$ the differences in OS-vs.-OD symmetry increased significantly, for 4 Zernike terms $(C[4,-4], C[5,+3], C[6,+6], C[7,-7])$ the differences in symmetry decreased significantly, and for 26 Zernike terms the OS-vs.-OD symmetry was preserved, compared to the preoperative baseline.

\section{B.4.5.2 Changes in bilateral symmetry of wavefront aberration as a result of refractive surgery \\ (Cambios en la simetría bilateral interocular provocados por la cirugía refractiva)}

Six months postoperatively, 3 patients (\#2, \#15, and \#22) lost significant OS-vs.-OD correlation symmetry, 1 patient (18) gained significant correlation symmetry, and 21 patients preserved OS-vs.-OD correlation symmetry, compared to the preoperative baseline.

6 months postoperatively, for 2 patients (\#6, \#15) the differences in OS-vs.OD symmetry increased significantly, for 1 patient (\#7) the differences in 
symmetry decreased significantly, and for 22 patients the OS-vs.-OD symmetry was preserved, compared to the preoperative baseline.

\section{Section B.5 DISCUSSION}

(Discusión)

\section{B.5.1 Aberration-free pattern \\ (Perfiles libres de aberración)}

Corneal refractive treatments typically induce a change in corneal asphericity. Recently, it has been argued that preserving the preoperative corneal asphericity after corneal refractive treatments might be positive, therefore, asphericity-based profiles have been developed. However, there is no clear evidence that asphericity is the variable that alone plays the major role in the visual process.

One problem using Q-factor customized ablation profiles are the severe difficulties to determine the $\mathrm{Q}$-factor to be targeted. The average asphericity of the human cornea is about $-0.28 .{ }^{170}$ Nevertheless, there are persons with Qfactor -0.25 and poor vision, and others with $Q$-factor +0.25 and supervision. Despite some remarkable theoretical works ${ }^{80,81,82}$, there is no proof that more negative quotients of asphericity provide better visual quality, or that an absolute optimum exists. When a patient is selected for non customized aspherical treatment, the global aim of the surgeon should be to leave all existing HOAb unchanged because the best corrected visual acuity, in this patient, has been unaffected by the pre-existing aberrations ${ }^{69}$. Hence, all factors that may induce HOAb, such as biomechanics, need to be taken into account prior to the treatment to ensure that the preoperative HOAb are unchanged after treatment.

Statistical analysis of a population of human corneas showed as an average result that the best fit aspherical surface had a Q-factor around 0.25. ${ }^{171,172}$ As a result, in general healthy human corneas show a "positive spherical aberration," which is balanced by the "negative spherical aberration" of the internal lens. ${ }^{55}$ As an average, human corneas manifest a corneal spherical aberration around $0.23 \mu \mathrm{m}$. One can say that the corneal-wavefront values are 
"overestimated" in the topographic systems compared to the ocular wavefront values.

As individuals are aging, the asphericity of the crystalline lens changes, reducing the amount of spherical aberration that can be balanced or even showing a certain amount of positive spherical aberration, whereas the corneal asphericity thus, corneal spherical aberration, remains relatively stable over time, disrupting the equilibrium between both.

However, in recent times there is a clear tendency of targeting a prolate postoperative anterior corneal surface as global optimum in refractive surgery. The intended meaning of the terms prolate and oblate is sometimes unclear. The confusion comes from the false usage of curvature and refractive power: As the average human cornea is prolate (Q-factor -0.25), the central part of the cornea has a stronger curvature than the periphery. However, refractive power is given by Snell's law. As the corresponding Cartesian-oval is the aberration-free surface (i.e. the only truly monofocal surface), and can be described by an aspherical surface with quotient of asphericity $-1 / \mathrm{n}^{2}$ (approx. -0.528 for human cornea), the average human cornea (Q-factor -0.25 ) is less prolate (so more oblate) than the corresponding Cartesian-oval, thus the refractive power of the outer corneal surface increases from central towards peripheral. In this way, the multifocality towards peripheral just answers the question whether the corneal spherical aberration is positive (refractive power increases towards peripheral) or negative (refractive power decreases towards peripheral) but not the question about the geometrical concept of prolate vs. oblate.

The first thing to be clarified is that even the amount of corneal spherical aberration and the asphericity are intrinsically related; the goal is always described in terms of change in spherical aberration ${ }^{158}$, because this is the factor related to the quality and sharpness of the retinal image. Then, in the treatments, the goals should be:

a) For aspherical treatments: Aberration-free profile, with no induced aberrations; a change in asphericity depending on the corrected defocus. 
b) For customized wavefront treatments: change in aberrations according to diagnosis; change in asphericity depending on the corrected defocus and on the $C(n, 0)$ coefficients applied.

The asphericity change using classical profiles is bigger than that using aberration-free profiles, and the asphericity change using aberration-free profiles it is intended in a controlled manner.

Please note that only a starting surface of a Cartesian-oval would lead to no corneal aberration, but the anterior cornea definitely is not a Cartesian-oval and posses corneal aberrations. However, with the proposed "aberration-free" concept the idea is to maintain the own aberrations of every individual cornea.

Even though the condition of stigmatism, that origins "free of aberration" verified for two points (object and image) and for a conicoid under limited conditions, is very sensitive to small deviations and decentrations (a question that usually arises in refractive surgery), the goal of these profiles is not to achieve an stigmatism condition postoperatively, but rather to maintain the original $\mathrm{HO}$ wavefront-aberration.

In wavefront guided ablation the objective is not only to reduce the induction moreover to reduce the aberration of the eye, although if we analyse the studies of wavefront guided ${ }^{186,190}$ the result is in some laser platforms that they induce less aberration than with standard profiles but cannot reduce the postoperative $\mathrm{HO}$ aberrations below the preoperative levels.

In our case, the theoretical model of aspheric Aberration-Free treatment tries to remodel the slope of the cornea to compensate for the attempted sphere and cylinder components, without inducing new aberrations. One of the most affecting aberrations after myopic LASIK is the spherical aberration. ${ }^{79}$

It is important to remark that the used Aberration-Free profiles intend to preserve preoperative aberrations and not preoperative asphericity.

Tuan and Chernyak ${ }^{190}$ analyzed the impact of corneal asphericity on wavefront-guided LASIK at six clinical sites and found no significant correlation between corneal shape and VA or contrast sensitivity. 
Pop and Payette ${ }^{191}$ studied the relationship between contrast sensitivity, Zernike wavefront-aberrations, and asphericity after LASIK to correct myopia. Contrast sensitivity was not correlated with asphericity but was correlated with wavefront-aberrations as expected. The change in asphericity was correlated with the refractive change and was predicted by the parabolic Munnerlyn equation.

Anera et al. ${ }^{177}$ (2003) analyzed the origin of the changes in the p-factor after LASIK and the effect of postsurgical asphericity on contrast sensitivity function. An increase in the p-factor after LASIK was higher than the predictions using the paraxial formula of Munnerlyn and coauthors.

Holladay and Janes (2002) determined the relationship between the spherical refractive change after myopic excimer laser surgery and the effective optical zone and corneal asphericity determined by corneal topography, which changed nonlinearly with the amount of treatment.

\section{B.5.2 Ablation centre}

(Centrado de la ablación)

We designed our centration strategies in two different centration references that can be detected easily and measured with currently available technologies. PC may be the most extensively used centration method for several reasons. First, the pupil boundaries are the standard references observed by the eyetracking devices. Moreover, the entrance pupil can be well represented by a circular or oval aperture, and these are the most common ablation areas. Centring on the pupil offers the opportunity to minimize the optical zone size. Because in LASIK there is a limited ablation area of about 9.25 millimetres (flap cap), the maximum allowable optical zone will be about 7.75 millimetres. Because laser ablation is a destructive tissue technique, and the amount of tissue removed is directly related to the ablation area diameter, the ablation diameter, maximum ablation depth, and ablation volume should be minimized. The planned optical zone should be the same size or slightly larger as the functional entrance pupil for the patients' requirements. 
The main HOAb effects (main parts of coma and spherical aberrations) arise from edge effects, i.e., strong local curvature changes from the optical zone to the transition zone and from the transition zone to the untreated cornea. It then is necessary to emphasize the use of a large optical zone (6.50 millimetres or more) to cover the scotopic pupil size, and a large and smooth transition zone.

However, there are several ways to determine the corneal vertex: the most extensively used one is to determine the coaxial corneal light reflex $\left(1^{\text {st }}\right.$ Purkinje image). Nevertheless, there is a problem using the coaxial light reflex because surgeons differ; for instance, the coaxial light reflex will be seen differently depending on surgeon eye dominance, surgeon eye balance, or the stereopsis angle of the microscope. For example, the LadarVision platform (Alcon) uses a coaxial photograph as reference to determine the coaxial light reflex, which is independent of the surgeons' focus. For that reason, in the current study, ablations were centred using the pupillary offset, the distance between the pupil centre and the normal $\mathrm{CV}$.

Considering this, for aberration-free profile, aspherical, or, in general, noncustomised treatments, we use minimum patient data (sphere, cylinder, and axis values) from the diagnosis. Therefore, we assume that the patient's optical system is aberration-free or that those aberrations are not clinically relevant (otherwise we would have planned a customised treatment). For those reasons, the most appropriate centring reference is the corneal vertex; we then modify the corneal asphericity with an aberration-free ablation profile, including loss of efficiency compensations. For customized wavefront treatments, change in aberrations according to diagnosis measurements, we use a more comprehensive data set from the patient diagnosis, including the aberrations, because the aberrations maps are described for a reference system in the centre of the entrance pupil. The most appropriate centring reference is the entrance pupil as measured in the diagnosis.

Providing different centring references for different types of treatments is not ideal, because it is difficult to standardize the procedures. Nevertheless, ray tracing indicates that the optical axis is the ideal centring reference. Because this is difficult to standardize and considering that the anterior corneal surface is the main refractive element of the human eye, the $\mathrm{CV}$, defined as the point of 
maximum elevation, will be the closest reference as proposed here. It shall be, however, noticed that on the less prevalent oblate corneas the point of maximum curvature (corneal apex) might be off centre and not represented by the corneal vertex.

However, it would be interesting to refer the corneal and/or ocular wavefront measurements to the optical axis or the $\mathrm{CV}$. This can be done easily for corneal wavefront analysis, because there is no limitation imposed by the pupil boundaries. However, it is not as easy for ocular wavefront analysis, because the portion of the cornea above the entrance pupil alone is responsible for the foveal vision. Moreover, in patients with corneal problems such as keratoconus/keratectasia, post-LASIK (pupil-centred), corneal warpage induced by contact lens wearing and other diseases causing irregularity on anterior corneal surface, the corneal vertex and the corneal apex may shift. In those cases, pupil centre is probably more stable. Moreover, since most laser systems are designed to perform multiple procedures besides LASIK, it is more beneficial that excimer laser systems have the flexibility to choose different centration strategies.

A deeper analysis of the induced ocular aberrations and the changes in asphericity showed significant differences favouring $\mathrm{CV}$ centration for the induction of coma and spherical ocular aberration and the changes in asphericity, and no significant differences for the induced ocular trefoil.

Due to the smaller angle kappa associated with myopes compared with hyperopes, centration issues are less apparent. However, we wanted to test whether the angle kappa in myopes was sufficiently large to show differences in results, because it is always desirable to achieve as much standardization as possible and not to treat the myopes using one reference, whereas the hyperopes use a different one.

Previous studies ${ }^{192}$ have reported that based on theoretical calculations with 7.0-mm pupils even for customized refractive surgery, that are much more sensitive to centration errors, it appears unlikely that optical quality would be degraded if the lateral alignment error did not exceed $0.45 \mathrm{~mm}$. In $90 \%$ of eyes, even accuracy of $0.8 \mathrm{~mm}$ or better would have been sufficient to achieve the goal $^{192}$. 
In our case, the pupillary offset averaged 0.29 millimetres and this moderate value seems to be sufficiently large to be responsible for differences in ocular aberrations, however, not large enough to correlate this difference in ocular aberrations with functional vision.

A limitation of this study is that we have used a comparison based upon different two groups of patients with different centrations used as reference. A direct comparison in a lateral/contralateral eye basis for the assignment of the centration reference would maybe reduce the variability of external uncontrollable effects (like flap cut, corneal response to the ablation, repeatability of the instruments, cooperation of the patients, etc...). However, such direct comparison may reduce patients' satisfaction, as patients may postoperatively observe differences among eyes due to the different centrations.

\section{B.5.3 Bilateral symmetry}

(Simetría bilateral)

The aim of this part was to evaluate the effects of laser corneal refractive surgery on the bilateral symmetry of the corneal wavefront aberration; in particular, following a treatment performed with the AMARIS system, which is based on an Aberration-Free ${ }^{\mathrm{TM}}$ ablation profile. The advantage of the AberrationFree $^{T M}$ ablation profile is that it aims to be neutral for HOAb, leaving the visual print of the patient as it was preoperatively with the best spectacle correction. If the aimed Aberration-Free concept would have been rigorously achieved, the bilateral symmetry between eyes would have been automatically obtained. In our group of patients, the aimed Aberration-Free concept does not hold rigorously true, but we had a very minor increase in corneal aberrations for a $6 \mathrm{~mm}$ pupil.

As shown from the data presented herein, non-customised femtosecond LASIK performed with the combination LDV and AMARIS platforms is safe, effective, and it preserves reasonably well the bilateral symmetry of the corneal wavefront aberration between eyes. This may be related to the advantages of profiles aiming to be neutral for HOAb or to the fact that the high-speed AMARIS 
system reduces variability from stromal hydration effects, which increase with the duration of treatment ${ }^{193},{ }^{194}$. Recognizing the high levels of defocus and astigmatism in this study, analysis of pre- and postoperative binocular vision ${ }^{195}$ would be of interest and is a partial limitation of this study ${ }^{196}$. Further analysis of bilateral symmetry as a function of the analysis diameter is also of interest. Longterm follow-up on these eyes will help determine the stability of these accurate results. Comparing similar outcomes from other lasers to see if any of the parameters we measured are really different for other lasers or microkeratomes and analyses to determine if these parameters are clinically relevant will help to determine the impact of this work.

Cuesta et al. ${ }^{197}$ found that even differences in corneal asphericity may affect the binocular visual function by diminishing the binocular contrast-sensitivity function. Jiménez et al. ${ }^{198}$ found that following LASIK, binocular function deteriorates more than monocular function, and that this deterioration increases as the interocular differences in terms of aberrations and corneal shape increase. They also found that interocular differences above $0.4 \mu \mathrm{m}$ of RMS for a $5 \mathrm{~mm}$ analysis diameter $(0.4 \mathrm{D})$ lead to a drop in binocular summation of more than $20 \%$.

In our study, only 4 out of 25 patients showed preoperative clinically relevant OS-vs.-OD differences (i.e., larger than $0.25 \mathrm{D}$ ), whereas 6 months postoperatively only 2 out of 25 patients showed clinically relevant OS-vs.-OD differences (i.e., larger than $0.25 \mathrm{D}$ ).

RMS $(\triangle H O A b)$ analysis for interocular differences accounts for the RMS of the differential corneal wavefront aberration and not for the difference of the corneal RMS(HOAb). RMS $(\triangle \mathrm{HOAb})$ is a rigorous analysis metric, because it accounts for any deviation (i.e. both inductions and reductions of the wavefront aberration, since both contribute positively to increase the RMS value). Furthermore, it can be mathematically demonstrated that:

$$
R M S(\triangle H O A b) \geq \triangle R M S(H O A b)
$$

Limitations of our study include the moderate number of eyes, limited follow-up and the lack of a control group. The method to determine whether or not symmetry is maintained consist of comparing individual terms in a variety of 
ad hoc ways both before and after refractive surgery, ignoring the fact that retinal image quality for any given individual is based on the sum of all terms. However, similar methodologies have been already used before. ${ }^{199}$ At this stage, we did not perform any specific visual tests on binocular vision; for example, stereotests. Some patients may not have good stereopsis but they may show good aberration symmetry. The analysis of bilateral symmetry should be related to the patients' binocular vision status. Despite these limitations, we were able to demonstrate that "aberration neutral" ablation profiles reasonably preserve the bilateral symmetry between eyes in terms of corneal wavefront aberration. The presented results cannot be extrapolated to patients with symptoms of amblyopia ${ }^{200}$, anisometropia, nystagmus, or aniseikonia ${ }^{201}$ without further studies.

This does not mean any "good or bad" point for binocular vision. Taking into account that we cannot precisely evaluate the role of aberrations monocularly (patients with a high level of aberrations can have an excellent visual acuity and vice versa), it is even more difficult to do it binocularly. The important question in binocular vision is "the role of interocular-differences," and if they can significantly influence binocular performance. Interocular-differences can be minor but significant for visual performance. Further studies shall help to determine the impact of this on binocular visual performance.

\title{
Section B.6 CONCLUSIONS
}

(Conclusiones)

\begin{abstract}
"Aberration-free" patterns for refractive surgery as defined here together with consideration of other sources of aberrations such as blending zones, eyetracking, and corneal biomechanics yielded results comparable to those of customisation approaches. CV-centred treatments performed better in terms of induced ocular aberrations and asphericity, but both centrations were identical in terms of photopic visual acuity. Aberration-Free Treatments with the SCHWIND AMARIS did not induce clinically significant aberrations, maintained the global OD-vs.-OS bilateral symmetry, as well as the bilateral symmetry between corresponding Zernike terms (which influences binocular summation). The induced corneal aberrations were less than compared with the classical profile or
\end{abstract}


other publications. Having close-to-ideal profiles should improve clinical outcomes decreasing the need for nomograms, and diminishing induced aberrations after surgery.

\section{Section B.7 OUTLOOK}

(Perspectiva)

In this study we have used aberration-free profiles as a basis for the simulations and clinical evaluations. We have learnt that aberration-free profiles may reduce the induction of aberrations below clinically relevant values. Since we are confident that on these grounds, induction of aberrations can be controlled, in further studies, wavefront guided profiles will be explored and analyzed in a similar way.

In this chapter, we have performed clinical evaluations in moderate levels of myopia and hyperopia. We have learnt that aberration-free profiles reduce the induction of aberrations below clinically relevant values, but yet induce some minor levels of aberrations. In further studies, higher levels of myopia and hyperopia will be analyzed to determine, to which extent induction of aberrations remains below clinically relevant values.

This chapter was limited to limit the induction of aberrations, further studies will attempt to manipulate the induction of aberrations in a controlled manner e.g. for presbyopic corrections. 

Topic C DECISION TREE ANALYSIS SYSTEM TO OPTIMISE REFRACTIVE SURGERY OUTCOMES

(Análisis por árbol de decisión para la optimización de resultados en cirugía refractiva)

\section{AUTHOR CONTRIBUTIONS}

Study concept and design (S.A.M.); data collection (M.C.A., M.C., D.O., J.G., I.M.); analysis and interpretation of data (S.A.M.); drafting (S.A.M.); critical revision (T.M., M.C.A., T.E., M.C., D.O., J.G., I.M.A.); statistical expertise (S.A.M.).

\section{Section C.1 ABSTRACT}

(Resumen)

PURPOSE: To assess a decision tree analysis system to further optimize refractive surgery outcomes.

METHODS: A 5-step decision tree, the Decision Assistant Wizard, based on previous experience with the SCHWIND AMARIS laser, was applied for selecting a customized refractive surgery treatment mode (aspheric aberration neutral, corneal wavefront-guided, or ocular wavefront-guided) to eliminate or reduce total aberration.

RESULTS: Using the Decision Assistant Wizard, 6467 LASIK treatments were performed over a 30-month period; 5262 and 112 for myopic and hyperopic astigmatism, respectively, using aspheric aberration neutral (AF) profiles, 560 using corneal wavefront-guided profiles, and 533 using ocular wavefront-guided profiles. Twenty-two $(0.3 \%)$ retreatments were performed overall; $18(0.3 \%)$ and $0(0 \%)$ after myopic and hyperopic astigmatism, respectively, using AF, $3(0.5 \%)$ after corneal wavefront-guided profiles, and $1(0.2 \%)$ after ocular wavefrontguided profiles. 
CONCLUSIONS: Decision assistant wizards may further optimize refractive surgical outcomes by providing the most appropriate ablation pattern based on an eye's anamnesis, diagnosis, and visual demands. The general principles may be applied to other laser systems; however, specifics will depend on manufacturers' specifications.

\section{Section C.2 INTRODUCTION}

(Introducción)

Many studies have proven that correction of ammetropiae with laser induces aberrations (most significant is the induction of spherical aberration). Corneal laser refractive surgery evolved from simple myopic ablations to the most sophisticated topography-guided, wavefront-driven, or aspheric patterns to preserve the preoperative level of high order aberrations. Some hypotheses state that there might be "good" aberrations and others that are to be avoided.

Reasonable reductions in $\mathrm{HOAb}$ after wavefront-guided treatments on aberrated eyes and reasonable changes in HOAb after wavefront-optimized treatments have been reported. However, a significant number of refractive surgery patients may not benefit from OW guided treatments as the induction of HOAb is related to baseline levels of HOAb. For example, HOAb tend to be induced in patients with less than $0.30 \mu \mathrm{m}$ and reduced in patients with greater than $0.30 \mu \mathrm{m}$ of HOAb. Physiologic aberrations may be required to maintain the visual quality of the eye.

Our definition of "Customisation" is conceptually different and can be stated as: "The planning of the optimum ablation pattern specifically for each individual eye based on its diagnosis and visual demands." It is often the case, that the best approach for planning an ablation is a sophisticated pattern, which can still be simply described in terms of sphere, cylinder, and orientation (axis).

We recently published a review of 18-month experience with AMARIS ${ }^{110}$ where we introduced the systematic use in our clinical routine of Decision-Tree analyses for selecting the most appropriate type of correction to be applied. Ablation profiles considered in the decision tree include the Aberration-Free ${ }^{\mathrm{TM}}$ 
(called aspheric aberration neutral in this chapter), OW-guided and CW-guided. During this time, some new findings have helped us to refine our strategies. A simplified, updated version of our current Decision-Tree is presented herein.

The Decision Assistant Wizard, which we present here, is based on our experience with the SCHWIND AMARIS laser. While the general principles of this Decision-Tree based planning (Figure 37) can basically be applied to any other laser platform offering aspheric and wavefront-guided profiles, some specific aspects concerning both diagnosis and treatments may depend on other manufacturers' specifications.

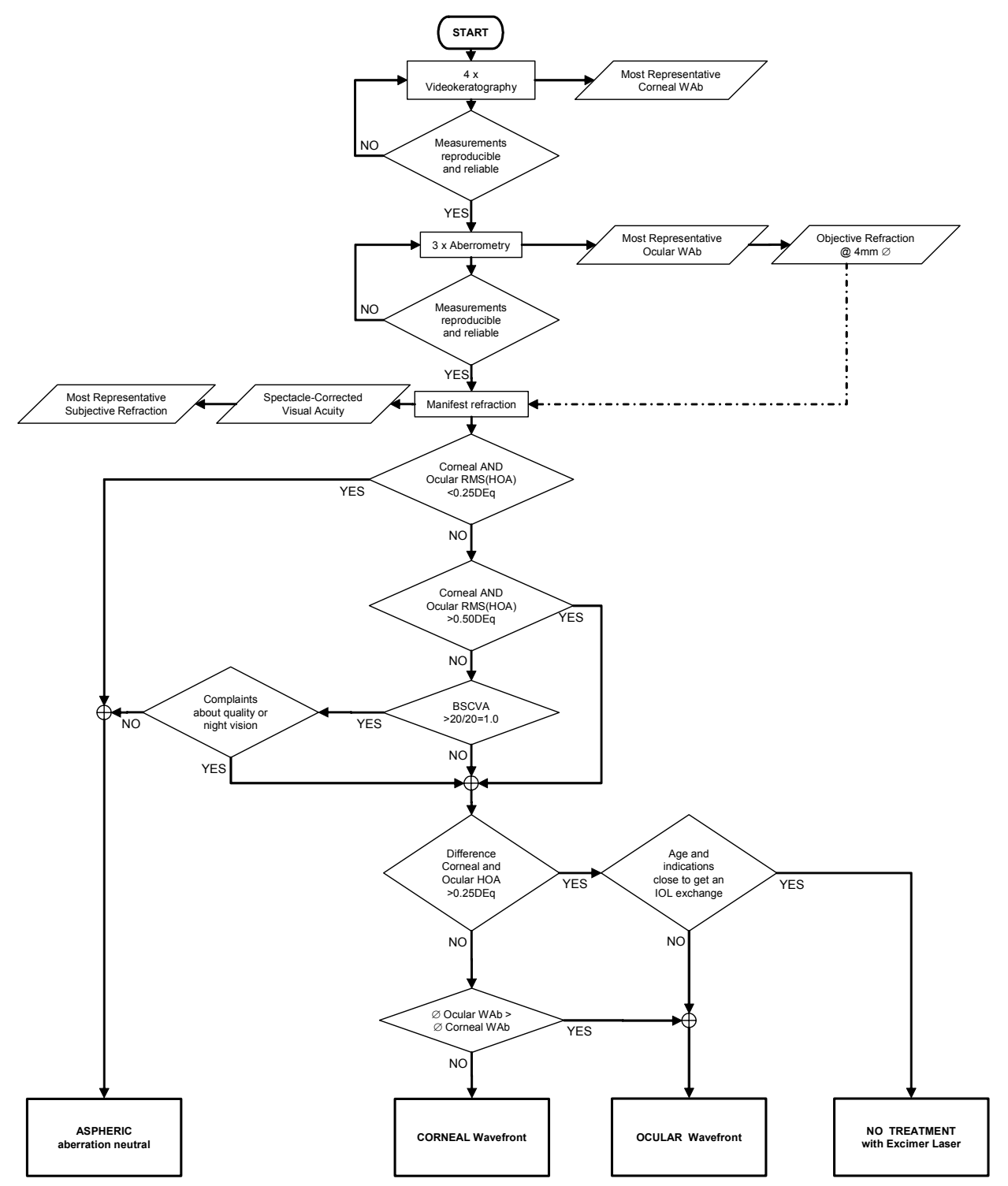

Figure 37: Decision-Tree applied for selecting the treatment mode (Aspheric aberration neutral, Corneal-Wavefront-Guided, or Ocular-Wavefront-Guided). 


\section{Section C.3 METHODS}

(Método)

\section{C.3.1 Videokeratoscopy \\ (Videoqueratoscopía)}

We begin by acquiring four corneal topographies (Corneal Wavefront Analyzer, SCHWIND eye-tech-solutions $\mathrm{GmbH} \&$ Co.KG, based on KeratronScout, OPTIKON2000, Rome, Italy) and derived CW analyses centred on the lineof-sight for each eye of the patient. We extract the mean, and discard the less representative one (the one with the poorest similarity to the mean). From those remaining three maps, we calculate the mean, and select the most representative one (the one with the highest similarity to the mean).

\section{C.3.2 Aberrometry}

\section{(Aberrometría)}

We continue acquiring, under non pharmacologically dilated pupils, noncycloplegic conditions, and natural dim light conditions (to avoid pharmacologically induced pupil shifts ${ }^{202},{ }^{203}$ ), 3 aberrometries (Ocular Wavefront Analyzer, SCHWIND eye-tech-solutions $\mathrm{GmbH} \&$ Co.KG, based on irx3, Imagine Eyes, Orsay, France) and objective refractions for each eye of the patient. To minimize the potential accommodative response of the patients, we ask them to "see-through-the-target" instead of "looking at the target." In this way, patients do not try to get a sharp image from the +1.5 D fogged target, since they were instructed to see-through-the-target. From those aberrometries, we calculate the mean, and select the most representative one (the aberrometry map with the highest similarity to the mean). 


\section{C.3.3 Manifest refraction \\ (Refracción manifiesta)}

We continue assessing subjective refraction based upon nonpharmacologic and non-cycloplegic conditions, under natural photopic illumination. We use the objective refraction analyzed for a sub-pupil of $4 \mathrm{~mm}$ diameter, as starting refraction for this step. This is particularly useful for determining the magnitude and orientation of the astigmatism ${ }^{138,142}$. We measure manifest refraction, uncorrected and best spectacle-corrected Snellen visual acuity $^{204}$ (UCVA and BSCVA, respectively). Further rules that we impose for accurately determining the manifest subjective refractions among equal levels of BSCVA are: taking the measurement with the least negative (the most positive) spherical equivalent (unmasking latent hyperopia), if several of them are equal in terms of spherical equivalent, we choose the measurement with the least amount of astigmatism (reducing the risk of postoperative shifts in the axis of astigmatism).

\section{C.3.4 Decision process \\ (Proceso de decisión)}

The decision process starts by estimating the global optical impairment resulting from the measured wave aberrations. This is done by objectively determining the actual clinical relevance of single terms in a Zernike expansion of the wave aberration.

In general, for the same magnitude of aberration, the optical blur produced by high order aberrations increases with increasing radial order and decreases with increasing angular frequencies. Based on this, the dioptric equivalent (DEq) was used.

If the global optical blur for both corneal and ocular wave-aberrations (CWAb and OWAb, respectively) are below $0.25 \mathrm{DEq}$ for both eyes, then the treatment to be applied is Aspheric aberration neutral. 
If the global optical blur for any corneal or ocular wave-aberrations is between $0.25 \mathrm{DEq}$ and $0.50 \mathrm{DEq}$ for any eye, then we check the BSCVA achieved during the manifest refraction. If the BSCVA is better than 20/20 for both eyes, then we ask the patient about complaints regarding night vision or, in general, quality of vision. If the patient does not report complaints, then the treatment to be applied is Aspheric aberration neutral.

If the patient reports complaints regarding quality of vision, the BSCVA is worse than 20/20 for any eye, or the global optical blur for both corneal and ocular wave-aberrations are above $0.50 \mathrm{DEq}$ for both eyes, then we compare corneal and ocular wave-aberrations. For this, we calculate the differential aberration (CWAb - OWAb, both centred at the line-of-sight) in terms of the Zernike expansion, and estimate the global optical difference.

If this global optical difference between corneal and ocular waveaberrations is below $0.25 \mathrm{DEq}$ for both eyes, we consider both corneal and ocular wave-aberrations as equivalent. In this case, the treatment to be applied depends on the available diameter of the wavefront maps and the scotopic pupil size. If the diameter of the Ocular- or Corneal-WAb map (the one providing the largest diameter) is at least as large as the scotopic pupil size (in natural dark conditions) reduced in $0.25 \mathrm{~mm}$, then Ocular- or Corneal-Wavefront-guided ablation is performed (the one providing the largest diameter), or Aspheric aberration neutral otherwise. Usually the size of the Ocular WAb maps is similar to the size of the scotopic pupils, whereas Corneal WAb maps are wider (up to $10 \mathrm{~mm}$ in diameter).

If the global optical difference between corneal and ocular waveaberrations is above $0.25 \mathrm{DEq}$ for any eye, we consider internal wave-aberration (IWAb) is relevant, then the treatment to be applied is Ocular-Wavefront-guided if the patient is neither in age nor in ophthalmic indications close to get an IOL exchange (due to e.g. lenticular opacities), otherwise no laser corneal refractive treatment is recommended (since IOL exchange is preferred). 


\begin{tabular}{|c|c|c|c|}
\hline Level of aberration & $\begin{array}{l}\text { Aspheric } \\
\text { aberration neutral }\end{array}$ & CW-guided & OW-guided \\
\hline $\begin{array}{l}\text { Corneal AND Ocular } \\
\text { Wavefronts < } \\
0.25 \text { Deq }\end{array}$ & Always & No & No \\
\hline $\begin{array}{l}\text { Corneal OR Ocular } \\
\text { Wavefront between } \\
0.25 \mathrm{DEq} \text { and } \\
0.50 \mathrm{Deq}\end{array}$ & $\begin{array}{l}\text { If BSCVA > 20/20 AND } \\
\text { no complaints about } \\
\text { quality or night vision }\end{array}$ & $\begin{array}{l}\text { If (BSCVA }<20 / 20 \text { OR complaints } \\
\text { about quality or night vision) AND } \\
\text { Internal Wavefront }<0.25 \mathrm{DEq}\end{array}$ & $\begin{array}{l}\text { If (BSCVA < 20/20 OR } \\
\text { complaints about quality or night } \\
\text { vision) AND no lenticular } \\
\text { problems }\end{array}$ \\
\hline $\begin{array}{l}\text { Corneal AND Ocular } \\
\text { Wavefronts > } \\
\text { 0.50Deq }\end{array}$ & $\begin{array}{l}\text { If wavefront maps smaller } \\
\text { than scotopic pupil }\end{array}$ & If Internal Wavefront $<0.25 \mathrm{DEq}$ & If no lenticular problems \\
\hline
\end{tabular}

Table 5: Indications chart.

\section{Section C.4 RESULTS}

(Resultados)

\section{C.4.1 Distribution of treatments \\ (Distribución de los tratamientos)}

In the 30 months we are using the SCHWIND AMARIS, we have performed 6467 LASIK treatments divided as: 5262 treatments (81\%) for myopicastigmatism using Aspheric aberration neutral profiles, 112 treatments (2\%) for hyperopic-astigmatism using Aspheric aberration neutral profiles, 560 treatments $(9 \%)$ using Corneal-Wavefront-guided profiles, and 533 treatments (8\%) using Ocular-Wavefront-guided profiles.

\section{C.4.2 Rate of retreatments \\ (Índice de retratamientos)}

From those, we have performed 22 re-treatments overall $(0.3 \%)$ : 18 $(0.3 \%)$ after myopic-astigmatism using AF profiles, no re-treatments $(0.0 \%)$ after hyperopic-astigmatism using AF profiles, 3 re-treatments $(0.5 \%)$ after $\mathrm{CW}$-guided profiles, and 1 re-treatment $(0.2 \%)$ after OW-guided profiles. 


\section{Section C.5 DISCUSSION}

(Discusión)

There are basically three types of approaches for planning a corneal refractive treatment. The first are those that have as their objective the elimination or reduction of the total aberrations of the eye. The main criticism to this approach argues that the goal "zero aberration" is inconsistent throughout the day due to accommodation, and little lasting, since aberrations change with age ${ }^{205}, 206,207$. The second approach is intended to correct all corneal aberrations, since corneal aberrations do not change with age 208 , 209. However, this concept might also be wrong considering corneal aberrations interact with internal aberrations, some of them being cancelled, and producing an aberration pattern of the total eye in general different from the aberration pattern of the cornea alone. Therefore, by only removing corneal aberration we might worsen the overall aberrations, since the internal aberration might not find a corneal aberration for compensation. In case that the corneal aberration is of the same sign as the internal aberration, the correction of the corneal aberration would be useful, as it would reduce the total aberration of the eye. A third approach tries not to induce aberrations. This type of treatment is not as ambitious, but much more simple to operate. The goal of the Aberration-Free ${ }^{T M}$ ablation profile is to provide a neutral HOAb ablation, ie to maintain the same HOAb profile both preoperatively with best spectacle correction and postoperatively without correction.

There is evidence of neural adaptation to the baseline wavefront profile. $^{69,72,73}$ The interaction between high-order-aberrations can be beneficial to visual quality regardless of the magnitude HOAb. ${ }^{187,188,189}$ Based on the random nature of the HOAb induction and current research, it may be beneficial to maintain the preoperative wavefront profile for a significant number of refractive surgery candidates.

We are not postulating that customized ablation algorithms in any form (ocular-wavefront-guided, corneal-wavefront-guided, topography-guided) are not be useful. Rather, that specific populations with specific demands deserve 
specific treatment solutions. Aspheric treatments aimed at preservation of the preoperative HOAb show their strengths in patients with preoperative BSCVA $20 / 20$ or better or in patients where the visual degradation cannot be attributable to the presence of clinically relevant HOAb (e.g. lens opacities).

The corneal wavefront customized approach shows its strength in cases where abnormal corneal surfaces are expected. Apart from the risk of additional ablation of corneal tissue, wavefront customized corneal ablation can be considered a safe and beneficial method.

Our experience suggests that wavefront customized treatments can only be successful, if pre-existing aberrations are greater than repeatability (e.g. repeatability of diagnostic ${ }^{210}$ and treatment devices) and biological noise (e.g. day-to-day variabilities in visual acuity, refraction, or aberration in the same subject).

Furthermore, coupling effects between different high order aberration terms, and between HOAb and manifest refraction have been found ${ }^{144},{ }^{211},{ }^{212}$ for example, between defocus and spherical aberration, or between $3^{\text {rd }}$ order aberrations and low order terms, between spherical aberration and coma, or between secondary and primary astigmatisms. These interactions may provide some relative visual benefits ${ }^{213}$, but may as well contribute as sources of uncertainty in the conversion of wavefront aberration maps to refractive prescriptions $^{137,139}$.

Notice that for comparing OWAb and CWAb, the analysis of the IWAb as CWAb - OWAb is mandatory since RMS(IWAb) accounts for any deviation (i.e. inductions and reductions of the wave-aberration both contribute positively to increase the RMS value).

The Decision Assistant Wizard presented here may theoretically be applied to any other laser platform offering aspheric, topography-guided, and wavefrontguided profiles, if appropriate analysis functions for CWAb, OWAb, and IWAb are available. Simplified versions with limited functionalities are also possible, if, for 
example, neither CW-analyses (i.e. no IWAb) nor topography-guided profiles are available.

\section{Section C.6 CONCLUSIONS}

(Conclusiones)

The desired outcome of non-wavefront-driven refractive surgery is to balance the effects on the wave-aberration, and, to provide normal eyes with perhaps the most natural unaltered quality of vision. While Ocular Wavefront treatments have the advantage of being based on Objective Refraction of the complete human eye system, whereas Corneal Wavefront treatments have the advantage of being independent from accommodation effects or light/pupil conditions; Aspheric treatments have the advantage of saving tissue, time and due to their simplicity offer better predictability.

\section{Section C.7 OUTLOOK}

(Perspectiva)

The clinical evaluations in this chapter were limited to correct the subjects' manifest refractions. However, in highly aberrated eyes, manifest refraction may become an art, a sort of guessing around the least blurred image. In further studies, systematic deviations from the measured manifest refractions, as well as other foreseeable couplings among Zernike coefficients will be evaluated. 


\title{
Topic D ANALYSIS OF THE LOSS OF ABLATION EFFICIENCY AT NON- NORMAL INCIDENCE \\ (Análisis de la pérdida de eficiencia de ablación para incidencia no-normal)
}

\begin{abstract}
AUTHOR CONTRIBUTIONS
Study concept and design (S.A.M.); data collection (S.A.M.); analysis and interpretation of data (S.A.M.); drafting (S.A.M.); critical revision (D.O., C.H., N.T., M.S.); statistical expertise (S.A.M.).
\end{abstract}

\section{Section D.1 ABSTRACT}

(Resumen)

A general method to analyze the loss of ablation efficiency at non-normal incidence in a geometrical way is provided.

The model is comprehensive and directly considers curvature, system geometry, applied correction, and astigmatism as model parameters, and indirectly laser beam characteristics and ablative spot properties.

The model replaces the direct dependency on the fluence by a direct dependence on the nominal spot volume and on considerations about the area illuminated by the beam, reducing the analysis to pure geometry of impact.

Compensation of the loss of ablation efficiency at non-normal incidence can be made at relatively low cost and would directly improve the quality of results.

\section{Section D.2 INTRODUCTION}

(Introducción)

The loss of ablation efficiency at non-normal incidence could explain, in part, many of the unwanted effects observed in refractive surgery, such as 
induction of spherical aberrations or high order astigmatism and consequently the extreme oblateness of postoperative corneas after myopic surgery.

Probably the earliest references related to the loss of ablation efficiency in laser refractive surgery refer to the observation of hyperopic postoperative refractions (hyperopic shifts) after negative cylinder ablation of the cornea. This hyperopic postoperative refraction had not been planned and depended on various factors, such as the laser system used, the amount of negative cylinder corrected, or the presence or absence of spherical terms in the ablation profile.

For the surgeons, it was difficult to adequately compensate this effect in their nomograms to achieve the desired refractive correction. According to some surgeons, some manufacturers introduced the concept of coupling factor, defined as the average sphere resulting from the application of one diopter of negative cylinder. Despite its empirical nature, this coupling factor enabled surgeons to plan their treatments with a reasonable degree of success.

One clue that relates this coupling factor to the loss of efficiency is analysis of the effect in the correction of simple negative astigmatisms (Figure 38). These cases revealed that the neutral axis became refractive, being less ablated in the periphery as compared to the centre. Similar experiences were observed using phototherapeutic keratectomy (PTK), but the results were not as conclusive, as cases where PTK is performed in large diameters are rare. 

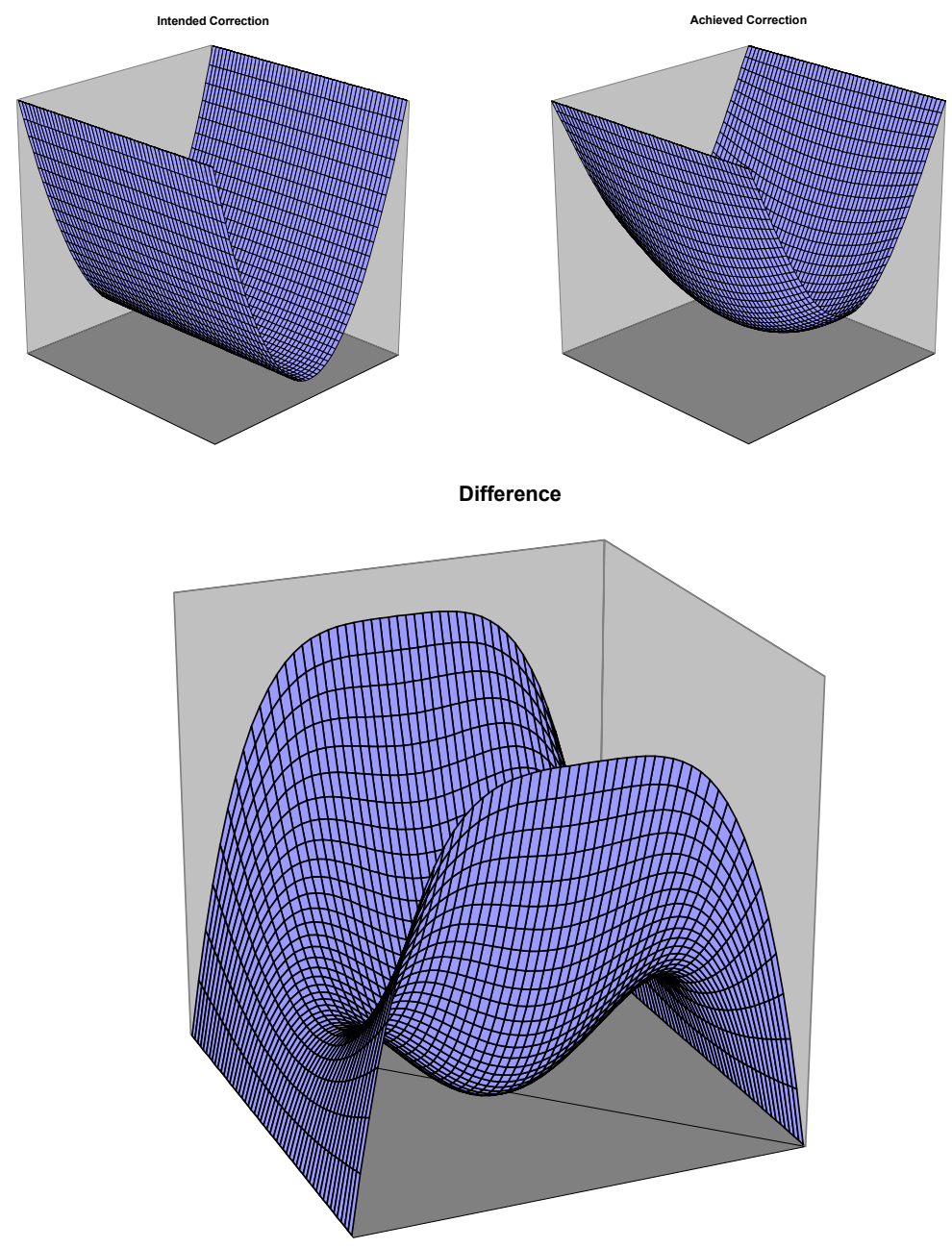

Figure 38: Hyperopic shift and coupling factor. Ablating a simple myopic astigmatism, the neutral axis became refractive, and the ablation depth in the periphery was smaller than in the centre.

\section{Section D.3 METHODS}

(Método)

D.3.1

Determination of the ablation efficiency at non-normal incidence

(Determinación de la eficiencia de la ablación para incidencia no-normal)

The issue of loss of ablation efficiency is composed of reflection losses and geometrical distortions (Figure 39). 

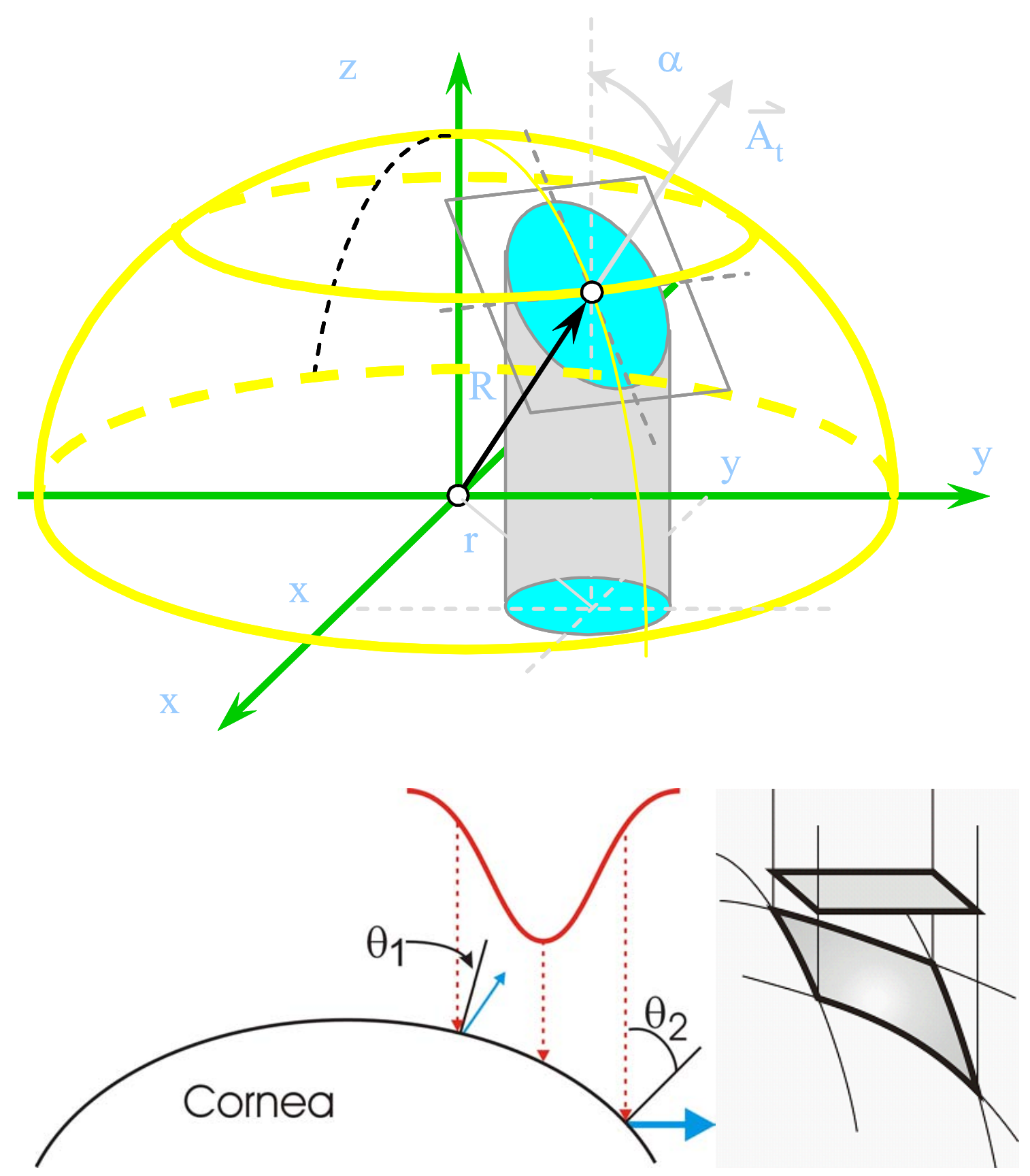

Figure 39: Loss on reflection (Fresnel's equations) dependent on the angle of incidence, and losses also dependent on the geometric distortion (angle of incidence).

The introduction of the concept of aberration-free profiles made it necessary to compensate for the induction of aberrations originating from deterministic and repeatable causes, thus minimizing the induction of aberrations to noise levels, so that a "new" model had to be developed. The aim in developing this model was to understand the mechanisms that govern the loss of 
ablation efficiency and to be able to predict their effect under different working conditions.

1 .- Considering the preoperative corneal curvature and asphericity as well as the intended refractive correction, the radius of curvature and asphericity the cornea will have after $50 \%$ of the treatment are estimated. (As the radius of corneal curvature changes during treatment, the efficiency also varies over treatment. The value at $50 \%$ of the treatment was chosen as a compromise to consider both the correction applied and the preoperative curvature, Figure 40).

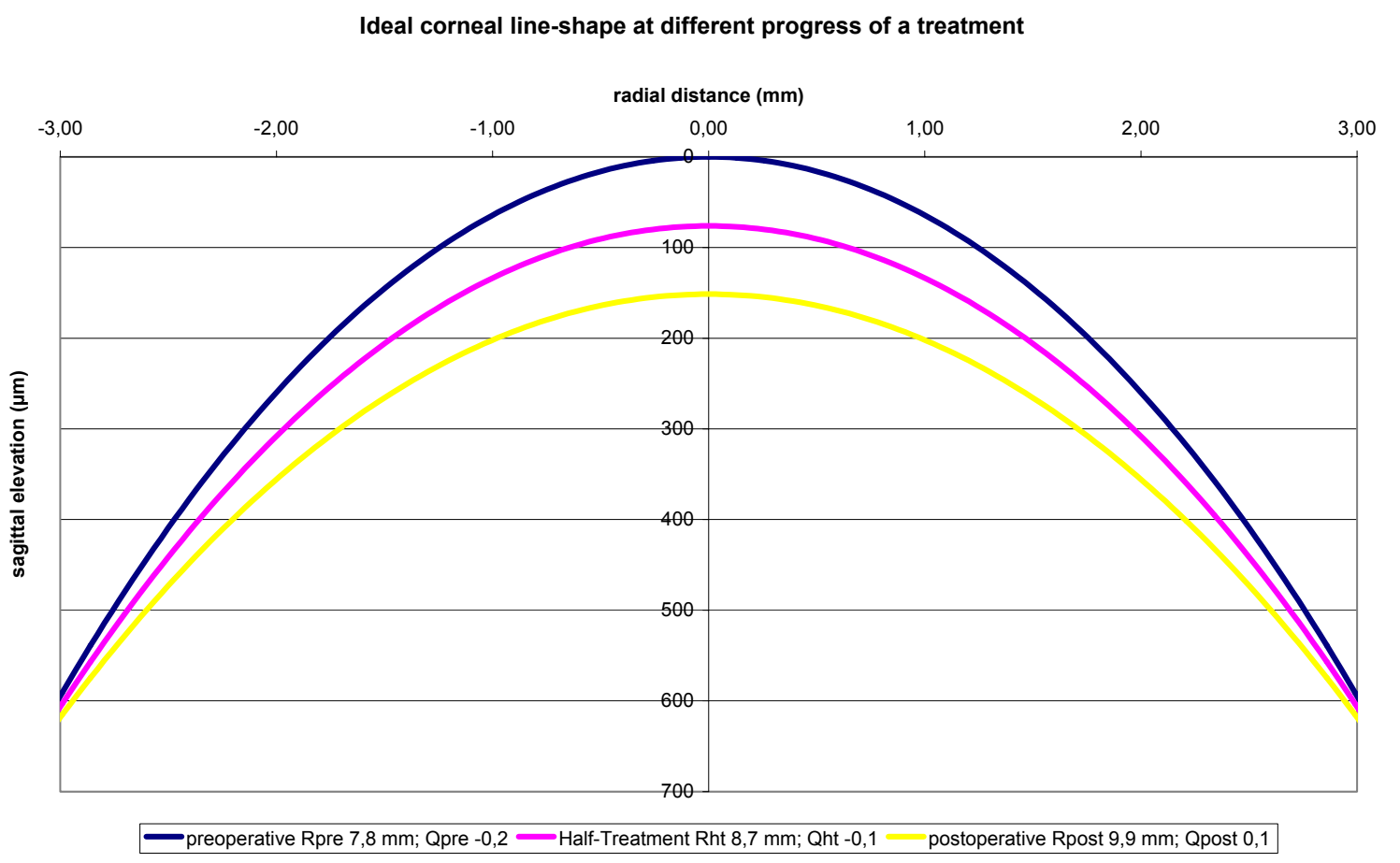

Figure 40: The radius of corneal curvature changes during treatment, efficiency also varies over treatment; the values at $50 \%$ of the treatment represent a reasonable compromise to consider both the correction applied and the preoperative curvature.

2 .- Considering the offset of the galvoscanners' neutral position compared to the system axis (Figure 41), the angle of incidence of the beam onto a flat surface perpendicular to the axis of the laser is calculated: 


$$
\alpha(x, y)=\arctan \left(\frac{\sqrt{\left(x-X_{G}\right)^{2}+\left(y-Y_{G}\right)^{2}}}{d_{G}}\right)
$$

where $\alpha$ is the angle of incidence on a "flat" surface, $X_{G}, Y_{G}$ the position of the galvoscanners, $x, y$ the radial positions of the incident beam, and $d_{G}$ the vertical distance from the last galvoscanner to the central point of the ablation.

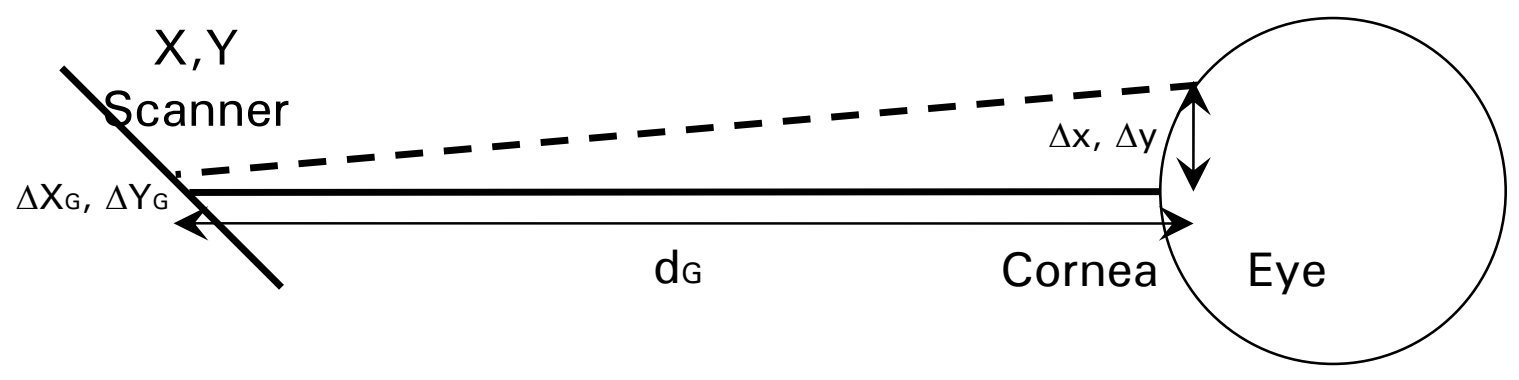

Figure 41: The offset of the galvoscanners from the axis of the system is considered in the calculation of the angle of incidence of the beam onto a flat surface perpendicular to the axis of the laser.

3 -- Considering the calculated curvature and asphericity at $50 \%$ of the treatment, the local angle of the cornea is calculated. Assuming the cornea as an ellipsoid, which satisfies Baker's equation, the following equation results:

$$
\begin{aligned}
& x^{2}+y^{2}+z^{2}\left(Q_{H T}+1\right)-2 z R_{H T}=0 \\
& \theta(x, y)=\arctan \left(\frac{\frac{\sqrt{x^{2}+y^{2}}}{R_{H T}}\left(Q_{H T}+1\right)}{Q_{H T}+\sqrt{1-\left(Q_{H T}+1\right) \times \frac{x^{2}+y^{2}}{R_{H T}^{2}}}}\right)
\end{aligned}
$$

where $\theta$ is the angle of the local tilt of a corneal location, and $R_{H T}$ and $Q_{H T}$ are the predicted radius of curvature and asphericity quotient at $50 \%$ of treatment progress.

4 .- To calculate the angle of incidence for each point on the corneal surface 


$$
\beta=\operatorname{ang}(\alpha, \theta)
$$

applies, where $\beta$ is angle of incidence.

5 -- The ablation efficiency is calculated by consideration of geometric distortions, reflections losses, and spot overlapping:

$$
\begin{aligned}
& I_{E f f}(x, y)=I(x, y) . \\
& \cdot \cos (\beta(x, y)) e^{2\left(\left(\frac{\left(x-x_{0}\right)^{2}+\left(y-y_{0}\right)^{2} \cos ^{2}(\beta(x, y))}{R_{0}^{2}}\right)^{N}-\left(\frac{\left(x-x_{0}\right)^{2}+\left(y-y_{0}\right)^{2}}{R_{0}^{2}}\right)^{N}\right)} . \\
& \cdot(1-R(x, y))
\end{aligned}
$$

where the factor $\cos (\beta(x, y)) e^{2\left(\left(\frac{\left(x-x_{0}\right)^{2}+\left(y-y_{0}\right)^{2} \cos ^{2}(\beta(x, y))}{R_{0}^{2}}\right)^{N}-\left(\frac{\left(x-x_{0}\right)^{2}+\left(y-y_{0}\right)^{2}}{R_{0}^{2}}\right)^{N}\right)}$ corresponds to the geometric distortions, the factor $(1-R(x, y))$ corresponds to the reflections losses, and $y$ is the radial direction along which angular projection occurs.

$$
\operatorname{Eff}(x, y)=\frac{\sum_{-m}^{m} \sum_{-n}^{n} d(x, y)}{\sum_{-m}^{m} \sum_{-n}^{n} d(0,0)}
$$

The sums represent the overlap and extent along the size of the impact.

Using the efficient radiant exposure and applying Lambert-Beer's law (blow-off model), we get:

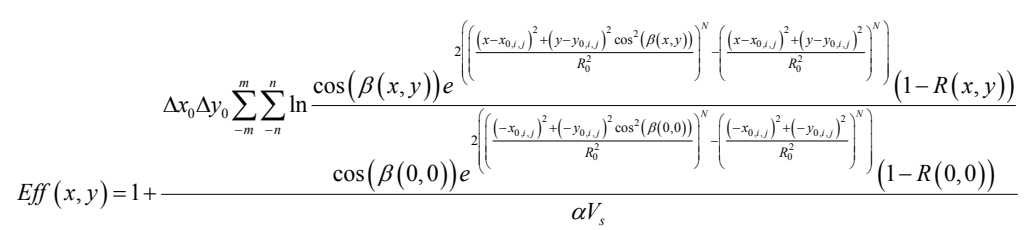

where $\Delta \mathrm{x}_{0}$ and $\Delta \mathrm{y}_{0}$ are the spot overlapping distances (i.e. the distance between two adjacent pulses) and $\mathrm{x}_{0, \mathrm{i}, \mathrm{j}}$ and $\mathrm{y}_{0, \mathrm{i}, \mathrm{j}}$ are the respective centres of the different spots contributing to the overlap at one corneal location.

If the galvoscanners are coaxial with the laser system: 


$$
\beta(x, y)=\alpha(x, y)+\theta(x, y)
$$

If the distance from the last mirror to the ablation plane is large:

$$
\begin{gathered}
d \gg r \\
\alpha(x, y) \rightarrow 0 \\
\beta(x, y) \simeq \theta(x, y) \\
\beta(0,0)=0 \\
E f f(x, y)=1+\frac{A_{S} \ln \left(\cos (\beta(x, y)) \frac{(1-R(x, y))\left(n_{t}+1\right)^{2}}{4 n_{t}}\right)}{\alpha V_{s}}+ \\
+\frac{2 \Delta x_{0} \Delta y_{0} \sum_{-m}^{m} \sum_{-n}^{n}\left(\left(\frac{\left(x-x_{0, i, j}\right)^{2}+\left(y-y_{0, i, j}\right)^{2} \cos ^{2}(\beta(x, y))}{R_{0}^{2}}\right)^{N}-\left(\frac{\left(x-x_{0, i, j}\right)^{2}+\left(y-y_{0, i, j}\right)^{2}}{R_{0}^{2}}\right)^{N}\right)}{\alpha V_{s}}
\end{gathered}
$$

If the spot overlapping is very tight and many pulses contribute to the ablation at each corneal location (i.e. $\Delta x_{0}, \Delta y_{0} \ll F P$ ), it further simplifies to:

$$
\begin{aligned}
& E f f(x, y)=1+\frac{A_{S} \ln \left(\cos (\beta(x, y)) \frac{(1-R(x, y))\left(n_{t}+1\right)^{2}}{4 n_{t}}\right)}{\alpha V_{s}}+ \\
& +\frac{2 \int_{-\frac{F P}{2}}^{+\frac{F P}{2}} \int_{\frac{F P}{2}}^{+\frac{F P}{2}}\left(\left(\frac{\left(x-x_{0}\right)^{2}+\left(y-y_{0}\right)^{2} \cos ^{2}(\beta(x, y))}{R_{0}^{2}}\right)^{N}-\left(\frac{\left(x-x_{0}\right)^{2}+\left(y-y_{0}\right)^{2}}{R_{0}^{2}}\right)^{N}\right) d x_{0} d y_{0}}{\alpha V_{s}}
\end{aligned}
$$

In this way, we removed the direct dependency on the fluence and replaced it by a direct dependence on the nominal spot volume and on considerations about the area illuminated by the beam, reducing the analysis to pure geometry of impact.

There are two opposing effects: the beam is compressed due to reflection and at the same time expands due to its projection angle.

6.- The compensation would be the inverse of efficiency: 


$$
\kappa_{i j}=1 / E f f_{i j}
$$

7 .- We can develop the efficiency (or the compensation) in power series:

$$
\begin{aligned}
& \text { Eff }=1-A(r / R)^{2}-B(r / R)^{4}+\ldots \\
& \kappa=1+C(r / R)^{2}+D(r / R)^{4}+\ldots
\end{aligned}
$$

Therefore, instead of using the radius at half of the treatment, we can calculate the overall effect of the variation in efficiency over treatment.

$$
\begin{aligned}
& \operatorname{Eff}\left(R_{i}, R_{f}, r\right)=\frac{\int_{R_{i}}^{R_{f}}(E f f) d R}{\int_{R_{i}}^{R_{f}} d R} \\
& \operatorname{Eff}\left(R_{i}, R_{f}, r\right) \approx 1-A \frac{r^{2}}{R_{i} R_{f}}-B \frac{r^{4}}{3 R_{i}^{3} R_{f}^{3}}\left(R_{i}^{2}+R_{i} R_{f}+R_{f}^{2}\right) \\
& \kappa\left(R_{i}, R_{f}, r\right)=\frac{\int_{R_{i}}^{R_{f}}(\kappa) d R}{\int_{R_{i}}^{R_{f}} d R} \\
& \kappa\left(R_{i}, R_{f}, r\right) \approx 1+C \frac{r^{2}}{R_{i} R_{f}}+D \frac{r^{4}}{3 R_{i}^{3} R_{f}^{3}}\left(R_{i}^{2}+R_{i} R_{f}+R_{f}^{2}\right)
\end{aligned}
$$

8 .- Returning to the concept of radius and asphericity at half of the treatment, we can further simplify the model by defining an averaged spot depth as if the energy profile of the beam were flat and apply the simple model:

$$
\begin{aligned}
& \bar{d}=\frac{\int_{0}^{2 \pi} \int_{0}^{\frac{F P}{2}} \frac{\ln \left(\frac{I_{0}}{I_{T h}}\right)-2\left(r / R_{0}\right)^{2 N}}{\alpha} r d r d \theta}{\int_{0}^{2 \pi} \int_{0}^{\frac{F P}{2}} r d r d \theta} \\
& \bar{d}=\frac{1}{\alpha} \frac{N}{N+1} \ln \left(\frac{I_{0}}{I_{T h}}\right)
\end{aligned}
$$


In general:

$$
\begin{aligned}
& \bar{d}=\frac{V_{S}}{A_{S}} \\
& E f f=1+\frac{\ln \left(\frac{\cos \beta(1-R)\left(n_{t}+1\right)^{2}}{4 n_{t}}\right)}{\alpha \bar{d}} \\
& E f f=1+\frac{A_{S} \ln \left(\frac{\cos \beta(1-R)\left(n_{t}+1\right)^{2}}{4 n_{t}}\right)}{\alpha V_{S}}
\end{aligned}
$$

If this model is applied to a spherical surface $(Q=0)$ and the depth per layer equals the depth per pulse or the spots do not overlap, it simplifies to the simple model.

9 .- Losses due to reflection are generally negligible. This is so because the highest reflection contribution occurs for normal incidence, as well, and this component is already normalized. Therefore, we can further simplify the model as below:

$$
\begin{aligned}
& \theta=\arcsin \left(\frac{\sqrt{X^{2}+Y^{2}}}{R_{H T}}\right) \\
& \text { Eff }=1+\frac{A_{S} \ln (\cos \theta)}{\alpha V_{S}}
\end{aligned}
$$

10 -- In the case of a strong astigmatic component, we can continue to calculate the $50 \%$ of treatment:

$$
D_{E f f}=D_{\varphi} \times \cos ^{2}(\delta-\varphi)+D_{\varphi+\pi / 2} \times \sin ^{2}(\delta-\varphi)
$$




$$
R_{E f f}=\frac{R_{\varphi} \times R_{\varphi+\pi / 2}}{R_{\varphi} \times \sin ^{2}(\delta-\varphi)+R_{\varphi+\pi / 2} \times \cos ^{2}(\delta-\varphi)}
$$

\section{Section D.4 RESULTS}

(Resultados)

As the radius of corneal curvature changes during treatment, the efficiency varies over treatment, as well. The ablation efficiency decreases steadily with increasing curvature, thus resulting in improvement of ablation efficiency during myopic corrections and increasing loss of ablation efficiency during hyperopic corrections (Figure 42).

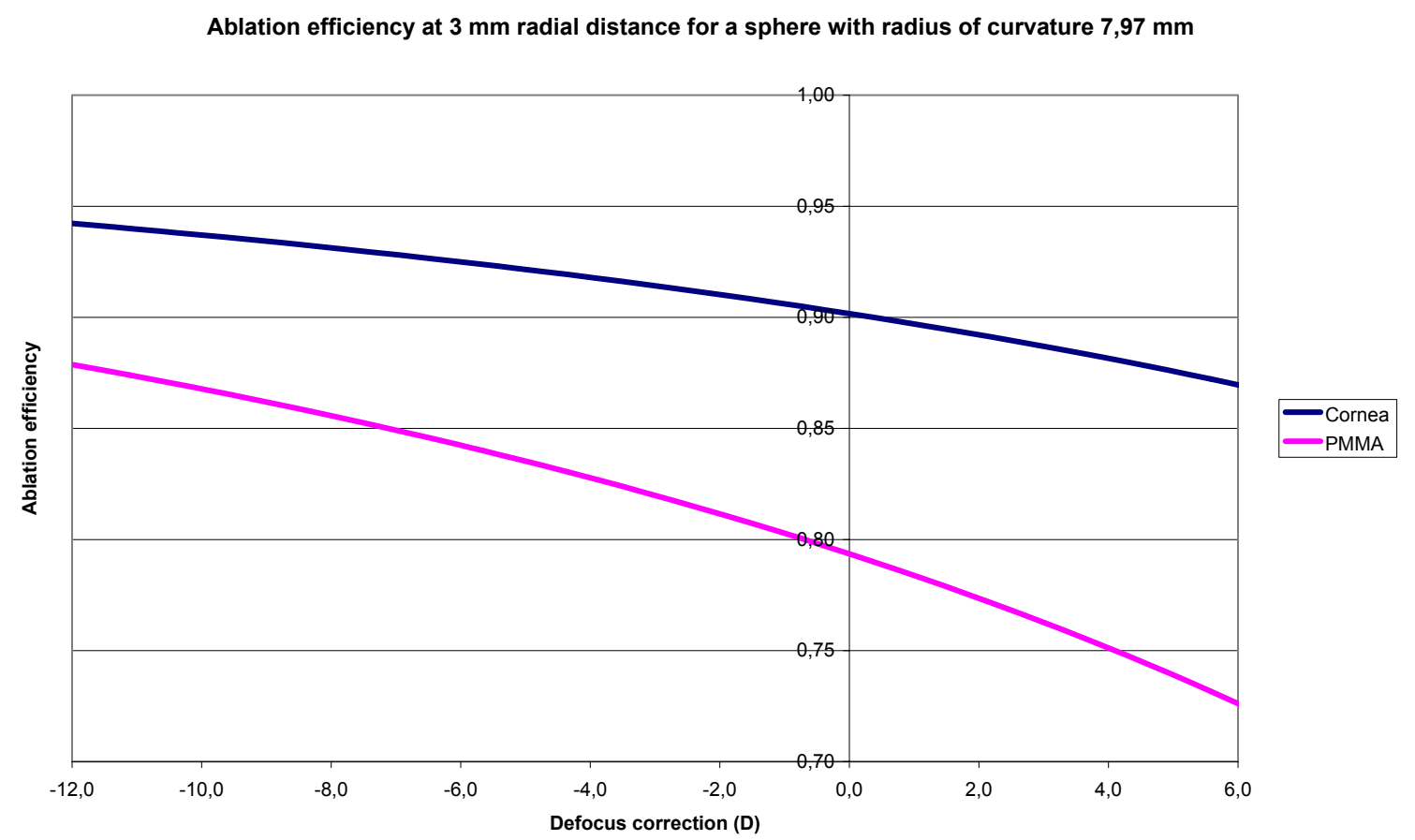

Figure 42: Ablation efficiency at $3 \mathrm{~mm}$ radial distance for a sphere with $7.97 \mathrm{~mm}$ radius of curvature. The ablation efficiency was simulated for an excimer laser with a peak radiant exposure of $120 \mathrm{~mJ} / \mathrm{cm}^{2}$ and a full-width-half-maximum (FWHM) beam size of $2 \mathrm{~mm}$. The radius of corneal curvature changes during 
treatment, accordingly also the efficiency varies over treatment. Note the improvement of ablation efficiency during myopic corrections as opposed to the increased loss of ablation efficiency during hyperopic corrections.

The model considers curvature based upon radius and asphericity. As expected, a parabolic surface provides higher peripheral ablation efficiency (due to prolate peripheral flattening) compared to an oblate surface (with peripheral steepening) (Figure 43).

Contribution of the asphericity quotient to the ablation efficicency

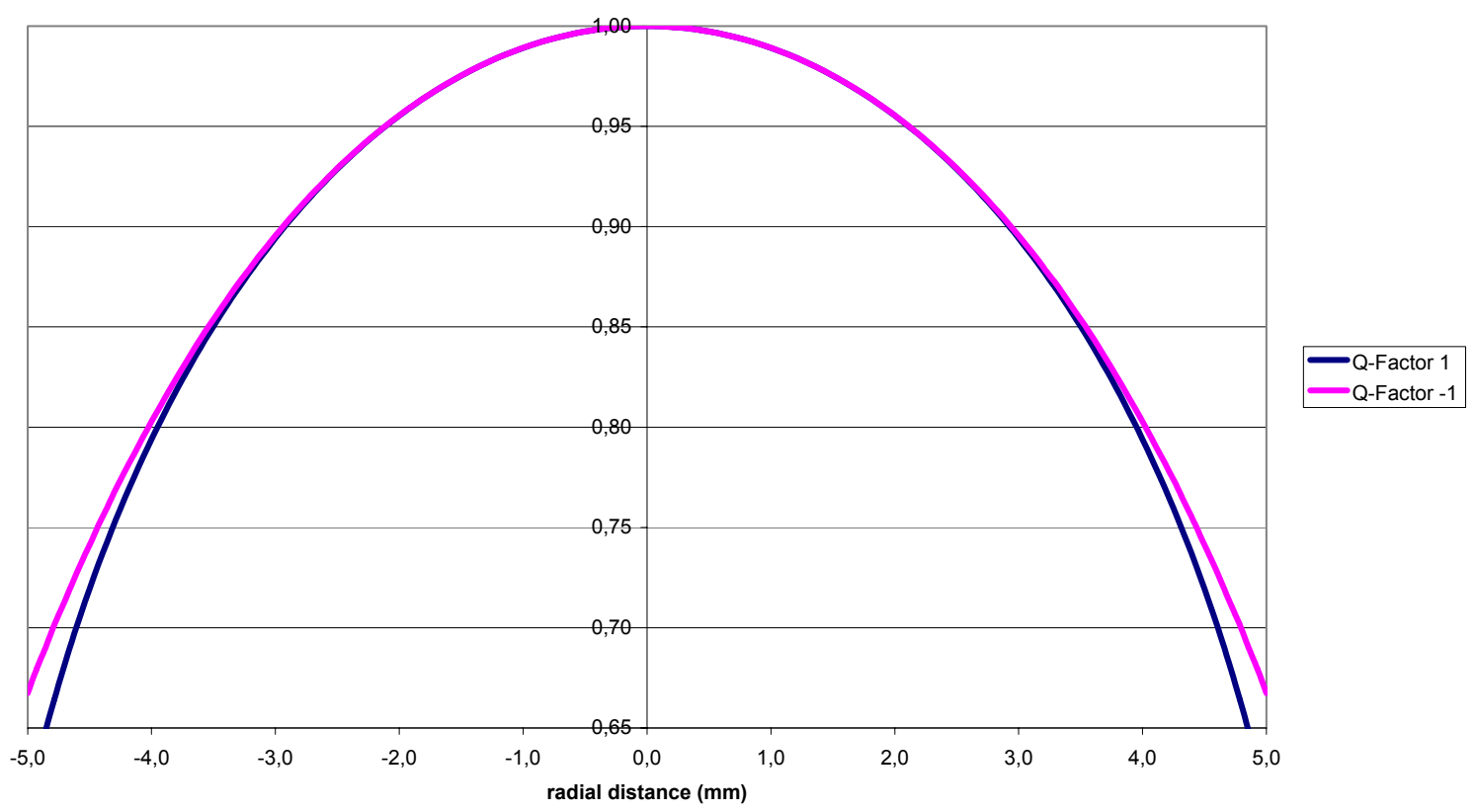

Figure 43: Contribution of the asphericity quotient to the ablation efficiency for a radius of $7.97 \mathrm{~mm}$ curvature. The ablation efficiency at the cornea was simulated for an excimer laser with a peak radiant exposure of $120 \mathrm{~mJ} / \mathrm{cm}^{2}$ and a beam size of $2 \mathrm{~mm}$ (FWHM). Note the identical ablation efficiency close to the vertex as opposed to differences in ablation efficiency at the periphery. A parabolic surface provides higher peripheral ablation efficiency (due to prolate peripheral flattening) compared to an oblate surface (with peripheral steepening). 
The model considers efficiency losses due to reflection losses, geometric distortions, and spot overlapping. Note that the reflection losses already exist for normal incidence and decrease by a very small amounts towards the periphery (Figure 44). Although normal reflection losses approximately amount to $5 \%$, they do not increase excessively for non-normal incidence. As our calculation defined ablation efficiency for a general incidence as the ratio between the spot volume for general incidence and the spot volume for normal incidence, it is evident that the so-defined efficiency equals 1 for normal incidences.

Contribution of the reflection and distortion losses to the ablation efficicency

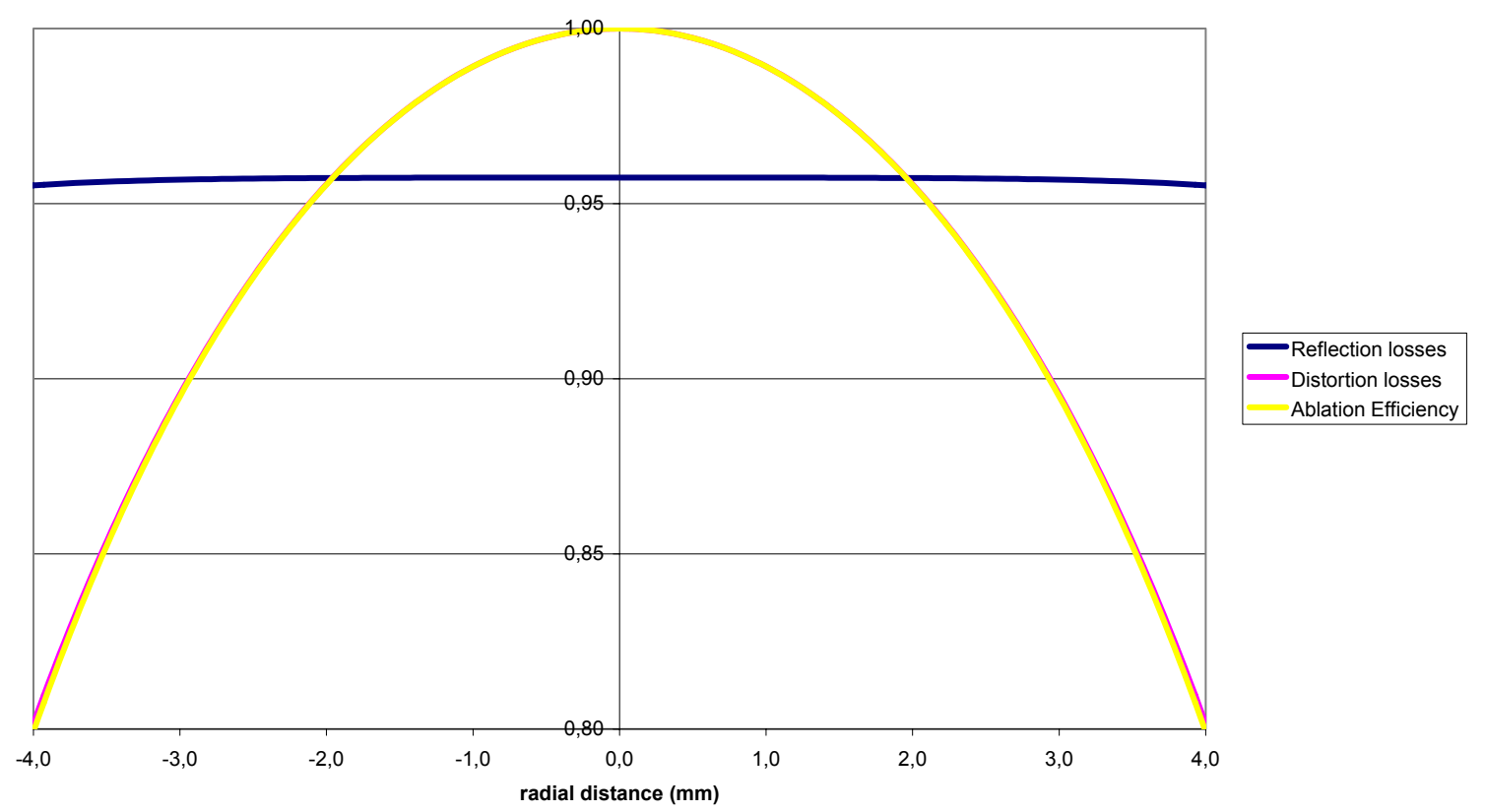

Figure 44: Contribution of the reflection and distortion losses to ablation efficiency for a sphere with $7.97 \mathrm{~mm}$ radius of curvature. Note that the reflection losses already exist with normal incidence and decrease very slightly towards the periphery. Although normal reflection losses approximately amount to $5 \%$, they do not increase excessively for non-normal incidence. As our calculation defined the ablation efficiency for a general incidence as the ratio between the spot volume for general incidence and the spot volume for normal incidence, it is evident that the so-defined efficiency equals 1 for normal incidences. 
Losses due to reflection are generally negligible, since the highest reflection contribution also occurs with normal incidence.

We removed the direct dependency on the fluence and replaced it by a direct dependence on the nominal spot volume and on considerations about the area illuminated by the beam, thus reducing the analysis to pure geometry of impact. Note that efficiency is very poor close to the ablation threshold and steadily increases with increasing radiant exposure approaching 100\% ablation efficiency (Figure 45). It should also be noted that the difference between efficiencies for cornea and PMMA increases with lowering radiant exposure (Figure 45).

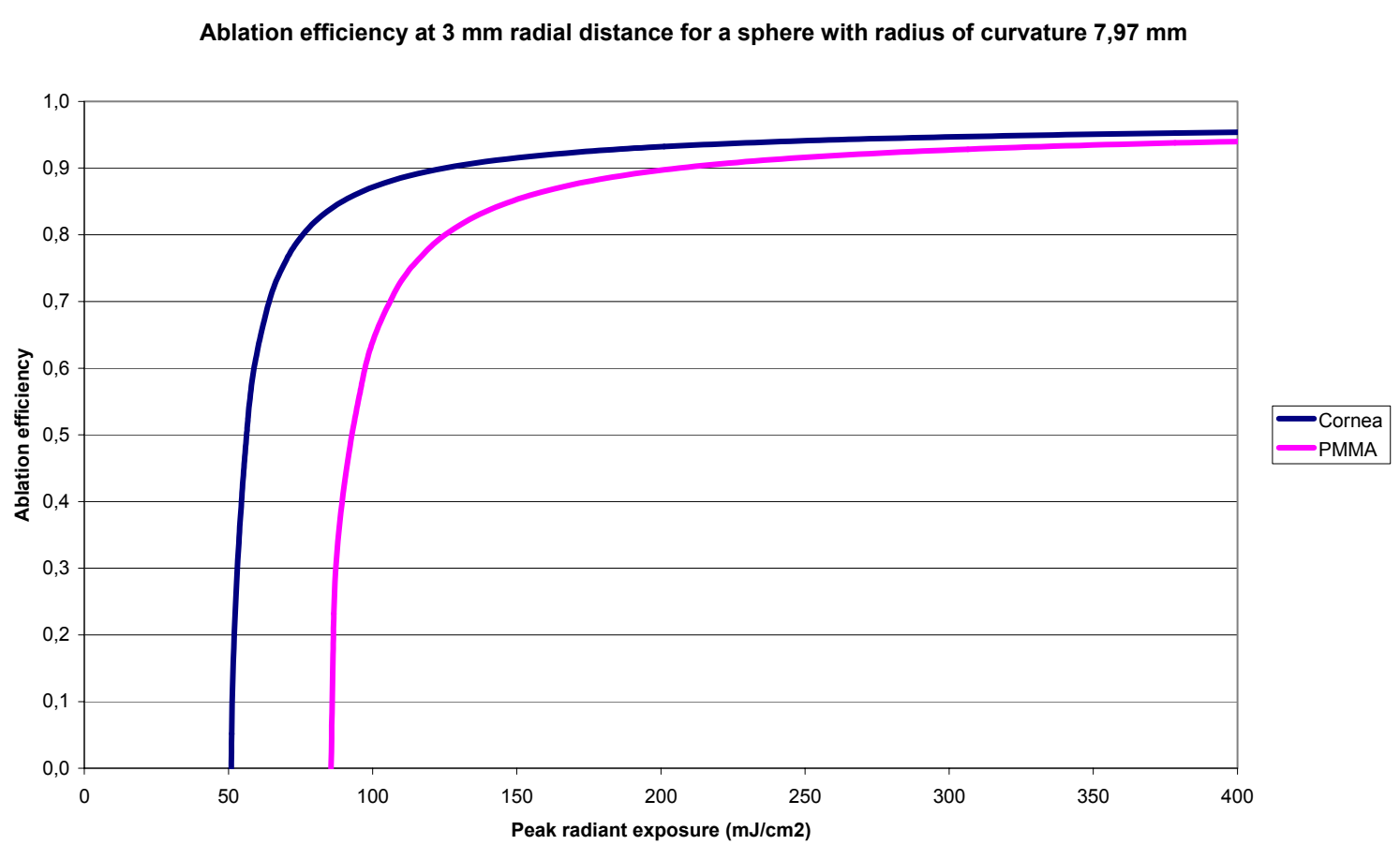

Figure 45: Ablation efficiency at $3 \mathrm{~mm}$ radial distance for a sphere with $7.97 \mathrm{~mm}$ radius of curvature. The ablation efficiency was simulated for an excimer laser with a peak radiant exposure up to $400 \mathrm{~mJ} / \mathrm{cm}^{2}$ and FWHM beam size of $2 \mathrm{~mm}$.

Finally, we compared the ablation efficiencies for cornea and PMMA for the spherical shapes prior to receiving any laser shot was evaluated, and evaluate the 
average ablation efficiencies for the surfaces during $a-12 \mathrm{D}$ and $\mathrm{a}+6 \mathrm{D}$ correction, respectively (Figure 46 to Figure 48). Again, note that the ablation efficiency decreases steadily with increasing curvature, resulting in an improvement of ablation efficiency during the $-12 \mathrm{D}$ correction as opposed to decreased ablation efficiency during the $+6 \mathrm{D}$ correction .

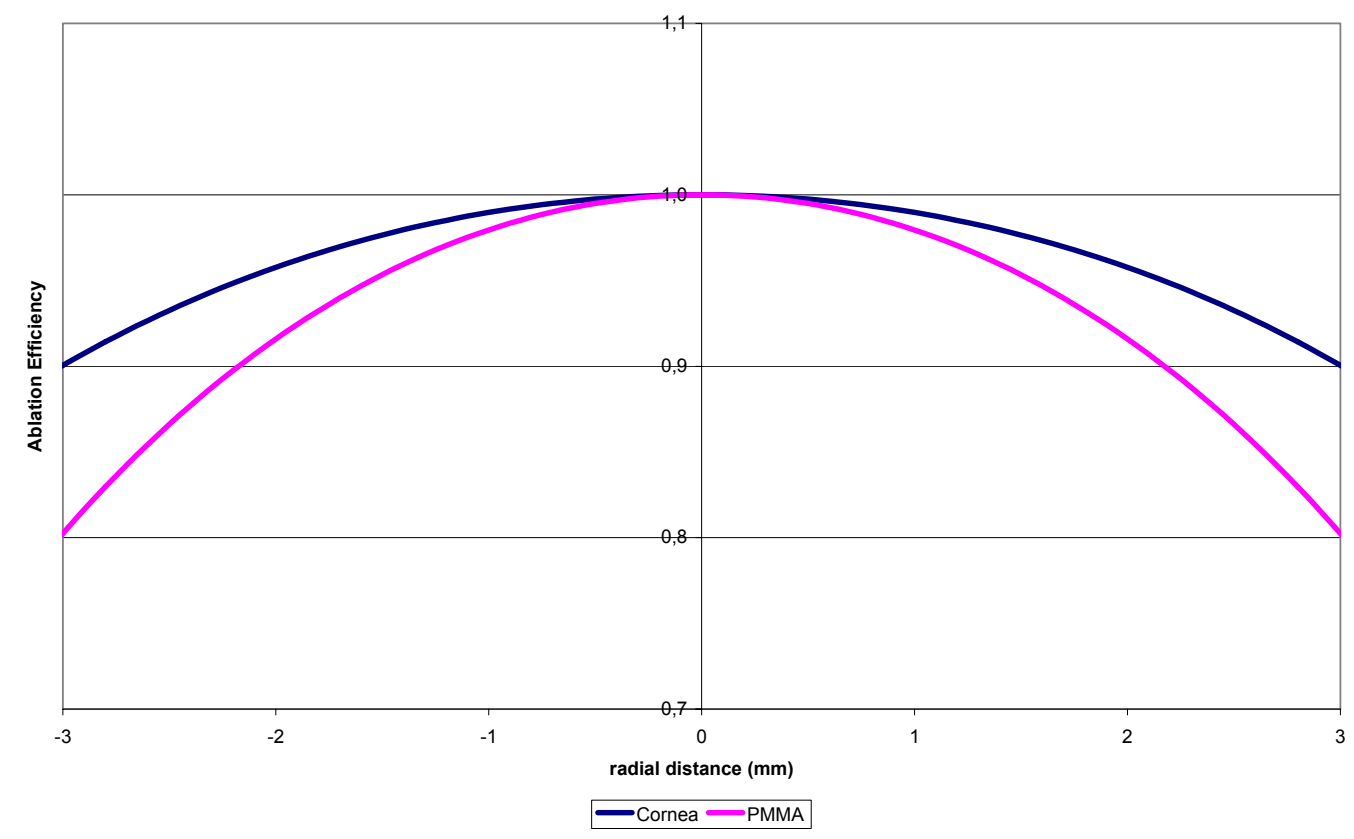

Figure 46: Efficiency obtained with the proposed model for the conditions reported by Dorronsoro et al. ${ }^{214}$ Ablation efficiency for a sphere with $7.97 \mathrm{~mm}$ radius of curvature. The ablation efficiency was simulated for an excimer laser with a peak radiant exposure of $120 \mathrm{~mJ} / \mathrm{cm}^{2}$ and FWHM beam size of $2 \mathrm{~mm}$. 


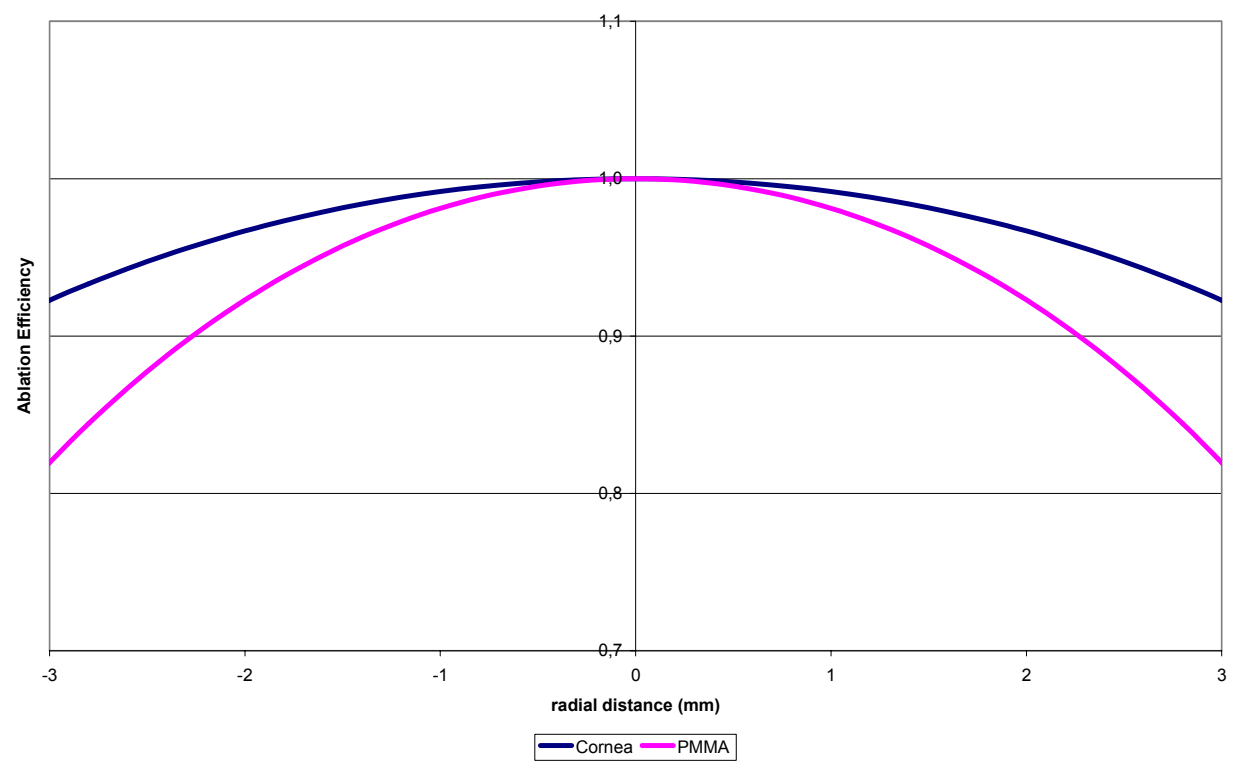

Figure 47: Efficiency obtained with the proposed model for the conditions reported by Dorronsoro et al. ${ }^{214}$ Average ablation efficiency for a sphere with 7.97 $\mathrm{mm}$ preoperative radius of curvature and a correction of $-12 \mathrm{D}$. The ablation efficiency was simulated for an excimer laser with a peak radiant exposure of 120 $\mathrm{mJ} / \mathrm{cm}^{2}$ and FWHM beam size of $2 \mathrm{~mm}$. The radius of corneal curvature changes during treatment, consequently, also the efficiency varies over treatment. Note the improvement of ablation efficiency.

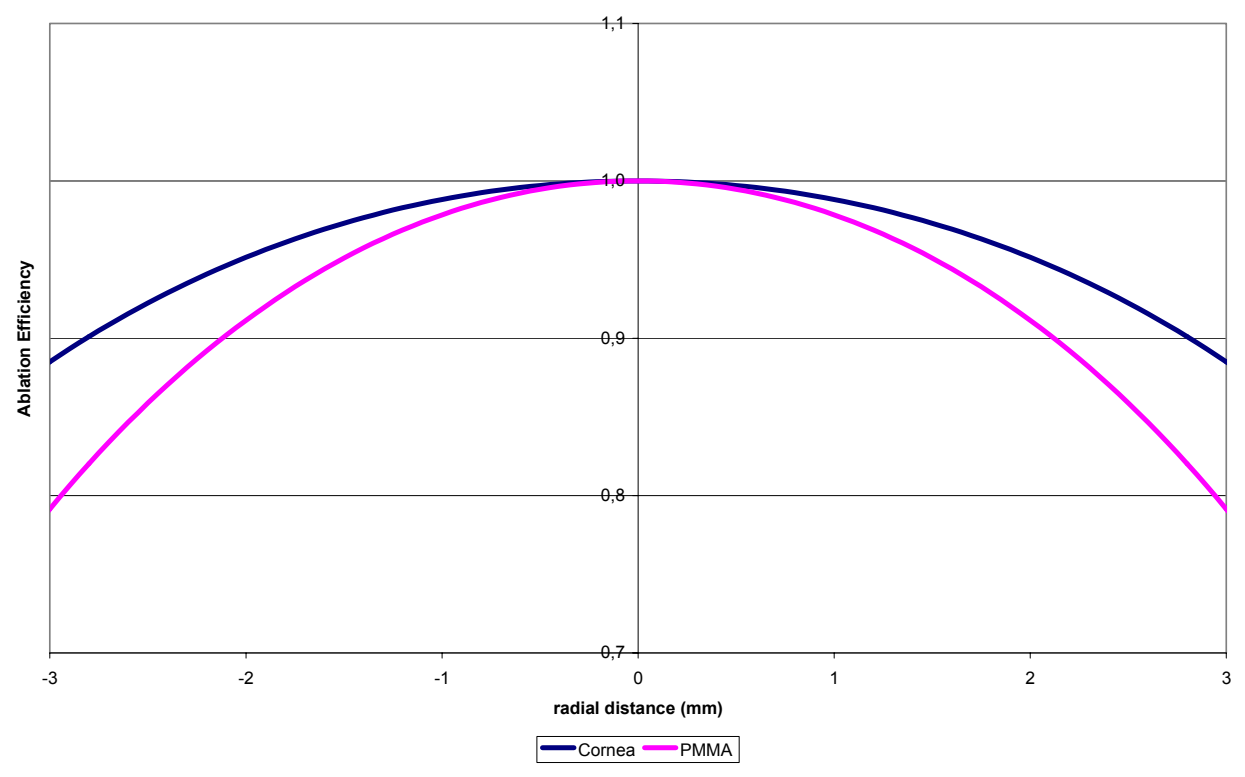

Figure 48: Efficiency obtained with the proposed model for the conditions reported by Dorronsoro et al. ${ }^{214}$ Average ablation efficiency for a sphere with 7.97 $\mathrm{mm}$ preoperative radius of curvature and a correction of $+6 \mathrm{D}$. The ablation 
efficiency was simulated for an excimer laser with a peak radiant exposure of 120 $\mathrm{mJ} / \mathrm{cm}^{2}$ and FWHM beam size of $2 \mathrm{~mm}$. The radius of corneal curvature changes during treatment, consequently also the efficiency varies over treatment. Note the increased loss of ablation efficiency during hyperopic corrections.

\section{Section D.5 DISCUSSION}

(Discusión)

Our approach reduces all calculations to geometrical analysis of the impact; the ablation efficiency does not primarily depend on the radiant exposure, but rather on the volume per single shot for the specific material and also on overlap and geometric considerations of the irradiated area per shot, supported by radiant exposure data. Different effects interact; the beam is compressed due to the loss of efficiency, but at the same time expands due to the angular "projection." Using this model for ablation efficiency at non-normal incidence in refractive surgery, up to $42 \%$ of the reported increase in spherical aberrations can be explained.

Applying this comprehensive loss of efficiency model to a pure myopia profile to get the achieved profile etched into the cornea, we observed that the profile "shrinks," steepening the average slope and then slightly increasing the myopic power of the profile as well as inducing spherical aberrations. The net effect can be expressed as an unintended positive spherical aberration and a small overcorrection of the spherical component. Applying this model to a pure hyperopia profile, we observed that the profile "softens," flattening the average slope and then decreasing the hyperopic power of the profile as well as inducing spherical aberrations. The net effect can be expressed as an undercorrection of the spherical component and a small amount of induced negative spherical aberration. Applying this model to a PTK profile, we observed that the flat profile becomes myopic due to the loss of efficiency, resulting in an unintended myopic ablation (hyperopic shift).

Corneal curvature and applied correction play an important role in the determination of the ablation efficiency and are taken into account for accurate results. As a compromise between accuracy and simplicity, we decided to use 
the predicted radius of corneal curvature after $50 \%$ of the treatment as curvature metric for determination the ablation efficiency. However, corneal toricity and applied astigmatism, even though easily computed using the comprehensive model, do not have a relevant impact as long as their values correspond to those of normal corneas. Only when toricity or astigmatism exceeds $3 \mathrm{D}$, their effects on ablation efficiency start to be significant.

System geometry is considered in this model using the offset of the galvoscanners' neutral position compared to the system axis as well as the distance from the last galvoscanner to the central point of the ablation. Nevertheless, usually the galvoscanners are coaxial with (or determine the axis of) the laser system, and the distance from the last galvo-mirror to the ablation plane used to be large.

We removed the direct dependency on the fluence and replaced it by a direct dependence on the nominal spot volume and on considerations about the area illuminated by the beam, reducing the analysis to pure geometry of impact in this way. We found that the efficiency is very poor close to the ablation threshold and steadily increases with increasing radiant exposure approaching $100 \%$ ablation efficiency. Also, differences between the efficiencies for the cornea and PMMA were observed to increase with lowering radiant exposure. Actually, the key factor is not the peak radiant exposure of the beam, but rather the average spot depth (i.e. the ratio spot volume to spot area).

The detailed model determines ablation efficiency considering geometric distortions, reflections losses, and spot overlapping. Geometric distortions are very important, because the angular projection expands the beam, thus spreading the beam energy over a wider area and flattening its radiant exposure. At the same time, spot overlapping is a major parameter, especially in flying-spot systems, where the spot spacing is small compared to the spot width and multiple spots overlap, all contributing to the ablation at each corneal location, whereas reflection losses can be neglected, because important reflection contribution already occurs in normal incidence and does not excessively increase in nonnormal incidence.

Surface asphericity before ablation, and especially after completion of $50 \%$ of the treatment, refines this comprehensive approach. Simulations, based on 
cornea and PMMA, for extreme asphericity values (from asphericity quotient of -1 to +1 ) showed minor effects with differences in ablation efficiency of $1 \%$ in the cornea and $2 \%$ in PMMA even at distances of $4 \mathrm{~mm}$ radially from the axis. Hence, for corneas with normal curvature and asphericity spherical geometry seems to be a reasonably simple approach for calculating the ablation efficiency at non-normal incidence.

The loss of efficiency in the ablation and non-normal incidence are responsible for much of the induction of spherical aberrations observed in the treatments as well as the excessive oblateness of postoperative corneas observed after myopic corrections (also part of some overcorrections observed in high myopias and many undercorrections observed in hyperopia) with major implications for treatment and optical outcome of the procedure. Compensation can be made at relatively low cost and directly affects the quality of results (after a correction of the profiles to avoid overcorrections or undercorrections in defocus and marginally in the cylinder).

Analyzing the different models available, the simple model of ablation efficiency at non-normal incidence bases its success on its simplicity, which forms the reason why it is still used by some trading houses. The problems arising from the simple model directly derive from its simplicity, and consequently the limitations of application as required by their implicit assumptions. The simple model does not consider the calculation or the asphericity of the cornea, or the energy profile of the beam, or the overlap of impacts, overestimating the ablation efficiency, underestimating its compensation.

The Jiménez-Anera mode ${ }^{160,161}$ provides an analytical expression for an adjustment factor to be used in photorefractive treatments, which includes both compensation for reflection and for geometric distortion, incorporating non-linear deviations of Lambert-Beer's law. It eliminates some problems of the simple model, because it considers the energy profile of the beam, overlapping, and losses by reflection. However, it does not consider the calculation nor asphericity of the cornea, it assumes that the energy profile is a Gaussian beam, assumes unpolarized light, it does not address the size or shape of the impact, it does not consider that the radius of curvature changes locally throughout the treatment, 
and accordingly the angle of incidence. Therefore, it often slightly overestimates the ablation efficiency, partially underestimating its compensation.

The Dorronsoro-Cano-Merayo-Marcos mode ${ }^{214}$ provides a completely new approach to the problem. It eliminates many of the problems of both the simple model and the model by Jiménez-Anera ${ }^{160,161}$, reducing the number of assumptions and using an empirical approach. Even so, it assumes that the reflection losses on cornea and PMMA are identical, it does not consider the local radius of corneal curvature, its asphericity or applied correction, it does not consider that the radius of curvature changes locally throughout the treatment, and accordingly the angle of incidence, and it does not consider the effects for different values of fluence.

The model described here eliminates the direct dependence on fluence and replaces it by direct considerations on the nominal spot volume and on the area illuminated by the beam, reducing the analysis to pure geometry of impact. The proposed model provides results essentially identical to those obtained with the model by Dorronsoro-Cano-Merayo-Marcos. ${ }^{214}$ Additionally, it offers an analytical expression including some parameters that were ignored (or at least not directly addressed) in previous analytical approaches. The good agreement of the proposed model with results reported in Dorronsoro's paper ${ }^{214}$ - to our knowledge the first study using an empirical approach to actually measure the ablation efficiency - may indicate that the used approach including the discussed simplifications is a reasonable description of the loss of efficiency effects. In so far, this model may complement previous analytical approaches to the efficiency problem and may sustain the observations reported by Dorronsoro et al. ${ }^{214}$

Even though a large number of detailed parameters are considered, this model is still characterized by a relatively low degree of complexity. In particular, the model could be further refined by incorporating non-linear deviations according to Lambert-Beer's law or by considering local corneal curvature directly from topographical measurements rather than modelling the best-fit surface elevation. 


\section{Section D.6 CONCLUSIONS}

(Conclusiones)

The loss of efficiency is an effect that should be offset in commercial laser systems using sophisticated algorithms that cover most of the possible variables. Parallelly, increasingly capable, reliable, and safer laser systems with better resolution and accuracy are required. The improper use of a model that overestimates or underestimates the loss of efficiency will overestimate or underestimate its compensation and will only mask the induction of aberrations under the appearance of other sources of error.

The model introduced in this study eliminates the direct dependence on fluence and replaces it by direct considerations on the nominal spot volume and on the area illuminated by the beam, thus reducing the analysis to pure geometry of impact and providing results essentially identical to those obtained by the model by Dorronsoro-Cano-Merayo-Marcos ${ }^{214}$, however, also taking into account the influence of flying spot technology, where spot spacing is small compared to the spot width and multiple spots overlap contributing to the same target point and the correction to be applied, since the corneal curvature changes during treatment, so that also the ablation efficiency varies over the treatment.

Our model provides an analytical expression for corrections of laser efficiency losses that is in good agreement with recent experimental studies, both on PMMA and corneal tissue. The model incorporates several factors that were ignored in previous analytical models and is useful in the prediction of several clinical effects reported by other authors. Furthermore, due to its analytical approach, it is valid for different laser devices used in refractive surgery.

The development of more accurate models to improve emmetropization and the correction of ocular aberrations in an important issue. We hope that this model will be an interesting and useful contribution to refractive surgery and will take us one step closer to this goal. 


\section{Section D.7 OUTLOOK}

(Perspectiva)

In further works, a comprehensive model to analyze the relative ablation efficiency at different materials (in particular human cornea and poly(methylmethacrylate) (PMMA)), which directly considers applied correction, including astigmatism, as well as, laser beam characteristics and ablative spot properties will be developed, providing a method to convert the deviations in achieved ablation observed in PMMA to equivalent deviations in the cornea.

We are developing, as well, a simple simulation model to evaluate ablation algorithms and hydration changes in laser refractive surgery. The model simulates different physical effects of an entire surgical process, and the shot-byshot ablation process based on a modelled beam profile. The model considers corneal hydration, as well as environmental humidity, as well as, laser beam characteristics and ablative spot properties. 
Topic E CLINICAL EFFECTS OF CYCLOTORSIONAL ERRORS DURING REFRACTIVE SURGERY

(Efectos clínicos de los errores de ciclotorsión durante cirugía refractiva)

\section{AUTHOR CONTRIBUTIONS}

Study concept and design (S.A.M.); data collection (D.O., M.C.A., I.M.A.); analysis and interpretation of data (S.A.M.); drafting (S.A.M.); critical revision (J.M., D.O., M.C.A., I.M.A.); statistical expertise (S.A.M.).

\section{Section E.1 ABSTRACT}

(Resumen)

PURPOSE. To describe the theoretical effects of cyclotorted ablations on induced aberrations and determine the limits of tolerance of cyclotorsional accuracy.

METHODS. We developed a method to determine the average cyclotorsion during refractive surgery without a cyclotorsion tracker. We simulated mathematical conditions to determine the optical, visual, and absolute benefits in 76 consecutive treatments performed on right eyes. The results were evaluated as Zernike expansion of residual wavefront aberrations.

RESULTS. Ablations based purely on Zernike decomposition but with cyclotorsion applied resulted in residual aberrations of the same Zernike modes of different magnitudes and orientations, indicating that the effect of cyclotorted compensation can be analyzed by single Zernike modes in magnitude and orientation. The effect on single Zernike modes depends only on angular frequency and not radial order. We obtained a mean value of $4.39^{\circ}$ of cyclotorsion. A theoretical optical benefit was achieved for $95 \%$ of treatments, a theoretical visual benefit in $95 \%$, and an absolute benefit in $93 \%$ compared with $89 \%, 87 \%$, and $96 \%$ of treatments achieving actual benefits, respectively. 
CONCLUSIONS. Residual aberrations resulting from cyclotorsion depend on aberrations included in the ablation and cyclotorsional error. The theoretical impact of cyclotorted ablations is smaller than decentred ablations or edge effects in coma and spherical aberrations. The results are valid within a single-failure condition of pure cyclotorsional errors, because no other sources of aberrations are considered. The leap from the mathematical model to the real world outcome cannot be extrapolated without further study.

\section{Section E.2 INTRODUCTION}

(Introducción)

Human eyes have six degrees of freedom to move: $X / Y$ lateral shifts, $Z$ levelling, horizontal/vertical rotations, and cyclotorsion (rotations around the optical axis).

Laser technology for refractive surgery allows corneal alterations to correct refractive errors ${ }^{15}$ more accurately than ever. Ablation profiles are based on the removal of tissue lenticules in the form of sequential laser pulses that ablate a small amount of corneal tissue to compensate for refractive errors. However, the quality of vision can deteriorate significantly, especially under mesopic and lowcontrast conditions. ${ }^{19}$

Induction of aberrations, such as spherical aberrations and coma, is related to loss of visual acuity (VA) ${ }^{70}$ and quality. Some aberrations, however, may be subject to neural adaptation. A study by Artal et al. $^{73}$ on the effects of neural compensation on vision indicated that visual quality in humans is superior to the optical quality provided by the human eye.

Measuring rotation when the patient is upright to when the refractive treatments are performed with the patient supine may lead to ocular cyclotorsion, 215 resulting in mismatching of the applied versus the intended profiles (Figure 49). Recently, some equipment can facilitate measurement of and potential compensation for static cyclotorsion occurring when the patient moves from upright to the supine position during the procedure. 

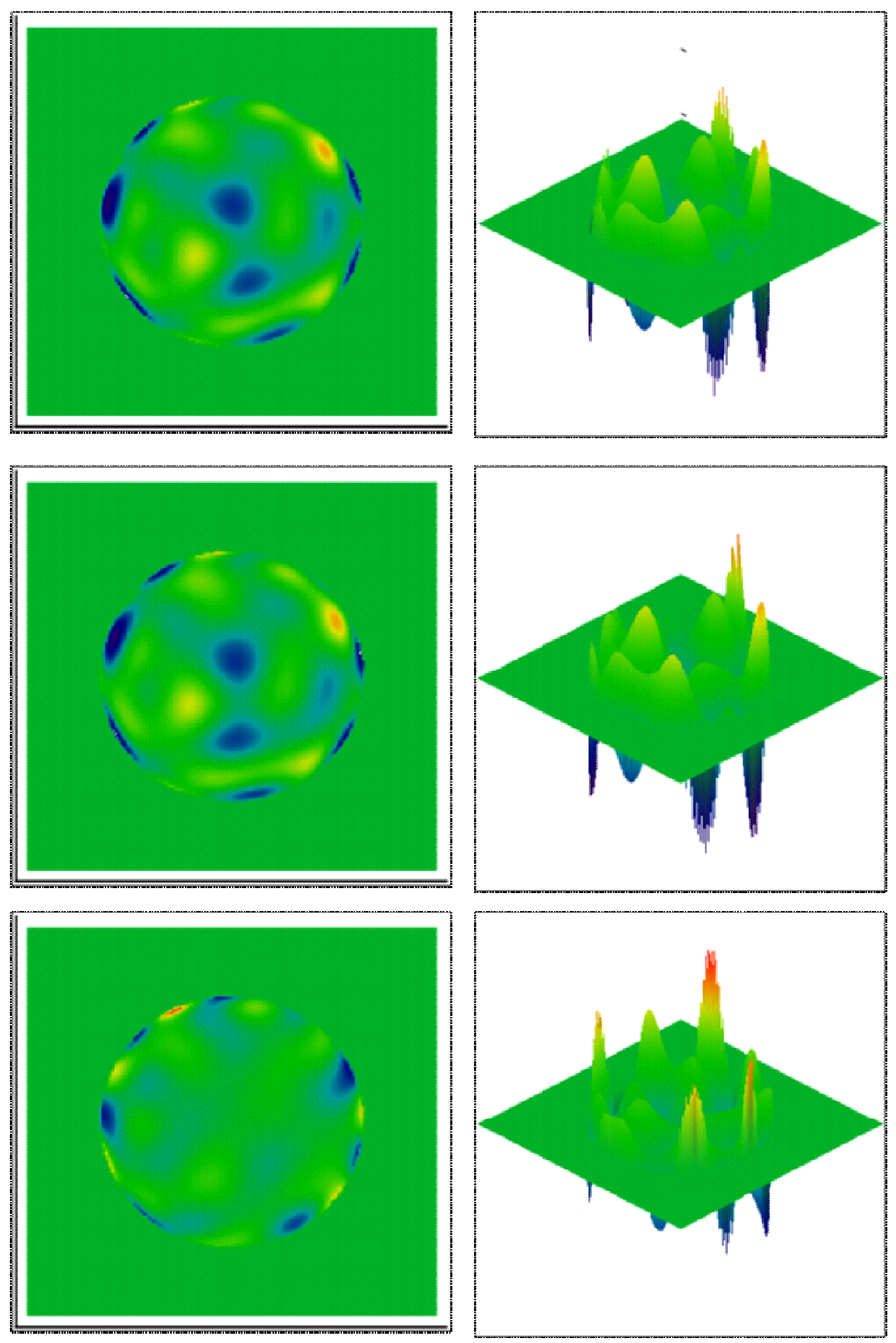

Figure 49: (Top) Original wavefront error, (middle) $15^{\circ}$ clockwise torted wavefront error, and (bottom) residual wavefront error (all in two-dimension and three-dimensions) 


\section{Section E.3 METHODS}

(Método)

\section{E.3.1 Determination of Cyclotorsion during Refractive Surgery \\ (Determinación de la ciclotorsión durante cirugía refractiva)}

We analyzed the topographies using the Keratron-Scout videokeratoscope (Optikon2000 S.p.A, Rome, Italy) preoperatively and 3-month after LASIK and measured the Maloney indices in 76 consecutive right eyes with myopic astigmatism. Using only the right eyes or only left eyes simplifies calculations because it directly avoids considering potential bilateral symmetry effects between eyes regarding cyclotorsion (i.e., cyclotorsional values in the left eye might be multiplied by -1$)$.

As reported previously, ${ }^{216}$ the achieved correction after refractive surgery can be calculated from the topographic changes. The vectorial differences in the astigmatic space between the postoperative and preoperative Maloney indices $^{216,217}$ were compared to the intended corrections (Figure 50), e.g., a preoperative topography of 41.6 dioptres (D) at $111^{\circ}$ and $41.2 \mathrm{D}$ at $21^{\circ}$ and a postoperative topography of $44.4 \mathrm{D}$ at $114^{\circ}$ and $43.5 \mathrm{D}$ at $24^{\circ}$ results in a spherical change of $+3.0 \mathrm{D}$ with a cylindrical component of $-0.5 \mathrm{D}$ at $117^{\circ}$ compared to the planned +3.0 D $-0.5 \mathrm{D} \times 110^{\circ}$ at the $12-\mathrm{mm}$ vertex distance, resulting in $7^{\circ}$ of counterclockwise cyclotorsion.

Maloney indices use the inner $3 \mathrm{~mm}$ zone, to fit this disk area best to a spherocylindrical surface in 3D. Cylinder orientation defines the two principal meridians, and sphere and cylinder provide the curvatures of the principal meridians. In "normal" corneas (without irregular astigmatism), sim-K and Maloney analyses provide very similar results. 


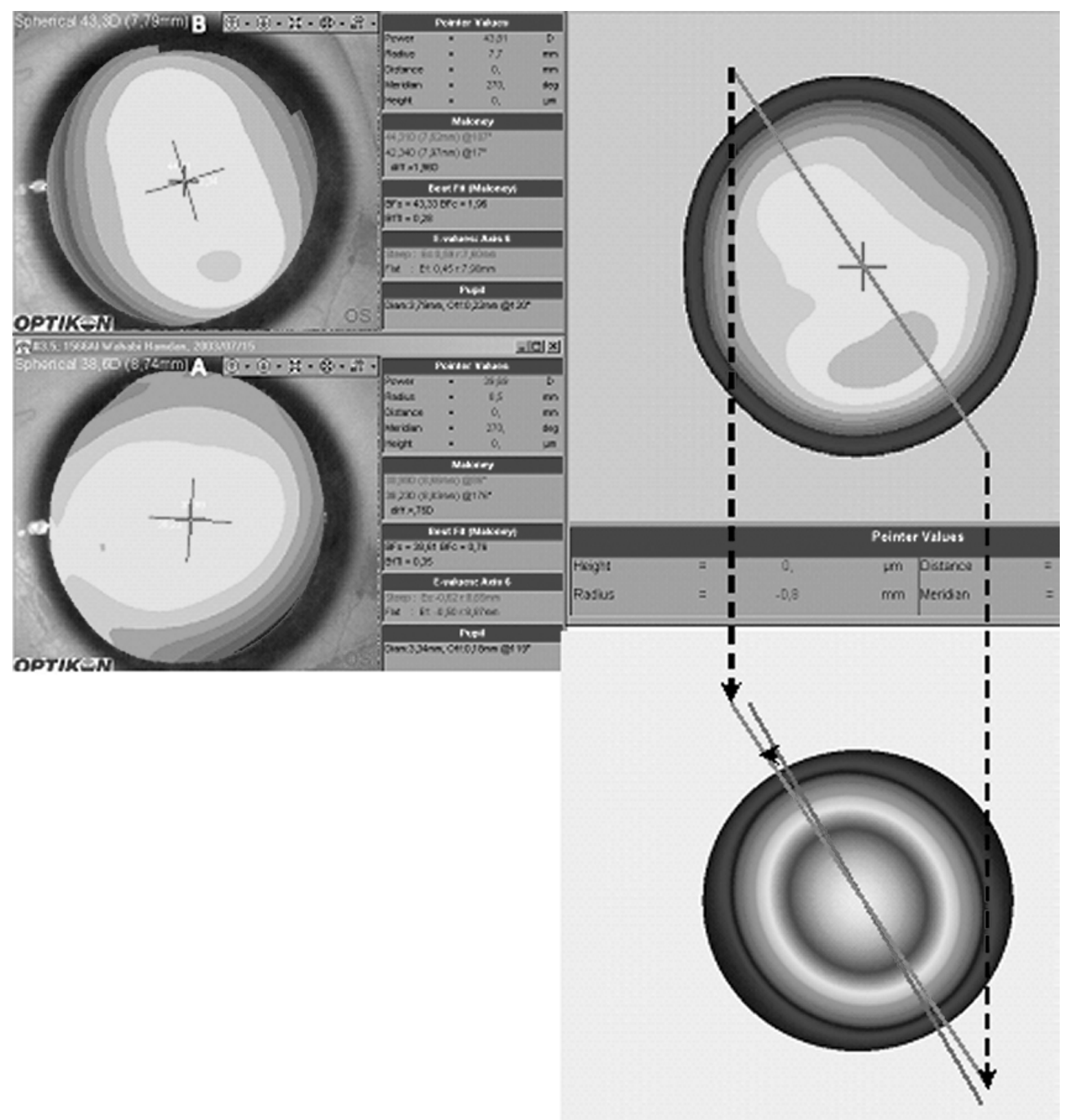

Figure 50: The difference between the postoperative and preoperative topographies compared to the intended correction (the difference in the orientation of the astigmatism defines the cyclotorsional error). (A) Preoperative topography. (B) Postoperative topography. (C) Differential topography. Planned correction. Counterclockwise torsion of the astigmatism can be seen 


\section{E.3.2}

Residual Aberration after Cyclotorsional Errors during Refractive Surgery

(Aberración residual tras errores de ciclotorsión durante cirugía refractiva)

When the rotation angle is 0 , the aberration and compensation patterns cancel each other, resulting in no residual aberration. Based on the definition of the Zernike polynomials ${ }^{36}(Z(n, m)$, where $n$ is a null or positive integer and $m$ is an integer ranging from $-n$ to $+n$, representing the radial and meridional orders, respectively) it is evident that the polynomials $Z(n, 0)$ are invariant under rotations around their centre. The only aberrations affected by cyclotorsional errors are those with a vector nature. For those, Zernike polynomials are structured in two complementary sets, governed by sine/cosine functions that avoid coupling of different aberration orders for rotations around their centre.

After rotation of the opposite of the Zernike components around their origin, the aberration mode still can be decomposed into two Zernike components:

$$
C_{n}^{\prime m}=-\left[C_{n}^{m} \cos (m \theta)+C_{n}^{-m} \sin (m \theta)\right]
$$

where $\mathrm{n}$ is the radial order, $\mathrm{m}$ the angular frequency, $\mathrm{C}_{\mathrm{n}}{ }^{\mathrm{m}}{ }^{\mathrm{m}}$ the rotated Zernike compensation, $\mathrm{C}_{\mathrm{n}}^{ \pm m}$ the original Zernike components, and $\theta$ the cyclotorsional angle.

The residual components are after compensating for the original pattern with rotated one:

$$
C_{n}^{\prime \prime m}=C_{n}^{m}[1-\cos (m \theta)]-C_{n}^{-m} \sin (m \theta)
$$

where $\mathrm{C} "{ }_{\mathrm{n}}^{\mathrm{m}}$ is the residual Zernike component.

Expressing each aberration in magnitude and orientation ${ }^{218}$ :

$$
\begin{gathered}
\left|C^{\prime \prime}{ }_{n m}\right|=\left|C_{n}^{ \pm m}\right| 2 \sin \left(\frac{m \theta}{2}\right) \\
\Delta \alpha=\alpha-\alpha_{0}=\frac{\theta}{2}-\frac{\pi}{2 m}
\end{gathered}
$$


Using the previous example, a planned correction of +3.0 D $-0.5 \mathrm{D} \times 110^{\circ}$ at the 12-mm vertex distance and an actual spherical change of $+3.0 \mathrm{D}$ with a cylindrical component of $-0.5 \mathrm{D}$ at $117^{\circ}$ results in $7^{\circ}$ of counterclockwise cyclotorsion and would lead to a postoperative refraction of $+0.07 \mathrm{D}-0.13 \mathrm{D} \times 69^{\circ}$.

The relative amount of residual aberrations depends only on cyclotorsional error (Figure 51 and Figure 52). Because the original aberration can be described as a linear combination of Zernike polynomials ${ }^{36}$ and each of these Zernike terms results in a residual Zernike term after partial compensatory rotation, the residual wavefront aberration then is the sum of all residual terms.

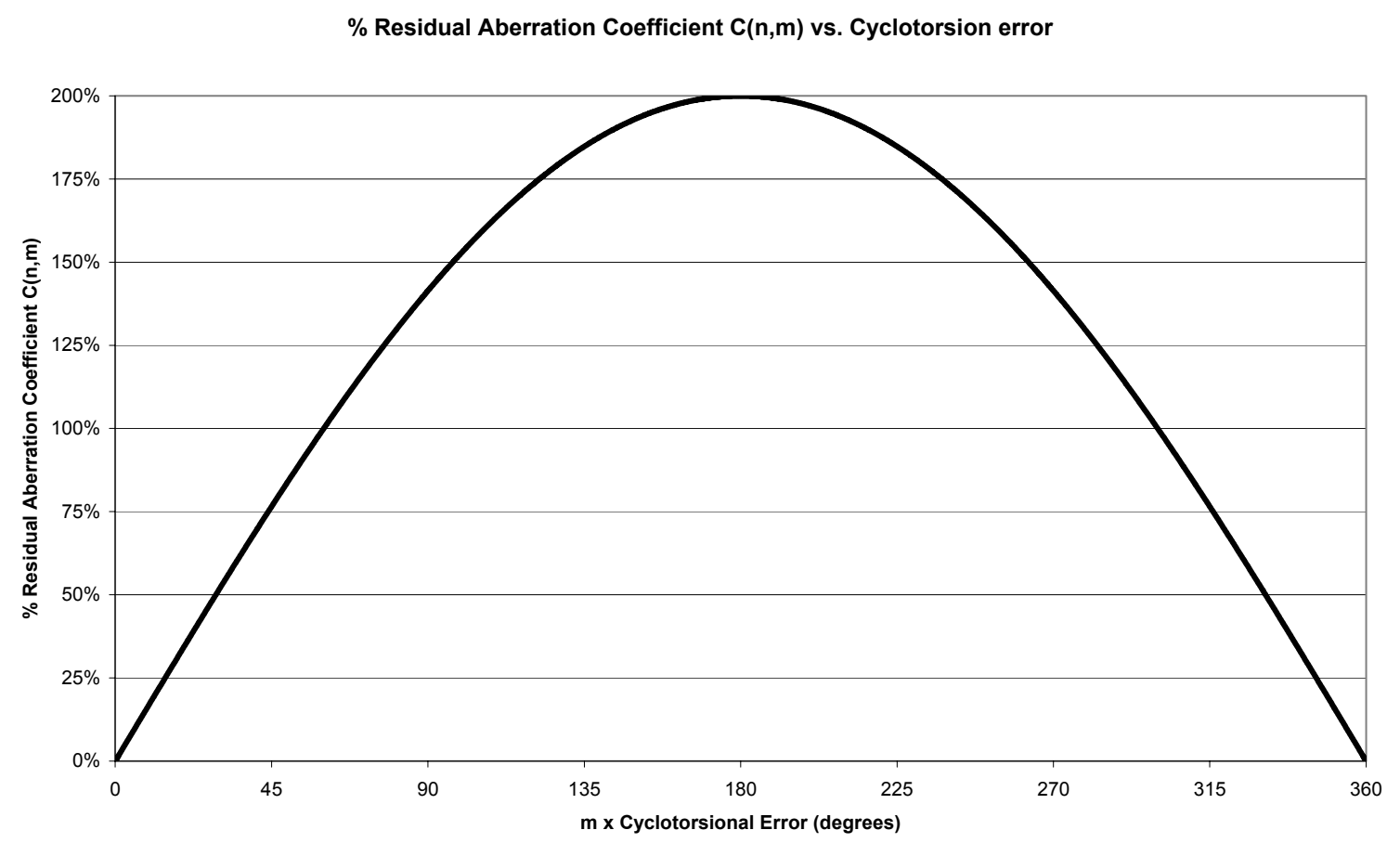

Figure 51: The percentage of residual aberrations vs. cyclotorsional error. Modulation of the cyclotorsional error by the angular frequency $(m)$ is seen; the higher the angular frequency, the faster the residual aberration varies. For $m=1$ (coma), the maximum residual error is achieved for $180^{\circ}$ torsion; for $\mathrm{m}=2$ (cylinder), the maximum residual error would be achieved for $90^{\circ}$ torsion; for $\mathrm{m}=3$ (trefoil), the maximum residual error would be achieved for $60^{\circ}$ torsion, and so on 
Relative Orientation residual aberration Coefficient $\mathrm{C}(\mathrm{n}, \mathrm{m})$ vs. Cyclotorsion error

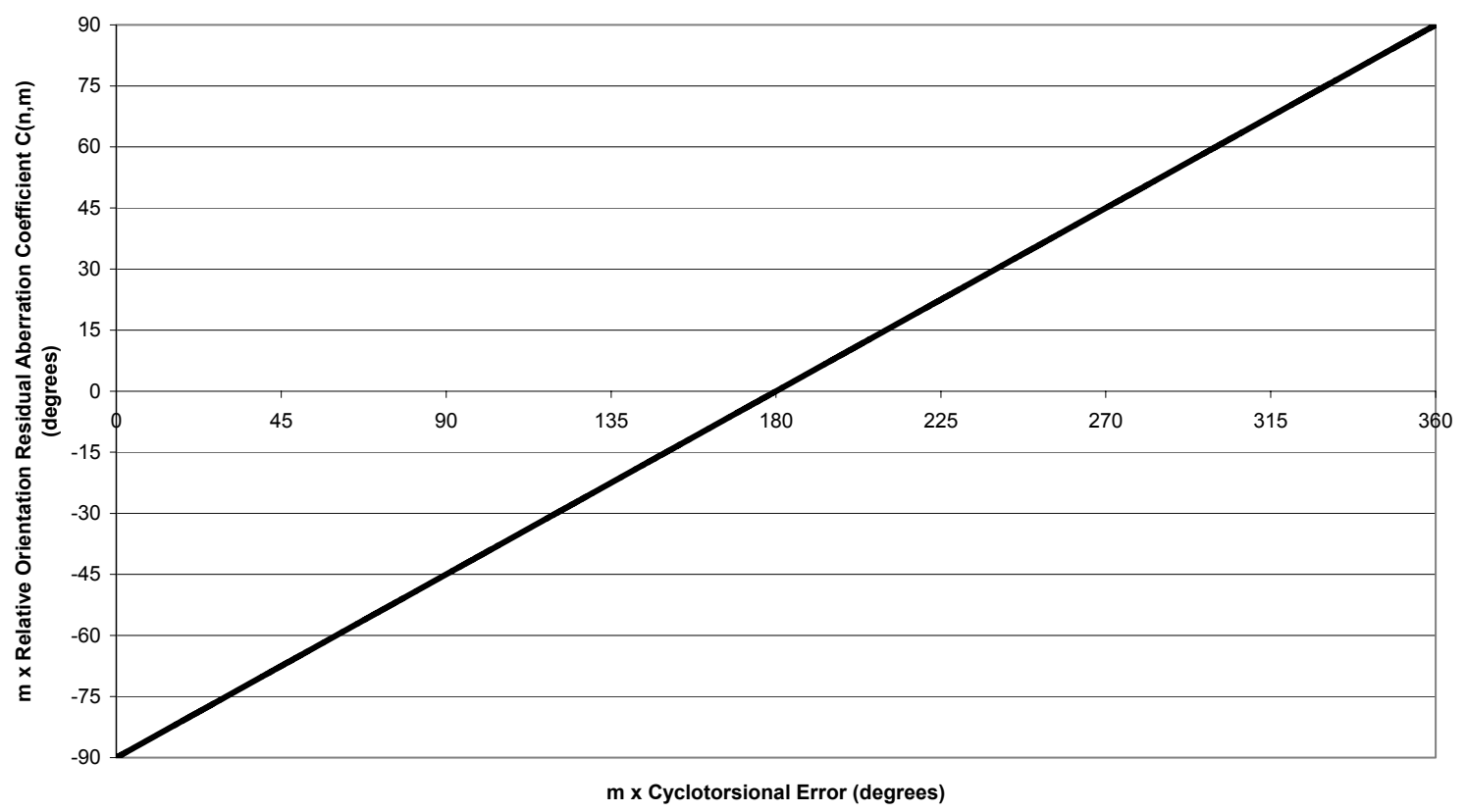

Figure 52: The relative orientation of residual aberrations vs. cyclotorsion error. Modulation of the cyclotorsional error and the relative orientation by the angular frequency $(m)$ are seen

\section{E.3.3 Derivation of a Mathematic Condition to Determine an Optical Benefit (Derivación de una condición matemática para determinar un beneficio óptico)}

A condition in which any postoperative aberration smaller than its preoperative magnitude was considered as positive, was called optical benefit:

$$
\begin{aligned}
& 2\left|\sin \left(\frac{m \theta}{2}\right)\right|<1 \\
& |m|<\frac{2 \arcsin (1 / 2)}{|\theta|}<\left|\frac{\pi}{3 \theta}\right|
\end{aligned}
$$

Using the previous example, $7^{\circ}$ of cyclotorsion would produce an optical benefit up to the octafoil angular frequencies.

Considering the cyclotorsional error and the preoperative astigmatism, we calculated how many treatments would theoretically achieve an optical benefit for 
the astigmatism component $(m=2)$. Because the treatments were planned as aberration-free profiles, and, therefore, only based on sphere, cylinder, and axis inputs, the astigmatism was the only vector nature aberration included. Moreover, astigmatism is in magnitude the major Zernike mode with a vector nature. We compared this value to the percentage of eyes that actually obtained a postoperative cylinder lower than the preoperative value.

E.3.4 Derivation of a Mathematic Condition to Determine a Visual Benefit (Derivación de una condición matemática para determinar un beneficio visual)

To distinguish between optical benefit (merely reducing the aberration magnitude) and visual performance (visual benefit), a model based on the findings of Artal et al. ${ }^{73}$ was adopted. In that study, equivalent human optical systems that differed only in the orientation of the aberration patterns (produced by adaptive optics) achieved different visual performances mainly due to neural compensation for the unique aberration pattern of each individual. For that reason, matching factor (MF) behaviour based on single aberrations was modelled. MF is maximum (equal to 1) for aberrations of the same orientation and minimum for aberrations of the opposite orientation in the Zernike space. The magnitude of aberration distribution was considered a decreasing exponential with the Zernike order as described by Thibos et al. ${ }^{149}$

Visual benefit was defined as a condition in which the postoperative aberration was smaller than its preoperative magnitude times the MF for that relative orientation:

$$
\begin{gathered}
2\left|\sin \left(\frac{m \theta}{2}\right)\right|<M F+(1-M F)|\cos (m \Delta \alpha)| \\
|m|<\frac{2 \arcsin \left(\frac{M F}{M F+1}\right)}{|\theta|}
\end{gathered}
$$

The arbitrary value of 0.625 was chosen as the MF generator; this value produces a maximum equal to 1 and a minimum equal to 0.25 (Figure 53). 


$$
\begin{aligned}
& M F=0.625 \\
& |m|<\frac{2 \arcsin (5 / 13)}{|\theta|}
\end{aligned}
$$

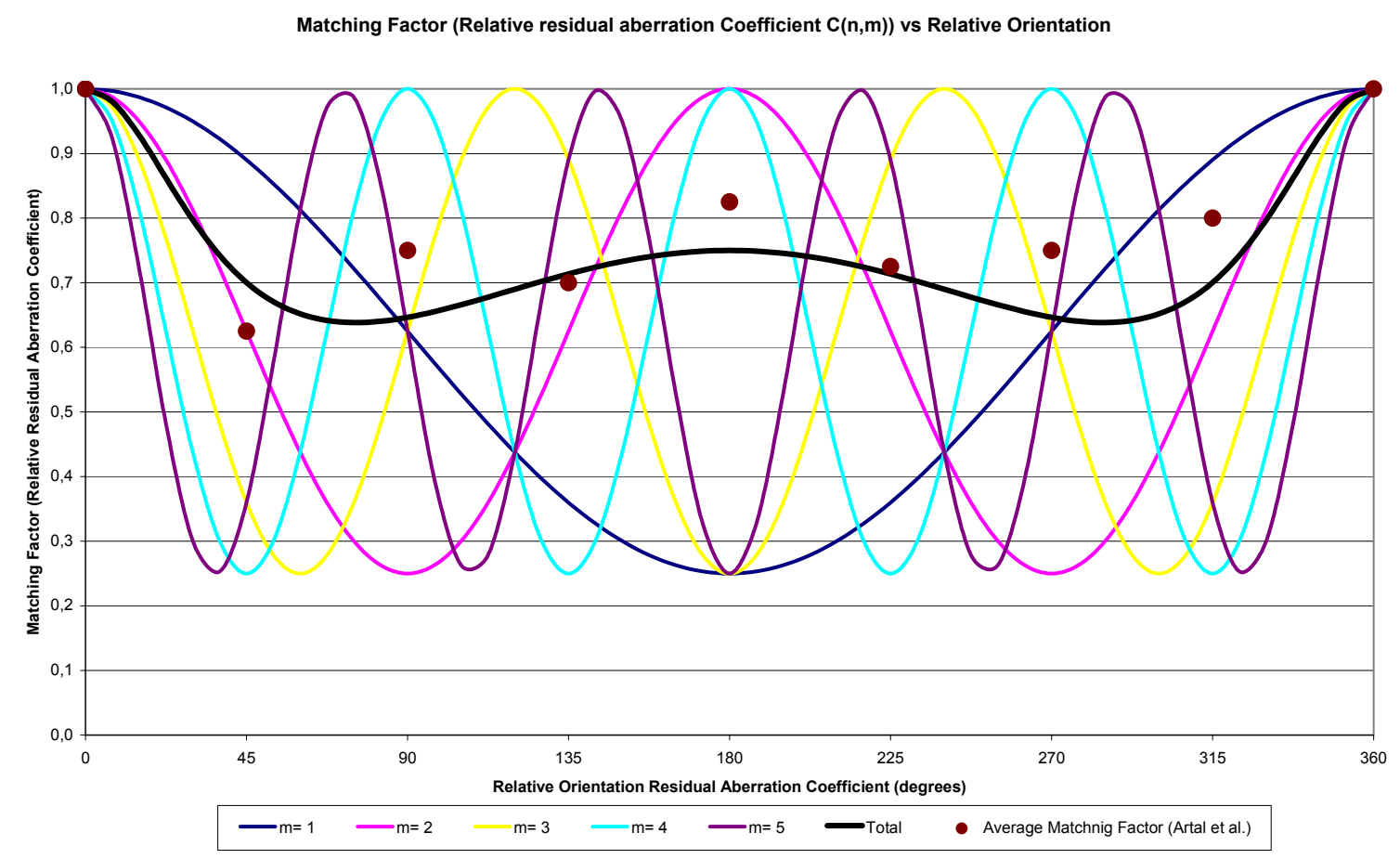

Figure 53: Matching factor vs. relative orientation of residual aberrations.

Using the previous example, $7^{\circ}$ of cyclotorsion would produce a visual benefit up to the hexafoil angular frequencies.

With the cyclotorsional error and the preoperative astigmatism, and assuming that cyclotorsional errors around the ablation centre were the only failure, the number of eyes was calculated that would have obtained a visual benefit for cylinder if the correction were correct but the axis was incorrect. We compared this value to the percentage of eyes that maintained or improved postoperative UCVA compared to the preoperative BSCVA and to the percentage of eyes with actually maintained or improved BSCVA. 
Derivation of a Mathematic Condition to Determine an Absolute Benefit

(Derivación de una condición matemática para determinar un beneficio absoluto)

The major ocular aberrations are defocus and primary astigmatism, which is the major aberration affected by a rotational error. The amount of tolerable residual astigmatism postoperatively cannot be defined as a percentage of the preoperative astigmatism, because the tolerance limit is set by the image-forming characteristics of the eye and so takes an absolute value. With simple spherical error, degradation of resolution begins for most people with errors of $0.25 \mathrm{D}$. A similar measure can be placed on the error due to cylinder axis error. ${ }^{219}$

The absolute benefit considers as positive any result for which the postoperative aberration pattern was smaller than an absolute limit of $0.50 \mathrm{DEQ}$ for the magnitude of each Zernike mode (i.e. a \pm 0.25 DEQ maximum deviation in one or several meridians).

The absolute benefit is ruled by the condition:

$$
\begin{array}{r}
\left|D E Q_{n m} 2 \sin \left(\frac{m \theta}{2}\right)\right|<0.50 \\
\left|D E Q_{n m}\right|<\frac{1}{4\left|\sin \left(\frac{m \theta}{2}\right)\right|}
\end{array}
$$

Using the previous example, $7^{\circ}$ of cyclotorsion, Zernike modes should not exceed 4.10 DEQ for coma, 2.05 DEQ for astigmatism, and, 1.37 DEQ for trefoil, for theoretically successful results.

With the torsional error and the preoperative astigmatism, and assuming that cyclotorsion around the ablation centre was the only failure, the number of eyes was calculated that would have obtained an absolute benefit for cylinder (postoperative magnitude, $\leq 0.50 \mathrm{D}$ ) if the cylindrical correction were correct but the axis was wrong. We compared this value to the eyes in which postoperative astigmatism was less than $0.50 \mathrm{D}$. 


\section{Section E.4 RESULTS}

(Resultados)

\section{E.4.1 Static Cyclotorsion during Laser Refractive Surgery \\ (Ciclotorsión estática durante cirugía refractiva laser)}

Preoperative and postoperative topographies were compared 3 months after treatment in 76 consecutives right eyes treated without adverse events at Augenzentrum Recklinghausen.

The preoperative spherical equivalent (SE) was $-3.56 \mathrm{D}$ with a standard deviation (SD) of $1.51 \mathrm{D}$ (range, -7.00 to $-1.25 \mathrm{D}$ ) and cylinder $0.82 \pm 0.66 \mathrm{D}(0.25$ to $3.00 \mathrm{D})$. $30 \%$ of the treatments $(n=23)$ had corrections of $0.25 \mathrm{D}$ of astigmatism, $20 \%(n=15)$ corrections of 0.50 D of astigmatism, $39 \% \quad(n=30)$ corrections between 0.75 and $1.50 \mathrm{D}$ of astigmatism, and $11 \%(n=8)$ corrections between 1.50 and $3.00 \mathrm{D}$ of astigmatism (Figure 54).

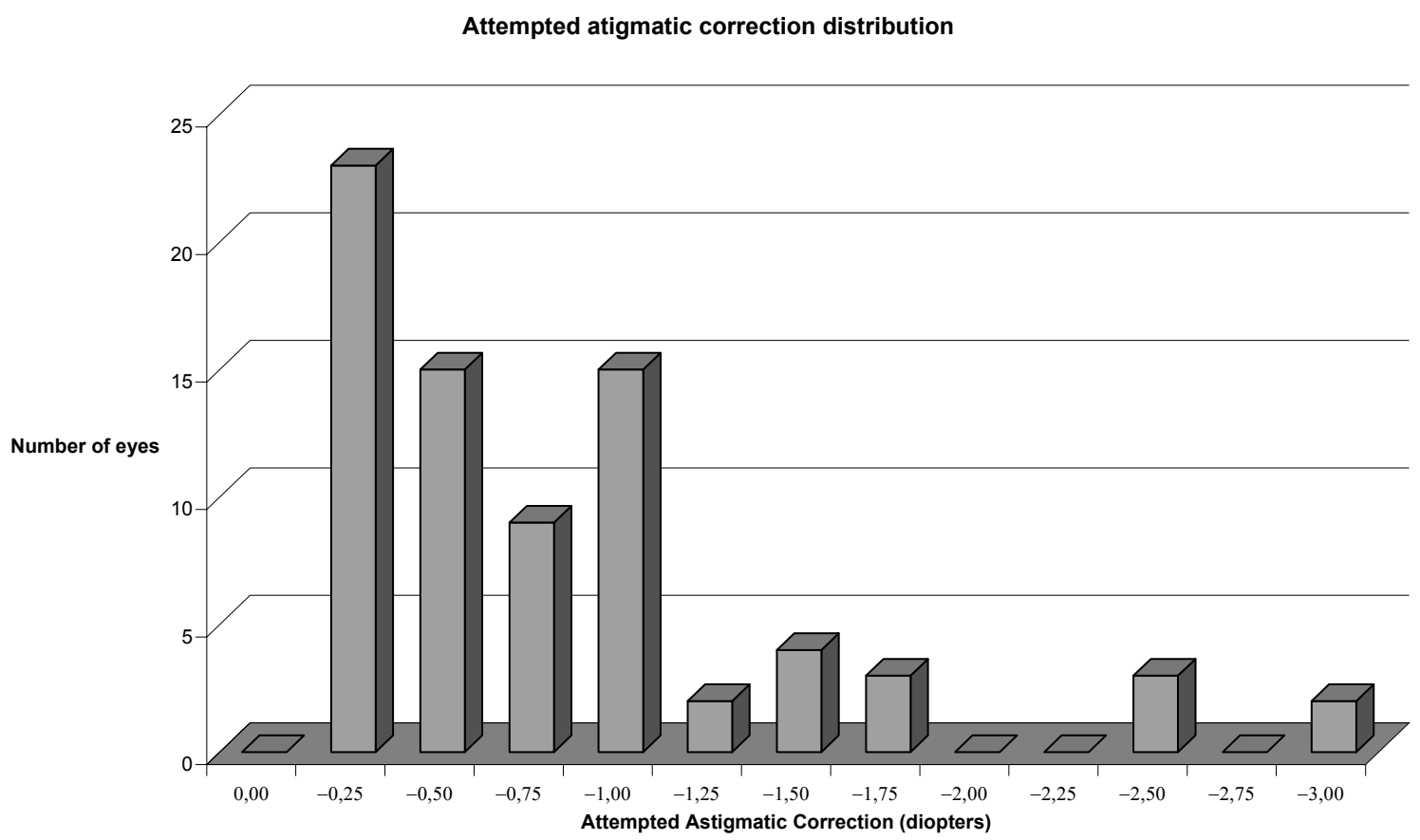

Figure 54: Distribution of the magnitudes of the attempted astigmatic correction 
At 3-month follow-up, the mean SE was $-0.14 \pm 0.30 \mathrm{D}(-1.00$ to $+0.25 \mathrm{D})$ and cylinder $0.17 \pm 0.26 \mathrm{D}(0.00$ to $1.25 \mathrm{D})$. Eighty-seven percent of the eyes $(n=66)$ were within $\pm 0.50 \mathrm{D}$ of the attempted correction, and $100 \%(n=76)$ were within $\pm 1.00 \mathrm{D}$.

The direct average of the cyclotorsional errors was $2.42^{\circ}$, whereas the absolute values averaged $4.39^{\circ}$. Seventy-one percent of the eyes $(n=54)$ had less than $2.5^{\circ}$ of cyclotorsion, $78 \%(n=59)$ less than $5.0^{\circ}$, and $87 \%(n=66)$ less than $10.0^{\circ}$ (Figure 55).

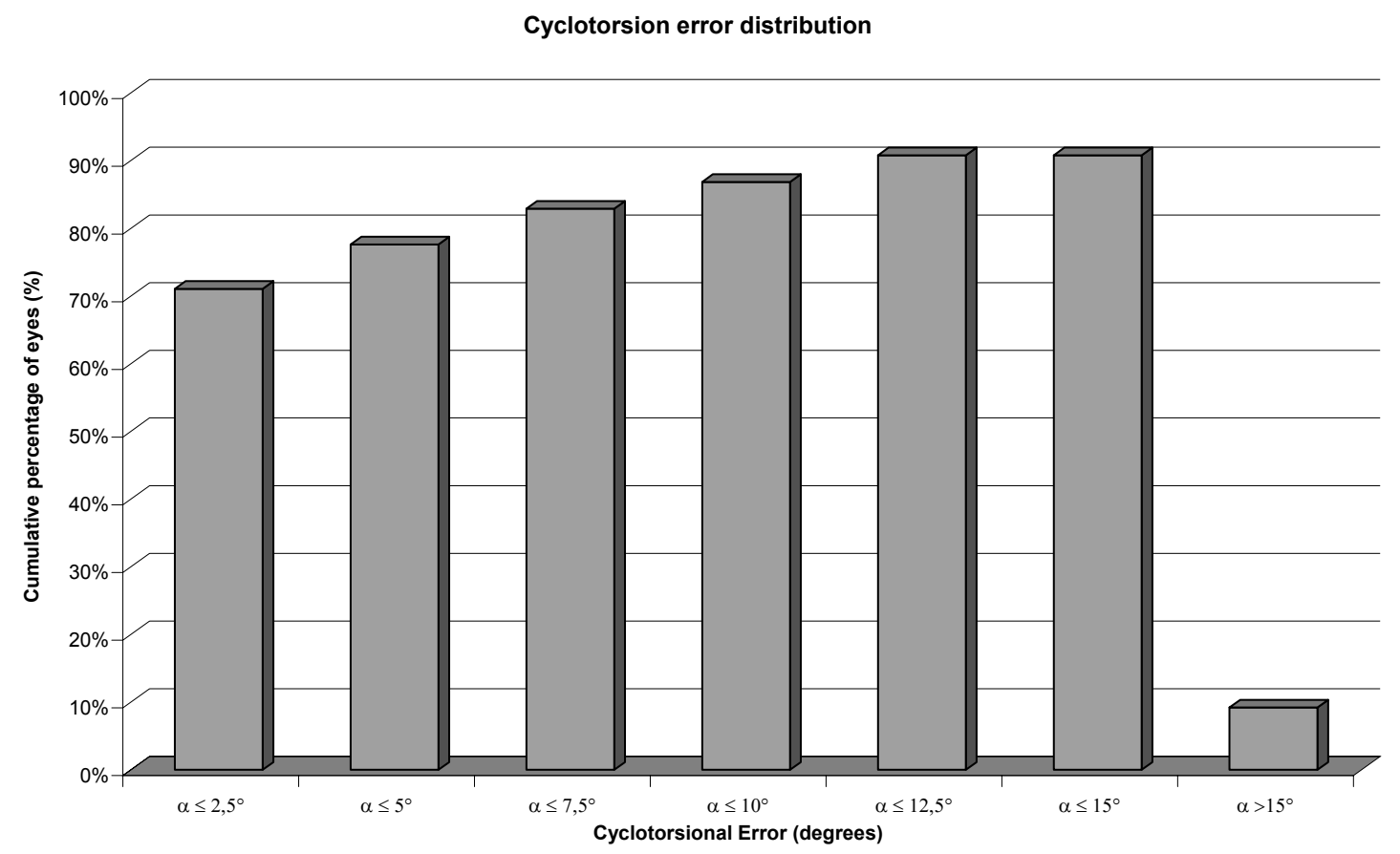

Figure 55: Distribution of the retrospectively calculated cyclotorsional errors

\section{E.4.2 Theoretical Ranges to Obtain Optical, Visual, and Absolute Benefits (Rangos teóricos para la obtención de beneficios ópticos, visuales o absolutos)}

The maximum angular frequency and Zernike mode magnitudes that fulfil these conditions were calculated for specific cyclotorsional errors (Figure 56, Table 6, and Table 7), but for the description of the magnitudes we focused on astigmatism, coma, and trefoil because these are the major Zernike modes with a vector nature. 
MAX Cyclotorsion error vs. Angular frequency

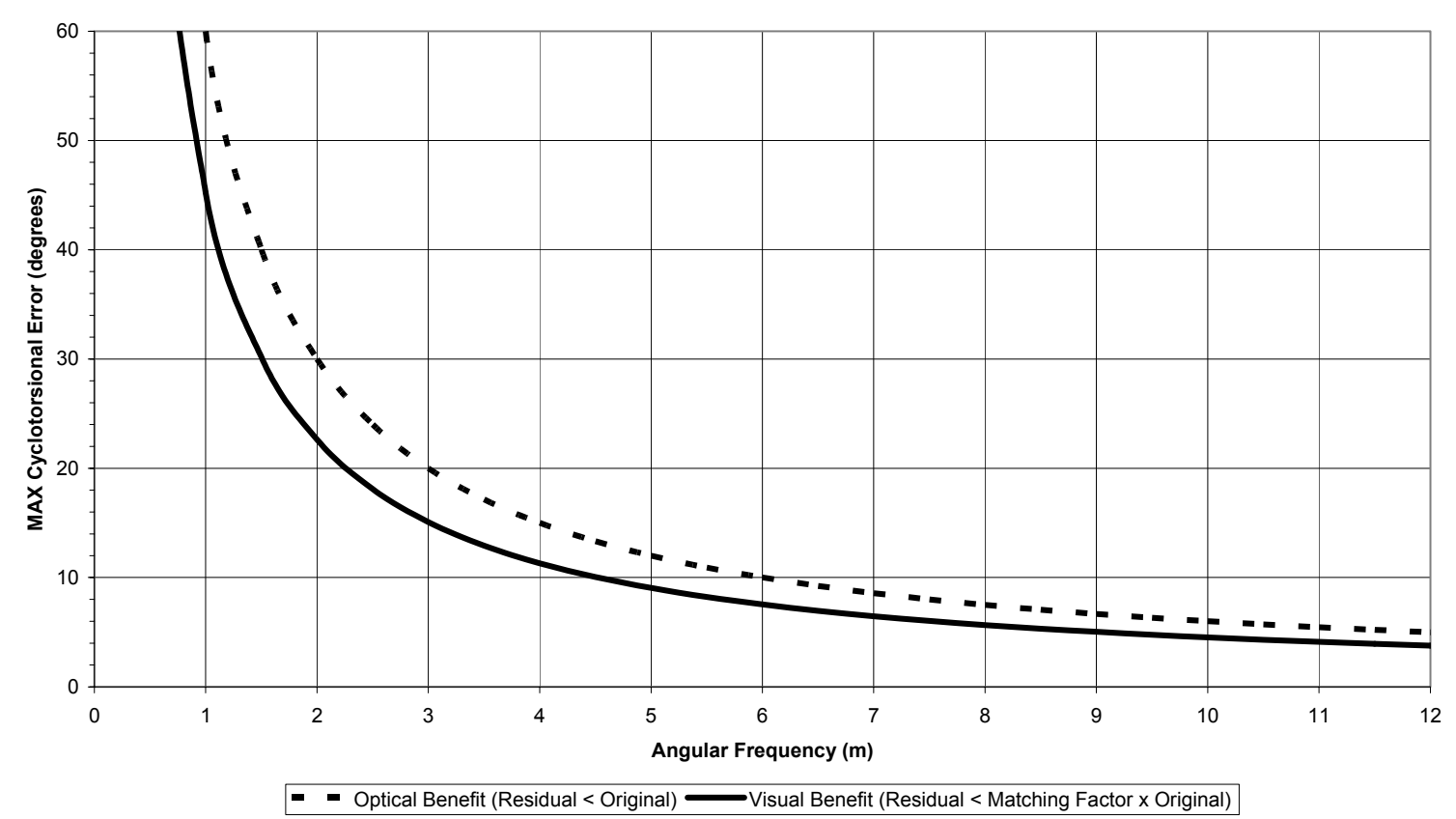

Figure 56: The maximum allowable cyclotorsional errors vs. angular frequency for different criteria

\begin{tabular}{|c|c|c|c|c|c|c|c|c|c|}
\hline Cyclotorsional Error & $\begin{array}{c}\text { Optical } \\
\text { benefit } \\
\text { angular } \\
\text { frequency } \\
\text { m }\end{array}$ & \begin{tabular}{|c|} 
Visual \\
benefit \\
angular \\
frequency \\
m
\end{tabular} & $\begin{array}{c}\text { Treated } \\
\text { coma } \\
\text { C(n,1) } \\
\text { (DEQ) }\end{array}$ & $\begin{array}{c}\text { Treated } \\
\text { cylinder } \\
\text { C(n,2) } \\
\text { (DEQ) }\end{array}$ & $\begin{array}{l}\text { Treated } \\
\text { trefoil } \\
\mathbf{C}(\mathbf{n}, 3) \\
\text { (DEQ) }\end{array}$ & $\begin{array}{l}\text { Treated } \\
\text { tetrafoil } \\
\text { C(n,4) } \\
\text { (DEQ) }\end{array}$ & $\begin{array}{l}\text { Treated } \\
\text { pentafoil } \\
\text { C(n,5) } \\
\text { (DEQ) }\end{array}$ & $\begin{array}{r}\text { Treated } \\
\text { hexafoil } \\
\text { C(n,6) } \\
\text { (DEQ) }\end{array}$ & $\begin{array}{r}\text { Treated } \\
\text { octafoil } \\
\text { C(n,8) } \\
\text { (DEQ) }\end{array}$ \\
\hline Torsion tracker 1.5 & 40 & 30 & 19.10 & 9.55 & 6.37 & 4.78 & 3.82 & 3.19 & 2.13 \\
\hline 2.5 & 24 & 18 & 11.46 & 5.73 & 3.82 & 2.87 & 2.30 & 1.92 & 1.28 \\
\hline Average torsion 4.0 & 15 & 11 & 7.16 & 3.58 & 2.39 & 1.80 & 1.44 & 1.20 & 0.81 \\
\hline 5.0 & 12 & 9 & 5.73 & 2.87 & 1.92 & 1.44 & 1.16 & 0.97 & 0.65 \\
\hline 7.5 & 8 & 6 & 3.82 & 1.92 & 1.28 & 0.97 & 0.78 & 0.65 & 0.45 \\
\hline 10.0 & 6 & 4 & 2.87 & 1.44 & 0.97 & 0.73 & 0.59 & 0.50 & 0.35 \\
\hline 12.5 & 4 & 3 & 2.30 & 1.16 & 0.78 & 0.59 & 0.48 & 0.41 & 0.30 \\
\hline $\begin{array}{r}\text { Maximum Torsion } \\
14.0\end{array}$ & 4 & 3 & 2.05 & 1.03 & 0.70 & 0.53 & 0.44 & 0.37 & 0.28 \\
\hline 15.0 & 4 & 3 & 1.92 & 0.97 & 0.65 & 0.50 & 0.41 & 0.35 & 0.27 \\
\hline
\end{tabular}

Table 6: Maximum Treatable Magnitude for Different Aberration Components and Different Cyclotorsional Errors for the $<0.50$ DEQ Criterion 


\begin{tabular}{|c|c|c|c|}
\hline Aberrations & $\overline{\mathbf{m}}$ & $\begin{array}{c}\text { Optical benefit } \\
\text { (residual < original) }\end{array}$ & $\begin{array}{c}\text { Visual benefit } \\
\text { (residual }<\text { matching factor) }\end{array}$ \\
\hline Coma & 1 & 60.0 & 45.2 \\
\hline Astigmatism & 2 & 30.0 & 22.6 \\
\hline Trefoil & 3 & 20.0 & 15.1 \\
\hline Tetrafoil & 4 & 15.0 & 11.3 \\
\hline Pentafoil & 5 & 12.0 & 9.0 \\
\hline Hexafoil & 6 & 10.0 & 7.5 \\
\hline Heptafoil & 7 & 8.6 & 6.5 \\
\hline Octafoil & 8 & 7.5 & 5.7 \\
\hline Eneafoil & 9 & 6.7 & 5.0 \\
\hline Decafoil & 10 & 6.0 & 4.5 \\
\hline Dodecafoil & 12 & 5.0 & 3.8 \\
\hline Pentadecafoil & 15 & 4.0 & 3.0 \\
\hline Icosafoil & 20 & 3.0 & 2.3 \\
\hline Triacontafoil & 30 & 2.0 & 1.5 \\
\hline Hexacontafoil & 60 & 1.0 & 0.8 \\
\hline
\end{tabular}

Table 7: Maximum Allowable Cyclotorsional Errors for Different Aberration Components and Different Criteria

For cyclotorsional errors up to $\pm 14^{\circ}$, it is possible to obtain a visual benefit for comatic, astigmatic, and trefoil angular frequencies and an optical benefit for tetrafoil angular frequencies, as well. It also is possible to control the creation of blur under an absolute limit whenever coma magnitudes do not exceed 2.05 DEQ, astigmatism $1.03 \mathrm{D}$, and trefoil $0.70 \mathrm{DEQ}$.

For maximum cyclotorsional errors up to $\pm 4^{\circ}$, the theoretical limit for visual benefit extends up to endecafoil (11-fold) angular frequencies and for optical benefit up to 15-fold (pentadecafoil) angular frequencies. The magnitudes for the major Zernike modes should not exceed 7.16 DEQ for coma, 3.58 DEQ for astigmatism, and 2.39 DEQ for trefoil. 
For cyclotorsional errors up to $\pm 1.5^{\circ}$, visual benefit extends up to triacontafoil (30-fold) angular frequencies and optical benefit even beyond these frequencies. Moreover, coma magnitudes below $19.10 \mathrm{DEQ}$, astigmatism up to 9.55 D, and trefoil up to 6.37 DEQ produce a postoperative blur under 0.50 DEQ.

For example, $1.00 \mu \mathrm{m}$ of trefoil at $30^{\circ}$ with a $5^{\circ}$ clockwise torsional error will result in $0.26 \mu \mathrm{m}$ of trefoil at $58^{\circ}$ as a postoperative residual error, or $3.00 \mathrm{DEQ}$ astigmatism at $75^{\circ}$ with a $10^{\circ}$ counterclockwise torsional error will result in 1.04 $\mathrm{DEQ}$ astigmatism at $35^{\circ}$ as the postoperative residual error.

\begin{tabular}{|c|c|c|c|c|c|c|c|}
\hline Cyclotorsional error & $\begin{array}{c}\text { Residual } \\
\text { coma } \\
\text { C(n,1) }\end{array}$ & $\begin{array}{c}\text { Residual } \\
\text { cylinder } \\
\text { C(n,2) }\end{array}$ & $\begin{array}{c}\text { Residual } \\
\text { trefoil } \\
\mathbf{C}(\mathbf{n}, \mathbf{3})\end{array}$ & $\begin{array}{c}\text { Residual } \\
\text { tetrafoil } \\
\text { C(n,4) }\end{array}$ & $\begin{array}{c}\text { Residual } \\
\text { pentafoil } \\
C(n, 5)\end{array}$ & $\begin{array}{c}\text { Residual } \\
\text { hexafoil } \\
\text { C(n,6) }\end{array}$ & $\begin{array}{c}\text { Residual } \\
\text { octafoil } \\
\text { C(n,8) }\end{array}$ \\
\hline Torsion tracker 1.5 & 3\%@271 & $5 \% @ 136^{\circ}$ & 8\%@91 & 10\%@68 & $13 \% @ 55^{\circ}$ & $16 \% @ 46^{\circ}$ & $24 \% @ 35^{\circ}$ \\
\hline 2.5 & $4 \% @ 271^{\circ}$ & $9 \% @ 136^{\circ}$ & $13 \% @ 91^{\circ}$ & $17 \% @ 69^{\circ}$ & $22 \% @ 55^{\circ}$ & $26 \% @ 46^{\circ}$ & $39 \% @ 35^{\circ}$ \\
\hline Average torsion 4.0 & $7 \% @ 272^{\circ}$ & $14 \% @ 137^{\circ}$ & $21 \% @ 92^{\circ}$ & $28 \% @ 70^{\circ}$ & $35 \% @ 56^{\circ}$ & $42 \% @ 47^{\circ}$ & $62 \% @ 36^{\circ}$ \\
\hline 5.0 & $9 \% @ 273^{\circ}$ & $17 \% @ 138^{\circ}$ & $26 \% @ 93^{\circ}$ & $35 \% @ 70^{\circ}$ & $43 \% @ 57^{\circ}$ & $52 \% @ 48^{\circ}$ & $77 \% @ 36^{\circ}$ \\
\hline 7.5 & $13 \% @ 274^{\circ}$ & $26 \% @ 139^{\circ}$ & $39 \% @ 94^{\circ}$ & $52 \% @ 71^{\circ}$ & $64 \% @ 58^{\circ}$ & $77 \% @ 49^{\circ}$ & $111 \% @ 38^{\circ}$ \\
\hline 10.0 & $17 \% @ 275^{\circ}$ & $35 \% @ 140^{\circ}$ & $52 \% @ 95^{\circ}$ & $68 \% @ 73^{\circ}$ & $85 \% @ 59^{\circ}$ & $100 \% @ 50^{\circ}$ & $141 \% @ 39^{\circ}$ \\
\hline 12.5 & $22 \% @ 276^{\circ}$ & $43 \% @ 141^{\circ}$ & $64 \% @ 96^{\circ}$ & $85 \% @ 74^{\circ}$ & $104 \% @ 60^{\circ}$ & $122 \% @ 51^{\circ}$ & $166 \% @ 40^{\circ}$ \\
\hline Maximum torsion 14.0 & $24 \% @ 277^{\circ}$ & $48 \% @ 142^{\circ}$ & $72 \% @ 97^{\circ}$ & $94 \% @ 75^{\circ}$ & $115 \% @ 61^{\circ}$ & $134 \% @ 52^{\circ}$ & $178 \% @ 41^{\circ}$ \\
\hline 15.0 & $26 \% @ 278^{\circ}$ & $52 \% @ 143^{\circ}$ & $77 \% @ 98^{\circ}$ & $100 \% @ 75^{\circ}$ & $122 \% @ 62^{\circ}$ & $141 \% @ 53^{\circ}$ & $185 \% @ 41^{\circ}$ \\
\hline
\end{tabular}

Table 8: Residual Aberration Ratios and Relative Orientations for Different Cyclotorsional Errors. The percentage is the amount of postoperative residual in magnitude, whereas the angle is the relative orientation of the postoperative residual

\section{E.4.3 Clinical Optical Benefit \\ (Beneficio óptico clínico)}

Considering the cyclotorsional error and the preoperative astigmatism, we calculated the number of treatments that would theoretically achieve an optical benefit for the astigmatism component $(\mathrm{m}=2)$. 
With these settings, $95 \%$ of the eyes $(n=72)$ would have obtained an optical benefit for the cylinder if the cylindrical correction were correct but the axis was wrong and if cyclotorsional errors occurring around the ablation centre were the only failures. This compared to $89 \%$ of the eyes $(n=68)$ that actually obtained a postoperative cylinder lower than preoperative value. The differences between the theoretical and the empirical results were marginally significant $(P=0.05)$.

\section{E.4.4 Clinical Visual Benefit \\ (Beneficio visual clínico)}

With the same settings as previously, $95 \%$ of the eyes $(n=72)$ would have obtained a visual benefit for the cylinder compared to $87 \%$ of the eyes $(n=66)$ that actually had a stable or improved postoperative UCVA compared to the preoperative BSCVA $(P<0.01)$ and to $91 \%$ of the eyes $(n=69)$ with a stable or improved BSCVA $(P=0.09)$.

\section{E.4.5 Clinical Absolute Benefit \\ (Beneficio absoluto clínico)}

With the same settings as previously, $93 \%$ of the eyes $(n=71)$ would have obtained an absolute benefit for the cylinder compared to $96 \%$ of the eyes $(n=73)$ in which the postoperative astigmatism was smaller than $0.50 \mathrm{D}(P=0.21)$.

\section{E.4.6 Clinical Ranges to Obtain Optical, Visual, and Absolute Benefits (Rangos clínicos para la obtención de beneficios ópticos, visuales o absolutos)}

Combining all success ratios to calculate the number of eyes that obtained (theoretically and empirically) optical, visual, and absolute benefits simultaneously, $89 \%$ of the eyes $(n=68)$ with theoretical global success vs. $79 \%$ of the eyes $(n=60)$ obtained a postoperative cylinder lower than the preoperatively, lower than $0.50 \mathrm{D}$, and a stable or improved BSCVA $(P<0.005)$. 
Considering the cyclotorsional error, we calculated a hypothetical case simulating for these patients up to which Zernike mode the treatments could have been planned to achieve optical and visual benefits (Table 9).

\begin{tabular}{|c|c|c|c|}
\hline $\mathbf{M}$ & Aberration & $\begin{array}{c}\text { Optical benefit } \\
\text { (residual }<\text { original) }\end{array}$ & $\begin{array}{c}\text { Visual benefit } \\
\text { (residual }<\text { matching factor) }\end{array}$ \\
\hline 1 & Coma & $97 \%$ & $96 \%$ \\
\hline 2 & Astigmatism & $95 \%$ & $95 \%$ \\
\hline 3 & Trefoil & $93 \%$ & $92 \%$ \\
\hline 4 & Tetrafoil & $92 \%$ & $89 \%$ \\
\hline 5 & Pentafoil & $89 \%$ & $87 \%$ \\
\hline 6 & Hexafoil & $87 \%$ & $83 \%$ \\
\hline 7 & Heptafoil & $84 \%$ & $78 \%$ \\
\hline 8 & Octafoil & $82 \%$ & $75 \%$ \\
\hline 9 & Eneafoil & $78 \%$ & $74 \%$ \\
\hline 10 & Decafoil & $75 \%$ & $71 \%$ \\
\hline & Dodecafoil & $71 \%$ & $66 \%$ \\
\hline & Pentadecafoil & $66 \%$ & $61 \%$ \\
\hline & Icosafoil & $61 \%$ & $58 \%$ \\
\hline 30 & Triacontafoil & $57 \%$ & $57 \%$ \\
\hline & Hexacontafoil & $55 \%$ & $55 \%$ \\
\hline
\end{tabular}

Table 9: Percentage of Treatments That Could Have Been Planned to Achieve an Optical and a Visual Benefit as a Function of the Highest Included Zernike Mode

\section{Section E.5 DISCUSSION}

(Discusión)

The method used in this study to determine the cyclotorsional error incurred during laser refractive surgery is indirect, because it calculates the torsional error retrospectively after the ablation procedures were performed. However, it is easy, straightforward, and does not require additional equipment or 
complicated algorithms. Its retrospective nature ensures that the calculated error corresponds to the average cyclotorsional error during the entire refractive surgery procedure. This way, the method could be used to validate the cyclotorsional errors obtained with other prospective methods.

This study had a limitation. Because this method considers that the difference between the planned astigmatism axis and the axis of the effectively achieved cylindrical correction is due only to cyclotorsional errors, it may be affected by other sources of unavoidable errors in laser refractive surgery, such as flap cuts, pattern decentration, blending zones, and corneal biomechanics. The results are valid in the absolute single-failure condition of pure cyclotorsional errors. Moreover, we assumed for the study that the torsion always occurred around the intended ablation centre. It usually happens that the pupil size and centre differ for the treatment compared to that during diagnosis. ${ }^{220}$ Then, excluding cyclotorsion, there is already a lateral displacement that mismatches the ablation profile. Further, cyclotorsion occurring around any position other than the ablation centre results in additional lateral displacement combined with cyclotorsion. ${ }^{221}$ Finally, this analysis considers the results in terms of the residual monochromatic wavefront aberration. However, the visual process is more complex than just an image-projection system and involves elements such as neural compensation and chromatic aberration, which were beyond the scope of this study. The cortical aspect of visual processing may affect the subjective symptoms associated with residual wavefront aberration.

With our indirect analysis of the cyclotorsional error, we obtained an average cyclotorsional error of $4.39^{\circ}$, which, despite the above mentioned limitations of the method, agrees with the observations of Ciccio et al., ${ }^{222}$ who reported $4^{\circ}$. In our sample, however, $13 \%$ of eyes had cyclotorsion exceeding 10 degrees. These patients would be expected to have at least $35 \%$ residual cylinder, 52\% residual trefoil, and higher residual errors of tetrafoil, pentafoil, and hexafoil. In addition, octafoil would be induced beginning at 7.5 degrees of cyclotorsion.

Due to the cyclic nature, the residual aberration error emanating from cyclotorsional error ranges from $0 \%$ to $200 \%$ of the original aberration. However, the induced aberrations emanating from lateral displacements always increase 
with decentration. ${ }^{223}$ If we also consider that in human eyes with normal aberrations the weight $\mathrm{C}(n, m)$ of the Zernike terms $Z(n, m)$ decreases with increasing Zernike order $(n)^{149}$, then the theoretical impact of cyclotorted ablations is smaller than decentred ablations or edge effects ${ }^{224}$ (coma and spherical aberration $\left.{ }^{225}\right)$. The results of the work of Guirao et al. ${ }^{221}$ and Bará et al., ${ }^{144,226}$ are confirmed by the current study with special emphasis on the independent nature of the cyclotorsional effect with the radial order.

We adopted three criteria based on the accuracy that can be achieved to overcome cyclotorsion: optical benefit provides the maximum angular frequency that can be included in the correction for which an objective improvement in the optical quality can be expected; visual benefit, the maximum angular frequency for which a subjective improvement in the visual performance can be expected; and absolute benefit, the maximum magnitudes for each Zernike mode for which an effective result can be expected.

When all criteria are met without other sources of aberrations, the result is expected to be successful. When only the terms allowed by the visual benefit condition are included, but any of their magnitudes exceed the limits imposed by the $<0.50 \mathrm{DEQ}$ condition, the visual performance is expected to improve, but it might not be successful. When terms beyond the limits set by the visual benefit condition are included, the risk that the patient will require time to readapt to the new aberration must be considered. When terms beyond the limits set by the optical benefit condition are included, the risk that the aberrations will worsen must be considered carefully.

Without eye registration technologies, ${ }^{227,228}$ considering that maximum cyclotorsion measured from the shift from the upright to the supine position does not exceed $\pm 14^{\circ},{ }^{222}$ it is theoretically possible to obtain a visual benefit up to the trefoil angular frequencies and an optical benefit up to the tetrafoil angular frequencies. This explains why "classical" spherocylindrical corrections in refractive surgery succeed without major cyclotorsional considerations. However, using our limit of absolute residual dioptric error smaller than DEQ 0.50 , only up to 2.05 DEQ coma, 1.03 DEQ astigmatism, and 0.70 DEQ trefoil can be corrected successfully. The limited amount of astigmatism that can be corrected effectively 
for this cyclotorsional error may explain partly some unsuccessful results reported in refractive surgery.

Considering that the average cyclotorsion resulting from the shift from the upright to the supine position is about $\pm 4^{\circ},{ }^{222}$ without an aid other than manual orientation, the theoretical limits for achieving a visual benefit extend up to the endecafoil (11-fold) angular frequencies and up to the pentadecafoil (15-fold) angular frequencies for optical benefit. Our limit of absolute residual dioptric error less than $0.50 \mathrm{DEQ}$ increases to 7.16 DEQ for coma, 3.58 DEQ for astigmatism, and 2.39 DEQ for trefoil. The extended limits confirm why spherocylindrical corrections in laser refractive surgery have succeeded.

With currently available eye registration technologies, which provide an accuracy of about $\pm 1.5^{\circ}$, it is theoretically possible to achieve a visual benefit up to the triacontafoil (30-fold) angular frequencies and an optical benefit even beyond these angular frequencies, and using our limit of absolute residual dioptric error less than $0.50 \mathrm{DEQ}$, up to $19.10 \mathrm{DEQ}$ coma, 9.55 DEQ astigmatism, and 6.37 DEQ trefoil can be corrected successfully. This opens a new era in corneal laser refractive surgery, because patients may be treated for a wider range of refractive problems with enhanced success ratios. However, this requires a higher resolution than technically achievable with currently available systems. ${ }^{164,165}$

To the best of our knowledge, currently available laser platforms for customized corneal refractive surgery include not more than the eighth Zernike order, which theoretically corresponds to a visual benefit range for cyclotorsional tolerance of $\pm 5.7^{\circ}$ and an optical benefit range for cyclotorsional tolerance of $\pm 7.5^{\circ}$, which covers most cyclotorsion occurring when shifting from the upright to the supine position. Thus, the aberration status and the visual performance of the patients are expected to improve. Moreover, the same $\pm 7.5^{\circ}$ cyclotorsional tolerance means that the magnitudes for the major Zernike modes should not exceed 3.82 DEQ for coma modes, 1.92 DEQ for astigmatic modes, and, 1.28 $\mathrm{DEQ}$ for trefoil modes for theoretically successful results.

Based on different criteria, Bueeler and co-authors ${ }^{229}$ also determined conditions and tolerances for cyclotorsional accuracy. Their OT criterion corresponds approximately to our optical benefit condition, and their results for 
the tolerance limits $\left(29^{\circ}\right.$ for $3-\mathrm{mm}$ pupils and $21^{\circ}$ for 7 -mm pupils) did not differ greatly from the optical benefit result for astigmatism, confirming that astigmatism is the major component to be considered.

In our study, the theoretical percentage of treatments that would achieve an optical benefit was significantly higher than the percentage of treatments that actually obtained a postoperative cylinder lower than preoperatively (95\% vs. $89 \% ; P=0.05)$. The percentage of treatments that theoretically would achieve a visual benefit was significantly higher than the percentage of treatments with a stable or improved postoperative UCVA compared to the preoperative BSCVA $(95 \%$ vs. $87 \% ; P<0.01)$. Both indicate that other sources of aberrations have substantial impact on the final results. The percentage of treatments that theoretically would achieve a visual benefit was higher than the percentage of treatments with a stable or improved BSCVA ( $95 \%$ vs. $91 \% ; P=0.09$ ). Because residual cylinder can be corrected with spectacles, this indicates that other factors induce aberrations and affect the final results. In discussing visual benefit, although VA data are helpful, there may be patients with 20/20 vision who are unhappy with their visual outcomes due to poor mesopic and low-contrast VA that were not addressed in the current study.

Interestingly, the percentage of treatments achieving a theoretical absolute benefit was $93 \%$, whereas the percentage of treatments that actually had postoperative astigmatism reduced to an absolute residual error smaller than 0.50 D was higher (96\%; $P=0.21)$.

Finally, the percentage of treatments that theoretically would achieve global success (optical, visual, and absolute benefits simultaneously) was significantly higher than the percentage of treatments that actually obtained a postoperative cylinder lower than the preoperative value, a stable or improved BSCVA, and decreased postoperative astigmatism to an absolute residual error less than 0.50 D ( $89 \%$ vs. $79 \% ; P<0.005)$. This confirms that cyclotorsion is not the only reason for differences between theory and practice; wound healing and surgical variation also are keys factors in the outcome. 


\section{Section E.6 CONCLUSIONS}

(Conclusiones)

In summary, the current study showed that cyclotorsional errors result in residual aberrations and that with increasing cyclotorsional error there is a greater potential for inducing aberrations. Thirteen percent of eyes had over $10^{\circ}$ of calculated cyclotorsion, which predicts approximately a $35 \%$ residual astigmatic error in these eyes. Because astigmatic error is generally the highest magnitude vectorial aberration, patients with higher levels of astigmatism are at higher risk of problems due to cyclotorsional error.

\section{Section E.7 OUTLOOK}

(Perspectiva)

Currently a prospective method for determining intraoperative cyclotorsion has been implemented at the SCHWIND AMARIS laser system. With this new setting we are evaluating intraoperative static and dynamic cyclotorsions, and postoperative outcomes on astigmatism and high-order-aberration, among astigmatic or aberrated eyes that underwent refractive surgery.

Similarly, a six-dimensional eye-tracking module is being developed for SCHWIND eye-tech-solutions, with this coming technology we will, as well, evaluate intraoperative static and dynamic eye movements in 6D, and postoperative outcomes on refraction, and high-order-aberration. 

Topic F THE EFFECTIVE OPTICAL ZONE AFTER REFRACTIVE SURGERY

(La zona óptica efectiva tras cirugía refractiva)

\section{AUTHOR CONTRIBUTIONS}

Study concept and design (S.A.M.); data collection (M.C.); analysis and interpretation of data (S.A.M.); drafting (S.A.M.); critical revision (M.C.); statistical expertise (S.A.M.).

\section{Section F.1 ABSTRACT}

(Resumen)

Purpose: To evaluate the Effective Optical Zone (EOZ) (the part of the ablation that receives full correction), among eyes that underwent LASEK/Epi-LASEK treatments for myopic astigmatism.

Methods: 20 LASEK/Epi-LASEK treatments with mean defocus $-5.49 \pm 2.35 \mathrm{D}$ performed using the SCHWIND AMARIS system were retrospectively evaluated at 6-month follow-up. In all cases, pre-/post-operative Corneal-Wavefront analyses using the Keratron-Scout (OPTIKON2000) were performed. EOZvalues were evaluated from the changes of Root-Mean-Square of High-Order Wavefront-Aberration ( $\triangle \mathrm{RMSho}$ ), Spherical Aberration $(\triangle \mathrm{SphAb})$, and RootMean-Square of the change of High-Order Wavefront-Aberration (RMS $(\triangle \mathrm{HOAb}))$. Correlations of EOZ with Planned Optical Zone (POZ) and Defocus correction (SEq) were analysed using a bilinear function, as well as, calculations of the isometric lines (IOZ) for which EOZ equals $\mathrm{POZ}$ and of the nomogrammed $\mathrm{OZ}$ (NOPZ) to achieve an intended EOZ (IEOZ).

Results: At six-month, defocus was $-0.05 \pm 0.43 \mathrm{D}$, ninety percent eyes were within $\pm 0.50 \mathrm{D}$ from emmetropia. Mean RMSho increased $0.12 \mu \mathrm{m}$, SphAb

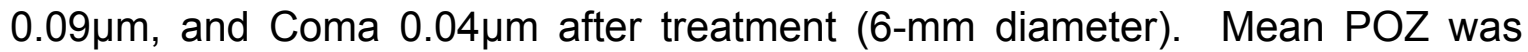
$6.76 \pm 0.25 \mathrm{~mm}$, whereas mean $\mathrm{EOZ}_{\triangle \mathrm{RMSh}} 6.74 \pm 0.66 \mathrm{~mm}$ (bilinear correlation $p<.005), E O Z_{\triangle S p h A b}$ was $6.83 \pm 0.58 \mathrm{~mm}$ (bilinear correlation $p<.0001$ ), 
$\mathrm{EOZ}_{\mathrm{RMS}(\triangle \mathrm{HOAb})} 6.42 \pm 0.58 \mathrm{~mm}$ (significantly smaller, $\mathrm{p}<.05$, bilinear correlation $p<.0005)$. EOZ positively correlates with $\mathrm{POZ}$ and declines steadily with SEq, depending stronger on $\mathrm{POZ}$ than on SEq. A treatment of $-5 \mathrm{D}$ in $6.00-\mathrm{mm} \mathrm{POZ}$ results in $5.75-\mathrm{mm} \mathrm{EOZ} \mathrm{(6.25-mm} \mathrm{NPOZ),} \mathrm{treatments} \mathrm{in} 6.50-\mathrm{mm} \mathrm{POZ}$ result in

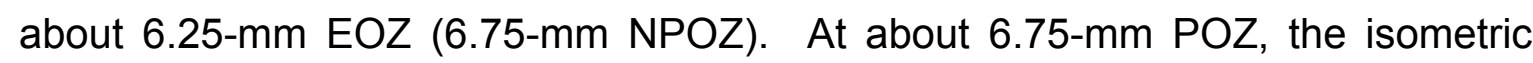
condition is met.

Conclusions: $E O Z_{\triangle R M S h o}$ and $E O Z_{\triangle S p h A b}$ were similar to $P O Z$, whereas $\mathrm{EOZ}_{\mathrm{RMS}(\triangle \mathrm{HOAb})}$ was significantly smaller. Differences between $\mathrm{EOZ}$ and $\mathrm{POZ}$ were larger for smaller POZ or larger Defocus corrections. POZ larger than $6.75-\mathrm{mm}$ result in EOZ, at least, as large as POZ. For OZ smaller than $6.75-\mathrm{mm}$, a nomogram for $\mathrm{OZ}$ could be applied.

\section{Section F.2 INTRODUCTION}

(Introducción)

The required ablation depth in corneal laser refractive surgery increases with the amount of ametropia to be corrected and the diameter of the optical zone selected $^{15}$. Therefore, the smallest diameter optical zone should be used compatible with normal physiologic optics of the cornea ${ }^{223}$.

Complaints of ghosting, blur, haloes, glare, decreased contrast sensitivity, and vision disturbance ${ }^{230}$ have been documented with small optical zones, especially when the scotopic pupil dilates beyond the diameter of the surgical optical zone $e^{231}$, and these symptoms may be a source of less patient satisfaction ${ }^{232}$. This is supported by clinical findings on night vision with small ablation diameters ${ }^{233},{ }^{234}$ as well as large pupil sizes ${ }^{231},{ }^{234}$ and attempted correction $^{235}$.

Laser refractive surgery generally reduces low order aberrations (defocus and astigmatism), yet high-order aberrations, particularly coma and spherical aberration, may be significantly induced ${ }^{64,224}$. 
In recent years, the increasing the size of the planned ablation zone and the use of new techniques to measure aberrations ${ }^{46}$ opened the possibility to correct, or at least reduce the induction, some of the high-order aberrations.

Methods for determining functional optical zones (FOZ) have been used previously. Independently developed ray-tracing programs ${ }^{234},{ }^{236}$ have been used to determined FOZ after refractive surgery. A direct approach to measure FOZ after refractive surgery has been proposed by manually determining the transition region between treated and untreated areas from corneal topography maps ${ }^{237}$.

In this study, we retrospectively evaluated three objective methods to determine the effective optical zone (EOZ) in eyes after keratorefractive surgery to provide assessment techniques to evaluate and compare keratorefractive surgical algorithms.

\section{Section F.3 METHODS}

(Método)

\section{F.3.1 Subjects \\ (Sujetos)}

The first consecutive 20 compound myopic astigmatism (MA) treatments (10 patients), treated by MC using the AMARIS Aberration-Free ${ }^{\mathrm{TM}}$ aspheric ablation with LASEK $^{26}$ or Epi-LASEK ${ }^{29}$ techniques, which completed $6 \mathrm{M}$ follow-up were retrospectively analysed.

Six-month follow-up was available in 20 of these eyes (100\%), and their preoperative data were as follows: mean manifest defocus refraction $-5.49 \pm 2.35$ $\mathrm{D}$ (range, -9.75 to $-2.25 \mathrm{D}$ ); mean manifest astigmatism magnitude $1.21 \pm 1.19 \mathrm{D}$ (range, 0.00 to $4.00 \mathrm{D}$ ). In all eyes, we measured corneal topography ${ }^{153}$ and derived corneal wavefront analyses (Keratron-Scout, OPTIKON2000, Rome, Italy), manifest refraction, and uncorrected and best spectacle-corrected Snellen visual acuity ${ }^{204}$ (UCVA and BSCVA, respectively). Measurements were performed preoperatively and at one, three, and six months after surgery. 


\section{F.3.2 Ablation profiles \\ (Perfiles de ablación)}

All ablations were non-customised based on "aberration neutral" profiles ${ }^{92}$ and calculated using the ORK-CAM software module version 3.1 (SCHWIND eyetech-solutions, Kleinostheim, Germany). Aspheric aberration neutral (AberrationFree $^{\mathrm{TM}}$ ) profiles are not based on the Munnerlyn proposed profiles ${ }^{15}$, and go beyond that by adding some aspheric characteristics ${ }^{238}$ to balance the induction of spherical aberration ${ }^{79,239}$ (prolateness optimization ${ }^{82,170}$ ). The ablations were performed using the AMARIS excimer laser (SCHWIND eye-tech-solutions, Kleinostheim, Germany).

\section{F.3.3 Ablation zones \\ (Zonas de ablación)}

Mean programmed optical zone (POZ) was $6.76 \pm 0.25 \mathrm{~mm}$ (range, 6.25 to $7.00 \mathrm{~mm}$ ) with a variable transition size (TZ) automatically provided by the laser related to the planned correction of $1.36 \pm 0.47 \mathrm{~mm}$ (range, 0.64 to $2.20 \mathrm{~mm}$ ) leading to a total ablation zone (TAZ) $8.13 \pm 0.31 \mathrm{~mm}$ (range, 7.64 to $8.70 \mathrm{~mm}$ ).

\section{F.3.4 Analysis of the effective optical zone (Análisis de la zona óptica efectiva)}

Definition of the optical zone $(\mathrm{OZ})$ reads "the part of the corneal ablation area that receives the full intended refractive correction" (Drum B. The Evolution of the Optical Zone in Corneal Refractive Surgery. $8^{\text {th }}$ International Wavefront Congress, Santa Fe, USA; February 2007). However, operational definition of the $\mathrm{OZ}$ consists of the part of the corneal ablation area that receives the treatment that is designed to produce the full intended refractive correction. Finally, Effective Optical Zone (EOZ) can be defined as the part of the corneal ablation area that actually conforms to the theoretical definition. However, the definition implies that the optical zone need not be circular. 
F.3.4.1 Change Of Root-Mean-Square Of Higher Order Wavefront Aberration Method

(Método del cambio de la raíz cuadrática media de la aberración de onda de alto orden)

By comparing postoperative and preoperative corneal wavefront aberrations analysed for a common diameter starting from 4-mm, we have increased the analysis diameter in $10 \mu \mathrm{m}$ steps and refit to Zernike polynomials up to the $7^{\text {th }}$ radial order, until the difference of the corneal RMSho was above $0.25 \mathrm{D}$ for the first time (Figure 57). This diameter minus $10 \mu \mathrm{m}$ was determining the EOZ:

$$
\triangle R M S h o(E O Z)=0.25 D
$$

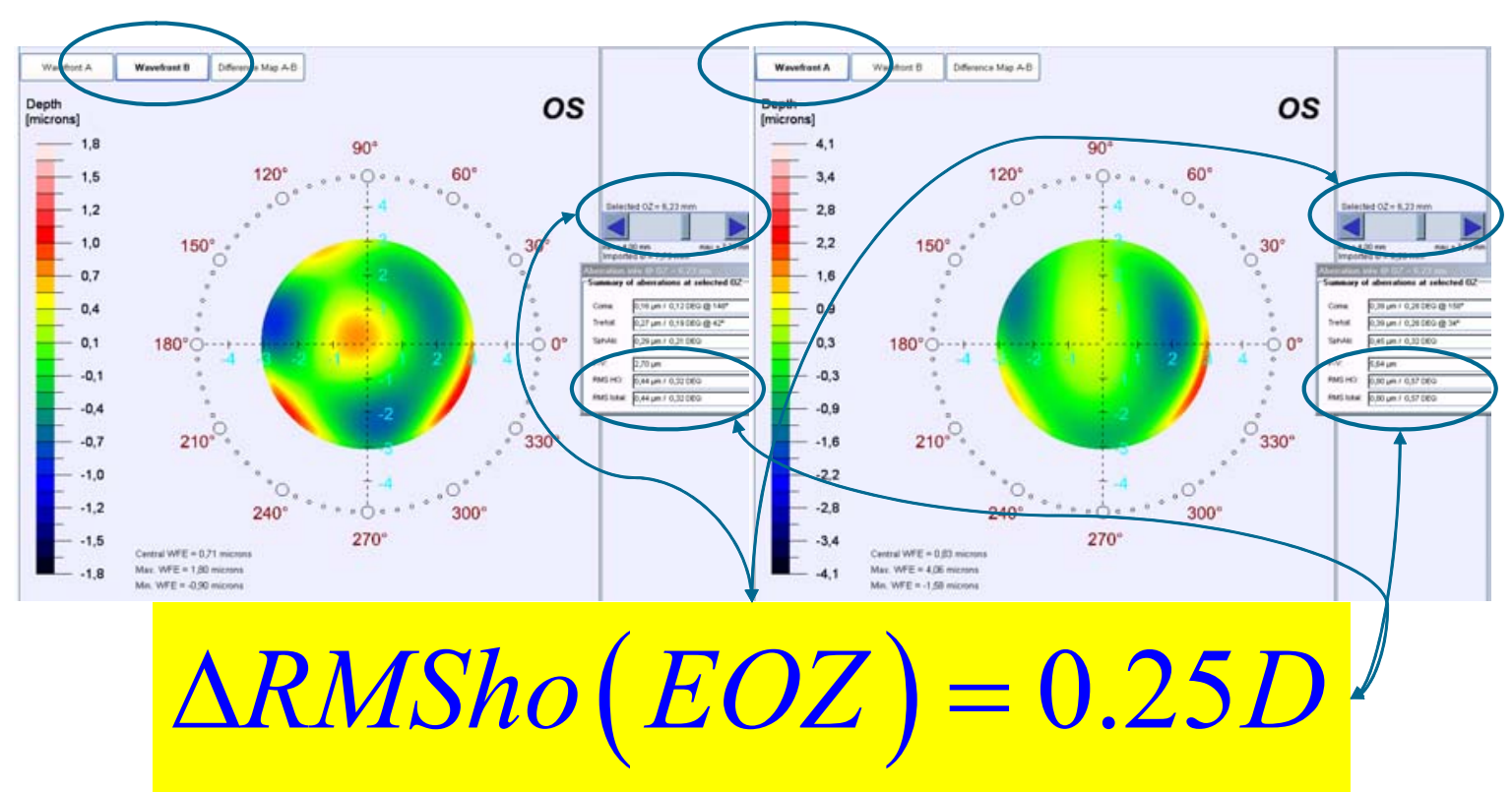

Figure 57: Concept of the $\triangle \mathrm{RMSho}$ method: By comparing postoperative and preoperative corneal wavefront aberrations analysed for a common diameter starting from 4-mm, we have increased the analysis diameter in $10 \mu \mathrm{m}$ steps, until the difference of the corneal RMSho was above $0.25 \mathrm{D}$ for the first time. This diameter minus $10 \mu \mathrm{m}$ was determining the EOZ.

\section{F.3.4.2 Change In Spherical Aberration Method \\ (Método del cambio de la aberración esférica)}

By analysing the differential corneal wavefront aberrations for a diameter starting from 4-mm, we have increased the analysis diameter in $10 \mu \mathrm{m}$ steps and 
refit to Zernike polynomials up to the $7^{\text {th }}$ radial order, until the differential corneal spherical aberration was above 0.25 D for the first time (Figure 58). This diameter minus $10 \mu \mathrm{m}$ was determining the EOZ:

$$
\triangle \operatorname{Sph} A b(E O Z)=0.25 D
$$

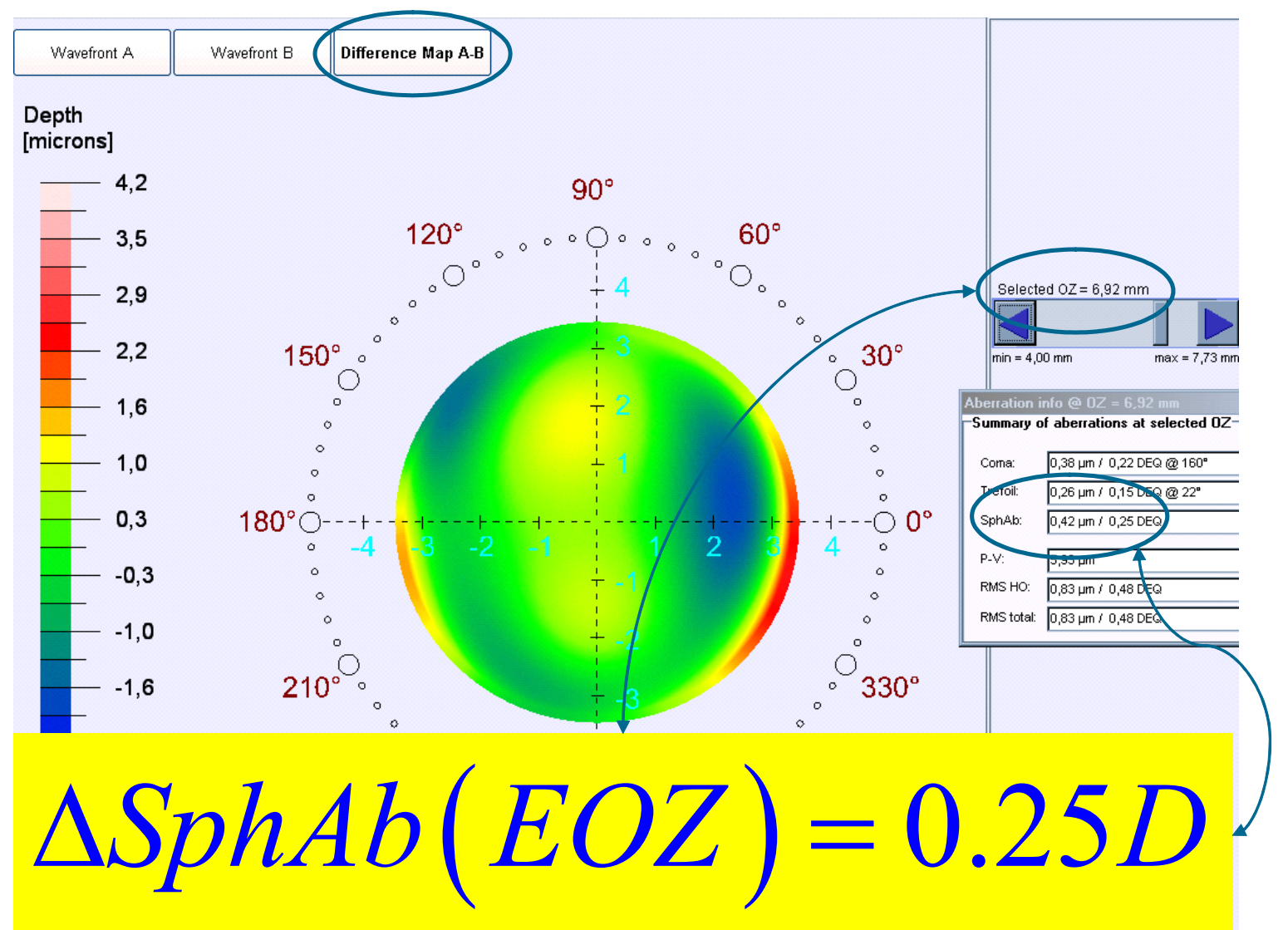

Figure 58: Concept of the $\triangle \mathrm{SphAb}$ method: By analysing the differential corneal wavefront aberrations for a diameter starting from 4-mm, we have increased the analysis diameter in $10 \mu \mathrm{m}$ steps, until the differential corneal spherical aberration was above $0.25 \mathrm{D}$ for the first time. This diameter minus $10 \mu \mathrm{m}$ was determining the EOZ.
F.3.4.3 Root-Mean-Square Of The Change Of Higher Order Wavefront Aberration Method
(Método de la raíz cuadrática media del cambio de la aberración de onda de alto orden)

By analysing the differential corneal wavefront aberrations for a diameter starting from 4-mm, we have increased the analysis diameter in $10 \mu \mathrm{m}$ steps and 
refit to Zernike polynomials up to the $7^{\text {th }}$ radial order, until the root-mean-square of the differential corneal wavefront aberration was above $0.25 \mathrm{D}$ for the first time (Figure 59). This diameter minus $10 \mu \mathrm{m}$ was determining the EOZ:

$$
R M S[\triangle H O A b(E O Z)]=0.25 D
$$

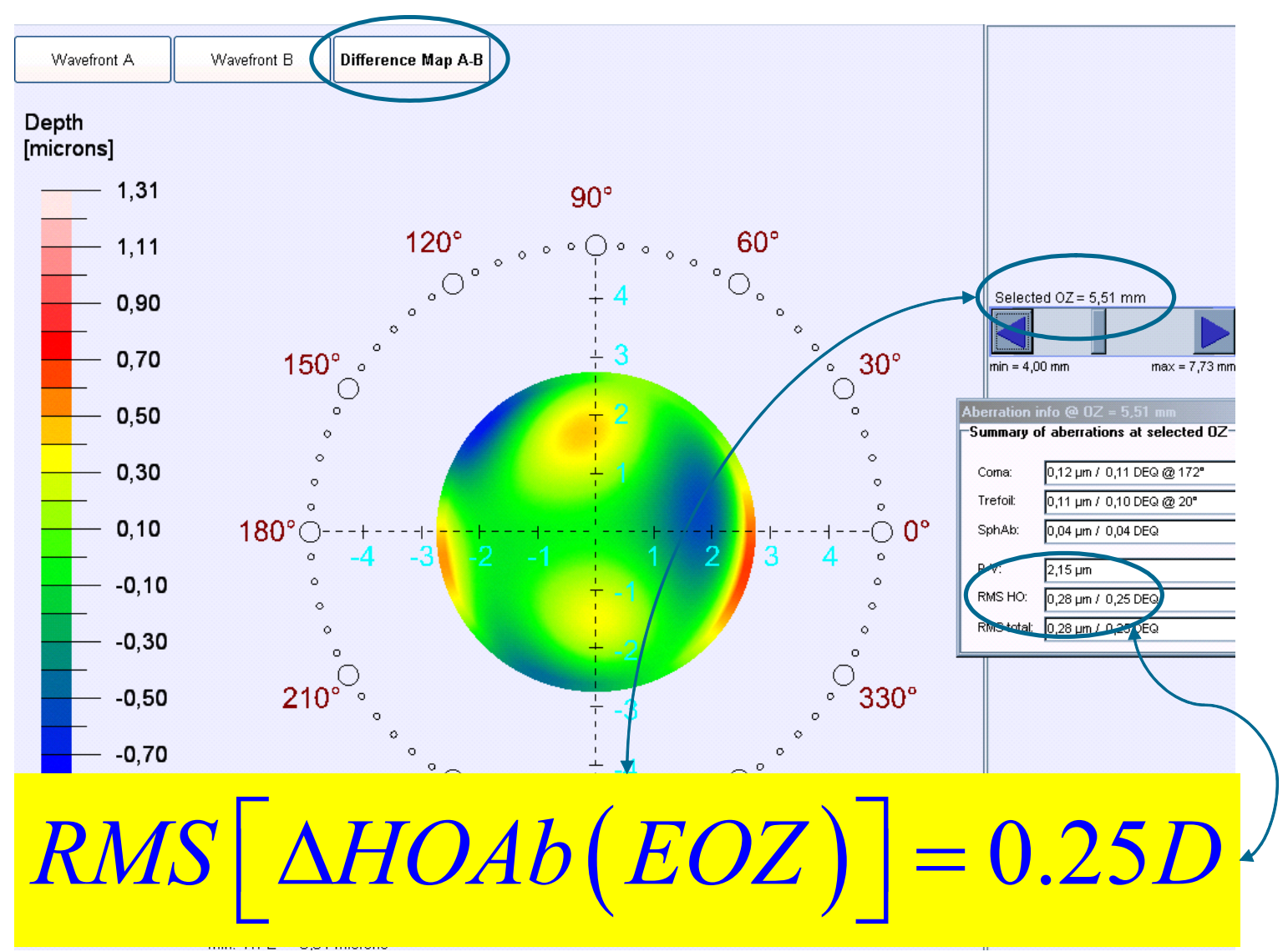

Figure 59: Concept of the RMS $(\triangle H O A b)$ method: By analysing the differential corneal wavefront aberrations for a diameter starting from 4-mm, we have increased the analysis diameter in $10 \mu \mathrm{m}$ steps, until the root-mean-square of the differential corneal wavefront aberration was above $0.25 \mathrm{D}$ for the first time. This diameter minus $10 \mu \mathrm{m}$ was determining the EOZ.

\section{F.3.5 Mean value analyses \\ (Análisis de valores promedio)}

We analysed the mean values of these metrics and assessed the statistical significance of the EOZ compared to the $\mathrm{POZ}$ using paired Student's T-tests. 


\section{F.3.6 Regression analyses \\ (Análisis de regresión)}

We have analysed the correlations of EOZ for each of the methods with POZ and with defocus correction, using a bilinear function (linear with POZ and defocus) of the form:

$$
E O Z=a+(b+c \cdot P O Z) \cdot(d+e \cdot S E q)
$$

where $a$ is a general bias term, $b$ a bias term for the linearity with POZ, $c$ the partial slope for the linearity with POZ, $d$ a bias term for the linearity with defocus, and $e$ the partial slope for the linearity with defocus. The ideal case is represented by the coefficients:

$$
\begin{aligned}
& a=0 \\
& b=0 \\
& c=1 \\
& d=1 \\
& e=0
\end{aligned}
$$

We assessed the statistical significance of the correlations using Student's T-tests, the Coefficient of Determination $\left(r^{2}\right)$ was used and the significance of the correlations has been evaluated considering a metric distributed approximately as $t$ with $\mathrm{N}-3$ degrees of freedom where $\mathrm{N}$ is the size of the sample.

\section{F.3.7 Calculation of isometric lines \\ (Cálculo de líneas isométricas)}

With the obtained parameters ( $a$ to $e$ ), we have calculated for each of the methods the isometric lines for optical zone (IOZ) for which the achieved effective optical zone equals the planned optical zone. The isometric lines fulfil the condition: 


$$
\begin{aligned}
& E O Z(P O Z, S E q)=P O Z \\
& I O Z=\frac{a+b \cdot(d+e \cdot S E q)}{1-c \cdot(d+e \cdot S E q)}
\end{aligned}
$$

\section{F.3.8 Calculation of proposed nomogram for $\mathrm{OZ}$}

(Cálculo de una propuesta de nomograma para ZO)

With the obtained parameters (a to $e$ ), we have calculated the nomogram planned OZ (NPOZ) required to achieve an intended EOZ (IEOZ):

$$
N P O Z=\frac{I E O Z-a-b \cdot(d+e \cdot S E q)}{c \cdot(d+e \cdot S E q)}
$$

Section F.4 RESULTS

(Resultados)

F.4.1 Adverse events

(Complicaciones)

Neither adverse events nor complications were observed intra- or postoperatively. No single eye needed or demanded a retreatment.

\section{F.4.2 Refractive Outcomes \\ (Resultados refractivos)}

Concerning refractive outcomes, we merely want to outline that both, the SEq and the cylinder were significantly reduced to subclinical values at 6 months postoperatively (mean residual defocus refraction was $-0.05 \pm 0.43 \mathrm{D}$ (range -1.00 to $+0.62 \mathrm{D})(p<.0001)$ and mean residual astigmatism magnitude $0.21 \pm 0.54 \mathrm{D}$ (range, 0.00 to $1.50 \mathrm{D})(\mathrm{p}<.001))$ and that $90 \%$ of eyes $(n=18)$ were within \pm 0.50 $\mathrm{D}$ of the attempted correction (Table 10). 


\begin{tabular}{|c|c|c|c|}
\hline & $\begin{array}{c}\text { Pre-op } \\
\text { (MeantStdDev) }\end{array}$ & $\begin{array}{l}\text { Post-op } \\
\text { ean } \pm \text { StdDev) }\end{array}$ & p-value \\
\hline Spherical Equivalent (D) & $-5.49 \pm 2.35$ & $-0.05 \pm 0.43$ & $<.0001^{*}$ \\
\hline Cylinder (D) & $1.21 \pm 1.19$ & $0.21 \pm 0.54$ & $<.001^{*}$ \\
\hline Predictability within $\pm 0.50 \mathrm{D}(\%)$ & --- & $90 \%$ & --- \\
\hline Predictability within $\pm 1.00 \mathrm{D}(\%)$ & --- & $100 \%$ & --- \\
\hline Coma Aberration at $6.00 \mathrm{~mm}(\mu \mathrm{m})$ & $0.26 \pm 0.23$ & $0.30 \pm 0.25$ & $<.05^{*}$ \\
\hline Spherical Aberration at $6.00 \mathrm{~mm}(\mu \mathrm{m})$ & $0.28 \pm 0.15$ & $0.38 \pm 0.24$ & $<.005^{*}$ \\
\hline High-Order Aberration at $6.00 \mathrm{~mm}(\mu \mathrm{m} R \mathrm{RMS})$ & $0.45 \pm 0.12$ & $0.56 \pm 0.28$ & $<.01^{*}$ \\
\hline
\end{tabular}

Table 10: Refractive outcomes and induced corneal aberrations after refractive surgery

\section{F.4.3 Changes in corneal wavefront aberration at 6-mm analysis diameter (Cambios en la aberración del frente de onda corneal analizado para 6- mm de diámetro)}

Preoperative corneal coma aberration $(C[3, \pm 1])$ was $0.26 \pm 0.23 \mu \mathrm{m} R \mathrm{RM}$, corneal spherical aberration $(\mathrm{C}[4,0])(\mathrm{SphAb})$ was $+0.28 \pm 0.15 \mu \mathrm{m}$, and corneal RMSho was $0.45 \pm 0.12 \mu \mathrm{m}$ RMS. Postoperatively, corneal coma magnitude changed to $0.30 \pm 0.25 \mu \mathrm{m}$ RMS $(p<.05)$, corneal SphAb to $+0.38 \pm 0.24 \mu \mathrm{m}$ $(p<.005)$, and corneal RMSho changed to $0.56 \pm 0.28 \mu \mathrm{m}$ RMS $(p<.01)$.

\section{F.4.4 Mean value analyses for EOZ \\ (Análisis de valores promedio para ZOE)}

We analysed the mean values of EOZ for each of the 3 methods and assessed the statistical significance of the EOZ compared to the POZ using paired Student's T-tests. $\mathrm{EOZ}_{\triangle \mathrm{RMSho}}$ and $\mathrm{EOZ}_{\triangle \mathrm{SphAb}}$ were similar to $\mathrm{POZ}$, whereas 
$E_{\mathrm{RMS}(\triangle \mathrm{HOAb})}$ was significantly $(\mathrm{p}<.05)$ smaller than $\mathrm{POZ}$ and the $\mathrm{EOZ}$ determined by the other two methods (Table 11).

\begin{tabular}{|c|c|c|c|c|c|c|c|}
\hline & Mean & StdDev & Min & Max & $\mathbf{p}$ & $\mathbf{R}^{2}$-corr & p-corr \\
\hline Planned OZ (mm) & 6.76 & 0.25 & 6.25 & 7.00 & --- & --- & --- \\
\hline $\mathrm{EOZ}_{\Delta \mathrm{RMSho}}(\mathrm{mm})$ & 6.74 & 0.66 & 5.81 & 7.81 & .4 & .5 & $<.005^{\star}$ \\
\hline $\mathrm{EOZ}_{\Delta \mathrm{SphAb}}(\mathrm{mm})$ & 6.83 & 0.58 & 5.91 & 7.53 & .3 & .7 & $<.00005^{\star}$ \\
\hline $\mathrm{EOZ}_{\mathrm{RMS}(\Delta \mathrm{HOAb})}(\mathrm{mm})$ & 6.42 & 0.58 & 5.51 & 7.31 & $<.05^{\star}$ & .7 & $<.0005^{*}$ \\
\hline
\end{tabular}

Table 11: Effective optical zone after refractive surgery vs. planned optical zone

\section{F.4.5 Regression analyses for EOZ \\ (Análisis de regresión de la zona óptica efectiva)}

We have analysed the correlations of EOZ for each of the methods with POZ and with defocus correction $\left(r^{2}=.5, p<.005\right.$ for the $\Delta$ RMSho method; $r^{2}=.7$, $p<.0001$ for the $\triangle$ SphAb method; and $r^{2}=.7, p<.0005$ for the RMS $(\triangle H O A b)$ method) (Figure 60). 
Effective Optical Zone diameter (mm) distribution from Laser Settings

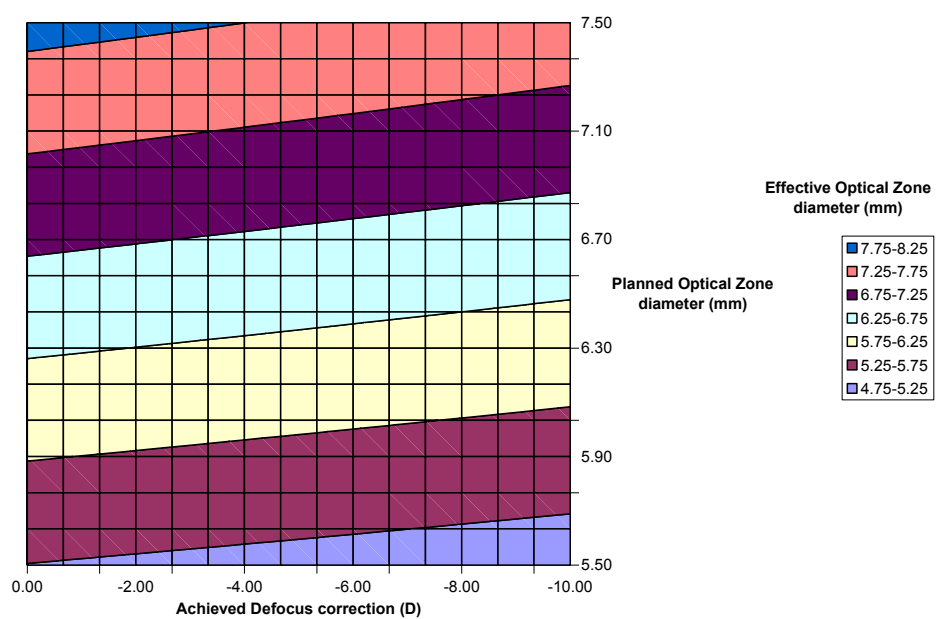

Effective Optical Zone diameter (mm) distribution from Laser Settings

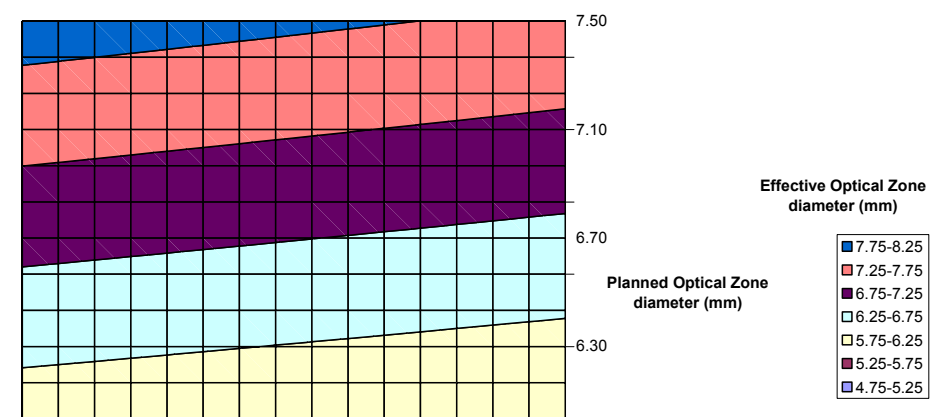

Effective Optical Zone diameter (mm) distribution from Laser Settings

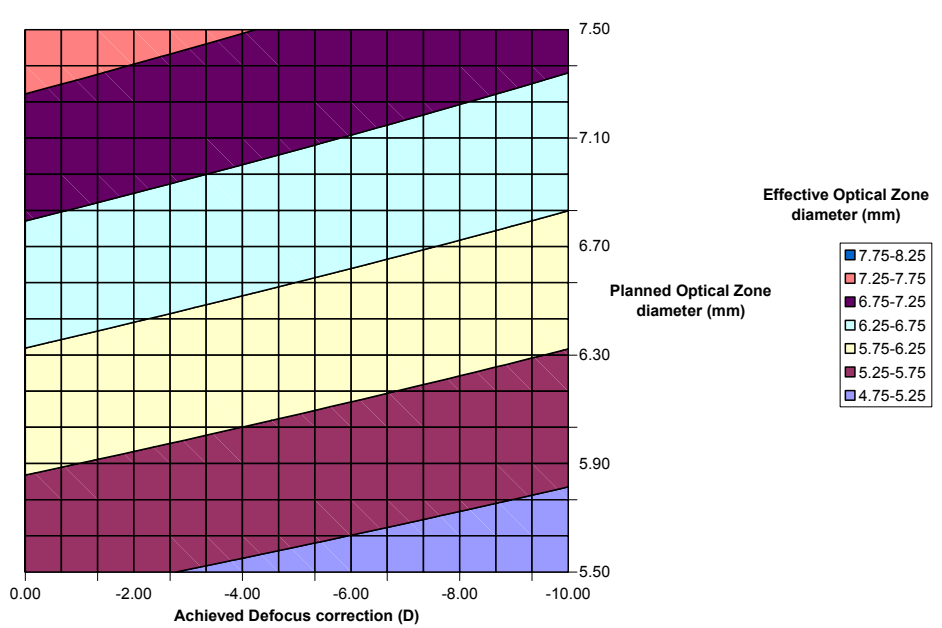

Figure 60: Bilinear regression analyses for the correlations of EOZ with $\mathrm{POZ}$ and with defocus correction for each of the methods: $\Delta$ RMSho method 
$\left(r^{2}=.5, \quad p<.005\right) \quad$ (top), $\quad \Delta$ SphAb method $\left(r^{2}=.7, \quad p<.0001\right) \quad$ (middle), and $\operatorname{RMS}(\triangle \mathrm{HOAb})$ method $\left(r^{2}=.7, p<.0005\right)$ (bottom). EOZ correlates positively with $\mathrm{POZ}$, and declines steadily with increasing defocus corrections. EOZ depends stronger on POZ than on SEq. Example of double-entry graphs: A treatment of $5 \mathrm{D}$ in $6.5 \mathrm{~mm} \mathrm{POZ}$ results in green when analysed with the $\triangle \mathrm{RMSho}$ and $\triangle S p h A b$ methods $(\sim 6.5 \mathrm{~mm} \mathrm{EOZ),} \mathrm{but} \mathrm{in} \mathrm{yellow} \mathrm{when} \mathrm{analysed} \mathrm{with} \mathrm{the}$ $\operatorname{RMS}(\triangle \mathrm{HOAb})$ method ( $6.0 \mathrm{~mm} \mathrm{EOZ})$.

EOZ correlates positively with $\mathrm{POZ}$, and declines steadily with increasing defocus corrections. EOZ depends stronger on POZ than on SEq.

On average, and simplifying the relationship to only EOZ and $\mathrm{POZ}$ we observed that planning an $\mathrm{OZ}$ of $5.50-\mathrm{mm}$ in diameter leads to an effective $\mathrm{OZ}$ of about $5.25-\mathrm{mm}$ in diameter, planning an $\mathrm{OZ}$ of $6.50-\mathrm{mm}$ in diameter leads to an effective $\mathrm{OZ}$ of about $6.25 \mathrm{-mm}$ in diameter, and planning an $\mathrm{OZ}$ of $7.50-\mathrm{mm}$ in diameter leads to an effective OZ of about $7.50-\mathrm{mm}$ in diameter (Table 12).

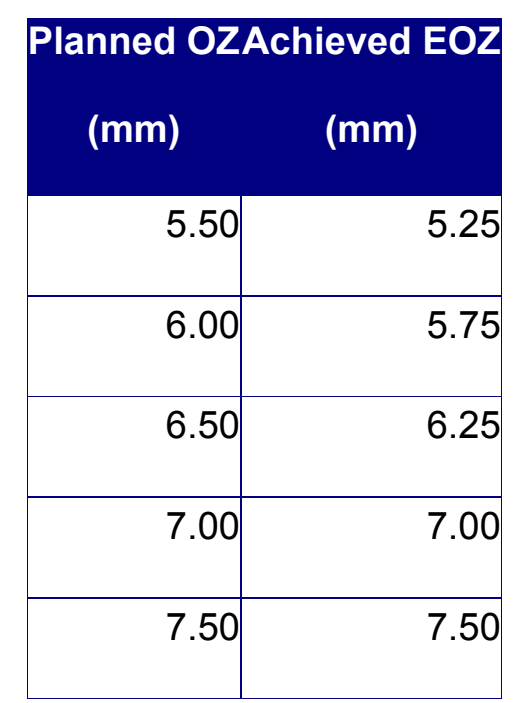

Table 12: Mean effective optical zone after refractive surgery vs. planned optical zone 
F.4.6

Isometric lines for $O Z$

(Lineas isométricas para ZO)

With the obtained parameters ( $a$ to $e$ ), we have calculated the isometric lines for optical zone (IOZ) for each of the methods (Figure 61) resulting that:

$$
\begin{aligned}
& P O Z<I O Z \Leftrightarrow E O Z<P O Z \\
& P O Z=I O Z \Leftrightarrow E O Z=P O Z \\
& P O Z>I O Z \Leftrightarrow E O Z>P O Z
\end{aligned}
$$

Isometric Optical Zone

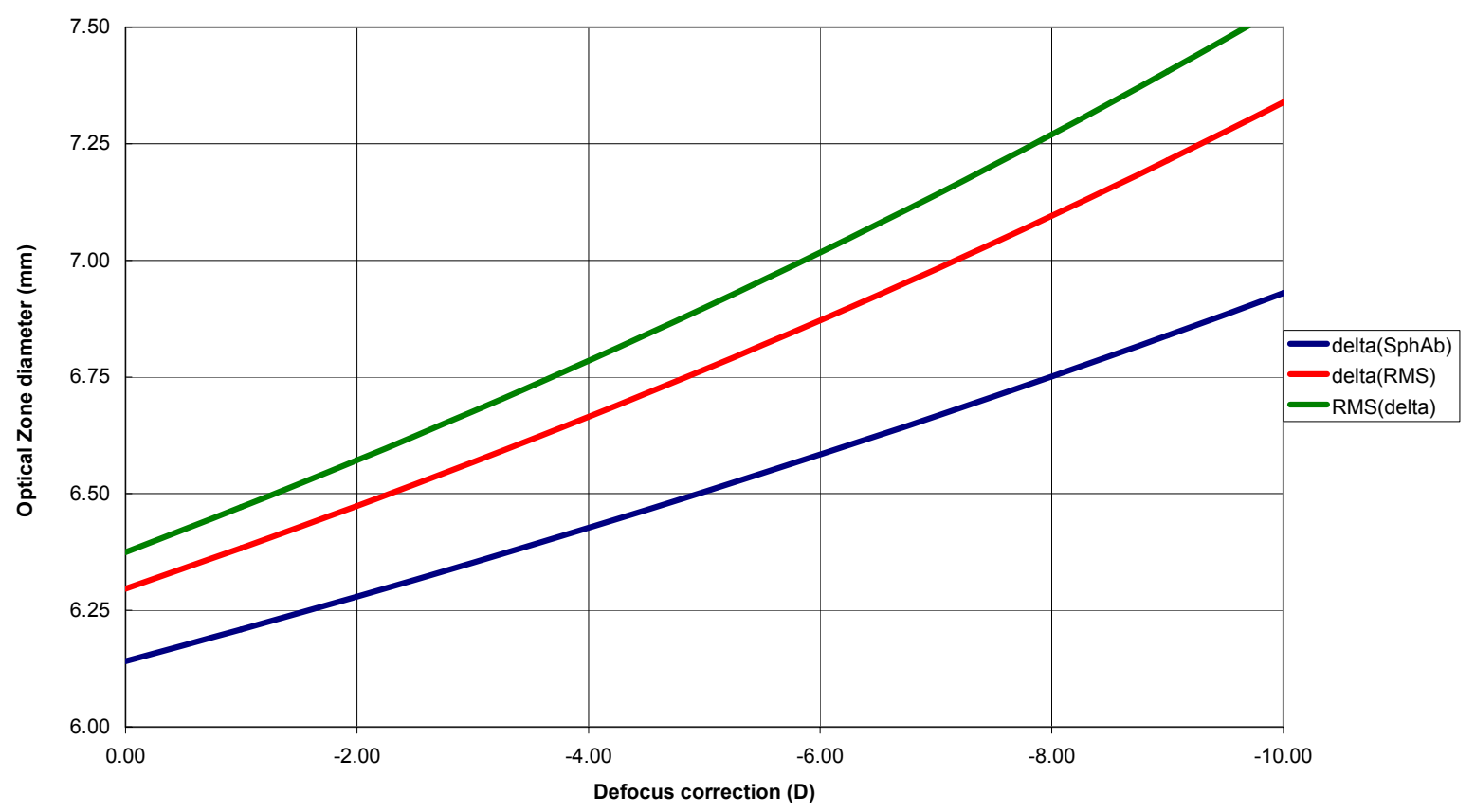

Figure 61: Isometric optical zones: $\triangle \mathrm{RMSho}$ method (red), $\triangle$ SphAb method (blue), and $\mathrm{RMS}(\triangle \mathrm{HOAb})$ method (green). For $P O Z<I O Z \Leftrightarrow E O Z<P O Z$, for $P O Z=I O Z \Leftrightarrow E O Z=P O Z$, and for $P O Z>I O Z \Leftrightarrow E O Z>P O Z$. POZ larger than 6.75 $\mathrm{mm}$ result in EOZ, at least, as large as $\mathrm{POZ}$.

\section{F.4.7}

Proposed nomogram for $O Z$

(Nomograma para $Z O$ )

With the obtained parameters ( $a$ to $e$ ), we have calculated the nomogram planned OZ (NPOZ) required to achieve an intended EOZ (IEOZ) (Figure 62). 
Nomogram for Optical Zone diameter (mm)

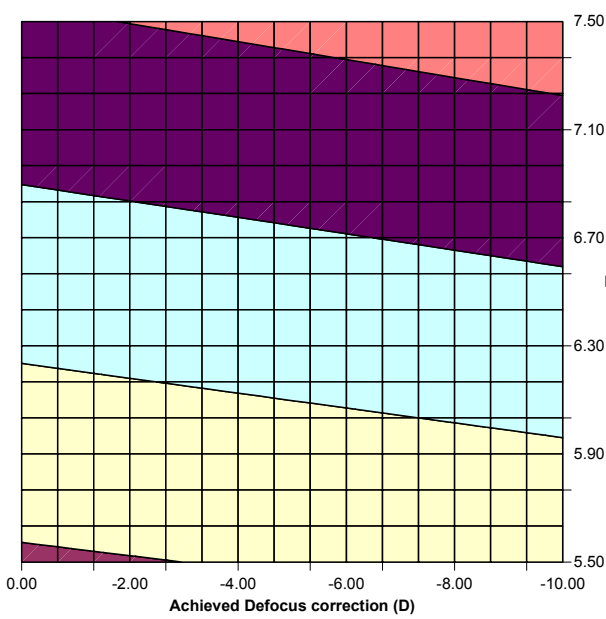

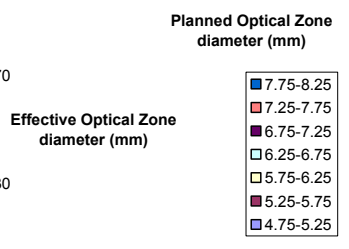

Nomogram for Optical Zone diameter $(\mathrm{mm})$

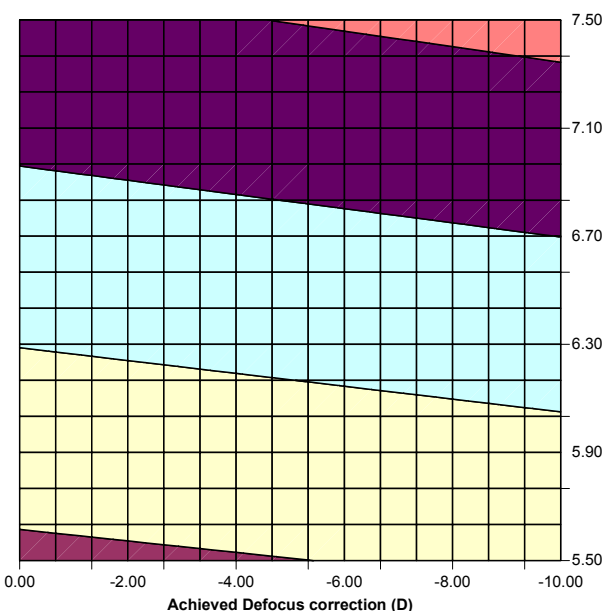

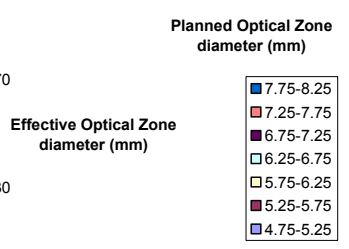

Nomogram for Optical Zone diameter $(\mathrm{mm})$
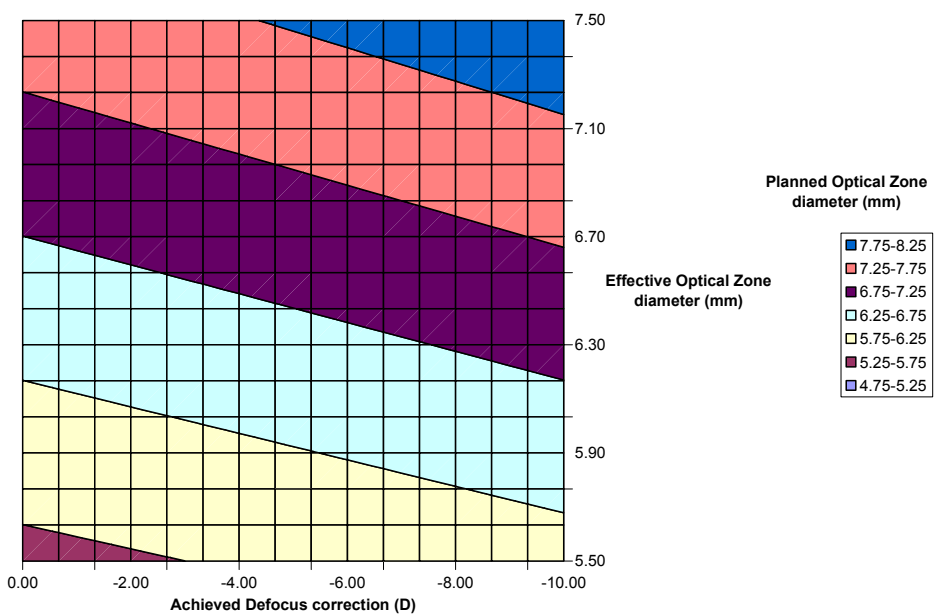

Figure 62: Calculated the nomogram planned OZ (NPOZ) required to achieve an intended EOZ (IEOZ) for defocus correction for each of the methods: 
$\Delta$ RMSho method (top), $\triangle$ SphAb method (middle), and RMS $(\triangle \mathrm{HOAb}$ ) method (bottom). Example of double-entry graphs: A treatment of $-5 \mathrm{D}$ with intended $E O Z$ of $6.5 \mathrm{~mm}$ results in green when planned for the $\triangle \mathrm{RMSho}$ and $\triangle \mathrm{Sph} A b$ methods ( $6.5 \mathrm{~mm}$ nomogrammed $\mathrm{OZ}$ ), but in yellow when planned for the $\operatorname{RMS}(\triangle \mathrm{HOAb})$ method ( 7.0 mm nomogrammed OZ).

On average, and simplifying the relationship to only IEOZ and NPOZ we observed that for achieving an EOZ of 5.50-mm in diameter, a POZ of about 5.75$\mathrm{mm}$ in diameter is required; for achieving an EOZ of 6.50-mm in diameter, a POZ of about 6.75-mm in diameter is required; and for achieving an EOZ of 7.50-mm in diameter, a POZ of about 7.50-mm in diameter is required (Table 13).

\begin{tabular}{|r|r|}
\multicolumn{1}{|c|}{$\begin{array}{r}\text { Intended EOZNomogrammed POZ } \\
(\mathbf{m m})\end{array}$} & 5.75 \\
\hline 5.50 & $\mathbf{( m m})$ \\
\hline 6.00 & 6.25 \\
\hline 6.50 & 6.75 \\
\hline 7.00 & 7.00 \\
\hline 7.50 & 7.50 \\
\hline
\end{tabular}

Table 13: Mean nomogrammed optical zone vs. intended effective optical zone

\section{Section F.5 DISCUSSION}

(Discusión)

To analyse EOZ in our treatments in a systematic way consistent with formal definitions, we decided to base our analysis upon previous knowledge. Since wavefront aberration describes properly optical quality, it seems adequate to use wavefront aberration for determining EOZ. Since we were applying the analysis to corneal laser refractive surgery, it seems adequate to use corneal 
wavefront aberration for determining EOZ. Since corneal refractive surgery increases wavefront aberration, it seems adequate to analyse the change of the corneal RMSho for determining EOZ ( $\triangle$ RMSho method). Since the most induced term is spherical aberration, it seems adequate to analyse the change of the corneal spherical aberration for determining EOZ ( $\triangle S p h A b$ method). Since AMARIS Aberration-Free profiles aim being neutral for $\mathrm{HOAb}$, it seems adequate to analyse the root-mean-square of the change of the corneal wavefront aberration for determining EOZ (RMS( $\triangle \mathrm{HOAb})$ method).

The measurement technique used in this study actually imposes restrictions on optical zone size that may underestimate it for decentrations. On the other hand, data not fit by the Zernike polynomials up to the $7^{\text {th }}$ radial order (36 Zernike coefficients). It is known that the residual irregularity of the cornea not fit by Zernike's may have a significant impact on visual quality. Ignoring this effect might bias the effective optical zone size determined leading to an overestimate that can be significant.

Uozato and Guyton ${ }^{223}$ were the first to calculate the optical zone area needed to obtain glare-free distance vision in emmetropia. They stated that, "for a patient to have a zone of glare-free vision centred on the point of fixation, the optical zone of the cornea must be larger than the entrance pupil (apparent diameter of the pupil)." Not only must this optical zone be without scarring and irregularity, but it must also be of uniform refractive power.

Biomechanical changes after MA treatments contribute to an oblate contour, increasing spherical aberration and shrinking the effective optical zone. Healing response ${ }^{240}$, radial ablation efficiency losses ${ }^{117,118}$ and biomechanical effects $^{241}$ all reduce the effective ablation in the outer portion of the nominal optical zone. These effects shrink the actual zone of full refractive correction, i.e., the effective optical zone. They also distort attempted cylindrical ablations by flattening the cornea along the astigmatic axis, introducing an unintended spherical correction component and reducing the cylindrical correction ${ }^{118}$.

The shrinking effect is larger for major corrections, i.e. larger optical zones should be used for major corrections, but larger optical zones result in deeper and longer ablations increasing the potential risks of keratectasia ${ }^{242}, 243$. 
Comparing the three methods in our study, we observed that EOZ correlated well with POZ and SEq for the 3 analysed methods. EOZ $\mathrm{ERMSho}_{\Delta \mathrm{B}}$ and $\mathrm{EOZ}_{\Delta \mathrm{SphAb}}$ were similar to $\mathrm{POZ}$, whereas $\mathrm{EOZ}_{\mathrm{RMS}(\triangle \mathrm{HOAb})}$ was significantly smaller than POZ and the results obtained by the other two methods.

$\Delta$ RMSho analysis accounts for all aberration terms from the perspective of the global optical quality of the cornea. A similar approach was used by Tabernero et al. ${ }^{244}$, applied in a different way. They analysed directly on the cornea the functional optical zone (FOZ) in patients pre and postoperatively, instead applied to the differential map. They wanted to determine the FOZ of the cornea, whereas we aimed to determine the EOZ of the treatments. But essentially the methods are equivalent.

$\triangle \mathrm{SphAb}$ analysis provides the largest EOZ, because only one Zernike term is analysed, i.e. decentration ${ }^{92}$ or cyclotorsion ${ }^{121}$ effects are not accounted for. Maloney ${ }^{231}$ described the consequences of a decentred optical zone and discussed methods to ensure centring.

$\mathrm{RMS}(\triangle \mathrm{HOAb})$ analysis provides the smallest EOZ, because it accounts for any deviation from the aimed Aberration-Free concept (i.e. both inductions and reductions of the wavefront aberration both contribute positively to increase the RMS value).

Caution must be taken with the results obtained by the $\mathrm{RMS}(\triangle \mathrm{HOAb})$ analysis, because it is the most sensitive of the presented analyses and it might have been affected by short term fluctuations of the wavefront aberration, which could explain, at least in part why the EOZ obtained by this method were significantly smaller than the EOZ obtained by the other two.

Multivariate correlation analysis of the EOZ versus POZ and attempted defocus correction showed that both contributions were statistically significant $(p<.001$ for POZ, $p<.01$ for defocus). Absolute and relative differences between $\mathrm{EOZ}$ and $\mathrm{POZ}$ were larger for smaller POZ or for larger Defocus corrections. EOZ correlates positively with POZ, declines steadily with increasing Defocus corrections; and EOZ depends stronger on POZ than on SEq. 
On average, and simplifying the relationship to only EOZ and $\mathrm{POZ}$ we observed that POZ larger than $6.75 \mathrm{~mm}$ result in EOZ, at least, as large as POZ. For OZ smaller than $6.75 \mathrm{~mm}$, a nomogram for OZ can be applied.

For our analysis the threshold value of $0.25 \mathrm{D}$ for determining EOZ was arbitrarily chosen, based upon the fact that with simple spherical error, degradation of resolution begins for most people with errors of $0.25 \mathrm{D}$. For all three methods, our search algorithm is an "increasing diameter" analysis, this ensures that the smallest EOZ condition is found. Finally, our search was set to start from 4-mm upwards, i.e. $3.99 \mathrm{~mm}$ is the smallest EOZ that could be found. We have done that because for very small analysis diameters, the Zernike fit seems to be less robust, mostly due to the decreasing sampling density within the unit circle.

Holladay and Janes (2002) determined the relationship between the spherical refractive change after myopic excimer laser surgery and the effective optical zone and corneal asphericity determined by corneal topography, which changed nonlinearly with the amount of treatment.

Mok and Lee ${ }^{245}$ reported that larger optical zones decrease postoperative high-order aberrations. They found the measured high-order aberrations to be less in eyes with larger optical zones. Assessing the quality of vision (rather than the quality of the optical zone) after a refractive procedure is a separate issue. The relationship between pupil size and vision after refractive surgery is critically important and this relationship cannot be evaluated accurately with a measurement of aberrations through a predetermined aperture with an aberrometer. Pupil sizes vary considerably among patients depending on light level and age $\mathrm{e}^{246}$. Mok and Lee have shown a strategy for planning optical zone size based on patient pupil size. However, an aberration analysis that takes into account variations in planned optical zone size may provide more insight as to the quality of the outcome obtained.

Partal and Manche ${ }^{247}$ using direct topographic readings observed over a large sample of eyes in moderate compound myopic astigmatism, a reduction from POZ of $6.50-\mathrm{mm}$ to EOZ of $6.00-\mathrm{mm}$. It is noteworthy and opposed to our findings that they did not find a greater contraction of EOZ for increasing myopic corrections. 
Qazi et al. ${ }^{248}$ using a different approach observed over a sample of eyes similar to ours, a reduction from $\mathrm{POZ}$ of $6.50-\mathrm{mm}$ to $\mathrm{EOZ}$ of $5.61-\mathrm{mm}$.

It is possible that the EOZ could be larger than the $\mathrm{POZ}$ if it encompasses some portions of the $T Z$, or even larger than the TAZ. Although POZ, TZ, and TAZ are parameters defined by the laser treatment algorithms, EOZ must be determined postoperatively (from the differences to the baseline) and may change with time because of healing and biomechanical effects. In the same way, it would be possible that the FOZ were larger postoperatively than it was preoperatively, or that the FOZ could be larger than the POZ or even than the TAZ.

Despite large defocus and astigmatism magnitudes, our study shows highorder aberrations are either minimally increased or unchanged after surgery with the AMARIS system, whereas EOZ is very similar to POZ (Tables 1 and 2). The EOZ obtained in this clinical setting show a trend toward slight undersize for smaller POZ or for larger Defocus corrections. On the other hand, the low standard deviation and the tight dispersion of the cluster of data demonstrate the consistency of the achieved results. Given the small deviation of the results, we believe that with some slight adjustment for the POZ, the EOZ results and the aberrometric analyses will improve significantly.

\section{Section F.6 CONCLUSIONS}

(Conclusiones)

In conclusion, our results suggest that wavefront aberration can be a useful metric for the analysis of the effective optical zones of refractive treatments or for the analysis of functional optical zones of the cornea or the entire eye by setting appropriate limit values. In particular, the method of analysis of the RMS $(\triangle \mathrm{HOAb})$ seems to be a rigorous analysis accounting for any deviation from the attempted target for the wavefront aberration.

In summary, this study demonstrated that "aberration neutral" profile definitions as implemented at the SCHWIND AMARIS system, which are not standard in refractive surgery, yield very good visual, optical, refractive results 
and EOZ for the correction of compound myopic astigmatism. "Aberration neutral" ablation profiles as demonstrated here, have, therefore, the potential to replace currently used standard algorithms for the non-customised correction of compound myopic astigmatism.

\section{Section F.7 OUTLOOK}

(Perspectiva)

Limitations of our study include that the clinical evaluation was performed over only 20 eyes, reducing the statistical power of the conclusions; and the lack of a control group. The clinical evaluation was limited to MA treatments, thus results cannot be extrapolated to hyperopic treatments without further clinical evaluations. Evaluation was limited to LASEK/Epi-LASEK techniques, thus results cannot be extrapolated to LASIK treatments without further clinical evaluations. Finally, in our sample, POZ significantly correlated with defocus $\left(r^{2}=.9, p<.0001\right)$, indicating that the two variables of the bilinear fit were interdependent.

To extend our methodology for the analysis of customised corrections can be quite simple if we consider that customised corrections in their intrinsic nature aim to reduce aberrations (either from the cornea only, or from the complete ocular system) to a zero level. In this way, the corresponding formulations would be:

$$
\begin{aligned}
& R M S h o_{C W}(E O Z)=0.25 D \\
& R M S h o_{O W}(E O Z)=0.25 D
\end{aligned}
$$

for corneal (CW) and ocular wavefront (OW) corrections, respectively.

Long-term follow-up on these eyes will help determine whether these accurate results also show improved stability compared to previous experiences. 

Topic G METHOD TO OBJECTIVELY MINIMISE THE ABLATED TISSUE OF A CUSTOMISED ABLATION BASED ON THE ZERNIKE EXPANSION OF THE WAVEFRONT ABERRATION

(Método para minimizar objetivamente la cantidad de tejido resecado en una ablación personalizada basada en la expansión de Zernike de la aberración del frente de onda)

\section{AUTHOR CONTRIBUTIONS}

Study concept and design (S.A.M.); data collection (D.O., M.R., C.V.); analysis and interpretation of data (S.A.M.); drafting (S.A.M.); critical revision (D.O., J.M., J.L.A., T.H., M.C.A.); statistical expertise (S.A.M.).

\section{Section G.1 ABSTRACT}

(Resumen)

The purpose of this work is to study the possibility of performing customized refractive surgery minimising the amount of ablated tissue without compromising visual quality and to evaluate the application of these methods for minimizing the ablated tissue upon objective minimization of depth and time of Zernike based customized ablations. A new algorithm for the selection of an optimized set of Zernike terms in customized treatments for laser corneal refractive surgery was developed. Its tissue-saving attributes have been simulated on 100 different wave aberrations at $6 \mathrm{~mm}$ diameter. Simulated outcomes were evaluated in terms of how much depth and volume was saved for each condition (in micrometers and in percentage), whether the proposed correction consists of either a full wavefront correction or an aberration-free treatment, and whether the proposed depth or volume was less than the one required for the equivalent aberration-free treatment. Clinical outcomes and tissue-saving attributes were evaluated on two groups (minimize depth: MD; and minimize volume: MV; 30 eyes each), plus a control group (corneal wavefront: $\mathrm{CW}, 30$ eyes) with conventional customized approach. Clinical outcomes were 
evaluated in terms of predictability, safety, and contrast sensitivity; and tissuesaving attributes in terms of saved depth and time for each condition (in micrometers, seconds and percentage), and whether minimized depth or time were less than required for equivalent non-customized treatments. Simulated outcomes showed an average saved depth of $5 \mu \mathrm{m}(0-16 \mu \mathrm{m})$, and an average saved volume of $95 \mathrm{nl}(0-127 \mathrm{nl})$ or $11 \%$ saved tissue $(0-66 \%$ saved tissue). Proposed corrections were always less deep than full wavefront corrections and in $59 \%$ of the cases were less deep than equivalent aberration-free treatments. For the case report, required ablation was reduced by approximately $15 \%$ compared to full customized correction. Refraction was corrected to subclinical levels, uncorrected distance visual acuity improved to 20/20, corrected distance visual acuity gained two lines, aberrations were reduced by approximately $40 \%$ compared to preoperative baseline levels, and the functional optical zone of the cornea was enlarged by approximately $40 \%$ compared to preoperative baseline levels. Trefoil, coma, spherical aberration, and the root-mean-square value of the higher order aberrations were reduced. In the clinical evaluation, $93 \%$ of treatments in CW group, 93\% in MD group, and 100\% in MV group were within $0.50 \mathrm{D}$ of SEq postoperatively. $40 \%$ of treatments in CW group, $34 \%$ in MD group, and $47 \%$ in MV group gained at least one line of BSCVA postoperatively. Tissue-saving attributes showed an average saved depth of $8 \mu \mathrm{m}(1-20 \mu \mathrm{m})$ and a saved time of $6 \mathrm{~s}(1-15 \mathrm{~s})$ in the MD group, and $6 \mu \mathrm{m}(0-20 \mu \mathrm{m})$ and $8 \mathrm{~s}(2-26 \mathrm{~s})$ in the MV group. Proposed corrections were always less deep and shorter than full wavefront corrections. In $43 \%$ of the MD cases were less deep, and in $40 \%$ of the MV cases were shorter than equivalent Aberration-Free treatments. Even though Zernike modes decomposition is a mathematical description of the aberration, it is not the aberration itself. Not all Zernike modes affect the optical quality in the same way. The eye does not see through Zernike decomposition but with its own aberration pattern. However, it seems feasible to efficiently perform laser corneal refractive surgery in a customized form minimising the amount of ablated tissue without compromising the visual quality. Eliminating all higher order aberrations may not optimize visual function in highly aberrated eyes. The new algorithm effectively reduced depth and time needed for ablation (up to a maximum of $50 \%$, and by $15 \%$ in average), without negatively affecting clinical outcomes 
postoperatively, yielding results equivalent to those of the full customization group.

\section{Section G.2 INTRODUCTION}

(Introducción)

There are different proposed approaches for minimising the tissue consumption in refractive surgery, among others: Multizonal treatments ${ }^{249}$, Smaller optical zone with bigger transition zones ${ }^{250}$, Smaller optical zone for the cylindrical component ${ }^{251}$, Boost slider method, Less optimisations in the profile, ZClipping method ${ }^{252}$, or Z-Shifting method ${ }^{252}$.

\section{G.2.1 Multizonal treatments \\ (Tratamientos multizonales)}

Minimisation by multizonal treatments is based on the concept of progressive decreasing corrections in different optical zones (Figure 63).

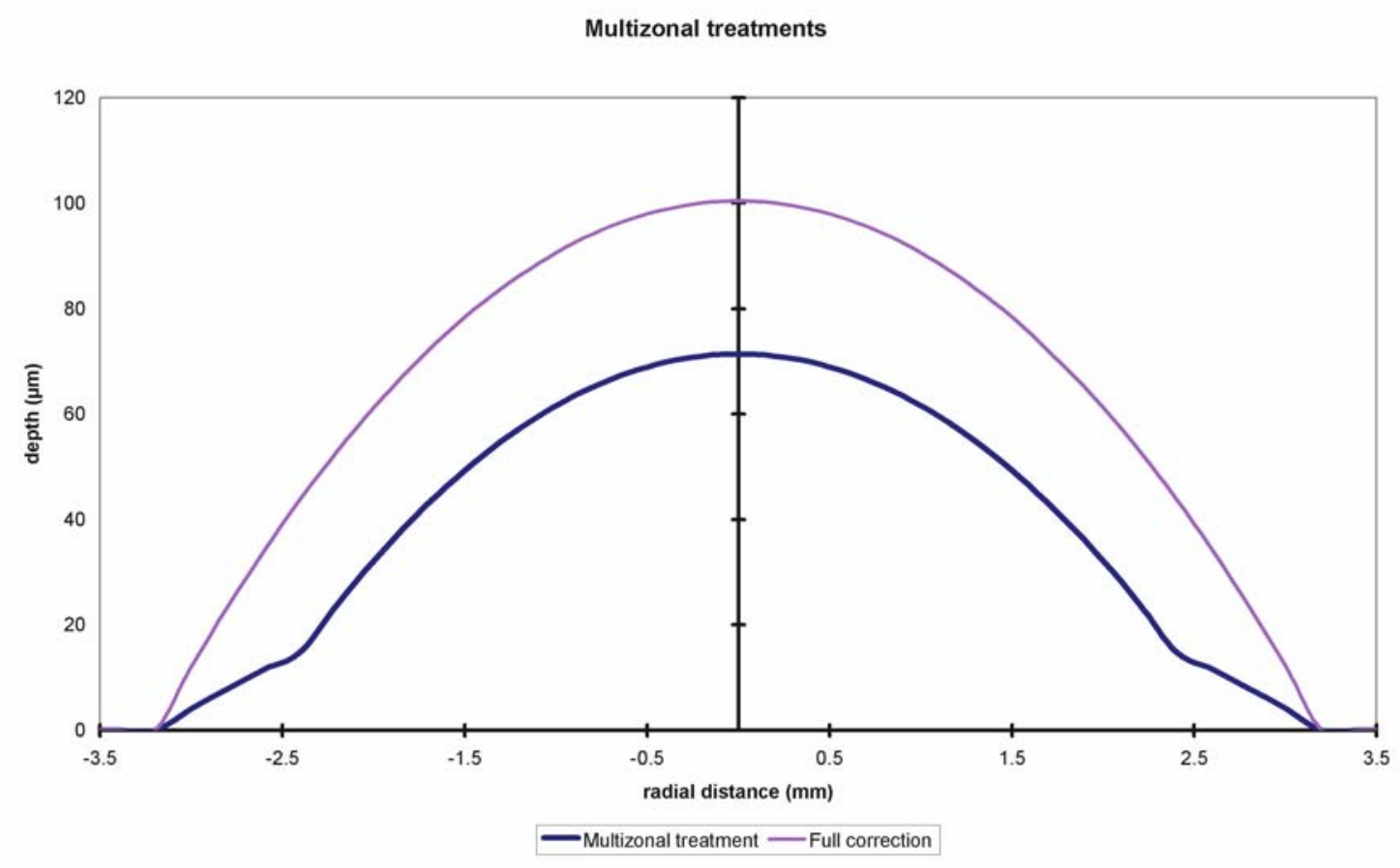

Figure 63: Minimisation by multizonal treatments 
G.2.2 Smaller optical zone treatments with large transition zone

(Tratamientos en menor zona óptica con mayor zona de transición)

Minimisation with smaller optical zone treatments with large transition zone is a variation of the multizone concept (Figure 64).

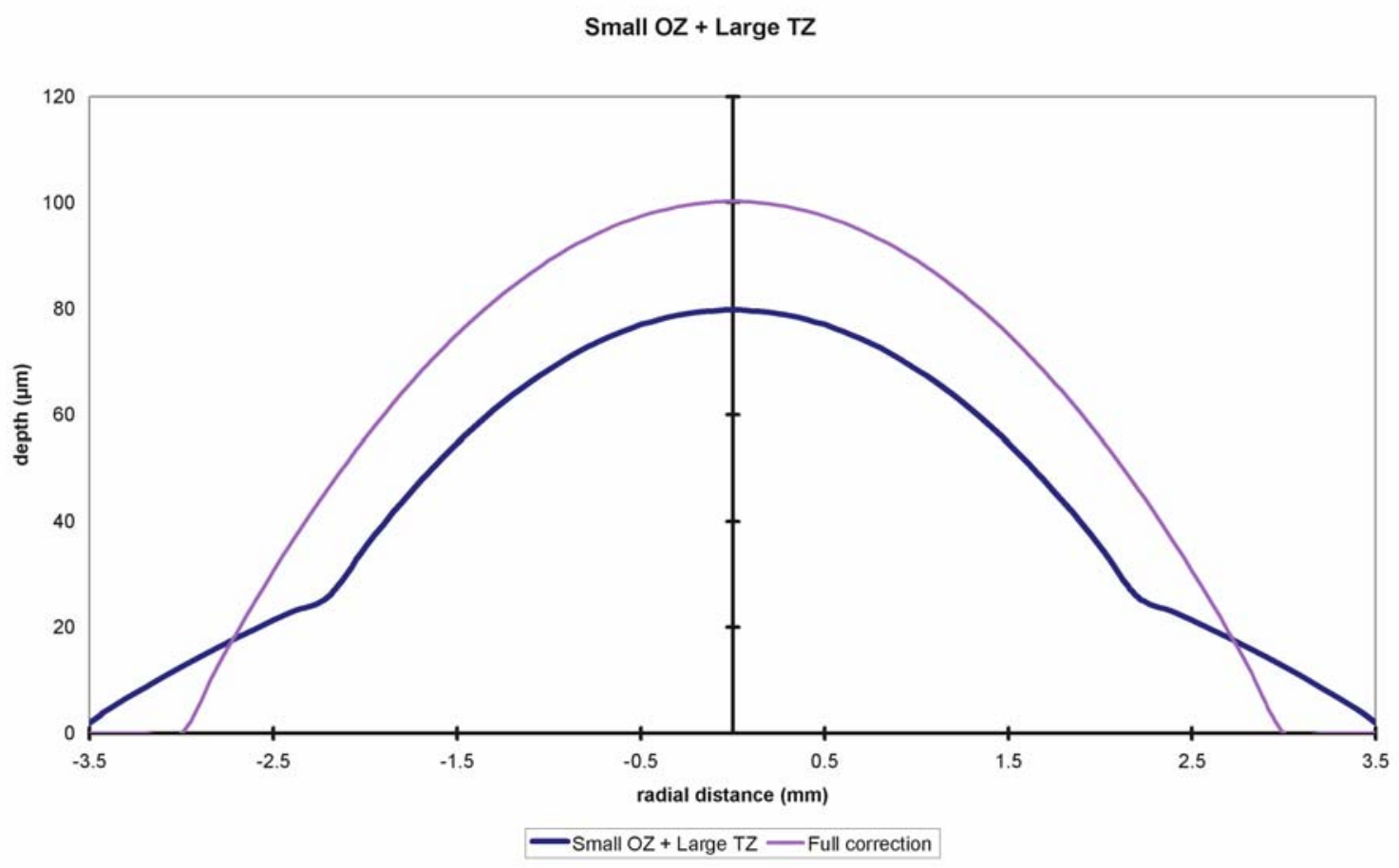

Figure 64: Minimisation with smaller optical zone treatments with large transition zone

\section{G.2.3 Smaller optical zone for the astigmatic correction \\ (Zonas ópticas menores para la corrección astígmata)}

Minimisation with smaller optical zone for the astigmatic correction is based upon the concept of the maximal depth being based on the lowest meridional refraction and the selected optical zone, and the effective optical zone of the highest meridional refraction is reduced to match the same maximal depth (Figure $65)$. 
Reduced $\mathrm{OZ}$ in highest meridional power

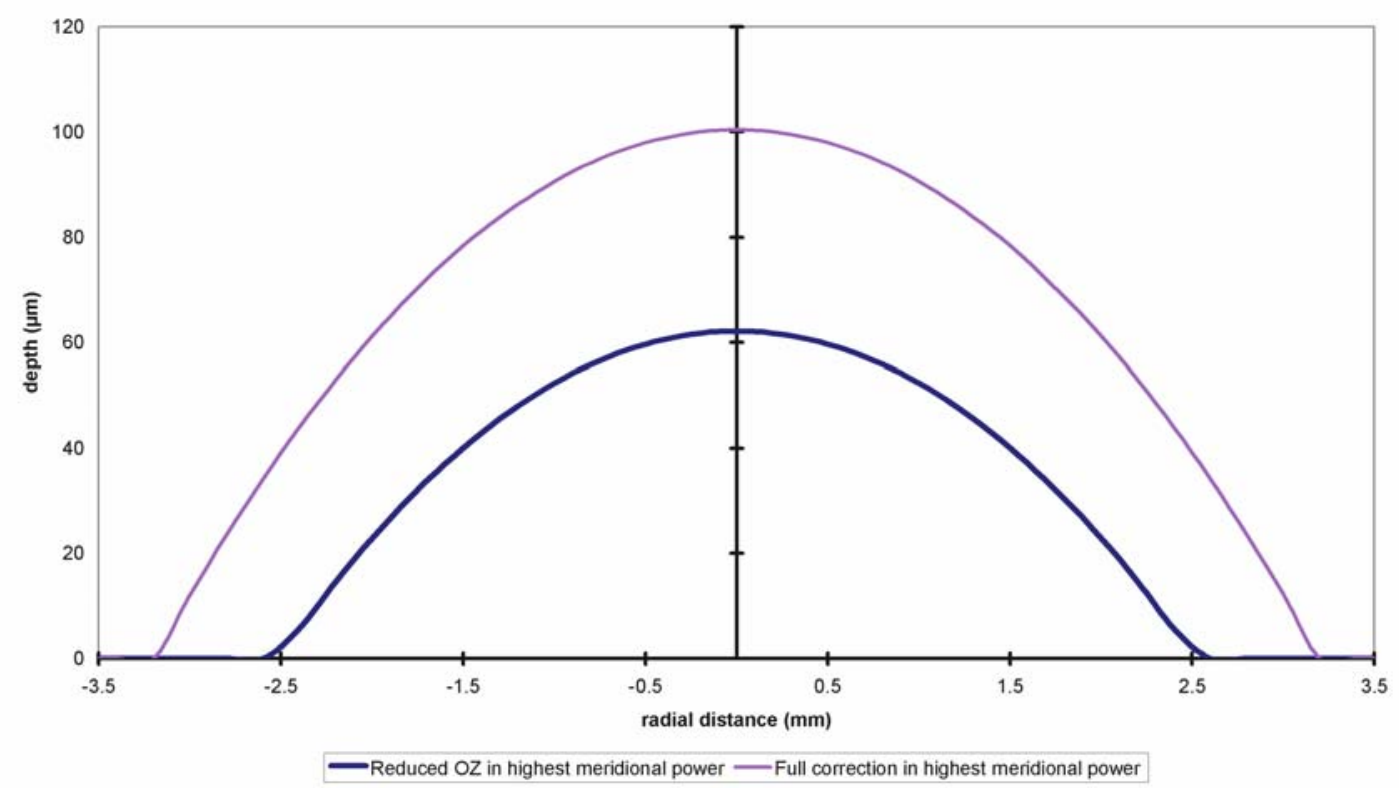

Figure 65: Minimisation with smaller optical zone for the astigmatic correction

G.2.4 Boost slider method

(El modulador incremental)

Minimisation by a boost method is a linear modulation of the volume (Figure 66).

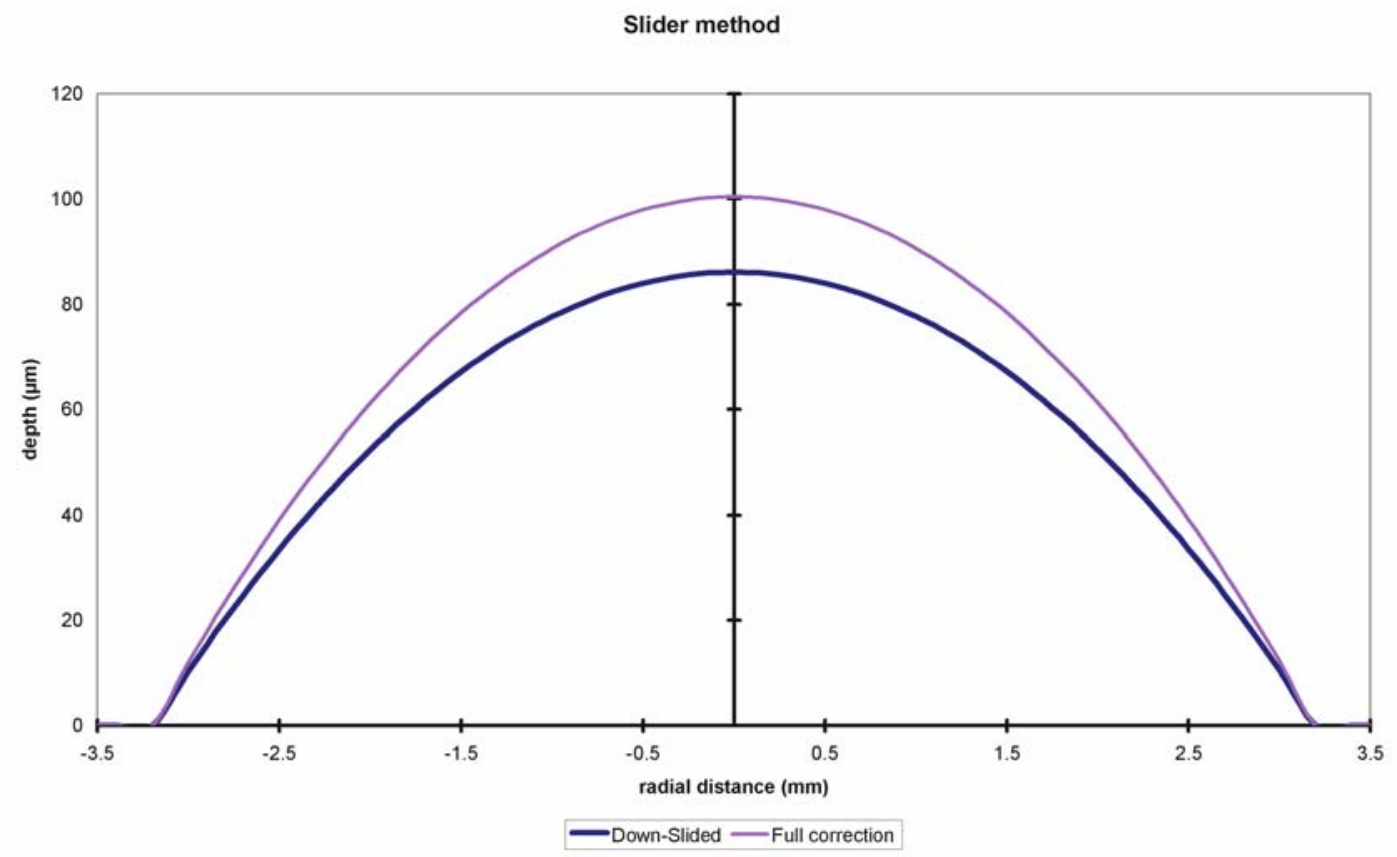

Figure 66: Minimisation by a boost slider (down-slided) 


\section{G.2.5 Simplified profile method \\ (El perfil simplificado)}

Minimisation by a simplified profile consists of compromising the expected quality of the profile by simplified assumptions.

\section{G.2.6 Z-Clipping method \\ (Método de la poda en Z)}

Minimisation by a Z-Clipping method consists of defining saturation for the ablated volume, all points planned to ablate deeper than the saturation value are ablated only by an amount equal to the saturation value (Figure 67).

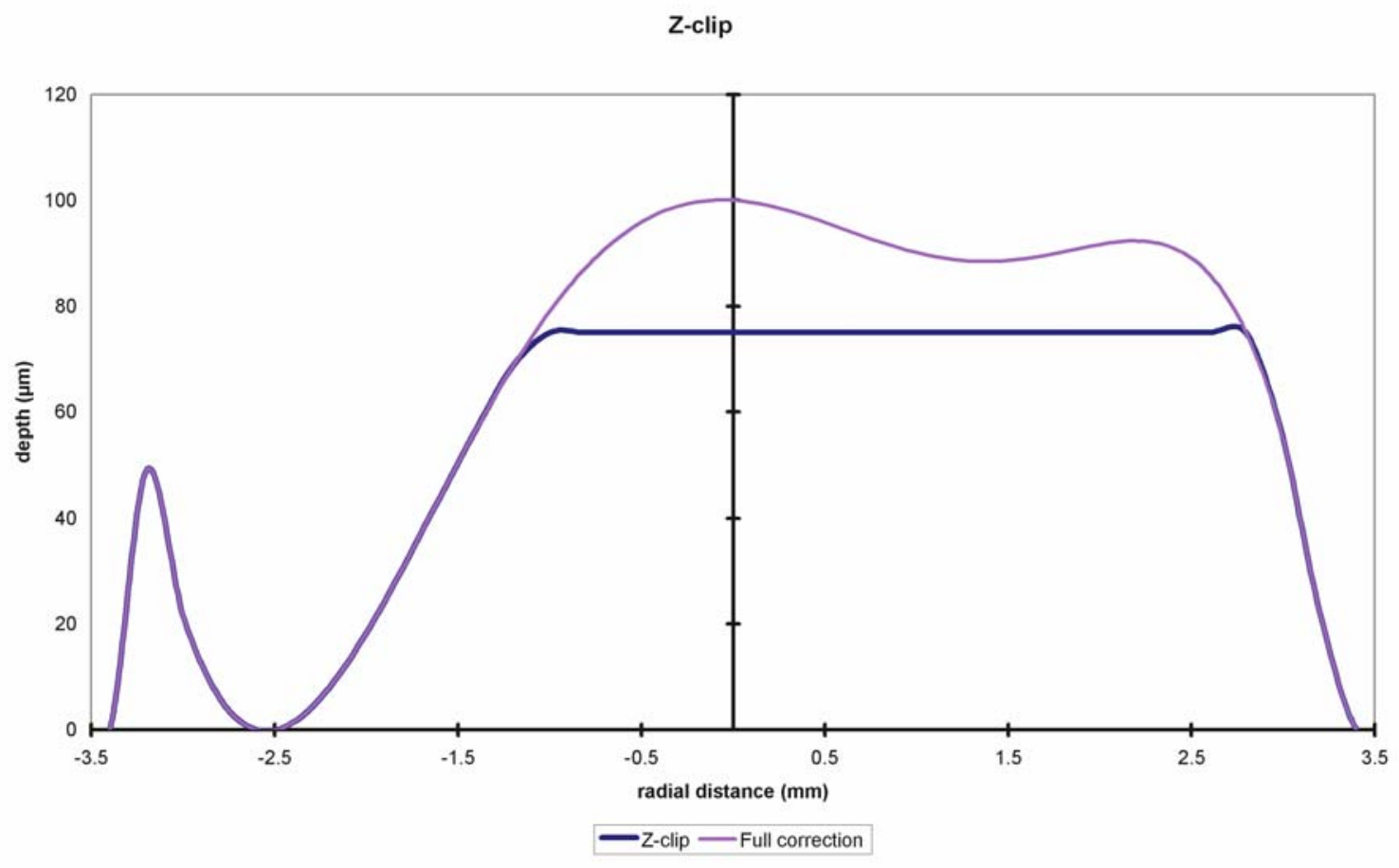

Figure 67: Minimisation by a Z-Clipping method 


\section{G.2.7}

Z-Shifting method

(Método del recorte en $Z$ )

Minimisation by a Z-Shifting method consists of defining a threshold value for the ablated volume, all points planned to ablate less than the threshold value are not ablated, the rest of the point as ablated by an amount equal to the original planned ablation minus the threshold value (Figure 68).

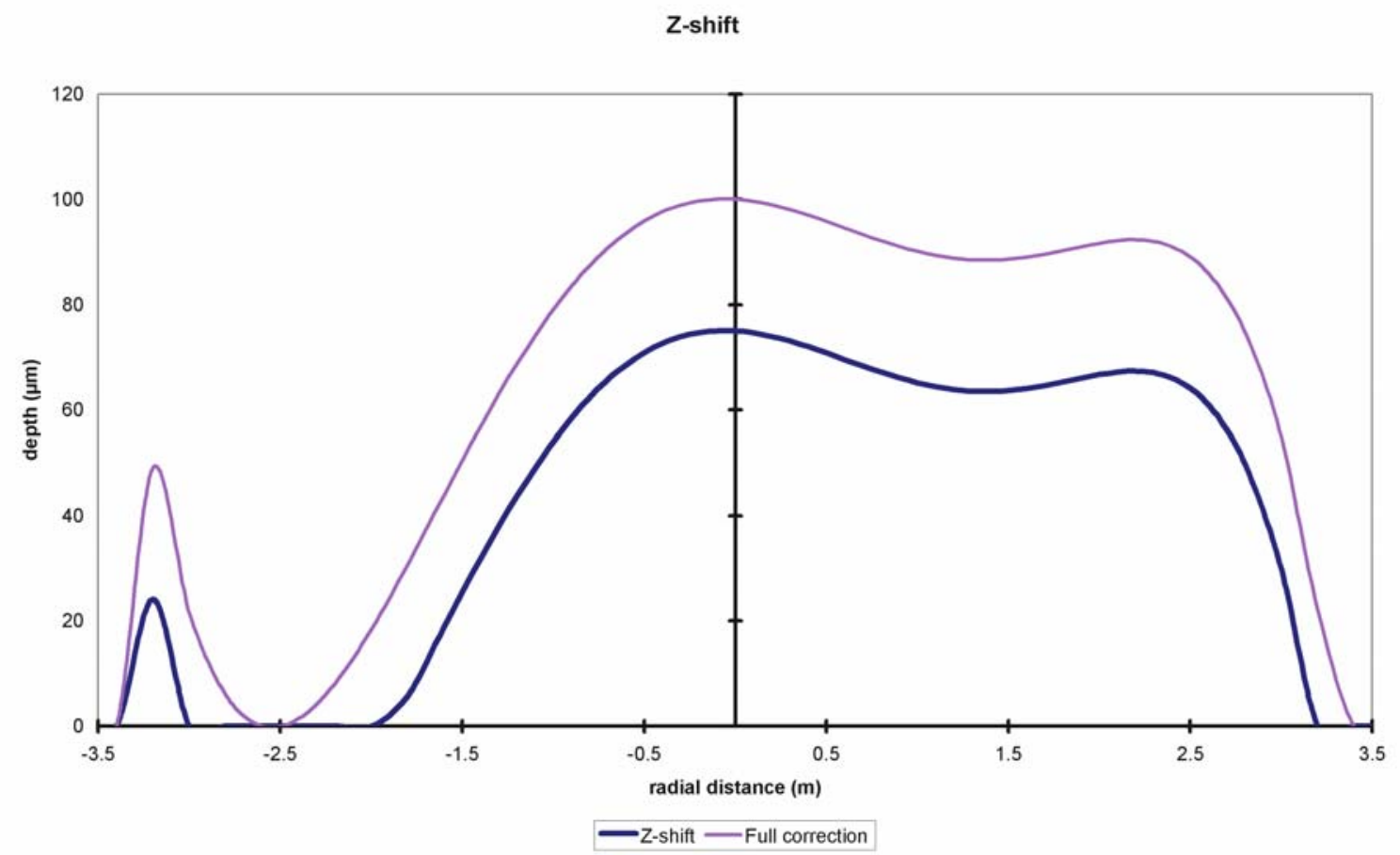

Figure 68: Minimisation by a Z-Shifting method

Section G.3 METHODS

(Método)

G.3.1

The "Minimise Depth" and "Minimise Depth+" functions

(Las funciones „Minimizar profundidad“ y ,Minimizar profundidad+“)

One of the minimisation approaches proposed in this work consists of simplifying the profile by selecting the subset of Zernike terms that minimises the 
necessary ablation depth while respecting the Zernike terms considered clinically relevant.

The "minimise depth" function analyses the Zernike pyramid described in the previous section and evaluates the resulting ablation depth for all those possible free combinations of Zernike terms that fulfil the following conditions:

- Only $3^{\text {rd }}$ or higher order terms can be disabled

- $\quad$ Only those terms whose optical blur dioptric equivalent is less than 0.25 $\mathrm{D}$ (in green) can be disabled

- For each subset of Zernike terms, the low order terms are recalculated using the Automatic Refraction Balance method described above

From this evaluation, the function selects the subset of Zernike terms for which the maximum ablation depth is minimal.

The "minimise depth +" function analyses, as well, the Zernike pyramid and evaluates the ablation depth of all possible free combinations of subsets of Zernike terms fulfilling the conditions:

- Only $3^{\text {rd }}$ or higher order terms can be disabled

- Only those terms whose optical blur dioptric equivalent is less than 0.50 D (in green or yellow) can be disabled

- For each subset of Zernike terms, the low order terms are recalculated using the Automatic Refraction Balance method described above

Again, the function selects the subset of Zernike terms for which the maximum ablation depth is minimal (Figure 72).

The rigorous formulation of these minimised-depth functions is to find a vector of values $E[n, m]$ ( 1 for enable, 0 for disable) that minimises the maximum ablation depth, conditioned to enabling the terms that have an optical blur dioptric equivalent above $0.25 \mathrm{D}$ or $0.50 \mathrm{D}$, respectively (in yellow or red).

$$
\operatorname{Abl}(\rho, \theta)=\frac{\sum_{n=0}^{\infty} \sum_{m=-n}^{+n} E_{n}^{m} C_{n}^{m} Z_{n}^{m}(\rho, \theta)-\min \left[\sum_{n=0}^{\infty} \sum_{m=-n}^{+n} E_{n}^{m} C_{n}^{m} Z_{n}^{m}(\rho, \theta)\right]}{n_{\text {Cornea }}-n_{\text {Air }}}
$$

This is equivalent to minimising the peak-to-valley value of the wavefront. 


$$
\operatorname{MaxAbl}=\frac{\max \left[\sum_{n=0}^{\infty} \sum_{m=-n}^{+n} E_{n}^{m} C_{n}^{m} Z_{n}^{m}(\rho, \theta)\right]-\min \left[\sum_{n=0}^{\infty} \sum_{m=-n}^{+n} E_{n}^{m} C_{n}^{m} Z_{n}^{m}(\rho, \theta)\right]}{n_{\text {Cornea }}-n_{\text {Air }}}(106)
$$

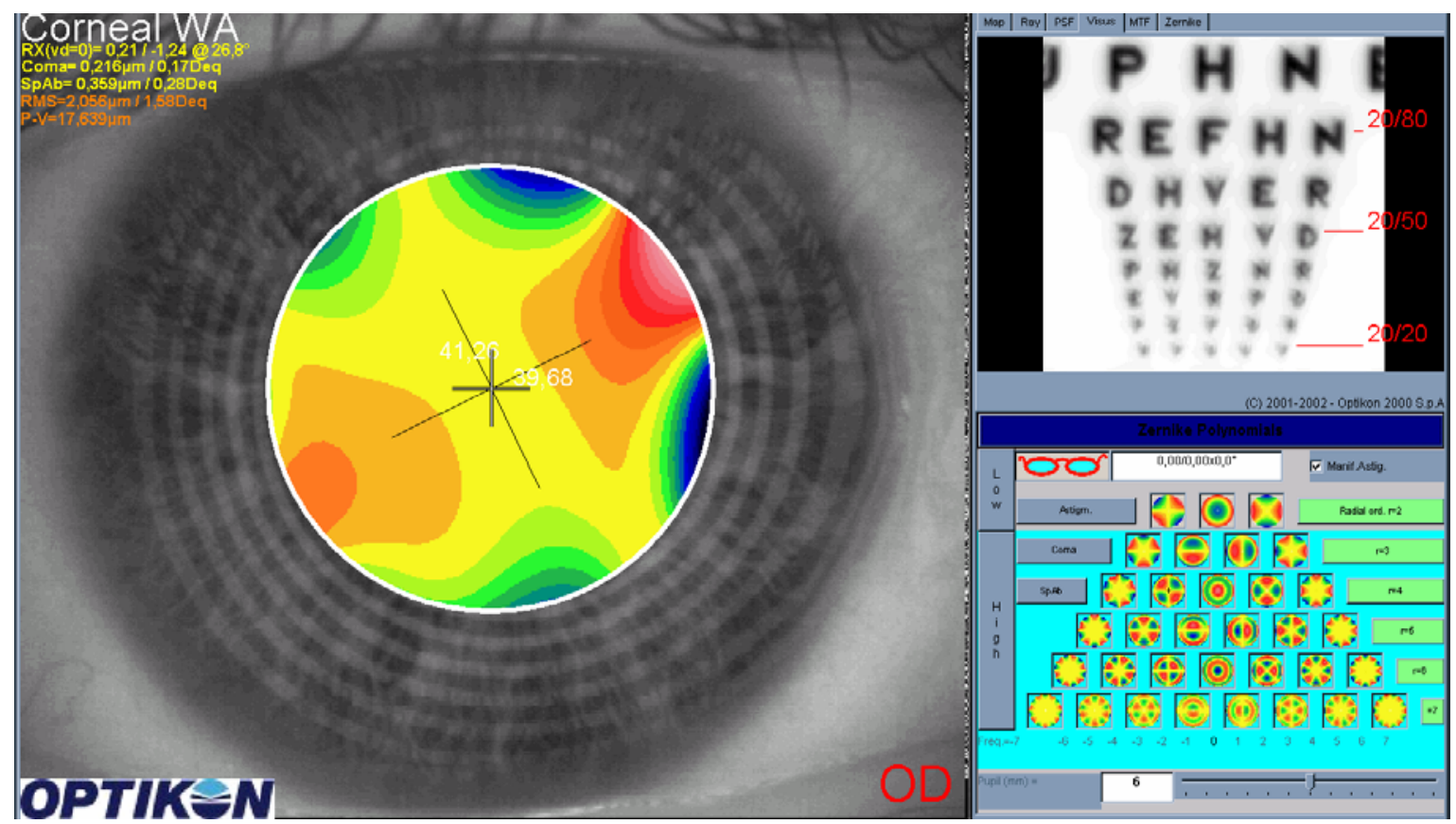

Figure 69: Example of a patient with a normal WFAb and his preoperative visus

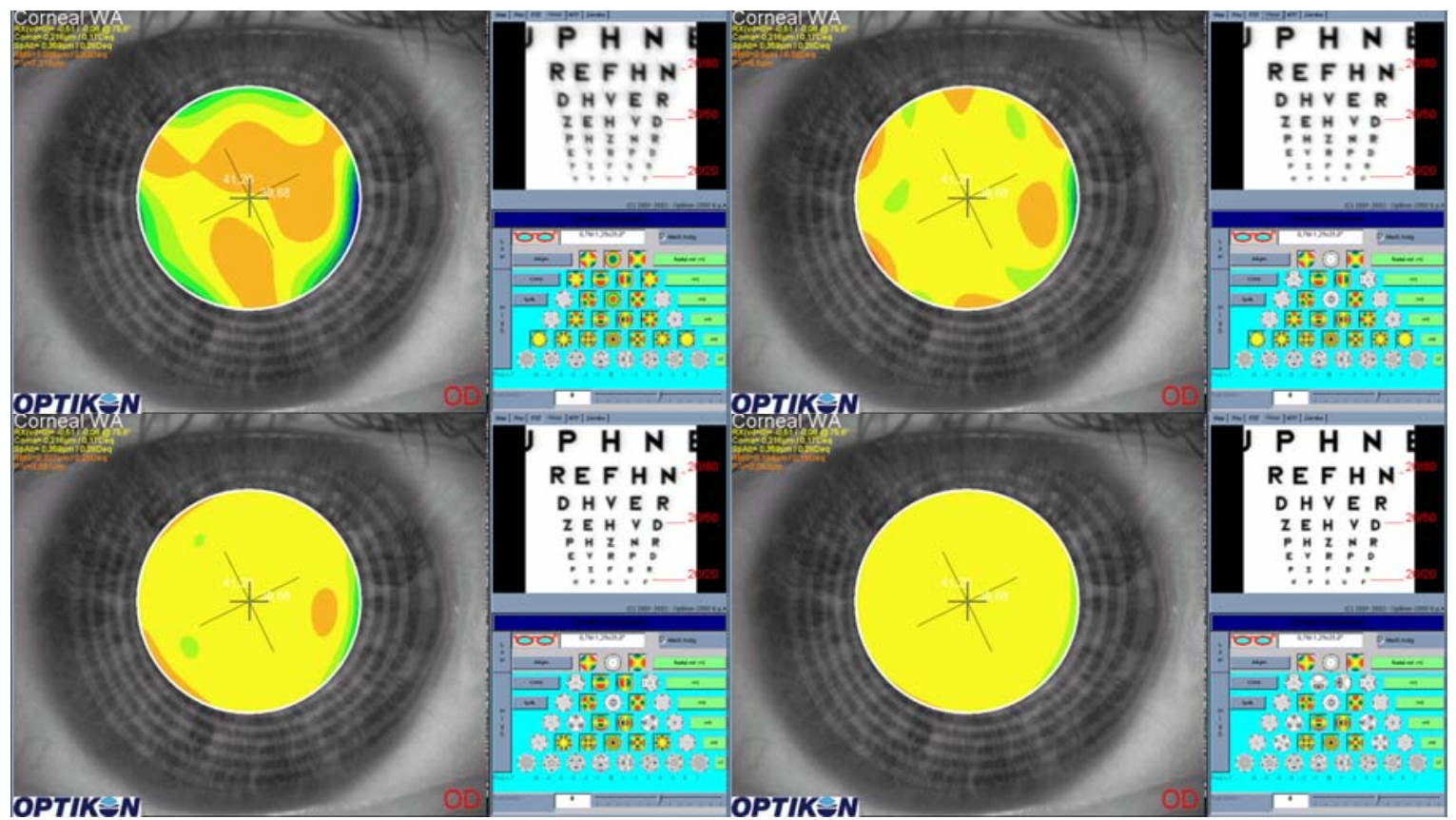

Figure 70: Manual analysis of the optical effects (visus) of the different aberration modes for the same WFAb 


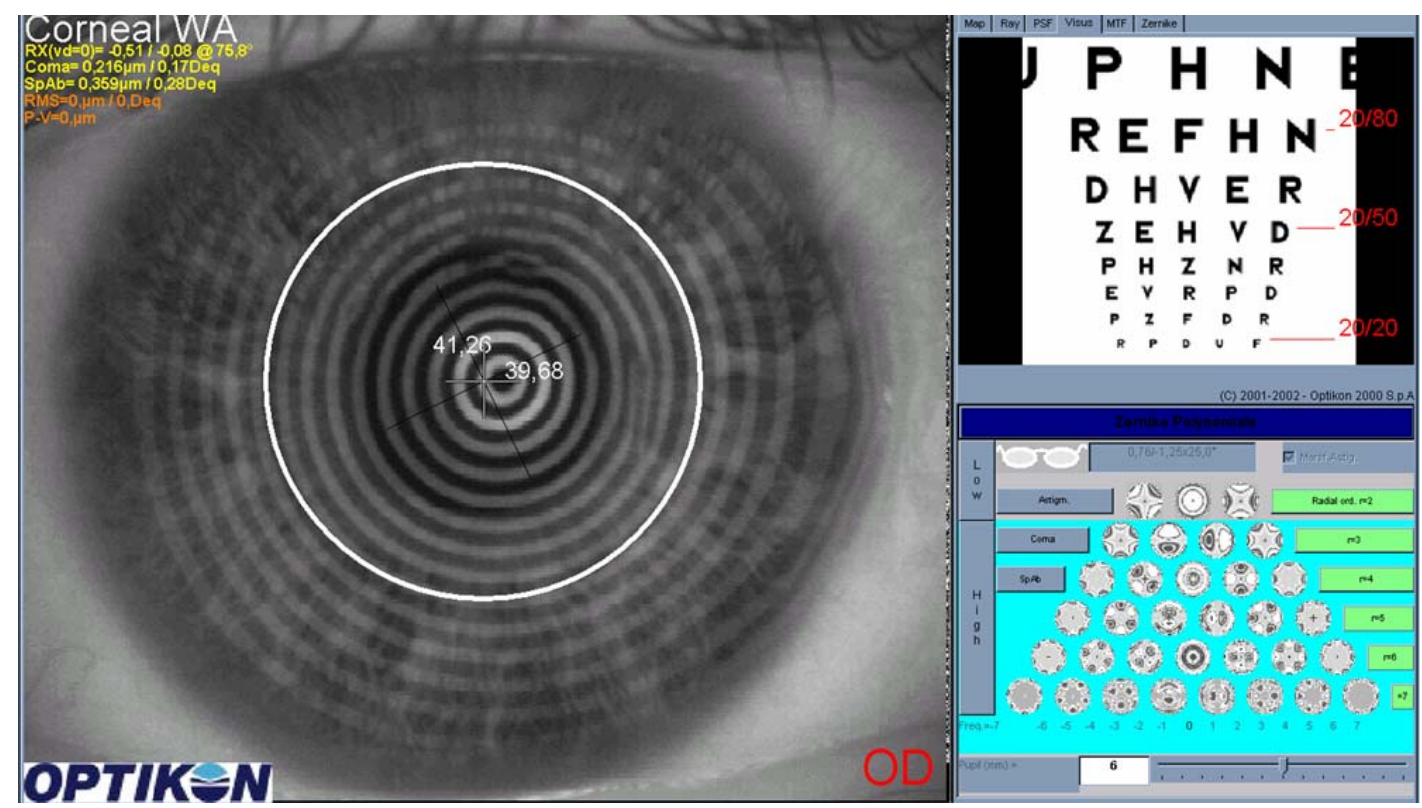

Figure 71: Diffraction limited visus (all aberration modes are corrected, ideal case)

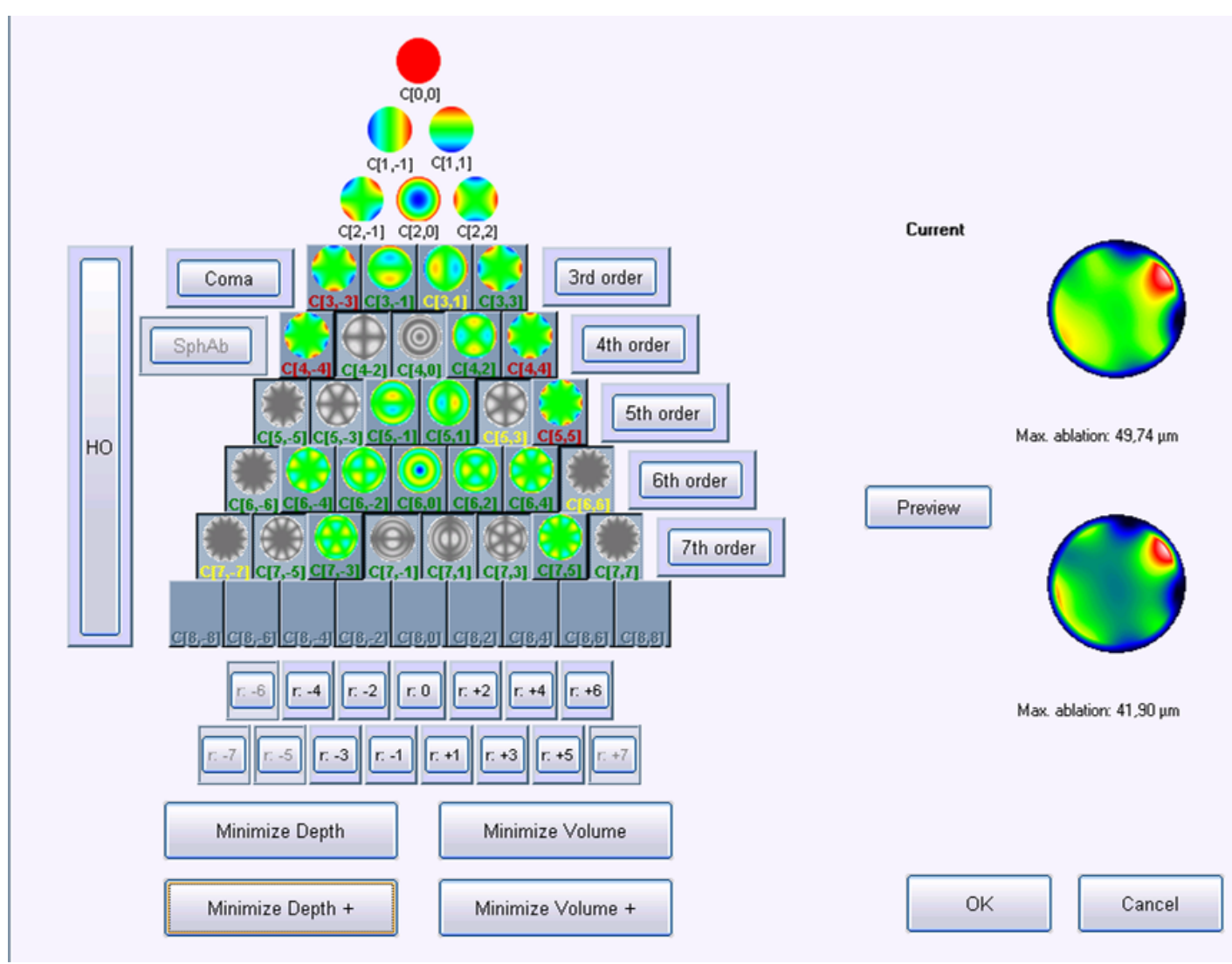

Figure 72: Objective analysis (Optimised Aberration modes selection) of the optical and ablative effects of the different aberration modes for the same WFAb: Notice that the aberration modes to be selected are not trivial: Not all the modes 
in green are unselected (not corrected) because some of them may help to save tissue. Not all aberration modes in yellow are selected (corrected) because some of them may have low impact on vision. Notice, as well, that $8 \mu \mathrm{m}$ tissue are saved ( $16 \%$ of the ablation), but that the overall shape of the ablation remains

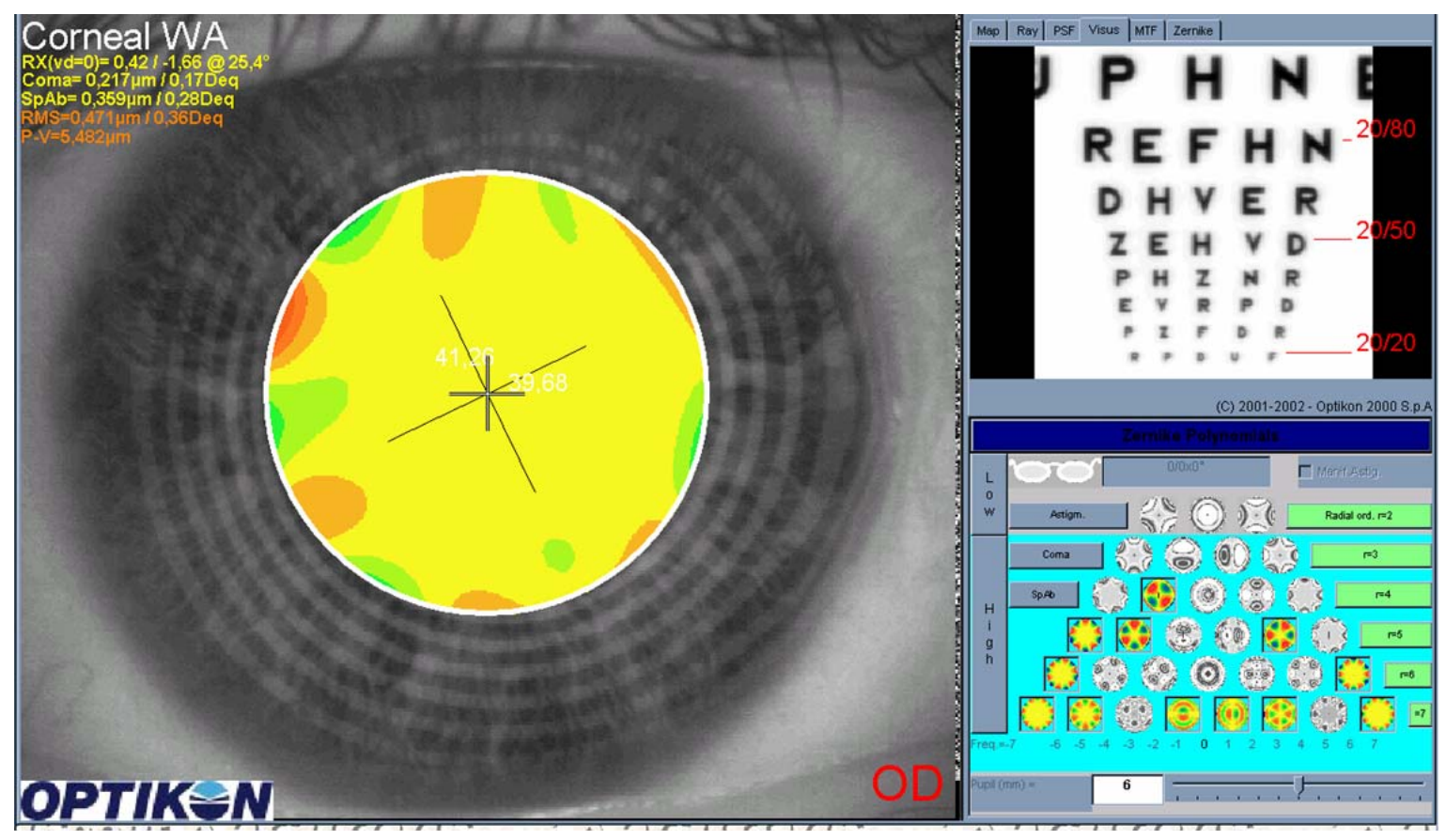

Figure 73: Analysis of the optical effects (visus) of the objective analysis (Optimised Aberration modes selection) for the same WFAb

\section{G.3.2 The "Minimise Volume" and "Minimise Volume+" functions (Las funciones ,, Minimizar volumen “ y ,Minimizar volumen+")}

The other minimisation approach proposed in this work consists of simplifying the profile by selecting the subset of Zernike terms that minimises the necessary ablation volume, while respecting those Zernike terms considered clinically relevant.

The "minimise volume" function analyses the Zernike pyramid described in the previous section and evaluates the required ablation volume for all those possible free combinations of Zernike terms that fulfil the following conditions:

- Only $3^{\text {rd }}$ or higher order terms can be disabled 
- Only those terms whose optical blur dioptric equivalent is less than 0.25 $\mathrm{D}$ (in green) can be disabled

- For each combination of subset of Zernike terms, the low order terms are recalculated using the Automatic Refraction Balance method described above

From this evaluation, the function selects the subset of Zernike terms for which the required ablated volume is minimal.

The "minimise volume +" function analyses, as well, the Zernike pyramid and evaluates the ablation depth of all possible free combinations of subsets of Zernike terms fulfilling the conditions:

- Only $3^{\text {rd }}$ or higher order terms can be disabled

- Only those terms whose optical blur dioptric equivalent is less than 0.50 $D$ (in green or yellow) can be disabled

- For each combination of subset of Zernike terms, the low order terms are recalculated using the Automatic Refraction Balance method described above

Again, the function selects the subset of Zernike terms for which the required ablated volume is minimal (Figure 74 and Figure 75 ).

The rigorous formulation of these minimised-volume functions is, again, to find a vector of values $E[n, m]$ ( 1 for enable, 0 for disable) that minimises the total ablation volume, conditioned to enabling those terms whose optical blur dioptric equivalent is above $0.25 \mathrm{D}$ or $0.50 \mathrm{D}$, respectively (in yellow or red).

$$
\begin{gathered}
A b l V o l=\int_{0}^{2 \pi} \int_{0}^{1} A b l(\rho, \theta) \rho d \rho d \theta \\
A b l V o l=\int_{0}^{2 \pi} \int_{0}^{1} \frac{\sum_{n=0}^{\infty} \sum_{m=-n}^{+n} E_{n}^{m} C_{n}^{m} Z_{n}^{m}(\rho, \theta)-\min \left[\sum_{n=0}^{\infty} \sum_{m=-n}^{+n} E_{n}^{m} C_{n}^{m} Z_{n}^{m}(\rho, \theta)\right]}{n_{\text {Correa }}-n_{\text {Air }}} \rho d \rho d \theta(10 d \theta
\end{gathered}
$$

Taking into account that: 


$$
\int_{0}^{2 \pi} \int_{0}^{1} Z_{n}^{m}(\rho, \theta) \rho d \rho d \theta=0
$$

This leads to:

$$
\text { AblVol }=\int_{0}^{2 \pi} \int_{0}^{1} \frac{-\min \left[\sum_{n=0}^{\infty} \sum_{m=-n}^{+n} E_{n}^{m} C_{n}^{m} Z_{n}^{m}(\rho, \theta)\right]}{n_{\text {Cornea }}-n_{\text {Air }}} \rho d \rho d \theta
$$

This is equivalent to maximising the minimum value of the wavefront.

$$
\text { AblVol }=\pi \frac{O Z^{2}}{4} \frac{-\min \left[\sum_{n=0}^{\infty} \sum_{m=-n}^{+n} E_{n}^{m} C_{n}^{m} Z_{n}^{m}(\rho, \theta)\right]}{n_{\text {Cornea }}-n_{\text {Air }}}
$$

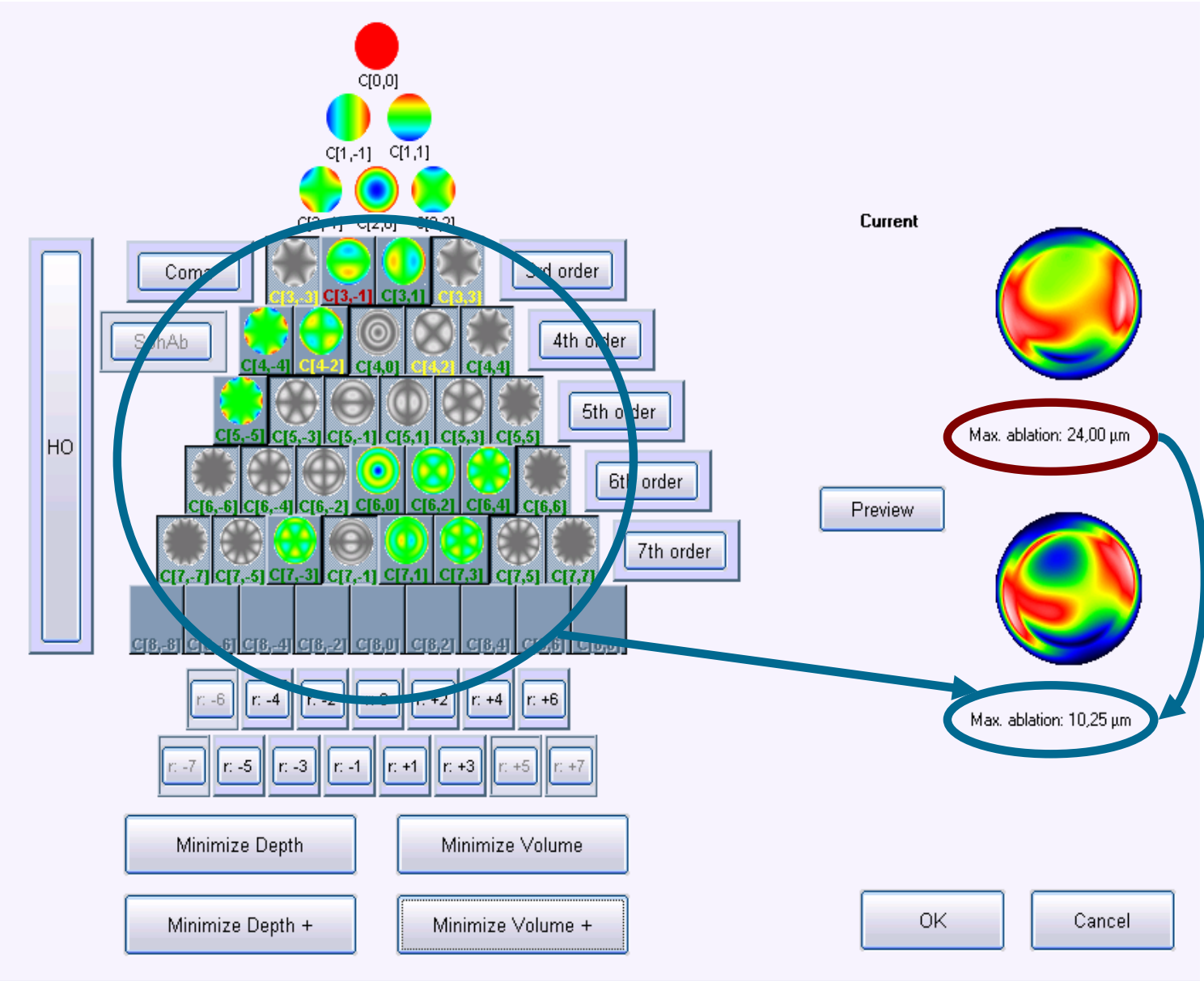

Figure 74: Optimised Aberration Modes Selection. Based on the wavefront aberration map, the software is able to recommend the best possible aberration modes selection to minimise tissue and time, without compromising the visual quality. Notice that the wavefront aberration is analysed by the software showing 
the original ablation for a full wavefront correction and the suggested set of aberration modes to be corrected. Notice the difference in required tissue, but notice as well that the most representative characteristics of the wavefront map are still presented in the minimised tissue selection.

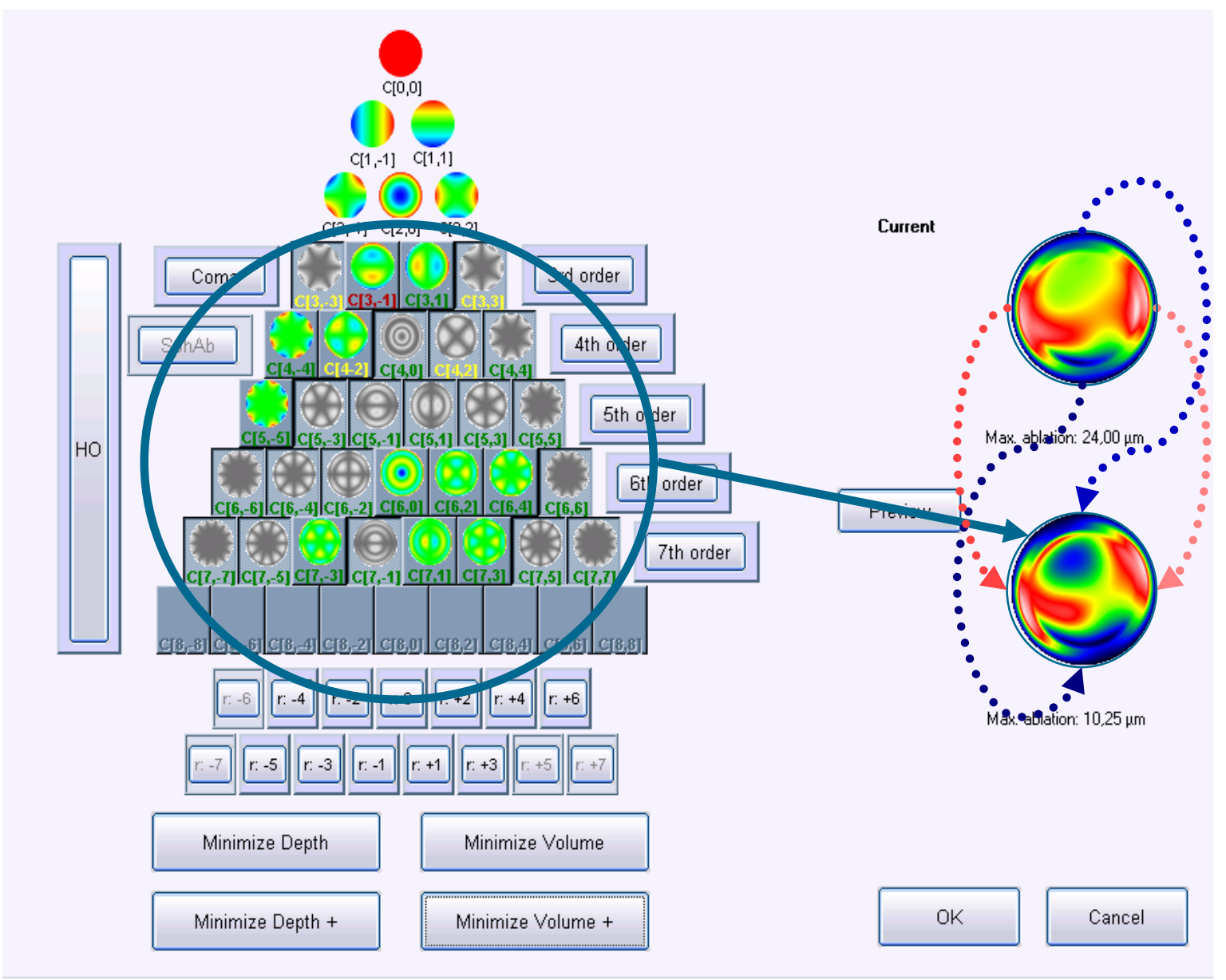

Figure 75: Optimised Aberration Modes Selection. Based on the wavefront aberration map, the software is able to recommend the best possible aberration modes selection to minimise tissue and time, without compromising the visual quality. Notice that the wavefront aberration is analysed by the software showing the original ablation for a full wavefront correction and the suggested set of aberration modes to be corrected. Notice the difference in required tissue, but notice as well that the most representative characteristics of the wavefront map are still presented in the minimised tissue selection. 
Simulation of the tissue-saving capabilities of such methods for minimising the required ablation tissue

(Simulación de la capacidad de ahorro de tejido de dichos métodos para minimizar la cantidad de tejido de ablación)

For each wave aberration map, for a $6 \mathrm{~mm}$ pupil, it has been simulated how deep and how much volume of tissue was it necessary to ablate for six different scenarios (Table 14):

- correction of the full wavefront

- $\quad$ minimising depth, minimising depth +

- $\quad$ minimising volume, minimising volume +

- equivalent Aberration-Free treatment

For each wave aberration, it has been calculated how much depth and volume of tissue was saved for each condition (in micrometers and in percentage, relative to the full wavefront correction), and it has been noted whether the proposed correction consists of either the full wavefront correction (with all Zernike terms included in the ablation) or the aberration-free treatment (without any Zernike term included in the ablation) and, finally, whether or not the proposed depth or volume was less than the one required for the equivalent aberration-free treatment.

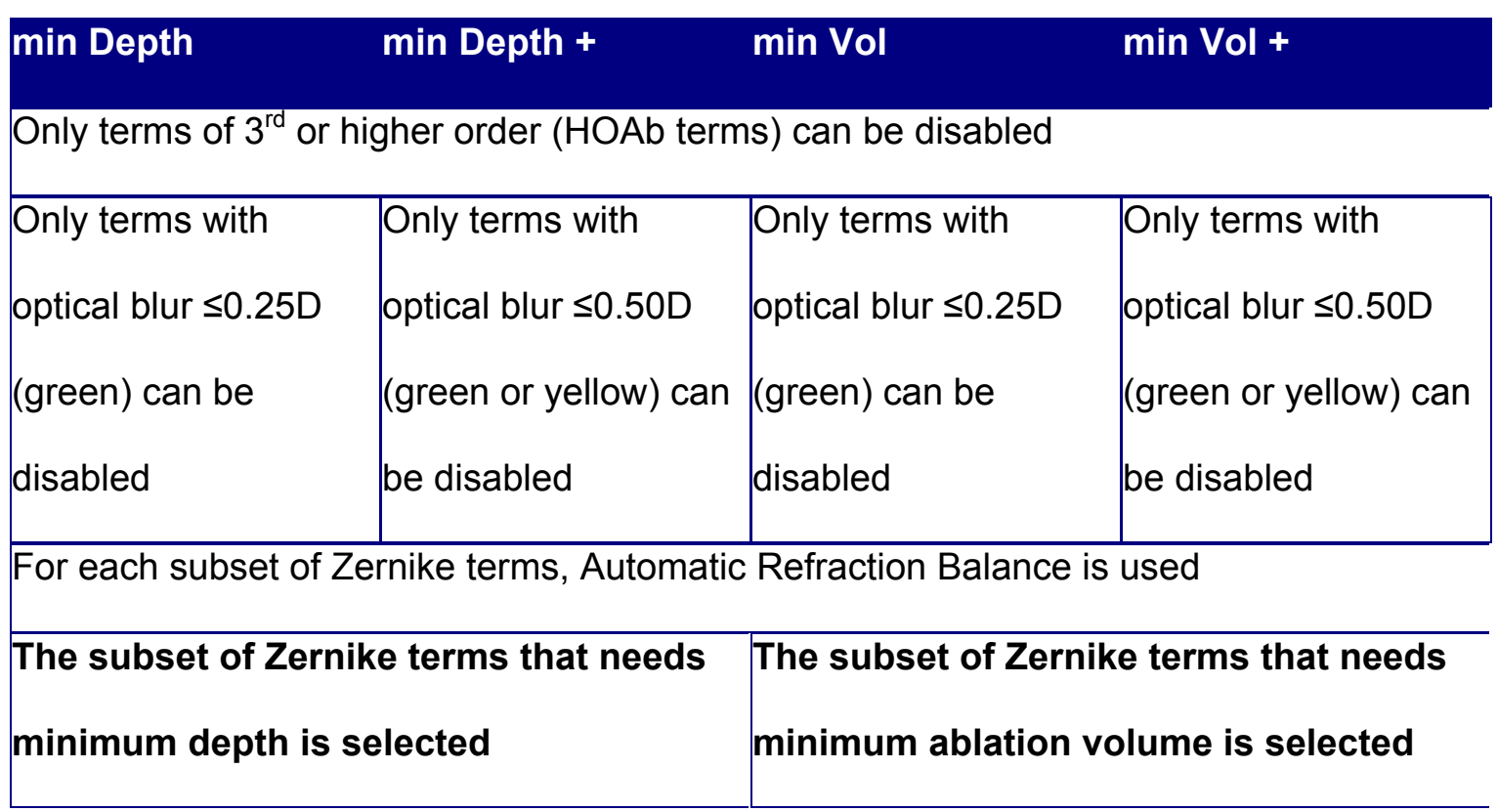

Table 14: Summary properties of the four minimisation approaches 
Once the data about tissue saving was computed for each wave aberration, to calculate the average tissue-saving for the different modalities over the sample of treatments we have used several methods:

a) direct average of the saved depth or volume

b) intercept with the axis in a correlation graph

c) direct average of the percentile saved depth or volume

d) intercept with the axis in a percentile correlation graph

\section{G.3.4 Evaluation of the clinical application of such methods for minimising the required ablation tissue \\ (Evaluación de la aplicación clínica de dichos métodos para minimizar la cantidad de tejido de ablación)}

Fourty-five patients (90 eyes) seeking laser correction at the Muscat Eye Laser Centre in the Sultanate of Oman were enrolled in this prospective study. Institutional Review Board approval was obtained and written informed consent was obtained from all the patients and the study conformed to tenets of Declaration of Helsinki.

The treatment plan was developed using CW customised aspheric profiles based on corneal ray tracing ${ }^{54}$. Using the Keratron Scout videokeratoscope ${ }^{153}$ (Optikon 2000 S.p.A, Rome, Italy), we analyzed the topographical surface and corneal wavefront (up to the $7^{\text {th }}$ order). The departure of the measured corneal topography from the theoretically optimal corneal surface was calculated for a balanced-eye model (Q-Val -0.25). Optical errors centred on the line-of-sight were described by the Zernike polynomials ${ }^{36}$ and the coefficients of the Optical Society of America (OSA) standard ${ }^{60}$.

Corneal Wavefront registers the type and size of each and every optical error generated on the anterior corneal surface, allowing a very selective correction. The defects are corrected at exactly the location where they occur - 
the anterior corneal surface. In this context, exact localization of defects is decisive in achieving optimal results in laser surgery. The corneal wavefront allows for a very precise diagnosis, thus providing an individual ablation of the cornea to obtain perfect results. With this treatment strategy, pupil dilation of the patient is not necessary for measurement, thus the pupil does not limit treatment zone, and accommodation does not influence measuring results.

Notice that in this way the treatments are not forcing a fixed asphericity quotient $(\mathrm{Q})$ on all eyes postoperatively but rather a postoperative expected asphericity quotient as:

$$
Q_{\text {exp }}=\frac{\frac{1}{n^{2}}-\frac{1}{4}}{\left(1+\frac{R \cdot S E q_{c p}}{n-1}\right)^{3}}-\frac{1}{n^{2}}
$$

where $Q_{\text {exp }}$ is the expected/predicted corneal asphericity quotient; $R$ the apical radius of curvature of the preoperative cornea, $\mathrm{SEq}_{\mathrm{cp}}$ the spherical equivalent to be corrected at the corneal plane; and $\mathrm{n}$ the refractive index of the cornea.

\section{G.3.4.1 Treatment selection criteria \\ (Criterios de selección de tratamiento)}

Only patients presenting aberrations $>0.325 \mu \mathrm{m}$ RMS $\mathrm{HO}$ at $6 \mathrm{~mm}$ analysis diameter measured by the OPTIKON Keratron Scout (both eyes) were enrolled in the study.

Exclusion criteria for enrolling the study were unstable refraction in the last six months, signs of keratoconus or abnormal corneal topography, collagen vascular, autoimmune or immunodeficiency diseases, severe local infective or allergic conditions, severe dry eye disease, monocularity or severe amblyopia, or cataracts.

The patients were sequentially assigned to three different groups ( $A, B$, and $C$ ). The rationale of the three groups were in group $A$ directly compare the full customised correction (CW) versus the minimum depth correction (MD), in group B directly compare the full customised correction (CW) versus the minimum 
volume (time) correction (MV), and in group C directly compare the minimum depth correction (MD) versus the minimum volume (time) correction (MV); all groups in a lateral/contralateral eye basis randomly assigned (coin toss) for the direct comparison.

This way, we got three patients groups (A, B, C) with 15 patients each; and 3 treatments groups (CW, MD and MV) with 30 patients each.

Preoperative topography and corneal aberrometry measurements were taken, and visual acuity, contrast sensitivity (CST 1800 digital, Vision Sciences Research Corporation, San Ramon, California, USA) and mesopic pupil size were measured. Each eye was planned according to the manifest refraction using the CAM Wavefront customised treatments and the corresponding minimization method. Immediately before the ablation, the laser was calibrated per manufacturer's instructions and the calibration settings were recorded.

All surgeries were performed by the same surgeon (M.C.A.). LASIK flaps were created with a superior hinge using a Carriazo-Pendular microkeratome ${ }^{154}$ (SCHWIND eye-tech-solutions $\mathrm{GmbH}$, Kleinostheim, Germany). The ablation was carried out with an ESIRIS excimer laser (SCHWIND eye-tech-solutions $\mathrm{GmbH}$, Kleinostheim, Germany). The ESIRIS laser system works at a repetition rate of $200 \mathrm{~Hz}$ and produces a spot size of $0.8 \mathrm{~mm}$ (Full Width at Half Maximum, FWHM) with a paraGaussian ablative flying-spot profile ${ }^{164,165}$. High-speed eyetracking with $330 \mathrm{~Hz}$ acquisition rate is accomplished with a 5-ms latency period $^{168}$.

All ablations were using the CAM (Customized Ablation Manager) on the ESIRIS without nomogram to plan the ablations. The CAM aspherical profiles were developed with the aim of compensating for the aberrations induction observed with other types of profile definitions ${ }^{158}$, some of those sources of aberrations are those related to the loss of efficiency of the laser ablation for nonnormal incidence ${ }^{159,160,161}$. Optimisation is realized by taking into account the loss of efficiency at the periphery of the cornea in relation to the centre as there is a tangential effect of the spot in relation to the curvature of the cornea ( $K$ 
(Keratometry) -reading). The software provides K-reading compensation, which considers the change in spot geometry and reflection losses of ablation efficiency. Real ablative spot shape (volume) is considered through a self-constructing algorithm. In addition, there is a randomised flying-spot ablation pattern, and controls for the local repetition rates to minimise the thermal load of the treatment ${ }^{163}$ (smooth ablation, no risk of thermal damage).

Pupil sizes averaged $6.00 \pm 0.58 \mathrm{~mm}$ in diameter (ranging from 5.00 to 7.00 $\mathrm{mm})$. Ablations were planned for an optical zone $(\mathrm{OZ})$ averaging $6.50 \pm 0.35 \mathrm{~mm}$ in diameter (ranging from 6.00 to $7.00 \mathrm{~mm}$ ), with a total ablation zone (TAZ) dynamically provided by the software averaging $7.75 \pm 0.45 \mathrm{~mm}$ in diameter (ranging from 7.00 to $8.75 \mathrm{~mm}$ ).

Outcomes at 3 months included preoperative and postoperative findings, auto-refractor measurements, manifest refraction; best spectacle corrected visual acuity (BSCVA), uncorrected visual acuity (UCVA), topography and corneal aberrometry as well as complications.

Evaluation of the tissue-savings of such methods for minimising the required ablation tissue

(Evaluación del ahorro de tejido de tales métodos para minimizar la cantidad de tejido de ablación)

For each treatment, it has been recorded how deep and how long time (how much volume) was required for three different conditions:

- correction of the full wavefront

- corresponding minimisation method (depth or volume)

- equivalent Aberration-Free treatment

For each wavefront aberration, it has been calculated how much depth and time was saved for each condition (in micrometers, time and percentage with respect to the full wavefront correction), and whether the proposed depth or time was less than the one required for the equivalent Aberration-Free treatment. 
The treatments with savings in depth higher than $9 \mu \mathrm{m}(\sim 0.75 \mathrm{D}$ using simplified Munnerlyn equation for $6 \mathrm{~mm}$ optical zone) or savings in time higher than $7 \mathrm{~s}(\sim 0.75 \mathrm{D}$ using ESIRIS) were accounted.

\section{G.3.4.3 Direct comparison \\ (Comparación directa)}

For each of the three groups contralateral eye direct comparison was evaluated with a subjective questionnaire. The questionnaire assess by direct answers from the patient subjective impression the differences between both eyes (with different treatment strategies) in terms of normal and dim light conditions (rating glare, halos, starbursts, blurry vision, shadows, ghost or double images, subjective visual quality indoors and outdoors, and preferred eye). 


\section{Subjective Enquiry}

\section{Patient Information}

\begin{tabular}{|l|c|}
\hline \multicolumn{1}{|c|}{ Concept } & Value \\
\hline Patient Id. & \\
\hline Patient Name & \\
\hline Pre-Op Status Questionnaire & 1 day \\
\hline & \\
Patient Follow-Up Time & 3 month \\
& Other: \\
\hline
\end{tabular}

Table 15: Patient information

\begin{tabular}{|l|l|l|}
\hline \multicolumn{2}{|c|}{ Concept } & \\
\hline Pre-Op Manifest Refraction & & \\
\hline Type of Surgery & & \\
\hline Initial / Enhancement & & \\
\hline Current Manifest Refraction & & \\
\hline Eye Dominance & & \\
\hline $\begin{array}{l}\text { Adverse Events / } \\
\text { Complications }\end{array}$ & & \\
\hline & & \\
\hline Comments & & \\
\hline
\end{tabular}

Table 16: Treatment information 


\section{Subjective Questionnaire}

\section{Normal Light Conditions}

The patients have to evaluate the appearance of the below listed topics under Normal Light Conditions.

$\diamond \quad$ The rating of questions $\mathrm{Nr}$. $1-5$ corresponds to the following scale:

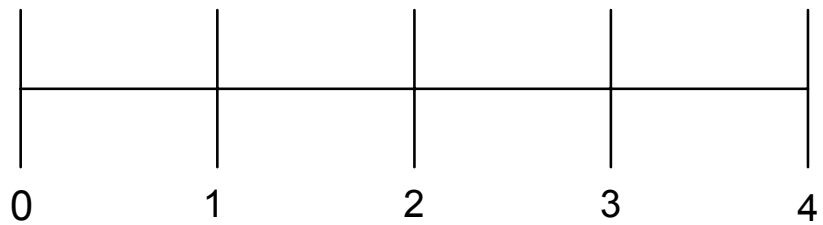

minimum

maximum

value

value

$\Rightarrow$ no complaints

$\Rightarrow$ highest complaints

\begin{tabular}{|r|l|l|l|}
\multicolumn{2}{|c|}{ Nr. Concept } & OD & OS \\
\hline 1 & Glare, Halos, Starbursts & & \\
\hline 2 & Blurry Vision & & \\
\hline 3 & Shadows, Ghost or Double Images & & \\
\hline
\end{tabular}

\begin{tabular}{|r|l|l|l|}
\hline 4 & $\begin{array}{l}\text { Subjective Visual Quality indoors } \\
(\text { Choose only 0, 2, or 4) }\end{array}$ & & \\
\hline 5 & $\begin{array}{l}\text { Subjective Visual Quality outdoors } \\
(\text { Choose only 0, 2, or 4) }\end{array}$ & & \\
\hline
\end{tabular}

\begin{tabular}{|l|l|l|}
\hline 6 & $\begin{array}{l}\text { Preferred Eye (OD/OS) } \\
\text { (Choose only OD or OS) }\end{array}$ & \\
\hline
\end{tabular}

Table 17: Normal light questionnaire 


\section{Dim Light Conditions}

The patients have to evaluate the appearance of the below listed topics under both normal and dimmed light conditions.

$\diamond \quad$ The rating of questions Nr. 8-11 corresponds to the following scale:

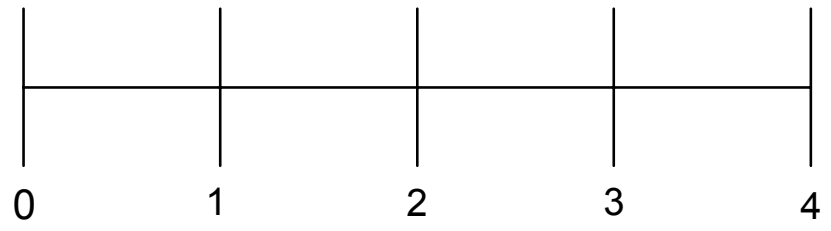

minimum

maximum

value

value

$\Rightarrow$ no complaints

$\Rightarrow$ highest complaints

\begin{tabular}{|r|l|l|l|}
\multicolumn{1}{|c|}{ Nr. Concept } & OD & OS \\
\hline 7 & Glare, Halos, Starbursts & & \\
\hline 8 & Blurry Vision & & \\
\hline 9 & Shadows, Ghost or Double Images & & \\
\hline
\end{tabular}

\begin{tabular}{|c|l|l|l|}
\hline 10 & $\begin{array}{l}\text { Subjective Visual Quality } \\
\text { (Choose only 0, 2, or 4) }\end{array}$ & & \\
\hline
\end{tabular}

11

Preferred Eye (OD/OS)

(Choose only OD or OS)

Table 18: Dim light questionnaire 


\section{Clinical Procedure}

1. Perform patient diagnosis (including PreOP Topography and Aberrometry; also Contrast Sensitivity Test)

\begin{tabular}{|l|l|c|}
\hline \multicolumn{2}{|c|}{ Pre-Op } \\
\hline Consent Form & \multicolumn{2}{|c|}{ X } \\
\hline Scout & Monocular (OD / OS) & $X$ \\
\hline COAS & Monocular (OD / OS) & $X$ \\
\hline Pupil size & Monocular (OD / OS) & $X$ \\
\hline UCVA & Monocular (OD / OS) & $X$ \\
\hline BCVA & Monocular (OD / OS) & $X$ \\
\hline Manifest Refraction & Monocular (OD / OS) & $X$ \\
\hline Pachymetry & Monocular (OD / OS) & $X$ \\
\hline
\end{tabular}

Table 19: Preoperative diagnosis

2. Input all data in ORK-CAM

3. Save project file

4. Save ocm file

5. Load ocm file into ESIRIS Laser

6. Printout Summary page

7. Import ocm file into ESIRIS Laser

8. Proceed with surgery

9. Perform patient follow-ups (including PostOP Topography and Aberrometry; also Contrast Sensitivity Test) 


\begin{tabular}{|c|c|c|c|c|c|}
\hline Test & Type & 1 WEEK & 1 MONTH & 3 MONTHS & 6 MONTHS \\
\hline Scout & $\begin{array}{l}\text { Monocular } \\
\text { (OD / OS) }\end{array}$ & --- & --- & $X$ & $\mathrm{X}$ \\
\hline COAS & $\begin{array}{l}\text { Monocular } \\
\text { (OD / OS) }\end{array}$ & --- & --- & $X$ & $X$ \\
\hline Pupil size & $\begin{array}{l}\text { Monocular } \\
\text { (OD / OS) }\end{array}$ & --- & --- & $X$ & -- \\
\hline UCVA & $\begin{array}{l}\text { Monocular } \\
\text { (OD / OS) }\end{array}$ & $\mathrm{x}$ & $x$ & $x$ & $x$ \\
\hline BCVA & $\begin{array}{l}\text { Monocular } \\
\text { (OD / OS) }\end{array}$ & --- & $x$ & $x$ & $\mathrm{X}$ \\
\hline Manifest Refraction & $\begin{array}{l}\text { Monocular } \\
\text { (OD / OS) }\end{array}$ & --- & $x$ & $x$ & $X$ \\
\hline Pachymetry & $\begin{array}{l}\text { Monocular } \\
\text { (OD / OS) }\end{array}$ & --- & --- & $X$ & --- \\
\hline Subjective Questionnaire & --- & $X$ & $x$ & $X$ & $X$ \\
\hline
\end{tabular}

Table 20: Scheduled diagnosis during follow-up 


\section{Descriptive statistics:}

Determination of minimal-, maximal-, and mean values, simple standard deviation. Statistics on how much tissue is saved by minimising, how often minimising goes below Aberration-Free profile depth or time.

For statistical analysis, paired $t$-tests were used to compare postoperative vs. preoperative results within each group, and the differences between the groups were analysed using ANOVA. For correlation tests, the Coefficient of Determination $\left(\mathrm{r}^{2}\right)$ was used and the significance of the correlations has been evaluated using $p$-values calculated considering a metric distributed approximately as $\mathrm{t}$ with $\mathrm{N}-2$ degrees of freedom where $\mathrm{N}$ is the size of the sample. For all tests, $p$ values of less than .05 were considered statistically significant.

Section G.4 RESULTS

(Resultados)

\section{G.4.1 Simulations \\ (Simulaciones)}

G.4.1.1 Objective determination of the actual clinical relevance of the single terms in a Zernike expansion of the wavefront aberration (Determinación objetiva de la relevancia clínica de términos individuales de la expansión de Zernike de la aberración del frente de onda)

The average root mean square for the high order wavefront aberration (RMS $\mathrm{HO}_{\text {) }}$ was $0.555 \pm 0.143 \mu \mathrm{m}$ for a $6 \mathrm{~mm}$ analysis diameter (from $0.327 \mu \mathrm{m}$ to $0.891 \mu \mathrm{m})$, whereas the average root mean square of the total wavefront aberration (RMS) was $3.955 \pm 2.715 \mu \mathrm{m}$ also for a $6 \mathrm{~mm}$ analysis diameter (from $0.741 \mu \mathrm{m}$ to $10.920 \mu \mathrm{m})$. 
The distribution of corneal aberration in Zernike terms seems to be normal (Figure 76).

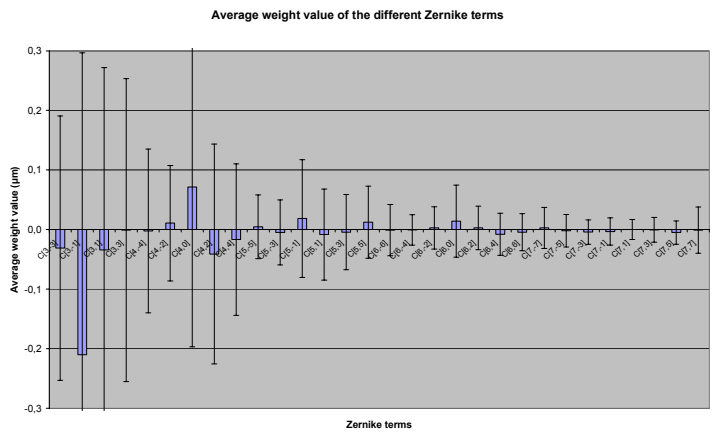

Zernike terms
Average of the weights in absolute value of the different Zernike term

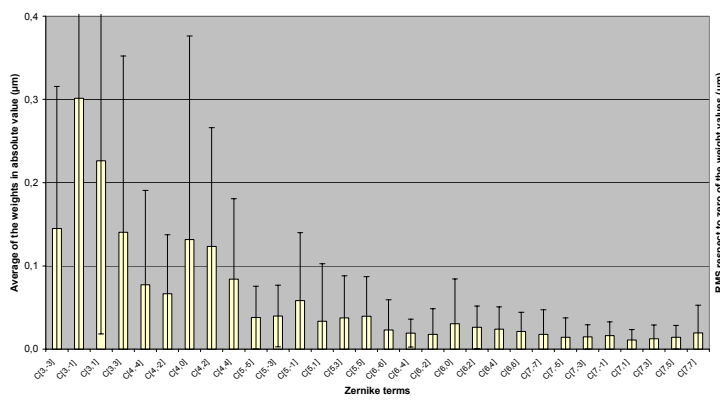

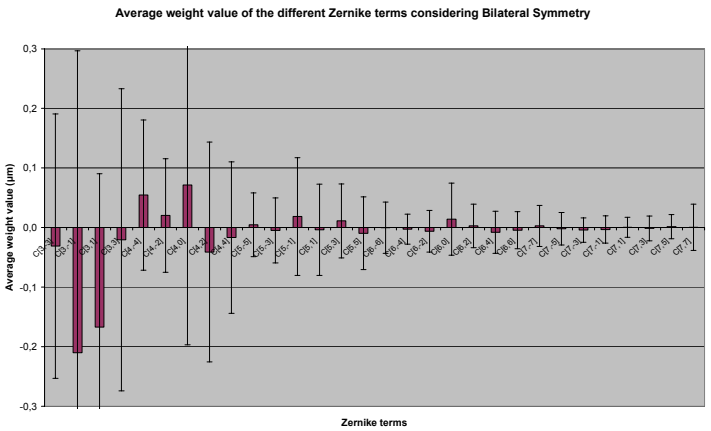

Zerikik terms
RMS respect to zero of the weight values of the different Zernike terms

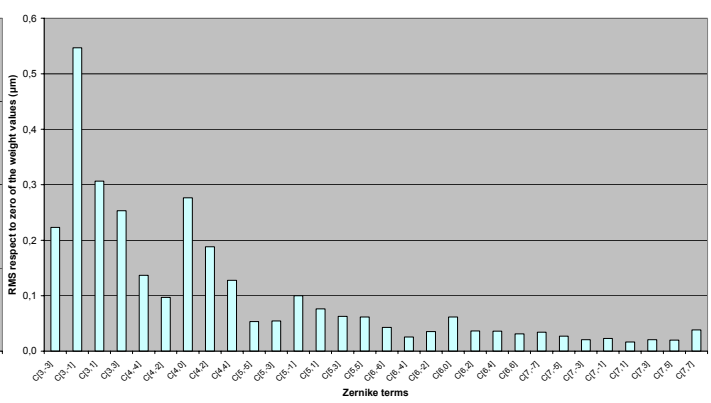

Figure 76: Zernike coefficients distribution for the sample population of wavefront maps: natural arithmetic average, average considering bilateral symmetry, average considering absolute value, root-mean-square respect to zero

Spherical aberration was $+0.107 \pm 0.205 \mu \mathrm{m}$ (from $-0.476 \mu \mathrm{m}$ to $+0.514 \mu \mathrm{m}$ ), coma aberration was $0.369 \pm 0.316 \mu \mathrm{m}$ (from $0.030 \mu \mathrm{m}$ to $1.628 \mu \mathrm{m}$ ), and trefoil aberration was $0.204 \pm 0.186 \mu \mathrm{m}$ (from $0.022 \mu \mathrm{m}$ to $1.118 \mu \mathrm{m}$ ) all of them refer to a $6 \mathrm{~mm}$ analysis diameter.

Spherical aberration was $+0.184 \pm 0.136 \mathrm{DEq}$ (from $0.000 \mathrm{DEq}$ to 0.511 $\mathrm{DEq}$ ), coma aberration was $0.232 \pm 0.199 \mathrm{DEq}$ (from $0.019 \mathrm{DEq}$ to $1.023 \mathrm{DEq}$ ), and trefoil aberration was $0.128 \pm 0.117 \mathrm{DEq}$ (from $0.014 \mathrm{DEq}$ to $0.703 \mathrm{DEq}$ ).

Out of all the wave aberration maps under study, $72 \%$ of them showed a spherical aberration below $0.25 \mathrm{DEq}, 23 \%$ of them showed a spherical aberration between $0.25 \mathrm{DEq}$ and $0.50 \mathrm{DEq}$, and only $5 \%$ of the maps showed a spherical aberration higher than $0.50 \mathrm{DEq}$. 
Regarding coma aberration, for $68 \%$ of the wavefront aberration maps it was below $0.25 \mathrm{DEq}$, for $23 \%$ of them it was between $0.25 \mathrm{DEq}$ and $0.50 \mathrm{DEq}$, and only for $9 \%$ of the maps was the coma aberration higher than $0.50 \mathrm{DEq}$.

Regarding trefoil aberration, for $87 \%$ of the wavefront aberration maps it was below $0.25 \mathrm{DEq}$, for $10 \%$ of them it was between $0.25 \mathrm{DEq}$ and $0.50 \mathrm{DEq}$, and only for $3 \%$ of the maps was the trefoil aberration higher than $0.50 \mathrm{DEq}$.

Objective minimisation of the maximum depth or volume of a customised ablation based on the Zernike expansion of the wavefront aberration (Minimización objetiva de la profundidad máxima o el volumen de ablación de tratamientos personalizados basados en la expansión de Zernike de la aberración del frente de onda)

Comparing the ablations planned to correct for the whole wave aberration with equivalent aberration-free ablations (designed to correct only for spherocylindrical refraction), we observed an average difference in maximum depth of $+8 \pm 8 \mu \mathrm{m}$ (range: from -4 to $+33 \mu \mathrm{m}$ ), and an average difference in volume of $+158 \pm 158 \mathrm{nl}$ (range: from $-127 \mathrm{nl}$ to $+664 \mathrm{nl}$ ); that is, $+32 \%$ (up to $+317 \%$ ), indicating that more tissue was necessary to ablate to achieve full customised corrections.

In $13 \%$ of the cases, the ablations designed to correct for the whole wave aberration needed to ablate less tissue than the equivalent aberration-free ablations.

Comparing the proposed "minimised-depth" ablations with the equivalent ablations designed to correct for the whole wave aberration, we observed an average difference in maximum depth of $-4 \pm 2 \mu \mathrm{m}$ (range: from $-10 \mu \mathrm{m}$ to -1 $\mu \mathrm{m}$ ), and an average difference in ablated volume of $-64 \pm 32 \mathrm{nl}$ (range: from $190 \mathrm{nl}$ to $0 \mathrm{nl}$ ); that is, $-8 \%$ (up to $-30 \%$ ), indicating that less tissue needs to be removed for the "minimised-depth" corrections.

In $43 \%$ of the cases, the proposed "minimised-depth" ablations resulted in less ablated tissue than the equivalent aberration-free ablations. 
Comparing the proposed "minimised-volume" ablations with the equivalent ablations devised to correct for the whole wave aberration, we observed an average difference in maximum depth of $-4 \pm 2 \mu \mathrm{m}$ (range: from $-10 \mu \mathrm{m}$ to $0 \mu \mathrm{m}$ ), and an average difference in volume of $-64 \pm 32 \mathrm{nl}$ (range: from -190 $\mathrm{nl}$ to $0 \mathrm{nl}$ ); that is $-7 \%$ (up to $-30 \%$ ), meaning less tissue removal for the "minimised volume" corrections.

In $39 \%$ of the cases, the proposed "minimised-volume" ablations required to remove less tissue than the equivalent aberration-free ablations (those devised to correct only for spherocylindrical refraction).

Comparing the proposed "minimised-depth+" ablations with the equivalent ablations designed to correct for the whole wave aberration, we observed an average difference in maximum depth of $-6 \pm 4 \mu \mathrm{m}$ (range: from $-16 \mu \mathrm{m}$ to -1 $\mu \mathrm{m}$ ), and an average difference in volume of $-127 \pm 95 \mathrm{nl}$ (range: from $-316 \mathrm{nl}$ to $0 \mathrm{nl}$ ) or $-15 \%$ (up to $-66 \%$ ); that is, less tissue removal was required for the "minimised-depth+" corrections.

In $80 \%$ of the cases, the proposed "minimised-depth+" ablations needed less tissue than equivalent aberration-free ablations planned to correct only spherocylindrical refraction.

Comparing the proposed "minimised-volume+" ablations with the equivalent ablations intended to correct for the whole wave aberration, we observed an average difference in maximum depth of $-6 \pm 4 \mu \mathrm{m}$ (range: from -15 $\mu \mathrm{m}$ to $0 \mu \mathrm{m}$ ), and an average difference in volume of $-127 \pm 64 \mathrm{nl}$ (range: from $316 \mathrm{nl}$ to $0 \mathrm{nl}$ ) or $-14 \%$ (up to $-63 \%$ ); that is, less tissue removal was needed for the "minimised-volume+" corrections.

In $75 \%$ of the cases, the proposed "minimised-volume+" ablations needed to remove less tissue than the equivalent aberration-free ablations. 
Maximum ablation depth

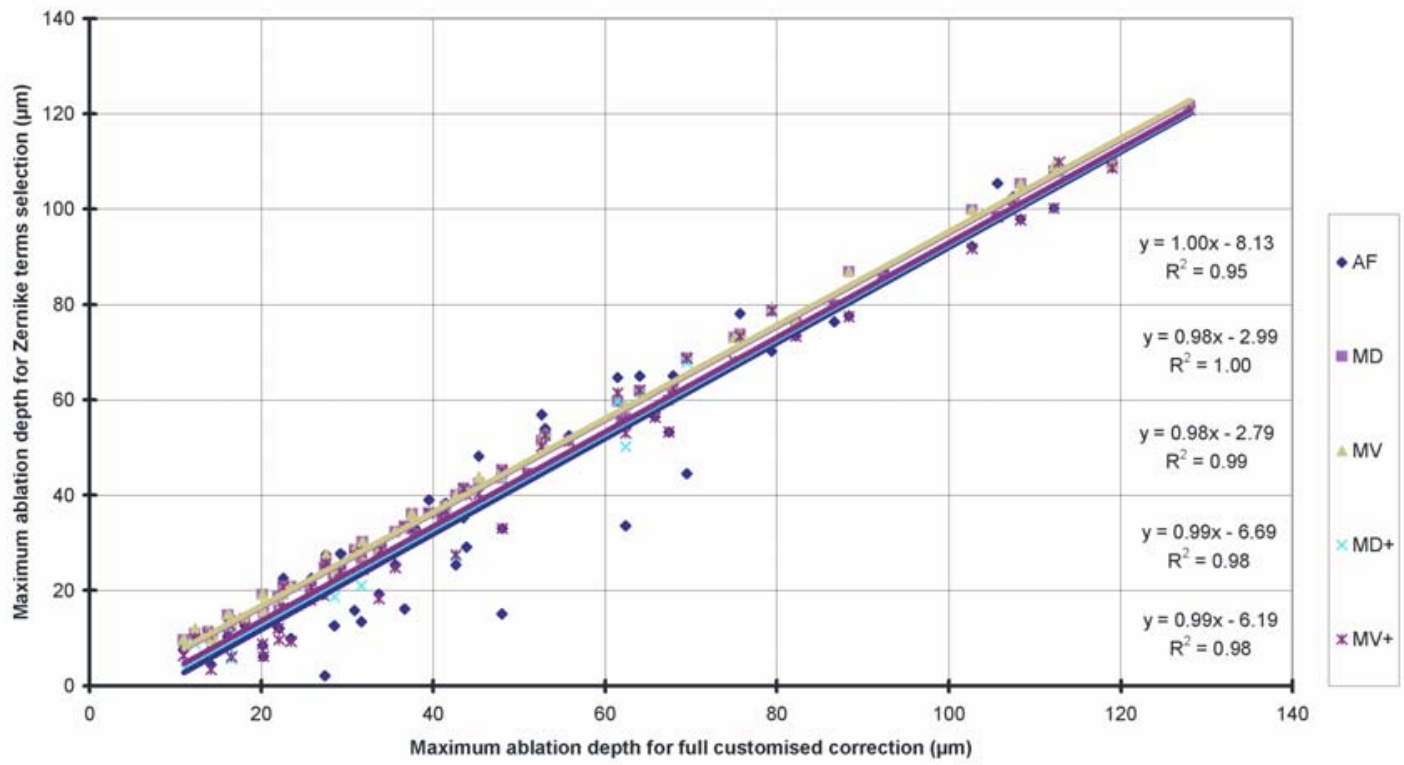

Figure 77: Ablation depth for OZTS vs. Ablation depth for full-customised correction for: Aberration-Free correction (all HOAb disabled) (in blue), minimise depth (in magenta), minimise volume (in yellow), minimise depth+ (in cyan), and minimise volume+ (in purple)

\section{Ablation time}

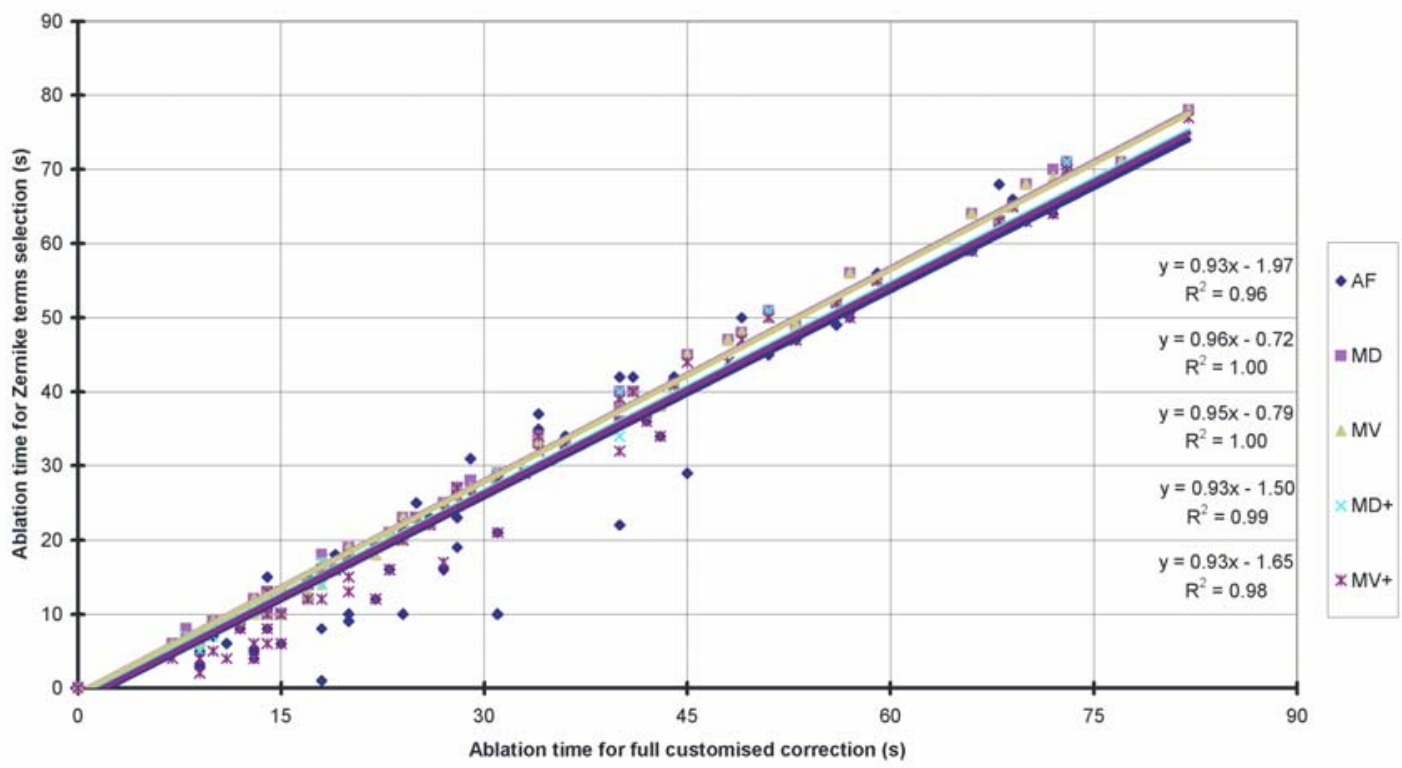

Figure 78: Ablation time for OZTS vs. Ablation time for full-customised correction for: Aberration-Free correction (all HOAb disabled) (in blue), minimise depth (in 
magenta), minimise volume (in yellow), minimise depth+ (in cyan), and minimise volume+ (in purple)

G.4.2 Evaluation of the clinical application of such methods for minimising the required ablation tissue

(Evaluación de la aplicación clínica de tales métodos para minimizar la cantidad de tejido de ablación)

Outcomes at 3 months included preoperative and postoperative findings, auto-refractor measurements, manifest refraction; best spectacle corrected visual acuity (BSCVA), uncorrected visual acuity (UCVA), topography and corneal aberrometry as well as complications.

\section{G.4.2.1 Case report \\ (Caso de estudio)}

In a pilot experience of the clinical application of such methods for minimising the required ablation tissue, the complete records with the clinical data of the very first eye of the first patient treated using this approach at the Augenzentrum Recklinghausen (Germany) were analysed in the form of a case report.

The purpose was to evaluate the clinical application of a method for minimizing the required ablation tissue based upon objective minimization of the depth, volume, and time of a customized ablation based on the Zernike expansion of the wavefront aberration.

The data correspond to the left eye of a female patient (K.S.) who was 59 years old at the time of retreatment (Table 21, Figure 79 to Figure 81). She had previous LASIK surgery for myopia (ex domo) resulting in undercorrection, induced astigmatism, and limited corneal thickness for retreatment, which may have been the cause of the corneal aberrations found.

Analyses were performed for uncorrected distance visual acuity (UDVA), corrected distance visual acuity (CDVA), refractive correction, and corneal wave 
aberration up to the seventh Zernike order at the $6.5-\mathrm{mm}$ diameter, functional optical zone, and tissue and time savings.

\begin{tabular}{|c|c|}
\hline Metric & Value \\
\hline UCVA & $20 / 125$ \\
\hline BSCVA & $20 / 25$ \\
\hline Manifest Refraction & $-1.50 \mathrm{D}-2.00 \mathrm{D} @ 155^{\circ}$ \\
\hline Corneal Pachymetry & $566 \mu \mathrm{m}$ \\
\hline \multicolumn{2}{|c|}{ Aberrations at $6.5-\mathrm{mm}$ analysis diameter } \\
\hline Coma & $0.33 \mu \mathrm{m}$ RMS \\
\hline Trefoil & $0.76 \mu \mathrm{m}$ RMS \\
\hline SphAb & $0.26 \mu \mathrm{m}$ \\
\hline $\mathrm{HOAb}$ & $0.90 \mu \mathrm{m}$ RMS \\
\hline \multicolumn{2}{|c|}{ Functional Optical Zone (FOZ) } \\
\hline $\mathrm{FOZ}(0.38 \mathrm{D})$ & $\varnothing 4.69 \mathrm{~mm}$ \\
\hline FOZ(0.50D) & $\varnothing 5.85 \mathrm{~mm}$ \\
\hline
\end{tabular}

Table 21: Preoperative data of the patient K.S. 


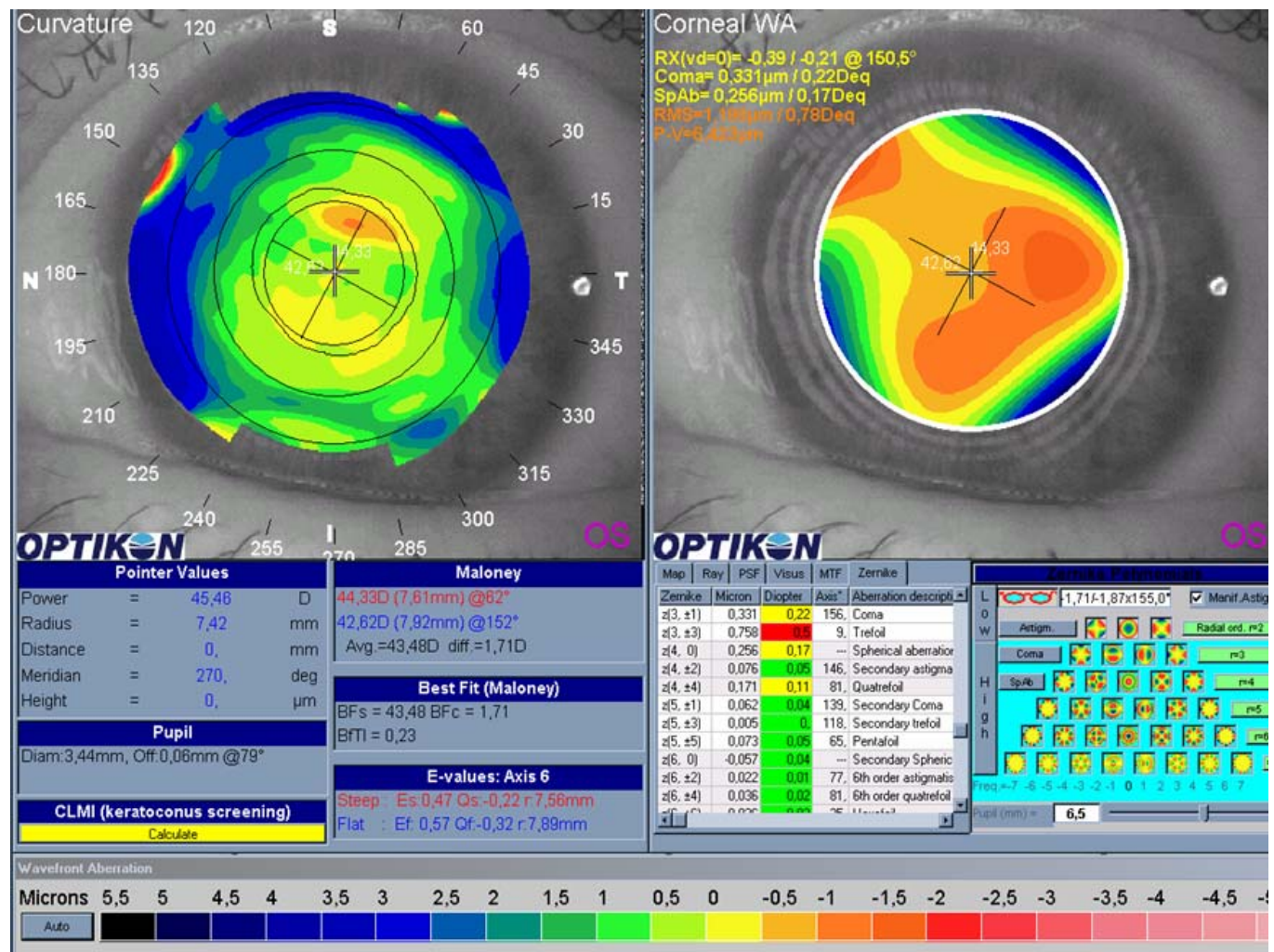

Figure 79: Preoperative topography and corneal wavefront maps of the patient K.S.

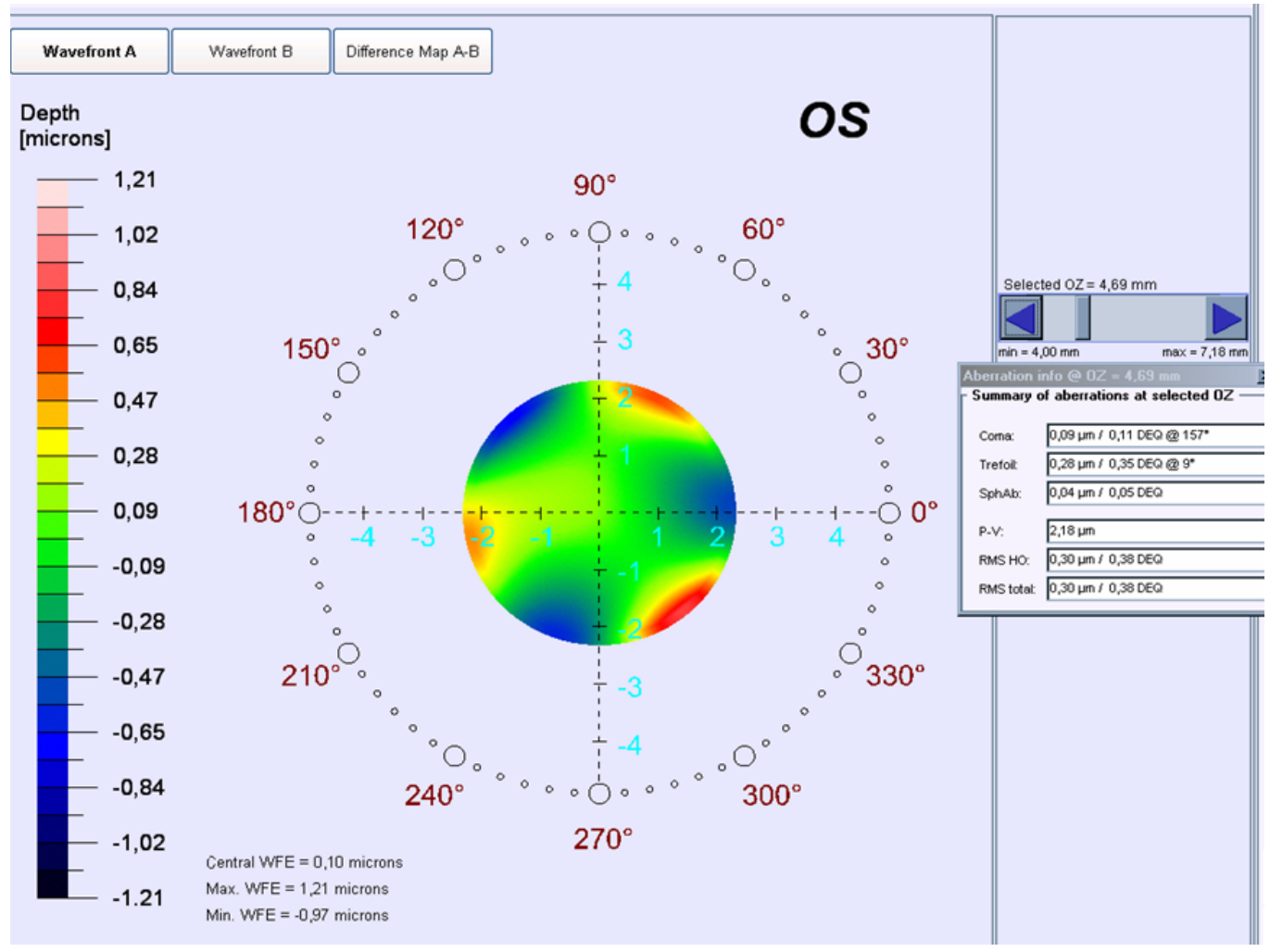

Figure 80: Preoperative corneal wavefront map of the patient K.S. determining a functional optical zone (with threshold of 0.38D) of $4.69 \mathrm{~mm} \varnothing$ 


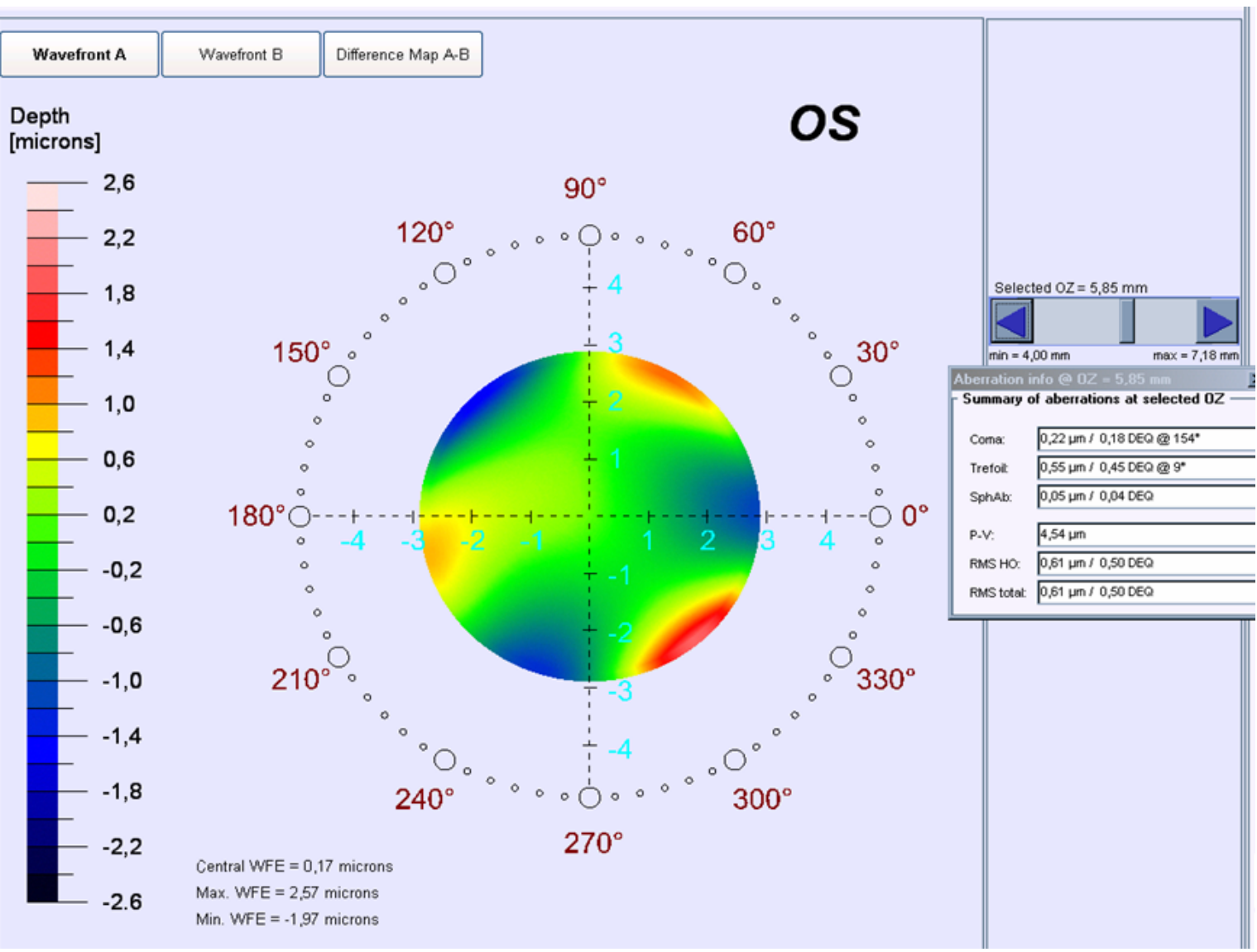

Figure 81: Preoperative corneal wavefront map of the patient K.S. determining a functional optical zone (with threshold of $0.50 \mathrm{D}$ ) of $5.85 \mathrm{~mm} \varnothing$

The planned retreatment was corneal wavefront guided laser epithelial keratomileusis (LASEK) over a 6.75-mm diameter optical zone. Planned laser settings for refractive correction were $-1.00-2.00 \times 155^{\circ}$ (Figure 82 and Table 22).

\begin{tabular}{|c|r|r|r|r|}
\hline \multicolumn{1}{|c|}{ Metric } & Full CW & Minimise Vol+ & Difference & \%Saving \\
\hline Ablation depth $(\mu \mathrm{m})$ & 64 & 57 & -7 & $-11 \%$ \\
\hline Ablation volume $(\mathrm{nl})$ & 2045 & 1757 & -288 & $-14 \%$ \\
\hline Ablation time $(\mathrm{s})$ & 67 & 52 & -15 & $-22 \%$ \\
\hline
\end{tabular}

Table 22: Comparative treatment plans and savings of the patient K.S. 

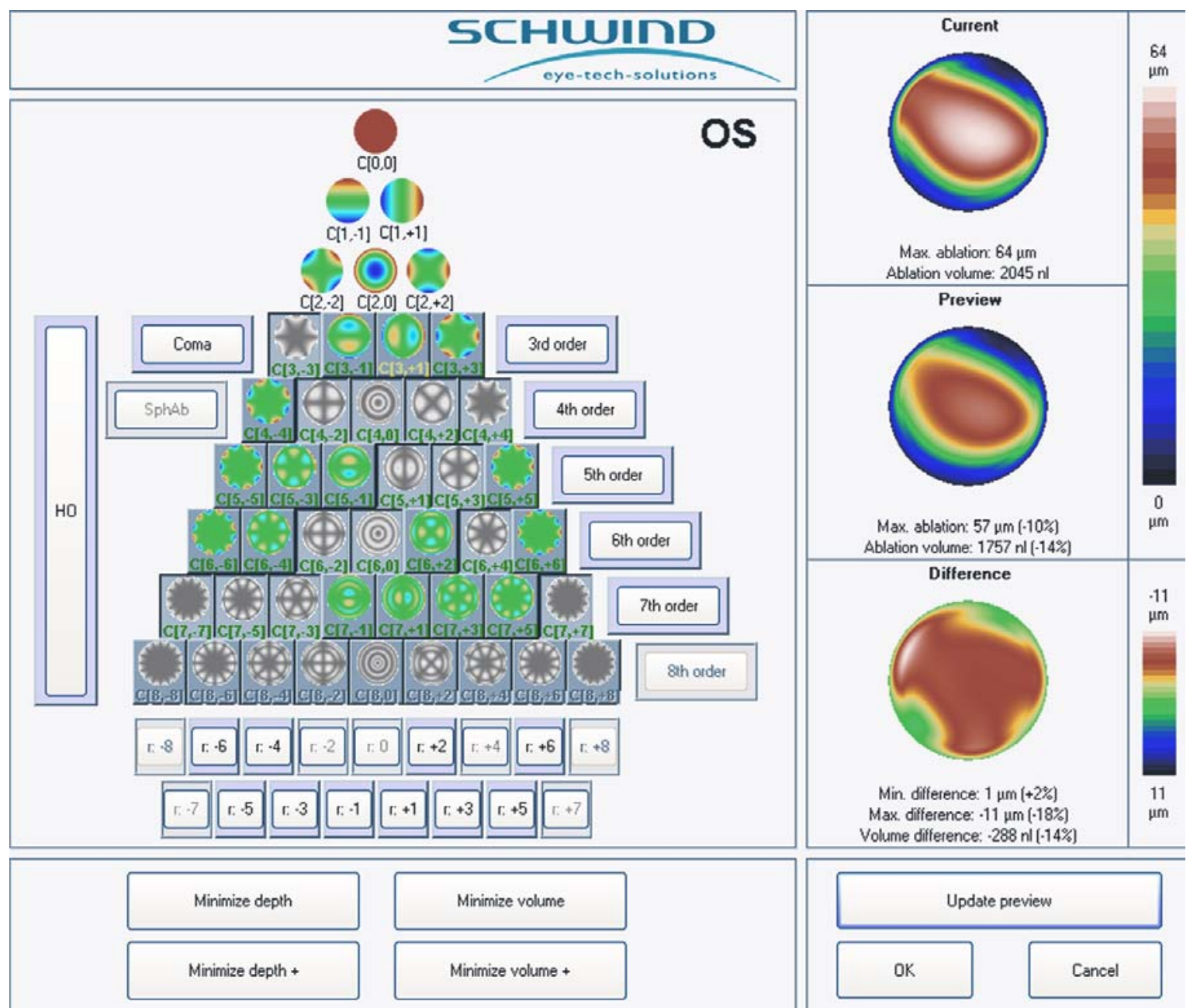

Figure 82: Comparative treatment plans and savings of the patient K.S.

A savings of approximately $15 \%$ in the minimized volume treatment plan is seen when compared to the full wave aberration correction.

Lower order aberrations are always enabled (included in the ablation pattern) as they refer to the classical ametropias. Higher order aberration terms are colour-coded. Higher order aberration terms are enabled (included in the ablation pattern) when they are in colour and disabled (discarded for the ablation pattern) when they are gray.

After uneventful refractive surgery, 3-month postoperative data are summarized in Table 23 and Figure 83 to Figure 87: 


\begin{tabular}{|c|c|}
\hline \multicolumn{2}{|l|}{ Metric } \\
\hline UCVA & 20/20 (+8 lines) \\
\hline BSCVA & 20/16 (+2 lines) \\
\hline Manifest Refraction & $-0.50 \mathrm{D}$ \\
\hline Corneal Pachymetry & $515 \mu \mathrm{m}$ \\
\hline \multicolumn{2}{|c|}{ Aberrations at $6.5-\mathrm{mm}$ analysis diameter } \\
\hline Coma & $0.13 \mu \mathrm{m}$ RMS $(-62 \%)$ \\
\hline Trefoil & $0.41 \mu \mathrm{m}$ RMS (-46\%) \\
\hline SphAb & $0.15 \mu \mathrm{m}(-39 \%)$ \\
\hline HOAb & $0.68 \mu \mathrm{m}$ RMS $(-24 \%)$ \\
\hline \multicolumn{2}{|c|}{ Functional Optical Zone (FOZ) } \\
\hline $\mathrm{FOZ}(0.38 \mathrm{D})$ & $\varnothing 5.64 \mathrm{~mm}(+45 \%)$ \\
\hline FOZ(0.50D) & Ø $6.85 \mathrm{~mm}(+37 \%)$ \\
\hline
\end{tabular}

Table 23: 3-month postoperative data of the patient K.S. 


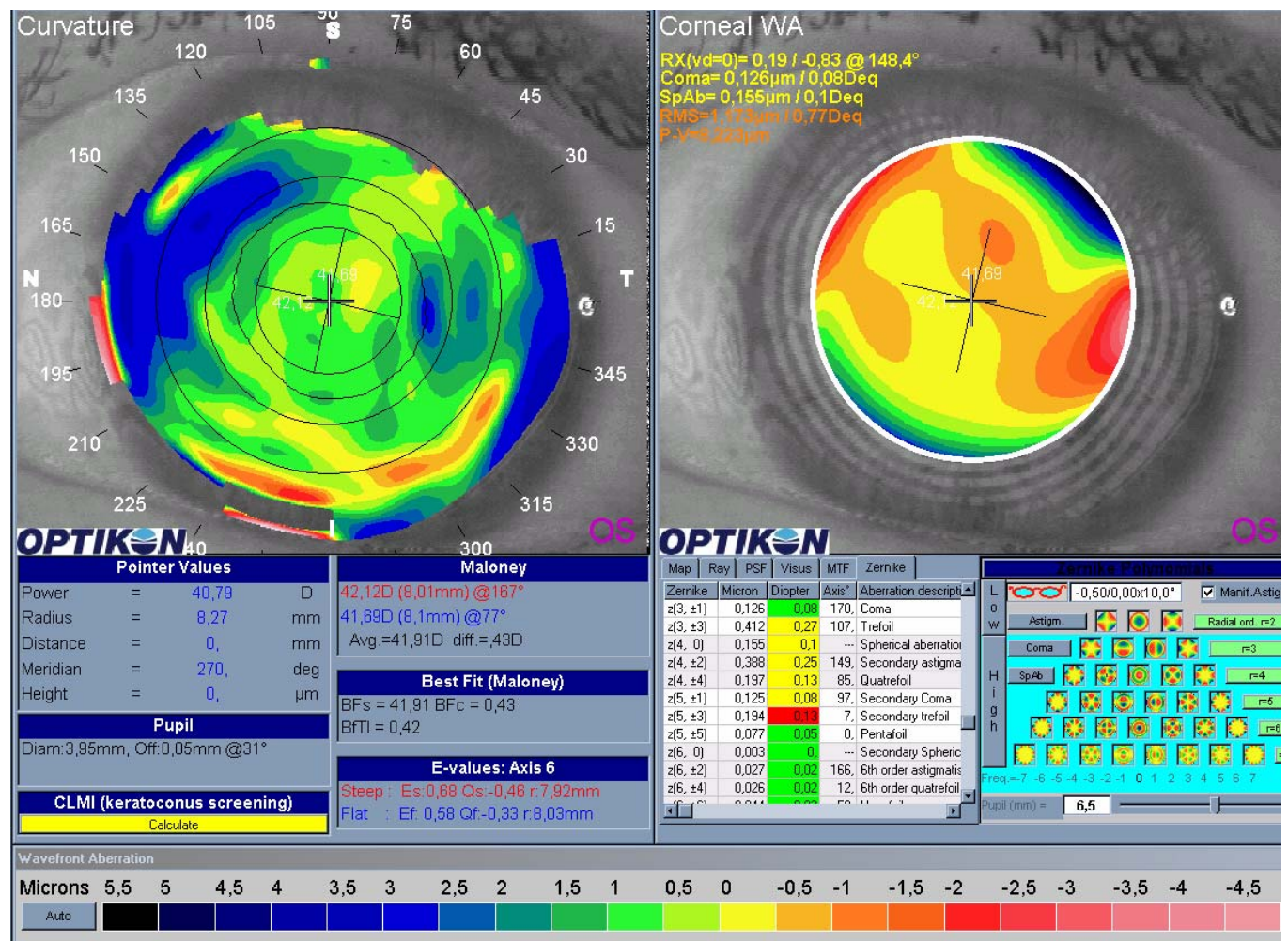

Figure 83: 3-month postoperative topography and corneal wavefront maps of the patient K.S.

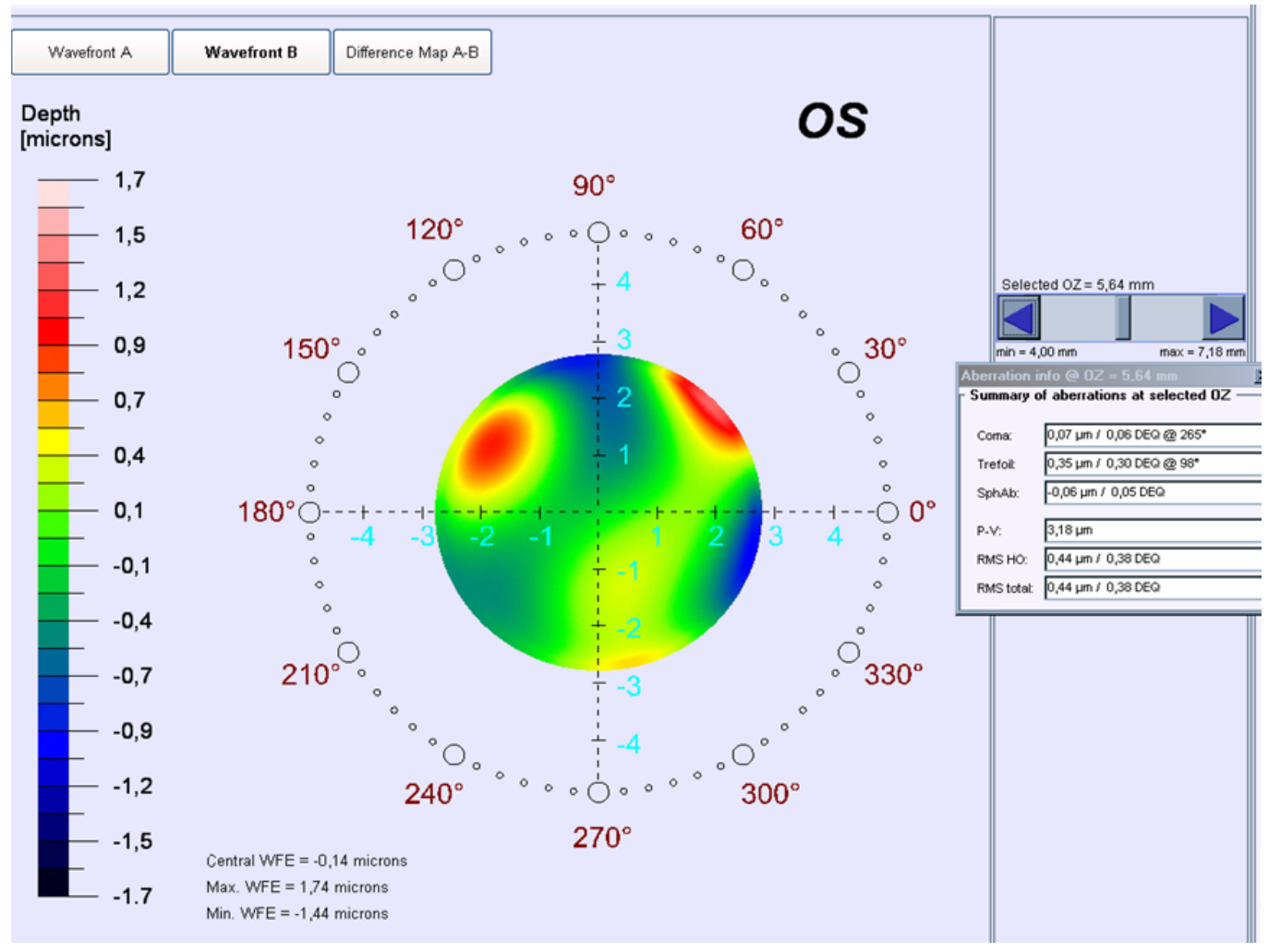

Figure 84: 3-month postoperative corneal wavefront map of the patient K.S. determining a functional optical zone (with threshold of $0.38 \mathrm{D}$ ) of $4.69 \mathrm{~mm} \varnothing$ 


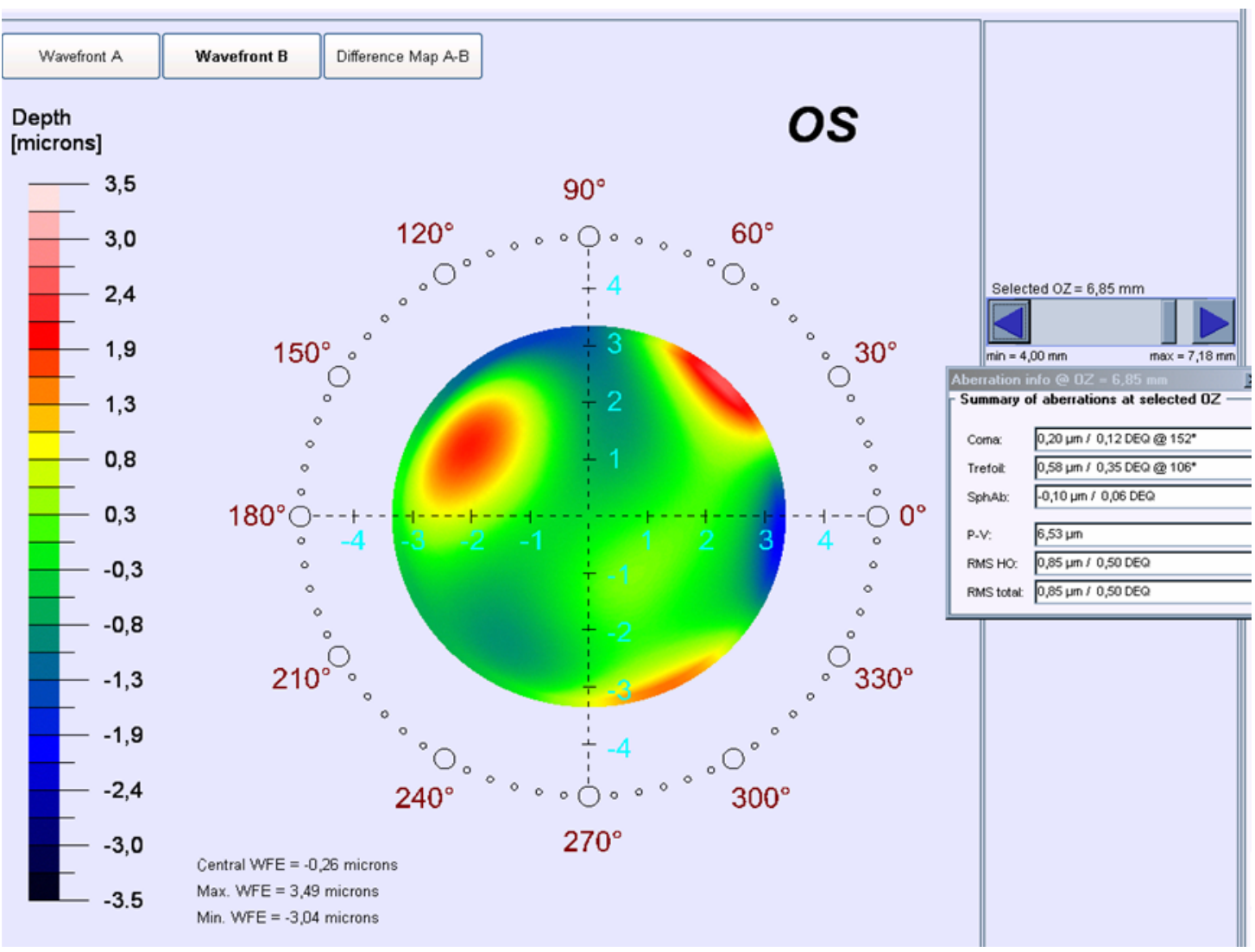

Figure 85: 3-month postoperative corneal wavefront map of the patient K.S. determining a functional optical zone (with threshold of $0.50 \mathrm{D}$ ) of $5.85 \mathrm{~mm} \varnothing$

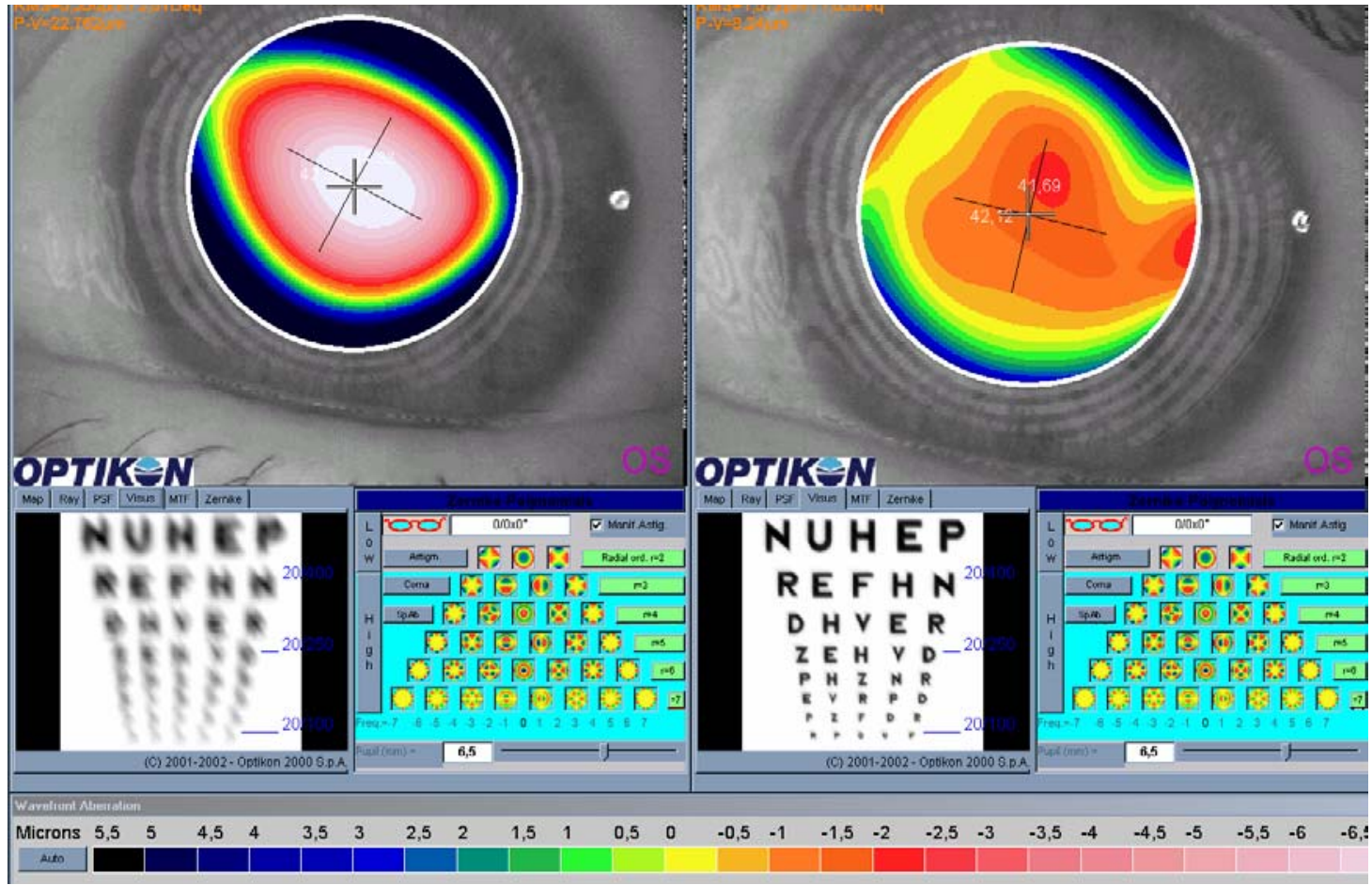

Figure 86: Comparative corneal wavefront maps of the patient K.S. simulating UCVA conditions 


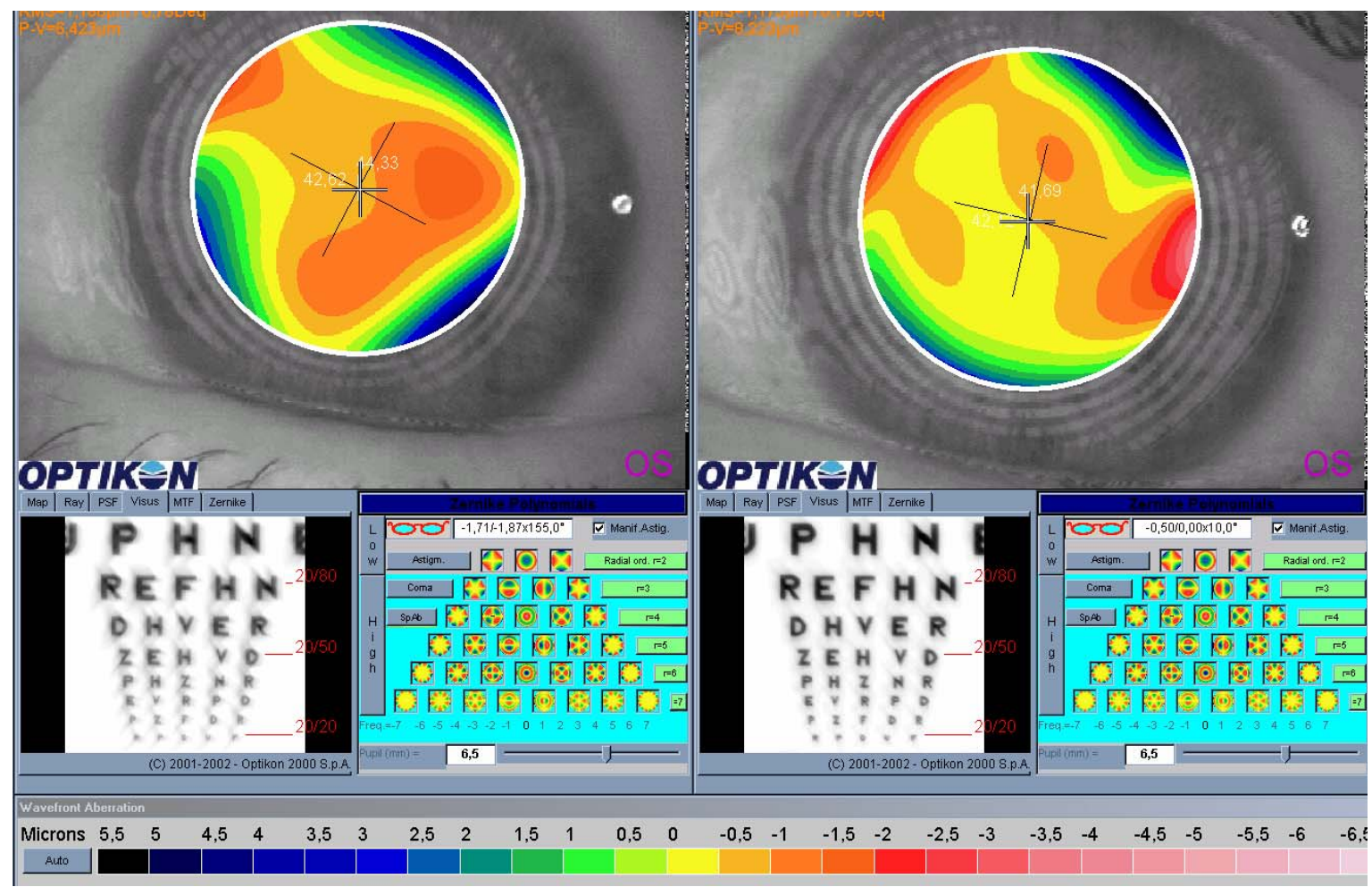

Figure 87: Comparative corneal wavefront maps of the patient K.S. simulating UCVA conditions

Comparative corneal wavefront maps at a 6.5-mm diameter, including the estimation of the optical effect of the wave aberration, show clear postoperative reductions in the corneal wave aberration as well as definite improvements in the optical simulation of UDVA and CDVA compared to the preoperative baseline levels.

CW group consisted of 30 eyes, 19 males and 11 females, 16 eyes OD and 14 eyes OS, with average age of 24 years old (18 to 35 years). Preoperative spherical equivalent (SEq) averaged $-2.80 \pm 1.25 \mathrm{D}(-5.50$ to $-1.00 \mathrm{D})$ and astigmatism $0.98 \pm 0.84 \mathrm{D}(0.00$ to $3.75 \mathrm{D})$. MD group consisted of 30 eyes, 14 males and 16 females, 17 eyes OD and 13 eyes OS, with average age of 29 years old (20 to 47 years old). Preoperative SEq averaged $-3.15 \pm 1.57 \mathrm{D}(-6.38$ to 
$-0.75 \mathrm{D})$ and preoperative astigmatism averaged $0.77 \pm 0.54 \mathrm{D}(0.00$ to $2.50 \mathrm{D})$. MV group consisted of 30 eyes, 17 males and 13 females, 12 eyes OD and 18 eyes OS, with average age of 27 years old (18 to 47 years old). Preoperative SEq averaged $-2.90 \pm 1.44 \mathrm{D}(-6.88$ to $-0.75 \mathrm{D})$ and preoperative astigmatism averaged $1.05 \pm 0.86 \mathrm{D}(0.25$ to $4.25 \mathrm{D})$. The differences between groups were not statistically significant (ANOVA test, $p=.1$ for SEq; ANOVA test, $p=.1$ for Astigmatism).

\section{Metric}

CW group

MD group

MV group

\begin{tabular}{|c|c|c|c|}
\hline Eyes (number) & 30 & 30 & 30 \\
\hline Age (years) & 24 & 29 & 27 \\
\hline (mean, range) & 18 to 35 & 20 to 47 & 18 to 47 \\
\hline Gender (Male / Female) & $19 / 11$ & $14 / 16$ & $17 / 13$ \\
\hline Eye (OD / OS) & $16 / 14$ & $17 / 13$ & $12 / 18$ \\
\hline Pre-op SEq (D) & $-2.80 \pm 1.25$ & $-3.15 \pm 1.57$ & $-2.90 \pm 1.44$ \\
\hline (mean \pm stddev, range) & -5.50 to -1.00 & -6.38 to -0.75 & -6.88 to -0.75 \\
\hline Post-op SEq (D) & $-0.22 \pm 0.23$ & $-0.22 \pm 0.23$ & $-0.26 \pm 0.21$ \\
\hline (mean \pm stddev, range) & -0.75 to +0.38 & -0.75 to +0.25 & -0.62 to +0.13 \\
\hline Pre-op Cyl (D) & $0.98 \pm 0.84$ & $0.77 \pm 0.54$ & $1.05 \pm 0.86$ \\
\hline (mean \pm stddev, range) & 0.00 to 3.75 & 0.00 to 2.50 & 0.25 to 4.25 \\
\hline Post-op Cyl (D) & $0.19 \pm 0.19$ & $0.16 \pm 0.19$ & $0.24 \pm 0.25$ \\
\hline (mean \pm stddev, range) & 0.00 to 0.50 & $0: 00$ to 0.50 & 0.00 to 0.75 \\
\hline
\end{tabular}

Table 24: Demographic data, preoperative and postoperative data for the three groups 
Mean residual SEq at 3-month follow-up was $-0.22 \pm 0.23 \mathrm{D}(-0.75$ to +0.38 $D$ ) in CW group (paired $t$-test, $p<.0001),-0.22 \pm 0.23 \mathrm{D}(-0.75$ to $+0.25 \mathrm{D})$ in MD group (paired $t$-test, $p<.0001)$, and $-0.26 \pm 0.21 \mathrm{D}(-0.62$ to $+0.13 \mathrm{D})$ in MV group (paired $t$-test, $\mathrm{p}<.0001$ ).

Mean residual astigmatism at 3-month follow-up was $0.19 \pm 0.19 \mathrm{D}(0.00$ to $0.50 \mathrm{D}$ ) in CW group (paired $t$-test, $p<.0001), 0.16 \pm 0.19 \mathrm{D}(0.00$ to $0.50 \mathrm{D})$ in $\mathrm{MD}$ group (paired $t$-test, $p<.0001$ ), and $0.24 \pm 0.25 \mathrm{D}(0.00$ to $0.75 \mathrm{D})$ in $\mathrm{MV}$ group (paired $t$-test, $p<.0001$ ).

The differences between groups were not statistically significant (ANOVA test, $p=.3$ for SEq; ANOVA test, $p=.1$ for Astigmatism).

In terms of predictability, $93 \%$ of the treatments in CW group, $93 \%$ in MD group and $100 \%$ in MV group were within $0.50 \mathrm{D}$ of SEq postoperatively (Figure 88). The difference favouring the MV group was not statistically significant (ANOVA test, $p=.3$ ).

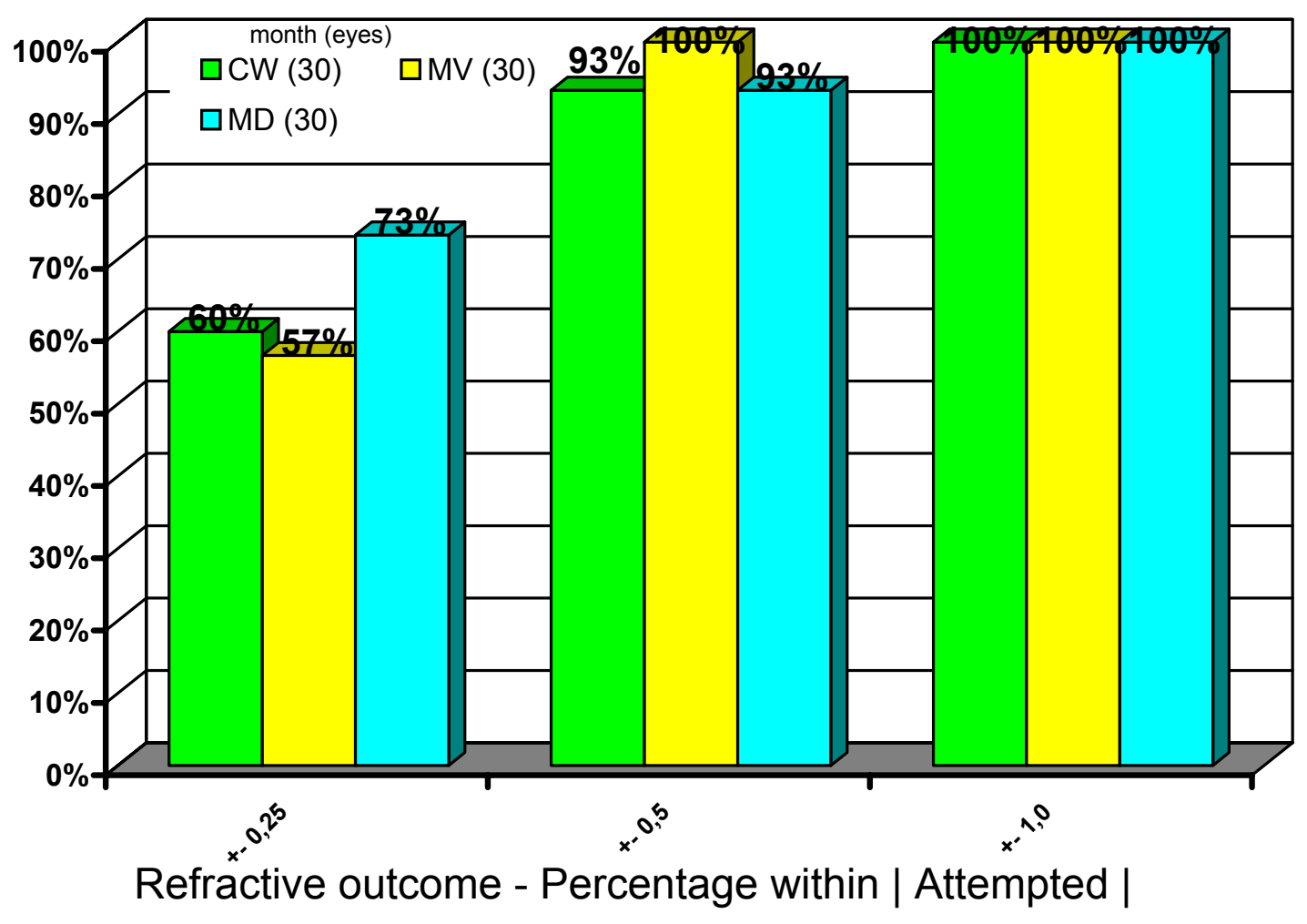

Figure 88: Comparison of the refractive outcome in SEq for CW group (green bars), MD group (blue bars), and MV group (yellow bars) 
Achieved correction was significantly correlated with intended correction for SEq for all three groups $\left(r^{2}=.97, p<.0001\right.$, slope of 1.00 for $C W ; r^{2}=.98, p<.0001$, slope of 0.95 for MD; $r^{2}=.98, p<.0001$, slope of 0.99 for MV, Figure 89).

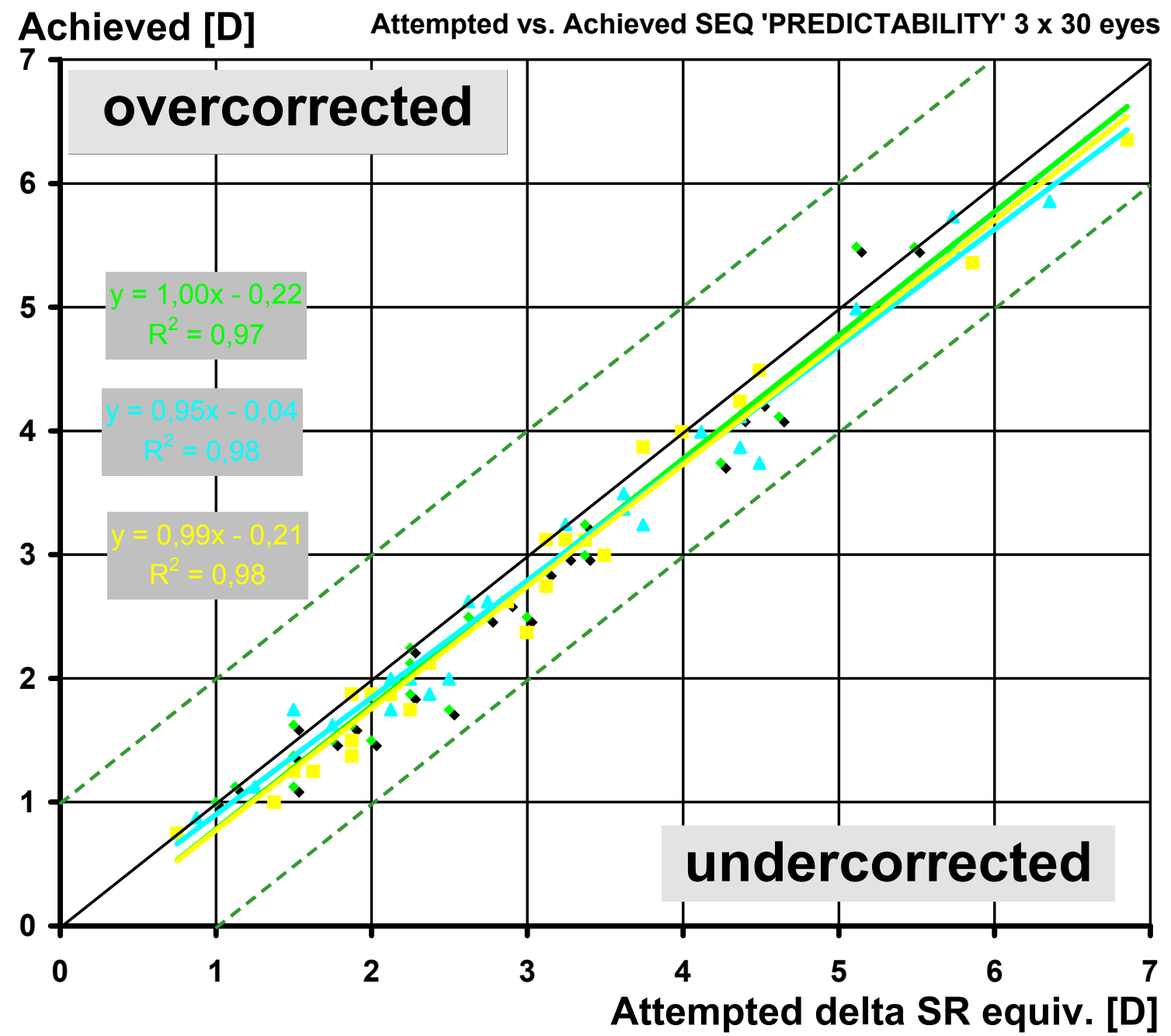

Figure 89: Comparison of the predictability scattergram for SEq for CW group (green diamonds), MD group (blue triangles), and MV group (yellow squares)

Achieved astigmatic correction was significantly correlated with intended astigmatic correction for all three groups (correlation test, $r^{2}=.94, p<.0001$, slope of 0.88 for $\mathrm{CW}$; correlation test, $\mathrm{r}^{2}=.91, \mathrm{p}<.0001$, slope of 0.94 for $\mathrm{MD}$; correlation test, $r^{2}=.92, p<.0001$, slope of 0.95 for MV, Figure 90 ). 


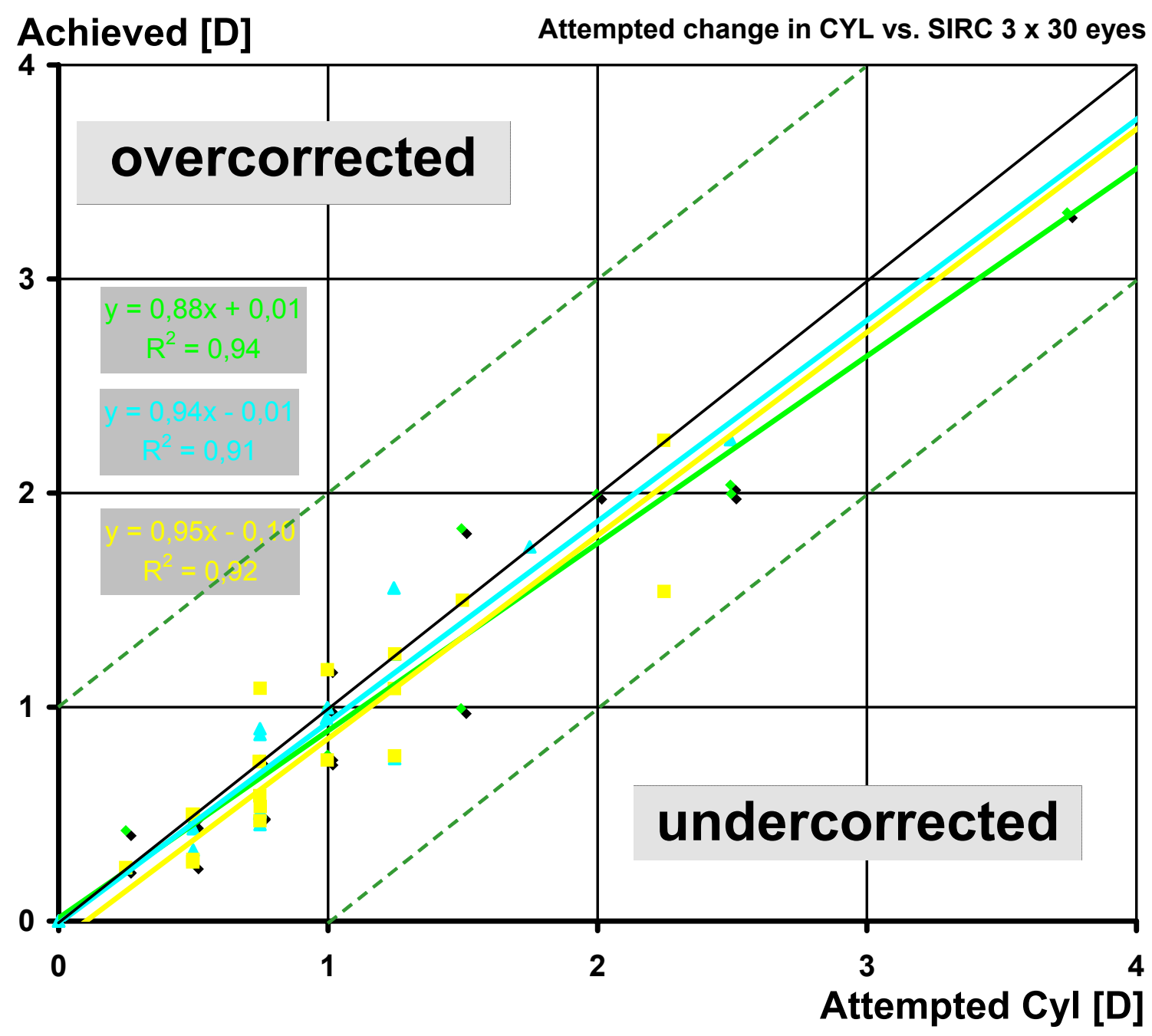

Figure 90: Comparison of the predictability for astigmatism for $\mathrm{CW}$ group (green diamonds), MD group (blue triangles), and MV group (yellow squares)

In terms of safety, $40 \%$ of treatments in CW group (paired $t$-test, $p<.05$ ), $34 \%$ in MD group (paired $t$-test, $p<.05$ ) and $47 \%$ in MV group (paired $t$-test, $p<.01$ ) gained at least one line of BSCVA postoperatively, and no single eye lost even one line of BSCVA (Figure 91). The difference favouring the MV group was not statistically significant (ANOVA test, $p=.2$ ). 


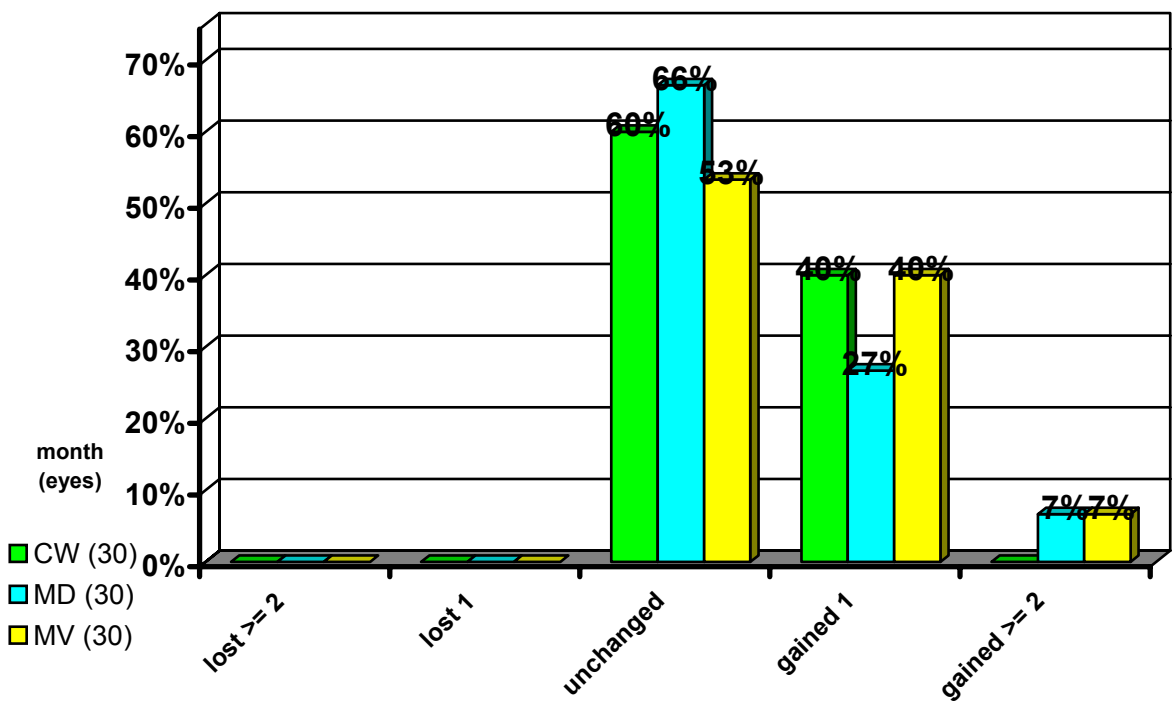

Change in BSCVA - Percentage 'SAFETY'

Figure 91: Comparison of the change in BSCVA (Safety) for CW group (green bars), MD group (blue bars), and MV group (yellow bars)

In terms of contrast sensitivity, results were very similar between all three groups and differences were not statistically significant (paired $t$-tests, $p=.1$; ANOVA test, $p=.3$, Figure 92).

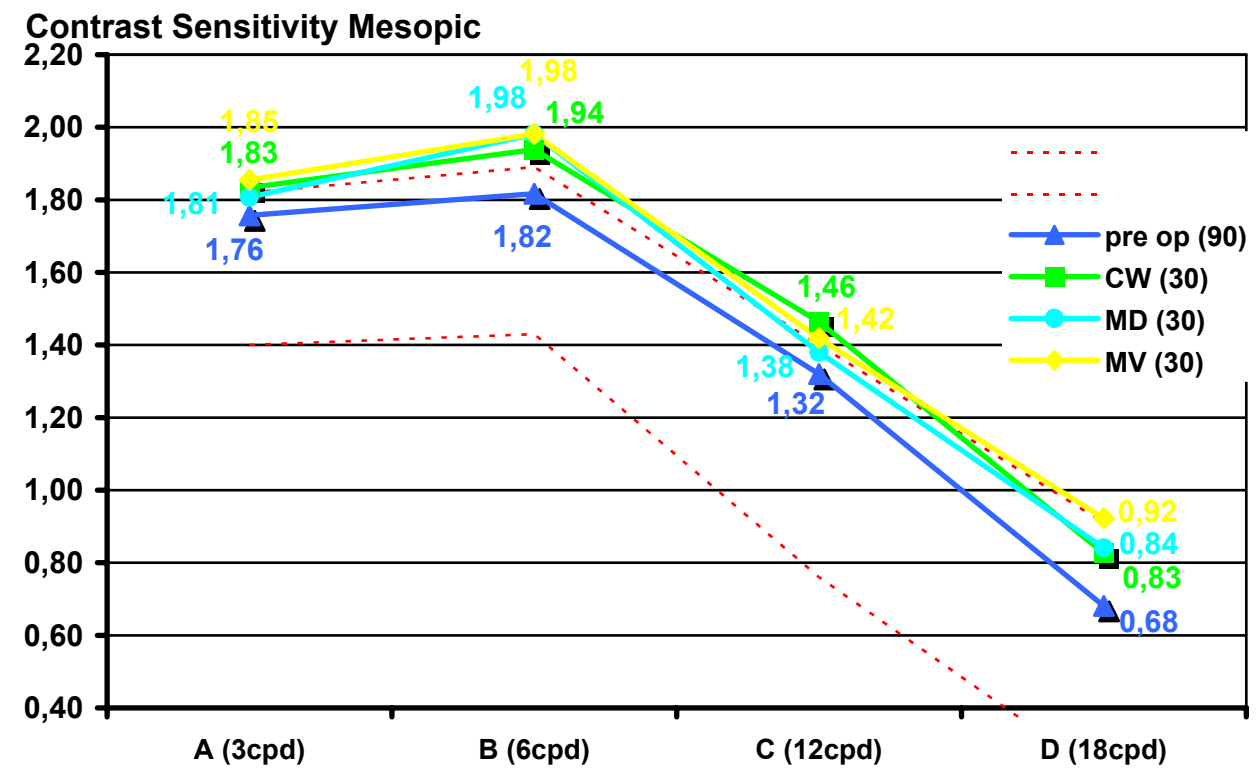

Spatial Frequency [cycles/degree]

Figure 92: Comparison of the contrast sensitivity for preoperative status (dark blue triangles), CW group (green squares), MD group (blue circles), and MV group (yellow diamonds) 
Comparative series: Evaluation of the tissue-saving capabilities for minimising the required ablation tissue

(Series comparativas: evaluación de la capacidad de ahorro de tejido para minimizar la cantidad de tejido de ablación)

Comparing the "minimise depth+" proposed ablations with equivalent ablations planned to correct the whole wavefront aberration, we observed an average difference in depth of $-8 \pm 4 \mu \mathrm{m}$ (from $-20 \mu \mathrm{m}$ to $-1 \mu \mathrm{m}$ ) or $-14 \%$ (up to $40 \%$ ), and an average difference in time of $-6 \pm 2 \mathrm{~s}$ (from $-15 \mathrm{~s}$ to $-1 \mathrm{~s}$ ) or $-16 \%$ (up to $-48 \%$ ) for "minimise depth+" corrections (Figure 93).

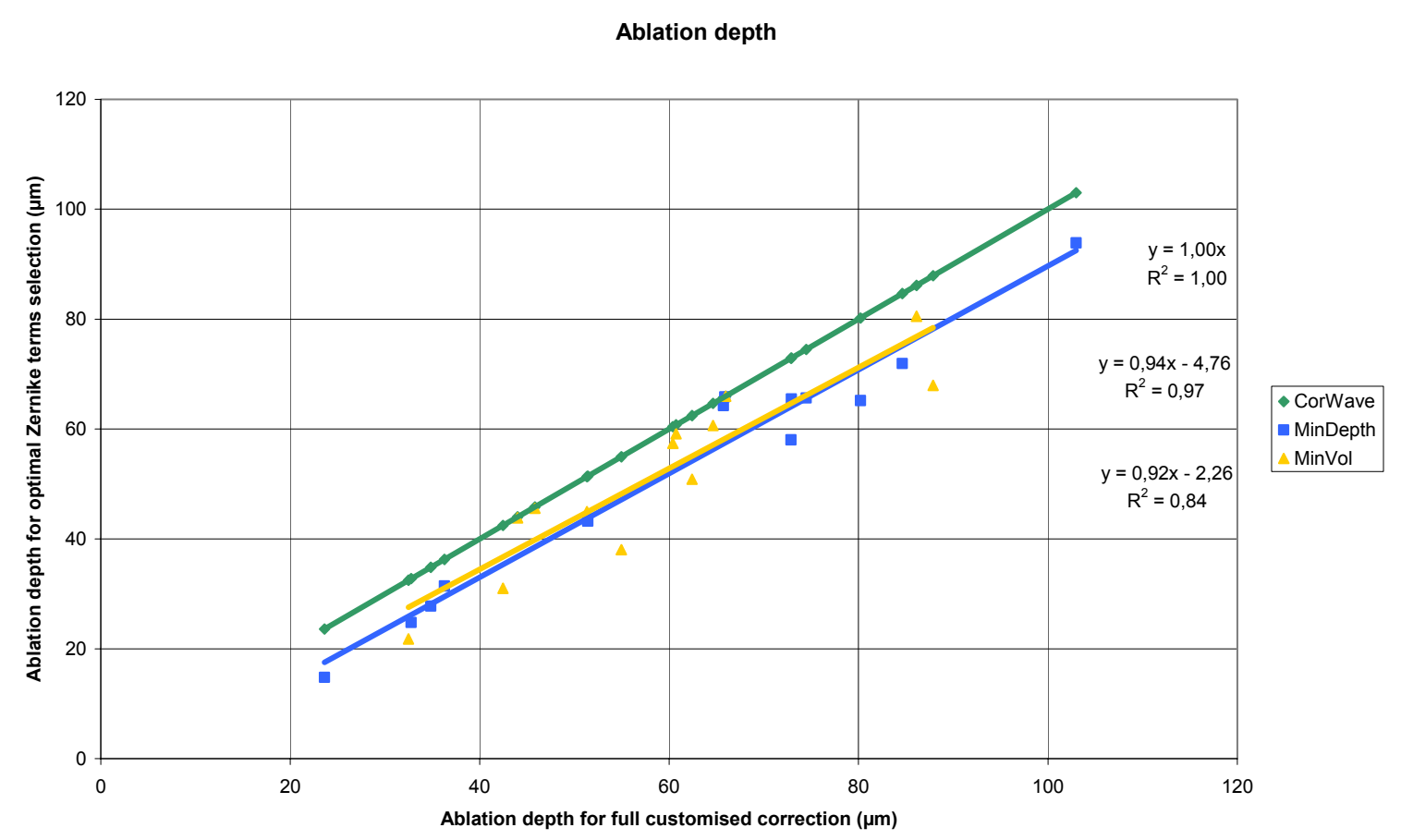

Figure 93: Comparison of the ablation depths for CW group (green diamonds), MD group (blue squares), and MV group (yellow triangles)

In $50 \%$ of the cases (15 treatments), the "minimise depth+" proposed ablations saved more than $9 \mu \mathrm{m}$ tissue, whereas in $43 \%$ of the cases (13 treatments), saved more than $7 \mathrm{~s}$ time.

In $43 \%$ of the cases (13 treatments), the "minimise depth+" proposed ablations needed less tissue than equivalent Aberration-Free ablations planned to correct only spherocylindrical refraction (Table 25). 


\begin{tabular}{lrrrr} 
& CW & MD & \multicolumn{1}{c}{ Diff } & \multicolumn{1}{c}{$\%$ Diff } \\
\hline Avg. Ablation Depth $(\mu \mathrm{m})$ & $48 \pm 32$ & $40 \pm 31$ & $-8 \pm 4$ & $-14 \%$ \\
\hline Ablation Depth range $(\mu \mathrm{m})$ & $24-103$ & $19-98$ & $-20--1$ & $-40 \%--1 \%$ \\
\hline Avg. Ablation Time $(\mathrm{s})$ & $39 \pm 20$ & $33 \pm 20$ & $-6 \pm 2$ & $-16 \%$ \\
\hline Ablation Time range $(\mathrm{s})$ & $17-76$ & $15-71$ & $-15--1$ & $-48 \%--4 \%$ \\
\hline min Depth $<$ Ab-Free $(\%)$ & --- & $43 \%(13)$ & --- & ---
\end{tabular}

Table 25: Savings in depth and time of the minimise depth approach

Comparing the "minimise volume+" proposed ablations with equivalent ablations planned to correct the whole wavefront aberration, we observed an average difference in depth of $-6 \pm 3 \mu \mathrm{m}$ (from $-20 \mu \mathrm{m}$ to $+9 \mu \mathrm{m}$ ) or $-10 \%$ (up to $45 \%$ ), and an average difference in time of $-8 \pm 2 \mathrm{~s}$ (from $-26 \mathrm{~s}$ to $-2 \mathrm{~s}$ ) or $-19 \%$ (up to $-50 \%$ ) for "minimise volume+" corrections (Figure 94 ).

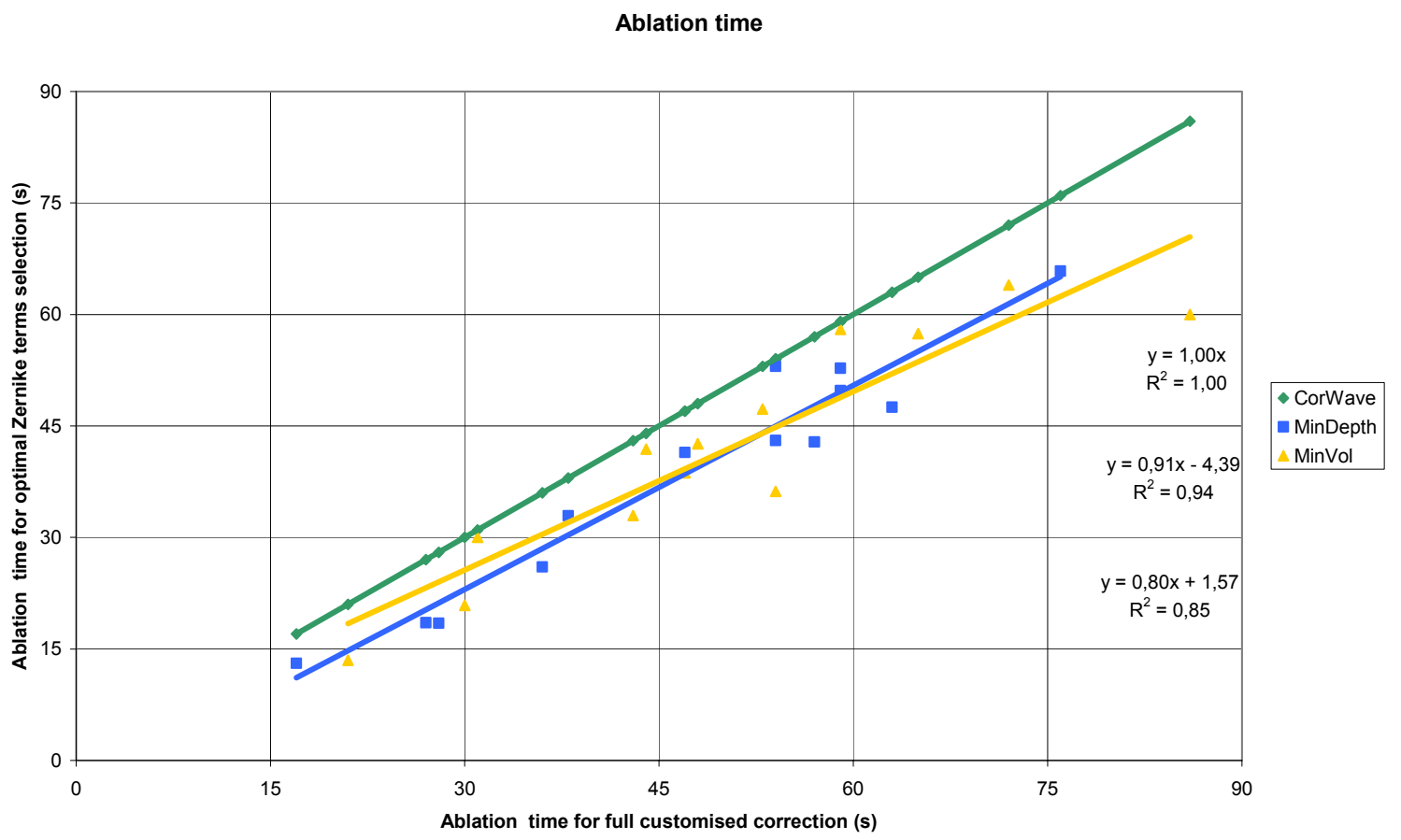

Figure 94: Comparison of the ablation times for CW group (green diamonds), MD group (blue squares), and MV group (yellow triangles) 
In $50 \%$ of the cases (15 treatments), the "minimise volume+" proposed ablations saved more than $9 \mu \mathrm{m}$ tissue, whereas in $57 \%$ of the cases (17 treatments), saved more than $7 \mathrm{~s}$ time.

In $40 \%$ of the cases (12 treatments), the "minimise volume+" proposed ablations needed shorter time than equivalent Aberration-Free ablations planned to correct only spherocylindrical refraction (Table 26).

\begin{tabular}{lccrr} 
& CW & MV & \multicolumn{1}{l}{ Diff } & \multicolumn{2}{l}{$\%$ Diff } \\
\hline Avg. Ablation Depth $(\mu \mathrm{m})$ & $48 \pm 32$ & $42 \pm 31$ & $-6 \pm 3$ & $-10 \%$ \\
\hline Ablation Depth range $(\mu \mathrm{m})$ & $32-88$ & $27-83$ & $-20-+9$ & $-45 \%-+9 \%$ \\
\hline Avg. Ablation Time $(\mathrm{s})$ & $40 \pm 20$ & $32 \pm 20$ & $-8 \pm 2$ & $-19 \%$ \\
\hline Ablation Time range $(\mathrm{s})$ & $21-86$ & $17-73$ & $-26--1$ & $-50 \%--1 \%$ \\
\hline min Vol < Ab-Free $(\%)$ & --- & $40 \%(12)$ & --- & ---
\end{tabular}

Table 26: Savings in depth and time of the minimise volume approach

\section{G.4.2.5 Comparative series: Direct comparison (Series comparativas: comparación directa)}

In group $\mathrm{A}(\mathrm{MD}$ vs. $\mathrm{CW}$ ), at 3-month follow-up, 1 patient $(7 \%)$ reported moderate levels of glare in normal light conditions in the MD treated eye, 2 patients $(13 \%)$ reported moderate levels of glare in dim light in the MD treated eye, and 1 patient $(7 \%)$ reported moderate levels of glare in dim light in both eyes. Three patients $(20 \%)$ reported the MD treated eye as their preferred eye. In total, three patients $(20 \%)$ reported some kind of disturbances.

In group B (MV vs. CW), at 3-month follow-up, 1 patient $(7 \%)$ reported minor levels of blur in normal light conditions in both eyes, and 1 patient $(7 \%)$ reported moderate levels of blur in normal light conditions in the CW treated eye. One patient $(7 \%)$ reported minor levels of glare in dim light conditions in both eyes, and three patients (20\%) reported moderate levels of glare in dim light conditions in both eyes. Two patients (13\%) reported minor levels of blur in dim light conditions in the $\mathrm{CW}$ treated eye, and one patient $(7 \%)$ reported moderate levels of blur in dim light conditions in both eyes. One patient (7\%) reported minor 
levels of shadows in dim light conditions in both eyes. Four patients $(27 \%)$ reported the MV treated eye as their preferred eye, whereas three patients $(20 \%)$ reported the CW treated eye as their preferred eye. In total, seven patients $(47 \%)$ reported some kind of disturbances.

In group C (MV vs. MD), at 3-month follow-up, 2 patients (13\%) reported minor levels of glare in normal light conditions in both eyes, 1 patient $(7 \%)$ reported minor levels of blur in normal light in the MV treated eye and moderate levels of blur in normal light in the MD treated eye, 1 patient $(7 \%)$ reported moderate levels of shadows in normal light in both eyes. 3 patients $(20 \%)$ reported minor levels of glare in dim light conditions in both eyes, 1 patient $(7 \%)$ reported minor levels of blur in dim light conditions in the MV treated eye and moderate levels of blur in dim light conditions in the MD treated eye, 1 patient (7\%) reported moderate levels of blur in dim light conditions in both eyes, 1 patient $(7 \%)$ reported moderate levels of shadows in dim light conditions in both eyes. Two patients $(13 \%)$ reported the MV treated eye as their preferred eye. In total, four patients $(27 \%)$ reported some kind of disturbances.

Overall, at the three groups, 14 patients $(31 \%)$ reported some kind of disturbances. No single patients at any group reported for any of the subjective impressions negative ratings of severe or extremely severe levels.

\section{Section G.5 DISCUSSION}

(Discusión)

G.5.1

Clinical relevance of wave aberrations

(Relevancia clínica de las aberraciones de onda)

We have used the proposed dioptric equivalent applied to each individual Zernike mode to compute its clinical relevance. It is important to bear in mind that the orientation of the vector-like modes is not taken into account in our proposal, and 1 dioptre of cardinal astigmatism (at $0^{\circ}$, for example) doesn't necessarily have the same effect as 1 dioptre of oblique astigmatism (at $45^{\circ}$, for example). Despite this, other studies have proved this assumption reasonable. ${ }^{253}$ 
Using common clinician limits, a classification, which represents the proposed objective determination of the actual clinical relevance of the single terms in a Zernike expansion of the wavefront aberration, was provided.

According to this classification, Zernike terms considered not clinically relevant $(\mathrm{DEq}<0.25 \mathrm{D})$ will be marked in green, Zernike terms that might be considered clinically relevant $(0.25 \mathrm{D}<\mathrm{DEq}<0.50 \mathrm{D})$ will be marked in yellow, and Zernike terms considered clinically relevant $(\mathrm{DEq}>0.50 \mathrm{D})$ will be marked in red.

One could use more sophisticated equations to model the equivalences between the optical blur produced by the different Zernike terms, but we have used a relatively simple approach driven primarily by the radial order.

\section{G.5.2 Minimisation of the ablated tissue (Minimización del tejido de ablación)}

Different approaches have been proposed for minimising tissue ablation in refractive surgery:

In multizonal treatments, the minimisation is based on the concept of progressively decreasing corrections in different optical zones. The problem comes from the aberrations that are induced (especially spherical aberration).

In the treatments designed having a smaller optical zone combined with bigger transition zones, the minimisation is a variation of the multizone concept. The problem comes, as well, from the aberrations that are induced (especially spherical aberration).

In the treatments designed having a smaller optical zone for the cylindrical component (or, in general, for the most powerful correction axis), the minimisation is based upon the concept of the maximal depth being based on the lowest meridional refraction and the selected optical zone, and the effective optical zone of the highest meridional refraction is reduced to match the same maximal depth. The problem comes again from the aberrations that are (especially high order astigmatism). 
In the boost-slider method, minimisation is achieved by a linear modulation of the ablated volume. The problem comes from the changes in refraction that are induced by the modulation.

In the Z-clip method, minimisation consists of defining a "saturation depth" for the ablated volume: in all those points where the ablation is designed to go deeper than the saturation value, the actual ablation depth is limited, being set to precisely that saturation value. The problem comes from the fact that this "saturation limit" may occur anywhere in the ablation area, compromising the refraction when those points are close to the ablation centre, and affecting the induction of aberrations in a complicated way.

In the Z-shift method, minimisation consists of defining a "threshold value" for the ablated volume, so that in those points where the ablated depth was designed to be less than that threshold value, no ablation is performed at all, and the rest of the points are ablated by an amount equal to the original planned ablation minus the threshold value. The problem comes from the fact that this "threshold value" may be reached anywhere in the ablation area, compromising the refraction when the below-threshold points are found close to the ablation centre, and the functional optical zone when they are found at the periphery.

The four minimisation approaches proposed in this work consists of simplifying the profile by selecting a subset of Zernike terms that minimises the necessary ablation depth or ablation volume while respecting the Zernike terms considered to be clinically relevant.

For each combination of Zernike terms, the low-order terms are recalculated using the Automatic Refraction Balance method described above, in such a way that the refractive correction is not compromised. Taking into account that the Zernike terms are planned either to be corrected or excluded, it does not compromise the visual performance because all those terms that are excluded (not planned to be corrected) are below clinical-relevance levels. The proposed approaches are safe, reliable and reproducible due to the objective foundation upon which they are based. In the same way, the selected optical zone will be used for the correction. 
It is important to remark that the selection of the Zernike terms to be included in the correction is not trivial. Only those Zernike terms considered not clinically relevant or of minor clinical relevance can be excluded from the correction, but they do not have to be necessarily excluded. Actually, individual Zernike terms considered to be not clinically relevant will only be used (or not) when they entail an extra amount of tissue for the ablation, and they will be enabled (included) when they help to save tissue for the ablation.

In this way, particular cases are represented by the full wavefront correction, by disabling all non-clinically relevant terms, or by disabling all highorder terms.

The selection process is completely automatic and driven by a computer, ensuring systematic results and a minimisation of the amount of tissue to be ablated. This automation also simplifies the foreseeable problems of manually selecting the adequate set of terms.

A criticism to this methodology can be that fact that we are not targeting diffraction-limited optical system. That means we are reducing the ablated tissue at the cost of accepting a "trade-off" in the optical quality. However, it is still not known precisely whether an "optically perfect eye" after surgery is better than preserving the aberrations that the eye had before surgery. Although the optical quality of the eye can be described in terms of the aberration of its wavefront, it was observed that those individuals with smaller aberration in their wavefront were not always those getting the best visual-quality scores. From that, the optical quality of the human eye does not determine in a one-to-one way its visual quality. The concept of neural compensation indicates that the visual quality we have is somewhat superior to the optical quality that our eye provides, because the visual system seems to be adapted to the eye's own aberration pattern.

The optical quality in an individual can be maximized for a given wavelength by cancelling the aberration of his wavefront and optimizing his defocus (for a single distance), but this has direct and dramatically negative implications for the optical quality for the rest of wavelengths (the greater the negative effect the more extreme is the wavelength). ${ }^{187}$ However, the optical quality of a person showing a certain degree of aberration of his wavefront 
decreases, relative to the maximum obtainable quality in the absence of aberration, but it has direct positive implications in the "stability" of the optical quality for a wide range of wavelengths (which covers the spectral sensitivity of the human eye).

The implications of this concept is very interesting because, for example, a patient corrected for his wave aberration represents a case in which despite having been improved his (monochromatic) optical quality in focus, his (polychromatic) visual quality is reduced. This confirms that it is not always advantageous or advisable to correct for all aberrations of an individual aspiring to obtain a monochromatically diffraction-limited optical system, as the chromatic blur would compromise his visual quality. Another positive implication that the wave aberrations may have on the visual function is that although it produces an overall blur, the wave aberration also brings depth of focus, i.e., some stability in terms of visual quality for a range of distances that can be considered simultaneously "in-focus." Lastly, moderate levels of wave aberration favour the stability of the image quality for wide visual fields. ${ }^{254}$

This way, there are at least five criteria (native aberrations, neural compensation, chromatic blur, depth of focus, wide field vision) favouring the option of leaving minor amounts of non-clinically relevant aberrations.

Besides, there are no foreseeable risks derived from the proposed minimisation functions because they propose ablation profiles that are simpler than the full-wavefront corrections.

However, some drawbacks and potential improvements may be hypothesised:

There may be a sort of "edge" problem, related to the fact that a Zernike term with $\mathrm{DEq}$ of $0.49 \mathrm{D}$ may be enabled or disabled, due to its expected minor clinical relevance, whereas a Zernike term with DEq of $0.51 \mathrm{D}$ needs to be corrected (according to our selection criteria).

It is controversial, as well, whether or not one can consider the clinical relevance of every Zernike term independently. 
The visual effect of an aberration does not only depend on it but also on the other aberrations that are present in the full pattern; for example, a sum of small, and previously considered clinically irrelevant aberrations, could involve a clear loss of overall optical quality.

A possible improvement comes from the fact that current selection strategy consists of a binary "ON/OFF" approach for each Zernike term. However, better corrections and higher amounts of tissue saving might be obtained by using a correcting factor $F[n, m]$ (range 0 to 1 ) for each Zernike correcting a wavefront of the form:

$$
A b l(\rho, \theta)=\sum_{n=0}^{\infty} \sum_{m=-n}^{+n} F_{n}^{m} C_{n}^{m} Z_{n}^{m}(\rho, \theta)
$$

However, this would come to a much higher computation cost.

Another possible improvement would be to consider possible aberration couplings, at least, between Zernike modes of the same angular frequency as a new evaluation parameter.

\section{G.5.3 Simulations \\ (Simulaciones)}

Based upon a sample population of 100 wavefront maps, the tissuessaving capabilities of this method to minimise the amount of required ablated tissue were simulated.

The wavefront maps that were used were derived only from corneal aberrations (from which defocus, for example, cannot be determined). Moreover, correcting corneal aberrations does not imply eliminating the correspondent for the eye, as it depends also on internal aberrations. However, the proposed methods try to minimize the amount of ablated tissue in a Zernike-based customized treatment irrespective of the origin of the wavefront map. 


\section{G.5.4 Clinical evaluations \\ (Evaluaciones clínicas)}

Eliminating all higher-order aberrations may not be the best strategy to optimize visual function. For example, some controlled aberrations can improve depth-of-focus with minimal degradation of image quality, as shown previously in a study of aspheric versus spherical intraocular lenses ${ }^{255}$.

The brain can adapt to long-term aberration patterns. In some cases, removing aberrations can therefore impair visual function. The importance of ocular aberrations has been addressed previously as the neural adaptation to ocular aberration ${ }^{256}$ or by the presence of high visual quality in patients with a normal amount of aberrations ${ }^{257}$.

The aim of optimization to improve overall optical quality should not be to obtain perfect corneal optics, but to fit it to internal ones ${ }^{258}$. An operational definition of the optimal optical zone can specify aberrations to be preserved as well as those to be removed.

Strictly speaking, one cannot consider the clinical relevance of every Zernike term independently without demonstrating it is exactly what occurs. The visual effect of an aberration does not only depend on it but also in the other possible aberration present; for example, a sum of small, and previously considered clinically irrelevant aberration, could suppose a clear loss of overall optical quality. The idea of approximating a distorted wavefront by an equivalent dioptric error is much controversial to be accepted without care.

Coupling effects between different high order aberration terms, and between HOAb and manifest refraction have been found ${ }^{144},{ }^{211,212}$ for example, between defocus and spherical aberration, or between third order aberrations and low order terms, between spherical aberration and coma, or between secondary and primary astigmatisms. These interactions may provide some relative visual benefits $^{213}$, but may as well contribute as sources of uncertainty in the conversion of wavefront aberration maps to refractive prescriptions ${ }^{137,139}$. 
In terms of predictability the two minimisation approaches resulted in outcomes, at least, equivalent to those of the full customised correction group (control group) with more than $57 \%$ treatments within $0.25 \mathrm{D}$ of SEq postoperatively, and $100 \%$ of the treatments within $1.00 \mathrm{D}$.

In terms of safety, the two minimisation approaches resulted in outcomes, at least, equivalent to those of the full-customised correction group (control group) with more than $34 \%$ of treatments gaining at least one line of BSCVA postoperatively, and no single eye lost even one line of BSCVA.

Contrast sensitivity confirmed the high quality of the CW treatments (with or without minimisation of ablation depth or time). The accuracy, predictability, and stability of the refractive power change, together with the minimal external impact of the CAM ablation profiles on the HOAb, lead to superior results in terms of visual acuities and improved contrast sensitivity compared with the preoperative status. This result was significantly better than previous reports, in which the postoperative contrast sensitivity decreased compared with the preoperative status, regardless of the amount of dioptres corrected ${ }^{177}$.

The standard parameters used to assess refractive surgery results (efficacy, predictability, refractive outcome, stability, and safety) were not sufficient to compare the different minimisation approaches (at least for this myopic astigmatism group).

Because the ablation procedures were performed in a physical world, they suffered from different types of unavoidable and inherent errors ${ }^{259}$ that led to aberrations $\mathrm{s}^{225}$, including biomechanical reactions due to the flap cut $^{30,260}$, blending zones, cyclotorsion ${ }^{192,215}$, centration errors ${ }^{221,223}$, spot size limitations ${ }^{164,165}$, active eye-tracking ${ }^{168,261}$ capabilities, and biomechanical reactions due to the ablation process itself $^{239}$.

Corneal wavefront customised treatments can only be successful if the preexisting aberrations are greater than the repeatability and the biological noise. Considerations such as treatment duration or tissue removal make it more difficult to establish a universal optimal profile. 
The "minimise depth" approach saved an average depth of $-8 \pm 4 \mu \mathrm{m}$ (from $20 \mu \mathrm{m}$ to $-1 \mu \mathrm{m}$ ), and an average time of $-6 \pm 2 \mathrm{~s}$ (from $-15 \mathrm{~s}$ to $-1 \mathrm{~s}$ ) or $-15 \%$ (up to $-48 \%$ ) less tissue removal and treatment duration for "minimise depth" corrections. In $43 \%$ of the cases (13 treatments), the "minimise depth" proposed ablations needed less tissue than equivalent Aberration-Free ablations planned to correct only spherocylindrical refraction.

The "minimise vol" approach saved an average depth of $-6 \pm 3 \mu \mathrm{m}$ (from -20 $\mu \mathrm{m}$ to $+9 \mu \mathrm{m}$ ), and an average time of $-8 \pm 2 \mathrm{~s}$ (from $-26 \mathrm{~s}$ to $-1 \mathrm{~s}$ ) or $-14 \%$ (up to $50 \%$ ) less tissue removal and treatment duration for "minimise vol" corrections. In $40 \%$ of the cases (12 treatments), the "minimise vol" proposed ablations needed shorter time than equivalent Aberration-Free ablations planned to correct only spherocylindrical refraction.

In this comparison, we have analysed the results splitted only by the minimisation approach used for the planning (full customised correction, minimize depth, minimize volume).

In direct comparison, we have used the three groups $(A, B, C)$ in a lateral/contralateral eye basis. This way, the variability of external uncontrollable effects (like flap cut, corneal response to the ablation, repeatability of the instruments, and cooperation of the patients) is kept to a minimum.

In all three direct comparison groups, the subjective questionnaire led to very similar results.

In group A (MD vs. CW), 3 patients $(20 \%)$ reported the MD treated eye as their preferred eye; in group B (MV vs. CW), 4 patients (27\%) reported the MV treated eye as their preferred eye, whereas 3 patients $(20 \%)$ reported the CW treated eye as their preferred eye; and in group C (MV vs. MD), 2 patients (13\%) reported the MV treated eye as their preferred eye.

The three groups compared here (CW for full customised correction, MD for minimising ablation depth, and $\mathrm{MV}$ for minimising ablation time) are predictable, safe, stable and accurate. 
The minimization techniques compared here can be used to reduce the depth and time needed for the ablation, and they effectively reduced ablation depth and time by up to a maximum of $50 \%$, and by $15 \%$ in average.

As per design, the MD group was actually optimised for minimum ablation depth, and showed the largest savings at this aim $(-8 \pm 4 \mu \mathrm{m}$, from $-20 \mu \mathrm{m}$ to -1 $\mu \mathrm{m})$, whereas the MV group was actually optimised for minimum ablation volume (time), and showed the largest savings at this aim $(-8 \pm 2 \mathrm{~s}$, from $-26 \mathrm{~s}$ to $-1 \mathrm{~s})$.

In this context, and as a rule-of-thumb, MD minimisation could be used in customised myopic treatments when reducing ablation depth is directly related to a decreased risk of keratectasia, whereas MV minimisation could be used in long customised treatments when reducing ablation time is directly related to a better maintenance of homogeneous corneal conditions.

This reduction in ablation depth and time, by correcting only a subset of the measured Zernike terms, did not affect negatively the clinical outcomes postoperatively. The two minimization techniques compared here yielded results equivalent to those of the full customization group.

\section{Section G.6 CONCLUSIONS}

(Conclusiones)

In this study, a method to objectively determine the actual clinical relevance of the single terms in a Zernike expansion of the wavefront aberration was described.

DEq $<0.25 \mathrm{D}$ determines that the considered Zernike term is not expected to be clinically relevant.

$0.25 \mathrm{D}<\mathrm{DEq}<0.50 \mathrm{D}$ determines that the considered Zernike term might be clinically relevant.

$\mathrm{DEq}>0.50 \mathrm{D}$ determines that the considered Zernike term is expected to be clinically relevant. 
A method to objectively minimise the maximum depth or the ablated volume of a customised ablation based on the Zernike expansion of the wavefront aberration was also provided within the frame of this work.

Based upon a sample population of 100 wavefront maps, the tissue-saving capabilities of such methods for minimising the required ablation tissue were simulated.

Finally, based upon a sample population of 90 treatments, the clinical application of such methods for minimising the required ablation tissue was evaluated.

Minimising the amount of ablated tissue in refractive surgery will still yield, for most of the cases, visual, optical, and refractive benefits comparable to the results obtained when compensating for the full wavefront aberration in refractive surgery. However, a marginal improved level of safety can be achieved under certain circumstances.

\section{Section G.7 OUTLOOK}

(Perspectiva)

In this study, we have used corneal wave aberration as a basis for the simulations and clinical evaluations. We have learnt that combination of subclinical Zernike terms determine the capabilities for saving tissue in an effective way. It is known, that ocular wave aberration use to show lower weighted coefficients. In further studies, the tissue savings based upon ocular wave aberration way will be explored, and compared with corneal wavefront ones.

This chapter was limited to a laser system (and ablation algorithm). However, both the laser platforms and the algorithms that they incorporate have evolved over the last years. In further studies, newer state-of-the-art laser systems and algorithms will be evaluated for tissue savings.

The clinical evaluations in this chapter were limited to correct the subjects' manifest refractions. In further studies, systematic deviations from the measured manifest refractions combined with the tissue saving algorithms will be evaluated. 


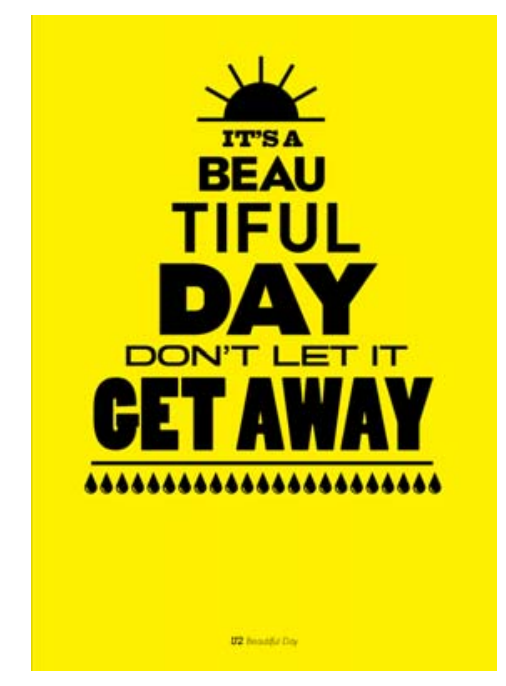

\section{CONCLUSIONS}

This thesis addresses physical aspects related to laser refractive surgery applied to the cornea to change its refractive state and consequently, to change the optical properties of the eye. In particular, this thesis has focused on better understanding corneal laser ablation mechanisms (and the changes induced on the cornea) and developing potential improvements to better control the ablative process. We have studied the changes induced in the geometry of the corneal surface, and the optical outcomes focused on optical aberrations. Measurements on real patients have allowed us to assess the influence and efficacy of the proposed improvements in a real clinical setting.

Laser corneal refractive surgery optimized with the improvements presented here helped reducing the complications and occurrence of adverse events during and after refractive surgery, improving the postoperative quality of vision, as well as reducing the ratio of retreatments and reoperations.

This dissertation demonstrated an improved application of laser cornea refractive surgical treatments by properly compensating the loss of ablation efficiency for non-normal incidences, enhancing the systems to track eye movements and optimising ablation profiles for customised refractive surgery. 
Methods to reduce the ablated tissue thickness (and, to a minor degree, to reduce the intervention time) have been proposed, evaluated and discussed.

The results and improvements derived out of this work have been implemented to the AMARIS laser system for corneal refractive surgery, as well as to the algorithms and computer programmes, which control and monitor the ablation procedures. 
WHENYOU

GOT

NOTHING

LOSE

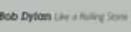

\section{ACHIEVED GOALS AND SPECIFIC CONCLUSIONS}

A. We have analysed the corneal asphericity using corneal wavefront and topographic meridional fits.

This study suggested that corneal wavefront alone is a useful metric to evaluate the optical quality of an ablation in refractive surgery, and a useful metric to evaluate corneal asphericity. Corneal wavefront can be used effectively to analyze laser refractive surgery, avoiding complicated non-linear effects in the analysis. On these grounds, this method has the potential to replace or perhaps supplement currently used methods of asphericity analysis based on simple averaging of asphericity values.

Corneal asphericity calculated from corneal wavefront aberrations represents a three-dimensional fit of the corneal surface; asphericity calculated from the main topographic meridians represents a two-dimensional fit of the principal corneal meridians. Postoperative corneal asphericity can be calculated from corneal wavefront aberrations with higher fidelity than from corneal topography of the principal meridians. Hyperopic treatments showed a greater accuracy than myopic treatments. 
B. We have provided a model of an aberration-free profile and evaluated the impact of treatments based upon these theoretical profiles in the post-operative cornea.

"Aberration-free" patterns for refractive surgery as defined here together with consideration of other sources of aberrations such as blending zones, eyetracking, and corneal biomechanics yielded results comparable to those of customisation approaches. CV-centred treatments performed better in terms of induced ocular aberrations and asphericity, but both centrations were identical in terms of photopic visual acuity. Aberration-Free treatments with the SCHWIND AMARIS did not induce clinically significant aberrations, maintained the global OD-vs.-OS bilateral symmetry, as well as the bilateral symmetry between corresponding Zernike terms (which influences binocular summation). The induced corneal aberrations were less than compared with the classical profile or other publications. Having close-to-ideal profiles should improve clinical outcomes decreasing the need for nomograms, and diminishing induced aberrations after surgery.

C. We have assessed a decision tree analysis system to further optimize refractive surgery outcomes.

The desired outcome of non-wavefront-driven refractive surgery is to balance the effects on the wave-aberration, and, to provide normal eyes with perhaps the most natural unaltered quality of vision. While Ocular Wavefront treatments have the advantage of being based on Objective Refraction of the complete human eye system, whereas Corneal Wavefront treatments have the advantage of being independent from accommodation effects or light/pupil conditions; Aspheric treatments have the advantage of saving tissue, time and due to their simplicity offer better predictability.

Decision assistant wizards may further optimize refractive surgical outcomes by providing the most appropriate ablation pattern based on an eye's anamnesis, diagnosis, and visual demands. The general principles may be 
applied to other laser systems; however, specifics will depend on manufacturers' specifications.

D. We have developed a geometrical analysis of the loss of ablation efficiency at non-normal incidence.

The loss of efficiency is an effect that should be offset in commercial laser systems using sophisticated algorithms that cover most of the possible variables. Parallelly, increasingly capable, reliable, and safer laser systems with better resolution and accuracy are required. The improper use of a model that overestimates or underestimates the loss of efficiency will overestimate or underestimate its compensation and will only mask the induction of aberrations under the appearance of other sources of error.

The model introduced in this study eliminates the direct dependence on fluence and replaces it by direct considerations on the nominal spot volume and on the area illuminated by the beam, thus reducing the analysis to pure geometry of impact and providing results essentially identical to those obtained by the model by Dorronsoro-Cano-Merayo-Marcos, however, also taking into account the influence of flying spot technology, where spot spacing is small compared to the spot width and multiple spots overlap contributing to the same target point and the correction to be applied, since the corneal curvature changes during treatment, so that also the ablation efficiency varies over the treatment.

Our model provides an analytical expression for corrections of laser efficiency losses that is in good agreement with recent experimental studies, both on PMMA and corneal tissue. The model incorporates several factors that were ignored in previous analytical models and is useful in the prediction of several clinical effects reported by other authors. Furthermore, due to its analytical approach, it is valid for different laser devices used in refractive surgery.

The development of more accurate models to improve emmetropization and the correction of ocular aberrations in an important issue. We hope that this 
model will be an interesting and useful contribution to refractive surgery and will take us one step closer to this goal.

E. We have analyzed the clinical effects of pure cyclotorsional errors during refractive surgery.

We have showed that cyclotorsional errors result in residual aberrations and that with increasing cyclotorsional error there is a greater potential for inducing aberrations. Thirteen percent of eyes had over 10 degrees of calculated cyclotorsion, which predicts approximately a 35\% residual astigmatic error in these eyes. Because astigmatic error is generally the highest magnitude vectorial aberration, patients with higher levels of astigmatism are at higher risk of problems due to cyclotorsional error.

Residual aberrations resulting from cyclotorsion depend on aberrations included in the ablation and cyclotorsional error. The theoretical impact of cyclotorted ablations is smaller than decentred ablations or edge effects in coma and spherical aberrations. The results are valid within a single-failure-condition of pure cyclotorsional errors, because no other sources of aberrations are considered. The leap from the mathematical model to the real world outcome cannot be extrapolated without further study.

F. We evaluated the effective optical zone after refractive surgery.

Our results suggested that wavefront aberration could be a useful metric for the analysis of the effective optical zones of refractive treatments or for the analysis of functional optical zones of the cornea or the entire eye by setting appropriate limit values. In particular, the method of analysis of the RMS( $\triangle \mathrm{HOAb})$ seems to be a rigorous analysis accounting for any deviation from the attempted target for the wavefront aberration. 
$\mathrm{EOZ}_{\Delta \mathrm{RMSho}}$ and $\mathrm{EOZ}_{\Delta S \text { phAb }}$ were similar to $\mathrm{POZ}$, whereas $\mathrm{EOZ}_{\mathrm{RMS}(\triangle \mathrm{HOAb})}$ was significantly smaller. Differences between EOZ and POZ were larger for smaller POZ or larger Defocus corrections. POZ larger than 6.75-mm result in EOZ, at least, as large as POZ. For OZ smaller than $6.75-\mathrm{mm}$, a nomogram for OZ could be applied.

G. We have developed a method to objectively minimise the ablated tissue of a customised ablation based on the Zernike expansion of the wavefront aberration.

Based upon a sample population of 100 wavefront maps, the tissue-saving capabilities of such methods for minimising the required ablation tissue were simulated. Finally, based upon a sample population of 90 treatments, the clinical application of such methods for minimising the required ablation tissue was evaluated.

Minimising the amount of ablated tissue in refractive surgery will still yield, for most of the cases, visual, optical, and refractive benefits comparable to the results obtained when compensating for the full wavefront aberration in refractive surgery. However, a marginal improved level of safety can be achieved under certain circumstances.

Even though Zernike modes decomposition is a mathematical description of the aberration, it is not the aberration itself. Not all Zernike modes affect the optical quality in the same way. The eye does not see through Zernike decomposition but with its own aberration pattern. However, it seems feasible to efficiently perform laser corneal refractive surgery in a customized form minimising the amount of ablated tissue without compromising the visual quality. Eliminating all higher order aberrations may not optimize visual function in highly aberrated eyes. The new algorithm effectively reduced depth and time needed for ablation (up to a maximum of $50 \%$, and by $15 \%$ in average), without negatively affecting clinical outcomes postoperatively, yielding results equivalent to those of the full customization group. 



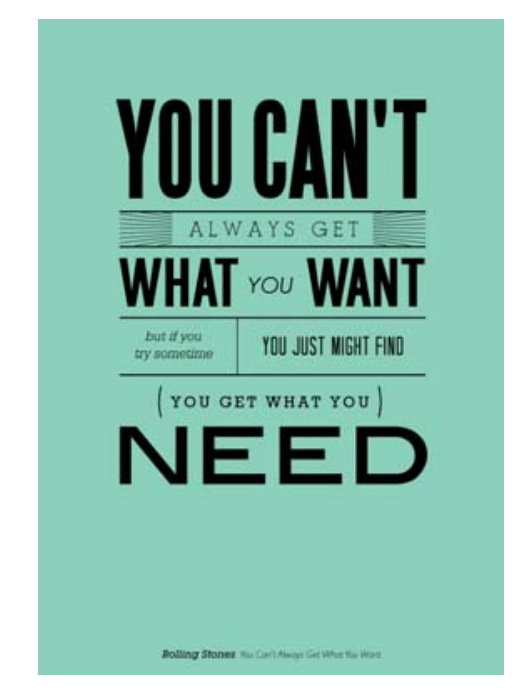

\section{LIST OF METHODOLOGICAL OUTPUTS}

A1. Method to calculate the corneal asphericity from the corneal wavefront.

A2. Method to predict the postoperative corneal asphericity from the corneal wavefront and the refractive correction.

B1. Definition of an aberration-free profile in mathematical terms.

B2. Method for mathematic compensation of the focus shift due to tissue removal.

B3. Proposal for a geometric reference (CV) used as ablation centre.

B4. Methods for evaluation of the bilateral symmetry between eyes.

C1. Proposal for selecting the most appropriate ablation profile for each specific surgical treatment based upon the corneal and ocular wave aberrations. 
D1. Methods to calculate geometry of the ablative spots.

D2. Method to analyze the loss of ablation efficiency at non-normal incidence.

E1. Method to determine the cyclotorsional error during refractive surgery.

E2. Method to determine the residual aberration due to cyclotorsional errors.

E3. Method to determine an optical benefit.

E4. Method to determine a visual benefit.

E5. Method to determine an absolute benefit.

F1. Methods to evaluate the optical zone after refractive surgery.

F2. Methods to determine dependencies among effective optical zone, planned optical zone, and refractive correction.

F3. Method to determine isometric optical zones.

F4. Method to determine a nomogram for the optical zone based upon planned optical zone and refractive correction. 
G1. Methods to objectively minimise the maximum ablation depth of a customised ablation based on the Zernike expansion of the wavefront aberration.

G2. Methods to objectively minimise the ablation volume of a customised ablation based on the Zernike expansion of the wavefront aberration. 



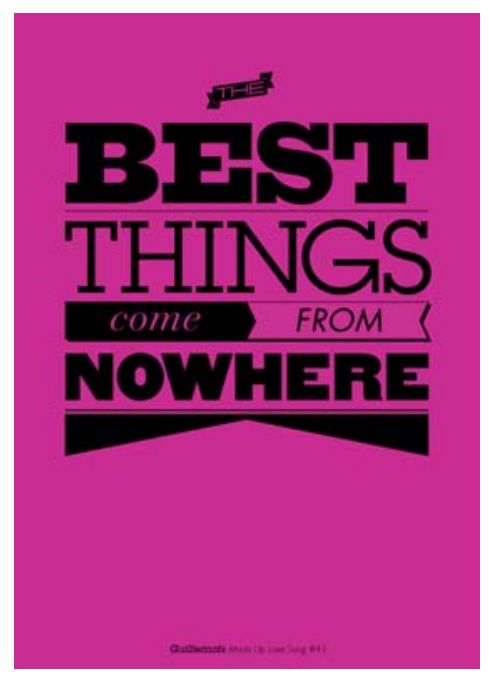

\section{IMPLICATIONS OF THIS RESEARCH}

1. The results reported in this thesis are of direct application in laser refractive surgery. The induction of aberrations is still a problem in today's LASIK surgery. We have demonstrated that most of the increase in aberrations can be explained by purely physical factors. We have obtained theoretical laser efficiency correction factors, which have been already applied in the ablation profiles of the SCHWIND AMARIS.

2. The results reported in this thesis demonstrate the great value of aberrometry (corneal and ocular) in the clinical practice. Similar protocols based on those followed in this thesis can be established to help identifying the most suitable ablation profile to each individual patient.

3. The results reported in this thesis on Aberration-Free profiles and Decision-Tree Analyses have important implications for the selection of ablative corrections or intraocular lenses. Providing the eye with the best visual performance is an extremely complex problem. We have provided evidence that not only the aberrations of individual eyes, but also the technical limitations of the correcting systems determine (and very often compromise) the final outcomes. Optical factors must be considered as well as effects related to neural adaptation. 



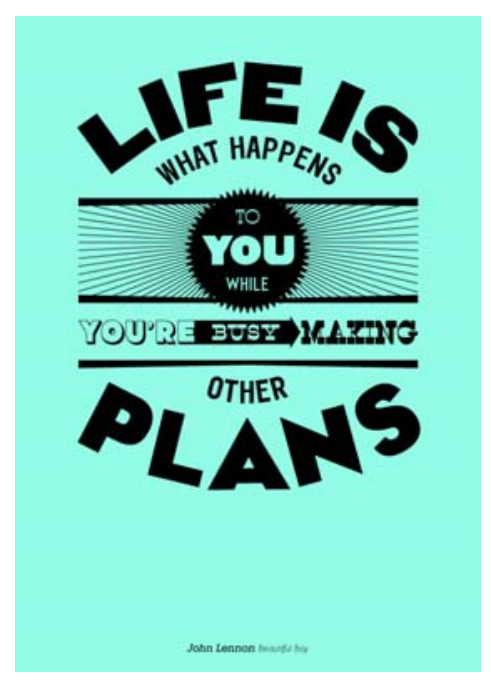

\section{FUTURE RESEARCH LINES}

The results can establish the basis for future research line directions:

A. In this study we have used corneal wave aberration as a basis for the determination of corneal asphericity. However, as the OSA recommends, corneal wave aberration was based on the line of sight. Thus, larger offsets between pupil centre and corneal vertex may have negatively affected the power of the correlations. In further studies, we will include the offsets between pupil centre and corneal vertex for improving the accuracy of the method.

This chapter was limited to a laser system (and ablation algorithm). However, both the laser platforms and the algorithms that they incorporate have evolved over the last years. In further studies, newer state-of-the-art laser systems and algorithms will be evaluated as well.

B. In this study we have used aberration-free profiles as a basis for the simulations and clinical evaluations. We have learnt that aberration-free profiles may reduce the induction of aberrations below clinically relevant values. Since we are confident that on these grounds, induction of aberrations can be 
controlled, in further studies, wavefront guided profiles will be explored and analyzed in a similar way.

In this chapter, we have performed clinical evaluations in moderate levels of myopia and hyperopia. We have learnt that aberration-free profiles reduce the induction of aberrations below clinically relevant values, but induce some minor levels of aberrations. In further studies, higher levels of myopia and hyperopia will be analyzed to determine, to which extent induction of aberrations remains below clinically relevant values. This chapter was limited to limit the induction of aberrations, further studies will attempt to manipulate the induction of aberrations in a controlled manner e.g. for presbyopic corrections.

C. The clinical evaluations in this chapter were limited to correct the subjects' manifest refractions. However, in highly aberrated eyes, manifest refraction may become an art, a sort of guessing around the least blurred image. In further studies, systematic deviations from the measured manifest refractions, as well as other foreseeable couplings among Zernike coefficients will be evaluated.

D. In further works, a comprehensive model to analyze the relative ablation efficiency at different materials (in particular human cornea and poly(methyl-methacrylate) (PMMA)), which directly considers applied correction, including astigmatism, as well as, laser beam characteristics and ablative spot properties will be developed, providing a method to convert the deviations in achieved ablation observed in PMMA to equivalent deviations in the cornea.

We are developing, as well, a simple simulation model to evaluate ablation algorithms and hydration changes in laser refractive surgery. The model simulates different physical effects of an entire surgical process, and the shot-byshot ablation process based on a modelled beam profile. The model considers corneal hydration, as well as environmental humidity, as well as, laser beam 
characteristics and ablative spot properties. Using pulse lists collected from actual treatments, we will simulate the gain of efficiency during the ablation process.

E. Currently a prospective method for determining intraoperative cyclotorsion has been implemented at the SCHWIND AMARIS laser system. With this new setting, we are evaluating intraoperative static and dynamic cyclotorsions, and postoperative outcomes on astigmatism and high-orderaberration, among astigmatic or aberrated eyes that underwent refractive surgery.

F. The clinical evaluation of the optical zone will be evaluated for hyperopic treatments as well as for LASIK treatments. Long-term follow-up on these eyes will help determine whether these accurate results also show improved stability compared to previous experiences.

G. In this study we have used corneal wave aberration as a basis for the simulations and clinical evaluations. We have learnt that combination of subclinical Zernike terms determine the capabilities for saving tissue in an effective way. It is known, that ocular wave aberration use to show lower weighted coefficients. In further studies, the tissue savings based upon ocular wave aberration way will be explored, and compared with corneal wavefront ones.

This chapter was limited to a laser system (and ablation algorithm). However, both the laser platforms and the algorithms that they incorporate have evolved over the last years. In further studies, newer state-of-the-art laser systems and algorithms will be evaluated for tissue savings. 



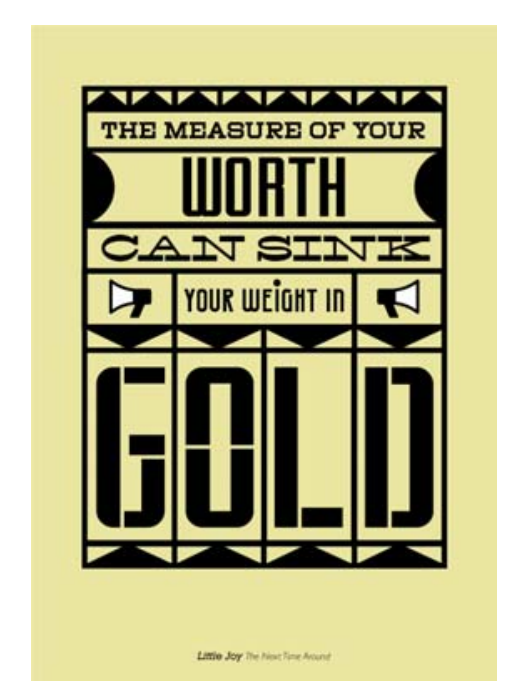

\section{LIST OF PUBLICATIONS AND PATENTS}

\section{- Peer-reviewed papers (Included as Annex)}

1. Arbelaez MC, Vidal C and Arba-Mosquera S. Six-month clinical outcomes in LASIK for high myopia with aspheric «aberration neutral» ablations using the AMARIS laser system. J Emmetropia 2010; 1: 111-116

2. Arba-Mosquera S, Hollerbach T. Ablation Resolution in Laser Corneal Refractive Surgery: The Dual Fluence Concept of the AMARIS Platform. Advances in Optical Technologies, vol. 2010, Article ID 538541, 13 pages, 2010. doi:10.1155/2010/538541

3. Arba-Mosquera S, Arbelaez MC, de Ortueta D. Laser corneal refractive surgery in the twenty-first century: a review of the impact of refractive surgery on high-order aberrations (and vice versa). Journal of Modern Optics, Volume 57 Issue 12, 1041-1074

4. de Ortueta D, Arba Mosquera S. Topographic Stability After Hyperopic LASIK. J Refract Surg 2010;26(8):547-554

5. Camellin M, Arba Mosquera S. Simultaneous aspheric wavefront-guided transepithelial photorefractive keratectomy and phototherapeutic keratectomy to correct aberrations and refractive errors after corneal surgery. J Cataract Refract Surg; 2010; 36: 1173-1180

6. Brunsmann U, Sauer U, Arba-Mosquera S, Magnago T, Triefenbach $\mathrm{N}$. Evaluation of thermal load during laser corneal refractive surgery using infrared thermography. Infrared Physics \& Technology 53 (2010) 342-347 
7. de Ortueta D, Arba Mosquera S, Häcker C. Theoretical considerations on the hyperopic shift effect observed when treating negative cylinder in laser refractive surgery. Journal of Emmetropia 2010; 1: 23-28

8. Arba Mosquera S, Merayo-Lloves J, de Ortueta D. Asphericity analysis using corneal wavefront and topographic meridional fits. J Biomed Opt; 2010; 15 (2): 028003

9. Brunsmann U, Sauer U, Dressler K, Triefenbach N, Arba Mosquera S. Minimisation of the thermal load of the ablation in high-speed laser corneal refractive surgery: the 'intelligent thermal effect control' of the AMARIS platform. J Modern Opt; 2010; 57: 466-479

10. Arba Mosquera S, Shraiki M. Analysis of the PMMA and cornea temperature rise during excimer laser ablation. J Modern Opt; 2010; 57: 400-407

11. Arbelaez MC, Vidal $C$ and Arba-Mosquera S. Bilateral Symmetry before and Six Months after Aberration-Free ${ }^{\mathrm{TM}}$ Correction with the SCHWIND AMARIS TotalTech Laser: Clinical Outcomes. J Optom 2010; 3: 20-28

12. Arba Mosquera S, de Ortueta D, Merayo-Lloves J. Tissue-Saving Zernike Terms Selection in Customized Treatments for Refractive Surgery. J Optom; 2009; 2: 182-196

13. Arbelaez MC, Vidal C, Arba Mosquera S. Clinical Outcomes of LASIK for Myopia Using the SCHWIND Platform With Ocular Wavefront Customized Ablation. J Refract Surg; 2009; 25: 1083-1090

14. Arbelaez MC, Vidal C, Al Jabri B, Arba Mosquera S. LASIK for Myopia With Aspheric "Aberration Neutral" Ablations Using the ESIRIS Laser System. J Refract Surg; 2009; 25: 991-999

15. Arbelaez MC, Vidal C, Arba Mosquera S. Excimer laser correction of moderate to high astigmatism with a non-wavefront-guided aberration-free ablation profile: Six-month results. J Cataract Refract Surg; 2009; 35: 17891798

16. Arbelaez MC, Vidal C, Arba Mosquera S. Central Ablation Depth and Postoperative Re-fraction in Excimer Laser Myopic Correction Measured With Ultrasound, Scheimpflug, and Optical Coherence Pachymetry. J Refract Surg; 2009; 25: 699-708

17. Arba Mosquera S, de Ortueta D. Analysis of optimized profiles for 'aberration-free' refractive surgery. Ophthalmic Physiol Opt;. 2009; 29: 535-548 
18. Arbelaez MC, Vidal C, Arba Mosquera S. Clinical outcomes of corneal wavefront customized ablation strategies with SCHWIND CAM in LASIK treatments. Ophthalmic Physiol Opt;. 2009; 29: 487-496

19. Arbelaez MC, Arba Mosquera S. The SCHWIND AMARIS total-tech laser as an all-rounder in refractive surgery. Middle East Afr J Ophthalmol; 2009; 16: 46-53 (Awarded "MEACO 2009 Best Paper of Session")

20. de Ortueta D, Arba Mosquera S, Baatz H. Comparison of Standard and Aberration neutral Profiles for Myopic LASIK With the SCHWIND ESIRIS Platform. J Refract Surg; 2009; 25: 339-349

21. de Ortueta $\mathrm{D}$, Arba Mosquera $\mathrm{S}$, Baatz H. Aberration-neutral Ablation Pattern in Hyperopic LASIK With the ESIRIS Laser Platform. J Refract Surg; 2009; 25: 175-184

22. Arbelaez MC, Vidal C, Arba Mosquera S. Clinical outcomes of corneal vertex versus central pupil references with aberration-free ablation strategies and LASIK. Invest Ophthalmol Vis Sci; 2008; 49: 5287-94

23. Arba-Mosquera S, Merayo-Lloves J, de Ortueta D. Clinical effects of pure cyclotorsional errors during refractive surgery. Invest Ophthalmol Vis Sci; 2008; 49: $4828-4836$

24. Arba Mosquera S, de Ortueta D. Geometrical analysis of the loss of ablation efficiency at non-normal incidence. Opt. Express; 2008; 16: 3877-3895

25. de Ortueta D, Arba Mosquera S. Mathematical properties of Asphericity: A Method to calculate with asphericities. J Refract Surg; 2008; 24: 119-121 (Letter to the Editor)

26. de Ortueta D, Arba Mosquera S. Topographic changes after hyperopic LASIK with the ESIRIS laser platform. J Refract Surg; 2008; 24: 137-144

27. de Ortueta D, Arba Mosquera S. Centration during hyperopic LASIK using the coaxial light reflex. J Refract Surg; 2007; 23: 11 (Letter to the Editor)

28. de Ortueta D, Arba Mosquera S, Magnago T. Q-factor customized ablations. J Cataract Refract Surg; 2006; 32: 1981-1982 (Letter to the Editor)

- Book chapters (Included as Annex)

1. Alió JL, Rosman M, Arba Mosquera S. Minimally invasive refractive surgery (pp. 97-123) in Minimally Invasive Ophthalmic Surgery, Springer publishers (2009)

2. de Ortueta D, Magnago T, Arba Mosquera S. Optimized Profiles for Aberration-Free Refractive Surgery in Laser Keratectomy: Approaches, Complications and Effectiveness, NOVA publishers (2009) 
3. Arba Mosquera S, Piñero D, Ortiz D, Alió JL. Customized LASIK: Aspherical Treatments with the ESIRIS Schwind platform (Chapter 40, pp. 378395) in Mastering the Techniques of Customised LASIK edited by Ashok Garg and Emanuel Rosen, Jaypee Medical International (2007)

4. Arbelaez MC, Magnago T, Arba Mosquera S. Customised LASIK: SCHWIND CAM-ESIRIS platform (Chapter 15, pp. 207-228) in Tips and Tricks in LASIK surgery edited by Shashi Kapoor and Ioannis G. Pallikaris, Jaypee Medical International (2007)

\section{- Presentations at international congresses}

1. Arbelaez MC, Arba Mosquera S. Six-month experience in 6D eye-tracking with the SCHWIND AMARIS Total-Tech Laser. Clinical Outcomes in ASCRS2010 (Free Paper; Awarded "ASCRS 2010: Best Paper of Session")

2. Arba Mosquera S. Investigar en empresas en Jornadas de Jóvenes Investigadores en Óptica Visual 2010 de la Ciencia Básica a la Transferencia Tecnológica (Invited Lecture)

3. Arbelaez MC, Arba Mosquera S. Six-month experience in aspheric correction of High-Astigmatism with the SCHWIND AMARIS TotalTech laser in ESCRS2009 (Free Paper)

4. Arba Mosquera S. Technical basis of the PresbyMAX® software: How it works, how it is created in ESCRS2009 (Invited Lecture)

5. Arbelaez MC, Arba Mosquera S. Three-month experience in customised Advanced Cyclotorsion Correction (ACC) with the SCHWIND AMARIS Total-Tech Laser. Clinical Outcomes in MEACO2009 (Free Paper; Awarded "MEACO 2009: Best Paper of Session")

6. Arba Mosquera S. Aspherical Optical Zones: The Effective Optical Zone with the SCHWIND AMARIS in SOI2008 (Invited Lecture)

7. Arbelaez MC, Arba Mosquera S. Six-Month Experience with the SCHWIND AMARIS Total-Tech Laser: Clinical Outcomes in Multicenter Experience with LASIK Treatments in ASCRS2008 (Free Paper; Awarded "ASCRS 2008 Best Paper of Session 3-H: KERATOREFRACTIVE Laser Platforms")

8. Arbelaez MC, Arba Mosquera S. Optimized Zernike Terms Selection in Customized Treatments for Laser Corneal Refractive Surgery: 3-Month Experience with LASIK Treatments in ASCRS2008 (Poster)

9. Arba Mosquera S. Representative Recent Improvements in the Perfect Refractive Package in ESOIRS2006 (Invited Lecture) 
10. Barraquer $\mathrm{C}$, Arba Mosquera S. Technical challenges that need to be overcome. SCHWIND eye-tech-solutions perspective in $7^{\text {th }}$ International Congress of Wavefront Sensing and Optimized Refractive Corrections (Invited Lecture)

- International Patents (Included as Annex)

1. Arba Mosquera S. Laser system for ablating the cornea in a patient's eye (EP2033605)

2. Hollerbach T, Grimm A, Arba Mosquera S. Laser system for ablating the cornea in a patient's eye (EP2030599)

3. Arba Mosquera S. Laser system to ablate corneal tissue of the eye of a patient (DE202008013344)

4. Arba Mosquera S, Klinner T. Method for controlling the location a laser pulse impinges the cornea of an eye during an ablation procedure (EP19335384)

5. Arba Mosquera S, Magnago T. Method for controlling a corneal laser ablation of an eye and associated system (EP1923027)

6. Grimm A, Arba Mosquera S, Klinner T. System for ablating the cornea of an eye (EP1923026) 



\section{RESÚMENES}

\section{Tema A ANÁLISIS DE LA ASFERICIDAD CORNEAL}

OBJETIVO: Evaluación de un método para calcular la asfericidad corneal y los cambios en asfericidad post cirugía refractiva.

MÉTODO: 60 ojos de 15 pacientes consecutivos miopes y 15 pacientes consecutivos hipermétropes ( $\mathrm{n}=30$ cada uno) fueron evaluados retrospectivamente. En el preoperatorio y a los tres meses postoperatorios se realizaron análisis topográficos y de la aberración frente de onda corneal mediante topografía corneal. Las ablaciones se realizaron con un láser con un perfil libre de aberraciones. Los cambios topográficos en la asfericidad de la córnea y en las aberraciones corneales se evaluaron para un diámetro de $6 \mathrm{~mm}$.

RESULTADOS: La inducción de aberración esférica corneal y los cambios de asfericidad correlacionaron con la corrección del desenfoque. Tanto pre- como postoperatoria, la asfericidad corneal calculada a partir de la asfericidad de los meridianos principales correlacionó con la asfericidad derivada del frente de onda corneal. Se obtuvo un fuerte correlación entre la asfericidad postoperatoria calculada y las predicciones teóricas calculadas a partir del frente de onda pero no de los meridianos. En tratamientos hipermétropes se obtuvo una mejor correlación que en los tratamientos de miopía.

CONCLUSIONES: La asfericidad corneal calculada a partir de las aberraciones de frente de onda corneal representa un ajuste tridimensional de la superficie corneal; mientras la asfericidad calculada a partir de los meridianos principales representa un ajuste de dos dimensiones. La asfericidad corneal postoperatoria puede ser calculada a partir de las aberraciones de frente de onda corneal con mayor fidelidad que de la topografía corneal de los meridianos principales. El método demostró una mayor exactitud en tratamientos de hipermetropía que en los tratamientos miopes. 



\section{Tema B MODELO DE UN PERFIL LIBRE DE ABERRACIÓN}

Objetivo: Proporcionar un modelo de un perfil libre de aberración y evaluar clínicamente los efectos en la córnea de tratamientos en base a estos perfiles teóricos, así como los resultados clínicos de tratamientos con los perfiles de ablación optimizados libres de aberración de las plataformas ESIRIS y AMARIS. Se incluye la comparación de resultados de la ablación centrados en el vértice corneal y el centro de pupilar, así como la comparación entre la aberración de frente de onda corneal inducida mediante perfiles de ablación asférica neutral frente a un perfil clásico basado en Munnerlyn.

Método: Los perfiles libres de aberración se dedujeron de la expansión Zernike de la diferencia entre dos óvalos cartesianos corneales. Se incorpora una compensación de los efectos de desplazamiento de foco provocados por la remoción de tejido de la córnea, mediante la preservación de la localización física del foco óptico de la superficie corneal anterior. La simulación de la eficacia quirúrgica del perfil se realizó por medio de simulación de trazado de rayos a través de una córnea descrita por su superficie anterior y paquimetría. Dos grupos clínicos (vértice corneal y centro de la pupila) con desplazamiento pupilar $>200$ micras fueron comparados. Los resultados clínicos fueron evaluados en términos de predictibilidad, resultados refractivos, seguridad, y aberración de frente de onda. La simetría bilateral OD / OS se evaluó en términos de la aberración de frente de onda corneal.

Resultados: La propuesta teórica de perfiles "libres de aberraciones" efectivamente preserva las aberraciones, y predice una asfericidad más oblata después de tratamientos miopes, y más prolata después de tratamientos hipermétropes. La inducción de aberraciones corneales en 6-mm estuvo por 
debajo de niveles clínicamente relevantes: $0.061 \pm 0.129 \mu \mathrm{m}$ de HO-RMS $(p$ $<.001$ ), $0.058 \pm 0.128 \mu \mathrm{m}$ la aberración esférica $(p<.001$ ) y $0.053 \pm 0.128 \mu \mathrm{m}$ para el coma $(p<.01)$, mientras la razón de cambio de aberraciones por dioptría de corrección fue $-0.042 \mu \mathrm{m} / \mathrm{D},-0.031 \mu \mathrm{m} / \mathrm{D}, \mathrm{y}-0.030 \mu \mathrm{m} / \mathrm{D}$ para HO-RMS, SphAb y coma respectivamente (todos $p$ <.001). Ningún otro modo de Zernike cambió de forma significativa. En $38 \%$ de los ojos CV la AV mejoró en comparación con el $24 \%$ de los ojos PC (comparación CV / PC P = 0.38). Coma ocular inducido fue en promedio 0.17 micrones para el grupo de $\mathrm{CV}$ y 0.26 micrones para el grupo de PC (comparación CV / PC P = 0.01 favoreciendo CV). La aberración esférica ocular inducida fue en promedio 0.01 micras para el grupo de CV y 0.07 micrones para el grupo de PC (comparación CV / PC P = 0.05 favoreciendo CV). En 6.0 $\mathrm{mm}$, las aberraciones corneales cambiaron en una cantidad más alta después de perfiles basados en Munnerlyn que después perfiles de aberración neutrales.

Conclusiones: Los perfiles "libres de aberración" para cirugía refractiva definidos aquí, junto con el examen de otras fuentes de aberraciones como las zonas de transición, eye-tracking, y la biomecánica corneal producen resultados comparables a los de tratamientos personalizados. Los tratamientos centrados en CV obtuvieron mejores resultados en términos de inducción de aberraciones oculares y esfericidad, pero ambos fueron idénticos en términos de agudeza visual fotópica. Los tratamientos libres de aberración con el SCHWIND AMARIS no inducen aberraciones clínicamente significativas, mantienen la simetría ODvs-OS (que influye en la visión binocular). Las aberraciones corneales fueron menos que en comparación con el perfil clásico u otras publicaciones. El uso de perfiles próximos al ideal debería mejorar los resultados clínicos disminuyendo la necesidad de nomogramas, y la disminución de las aberraciones inducidas después de la cirugía. 
Tema C ANÁLISIS POR ÁRBOL DE DECISIÓN PARA OPTIMIZAR RESULTADOS EN CIRUGÍA REFRACTIVA

OBJETIVO: Evaluar un sistema de análisis de árbol de decisiones para optimizar los resultados de la cirugía refractiva.

MÉTODO: Un árbol de decisión de 5 pasos, el Asistente de Decisión, basado en la experiencia anterior con el láser SCHWIND AMARIS, se aplicó para la selección de modos personalizados de tratamiento en cirugía refractiva (aberración asférica neutral, guiado por frente de onda corneal, o guiado por frente de onda ocular) para eliminar o reducir la aberración total.

RESULTADOS: Se realizaron 6467 tratamientos LASIK con el Asistente de Decisión durante un período de 30 meses; 5262 y 112 para tratamientos miópicos e hipermetrópicos con astigmatism, respectivamente, con los perfiles de aberración asférica neutral (AF), 560 utilizando perfiles guiadas por frente de onda corneal, y 533 utilizando perfiles guiados por frente de onda ocular. Se realizaron veinte y dos $(0.3 \%)$ retratamientos general, $18(0.3 \%)$ y $0(0 \%)$ después de astigmatismo miópico e hipermetrópico, respectivamente, con AF, 3 $(0.5 \%)$ después de perfiles guiados por frente de onda corneal, y $1(0.2 \%)$ después de perfiles guiados por frente de onda ocular.

CONCLUSIONES: Los asistentes de decisión pueden optimizar aún más los resultados quirúrgicos en cirugía refractiva proporcionando el patrón de ablación más apropiado basado en la anamnesis de un ojo, el diagnóstico y las demandas visuales. Los principios generales pueden aplicarse a otros sistemas láser, sin embargo, los detalles dependerán de las especificaciones del fabricante. 

Tema D ANÁLISIS DE LA PÉRDIDA DE EFICIENCIA DE ABLACIÓN EN INCIDENCIA NO-NORMAL

Se propone un método general para analizar de una forma geométrica la pérdida de eficacia de ablación en incidencia no normal.

El modelo considera la curvatura, la geometría del sistema, la corrección aplicada, y el astigmatismo como parámetros directos, e indirectamente las características y propiedades del haz láser de ablación.

El modelo sustituye la dependencia directa de la exposición radiante por una dependencia directa sobre el volumen nominal de tejido y por consideraciones sobre el área iluminada por el haz, lo que reduce el análisis a la geometría pura del impacto.

La pérdida de eficacia de ablación en la incidencia no normal se puede compensar a un costo relativamente bajo y así directamente mejorar la calidad de los resultados. 



\section{Tema E EFECTOS CLÍNICOS DE LOS ERRORES DE CICLOTORSIÓN DURANTE CIRUGÍA REFRACTIVA}

OBJETIVO. Describir los efectos teóricos en las aberraciones de ablaciones realizadas con ciclotorsión y determinar los límites de tolerancia de la precisión en la compensación de los errores por ciclotorsión.

MÉTODO. Hemos desarrollado un método para determinar la ciclotorsión promedio durante cirugía refractiva, sin necesidad de un tracker de ciclotorsión. Se proponen condiciones matemáticas teóricas y simuladas para determinar los beneficios ópticos, visuales y absolutos en 76 tratamientos consecutivos realizados en ojos derechos. Los resultados se evaluaron como la expansión de Zernike de las aberraciones del frente de onda residual.

RESULTADOS. Ablaciones basadas en descomposición de Zernike que sufran errores de ciclotorsión resultan en aberraciones residuales de los mismos modos de Zernike con diferentes magnitudes y orientaciones. El efecto solo depende de la frecuencia angular y no del orden radial. Se obtuvo un valor promedio de $4.39^{\circ}$ de ciclotorsión. En el $95 \%$ de los tratamientos se habría obtenido teóricamente un beneficio óptico, un beneficio visual teórico en el 95\% y un beneficio absoluto en el $93 \%$ en comparación con $89 \%, 87 \%$ y $96 \%$ de los tratamientos que alcanzaron beneficios reales, respectivamente.

CONCLUSIONES. La aberraciones residuales derivadas de errores por ciclotorsión dependen de las aberraciones incluidas y del error ciclotorsional. El impacto de ablaciones que sufren ciclotorsión es menor que el efecto de descentrajes o los efectos de borde en coma y aberraciones esféricas. Los resultados son válidos dentro de una condición de fallo único, ya que no se consideraron otras fuentes de aberraciones. El salto del modelo matemático de los resultados del mundo real no pueden extrapolarse sin más estudios. 



\section{Tema F LA ZONA ÓPTICA EFECTIVA EN CIRUGÍA REFRACTIVA}

OBJETIVO: Evaluar la Zona Óptica Efectiva (EOZ) (la parte de la ablación que recibe la corrección completa), entre ojos que se sometieron a tratamientos LASEK / Epi-LASEK para la corrección de miopía con astigmatismo.

MÉTODO: A los 6 meses de seguimiento se evaluaron retrospectivamente 20 tratamientos LASEK / Epi-LASEK con una media de $-5.49 \pm 2.35 \mathrm{D}$ de desenfoque utilizando el sistema SCHWIND AMARIS. En todos los casos se llevaron a cabo análisis pre-/post-operatorios del frente de onda corneal utilizando el Keratron-Scout (OPTIKON2000). EOZ se evaluó en función del cambio del RMS de la aberración del frente de onda corneal de orden superior

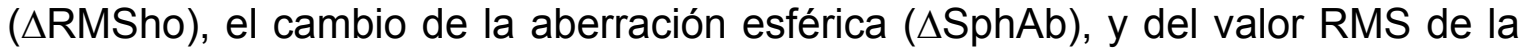
aberración del frente de onda corneal de orden superior (RMS $(\triangle \mathrm{HOAb})$ ). Las correlaciones de EOZ con la Zona Óptica Planeada (POZ) y la corrección de Desenfoque (SEQ), se analizaron utilizando una función bilineal, así como, los cálculos de las líneas isométricas (IOZ) para los que EOZ es igual a POZ y del nomograma para la Zona Óptica (NOPZ).

RESULTADOS: A los seis meses, el desenfoque fue $-0.05 \pm 0.43 \mathrm{D}$, el noventa por ciento de los ojos estaban dentro de $\pm 0.50 \mathrm{D}$ de emetropía. Después del tratamiento, la media de aumento de RMSho fue $0.12 \mu \mathrm{m}, 0.09 \mu \mathrm{m}$ para SphAb y $0.04 \mu \mathrm{m}$ para Coma (6-mm de diámetro). La POZ promedio fue de $6.76 \pm 0.25$ $\mathrm{mm}$, mientras que la EOZ promedio fue para $\triangle \mathrm{RMSho} 6.74 \pm 0.66 \mathrm{~mm}$ (correlación bilineal $\mathrm{p}<.005$ ), EOZ para $\triangle \mathrm{SphAb}$ fue $6.83 \pm 0.58 \mathrm{~mm}$ (correlación bilineal $p<.0001$ ), EOZ para RMS $(\triangle \mathrm{HOAb})$ fur $6.42 \pm 0.58 \mathrm{~mm}$ (significativamente menor, $p<.05$, correlación bilineal $p<.0005)$. EOZ correlaciona positivamente con 
POZ y disminuye de manera constante con la SEq. Un tratamiento de-5D en POZ 6.00 milímetros resulta en EOZ 5.75 milímetros (6.25 mm NPOZ), los tratamientos de POZ 6.50 milímetros resultan en cerca de 6.25 milímetros EOZ (6.75 mm NPOZ). La condición isométrica se cumple para POZ alrededor de 6.75 milímetros.

CONCLUSIONES: $\triangle$ RMSho y $\triangle$ SphAb proporcionaron EOZ similares a $\mathrm{POZ}$, mientras que EOZ para $\mathrm{RMS}(\triangle \mathrm{HOAb})$ fue significativamente menor. Las diferencias entre EOZ y POZ fueron mayores para pequeñas $\mathrm{POZ}$ o grandes correcciones. POZ mayor que $6.75 \mathrm{~mm}$ resulta en EOZ, al menos, tan grande como POZ. Para OZ menor que $6.75 \mathrm{~mm}$, se podría aplicar un nomograma para OZ. 
Tema G MÉTODO PARA REDUCIR OBJETIVAMENTE EL TEJIDO DE ABLACIÓN DE UNA TRATAMIENTOS PERSONALIZADOS BASADOS EN LA EXPANSIÓN DE ZERNIKE DE LA ABERRACIÓN DEL FRENTE DE ONDA

El propósito de este trabajo es estudiar la posibilidad de realizar cirugía refractiva personalizada reduciendo al mínimo la cantidad de tejido extirpada sin comprometer la calidad visual, así como evaluar la aplicación de estos métodos para reducir al mínimo el tejido ablación a la minimización objetivo de profundidad y el tiempo de ablaciones personalizadas.

Se desarrolló un nuevo algoritmo para la selección de un conjunto de términos de Zernike optimizado en los tratamientos personalizados para cirugía láser refractiva corneal. Sus atributos de ahorro de tejido se han simulado en 100 aberraciones de onda diferentes en $6 \mathrm{~mm}$ de diámetro. Los resultados de la simulación se evaluaron en términos de cuánta profundidad y volumen se redujo para cada condición (en micras y en porcentaje), si la corrección propuesta consiste en una corrección completa de frente de onda o de un tratamiento libre de aberración, y si la profundidad o el volumen propuesto fue menor que la requerida para el tratamiento equivalente libre de aberración. Los resultados clínicos y los atributos de ahorro de tejido fueron evaluados en dos grupos (minimizar la profundidad: MD, y minimizar el volumen: MV; 30 ojos cada uno), más un grupo control (frente de onda corneal: CW, 30 ojos). Los resultados clínicos fueron evaluados en términos de previsibilidad, seguridad y sensibilidad al contraste.

Los resultados de la simulación mostró una profundidad media ahorrada de $5 \mu \mathrm{m}(0-16 \mu \mathrm{m})$, y un volumen medio salvado de $95 \mathrm{nl}(0-127 \mathrm{nl})$ o una reducción de $11 \%$ en tejido (0-66\% ahorro de tejido). La correcciones propuestas siempre 
fueron menos profundas que las correcciones de frente de onda completo y en el $59 \%$ de los casos fueron menos profundos que los tratamientos equivalentes libres de aberraciones. En un primer caso, la ablación se redujo significativamente en un $15 \%$ en comparación con la corrección personalizada completa. La refracción se corrigió a niveles subclínicos, la agudeza visual sin corrección mejoró a 20/20, la agudeza visual mejor corregida aumentó en 2 líneas, las aberraciones se redujeron aproximadamente un $40 \%$ en comparación con los niveles basales preoperatorios, y la zona óptica funcional de la córnea se amplió en aproximadamente un $40 \%$ en comparación con los niveles basales preoperatorios. Se redujeron trébol, coma, aberración esférica, y RMS de las aberraciones de orden superior. En la evaluación clínica, el $93 \%$ de los tratamientos CW, el $93 \%$ en el grupo MD y $100 \%$ en el grupo MV se encontraron dentro de $0,50 \mathrm{D}$ de la SEq después de la operación. El $40 \%$ de los tratamientos CW, el $34 \%$ en el grupo MD y $47 \%$ en el grupo MV mejoró por lo menos una línea de AVMC después de la operación. El ahorro de tejido arrojó una reducción media de $8 \mu \mathrm{m}(1-20 \mu \mathrm{m})$ y un ahorro de tiempo de $6 \mathrm{~s}$ (1-15s) en el grupo de MD, y $6 \mu \mathrm{m}(0-20 \mu \mathrm{m})$ y el $8(2-26 \mathrm{~s})$ en el grupo MV.

A pesar de que la descomposición Zernike modos es una descripción matemática de la aberración, no es la aberración en sí misma. No todos los modos de Zernike afectan a la calidad óptica de la misma manera. El ojo no ve a través de la descomposición Zernike sino con su patrón propio de aberración. Sin embargo, parece factible de realizar con eficacia la cirugía láser refractiva corneal en una forma personalizada reduciendo al mínimo la cantidad de tejido extirpada sin comprometer la calidad visual. La eliminación de todas las aberraciones de orden superior puede no optimizar la función visual en ojos con gran aberración. El nuevo algoritmo reduce efectivamente la profundidad y el tiempo necesarios para la ablación (hasta un máximo del $50 \%$ y un $15 \%$ en promedio), sin afectar negativamente los resultados clínicos después de la operación, con resultados equivalentes a los del grupo de personalización completa. 


\section{CONCLUSIONES}

Esta tesis aborda los aspectos físicos relacionados con la cirugía refractiva con láser aplicado a la córnea para cambiar su estado refractivo y, en consecuencia, para cambiar las propiedades ópticas del ojo. En particular, esta tesis se ha centrado en una mejor comprensión de los mecanismos de ablación corneal por láser (y los cambios inducidos en la córnea) y el desarrollo de las mejoras potenciales para controlar mejor el proceso de ablación. Se han estudiado los cambios inducidos en la geometría de la superficie corneal, los resultados ópticos se describieron en forma de aberraciones ópticas. Las mediciones realizadas en pacientes reales nos han permitido evaluar la influencia y la eficacia de las mejoras propuestas en un entorno clínico real.

La cirugía refractiva corneal por laser optimizada con las mejoras que se presentan aquí ayudó a reducir las complicaciones y la aparición de eventos adversos durante y después de la cirugía refractiva, la mejora de la calidad de la visión postoperatoria, así como la reducción de la proporción de retratamientos.

Esta tesis ha demostrado una mejor aplicación de los tratamientos láser quirúrgicos refractivos en la córnea, compensando adecuadamente la pérdida de eficacia de la ablación para incidencia no normal, la mejora de los sistemas para rastrear los movimientos del ojo y la optimización de los perfiles de ablación para personalizar la cirugía refractiva.

Diferentes métodos para reducir el espeso de tejido de ablación ( $\mathrm{y}$, en menor medida, para reducir el tiempo de intervención) han sido propuestos, evaluados y discutidos.

Los resultados derivados de este trabajo se han aplicado en el sistema AMARIS para cirugía refractiva corneal, así como a los algoritmos y programas informáticos que controlan y supervisan los procedimientos de ablación. 



\title{
LOGROS ALCANZADOS Y CONCLUSIONES ESPECÍFICAS
}

\begin{abstract}
A. Se ha analizado la asfericidad corneal mediante frente de onda corneal y mediante análisis meridional.

Este estudio sugiere que el único frente de onda corneal representa una métrica útil para evaluar tanto la calidad óptica de una ablación en cirugía refractiva, como la asfericidad corneal, evitando complicados efectos no lineales en el análisis. Por estos motivos, este método tiene el potencial para reemplazar o al menos complementar los métodos de análisis de asfericidad que se utilizan actualmente basados en un promedio simple de los valores de asfericidad.
\end{abstract}

La asfericidad corneal calculada a partir de las aberraciones de frente de onda corneal representa un ajuste tridimensional de la superficie corneal; la asfericidad calcula a partir de los meridianos topográficos principales representa un ajuste de dos dimensiones de los meridianos principales corneales. La asfericidad corneal postoperatoria puede ser calculada a partir de las aberraciones de frente de onda corneal con mayor fidelidad que de la topografía corneal de los meridianos principales. En tratamientos de hipermetropía se demostró una exactitud mayor que los tratamientos miopes.

B. Se ha proporcionado un modelo de un perfil libre de aberración y evaluado el impacto de los tratamientos en base a estos perfiles teóricos en la córnea después de la operación.

La aplicación de patrones libres de aberración para cirugía refractiva como se definen aquí, junto con el examen de otras fuentes de aberraciones como las zonas de transición, eye-tracking, y la biomecánica corneal ha producido resultados comparables a los de los tratamientos personalizados. Los 
tratamientos centrados en CV obtuvieron mejores resultados en términos de la inducción de aberraciones oculares y la esfericidad, pero ambos fueron idénticos en términos de agudeza visual fotópica. Los tratamientos libres de aberración incluidos en SCHWIND AMARIS no indujeron aberraciones clínicamente significativas, se mantuvo la simetría bilateral (que influye en la visión binocular). Las aberraciones corneales fueron menores que en comparación con el perfil clásico u otras publicaciones. La existencia de perfiles próximos al ideal debería mejorar los resultados clínicos disminuyendo la necesidad de nomogramas, y la disminución de las aberraciones inducidas después de la cirugía.

C. Se ha evaluado un análisis por árbol de decisión para optimizar aún más los resultados de la cirugía refractiva.

El resultado deseado de la cirugía refractiva es proporcionar a los pacientes la calidad óptima de visión funcional. Si bien los tratamientos guiados por frente de onda ocular tienen la ventaja de estar basados en la refracción objetiva del sistema ojo humano completo, mientras que los tratamientos basados en el frente de onda corneal tienen la ventaja de ser independientes de los efectos de acomodación o de las condiciones de luz y pupila; los tratamientos asféricos tienen la ventaja de ahorrar tejido, tiempo.

El uso de asistentes de decisión puede optimizar aún más los resultados quirúrgicos de proporcionando el patrón de ablación más adecuada teniendo en la anamnesis de un ojo, el diagnóstico y las demandas visuales. Los principios generales pueden aplicarse a otros sistemas láser, sin embargo.

D. Se ha desarrollado un análisis geométrico de la pérdida de eficacia de ablación en incidencia no normal.

La pérdida de eficiencia es un efecto que debe ser compensado en los sistemas láser comerciales mediante sofisticados algoritmos para cubrir la mayoría de las variables posibles. Paralelamente, se requiren sistemas láser 
cada vez más capaces, confiables, seguros y con mejor resolución y precisión. El uso inadecuado de un modelo que sobreestime o subestime la pérdida de eficiencia, sobreestimará o subestimará su compensación y sólo enmascarará la inducción de aberraciones bajo la apariencia de otras fuentes de error.

El modelo presentado en este estudio elimina la dependencia directa de la exposición radiante y la sustituye por consideraciones del volumen nominal de tejido retirado por imacto y en la zona iluminada por el haz, lo que reduce el análisis a la geometría pura de impacto y con resultados idénticos a los obtenidos por el modelo Dorronsoro-Cano-Merayo-Marcos, sin embargo, teniendo también en cuenta la influencia de la tecnología de punto volante, donde el espaciamiento es pequeño en comparación con la anchura del impacto y múltiples impactos se superponen contribuyendo al mismo punto de ablación y la corrección aplicada, ya que los cambios en la curvatura de la córnea durante el tratamiento varían del mismo modo la eficacia de ablación a lo largo del tratamiento.

Nuestro modelo proporciona una expresión analítica para la corrección de las pérdidas de eficiencia láser que está en buen acuerdo con los recientes estudios experimentales, tanto en PMMA y en tejido corneal. El modelo incorpora varios factores que fueron ignorados en los anteriores modelos analíticos y es útil en la predicción de varios efectos clínicos reportados por otros autores. Además, debido a su enfoque analítico, es válido para diferentes dispositivos láser usados en cirugía refractiva.

El desarrollo de modelos más precisos para mejorar emetropización y la corrección de las aberraciones oculares en un tema importante. Esperamos que este modelo será un aporte interesante y útil para la cirugía refractiva y nos llevará un paso más cerca a este objetivo.

E. Se han analizado los efectos clínicos de errores por ciclotorsión durante cirugía refractiva. 
Hemos mostrado que los errores por ciclotorsión resultan aberraciones residuales y que con el aumento de error por ciclotorsión existe un mayor potencial para la inducción de aberraciones. El trece por ciento de los ojos mostró más de 10 grados de ciclotorsión, que predice aproximadamente un $35 \%$ de error residual astigmático en esos ojos. Debido a que el error astigmático es generalmente la magnitud más alta de aberración vectorial, los pacientes con niveles más altos de astigmatismo se encuentran en mayor riesgo de problemas debido a un error ciclotorsional.

Las aberraciones residuales derivadas de errores por ciclotorsión dependen de las aberraciones incluidas en la ablación y del error ciclotorsional. El impacto teórico de errores por ciclotorsión es más pequeño que el de las ablaciones descentradas o los efectos de borde en coma y aberraciones esféricas. Los resultados son válidos dentro de una condición de fallo único, porque no se consideran otras fuentes de aberraciones.

F. Se ha evaluado la zona óptica efectiva después de cirugía refractiva.

Nuestros resultados sugieren que la aberración de frente de onda puede ser una métrica útil para el análisis de las zonas ópticas efectivas o para el análisis de las zonas ópticas funcionales de la córnea o de todo el ojo mediante el establecimiento de valores límite adecuados. En particular, el método de análisis de la $\mathrm{RMS}(\triangle \mathrm{HOAb})$ parece ser un riguroso análisis de cualquier desviación de la meta tentativa para la aberración de frente de onda.

EOZ obtenidas mediante los métodos $\triangle \mathrm{RMSho} \mathrm{y} \triangle \mathrm{SphAb}$ fueron similares a POZ, mientras que con el método RMS $(\triangle \mathrm{HOAb})$ fue significativamente menor. Las diferencias entre EOZ y $\mathrm{POZ}$ fueron mayores para $\mathrm{POZ}$ pequeñas o más grandes correcciones. POZ mayor que $6.75 \mathrm{~mm}$ resulta en EOZ, al menos, tan grande como POZ. Para OZ menor que $6.75 \mathrm{~mm}$, se podría aplicar un nomograma para $\mathrm{OZ}$. 
G. Se ha desarrollado un método para minimizar objetivamente la ablación de tejido de un tratamiento personalizado basado en la expansión de Zernike de la aberración de frente de onda.

Con base a una muestra poblacional de 100 mapas de frente de onda, se simuló la capacidad de ahorro de tejido de estos métodos para reducir al mínimo la ablación del tejido requerido.

Por último, se evaluó la aplicación clínica de estos métodos para reducir al mínimo la ablación de tejido requerido en base a una muestra poblacional de 90 tratamientos.

Los métodos de reducción de tejido extirpado en cirugía refractiva demostraron beneficios visuales, ópticos y refractivos comparables a los resultados obtenidos en la compensación completa de la aberración de frente de onda en cirugía refractiva. Sin embargo, bajo ciertas circunstancias, se podría alcanzar un nivel marginal de mejora de la seguridad.

A pesar de que la descomposición en modos de Zernike es una descripción matemática de la aberración, no es aberración en sí misma. No todos los modos de Zernike afectan a la calidad óptica de la misma manera. El ojo no ve a través de la descomposición de Zernike sino con su propio patrón de aberración. Sin embargo, parece factible realizar cirugía láser refractiva corneal con eficacia en una forma personalizada reduciendo al mínimo la cantidad de tejido extirpada sin comprometer la calidad visual. La eliminación de todas las aberraciones de orden superior puede no optimizar la función visual en ojos que presenten gran aberración. El nuevo algoritmo redujo de fporma efectiva la profundidad y el tiempo necesarios para la ablación (hasta un máximo del 50\% y un $15 \%$ en promedio), sin afectar negativamente los resultados clínicos, con resultados equivalentes a los del grupo de personalización completa. 



\section{LISTA DE RESULTADOS METODOLÓGICOS}

A1. Método para el cálculo de la asfericidad corneal a partir del frente de onda corneal.

A2. Método para predecir la asfericidad corneal post-operatoria a partir del frente de onda corneal y de la corrección refractiva.

B1. Definición en términos matemáticos de un perfil de libre de aberración.

B2. Método matemático para la compensación del cambio de foco debido a la remoción de tejido.

B3. Propuesta de una referencia geométrica $(\mathrm{CV})$, utilizada como centro de ablación.

B4. Métodos para la evaluación de la simetría bilateral entre ojos.

C1. Propuesta para la selección del perfil de ablación más adecuado para cada tratamiento quirúrgico específico basado en las aberraciones de onda corneal y ocular.

D1. Métodos para el cálculo de la geometría de los impactos de ablación.

D2. Método para analizar la pérdida de eficacia de ablación en incidencia no normal. 
E1. Método para determinar el error ciclotorsional durante cirugía refractiva.

E2. Método para determinar la aberración residual debido a errores por ciclotorsión.

E3. Método para determinar el beneficio óptico.

E3. Método para determinar el beneficio visual.

E4. Método para determinar el beneficio absoluto.

F1. Métodos para evaluar la zona óptica después de la cirugía refractiva.

F2. Métodos para determinar las dependencias entre zona óptica efectiva, zona óptica planificada, y la corrección refractiva.

F3. Método para determinar las zonas ópticas isométricas.

F4. Método para determinar un nomograma para la zona óptica basada en la zona óptica requerida y la corrección de refractiva.

G1. Métodos para reducir al mínimo objetivamente la profundidad de ablación máximo de un tratamiento personalizado basado en la expansión de Zernike de la aberración de frente de onda.

G2. Métodos para minimizar objetivamente el volumen de ablación de un tratamiento personalizado basado en la expansión de Zernike de la aberración de frente de onda. 


\section{IMPLICACIONES DE ESTA INVESTIGACIÓN}

1. Los resultados de esta tesis son de aplicación directa en la cirugía refractiva con láser. La inducción de aberraciones sigue siendo un problema en la cirugía LASIK de hoy. Hemos demostrado que la mayor parte del aumento de las aberraciones se puede explicar por factores puramente físicos. Hemos obtenido los factores teóricos de corrección de eficiencia, que han sido ya aplicados en los perfiles de ablación del SCHWIND AMARIS.

2. Los resultados de esta tesis permiten demostrar el gran valor de la aberrometría (corneal y ocular) en la práctica clínica. Protocolos similares basados en los que se siguen en esta tesis pueden ser establecidos para ayudar a identificar el perfil de ablación más adecuado para cada paciente individual.

3. Los resultados de esta tesis en cuanto a perfiles libres de aberración y al análisis por árbol de decisión tienen implicaciones importantes para la selección de correcciones ablativas o lentes intraoculares. Proporcionar a los ojos con el mejor rendimiento visual es un problema extremadamente complejo. Hemos presentado pruebas de que no sólo las aberraciones de ojos individuales, sino también las limitaciones técnicas de la corrección de los sistemas determinan (y muy a menudo limitan) los resultados finales. Factores ópticos deben ser considerados, así como efectos relacionados con la adaptación neural. 



\section{LÍNEAS DE INVESTIGACIÓN FUTURA}

En base a estos resultados se pueden establecer las bases para futuras líneas de investigación:

A. En este estudio hemos utilizado la aberración de onda corneal como base para la determinación de la asfericidad corneal. Sin embargo, como recomienda la OSA, la aberración de onda corneal se basó en la línea de visión. Así, desviaciones grandes entre el centro pupilar y el vértice corneal pueden haber afectado negativamente a la potencia de las correlaciones. En estudios adicionales, se incluirán las desviaciones entre el centro pupilar y el vértice corneal para mejorar la exactitud del método.

Este capítulo se limita a un sistema láser (y a un algoritmo de ablación). Sin embargo, tanto las plataformas láser como los algoritmos que incorporan han evolucionado en los últimos años. En estudios adicionales, los nuevos estado de la técnica de sistemas láser y algoritmos serán evaluados también.

B. En este estudio se han utilizado perfiles libre de aberraciones, como base para las simulaciones y evaluaciones clínicas. Hemos aprendido que los perfiles libres de aberración puede reducir la inducción de aberraciones por debajo de los valores clínicamente relevantes. Como estamos seguros de que por estos motivos, la inducción de aberraciones se puede controlar, en otros estudios, se explorarán los perfiles guiados por frente de onda que serán analizados de una manera similar.

En este capítulo, se han realizado evaluaciones clínicas en niveles moderados de miopía e hipermetropía. Hemos aprendido que los perfiles libre de aberraciones reducir la inducción de aberraciones debajo de los valores clínicamente relevante, pero sin embargo, todavía se inducen algunos niveles 
menores de aberraciones. En estudios adicionales, se analizarán niveles mayores de miopía e hipermetropía para determinar, si la inducción de aberraciones medida se mantiene por debajo clínicamente relevantes valores.

Este capítulo se limita a reducir la inducción de aberraciones, en otros estudios se intentará manipular la inducción de aberraciones de una manera controlada, por ejemplo para correcciones de presbicia.

C. Las evaluaciones clínicas en este capítulo se limitan a corregir las refracciones que los sujetos manifiestan. Sin embargo, en ojos con gran aberración, determinar la refracción manifiesta puede llegar a ser un arte, una especie de adivinar la imagen menos borrosa. En estudios adicionales, se analizarán desviaciones sistemáticas de las refracciones manifiestas medias, de manera que se evalúen los efectos de acoplamiento entre modos de Zernike.

D. Estamos desarrollando un modelo de simulación simple para evaluar los algoritmos de ablación y los cambios de hidratación en cirugía refractiva con láser. El modelo simula diferentes efectos físicos de un proceso quirúrgico, y el proceso de ablación impacto por impacto, sobre la base de un perfil de haz modelado. El modelo considera la hidratación corneal, así como la humedad del ambiente, las características y propiedades del haz láser ablativo. Utilizando listas de pulsos tomadas de tratamientos reales, se simulará la ganancia de eficiencia durante el proceso de ablación.

E. En la actualidad se ha implementado un método prospectivo para determinar la ciclotorsión intraoperatoria en el sistema láser SCHWIND AMARIS. Con este nuevo escenario estamos evaluando la torsión intraoperatoria estática y dinámica, y los resultados postoperatorios de astigmatismo y aberraciones de alto orden. 
Del mismo modo, un módulo de eye-tracking de seis dimensiones está siendo desarrollado por ojo SCHWIND eye-tech-solutions. Con esta tecnología, se podrán evaluar de forma intraoperatoria los movimientos estáticos y dinámicos de los ojos en 6D.

F. La evaluación clínica de la zona óptica será evaluada para tratamientos hipermétropes, así como para tratamientos LASIK.

Un mayor plazo el seguimiento de estos ojos permitirá determinar si estos resultados muestran asimismo una mayor estabilidad en comparación con experiencias anteriores.

G. En este estudio hemos utilizado la aberración de onda corneal como base para las simulaciones y evaluaciones clínicas. Hemos aprendido que la combinación de términos de Zernike con valores subclínicos puede determinar las capacidades para el ahorro de tejido de forma eficaz. Se sabe que la aberraciones oculares suelen mostrar menores coeficientes de ponderación. En estudios adicionales, el ahorro de tejido basado en la aberración de onda ocular será explorado y comparado con los del frente de onda corneal.

Este capítulo se limita a un sistema láser (y a un algoritmo de ablación). Sin embargo, tanto las plataformas láser como los algoritmos que incorporan han evolucionado en los últimos años. En estudios adicionales, los nuevos estado de la técnica de sistemas de láser y algoritmos serán evaluados para el ahorro de tejido. 



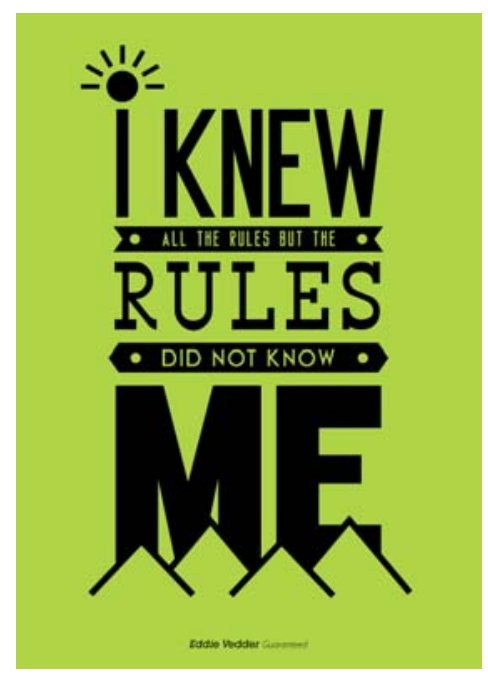

\section{REFERENCES}

(Bibliografía)

${ }^{1}$ Trokel SL, Srinivasan R, Braren B. Excimer laser surgery of the cornea. Am J Ophthalmol. 1983; 96: 710-715.

${ }^{2}$ Antón A, Andrada MT, Mayo A, Portela J, Merayo J. Epidemiology of refractive errors in an adult european population: The Segovia study. Journal of Ophthalmic Epidemiology 2009; 16: 231-237.

${ }^{3}$ GOMEZ S, HERRERAS J, MERAYO-LLOVES JM, GARCÍA M, ARGUESO P, CUEVAS J. Effect of hyaluronic acid on corneal haze in a photorefractive keratectomy experimental model. Journal of Refractive Surgery 2001; 17: 549554.

4 TORRES RM, MERAYO-LLOVES JM, BLANCO-MEZQUITA JT, GÜNTHER CP, RODRÍGUEZ-ZARZUELO G, MARTÍNEZ-GARCÍA C, GUTIERREZ R. Experimental Model of Laser Assisted in Situ Keratomileusis in Hens. Journal Refractive Surgery. 2005; 21: 392-398.

${ }^{5}$ Kling S, Remon L, Pérez-Escudero A, Merayo-Lloves J, Susana Marcos S. Corneal biomechanical changes after collagen cross-linking from porcine eye inflation experiments. Investigative Ophthalmology Visual Science 2010; 51. 
${ }^{6}$ ALIÓ JL, HELMY SHABAYEK M, MONTES-MICO R, MULET ME, GALAL A, AHMED A, MERAYO-LLOVES JM. Intracorneal hydrogel lenses and corneal aberrations. Journal of Refractive Surgery 2005; 21: 247-252.

${ }^{7}$ MARTÍNEZ GARCÍA C, MERAYO-LLOVES JM, BLANCO MEZQUITA T, MAR SARDAÑA S. Wound Healing following refractive surgery in hens. Experimental Eye Research 2006, 83(4): 728-735.

8 DEL VAL J, BARRERO S, YAÑEZ B, MERAYO-LLOVES JM, APARICIO J, GONZÁLEZ V, PASTOR JC, MAR S. Experimental measurement of corneal haze after excimer laser keratectomy. Applied Optics 2001; 40: 1727-1731.

9 MERAYO-LLOVES JM, YAÑEZ BP, MAYO A, MARTÍN R, PASTOR JC. Experimental model of corneal haze in chickens. Journal of Refractive Surgery 2001; 17: 696-699.

10 Merayo-LLoves, J, Blanco Mezquita T, Ibares-Frías, L, Fabiani, L, AlvarezBarcia, A, Martinez-García C, Induction of controlled wound healing with PMMA segments in the deep stroma in corneas of hens. European Journal of Ophthalmology. 2010;20:62-70.

${ }^{11}$ BARBERO S, MARCOS S, MERAYO-LLOVES JM. Corneal and total optical aberrations in a unilateral aphakic patient. Journal of Cataract Refractive Surgery 2002; 28: 1594-600.

${ }^{12}$ Gould G. Laser. US patent: US19590804539 19590406; 1959.

13 Schawlow AL, Townes $\mathrm{CH}$. Infrared and Optical Masers. Physical Review; 1958; 112: 1940-1949.

14 Swinger CA. Refractive surgery for the correction of myopia. Trans Ophthalmol Soc U K; 1981; 101: 434-439.

15 Munnerlyn CR, Koons SJ, Marshall J. Photorefractive keratectomy: a technique for laser refractive surgery. J Cataract Refract Surg; 1988; 14: 46-52.

${ }^{16}$ Krueger RR, Trokel SL. Quantitation of corneal ablation by ultraviolet laser light. Arch Ophthalmol. 1985; 103: 1741-1742.

17 Pettit GH, Ediger MN, Weiblinger RP. Excimer laser corneal ablation: absence of a significant "incubation" effect. Lasers Surg Med; 1991; 11: 411-418. 
18 Seiler T, Genth U, Holschbach A, Derse M. Aspheric photorefractive keratectomy with excimer laser. Refract Corneal Surg; 1993; 9: 166-172.

19 Mastropasqua L, Toto L, Zuppardi E, Nubile M, Carpineto P, Di Nicola M, Ballone E. Photorefractive keratectomy with aspheric profile of ablation versus conventional photorefractive keratectomy for myopia correction: six-month controlled clinical trial. J Cataract Refract Surg; 2006; 32: 109-116.

20 Kirwan C, O'Keefe M. Comparative study of higher-order aberrations after conventional laser in situ keratomileusis and laser epithelial keratomileusis for myopia using the technolas $217 z$ laser platform. Am J Ophthalmol; 2009 ; 147: 77-83.

21 McDonnell PJ, Moreira H, Garbus J. Photorefractive keratectomy to create toric ablations for correction of astigmatism. Arch Ophthalmol; 1991; 109: 710713.

${ }^{22}$ Buratto L, Ferrari M, Rama P. Excimer laser intrastromal keratomileusis. Am J Ophthalmol; 1992; 113: 291-295.

23 Pallikaris IG, Siganos DS. Excimer laser in situ keratomileusis and photorefractive keratectomy for correction of high myopia. J Refract Corneal Surg; 1994; 10: 498-510.

${ }^{24}$ Ren Q, Simon G, Legeais JM, Parel JM, Culbertson W, Shen J, Takesue Y, Savoldelli M. Ultraviolet solid-state laser (213-nm) photorefractive keratectomy. In vivo study. Ophthalmology; 1994; 101: 883-889.

${ }^{25}$ Gobbi PG, Carones F, Brancato R, Carena M, Fortini A, Scagliotti F, Morico A, Venturi E. Automatic eye tracker for excimer laser photorefractive keratectomy. $J$ Refract Surg. 1995; 11: S337-S342.

${ }^{26}$ Camellin M. Laser epithelial keratomileusis for myopia. J Refract Surg; 2003; 19: $666-670$.

27 Simon G, Huang C-H. Laser beam ophthalmological surgery method and apparatus. WO9717903. 1997 
28 Pallikaris IG, Naoumidi II, Kalyvianaki MI, Katsanevaki VJ. Epi-LASIK: comparative histological evaluation of mechanical and alcohol-assisted epithelial separation. J Cataract Refract Surg; 2003; 29: 1496-1501.

${ }^{29}$ Camellin M, Wyler D. Epi-LASIK versus epi-LASEK. J Refract Surg; 2008; 24 : S57-S63.

${ }^{30}$ Tran DB, Sarayba MA, Bor Z, Garufis C, Duh YJ, Soltes CR, Juhasz T, Kurtz RM. Randomized prospective clinical study comparing induced aberrations with IntraLase and Hansatome flap creation in fellow eyes: potential impact on wavefront-guided laser in situ keratomileusis. J Cataract Refract Surg; 2005; 31: 97-105.

31 Durrie DS, Slade SG, Marshall J. Wavefront-guided excimer laser ablation using photorefractive keratectomy and sub-Bowman's keratomileusis: a contralateral eye study. J Refract Surg; 2008; 24: S77-S84.

32 Mearza AA, Aslanides IM. Uses and complications of mitomycin $\mathrm{C}$ in ophthalmology. Expert Opin Drug Saf; 2007; 6: 27-32.

${ }^{33}$ Fisher BT, Hahn DW. Measurement of small-signal absorption coefficient and absorption cross section of collagen for 193-nm excimer laser light and the role of collagen in tissue ablation. Appl Opt; 2004; 43: 5443-5451.

${ }^{34}$ Fisher BT, Hahn DW. Development and numerical solution of a mechanistic model for corneal tissue ablation with the $193 \mathrm{~nm}$ argon fluoride excimer laser. $J$ Opt Soc Am A Opt Image Sci Vis; 2007; 24: 265-277.

${ }^{35}$ Kwon Y, Choi M, Bott S. Impact of ablation efficiency reduction on post-surgery corneal asphericity: simulation of the laser refractive surgery with a flying spot laser beam. Opt Express; 2008; 16: 11808-11821.

${ }^{36}$ Zernike F. Diffraction theory of the knife-edge test and its improved form, the phase-contrast method. Monthly Notices of the Royal Astronomical Society; 1934; 94: 377-384.

${ }^{37}$ Mrochen M, Kaemmerer M, Seiler T. Clinical results of wavefront-guided laser in situ keratomileusis 3 months after surgery. J Cataract Refract Surg; 2001; 27: 201-207. 
38 Smirnov MS. Measurement of the wave aberration of the human eye. Biofizika. 1961; 6: 687-703.

39 Thibos L, Hong X, Bradley A, Applegate RA. Accuracy and precision of objective refraction from wavefront aberrations. J Vis; 2004; 4: 329-351.

40 Burns SA. The spatially resolved refractometer. J Refract Surg; 2000; 16 : S566-S569.

41 Tscherning M. Die monochromatischen Aberratoinen des menschlichen. Auges Z Psychol Physiol Sinn; 1894; 6: 456-471.

42 Mrochen M, Kaemmerer M, Mierdel P, Krinke HE, Seiler T. Principles of Tscherning aberrometry. J Refract Surg; 2000; 16: S570-S571.

43 Hartmann J. Bemerkungen ueber den Bau und die Justierung von Spktrographen. Zeitschrift fuer Instrumentenkunde; 1900; 20: 47.

44 Shack RB, Platt BC. Production and use of a lenticular Hartmann screen. $J$ Opt Soc Am; 1971; 61:656.

${ }^{45}$ MacRae S, Fujieda M. Slit skiascopic-guided ablation using the Nidek laser. $J$ Refract Surg; 2000; 16: S576-S580.

46 Liang J, Grimm B, Goelz S, Bille JF. Objective measurement of wave aberrations of the human eye with the use of a Hartmann-Shack wave-front sensor. J Opt Soc Am A Opt Image Sci Vis; 1994; 11: 1949-1957.

${ }^{47}$ Babcock HW. Roddier Wavefront Sensor. Science; 1990; 250: 192.

48 Moreno-Barriuso E, Marcos S, Navarro R, Burns SA. Comparing laser ray tracing, the spatially resolved refractometer, and the Hartmann-Shack sensor to measure the ocular wave aberration. Optom Vis Sci; 2001; 78: 152-156.

49 Carvalho LA, Castro JC. The placido wavefront sensor and preliminary measurement on a mechanical eye. Optom Vis Sci; 2006; 83: 108-118.

50 Díaz-Doutón F, Benito A, Pujol J, Arjona M, Güell JL, Artal P. Comparison of the retinal image quality with a Hartmann-Shack wavefront sensor and a doublepass instrument. Invest Ophthalmol Vis Sci; 2006; 47: 1710-1716. 
51 Díaz-Doutón F, Pujol J, Arjona M, Luque SO. Curvature sensor for ocular wavefront measurement. Opt Lett; 2006; 31: 2245-2247.

${ }^{52}$ Corbett AD, Wilkinson TD, Zhong JJ, Diaz-Santana L. Designing a holographic modal wavefront sensor for the detection of static ocular aberrations. J Opt Soc Am A Opt Image Sci Vis; 2007; 24: 1266-1275.

${ }^{53}$ Warden L, Liu Y, Binder PS, Dreher AW, Sverdrup L. Performance of a new binocular wavefront aberrometer based on a self-imaging diffractive sensor. $J$ Refract Surg; 2008; 24: 188-196.

${ }^{54}$ Salmon TO. Corneal contribution to the Wavefront aberration of the eye. PhD Dissertation; 1999: 70.

${ }^{55}$ Mrochen M, Jankov M, Bueeler M, Seiler T. Correlation Between Corneal and Total Wavefront Aberrations in Myopic Eyes. J Refract Surg; 2003; 19: 104-112.

${ }^{56}$ Alio JL, Belda JI, Osman AA, Shalaby AM. Topography-guided laser in situ keratomileusis (TOPOLINK) to correct irregular astigmatism after previous refractive surgery. J Refract Surg; 2003; 19: 516-527.

57 Mrochen M, Donetzky C,Wüllner C, Löffler J. Wavefront-optimized ablation profiles: Theoretical background. J Cataract Refract Surg; 2004; 30: 775-785.

${ }^{58}$ Reinstein DZ, Neal DR, Vogelsang H, Schroeder E, Nagy ZZ, Bergt M, Copland J, Topa D. Optimized and wavefront guided corneal refractive surgery using the Carl Zeiss Meditec platform: the WASCA aberrometer, CRS-Master, and MEL80 excimer laser. Ophthalmol Clin North Am; 2004; 17: 191-210.

${ }^{59}$ Koller T, Iseli HP, Hafezi F, Mrochen M, Seiler T. Q-factor customized ablation profile for the correction of myopic astigmatism. J Cataract Refract Surg; 2006; 32: $584-589$.

60 Thibos LN, Applegate RA, Schwiegerling JT, Webb R, VSIA Standards Taskforce Members. Standards for Reporting the Optical Aberrations of Eyes. $J$ Refract Surg; 2002; 18: S652-S660.

${ }^{61}$ Ditzen K, Huschka H, Pieger S. Laser in situ keratomileusis for hyperopia. J Cataract Refract Surg; 1998; 24: 42-47. 
62 el Danasoury MA, Waring GO 3rd, el Maghraby A, Mehrez K. Excimer laser in situ keratomileusis to correct compound myopic astigmatism. J Refract Surg; 1997; 13: 511-520.

63 De Ortueta D, Haecker C. Laser in situ keratomileusis for mixed astigmatism using a modified formula for bitoric ablation. Eur J Ophthalmol. 2008; 18: 869-76. ${ }^{64}$ Moreno-Barriuso E, Lloves JM, Marcos S. Ocular Aberrations before and after myopic corneal refractive surgery: LASIK-induced changes measured with LASER ray tracing. Invest Ophthalmol Vis Sci; 2001; 42: 1396-1403.

65 Seiler T, Kaemmerer M, Mierdel P, Krinke H-E. Ocular optical aberrations after PRK for myopia and myopic astigmatism. Arch Ophthalmol; 2000; 118: 17-21.

66 Buzzonetti L, larossi G, Valente P, Volpi M, Petrocelli G, Scullica L. Comparison of wavefront aberration changes in the anterior corneal surface after LASEK and LASIK: preliminary study. J Cataract Refract Surg; 2004; 30: 19291933.

${ }^{67}$ Cheng ZY, He JC, Zhou XT, Chu RY. Effect of flap thickness on higher order wavefront aberrations induced by LASIK: a bilateral study. J Refract Surg; 2008; 24: 524-529.

${ }^{68}$ Aslanides IM, Tsiklis NS, Astyrakakis NI, Pallikaris IG, Jankov MR. LASIK flap characteristics using the Moria M2 microkeratome with the 90-microm single use head. J Refract Surg; 2007; 23: 45-49.

${ }^{69}$ Levy Y, Segal O, Avni I, Zadok D. Ocular higher-order aberrations in eyes with supernormal vision. Am J Ophthalmo.; 2005; 139: 225-228.

${ }^{70}$ Applegate RA, Howland HC. Refractive surgery, optical aberrations, and visual performance. J Refract Surg; 1997; 13: 295-299.

71 Gambra E, Sawides L, Dorronsoro C, Marcos S. Accommodative lag and fluctuations when optical aberrations are manipulated. J Vis; 2009; 9: 1-15.

72 Held R. The rediscovery of adaptability in the visual system: effects of extrinsic and intrinsic chromatic dispersion. In Harris CS, ed. Visual coding and Adaptability. Hillsdalle, NJ: Lawrence Erbaum Associates; 1980. 
${ }^{73}$ Artal P, Chen L, Fernandez EJ, Singer B, Manzanera S, Williams DR. Neural compensation for the eye's optical aberrations. J Vis; 2004; 4: 281-287.

74 Lohmann CP, Gartry DS, Muir MK, Timberlake GT, Fitzke FW, Marshall J. Corneal haze after excimer laser refractive surgery: objective measurements and functional implications. Eur J Ophthalmol; 1991; 1: 173-180.

75 Duffey RJ, Leaming D. US trends in refractive surgery: 2002 ISRS survey. J Refract Surg; 2003; 19: 357-363.

76 Cui M, Chen XM, Lü P. Comparison of laser epithelial keratomileusis and photorefractive keratectomy for the correction of myopia: a meta-analysis. Chin Med J (Engl); 2008; 121: 2331-2335.

77 Gamaly TO, El Danasoury A, El Maghraby A. A prospective, randomized, contralateral eye comparison of epithelial laser in situ keratomileusis and photorefractive keratectomy in eyes prone to haze. J Refract Surg; 2007; 23: S1015-S1020.

${ }^{78}$ O'Doherty M, Kirwan C, O'Keeffe M, O'Doherty J. Postoperative pain following epi-LASIK, LASEK, and PRK for myopia. J Refract Surg; 2007 ; 23: 133-138.

79 Hersh PS, Fry K, Blaker JW. Spherical aberration after LASIK and PRK. Clinical results and theoretical models of etiology. J Cataract Refract Surg; 2003; 29: 2096-2104.

${ }^{80}$ Klein SA. Optimal corneal ablation for eyes with arbitrary Hartmann-Shack aberrations. J Opt Soc Am A Opt Image Sci Vis; 1998; 15: 2580-2588.

81 Patel S, Marshall J, Fitzke FW 3rd. Model for predicting the optical performance of the eye in refractive surgery. Refract Corneal Surg; 1993; 9: 366375.

${ }^{82}$ Gatinel D, Malet J, Hoang-Xuan T, Azar DT. Analysis of customized corneal ablations: theoretical limitations of increasing negative asphericity. Invest Ophthalmol Vis Sci; 2002; 43: 941-948.

83 Perez-Escudero A, Dorronsoro C, Sawides L, Remon L, Merayo-Lloves J, Marcos S. Minor influence of Myopic Laser In Situ Keratomileusis on the 
Posterior Corneal Surface. Invest Ophthalmol Vis Sci. 2009 Apr 22. [Epub ahead of print].

84 Patel S, Aslanides IM. Main causes of reduced intraocular pressure after excimer laser photorefractive keratectomy. J Refract Surg. 1996; 12: 673-674.

85 Arbelaez MC, Vidal C, Arba Mosquera S. Clinical Outcomes of LASIK for Myopia Using the SCHWIND Platform With Ocular Wavefront Customized Ablation. J Refract Surg; 2009; 25: 1083-1090.

86 Arbelaez MC, Vidal C, Arba Mosquera S. Clinical Outcomes of Corneal Wavefront Customized Ablation Strategies with SCHWIND CAM in LASIK Treatments. Opthalmic Physiol Opt; 2009; 16: 487-496.

${ }^{87}$ de Ortueta D, Arba Mosquera S, Magnago T. Q-factor customized ablations. J Cataract Refract Surg; 2006; 32: 1981-1982.

${ }^{88}$ de Ortueta D, Arba Mosquera S. Mathematical properties of Asphericity: A Method to calculate with asphericities. J Refract Surg; 2008; 24: 119-121.

${ }^{89}$ Arba-Mosquera S, Merayo-Lloves J, de Ortueta D. Asphericity analysis using corneal wavefront and topographic meridional fits. J Biom Opt; 2010; 15 (2): 028003

${ }^{90}$ Arba Mosquera S, Piñero D, Ortiz D, Alió JL. Customized LASIK: Aspherical Treatments with the ESIRIS SCHWIND platform (Chapter 40, pp. 378-395) in Mastering the Techniques of Custom-ized LASIK edited by Ashok Garg and Emanuel Rosen, Jaypee Medical International (2007).

91 Arbelaez MC, Vidal C, Arba Mosquera S. Three-month clinical outcomes with Aberration-Free ablations in LASEK treatments using the AMARIS laser system. Highlights of Ophthalmology; 2008: 36: 4: 6-9.

92 Arbelaez MC, Vidal C, Arba-Mosquera S. Clinical outcomes of corneal vertex vs central pupil references with aberration-free ablation and LASIK. Invest Ophthalmol Vis Sci; 2008; 49: 5287-5294.

${ }^{93}$ Arbelaez MC, Arba Mosquera S. Clinical Pearls for Success in Femto-LASIK Refractive Surgery. Cataract Refract Surg Today; 2009; Feb: 37-43. 
${ }^{94}$ Arba Mosquera S, de Ortueta D. Analysis of Optimized Profiles for "AberrationFree" Refractive Surgery. Opthalmic Phys Opt; 2009; 29: 535-548.

${ }^{95}$ de Ortueta D, Arba Mosquera S, Baatz H. Aberration-neutral ablation pattern in hyperopic LASIK with the ESIRIS laser platform. J Refract Surg. 2009; 25: 17584.

96 de Ortueta D, Arba Mosquera S, Baatz H. Comparison of Standard and Aberration-neutral Profiles for Myopic LASIK With the SCHWIND ESIRIS Platform. J Refract Surg; 2009; 25: 339-349.

97 Arbelaez MC, Vidal C, Al Jabri B, Arba Mosquera S. LASIK for Myopia With Aspheric "Aberration Neutral" Ablations Using the ESIRIS Laser System. J Refract Surg; 2009; 25: 991-999.

${ }^{98}$ Arbelaez MC, Vidal C, Arba Mosquera S. Excimer laser correction of moderate to high astigmatism with a non-wavefront-guided aberration-free ablation profile: Six-month results. J Cataract Refract Surg; 2009; 35: 1789-1798.

99 de Ortueta D, Magnago T, Arba Mosquera S. Optimized Profiles for Aberration-Free Refractive Surgery in Laser Keratectomy: Approaches, Complications and Effectiveness, NOVA publishers (2009).

${ }^{100}$ Arbelaez MC, Vidal C, Arba Mosquera S. Bilateral Symmetry before and sixmonth after Aberration-FreeTM correction with the SCHWIND AMARIS TotalTech laser: Clinical outcomes. J Optom; 2010; 3: 20-28.

101 Arbelaez MC, Vidal C, Arba Mosquera S. Comparison of LASEK and LASIK with thin and ultrathin flap after excimer ablation with aspheric ablation profile. $J$ Refract Surg; 2010; in press

102 Gatell J, Arba-Mosquera S. Comparison of the induced corneal spherical and comatic aberrations between the classic profiles of the ESIRIS system and the aspheric profiles of the AMARIS system. J Emmetropia. 2010; (submitted).

103 Arba-Mosquera S, de Ortueta D. Theoretical influence of decentred ablations on induced Coma aberrations. J Emmetropia. 2010; (submitted).

${ }^{104}$ Arba-Mosquera S, Arbelaez MC. Validating the Aberration-Free concept with AMARIS. J Ref Surg. 2010; (submitted). 
105 Arba-Mosquera S, Arbelaez MC. Hyperopia and hyperopic astigmatism correction with the SCHWIND ESIRIS: 1-year results. J Emmetropia. 2010; (accepted).

${ }^{106}$ Arba-Mosquera S, Arbelaez MC. Six-month experience Hyperopic correction with the SCHWIND AMARIS Total-Tech Laser: Clinical Outcomes. J Optom. 2010; (submitted).

${ }^{107}$ Arbelaez MC, Vidal C and Arba-Mosquera S. Six-month clinical outcomes in LASIK for high myopia with aspheric "aberration neutral» ablations using the AMARIS laser system. J Emmetropia 2010; 1: 111-116

108 Arba-Mosquera S, de Ortueta D. Correlation Among Ocular Spherical Aberration, Corneal Spherical Aberration, and Corneal Asphericity Before and After LASIK for Myopic Astigmatism With the SCHWIND Amaris Platform. J Refract Surg. 2010; (in press).

${ }^{109}$ Arbelaez MC, Magnago T, Arba Mosquera S. Customised LASIK: SCHWIND CAM-ESIRIS platform (Chapter 15, pp. 207-228) in Tips and Tricks in LASIK surgery edited by Shashi Kapoor and Ioannis G. Pallikaris, Jaypee Medical International (2007).

${ }^{110}$ Arbelaez MC, Arba Mosquera S. The SCHWIND AMARIS total-tech laser as an all-rounder in refractive surgery. Middle East Afr J Ophthalmol; 2009; 16: 4653

111 Arbelaez MC, Ewering T, Arba Mosquera S. Decision Assistant Wizard to Standardize Optimal Outcomes in Excimer Laser Refractive Corneal Surgery. J Refract Surg; 2010; (in press)

112 Camellin M, Arba Mosquera S. Simultaneous aspheric wavefront-guided transepithelial photorefractive keratectomy and phototherapeutic keratectomy to correct aberrations and refractive errors after corneal surgery. J Cataract Refract Surg; 2010; 36: 1173-1180

113 Arba-Mosquera S, Arbelaez MC, de Ortueta D. Laser corneal refractive surgery in the twenty-first century: a review of the impact of refractive surgery on high-order aberrations (and vice versa). Journal of Modern Optics, Volume 57 Issue 12, 1041-1074 
114 Gatell J, Arba Mosquera S. High-aberrations correction with the SCHWIND AMARIS: A Case Report. J Emmetropia; 2010: (submitted).

${ }^{115}$ Aslanides IM, Arba Mosquera S. 4y CW efficacy and stability with ESIRIS. J Refract Surg; 2010: (submitted).

116 Arba Mosquera S, Ewering T. A new cooking recipe for retreatments in a systematic 15 steps methodology. Inv Ophth Vis Sci; 2010: (submitted).

117 Arba Mosquera S, de Ortueta D. Geometrical analysis of the loss of ablation efficiency at non-normal incidence. Opt. Express; 2008; 16: 3877-3895.

118 de Ortueta D, Arba Mosquera S, Häcker C. Theoretical considerations on the hyperopic shift effect observed when treating negative cylinder in laser refractive surgery. J Emmetropia; 2010; 1: 23-28.

119 Arba Mosquera S, Triefenbach N. Analysis of the cornea-to-PMMA ablation ratio. Optom Vis Sci; 2010; submitted.

120 Arba Mosquera S, Shraiki M. Analysis of the impact of the gain of ablation efficiency due to hydration changes during cornea refractive surgery. $J$ Refract Sug; 2010; submitted.

${ }^{121}$ Arba-Mosquera S, Merayo-Lloves J, de Ortueta D. Clinical effects of pure cyclotorsional errors during refractive surgery. Invest Ophthalmol Vis Sci; 2008; 49: 4828-4836.

122 Arba-Mosquera S, Arbelaez MC. Three-month clinical outcomes with Static and Dynamic Cyclotorsion Correction with the SCHWIND AMARIS Total-Tech Laser. in ESCRS2009 (Free paper).

123 Arba-Mosquera S, Arbelaez MC. Three-month clinical outcomes with Static and Dynamic Cyclotorsion Correction with the SCHWIND AMARIS Total-Tech Laser. Cornea; 2010; (submitted).

${ }^{124}$ Arbelaez MC, Arba-Mosquera S. Six-month experience in Ocular-Wavefrontguided customised with the SCHWIND AMARIS Total-Tech Laser 6D EyeTracker: Clinical Outcomes. ASCRS; 2010; (free paper).

125 Arba-Mosquera S, Aslanides IM. Turbo eye tracker analysis. J Mod Opt; 2010; (submitted). 
${ }^{126}$ Aslanides IM, Arba-Mosquera S. Intraoperative cyclotorsion analysis. Ant Eye Contact Lens; 2010; (submitted).

127 Arba Mosquera S. Aspherical Optical Zones: The Effective Optical Zone with the SCHWIND AMARIS in SOI2008 (Invited Lecture).

128 Camellin M, Arba Mosquera S. Aspherical Optical Zones: The Effective Optical Zone with the SCHWIND AMARIS. J Refract Surg; 2010; in press.

${ }^{129}$ Camellin M, Arba Mosquera S. Aspherical Optical Zones in hyperopia with the SCHWIND AMARIS. Opt Vis Sci; 2010; submitted.

${ }^{130}$ Arba Mosquera S. Effect of High-Order-Aberrations on the Manifest Refraction in SCHWIND eye-tech-solutions User Meeting 2008 (Invited Lecture).

131 Arba Mosquera S, de Ortueta D, Merayo-Lloves J. Tissue-Saving Zernike terms selection in customised treatments for refractive surgery. J Optom; 2009; 2: $182-196$.

132 Alió JL, Rosman M, Arba Mosquera S. Minimally invasive refractive surgery (pp. 97-123) in Minimally Invasive Ophthalmic Surgery, Springer publishers (2009).

133 Arba-Mosquera S, Hollerbach T. Ablation Resolution in Laser Corneal Refractive Sur-gery: The Dual Fluence Concept of the AMARIS Platform. Advances in Optical Technologies, vol. 2010, Article ID 538541, 13 pages, 2010. doi:10.1155/2010/538541

134 Arba Mosquera S, de Ortueta D. Optimized Zernike Term Selection in Customized Treatments for Laser Corneal Refractive Surgery: Case Report. J Refract Surg; 2010; (in press)

135 Arbelaez MC, Vidal C, Arba Mosquera S. Optimized Zernike Terms Selection in Customized Treatments for Laser Corneal Refractive Surgery: 3-Month Experience with LASIK Treatments in ASCRS2008 (Poster).

136 Arba Mosquera S, Arbelaez MC, Merayo-Llovés J. Six-month clinical outcomes of customised treatments minimized for depth and time in laser corneal refractive surgery. Cornea; 2010; (in press). 
${ }^{137}$ Cheng X, Bradley A, Thibos LN. Predicting subjective judgment of best focus with objective image quality metrics. J Vis; 2004; 4: 310-321.

138 Marsack JD, Thibos LN, Applegate RA. Metrics of optical quality derived from wave aberrations predict visual performance. J Vis; 2004; 4: 322-328.

139 Thibos LN. Unresolved issues in the prediction of subjective refraction from wavefront aberration maps. J Refract Surg. 2004 Sep-Oct;20(5):S533-6.

140 Watson AB, Ahumada AJ Jr. Predicting visual acuity from wavefront aberrations. J Vis; 2008; 8: 1-19.

141 Marcos S, Sawides L, Gambra E, Dorronsoro C. Influence of adaptive-optics ocular aberration correction on visual acuity at different luminances and contrast polarities. J Vis. 2008; 8: 1-12.

142 Salmon TO, West RW, Gasser W, Kenmore T. Measurement of refractive errors in young myopes using the COAS Shack-Hartmann aberrometer. Optom Vis Sci; 2003; 80: 6-14.

143 Chateau N, Harms F, Levecq X. Refractive representation of primary wavefront aberrations. $5^{\text {th }}$ International Congress of Wavefront Sensing \& Optimized Refractive Corrections; Whistler, Canada; 2004.

144 Bará S, Arines J, Ares J, Prado P. Direct transformation of Zernike eye aberration coefficients between scaled, rotated, and/or displaced pupils. J Opt Soc Am A Opt Image Sci Vis; 2006; 23: 2061-2066.

145 Nam J, Thibos LN, Iskander DR. Describing ocular aberrations with wavefront vergence maps. Clin Exp Optom; 2009; Epub ahead of print.

${ }^{146}$ Applegate RA, Marsack JD, Thibos LN. Metrics of retinal image quality predict visual performance in eyes with $20 / 17$ or better visual acuity. Optom Vis Sci; 2006; 83: 635-640.

147 Ravikumar S, Thibos LN, Bradley A. Calculation of retinal image quality for polychromatic light. J Opt Soc Am A Opt Image Sci Vis; 2008; 25: 2395-2407.

${ }^{148}$ Hartridge $\mathrm{H}$. Chromatic aberration and resolving power of the eye. J Physiol; 1918; 52: 175-246. 
149 Thibos LN, Hong X, Bradley A, Cheng X. Statistical variation of aberration structure and image quality in a normal population of healthy eyes. J Opt Soc Am A; 2002; 19: 2329-2348.

150 Jiang B, Liu Y. An analysis on the equivalence of the eye to a system with aberration. Sci Sin [B]; 1982; 25: 970-980.

151 Roorda A. A review of optics. $7^{\text {th }}$ International Congress of Wavefront Sensing \& Optimized Refractive Corrections; Paradise Island, Bahamas; 2006.

152 Thibos LN, Wheeler W, Horner D. Power vectors: an application of Fourier analysis to the description and statistical analysis of refractive error. Optom Vis Sci; 1997; 74: 367-375.

153 Mattioli R, Tripoli NK. Corneal Geometry Reconstruction with the Keratron Videokeratographer. Optometry and Vision Science; 1997; 74: 881-894.

154 De Ortueta D. Planar flaps with the Carriazo-Pendular microkeratome. $J$ Refract Surg; 2008; 24: 322.

155 Dorronsoro C, Siegel J, Remon L, Marcos S. Suitability of Filofocon A and PMMA for experimental models in excimer laser ablation refractive surgery. Opt Express; 2008; 16: 20955-20967.

156 Arbelaez MC et al. LASIK for myopia and astigmatism using the SCHWIND AMARIS excimer laser. J Refract Surg; 2010 (in press).

157 Mearza AA, Muhtaseb M, Aslanides IM. Visual and refractive outcomes of LASIK with the SCHWIND ESIRIS and WaveLight ALLEGRETTO WAVE Eye-q excimer lasers: a prospective, contralateral study. J Refract Surg; 2008; 24: 885890.

158 Marcos S, Cano D, Barbero S. Increase in corneal asphericity after standard laser in situ keratomileusis for myopia is not inherent to the Munnerlyn algorithm. J Refract Surg; 2003; 19: S592-S596.

159 Mrochen M, Seiler T. Influence of Corneal Curvature on Calculation of Ablation Patterns used in photorefractive Laser Surgery. J Refract Surg; 2001; 17: 584-587. 
160 Jiménez JR, Anera RG, Jiménez del Barco L, Hita E. Effect on laser-ablation algorithms of reflection losses and nonnormal incidence on the anterior cornea. Applied Physics Letters; 2002; 81: 1521-1523.

161 Jiménez JR, Anera RG, Jiménez del Barco L, Hita E, Pérez-Ocón F. Correlation factor for ablation agorithms used in corneal refractive surgery with gaussian-profile beams. Optics Express; 2005; 13: 336-343.

162 Arba Mosquera S. Laser system for ablating the cornea in a patient's eye (EP2033605).

163 Bende T, Seiler T, Wollensak J. Side effects in excimer corneal surgery. Corneal thermal gradients. Graefes Arch Clin Exp Ophthalmol; 1988; 226: 277280.

164 Huang D, Arif M. Spot size and quality of scanning laser correction of higherorder wavefront aberrations. J Cataract Refract Surg; 2002; 28: 407-416.

165 Guirao A,Williams D, MacRae S. Effect of beam size on the Expected benefit of customized laser refractive surgery. J Refract Surg; 2003; 19: 15-23.

166 Grimm A, Arba Mosquera S, Klinner T. System for ablating the cornea of an eye (EP1923026).

167 Arba Mosquera S, Klinner T. Method for controlling the location a laserpulse impinges the cornea of an eye during an ablation procedure (EP19335384).

168 Bueeler M, Mrochen M. Simulation of eye-tracker latency, spot size, and ablation pulse depth on the correction of higher order wavefront aberrations with scanning spot laser systems. J Refract Surg; 2005; 21: 28-36.

${ }^{169}$ Gatinel D, Haouat M, Hoang-Xuan T. A review of mathematical descriptors of corneal asphericity. J Fr Ophtalmol 2002; 25: 81-90

${ }^{170}$ Calossi A. Corneal asphericity and spherical aberration. J Refract Surg 2007; 23: $505-514$

171 Kiely PM, Smith G, Carney LG. The mean shape of the human cornea. Optica Acta. 1982; 29: 1027-1040 
172 Sheridan M, Douthwaite WA. Corneal asphericity and refractive error. Ophthal Physiol Opt 1989; 9: 235-238

173 Stojanovic A, Wang L, Jankov MR, Nitter TA, Wang Q. Wavefront optimized versus custom- $Q$ treatments in surface ablation for myopic astigmatism with the WaveLight ALLEGRETTO laser. J Refract Surg 2008; 24: 779-789

174 Jiménez JR, Anera RG, Díaz JA, Pérez-Ocón F. Corneal asphericity after refractive surgery when the Munnerlyn formula is applied. J Opt Soc Am A Opt Image Sci Vis 2004; 21: 98-103

175 Vinciguerra P, Camesasca FI. Treatment of hyperopia: a new ablation profile to reduce corneal eccentricity. J Refract Surg 2002; 18: S315-317

${ }^{176}$ Gatinel D, Hoang-Xuan T, Azar DT. Determination of corneal asphericity after myopia surgery with the excimer laser: a mathematical model. Invest Ophthalmol Vis Sci 2001; 42: 1736-1742

177 Anera RG, Jiménez JR, Jiménez del Barco L, Bermúdez J, Hita E. Changes in corneal asphericity after LASER in situ keratomileusis, J Cataract Refract Surg 2003; 29: 762-768

178 ISO19980:2005

179 Altman DG, Bland JM. Measurement in Medicine: the Analysis of Method Comparison Studies. The Statistician; 1983; 32: 307-317

${ }^{180}$ Pérez-Escudero, A., Dorronsoro, C. and Marcos, S. An artifact in fits to conicbased surfaces. E-pub only: arXiv:0905.0814v1 (2009)

${ }^{181}$ Navarro R, González L, Hernández JL. Optics of the average normal cornea from general and canonical representations of its surface topography. J Opt Soc Am A 2006; 23: 219-232

182 Preussner PR, Wahl J. Simplified mathematics for customized refractive surgery. J Cataract Refract Surg 2003; 29: 462-470

183 Preussner PR, Wahl J, Kramann C. Corneal model. J Cataract Refract Surg 2003; 29: 471-477 
184 Binder PS, Rosenshein J. Retrospective comparison of 3 laser platforms to correct myopic spheres and spherocylinders using conventional and wavefrontguided treatments. J Cataract Refract Surg; 2007; 33: 1158-76

185 Hori-Komai Y, Toda I, Asano-Kato N, Ito M, Yamamoto T, Tsubota K. Comparison of LASIK using the NIDEK EC-5000 optimized aspheric transition zone (OATz) and conventional ablation profile. J Refract Surg; 2006; 22: 546-55

186 Subbaram MV, MacRae SM. Customized LASIK treatment for myopia based on preoperative manifest refraction and higher order aberrometry: the Rochester nomogram. J Refract Surg; 2007; 23: 435-41

187 McLellan JS, Marcos S, Prieto PM, Burns SA. Imperfect optics may be the eye's defence against chromatic blur. Nature; 2002; 417: 174-6

188 Applegate RA, Sarver EJ, Khemsara V. Are all aberrations equal? J Refract Surg; 2002; 18: S556-62

189 Applegate RA, Marsack JD, Ramos R, Sarver EJ. Interaction between aberrations to improve or reduce visual performance. J Cataract Refract Surg; 2003; 29: 1487-95

190 Tuan KA, Somani S, Chernyak DA. Changes in wavefront aberration with pharmaceutical dilating agents. J Refract Surg; 2005; 21: S530-534

191 Pop M, Payette Y. Photorefractive keratectomy versus laser in situ keratomileusis: a control-matched study. Ophthalmology. 2000; 107: 251-257.

192 Bueeler M, Mrochen M, Seiler T. Maximum permissible lateral decentration in aberration-sensing and wavefront-guided corneal ablations. J Cataract Refract Surg 2003; 29: 257-263

193 Kim WS, Jo JM. Corneal hydration affects ablation during laser in situ keratomileusis surgery. Cornea. 2001; 20: 394-397.

194 Dougherty PJ, Wellish KL, Maloney RK. Excimer laser ablation rate and corneal hydration. Am J Ophthalmol. 1994; 118: 169-176.

195 Fam HB, Lim KL. Effect of higher-order wavefront aberrations on binocular summation. J Refract Surg. 2004; 20: S570-5 
196 Nelson-Quigg JM, Cello K, Johnson CA. Predicting binocular visual field sensitivity from monocular visual field results. Invest Ophthalmol Vis Sci. 2000; 41: $2212-21$

197 Cuesta JR, Anera RG, Jiménez R, Salas C. Impact of interocular differences in corneal asphericity on binocular summation. Am J Ophthalmol. 2003; 135: $279-84$

198 Jiménez JR, Villa C, Anera RG, Gutiérrez R, del Barco LJ. Binocular visual performance after LASIK. J Refract Surg. 2006; 22: 679-88

${ }^{199}$ Marcos S, Burns SA. On the symmetry between eyes of wavefront aberration and cone directionality. Vision Res. 2000; 40: 2437-47

200 Mansouri B, Thompson B, Hess RF. Measurement of suprathreshold binocular interactions in amblyopia. Vision Res. 2008 Oct 31. [Epub ahead of print]

201 Jiménez JR, Ponce A, Anera RG. Induced aniseikonia diminishes binocular contrast sensitivity and binocular summation. Optom Vis Sci. 2004; 81: 559-62

202 Yang Y, Wu F. Technical note: Comparison of the wavefront aberrations between natural and pharmacological pupil dilations. Ophthalmic Physiol Opt; 2007; 27: 220-223

${ }^{203}$ Erdem U, Muftuoglu O, Gundogan FC, Sobaci G, Bayer A. Pupil center shift relative to the coaxially sighted corneal light reflex under natural and pharmacologically dilated conditions. J Refract Surg; 2008; 24: 530-538

204 Snellen H. Letterproeven tot Bepaling der Gezichtsscherpte. Utrecht, Weyers, 1862

205 Radhakrishnan H, Charman WN. Age-related changes in ocular aberrations with accommodation. J Vis. 2007; 7: 11.1-21

206 López-Gil N, Fernández-Sánchez V, Legras R, Montés-Micó R, Lara F, Nguyen-Khoa JL. Accommodation-related changes in monochromatic aberrations of the human eye as a function of age. Invest Ophthalmol Vis Sci. 2008; 49: 1736-43 
207 lida Y, Shimizu K, Ito M, Suzuki M. Influence of age on ocular wavefront aberration changes with accommodation. J Refract Surg. 2008; 24: 696-701 ${ }^{208}$ He JC, Gwiazda J, Thorn F, Held R, Huang W. Change in corneal shape and corneal wave-front aberrations with accommodation. J Vis. 2003; 3:456-63

209 Atchison DA, Markwell EL, Kasthurirangan S, Pope JM, Smith G, Swann PG. Age-related changes in optical and biometric characteristics of emmetropic eyes. J Vis. 2008; 8: 29.1-20

210 Holzer MP, Sassenroth M, Auffarth GU. Reliability of corneal and total wavefront aberration measurements with the SCHWIND Corneal and Ocular Wavefront Analyzers. J Refract Surg; 2006; 22: 917-920.

${ }^{211}$ MacRae S. Aberration Interaction In Wavefront Guided Custom Wavefront Guided Custom Ablation. Wavefront Congress 2007.

212 Bühren J, Yoon GY, Kenner S, Artrip S, MacRae S, Huxlin K. The effect of decentration on lower- and higher-order aberrations after myopic photorefractive keratectomy (PRK) in a cat model. Wavefront Congress 2007.

213 McLellan JS, Prieto PM, Marcos S, Burns SA. Effects of interactions among wave aberrations on optical image quality. Vision Res; 2006; 46: 3009-3016.

${ }^{214}$ Dorronsoro C, Cano D, Merayo-Lloves J, Marcos S. Experiments on PMMA models to predict the impact of refractive surgery on corneal shape. Opt. Express; 2006; 14: 6142-6156

215 Smith EM Jr, Talamo JH. Cyclotorsion in the seated and the supine patient. $J$ Cataract Refract Surg. 1995; 21: 402-403.

216 de Ortueta D, Arba Mosquera S, Baatz H. Topographic changes after hyperopic LASIK with the ESIRIS laser platform. J Refract Surg. 2008; 24: 137 144.

217 Rosa N, Furgiuele D, Lanza M, Capasso L, Romano A. Correlation of Changes in Refraction and Corneal Topography After Photorefractive Keratectomy. J Refract Surg. 2004; 20: 478-483.

${ }^{218}$ Campbell CE. A new method for describing the aberrations of the eye using Zernike polynomials. Optom Vis Sci. 2003; 80: 79-83. 
${ }^{219}$ Campbell CE. A method to analyze cylinder axis error. Optom Vis Sci. 1999; 76: $254-255$.

220 Yang Y, Thompson K, Burns S. Pupil location under mesopic, photopic and pharmacologically dilated conditions. Invest Ophthalmol Vis Sci. 2002; 43: 25082512.

${ }^{221}$ Guirao A, Williams D, Cox I. Effect of rotation and translation on the expected benefit of an ideal method to correct the eyes higher-order aberrations. J Opt Soc Am A. 2001; 18: 1003-1015.

222 Ciccio AE, Durrie DS, StahI JE, Schwendeman F. Ocular cyclotorsion during customized laser ablation. J Refract Surg. 2005; 21: S772-S774.

223 Uozato H, Guyton DL. Centering corneal surgical procedures. Am J Ophthalmol. 1987; 103: 264-275.

${ }^{224}$ Marcos S, Barbero S, Llorente L, Merayo-Lloves J. Optical response to LASIK surgery for myopia from Total and Corneal Aberration Measurements. Invest Ophthalmol Vis Sci. 2001; 42: 3349-3356.

${ }^{225}$ Marcos S. Aberrations and visual performance following standard Laser vision correction. J Refract Surg. 2001; 17: S596-S601.

226 Bará S, Mancebo T, Moreno-Barriuso E. Positioning tolerances for phase plates compensating aberrations of the human eye. Appl Opt. 2000; 39: 34133420.

${ }^{227}$ Chernyak DA. Iris-based cyclotorsional image alignment method for wavefront registration. IEEE Transactions on Biomedical Engineering. 2005; 52: 20322040.

228 Schruender S, Fuchs H, Spasovski S, Dankert A. Intraoperative corneal topography for image registration. J Refract Surg. 2002; 18: S624-S629.

${ }^{229}$ Bueeler M, Mrochen M, Seiler T. Maximum permissible torsional misalignment in aberration-sensing and wavefront-guided corneal ablation. J Cataract Refract Surg. 2004; 30: 17-25. 
230 Hersh PS, Steinert RF, Brint SF; Summit PRK-LASIK Study Group. Photorefractive keratectomy versus laser in situ keratomileusis: a comparison of optical side effects. Ophthalmology. 2000; 107: 925-933.

231 Maloney RK. Corneal topography and optical zone location in photorefractive keratectomy. Refract Corneal Surg 1990; 6: 363-371.

232 Hersh PS, Schwartz-Goldstein BH; Summit Photorefractive Keratectomy Topography Study Group. Corneal topography of phase III excimer laser photorefractive keratectomy: characterization of clinical effects. Ophthalmology. 1995; 102: 963-978.

233 O’Brart DPS, Gartry DS, Lohmann CP, Kerr Muir MG, Marshall J. Excimer laser photorefractive keratectomy for myopia: comparison of 4.00- and 5.00millimeter ablation zones. J Refract Corneal Surg. 1994; 10: 87-94.

${ }^{234}$ Roberts CW, Koester CJ. Optical zone diameters for photorefractive corneal surgery. Invest Ophthalmol Vis Sci. 1993; 34: 2275-2281.

235 Halliday BL. Refractive and visual results and patient satisfaction after excimer laser photorefractive keratectomy for myopia. $\mathrm{Br} J$ Ophthalmol. 1995; 79: 881-887.

${ }^{236}$ Nepomuceno RL, Boxer Wachler BS, Scruggs R. Functional optical zone after myopic LASIK as a function of ablation diameter. J Cataract Refract Surg. 2005; 31: 379-384.

237 Rojas MC, Manche EE. Comparison of videokeratographic functional optical zones in conductive keratoplasty and LASIK for hyperopia. J Refract Surg. 2003; 19: 333-337.

${ }^{238}$ Mrochen M, Büeler M. Aspheric optics: fundamentals. Ophthalmologe 2008; 105: 224-33.

239 Yoon G, MacRae S, Williams DR, Cox IG. Causes of spherical aberration induced by laser refractive surgery. J Cataract Refract Surg; 2005;31:127-135

240 Reinstein DZ, Silverman RH, Sutton HF, Coleman DJ. Very high frequency ultrasound corneal analysis identifies anatomic correlates of optical complications 
of lamellar refractive surgery: anatomic diagnosis in lamellar surgery. Ophthalmology. 1999; 106: 474-482.

241 Dupps WJ Jr, Roberts C. Effect of acute biomechanical changes on corneal curvature after photokeratectomy. J Refract Surg. 2001; 17: 658-669.

${ }^{242}$ Wang Z, Chen J, Yang B. Posterior corneal surface topographic changes after LASIK are related to residual corneal bed thickness. Ophthalmology; 1999; 106: 406-9

${ }^{243}$ Binder PS. Analysis of ectasia after laser in situ keratomileusis: risk factors. $J$ Cataract Refract Surg; 2007; 33: 1530-8

244 Tabernero J, Klyce SD, Sarver EJ, Artal P. Functional optical zone of the cornea. Invest Ophthalmol Vis Sci. 2007; 48: 1053-60.

${ }^{245}$ Mok KH, Lee VW. Effect of optical zone ablation diameter on LASIK-induced higher order optical aberrations. J Refract Surg. 2005; 21: 141-143.

246 Netto MV, Ambrosio R Jr, Wilson SE. Pupil size in refractive surgery candidates. J Refract Surg. 2004;20:337-342.

247 Partal AE, Manche EE. Diameters of topographic optical zone and programmed ablation zone for laser in situ keratomileusis for myopia. $J$ Refract Surg. 2003; 19: 528-33

248 Qazi MA, Roberts CJ, Mahmoud AM, Pepose JS. Topographic and biomechanical differences between hyperopic and myopic laser in situ keratomileusis. J Cataract Refract Surg. 2005; 31: 48-60

${ }^{249} \mathrm{Kim}$ HM, Jung HR. Multizone photorefractive keratectomy for myopia of 9 to 14 diopters. J Refract Surg; 1995; 11: S293-S297.

250 Kermani O, Schmiedt K, Oberheide U, Gerten G. Early results of nidek customized aspheric transition zones (CATz) in laser in situ keratomileusis. $J$ Refract Surg; 2003; 19: S190-S194.

251 Kezirian GM. A Closer Look at the Options for LASIK Surgery. Review of Ophthalmology; 2003; 10: 12. 
252 Goes FJ. Customized topographic repair with the new platform: ZEiSS MEL80/New CRS Master TOSCA II (Chapter 18, pp. 179-193) in Mastering the Techniques of Customised LASIK edited by Ashok Garg and Emanuel Rosen, Jaypee Medical International (2007)

${ }^{253}$ Remón L, Tornel M, Furlan WD. Visual Acuity in Simple Myopic Astigmatism: Influence of Cylinder Axis. Optom Vis Sci; 2006; 83: 311-315.

254 Bará S, Navarro R. Wide-field compensation of monochromatic eye aberrations: expected performance and design trade-offs. J. Opt. Soc. Am. A 2003; 20: 1-10.

255 Rocha KM, Soriano ES, Chamon W, Chalita MR, Nose W. Spherical aberration and depth of focus in eyes implanted with aspheric and spherical intraocular lenses: a prospective randomized study. Ophthalmology; 2007; 114: 2050-2054.

${ }^{256}$ Chen L, Artal P, Gutierrez D, Williams DR. Neural compensation for the best aberration correction. J Vis; 2007; 7: 1-9.

257 Villegas EA, Alcón, E, Artal P. Optical quality of the eye in subjects with normal and excellent visual acuity. Invest Opthalmol Vis Sci; 2008; 49: 46884696.

258 Benito A, Redondo M, Artal P. Laser In Situ Keratomileusis disrupts the aberration compensation mechanism of the human eye. Am J Ophthalmol; 2009; 147: 424-431.

259 Lipshitz I. Thirty-four challenges to meet before excimer laser technology can Achieve super vision. J Refract Surg; 2002; 18: 740-743.

260 Durrie DS, Kezirian GM. Femtosecond laser versus mechanical keratome flaps in wavefront-guided laser in situ keratomileusis. J Cataract Refract Surg; 2005; 31: 120-126.

261 Tsai YY, Lin JM. Ablation centration after active eye-tracker-assisted photorefractive keratectomy and laser in situ keratomileusis. J Cataract Refract Surg; 2000; 26: 28-34. 